\title{
RECENT PROGRESS IN
}

\section{HORMONE RESEARCH}

\author{
Edited by \\ ANTHONY R. MEANS
}

VOLUME 56

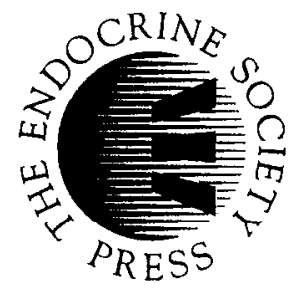

"Recent Progress in Hormone Research" is an annual publication of The Endocrine Society that is published under the editorial auspices of Endocrine Reviews.

The Endocrine Society

4350 East West Highway, Suite 500

Bethesda, Maryland 20814 
Copyright 2001 by The Endocrine Society All Rights Reserved

The reproduction or utilization of this work in any form or in any electronic, mechanical, or other means, now known or hereafter invented, including photocopying or recording and in any information storage or retrieval system, is forbidden, except as may be expressly permitted by the 1976 Copyright Act or by permission of the publisher.

ISBN 0-879225-41-7 


\section{CONTENTS}

Senior Author Correspondence Information $v$

1. Protein Glycation, Diabetcs, and Aging 1

Peter Ulrich and Anthony Cerami

2. BETA2 and Pancreatic Islet Development

Khoi Chu, Eric Nemoz-Gaillard, and Ming-Jer Tsai

3. Of Mice and Men: $K_{A T P}$ Channels and Insulin Secretion

Lydia Aguilar-Bryan, Joseph Bryan, and Mitsuhiro Nakazaki

4. Genetics of Type 1A Diabetes

Maria I. Redondo, Pamela R. Fain, and George S. Eisenbarth

Gino M. Salituro, Fernando Pelaez, and Bei B. Zhang

of the MAP Kinase Cascade

Joseph Avruch, Andrei Khokhlatchev, John M. Kyriakis, Zhijun Luo,

Guri Tzivion, Demetrios Vavvas, and Xian-feng Zhang

8. The Role of Protein Phosphatase-1 in Insulin Action

Matllew J. Brady and Alan R. Salticl

9. Intracellular Organization of Insulin Signaling and GLU'J'4 Translocation

Robert T. Watson and Jeffrey E. Pessin

10. Cell-specific Roles of Glucokinase in Glucose Homeostasis

Catherine Postic, Masakazu Shiota, and Mark A. Magnuson

11. Nuclear Magnetic Resonance Studies of Hepatic Glucose Metabolism in Humans Michael Roden, Kitt Falk Petersen, and Gerald I. Shulman 
12. Peroxisome Proliferator-Activated Receptors: From Genes to Physiology

Steven A. Kliewer, H. Eric Xu, Millard H. Lambert, and Timothy M. Willson

13. Insulin Resistance and Its Treatment by Thiazolidinediones

Harold E. Lebovitz and Mary Ann Banerji

14. Insulin Resistance in Pulycystic Ovary Syndronle: Progress and Paradoxes Aradhana M. Venkatesan, Andrea Dunaif, and Anne Corbould

15. The B-Adrenergic Receptors and the Control of Adipose Tissue Metaholism

and Thermogenesis

Sheila Collins and Richard S. Surwit

16. The Adipocyte as a Secretory Organ: Mechanisms of Vesicle Transport and Secretory Pathways

Richard L. Bradley, Kelly A. Cleveland, and Bentley Cheatham

17. Central Melanocortins and the Regulation of Weight During Acute and Chronic Disease Daniel L. Marks and Roger D. Cone

18. Glucagon-Like Peptide-1

Máire E. Doyle and Josephine M. Egan

19. Leptin Controls Bone Formation Through a Hypothalamic Relay

Gerard Karsenty

20. Mechanisms of Inhibin Signal Transduction

Daniel J. Bernard, Stacey C. Chapman, and Teresa K. Woodruff

Author Index

Subject Index 


\section{SENIOR AUTHOR CORRESPONDENCE INFORMATION}

Lydia Aguilar-Bryan

Department of Medicine

Division of Endocrinology

Baylor College of Medicine

One Baylor Plaza, 537E

Houston, TX 77030

Phone: $713-798-3462$

Fax: 713-798-4585

E-mail: Jbryan@bcm.tmc.edu

Joseph Avruch

Diabetes Research Laboratory

Department of Molecular Biology

Massachusetts General Hospital

50 Blossom Street, Wellman 8

Boston, MA 02114

Phone: 617-726-6909

Fax: 617-726-5649

E-mail: avruch@helix.mgh.harvard.edu

\section{Anthony Cerami}

Kenneth S. Warren Laboratories

765 Old Saw Mill River Road

Tarrytown, NY 10591

Phone: 914-345-3478

Fax: 914-345-3795

E-mail: accerami@aol.com

\author{
Bentley Cheatham \\ Joslin Diabetes Center \\ One Joslin Place \\ Boston, MA 02115 \\ Phone: 617-732-2629 \\ Fax: 617-735-1970 \\ E-mail: Bentley.Cheatham(ajoslin.harvard.edu
}

Roger D. Cone
Vollum Institute
Oregon Health Sciences University
3181 SW Sam Jackson Park Road, L474
Portland, OR 97201-3098
Phone: 503-494-4668
Fax: 503-494-4534
E-mail: cone@ohsu.edu 
Andrea Dunaif

Division of Women's Health

Brigham and Women's Hospital

75 Francis Street, PBB5

Boston, MA 02115

Phone: 617-732-8798

Fax: 617-264-5210

E-mail: adunaif@partners.org

Josephine M. Egan

Diabetes Section, \#23

National Institute on Aging

National Institutes of Health

5600 Nathan Shock Drive

Baltimore, MD 21224

Phone: 410-558-8414

Fax: 410-558-8381

E-mail: eganj@vax.grc.nia.nih.gov

George S. Eisenbarth

Barbara Davis Center for Childhood Diabetes

University of Colorado Health Sciences Center

Box B140, 4200 East 9th Avenue

Denver, CO 80262

Phone: 303-315-4891

Fax: 303-315-4892

E-mail: george.eisenbarth@UCHSC.edu

Philippe Froguel

Departement de Genetique Humaine

CNRS 8090

Institut Pasteur de Lille

1 rue Calmette

59000 Lille France

Phone: 011-33-3-20-87-79-54

Fax: 011-33-3-20-87-72-29

E-mail:Philippe.Froguel@mail-good.pasteur-

lille.fr

\section{Gerard Karsenty}

Department of Molecular and Human Genetics

Baylor College of Medicine

One Baylor Plaza, Room S930A

Houston, TX 77030

Phone: 713-798-5489

Fax: 713-798-1465

E-mail: karsenty@bcm.tmc.edu

Steven A. Kliewer

Glaxo Wellcome

5 Moore Drive

Research Triangle Park, NC 27709

Phone: 919-483-5601

Fax: 919-483-3895

E-mail: sak15922@glaxowellcome.com

Harold E. Lebovitz

State University of New York

Health Science Center at Brooklyn

Box 50

450 Clarkson Avenue

Brooklyn, NY 11203

Phone: 718-816-4638

Fax: 718-447-1558

E-mail: hlebovitz@attglobal.net

Mark A. Magnuson

Department of Molecular Physiology

\& Biophysics

Vanderbilt University School of Medicine

747 Light Hall

Nashville, TN 37232-0615

Phone: 615-322-7006

Fax: 615-343-3173

E-mail: mark.magnuson@mcmail.

vanderbilt.edu 
Jeffrey E. Pessin

Department of Physiology and Biophysics

University of Iowa

BSB - Room 5-530

51 Newton Road

lowa City, Iowa 52242

Phone: 319-335-7823

Fax: $319-335-7330$

E-mail: Jeffrey-Pessin@uiowa edu

\author{
Alan R. Saltiel \\ Parke-Davis Pharmaceutical Division \\ Warner Lambert Company \\ 2800 Plymouth Road \\ Ann Arbor, MI 48105-2430 \\ Phone: 734-622-3960 \\ Fax: 734-622-5668 \\ E-mail: alan.saltiel@wl.com
}

\section{Gerald I. Shulman}

Howard Hughes Medical Institute Boyer Center for Molecular Medicine Yale University School of Medicine P.O. Box 9812

New Haven, CT 96536-8012

Phone: 203-785-5447

Fax: 203-737-4059

E-mail: gerald.shulman@yale.edu

\author{
Ming-Jer Tsai \\ Department of Molecular and Cellular Biology \\ Baylor College of Medicine \\ One Baylor Plaza \\ Room M734A \\ Houston, TX 77030 \\ Phone: 713-798-6253 \\ Fax: 713-798-8227 \\ E-mail: mtsai@bcm.tmc.edu
}

Teresa K. Woodruff

Department of Neurobiology and Physiology

Northwestern University

OT Hogan 4-150

2153 N. Campus Drive

Evanston, IL 60208

Phone: 847-491-2666

Fax: 847-491-2224

E-mail: tkw@northwestern.edu

Bei B. Zhang

Merck Research Laboratories

R80W250, P.O. Box 2000

$126 \mathrm{E}$. Lincoln Avenue

Rahway, NJ 07065

Phone: 732-594-1926

Fax: 732-594-1169

E-mail: bei_zhang@merck.com 



\title{
Protein Glycation, Diabetes, and Aging
}

\author{
Peter Ulrich and Anthony Cerami \\ The Kenneth S. Warren Laboratories, 765 Old Saw Mill River Road, \\ Tarrytown, New York 10591
}

\begin{abstract}
Biological amines react with reducing sugars to form a complex family of rearranged and dehydrated covalent adducts that are often yellow-brown and/or fluorescent and include many crosslinked structures. Food chemists have long studied this process as a source of flavor, color, and texture changes in cooked, processed, and stored foods. During the 1970s and 1980s, it was realized that this process, called the Maillard reaction or advanced glycation, also occurs slowly in vivo. Advanced glycation endproducts (AGEs) that form are implicated, causing the complications of diabetes and aging, primarily via adventitious and crosslinking of proteins. Long-lived proteins such as structural collagen and lens crystallins particularly are implicated as pathogenic targets of AGE processes. AGE formation in vascular wall collagen appears to be an especially deleterious event, causing crosslinking of collagen molecules to each other and to circulating proteins. This leads to plaque formation, basement membrane thickening, and loss of vascular elasticity. The chemistry of these later-stage, glycation-derived crosslinks is still incompletely understood but, based on the hypothesis that AGE formation involves reactive carbonyl groups, the authors introduced the carbonyl reagent aminoguanidine hydrochloride as an inhibitor of AGE formation in vivo in the mid 1980s. Subsequent studies by many researchers have shown the effectiveness of aminoguanidine in slowing or preventing a wide range of complications of diabetes and aging in animals and, recently, in humans. Since, the authors have developed a new class of agents, exemplified by 4,5-dimethyl-3-phenacylthiazolium chloride (DPTC), which can chemically break already-formed AGE protein-protein crosslinks. These agents are based on a new theory of AGE crosslinking that postulates that $\alpha$-dicarbonyl structures are present in AGE protein-protein crosslinks. In studies in aged animals, DPTC has been shown to be capable of reverting indices of vascular compliance to levels seen in younger animals. Human clinical trials are underway.
\end{abstract}

\section{Introduction}

The nonenzymatic reaction of the amino groups of amino acids, peptides, and proteins with reducing sugars, ultimately resulting in the formation of complex brown pigments and protein-protein crosslinks, was first studied under defined conditions by L.C. Maillard in the early 1900s (Maillard and Gautier, 1912). Thus, it came to be known as the Maillard reaction. Ironically, Maillard's speculation that this process might be important in diabetes was an insight too far ahead of the technology capable of demonstrating it. Food chemists soon recognized the 
practical importance of the Maillard reaction in explaining brown color formation and loss of protein quality in cooked and stored foods. However, realization of the importance of Maillard-like reactions in vivo began in the mid-1970s. This started with our studics of hemoglobin Alc ( $\mathrm{HbAlc}$ ), a naturally occurring minor human hemoglobin that is elevated in diabetics. HbAlc was known to be a post-translational adduct of glucose with the $\mathrm{N}$-terminal valine amino group of the $\beta$ chain of hemoglobin, in which the glucose was thought to be attached via a nonenzymatically formed Schiff base structure. We found that measurement of the elevation of $\mathrm{HbAlc}$ in diabetics allowed assessment of the degree of glucose control integrated over several weeks (Koenig et al., 1976). However, the idea that $\mathrm{HbAlc}$ was a Schiff base was troubling, since Schiff bases are generally hydrolytically unstable. Subsequent work (Koenig et al., 1977) revealed that the carbohydrate in $\mathrm{HbAlc}$ was attached as a 1-deoxy-1-fructosyl residue to the $\mathrm{N}$-terminal valine nitrogen, derived from an initially formed Schiff base via a chemical transformation known as the Amadori rearrangement (Figure 1) (Hodge, 1955).

It was not until around 1980 that researchers began recognizing the significance of the complex, late-stage Maillard processes as mediators of the complications of diabetes (Bunn et al., 1978) and aging (Monnier and Cerami, 1981). Subsequently, proteins bearing Amadori product have come to be referred to as glycated proteins (distinguishing them from enzymatically glycosylated proteins), while the process of Amadori product formation is termed glycation. The laterstage, complex pigments and crosslinks formed from glycated protein during the in vivo Maillard reaction have come to be known as advanced glycation end-products, or AGEs (Bucala et al., 1992). Throughout the 1980s and 1990s, a large body of evidence has accumulated implicating AGEs as mediators of various complications of diabetes and aging. Pharmacological agents have been developed and placed in clinical trials to combat AGE processes in man. This chapter will summarize developments in this field and consider its future direction.

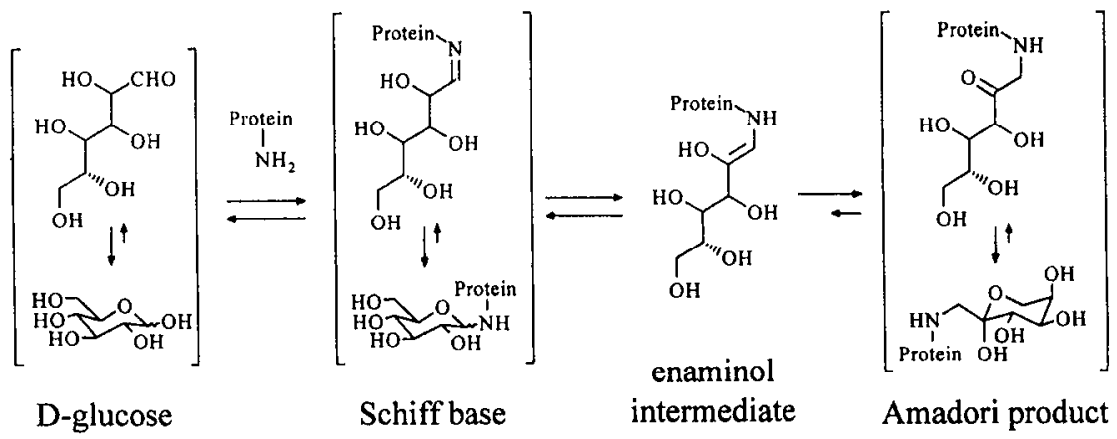

FIG. 1. Formation of the glucose-protein Schiff base and the Amadori rearrangement. 


\section{Chemical Background of Glycation and AGE Formation Under Physiological Conditions}

\section{A. THE MAILLARD REACTION}

Reducing sugars (e.g, glucose, fructose, galactose, mannose, ribose) and certain other carbohydrate relatives (e.g., ascorbic acid) are inherently reactive toward nucleophiles. Their reactivity toward nucleophilic nitrogen bases in protein underlies the Maillard reaction. Glucose is, in fact, the least reactive of the common sugars, perhaps leading to its evolutionary selection as the principal free sugar in vivo (Bunn et al., 1978). Nevertheless, glucose can react with a free amino group, such as the $\varepsilon$-amino group of a protein lysine residue, to form an adduct commonly referred to as a Schiff base. In a Schiff base, the aldehydic carbon-oxygen double bond of the sugar is converted to a carbon-nitrogen double bond with the amine (Figure 1). As is the case with free sugars, the open-chain, doublebonded form of the Schiff base adducts of hexoses or pentoses are thermodynamically disfavored with respect to the equilibrium cyclized pyranose or furanose forms, or glycosylamines (Paulsen and Pflughaupt, 1980) (Figure 1). To simplify mechanistic discussions of subsequent reactions, the adducts are frequently depicted as the more-reactive, open-chain forms. This convention will generally be followed here.

In the 1920s, Amadori showed that glucose-aniline Schiff bases could be converted to isomeric products that were not glycosylamine anomers (Amadori, 1925) but were subsequently found to be 1-anilino-1-deoxyfructose derivatives. It was not until the $1950 \mathrm{~s}$ that it was realized that Amadori products can form from aliphatic amines such as amino acids, not just from aromatic amines, and that the Amadori rearrangement was a key early step in the Maillard reaction (Hodge, 1955). The Amadori rearrangement of the Schiff base to the Amadori product is believed to occur via an intermediate, open-chain enol form (Figure 1).

Formation of the Schiff base from sugar and amine is relatively fast and highly reversible. Formation of Amadori product from the Schiff base is slower but much faster than the reverse reaction, so that the Amadori glycation product tends to accumulate on proteins. The Amadori rearrangement of a lysine-glucose Schiff base is thought to be facilitated if there is a histidine side-chain or another lysine amino group about $5 \AA$ from the amino group on which the Schiff base has formed, due to localized acid-base catalysis (Acosta et al., 2000). It is subsequent to the Amadori rearrangement that the less-well-understood, late-stage, advanced glycation processes begin. Despite considerable research by many laboratories, these slow, in vivo, AGE-forming processes are incompletely understood. It is known that the products include protein-protein crosslinking structures as well as various noncrosslinked structures. Many, but not all, of these products incorporate ultraviolet-visible and/or fluorescent chromophores. 
In recent years, it has become increasingly clear that $\alpha$-dicarbonyl compounds are key intermediates in the formation of AGEs. The Amadori product can break down via its enol form to reactive, free $\alpha$-dicarbonyl glyoxal compounds such as 3-deoxyglucosone, methylglyoxal, and glyoxal (Figure 2) (Thomalley et al., 1999, and references therein). These compounds are known to crosslink proteins and have been detected in vivo. The Amadori product also can dehydrate at the 4-position to yield a 1-amino-4-deoxy-2,3-dione (Amadori dione) (Figure 3) (Huber and Ledl, 1990; Chen and Cerami, 1993). This substance can further dehydrate at the 5-position to yield an unsaturated dione, the Amadori ene-dione (Figure 3) (Estendorfer $e t$ al., 1990). Evidence for crosslinking of proteins by the Amadori ene-dione, as depicted in Figure 3, will be discussed.

\section{B. FLUORESCENT AGE CROSSLINKS}

Reactions of $\alpha$-dicarbonyl compounds with basic amino acid residues or with Amadori intermediates are thought to be involved in formation of many of the known or suspected AGE crosslinking structures shown in Figure 4. Protein-protein crosslinks by such structures in vivo are thought to be responsible for a major share of the deleterious effects of AGEs in diabetes and aging. Along with brown color, fluorescence is one of the qualitative properties classically used to estimate<smiles>O=CC=O</smiles>

glyoxal<smiles>CC(=O)C=O</smiles>

methylglyoxal<smiles>O=CC(=O)CC(O)[C@H](O)CO</smiles>

3-deoxyglucosone

FIG. 2. Some $\alpha$-dicarbonyl glyoxal derivatives formed during glycation.

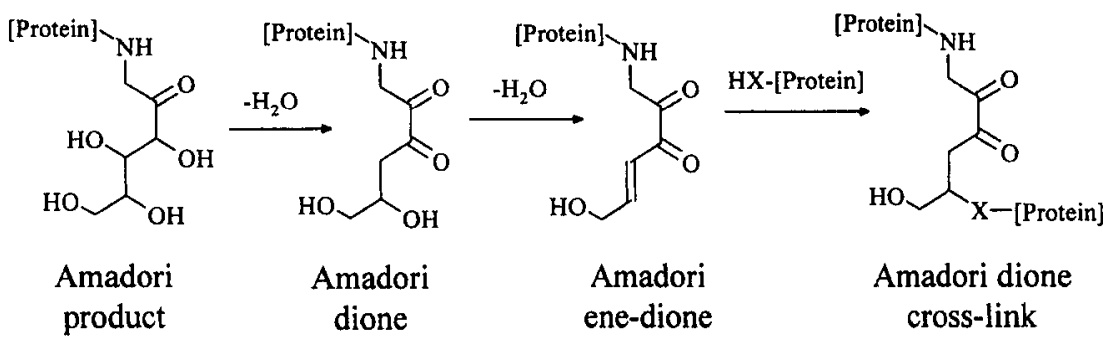

FIG. 3. Dehydration of the Amadori product to form Amadori dione and Amadori ene-dione and conjugate addition of a protein nucleophile to form a protein-protein crosslink. 
AGE formation. Many of the AGEs in Figure 4 were detected and isolated based on their fluorescent properties. The fluorescent AGE crosslink pentosidine was first isolated and identified from dura mater collagen and has since been identified in many tissues (Sell et al., 1991). Pentosidine is unusual in that it can be formed by the reaction of lysine and arginine with any of several carbohydrate precursors, including glucose, ribose, ascorbic acid, and 3-deoxyglucosone. Workers in a number of laboratories have isolated a variety of other fluorescent crosslinks in model reactions under physiological conditions: crosslines $A$ and $B$, vesperlysines A, B, and C; AGE-X1; pentodilysine; and pyrropyridine or FPPC. Of these, the crosslines (Obayashi et al., 1996) and vesperlysines (Tessier et al., 1999) have been detected in vivo.

\section{NONFLUORESCENT AGE CROSSLINKS}

Although their ease of detection makes them useful markers of AGE formation, the fluorescent AGE crosslinks are thought to account for only one percent or less of the total crosslinking structures formed under physiological conditions (Dyer et al., 1991). Thus, the major AGE structures responsible for protein-protein crosslinking in vivo are nonfluorescent structures that have not yet been conclusively identified. Recently, criteria other than fluorescence have been utilized to find new, nonfluorescent crosslinking AGE candidates that might represent the major in vivo AGE crosslink(s). These are depicted in Figure 5 and will be discussed individually in the following paragraphs.

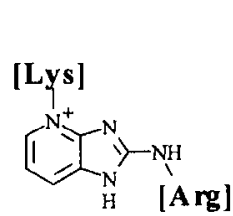

pentosidine

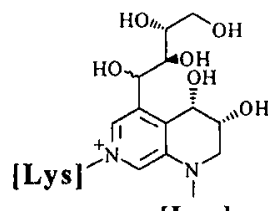

[Lys]

crossline

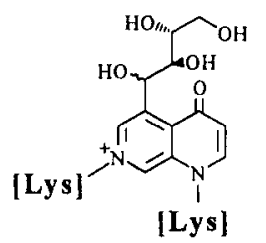

AGE-X1<smiles>O=C1CNc2cc[n+]([Al][Al])cc2C1</smiles>

[Lys]<smiles>Cc1c(O)cc2c(ccn2[Al])c1[N+]([AlH2])=[V]</smiles>

vesperlysine $A, B$<smiles></smiles>

vesperlysine $\mathrm{C}$<smiles>OCC(O)C1CN([AlH2])C2OC(N([AlH2])[AlH2])C2(O)C1</smiles>

FPPC

FIG. 4. Fluorescent AGE crosslinks formed under physiological conditions. [Lys] represents an E-norleucyl group (a desamino-lysine residue); [Arg] represents a $\delta$-norvalyl group (a desguanidinoarginine residue). 


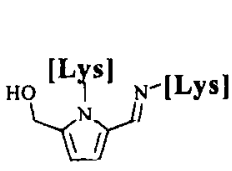

pyrraline imine

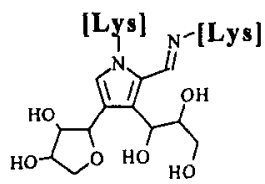

AFGP imine
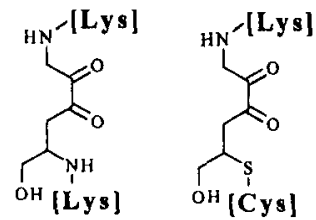

Some Possible Amadori dione cross-links

[His]

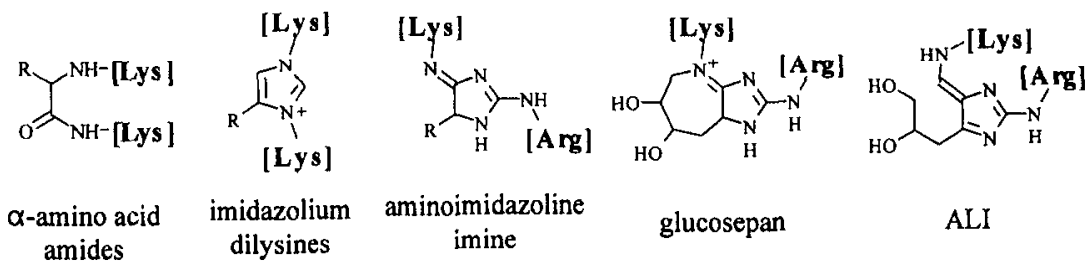

HIG. 5. Known and suspected nonfluorescent AGE crosslinks of physiological relevance. [Lys] represents an $\varepsilon$-norleucyl group (a desamino-lysine residue); [Arg] represents a $\delta$-norvalyl group (a desguanidino-arginine residue). [Cys] and [His] represent $\beta$-substituted alanine portions of cysteine and histidine residues, respectively.

\section{Imidazolium Dilysine Crosslinks}

Nonfluorescent imidazolium dilysine crosslinks, also known as GOLD/MOLD crosslinks, have been isolated from reaction of two molecules of glyoxal derivative with two lysine residues in vitro and also have been detected in vivo (Brinkmann Frye et al., 1998, and references therein). A related 2-acylimidazolium dilysine crosslink was proposed some years ago by our group, based on the isolation of presumed breakdown product 4-furanyl-2-furoylimidazole (FFI) from acid hydrolysis of AGE-modified bovine serum albumin (Pongor et al., 1984). Formation of FFI under these conditions is now thought to occur during isolation (Njoroge et al, 1988). However, AGEs of unknown structure crossreactive with FFI are detectable in AGE proteins in vitro and in vivo (Chang et al., 1985; Nakayama et al., 1988; Palinski et al., 1995). Such 2-acylimidazolium compounds are now known to be unstable toward hydrolytic loss of the acyl group, yielding 2-unsubstituted imidazolium derivatives, and thus are probable precursors of the imidazolium dilysine crosslinks (Al-Abed et al, 1996). The requirement of two moles of a glyoxal derivative for formation of a single imidazolium crosslink represents a mechanistic limitation on the role of imidazolium dilysine crosslinks in vivo. Moreover, formation of an imidazolium dilysine crosslink requires intermediacy of a double Schiff base of a glyoxal derivative with two lysine residues, which may alternatively rearrange to an $\alpha$-amino amide crosslink in a competing reaction (see below). Nevertheless, the imidazole dilys- 
ines are reported to be present at levels 10 - to 50 -fold higher than the fluorescent crosslink pentosidine in tissue proteins (Degenhardt et al., 1998).

\section{Alkyl Formyl Glycosyl Pyrrole (AFGP) Crosslinks}

With James Farmar, we isolated AFGPs (alkyl formyl glycosyl pyrroles) as noncrosslinking AGEs that form in the reaction of two sugar molecules with one alkylamine molecule representing a lysine residue (Farmar et al., 1988). The $\alpha$ positions of the side-chains attached to pyrrole ring carbons in AFGPs are susceptible to nucleophilic attack by thiols (Klein et al., 1992) and by lysine amino groups (J. Farmar and A. Cerami, unpublished data) (Figure 5), indicating that AFGPs are likely to be able to crosslink proteins. The requirement of two moles of sugar to form the AFGP structure and the fact that AFGPs are minor products in the absence of sulfite as a trapping agent (Farmar et al., 1988) suggest that AFGP crosslinks may be of limited importance in vivo.

\section{Pyrraline Crosslinks}

$N$-Alkyl-5-hydroxymethyl-2-pyrrolaldehyde, or pyrraline, is a monomeric AGE that may form on lysine residues in vivo (see below). The aldehyde of pyrraline can form a Schiff base with another amino group (Nissl et al., 1995) (Figure 5), which might lead to lysine-lysine crosslinking in vivo. However, this has not been demonstrated. Such crosslinks might be of limited stability with respect to hydrolytic reversion to their precursors.

\section{4. $\alpha$-Amino Acid Amide Crosslinks}

Sugars and Amadori compounds have been shown to react with primary amines to form $\mathrm{N}, \mathrm{N}^{\prime}$-dialkyl-alanine or -glycine amides (Figure 5). These represent a mode of lysine-lysine crosslinking that may occur in vivo; glyoxal derivatives from Amadori product breakdown are probable intermediates in their formation (Büttner et al., 1997). Such $\alpha$-amino amides may be difficult to isolate from proteins because they lack distinctive chromophores and are likely to be cleaved under protein hydrolysis conditions to give products (carboxymethyllysine and carboxyethyllysine) that are already known to form via other AGE-related processes in vivo (see below). Nevertheless, $\alpha$-amino acid amides are interesting contenders for a significant role in protein crosslinking in vivo.

\section{Aminoimidazoline Imine Crosslinks}

A new class of aminoimidazoline imine AGE protein crosslinks (Figure 5) has recently been reported. These are thought to be derived from reaction of arginine and an $\alpha$-oxoaldimine Schiff base of a lysine residue and glyoxal or methylglyoxal or 3-deoxyglucosone (Lederer and Klaiber, 1999, and references 
therein). The latter product can cyclize to a bicyclic crosslink the authors named glucosepan (Figure 5). These nonfluorescent crosslinks are potentially significant players in AGE crosslinking in vivo, as they require only one mole of carbohydrate-derived dicarbonyl component for their formation and they form relatively efficiently in model systems.

\section{Arginine-Lysine-Imidazole (ALI) Crosslink}

This recently described, nonfluorescent crosslink (Al-Abed and Bucala, 2000) (Figure 5) presumably derives from reaction of the Amadori dione (Figure 3) with an arginine residue. 'The ALI structure was isolated in a model system employing a model dipeptide (Arg-Lys), so that formation of the crosslink results in closure of a macrocyclic ring. ALI has not been reported as an intermolecular crosslink. It contains an enamine-like attachment that might be hydrolyzable. On the other hand, the authors showed that ALI has a potentially close immunochemical relationship with AGE structures on glucose-modified bovine serum albumin using an anti-AGE antibody that has been well characterized in prior studies in our laboratory (Makita et al., 1992). Thus, it is possible that ALI may be more important as an entropically stabilized, intramolecular protein crosslink than as an intermolecular crosslink; this question awaits further research.

\section{Amadori Dione Crosslink}

Conjugate addition of a nucleophilic protein side-chain to a protein-bound Amadori ene-dione would result in a protein-protein crosslink containing an $\alpha$-diketone structure in the linker (Figure 3). Figure 5 shows possible adducts for lysine, cysteine, and histidine. Precedent for conjugate addition of these amino acid residues to such an activated double bond is seen in the adduction of lysine, cysteine, and histidine to a structurally analogous site in the lipid peroxidation product 4-hydroxynonenal (Nadkarni and Sayre, 1995). We have postulated the existence of such an $\alpha$-dione-containing AGE crosslinking structure as shown in Figure 3. The current evidence for the existence of such crosslinks is that certain compounds we designed to destroy $\alpha$-dicarbonyl AGE intermediates were found to break a substantial proportion of the AGE crosslinks themselves, returning proteins experimentally crosslinked with AGEs to a noncrosslinked state (Vasan et al., 1996). The agents that do this are thiazolium salts, whose mechanism of action is based on the catalytic chemistry seen for the co-enzyme vitamin, thiamin, which has a thiazolium structure. These agents and their therapeutic effects will be discussed in detail later. One such agent, 4,5-dimethyl-3-phenacylthiazolium chloride, or DPTC, has been found to reverse certain sequelae of diabetes and aging in vivo (Wolffenbuttel et al., 1998; Asif et al., 2000). Other than the activity of agents like DPTC, there is as yet no direct chemical demonstration of the existence of the Amadori dione crosslink. It is also possible that agents such as 
DPTC might break other types of AGE crosslinks by as-yet-unknown mechanisms.

\section{NONCROSSLINKING, PROTEIN-BOUND AGE STRUCTURES OF IMPORTANCE IN VIVO}

Besides the crosslinking AGEs, which have profound effects on protein structure and function, a number of noncrosslinking AGE structures are found under physiological conditions. These may have deleterious effects as precursors of crosslinks or as biological receptor ligands inducing a variety of adverse cellular and tissue changes. [Such activities will be discussed in Section II.] Some of the more-important, noncrosslinking AGEs, shown in Figure 6, are described in the following paragraphs:

\section{Pyrraline}

Pyrraline is a pyrrole aldehyde AGE reportedly found in vivo (Hayase et al., 1989; Niwa, 1999). Pyrraline is derived from reaction of 3-deoxyglucosone with lysine residues. Pyrraline may form crosslinks between proteins, as discussed earlier.

\section{1-Carboxyalkyllysines}

AGEs in which a 1-carboxyalkyl group is attached to a free amino group of an amino acid residue, such as $N^{\varepsilon}$-(carboxymethyl)lysine (CML) and $N^{\varepsilon}$-(1-carboxyethyl)lysine (CEL), are found in proteins and in free form in vivo (Liardon et al., 1987; Krook et al., 1993; Ahmed et al., 1997). They may arise either from reaction of lysine residues with glyoxal derivatives (Glomb and Monnier, 1995) or from autoxidation of early-stage AGEs such as the Amadori product (Brinkmann Frye et al., 1998). Carboxymethyl derivatives of $\alpha$-amino groups of N-terminal amino acids are also possible, such as $\mathrm{N}$-carboxymethylvaline in hemoglobin (Cai and Hurst, 1999). CML formation seems to be a major pathway in vivo,

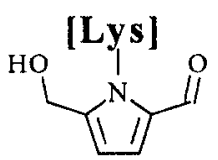

pyrraline<smiles>[R]C(N[AlH2])C(=O)O</smiles>

1 -carboxyalkyllysine

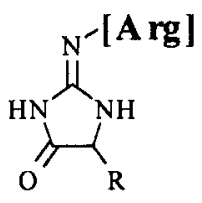

imidazolone A<smiles>[R]C1=N/C(=N\[141I][Na])NC1=O</smiles>

imidazolone B

FIG. 6. Some noncrosslinking AGEs of physiological relevance. [Lys] represents an $\varepsilon$-norleucyl group (a desamino-lysine residue); $|\mathbf{A r g}|$ represents a $\delta$-norvalyl group (a desguanidino-arginine residue). 
as urinary levels of CML in humans average 30 to 40 percent of levels of the lysine Amadori product (Knecht et al., 1991).

\section{Imidazolones}

The reaction of glyoxal or methylglyoxal (Paul et al., 1998, and references therein) or 3-deoxyglucosone (Niwa et al., 1997, and references therein) with the guanidino group of arginine can lead to formation of AGEs called imidazolones (Figure 6). These are structurally related to, but are probably not precursors of, aminoimidazoline imine crosslinks. Imidazolones of type A (Figure 6) undergo slow oxidation to imidazolones of type B. 3-Deoxyglucosone-derived imidazolone $\mathrm{A}$ and traces of $\mathrm{B}$ are found in vivo.

\section{E. FORMATION OF AGES ON DNA}

Like protein, DNA contains amino groups. The 2-amino group of guanosine is the most reactive. Reaction of glucose with guanosine yields $N^{2}$-glucopyranosylguanosine (Knerr et al., 1994) and a 1-carboxyalkyl derivative of $\mathrm{N}-2$ from glucose-derived 3-deoxyglucosone (Knerr and Severin, 1993). A 1-carboxyethyl $\mathrm{N}-2$ adduct of guanosine results from reaction with methylglyoxal (Papoulis et al., 1995) or glucose. Glyoxal and its derivatives also can form five-membered ring cyclic derivatives bridging $\mathrm{N}-1$ and the $\mathrm{C}-2$ amino of guanosine crosslink (Shapiro et al., 1969). None of these have been identified in in vivo sources. However, lysine and glucose-6-phosphate have been noted to form a reactive intermediate, not yet identified, that binds covalently to DNA (Lee and Cerami, 1987). Labeling studies show that the bound species contains the lysine component. The presence of AGEs on DNA causes unusual transpositional rearrangements (Lee and Cerami, 1990; Bucala et al., 1993a) [see Section II].

\section{F. ROLE OF OXIDATION IN FORMATION OF AGES}

Both the Amadori product and later-stage AGEs undergo autoxidation and have pro-oxidant effects on other molecules (Baynes and Thorpe, 1999). Enol structures such as the enaminol precursor of the Amadori product (Figure 1) or the 2,3-enol of the Amadori product may undergo one-electron oxidation to a radical species. Further oxidation of the radical can lead to fragmentation products, such as carboxymethyllysine (Brinkmann Frye et al., 1998). Alternatively, the AGE radical may abstract a hydrogen atom from a nearby biomolecule, converting it to a radical, leading to its autoxidation. The latter process is well demonstrated by the effect of glycation on lipoprotein complexes such as LDL (Bucala et al., 1994,1995). These effects will be discussed in more detail in Section II. 


\section{Biological Aspects of AGE Formation}

AGE precursors were first discovered in vivo on a hemoglobin. The glycation of hemoglobin increases its oxygen affinity and makes it more susceptible to oxidation (Bucala et al, 1992). However, effects of this type on soluble proteins are generally small and limited to the percentage of protein molecules actually glycated, which, in turn, depends on the half-life of the protein. Thus, most of the primary effects of AGEs leading to the complications of diabetes and aging are due to AGE formation on long-lived proteins. Collagen has a very low turnover rate and is a prime target. Especially damaging are the effects of AGEs on vascular collagen, which may contribute to atherosclerosis and coronary disease, kidney damage, retinal pathology, poor peripheral circulation, and other lesions. Lens crystallins are also a long-lived target, leading to cataracts. AGE damage to DNA may cause birth defects.

\section{A. EFFECTS OF AGE FORMATION ON CONNECTIVE TISSUE}

During formation of normal collagen fibrils, some collagen lysine residues and hydroxylysine residues are oxidized by lysyl oxidase, which converts their $\varepsilon$-amino groups to aldehydes. These aldehydes then undergo crosslinking reactions with lysine or hydroxylysine residues in adjacent collagen molecules in the fibrils. This process is self-limiting in that lysines in closely crosslinked collagen molecules are no longer sterically accessible to lysyl oxidase. Evolution has tailored this process to produce collagen fibrils with an optimal balance of strength and flexibility. Unfortunately, AGE crosslinking, being mediated by small molecules, is not subject to such limitations and can proceed even in mature collagen molecules. AGE-mediated overcrosslinking of connective tissue molecules (e.g., collagen molecules) to each other causes loss of bulk elasticity and flexibility and increased brittleness. Another example of damage to connective tissue by AGEs is the crosslinking of exogenous molecules to collagen, which can lead to pathological surface changes and overactivation of tissue repair mechanisms.

\section{Effects of AGE Formation on Vascular Connective Tissue}

Collagen has been shown to react with other collagen molecules or with other plasma proteins (e.g., albumin, immunoglobulin, low-density lipoprotein (LDL)), presumably through AGE crosslinking reactions (Brownlee et al., 1983). AGE crosslink formation in collagen contributes to diabetic circulatory complications such as vascular stiffening (Sims et al., 1996) and myocardial dysfunction (Avendano et al., 1999). The accumulation of the plasma proteins on structural proteins is believed to be a factor leading to the thickening of basement membranes and the development of atherosclerotic lesions (Bucala and Cerami, 1995). The possible involvement of AGE-modified LDL in this process is discussed below. 


\section{Effects of AGE Formation on Nonvascular Connective Tissue}

In both animals and humans, there is a significant correlation between the stiffening of structural collagen (e.g., tendon collagen and dura collagen) with age, the development of yellow-brown discoloration and fluorescence, and the levels of AGEs present. This can be mimicked by incubation of collagen sample with sugars in vitro (Yue et al., 1983; Kohn et al., 1984). These changes are accelerated in diabetes.

\section{B. EFFECTS OF AGE FORMATION ON SOLUBLE PROTEINS}

\section{Effects of AGE Formation in Lens Proteins}

Human lens crystallin proteins are very long lived, possibly lasting an entire lifetime. Although crystallins have evolved to retain optical clarity for an extended period, yellow-brown pigments with the spectral and fluorescent properties of AGFs do form in the lens as a function of age (Monnier and Cerami, 1983). These pigments can become so dark as to form cataracts, leading to blindness. In the normal lens, individual lens proteins associate via hydrophobic interactions to form aggregates of several million daltons. Substances such as glucose or cyanate can modify amino groups in crystallins, causing conformational changes. These changes expose previously buried sulfhydryl groups, which can then autoxidize to form intermolecular disulfide bonds (Monnier et al., 1979). The resulting disulfide-linked superaggregates of the aggregated lens crystallins can be of sufficient size to scatter light and produce a cataract. The importance of disulfide bonds in human cataract formation is indicated by studies showing that light scattering by cataracts in lens from older individuals can be significantly decreased by addition of reducing agents. However, a residual amount of protein crosslinking is present that is not disulfide related (Harding, 1973). AGEs may be directly implicated in this crosslinking.

\section{Effects of AGE Formation on Lipoproteins}

AGE modification of LDL can occur both on amino groups of the apoprotein component (Bucala et al., 1995) and on aminolipid components such as phosphatidylethanolamine (Bucala et al., 1993b). AGE formation on the apoprotein component can lead to crosslinking of LDL to the collagen layer of the blood vessel wall (Brownlee et al., 1983) and can increase the half-life of LDL in serum by blocking the recognition site for its receptor-mediated uptake (Bucala et al., 1995). This increases the probability of autoxidation of the lipid component (Bucala et al., 1993b). Like AGE formation, such oxidation of LDL can lead to loss of recognition by cellular LDL receptors and can induce its preferential uptake by macrophage scavenger receptors (Witztum and Steinberg, 1991). Macrophages 
attracted to AGEs on vessel wall collagen may thus accumulate modified LDL, leading to their conversion to foam cells that are thought to be central to the atherosclerotic process. The ability of AGEs present on LDL to initiate free-radical reactions (Bucala et al., 1993b) is of considerable importance because it provides an alternative to the hypothesis that metals are responsible for LDL autoxidation. Previous work on the initiation of LDL oxidation generally has implicated metal ions (e.g., iron, copper) as oxidation initiators. Unactivated oxygen does not normally react with most organic compounds. Through one-electron redox chemistry, iron or copper ions can induce the radical addition of oxygen to the olefinic bonds of unsaturated fatty acids to form hydroperoxides. Further reactions of these hydroperoxides with metals can form hydroxyl- and peroxyradicals that eventually cleave the fatty acids to form aldehydes. However, free (unchelated) metals usually are not present in significant amounts in vivo. The source of oxidation initiation for substrates like LDL has therefore been puzzling. The ability of AGEs to initiate such oxidative reactions in the absence of metals provides a new explanation for lipoprotein lipid peroxidation in vivo (Bucala et al., 1994).

\section{AGE Formation in $\beta$-Amyloid Plaques}

There is evidence that AGEs play a role in abnormal amyloid aggregation in Alzheimer's disease (AD). Amyloid plaque material like that from AD patients is also found in low quantity in age-matched human controls. Analysis of plaque material from both sources revealed that the plaque from AD patients had three times the AGE content per milligram amyloid as the amyloid from controls (Vitek et al., 1994). Since we know that AGE levels increase over time in normoglycemic individuals, one interpretation is that, in AD patients, a mechanism for removal of amyloid plaque is defective. The persisting plaque undergoes AGE crosslinking, gaining further resistance to proteolytic degradation and removal processes. In vitro studies have found that formation of insoluble, fibrillar aggregates from AGE-modified $\beta$-amyloid peptide was much faster than for control $\beta$-amyloid peptide.

\section{AGE-INDUCED OXIDANT DAMAGE}

The role of oxidative processes in the formation of carboxymethyllysine from the Amadori product, and the pro-oxidant effect of AGE formation on LDL, have been discussed in previous sections. These are part of a larger area of what have been termed "glycoxidation" reactions (Baynes and Thorpe, 1999) in which AGE formation processes and oxidative processes synergistically interact to produce deleterious tissue modifications, with the possible involvement of redox-active metals. These processes have some overlap with lipid peroxidation processes. The case of LDL oxidation already has been discussed, as has the chemical similarity 
of the Amadori ene-dione to the lipid oxidation product, 4-hydroxynonenal, both of which may crosslink proteins. Furthermore, glycoxidation and lipid peroxidation both can form glyoxal and other similar, small-molecule crosslink precursors.

\section{AGE-INDUCED DNA REARRANGEMENT}

As noted earlier, AGEs can form on DNA, particularly on guanine residues. The most-dramatic effect of AGE formation on DNA reported to date is the induction of unusual DNA rearrangements (Lee and Cerami, 1990,1991; Bucala et al., 1993a). Strains of $E$. coli that accumulate high levels of glucose-6-phosphate, which is particularly active in forming AGEs, demonstrate increased levels of the gamma delta transposition, which is the relocation of the transposable element gamma delta (Lee and Cerami, 1991). In mammalian cells, evidence has been found that AGE formation on DNA may be responsible for insertions containing repetitive sequences of the Alu family that have been found to disrupt human genes (Bucala et al., 1993a). The possibility that AGEs may induce genetic rearrangements in vivo has important implications; for example, as a possible cause of congenital malformations in infants of poorly controlled, insulin-dependent, diabetic mothers (Lee et al., 1995).

\section{E. EFFECTS OF AGEs IN FOOD}

Early studies showed that intravenous administration of exogenous, AGEmodified rat serum albumin (AGE-RSA) to rats caused complex vascular dysfunction, compared to rats receiving control RSA. These effects included significant increase in vascular permeability, enhanced mononuclear cell migration into subendothelial and periarteriolar spaces, and defective vasodilatory responses (Vlassara et al., 1992). Although ingestion of AGEs formed during the cooking or processing of food could be a potential source of exogenous AGEs, the possible importance of this was discounted due to the presumed poor absorption of food AGEs. However, it has since been found that food AGEs can be absorbed after eating. In diabetics with impaired renal function, food-derived AGEs with crosslinking activity can accumulate in serum to a significant degree (Koschinsky et al., 1997), implying that normal kidney function is important to protect from dietary AGEs.

\section{F. RECEPTORS FOR AGES AND THEIR POSSIBLE ROLE IN NORMAL REMOVAL OF AGES}

Scavenger receptors with an affinity for AGEs have been found on many cells such as macrophages, lymphocytes, and barrier cells (e.g., endothelial cells, mesangial cells) (Esposito et al., 1989; Skolnik et al., 1991). Phagocytic cells expressing these receptors can internalize and digest senescent, AGE-modified pro- 
teins, releasing AGE-modified peptides similar to those absorbable from food and normally excreted by the kidneys. The uptake of AGE proteins can signal the synthesis and release of certain cytokines and growth factors that can stimulate re-synthesis of proteins that have been phagocytically removed (Vlassara et al., 1988). If this process is not well regulated, serious problems can develop (e.g., tissue overgrowth). High levels of AGEs on matrix proteins in the diabetic kidney can induce mesangial cells to produce excessive amounts of matrix proteins, resulting in a thickened basement membrane incapable of sustaining normal kidney filtration processes (Makita et al., 1991).

\section{Pharmacological Intervention Against AGE Formation in Vivo}

The development of agents against AGE-related complications has followed two main approaches (Figure 7). First, inhibition of AGE formation processes by carbonyl-blocking agents such as aminoguanidine, and second, more recently, the cleavage of already formed AGE protein-protein crosslinks by dicarbonyl-breaking compounds like DPTC.

\section{A. PHARMACOLOGICAL INHIBITION OF AGE CROSSLINK FORMATION}

In the early 1980 s, when it became clear that advanced glycation reactions were a potential cause of complications of diabetes and aging, we began to think

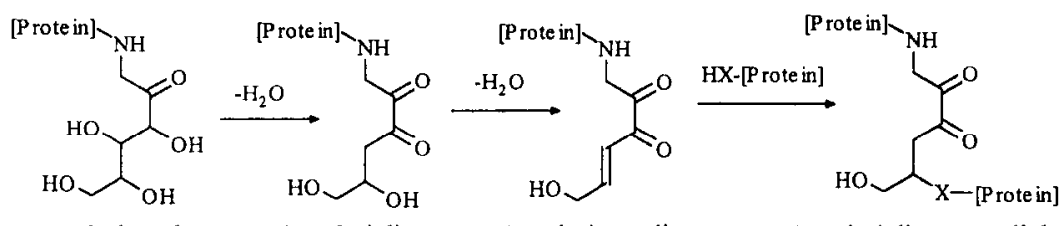

Amadori product (glycated protein)

Amadori dione

Amadori ene-dione
Amadori dione crosslink

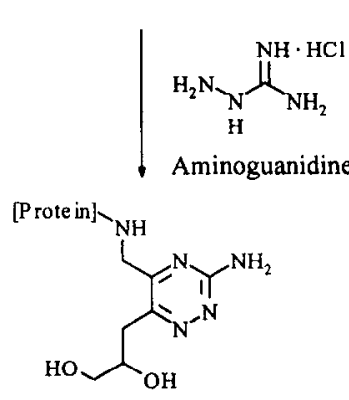

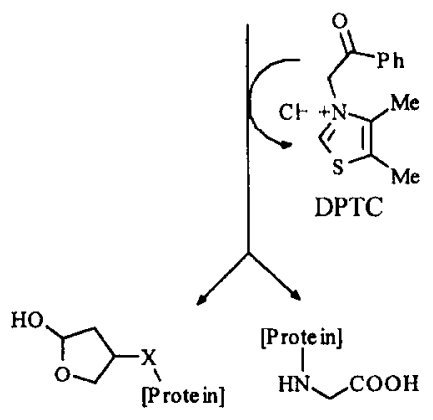

FIG. 7. Possible sites of post-Amadori crosslink prevention by aminoguanidine and crosslink cleavage by DPTC (ALT-711). 
about ways to interfere with this process pharmacologically. Unfortunately, little was known at that time about the pathways of AGE formation in the Maillard reaction in vivo. We focussed our attention on the structure of the Amadori product. We reasoned that the ketone group of the 1-amino-1-deoxyfructose residue in the Amadori product was the key to subsequent AGE-forming reactions and that chemical deactivation of this ketone group would prevent AGE formation. We therefore sought to identify an agent that would react rapidly with ketones, rendering them chemically inert, while being relatively nontoxic. A number of factors focussed our attention on aminoguanidine. It is a low-molecular-weight, highly nucleophilic compound that very readily reacts with ketones and aldehydes. There are some published reports of its experimental use in humans for unrelated purposes, without significant side-effects. We therefore selected aminoguanidine for preclinical study (Brownlee et al, 1986). In the intervening years, research in our laboratory and in others around the world has shown the effectiveness of aminoguanidine in inhibiting AGE formation in vivo in a wide variety of systems and tissues (Table I). As discussed earlier, $\alpha$-dicarbonyl intermediates, and not the initial Amadori product, are now believed to be the targets of aminoguanidine inhibition.

TABLE I

Effect of Aminoguanidine on Diabetes-related Complications or AGE Formation in Vivo

Nephropathy

- Inhibition of basement membrane thickening (Ellis and Good, 1991)

- Prevention of albuminuria (Soulis-Liparota et al., 1991)

- Stabilization of IGF system (Bach et al., 2000).

Retinopathy

- Normalization of endothelial cell proliferation (Hammes et al., 1991)

- Restoration of retinal nNOS neurons (Roufail et al., 1998)

Vasculopathy

- Reduction of vasodilatory impairment (Vlassara et al., 1992)

- Preservation of vascular elasticity (Huijberts et al., 1993)

- Improvement of erythrocyte deformability (Brown et al., 1993)

- Anti-atherogenic effect (Panagiotopoulos et al., 1998)

Neuropathy

- Retention of nerve conduction velocity (Kihara et al., 1991; Miyauchi et al., 1996) 
A phase III clinical study of aminoguanidine (also known by the generic name, pimagedine) in diabetic patients was completed recently. Patients in the study showed significantly decreased LDL levels, decreased urinary albumin levels (an important indicator of diabetic nephropathy), decreased progression of retinal degeneration, and a trend toward prevention of loss of kidney function. Further trials will be necessary before aminoguanidine can be approved for clinical use.

\section{B. PHARMACOLOGICAL CLEAVAGE OF AGE CROSSLINKS}

Like the outcome of the Diabetes Control \& Complications Trial, the activity of aminoguanidine against diabetic complications is another important piece of evidence supporting the AGE theory of diabetic complications in humans. However, there is a major limitation to the utility of AGE formation inhibitors like aminoguanidine: they cannot affect already-formed AGE damage, particularly crosslinking. A drug that could break already-formed crosslinks is a more desirable goal, as it would allow direct restoration of AGE-damaged tissue to its original functionality, without relying on the body's much-slower, more-problematical removal and repair mechanisms. In the absence of a clear understanding of the specific chemical mechanisms of AGE crosslink formation, developing such a drug seemed an unattainable goal. However, in the course of designing a new class of AGE inhibitors, we devised a new theory of AGE crosslinking that suggested the possible existence of crosslinking structures that could be chemically broken.

Alpha-dicarbonyl compounds such as $\alpha$-oxoaldehydes (glyoxal derivatives) (Figure 2) and their Schiff bases are thought by many researchers to be key reactive intermediates in AGE formation in vivo. Somewhat less studied have been $\alpha$-dicarbonyl (diketone) intermediates of the $\Lambda$ madori dione pathway, resulting from dehydration of the Amadori product with the amino acid still attached (Figure 3). We noticed that the Amadori ene-dione contained a structural motif that would allow it to form a protein-protein crosslink via conjugatc addition of another nucleophilic side-chain to the carbon-carbon double bond in a Michael reaction (Figure 3). Such a conjugate addition by lysine, histidine, and cysteine residues has been reported for a chemically related structural motif in the lipid peroxidation product, 4-hydroxynonenal (Nadkarni and Sayre, 1995). The resulting Amadori dione AGE crosslink structure (Figures 3 and 5) would contain an $\alpha$-diketone.

We designed a new class of anti-AGE agents that contained a thiazolium structure, which could chemically break $\alpha$-dicarbonyl compounds (e.g., glyoxals, $\alpha$-diketones) by cleaving the carbon-carbon bond between the carbonyls. We thought that these agents would inhibit $A G E$ processes by destroying glyoxal derivatives and the Amadori diones. However, it was difficult to assess the 
prospects for a major physiological role for $\alpha$-dicarbonyl-containing crosslink structures that would be cleavable by these agents. Thus, it was a pleasant surprise when we found that the thiazolium agent 3-phenacylthiazolium bromide (PTB) was able to cleave a major proportion of AGE crosslinks formed under physiological conditions (Vasan et al., 1996). Subsequently, we have developed a more-stable and more-active derivative, 4,5-dimethyl-3-phenacylthiazolium chloride (DPTC), which has shown a variety of promising effects in preclinical studies. For example, at a dose of $1 \mathrm{mg} / \mathrm{kg} /$ day for $1-3$ weeks in streptozocin-diabetic rats, DPTC reversed the large artery stiffness resulting from diabetes, as assessed by the criteria of systemic arterial compliance, aortic impedance, and carotid artery compliance. DPTC also reduced the diabetes-related increased crosslinking of tail tendon collagen, as measured by susceptiblilty to proteolytic degradation (Wolffenbuttel et al., 1998). In a more-recent study in aged dogs, invasive monitoring was used to measure end diastolic pressure and volume pre- and post-treatment. Treatment with DPTC at $1 \mathrm{mg} / \mathrm{kg} /$ day for 4 weeks led to a 40 percent lowering of left ventricular stiffness, essentially restoring the elasticity of the heart in aged animals to levels seen in much-younger ones (Asif et al., 2000).

The finding that already-formed AGE crosslinks can be pharmacologically severed and attendant pathology thereby reversed has broad implications for the therapeutics of the complications of diabetes and diseases of aging. The prospect of reversing the effects of AGEs was previously only contemplated in the context of stimulating the body's own removal and repair mechanisms. Because of the unique mechanistic aspects of agents like DPTC, further chemical, preclinical, and clinical studies with these AGE-crosslink-breaking agents has the potential to establish new paradigms in the management of diabetes and aging diseases. Such research also will increase the understanding of how AGEs cause pathology and the extent of AGE involvement in the various complications of diabetes and aging, compared to other mechanisms proposed for these processes.

\section{REFERENCES}

Acosta, J., Hettinga, J., Fluckiger, R., Krumrei, N., Goldfine, A., Angarita, L., and Halperin, J. (2000). Proc. Natl. Acad. Sci. U.S.A. 97, 5450-5455.

Ahmed, M.U., Brinkmann Frye, E., Degenhardt, T.P., Thorpe, S.R., and Baynes, J.W. (1997). Biochem. J. 324, 565-570.

Al-Abed, Y., and Bucala, R. (2000). Bioconjug. Chem. 11, 39-45.

Al-Abed, Y., Mitsuhashi, T., Ulrich, P. and Bucala, R. (1996). Bioorg. Med. Chem. Lett. 6, 1577-1578. Antudori, M. (1925). Atti real. accad. naz. Lincei 2, 337.

Asif, M., Egan, J., Vasan, S., Jyothirmayi, G.N., Masurekar, M.R., Lopez, S., Williams, C., Torres, R.L., Wagle, D., Ulrich, P., Cerami, A., Brines, M., and Regan, T.J. (2000). Proc. Natl. Acad. Sci. U.S.A. 97, 2809-2813.

Avendano, G.F., Agarwal, R.K., Bashey, R.I., Lyons, M.M., Soni, B.J., Jyothirmayi, G.N., and Regan, T.J. (1999). Diabetes 48, 1443-1447.

Bach, L.A., Dean, R., Youssef, S., and Cooper, M.E. (2000). Nephrol. Dial. Transplant. 15, 347-354. 
Baynes, J.W., and Thurpe, S.R. (1999). Diabetes 48, 1-9.

Brinkmann Frye, E., Degenhardt, T.P., Thorpe, S.R., and Baynes, J.W. (1998). J. Biol. Chem. 273, 18714-18719.

Brown, C.D., Zhao, Z.H., De Alvaro, F., Chan, S., and Friedman, E.A. (1993). Diabetes 42, 590-593.

Brownlee, M., Pongor, S., and Cerami, A. (1983). J. Exp. Med. 158, 1739-1744.

Brownlee, M., Vlassara, H., Kooney, A., Ulrich, P., and Cerami, A. (1986). Science 232, 1629-1632.

Bucala, R., and Cerami, A. (1995). In "Multiple Risk Factors in Cardiovascular Disease : Vascular and Organ Protection" (A.M. Gotto, Jr. et al., eds.), pp. 155-163. Kluwer Acadernic Publishers, Dordrecht, Netherlands.

Bucala, R., Vlassara, H., and Cerami, A. (1992). In "Post-Translation Modification of Proteins" (J.J. Harding and M.J.C. Crabbe, eds.), pp. 53-79. CRC Press, Inc, Boca Raton, Fla.

Bucala, R., Lee, A.T., Rourke, L., and Cerami, A. (1993a). Proc. Natl. Acad. Sci. U.S.A. 90, 2666-2670

Bucala, R., Makita, Z., Koschinsky, T., Cerami, A., and Vlassara, H. (1993b). Proc. Natl. Acad. Sci. U.S.A. 90, 6434-6438.

Bucala, R., Makita, Z., Koschinsky, T., Cerami, A., and Vlassara, H. (1994). Spec. Publ. R. Soc. Chem. 151, 286-291.

Bucala, R., Mitchell, R., Arnold, K., Innerarity, T., Vlassara, H., and Cerami, A. (1995). J. Biol. Chem. 270, 10828-10832.

Bunn, H.F., Gabbay, K.H., and Gallop, P.M. (1978). Science 200, $21-27$.

Buttner, U., Gerum, F., and Severin, T. (1997). Carbohydr. Res. 300, 265-269.

Cai, J., and Hurst, H.E. (1999). J. Mass Spectrom. 34, 537-543.

Chang, J.C., Ulrich, P.C., Bucala, R., and Cerami, A. (1985). J. Biol. Chem. 260, 7970-7974.

Chen, H.J.C., and Cerami, A. (1993). J. Carbohydr. Chem. 12, 731-742.

Degenhardt, T.P., Thorpe, S.R., and Baynes, J.W. (1998). Cell. Mol. Biol. (Noisy-le-grand) 44, 1139-1145.

Dyer, D.G., Blackledge, J.A., Katz, B.M., Hull, C.J., Adkisson, H.D., Thorpe, S.R., Lyons, T.J., and Baynes, J.W. (1991). Zeitschr. Ernahrungswiss. 30, 29-45.

Ellis, E.N., and Good, B.H. (1991). Metabolism 40, 1016-1019.

Esposito, C., Gerlach, H., Brett, J, Stern, D., and Vlassara, H. (1989). J. Exp. Med. 170, 1387-1407.

Estendorfer, S., Ledl, F, and Severin, T. (1990). Angew. Chem. Int. Ed. Eng 29, 536-537.

Farmar, J.G., Ulich, P.C., and Cerami, A. (1988). J. Org. Chem. 53, 2346-2349.

Glomb, M.A., and Monnier, V.M. (1995). J. Biol. Chem. 270, 10017-10026.

Hammes, H.P., Martin, S., Federlin, K., Geisen, K., and Brownlee, M. (1991). Proc. Natl. Acad. Sci. U.S.A. 88, $11555-11558$.

Harding, J.J. (1973). Exp. Eye Res. 17, 377-383

Hayase, F., Nagaraj. R.H., Miyata, S., Njoroge, F.G., and Monnier, V.M. (1989). J. Biol. Chem. 264, 3758-3764.

Hodge, J.E. (1955). Adv. Carbohydr. Chem. 10, 169-205.

Huber, B., and Ledl, F. (1990). Carbohydr. Res. 204, 215-220.

Huijberts, M.S., Wolffenbuttel, B.H., Boudier, H.A., Crijns, F.R., Kruseman, A.C., Poitevin, P., and Levy, B.I. (1993). J. Clin. Invest. 92, 1407-1411.

Kihara, M., Schmelzer, J.D., Poduslo, J.F., Curran, G.L., Nickander, K.K., and Low, P.A. (1991). Proc. Natl. Acad. Sci. U.S.A. 88, 6107-6111.

Klein, E., Ledl, F, Bergmuller, W., and Severin, T. (1992). Z. Lebensm. Unters. Forsch. 194, 556-560.

Knecht, K.J., Dunn, J.A., McFarland, K.F., McCance, D. R., Lyons, T.J., Thorpe, S.R., and Baynes, J.W. (1991). Diabetes 40, 190-196.

Knerr, T., and Severin, T. (1993). Tetrahedron Lett. 46, 7389-7390.

Knerr, T., Ochs, S., and Severin, T. (1994). Carbohydr. Res. 256, 177-183.

Koenig, R.J., Peterson, C.M., Jones, R.L., Saudek, C., Lehrman, M., and Cerami, A. (1976). N. Engl. J. Med. 295, 417-420. 
Koenig, R.J., Blobstein, S.H., and Cerami, A. (1977). J. Biol. Chem. 252, 2992-2997.

Kohn, R.R., Cerami, A., and Monnier, V.M. (1984). Diabetes 33, 57-59.

Koschinsky, T., He, C.J., Mitsuhashi, T., Bucala, R., Liu, C., Buenting, C., Heitmann, K., and Vlassara, H. (1997). Proc. Natl. Acad. Sci. U.S.A. 94, 6474-6479.

Krook, M., Ghosh, D., Stromberg, R., Carlquist, M., and Jornvall, H. (1993). Proc. Natl. Acad. Sci. U.S.A. 90, 502-506.

Lederer, M.O., and Klaiber, R.G. (1999). Bioorg. Med. Chem. 7, 2499-2507.

Lee, A.T., and Cerami, A. (1987). Mutat. Res. 179, 151-158.

Lee, A.T., and Cerami, A. (1990). Mutat. Res. 238, 185-191.

Lee, A.T., and Cerami, A. (1991). Mutat. Res. 249, 125-133.

Lee, A.T., Plump, A., DeSimone, C., Cerami, A., and Bucala, R. (1995). Diabetes 44, $20-24$.

l.iardon, R., de Weck-Gaudard, D., Philippossian, G., and Finot, P.A. (1987). J. Agric. Food Chem. 35, 427-431.

Maillard, L.C., and Gautier, M.A. (1912). C. R. Seances Acad. Sci. III 154, 66-68.

Makita, Z., Radoff, S., Rayfield, E.J., Yang, Z., Skolnik, E., Delaney, V., Friedman, E.A., Cerami, A., and Vlassara, H. (1991). N. Engl. J. Med. 325, 836-842

Makita, Z., Vlassara, H., Cerami, A., and Bucala, R. (1992). J. Biol. Chem. 267, 5133-5138.

Miyauchi, Y., Shikama, H., Takasu, T., Okamiya, H., Umeda, M., Hirasaki, E., Ohhata, I., Nakayama, H., and Nakagawa, S. (1996). Eur. J. Endocrinol. 134, 467-473.

Monnier, V.M., and Cerami, A. (1981). Science 211, 491-493.

Monnier, V.M., and Cerami, A. (1983). Biochim. Biophys. Acta 760, 97-103.

Monnier, V.M., Stevens, V.J., and Cerami, A. (1979). J. Exp. Med. 150, 1098-1107.

Nadkarni, D.V., and Sayre, L.M. (1995). Chem. Res. Toxicol. 8, 284-291.

Nakayama, H., Kato, M., Makita, Z., Aoki, S., Kuroda, Y., Misawa, K., Yanagisawa, K., Nakagawa, S., and Ikeda, K. (1988). J. Immunol. Meth. 112, 57-61.

Nissl, J., Pischetsrieder, M., Klein, E., and Severin, T. (1995). Carbohydr. Res. 270, C1-C5.

Niwa, T. (1999). J. Chromatogr. B Biomed. Sci. Appl. 731, 23-36.

Niwa, T., Katsuzaki, T., Ishizaki, Y., Hayase, F., Miyazaki, T., Uematsu, T., Tatemichi, N., and Takei, Y. (1997). FEBS Lett. 407, 297-302.

Njoroge, F.G., Fernandes, A.A., and Monnier, V.M. (1988). J. Biol. Chem. 263, 10646-10652

Obayashi, H., Nakano, K., Shigeta, H., Yamaguchi, M., Yoshimori, K., Fukui, M., Fujii, M., Kitagawa, Y., Nakamura, N., Nakamura, K., Nakazawa, Y., lenaga, K., Ohta, M., Nishimura, M., Fukui, 1., and Kondo, M. (1996). Biochem. Biophys. Res. Commun. 226, 37-41.

Palinski, W., Koschinsky, T., Butler, S. W., Miller, E., Vlassara, H., Cerami, A., and Witztum, J. L. (1995). Arterioscler. Thromb. Vasc. Biol. 15, 571-582.

Panagiotopoulos, S., O'Brien, R.C., Bucala, R., Cooper, M.E., and Jerums, G. (1998). Atherosclerosis 136, 125-131.

Papoulis, A., Al-Abed, Y., and Bucala, R. (1995). Biochemistry 34, 648-655.

Paul, R.G., Avery, N.C., Slatter, D.A., Sims, T.J., and Bailey, A.J. (1998). Biochem. J. 330, 1241-1248.

Paulsen, H., and Pflughaupt, K.W. (1980). In "The Carbohydrates: Chemistry and Biochemistry," edit. 2, vol lB (W. Pigman and D. Horton, eds.), pp. 881-927. Academic Press, New York.

Pongor, S., Ulrich, P.C., Bencsath, F.A., and Cerami, A. (1984). Proc. Natl. Acad. Sci. U.S.A. 81, 2684-2688.

Roufail, E., Soulis, T., Boel, E., Cooper, M.E., and Rees, S. (1998). Diabetologia 41, 1419-1425.

Sell, D.R., Nagaraj, R.H., Grandhee, S.K., Odetti, P., Lapolla, A., Fogarty, J., and Monnier, V.M. (1991). Diabetes Metab. Rev. 7, 239-251

Shapiro, R., Cohen, B.I., Shiuey, S.J., and Maurer, H. (1969). Biochemistry 8, 238-245.

Sims, T.J., Rasmussen, L.M., Oxlund, H., and Bailey, A.J. (1996). Diabetologia 39, 946-951

Skolnik, E.Y., Yang, Z., Makita, Z., Radoff, S., Kirstein, M., and Vlassara, H. (1991). J. Exp. Med. 174, 931-939. 
Soulis-Liparota, I., Cooper, M., Papazoglou, D., Clarke, B., and Jerums, G. (1991). Diabetes 40, 1328-1334.

Tessier, F., Obrenovich, M., and Monnier, V.M. (1999). J. Biol. Chem. 274, 20796-20804.

Thornalley, P.J., I anghorg, A, and Minhas, H.S (1999). Biochem. J. 344, 109-116.

Vasan, S., Zhang, X., Kapurniotu, A., Bernhagen, J., Teichberg, S., Basgen, J., Wagle, D., Shih, D., Terlecky, I., Bucala, R., Cerami, A., Egan, J, and Ulrich, P. (1996). Nature 382, 275-278.

Vitek, M.P., Bhattacharya, K., Glendening, J.M., Stopa, E., Vlassara, H., Bucala, R., Manogue, K., and Cerami, A. (1994). Proc. Natl. Acad. Sci. U.S.A. 91, 4766-4770.

Vlassara, H., Brownlee, M., Manogue, K.R., Dinarello, C.A., and Pasagian, A. (1988). Science 240, 1546-1548.

Vlassara, H., Fuh, H., Makita, Z., Krungkrai, S., Cerami, A., and Bucala, R. (1992). Proc. Natl. Acad Sci. U.S.A. 89, $12043-12047$.

Witztum, J.L., and Steinberg, D. (1991). J. Clin. Invest. 88, 1785-1792.

Wolffenbuttel, B.H., Boulanger, C.M., Crijns, F.R., Huijberts, M.S., Poitevin, P., Swennen, G.N., Vasail, S., Egan, J.J., Ulrich, P., Cerami, A., and Levy, B.I. (1998), Proc. Natl. Acad. Sci. U.S.A. 95, 4630-4634.

Yue, D.K., McLennan, S., Delbridge, L., Handelsman, D.J., Reeve, T., and Turtle, J.R. (1983). Diabetologia 24, 282-285. 



\title{
BETA2 and Pancreatic Islet Development
}

\author{
Khol Chu, Eric Nemoz-Gaillard, and Ming-Jer Tsaj \\ Department of Molecular and Cellular Biology, Baylor College of Medicine, \\ I Baylor Plaza, Houston, Texas 77030
}

\begin{abstract}
The pancreas is essential for digestion and glucose homeostasis. Diseases associated with the pancreas (e.g., pancreatitis, pancreatic cancer, diabetes) are generally debilitating for the patient. Diabetes is particularly prominent in the United States, affecting nearly 6 percent of the population, with associated annual health costs in the billions of dollars. Pancreas development is a complex process that requires the timely expression of numerous factors. Among them, a basic Helix-LoopHelix factor, BETA2, was shown to be important for terminal differentiation of islet cells including insulin- and glucagon-producing cells. Expression studies demonstrated the presence of BETA2 in islet cells and specific neurons. Targeted deletion of the BETA2 gene in mice revealed its significance in pancreas development. In addition, BETA2 is important in granule cell development of the hippocampus and cerebellum. This chapter will focus on the role of BETA2 in pancreas physiology, neuronal development, and its molecular biology.
\end{abstract}

\section{Introduction}

According to the American Diabetes Association (ADA), 15.7 million Americans (10.3 million diagnosed and 5.4 million undiagnosed), representing 5.9 percent of the U.S. population, suffer from diabetes. Diabetes mellitus, whether type 1 (insulin-dependent diabetes mellitus (IDDM), 5-10 percent of diabetes) or type 2 (non-insulin-dependent diabetes mellitus (NIDDM), 90-95 percent of diabetes), results from an impaired glucose homeostasis by the hormone insulin. In the former, a complex autoimmune process leads to the loss of insulin-secreting cells of the pancreas, while the latter is characterized by a failure to compensate for peripheral insulin resistance.

An increasing number of factors have been implicated in the etiology of diabetes, pointing to both environmental and genetic determinants in the development of this disease. Over the past few years, the efficiency of modern molecular biology techniques, with the generation and analysis of numerous transgenic and knockout animals, provoked an explosion of the number of publications on the development of the pancreas and, more specifically, of islet cells. The results obtained from these studies have revealed some of the complex mechanisms 
governing the ontogeny of pancreatic endocrine cells. Indeed, an elaborate developmental program was expected in order to maintain the integrity and functionality of the pancreas.

Among the important molecular factors involved in the proper formation of the pancreas, our group described the transcription factor BETA2 to be critical for the terminal differentiation of pancreatic islets (Naya et al., 1995,1997). In addition, BETA2 plays a significant role in the development of various structures in the nervous system (Lee et al., 1995; Liu et al., 2000a,2000b; Miyata et al., 1999).

This chapter will focus mainly on the role of BETA2 in pancreas development and its molecular biology. Its function outside the pancreas also is described, to profile the multifaceted role of this differentiation factor.

\section{A. PANCREAS}

The mature pancreas is comprised of three main type of cells: duct epithelium, endocrine, and exocrine cells (Pictet et al., 1972; Slack, 1995). The exocrine cells, which represent the major cell type in the pancreas, secrete digestive enzymes into the duodenum through a branched ductal system. In contrast, endocrine cells represent 1 to 2 percent of the mature pancreas mass. These cells are arranged as compact, spheroidal clusters of hormone-producing cells, dispersed throughout the pancreas, named the islets of Langerhans. An adult islet is composed of four major cell types $-\alpha, \beta, \delta$, and pancreatic polypeptide (PP) cells - that synthesize glucagon, insulin, somatostatin, and pancreatic polypeptide, respectively. In addition to the glandular component, islets have a rich blood supply through a sophisticated, intra-islet portal system. Importantly, it appears that the directional flow in the islet portal system may be a major determinant of $\beta$-cell function and therefore is pertinent to subsequent functioning of the organ.

In humans, the pancreas is a well-defined, elongated, triangular organ of 15-25 centimeters weighing about 70-100 grams. In rodents, the shape of the pancreas is less well defined, appearing as a loose mass of tissue attached to the stomach and small intestine. In rodents, the islets are normally rounded, with a central core of $\beta$ cells surrounded by a mantle, primarily composed of glucagon cells intermingled with $\delta$ and PP cells. In humans, islets are lobulated and the segregation of $\beta$ cells vs. $\alpha, \delta$, and PP cells is not as well defined.

\section{B. PANCREAS DEVELOPMENT}

Despite some discrepancies between species in the overall structural or substructural pancreatic organization, the developmental program of the pancreas is essentially equivalent in mammals and, so far, is known to involve the same molecular components (Grapin-Botton and Melton, 2000; Kim et al., 1997; Wells 
and Melton, 1999). Upon the appropriate inductive signals, two pancreatic anlaga bud from the gut endoderm into the surrounding splanchnic mesoderm (Pictet et al., 1972). As these buds grow, they rapidly form new protrusions, leading to a highly branched structure. These two primitive outgrowths develop independently, forming both endocrine and exocrine tissues, and finally merge to form the pancreas. In the developing pancreas, a subset of cells adjacent to the nascent pancreatic duct has been identified as progenitor cells for the pancreatic islets. These progenitor cells actively proliferate, then differentiate through a series of maturation steps as they migrate away from the duct to finally aggregate into mature, properly organized islets. The identity of the endocrine progenitor cells remains elusive and is the object of active research by both academic and pharmaceutical scientists. Overall, the development of the pancreas appears as a complex process involving many factors whose identities and functions are now being uncovered.

Several transcription factors from the homeodomain, basic Helix-Loop-Helix (bHLH), and winged-helix families have been identified to play a role at various stages or decision branchpoints of pancreas development. These include Isletl, PDX1, Pax4, Pax6, Nkx2.2, Nkx6.1, HB9, and hepatocyte nuclear factor (HNF)6 in the homeodomain family (Ahlgren et al., 1996,1997; Gannon et al., 2000; Harrison et al., 1999; Jacquemin et al., 2000; Jonsson et al., 1994; Li et al., 1999; Offield et al., 1996; Sosa-Pineda et al., 1997; St-Onge et al., 1997); HNF3 $\alpha$ and HNF3 $\beta$ in the winged-helix family (Ang and Rossant, 1994; Kaestner et al., 1999; Weinstein et al., 1994); and BETA2, Neurogenin3 (NGN3), p48, and hairy and enhancer of split l (HES1) in the bHLH family (Gradwohl et al, 2000; Ishibashi et al., 1995; Jensen et al., 2000b; Krapp et al., 1998; Naya et al., 1995,1997). Among these, PDX1, NGN3, and HES1 seem to interact, physically or functionally, with BETA2, in a direct or indirect manner. Since it is beyond the scope of this chapter to discuss or dissect the role of other transcription factors involved in pancreas development, readers are invited to consult recent reviews (Bramblett et al., 2000; Edlund, 1998, 1999).

\section{PDXI}

PDX1 - a homeobox gene also known as IPF-1, STF-1, IDX-1, and $\mathrm{XIHbox} 8$ - was shown to be involved in islet hormone gene transcription and expressed in pancreatic tissue during development and in the adult (Guz et al., 1995; Leonard et al., 1993; Miller et al., 1994; Peshavaria et al., 1994; Serup et al., 1995). During embryonic development, PDX1 is expressed in a domain that includes the pancreatic buds and expands from the antral part of the stomach to the proximal duodenum (Ahlgren et al., 1996; Guz et al., 1995; Jonsson et al., 1995; Offield et al., 1996; Serup et al., 1995). As differentiation proceeds, PDX1 expression is progressively restricted to $\beta$ cells and some $\delta$ cells. In mice and 
humans harboring a defective mutation of PDX1, the pancreas is absent (Jonsson et al., 1994; Offield et al., 1996; Stoffers et al., 1999). This dramatic phenotype suggests that PDX1 is required for the proliferation and differentiation of the presumptive pancreatic epithelium from the foregut. Studies in $\mathrm{PDXN}^{\%}$ mice indicated that both insulin- and glucagon-producing cells are present in the pancreatic buds, suggesting that regulation of the insulin and glucagon genes is complex and requires many factors besides PDX1 (Offield et al., 1996). In order to study the role of PDX1 in adult mice, the PDX1 gene was specifically disrupted in $\beta$ cells by using the CRE-LoxP system under the control of the insulin promoter (Ahlgren et al., 1998). PDX1 was shown to be required for the maintenance of $\beta$ cells and to positively regulate insulin expression (Ahlgren et al., 1998). Overall, in addition to its early involvement in the patterning of the pancreatic primordia, PDX1 appears to participate in the regulation of insulin gene expression in mature pancreatic $\beta$ cells.

\section{Neurogenin 3}

The neurogenins (NGNs) are bHLHs of the Atonal subfamily, an important pro-neural gene in Drosophila. Consistent with such a role, targeted ablation of NGN1 or NGN2 results in the absence of specific neurons (Fode et al., 1998,2000; Ma et al., 1996,1998,1999; Perez et al., 1999). NGN3 - also named Math4B or Relax - is specifically expressed during embryogenesis in the pancreas, the developing neural tube, and the hypothalamus (Ma et al., 1996). In the developing pancreas, NGN3 expression can be observed very early in a speckled pattern (e9.0 in the mouse, i.e., when pancreatic buds are starting to form). These cells have been considered to represent pancreatic islet progenitors (Gradwohl et al., 2000; Schwitzgebel et al., 2000). Consistent with this idea, NGN3 expression is transient in the developing pancreas; increasing levels of NGN3 are observed from e9.5 to e15.5 and decline thereafter. Interestingly, BETA2 expression is superficially similar to NGN3, suggesting a possible regulation of BETA2 by NGN3 (Figure 1) (Huang et al., 2000). Indeed, NGN3 expression is restricted to a subset of proliferating cells associated with the nascent duct epithelium and mostly overlaps with those of the early islet differentiation factors PDX1, Nkx6.1, and Nkx2.2 (Jensen et al, 2000a; Schwitzgebel et al., 2000). Interestingly, targeted disruption of the NGN3 gene results in a total loss of pancreatic endocrine cells, highlighting the crucial role played by NGN3 in pancreatic islet cell formation (Gradwohl et al., 2000). Furthermore, in a gain-of-function study, NGN3 induced premature differentiation of pancreatic precursor cells into endocrine cells, to the detriment of the exocrine tissue when ectopically expressed in the pancreatic bud under control of the PDX1 promoter (Apelqvist et al., 1999; Schwitzgebel et al., 2000). These results are consistent with the pro-endocrine role of the NGN3 gene (Figure 1) and are compatible with the pro-neural function of Atonal in Drosophila. 


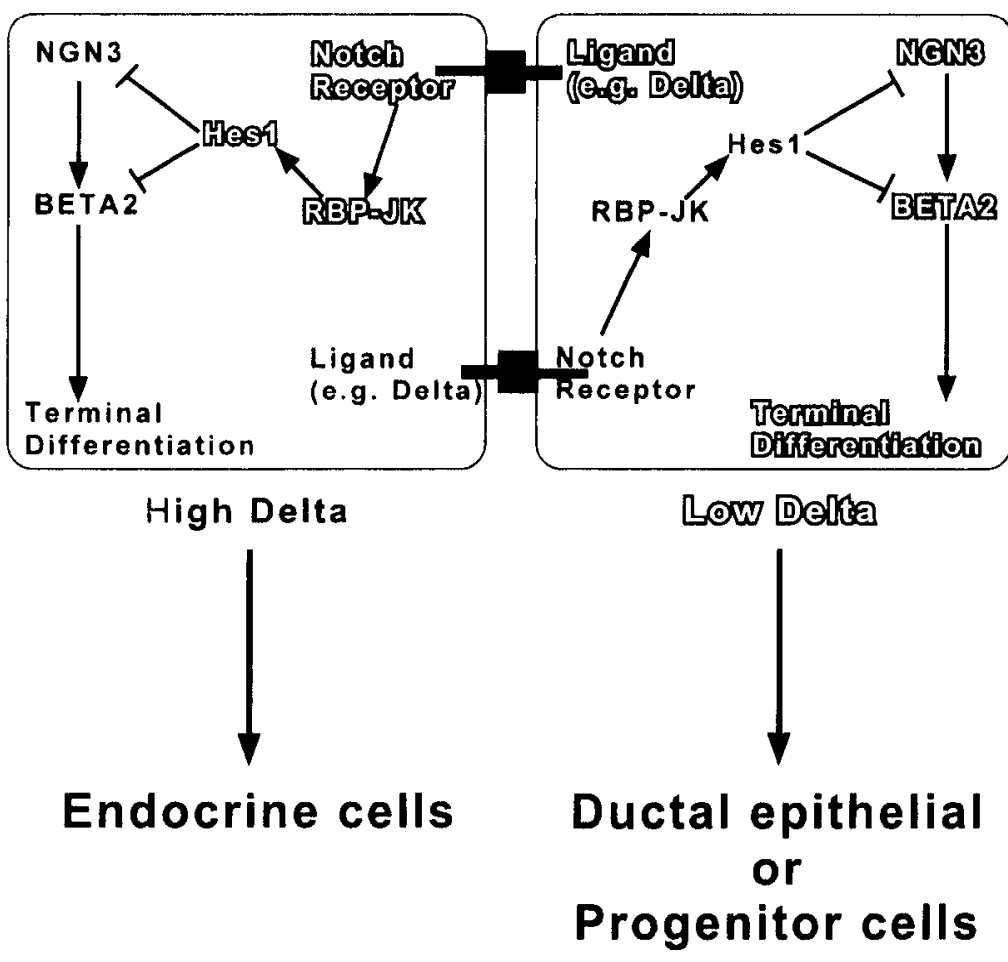

FIG. 1. Lateral inhibition. In lateral inhibition, signaling requires direct cell-cell contact. Notch signaling is pivotal for lateral inhibition to operate. In a homogenous cell population, there may be small variation in the concentration of ligands and receptors among cells. The initial changes are amplified, so that the cell adopting one fate (e.g., endocrine) expresses high levels of ligand and low levels of receptor, while the neighboring cell that takes another fate (e.g., ductal epithelial or progenitor) expresses low levels of ligand and high levels of receptor.

\section{HESI}

Hairy, otherwise known as enhancer of split-1 (HES1), is a bHLH protein that encodes a transcriptional repressor, which generally antagonizes positive bHLH proteins such as Mash1 (Guillemot, 1999). HES1 is important for normal embryonic development. Deletion of the HES1 locus in mice results in embryonic death (Guillemot, 1999; Ishibashi et al., 1995). Many defects are observed and include a small pancreas and the lack of brain, eyes, and thymus. The neuronal phenotype was attributed to a depletion of precursor cells due to premature differentiation. The proneural gene Mash 1 was upregulated in neuronal precursor cells, which suggests a potential role of HESl in the control of positive bHLHs such as Mash1. In analogy to the phenotype observed in the brain, the develop- 
ment of the pancreas is pcrturbed and premature differentiation of endocrine precursor cells also is observed (Jensen et al., 2000b). HES1 is expressed in PDX1-expressing ductal epithelial cells at e12.5 and also in the adjacent mesenchymal cells (Jensen et al., 2000b). In HES1 $1^{-/}$embryos, the expression of NGN3 and BETA2 is upregulated in endoderm-derived tissues such as the stomach and intestine. Similar studies were not performed with pancreatic tissues due to the severe disruption of normal pancreatic structure in HES1\% embryos. Nevertheless, the HES1 pancreatic phenotype is likely to involve the NGN3-BETA2 pathway (Figure 1), as will be discussed.

HES- 1 is an important mediator of the Notch signaling pathway, which plays a pivotal role in lateral inhibition, a mechanism mediating asymmetric signaling between adjacent cells (Jarriault et al., 1995,1998). The lateral inhibition model supposes, in the simplest example, a group of equivalent cells expressing both the receptor Notch and the ligand Delta. Notch-Delta interaction leads to Notch activation by its proteolytic cleavage and release of the Notch intracellular domain, which associates with the DNA binding protein RBP-JK, and the transcription of genes such as HES1. HES1 then represses downstream target genes such as the neurogenins as well as the Notch ligand Delta. Consequently, due to normal variation in expression among cells, a cell that produces more ligand will cause its neighbors to produce less. This enables the cell to increase its ligand production even further because it receives a weakened inhibitory signal from its neighbors. The effect of this feedback loop is to drive neighboring cells into distinct cell fates; any initial difference between them is intensified and maintained.

In the pancreas, most epithelial cells express both Notch1 and Notch2, while Notch 3 is detected in endothelial cells (Apelqvist et al., 1999; Jensen et al., 2000b; Lammert et al., 2000). The ligand Delta-like I is detected in scattered ductal epithelial cells, which suggests a potential role for it as a determinant of cell fate (Lammert et al, 2000). Other experimental models have implicated the lateral inhibition mechanism in the pancreas (Figure 1). Indeed, knockout of important players of the Notch signaling pathway - such as the Delta-like gene I, the intracellular mediator RBP-JK, and the repressor HES1 - will lead to an accelerated differentiation of pancreatic endocrine cells and the subsequent depletion of the pancreatic precursor pool (Apelqvist et al., 1999; Jensen et al., 2000b). In addition, expression of a dominant-negative form of Notch3 in ductal epithelial cells showed a similar premature differentiation phenotype (Apelqvist et al., 1999). This phenotype is associated with an increase of the expression of positive regulators of endocrine cell lineage such as NGN3 and BETA2, suggesting that the overexpression of NGN3 and BETA2 should result in a similar phenotype. Indeed, overexpression of the activators NGN3 and BETA2 in ductal epithelial cells induces premature endocrine differentiation by depleting the initial pool of endocrine cell precursors (Apelqvist et al., 1999; Schwitzgebel et al., 2000). 
Taken together, these results indicate an important role for the Notch signaling pathway in pancreatic development.

\section{Molecular Biology of BETA2}

\section{A. CLONING OF BETA2}

Most of the transcription factors discovered to play a key role during pancreatic development have been initially characterized as factors involved in the regulation of pancreatic hormone gene expression. This reverse genetic approach has been used successfully as a paradigm to clone transcription factors acting in the pancreatic differentiation process (e.g., BETA2) by our group, after many years of intense classical molecular biology research. The insulin promoter RIPE3 DNA element was identified by us and others as an important DNA element for insulin gene transcription (Crowe et al., 1988; Crowe and Tsai, 1989; Hwung et al., 1990; Robinson et al., 1994; Shieh and Tsai, 1991). The RIPE3 element consists of two cooperative subelements, RIPE3a and RIPE3b. Further examination of the RIPE3a sequence revealed a close resemblance to the consensus DNA element E-box, a known target of the bHLH family of transcription factors. In addition, RIPE3a binding activity is detected by gel-shift assays in insulin-expressing but not in nonexpressing cell lines (e.g., the hamster insulinoma cell line HIT vs. the kidney-derived cell line BHK) (Shieh and Tsai, 1991). Thus, the factor that binds to the RIPE3a element appears to be present only in insulin-producing lines. Taken together, these observations indicated that a tissue-restricted transcription factor of the bHLH family was a likely candidate for the RIPE3a binding activity.

The bHLH family of transcription factors is a very-well-conserved protein family with members present from yeast to higher eukaryotes. Most bHLH proteins bind to the consensus sequence CANNTG (Blackwell et al., 1993; Blackwell and Weintraub, 1990; Murre et al., 1989a). Three-dimensional studies of bHLH proteins have revealed the presence of two conserved helices separated by a loop or spacer region (Ellenberger et al., 1994; Ferre-D'Amare et al., 1993,1994; Ma et al., 1994). The basic region of bHLH factors is located N-terminal of the first helix and is required to interact with the DNA (Murre et al., 1989b; Voronova and Baltimore, 1990). The dimerization interface involves both helices 1 and 2 and is stabilized by van der Waals interactions and hydrogen bonds.

The bHLH family of transcription factors can be divided into three classes. The first, class A bHLHs, generally are characterized by an ubiquitous tissue distribution and an ability to bind DNA as homodimers or heterodimers with class $B$ members. The class B factors are tissue specific and bind DNA as a heterodimer with a class $A$ member. The class $C$ proteins are characterized by a leucine zipper motif located C-terminal to the bHLH. The latter class seems to dimerize via the 
leucine zipper and thus does not interact with either class A or B. The tissue-restricted class B bHLHs are believed to play important functions as developmental regulators. Hence, they are involved in the morphogenesis of various organs, ranging from muscle formation, with the $\mathrm{MyoD}$ family, to neurogenesis, with the Atonal and Achaete-Scute family.

Taking advantage of the requirement of class B or tissue-specific bHLH factors to dimerize with class A members, a modified yeast two-hybrid system was used to clone the RIPE3a binding activity (Naya et al., 1995). The ubiquitous bHLH E47 without the activation domain was chosen as the bait, since it was previously shown to be present in the RIPE3a complex. The library was derived from the insulinoma HIT cell line, where the RIPE3a activity can be detected. A clone was selected and named BETA2. The same protein was cloned as an interacting partner of the ubiquitous Drosophila bHLH Daughterless using a very similar yeast-based assay (Lee et al., 1995). This clone was termed NeuroD for its ability to induce neuronal differentiation when ectopically expressed in ectodermal cells. For the purpose of simplicity, the BETA2 nomenclature is used here, since this review is focused on the pancreas.

\section{B. BETA2}

As deduced from the cloned cDNA sequence, the hamster BETA2 encodes a $42 \mathrm{kD}$-protein of 355 amino acids with a bHLH domain located between residues 100 to 155 . In addition to its restricted expression pattern (see below), the presence of a characteristic tyrosine in the second helix indicates that it belongs to the class B subfamily. Sequence alignment of BETA2 proteins from different species reveals an overall strong homology between mouse, rat, human, chicken, frog, and zebrafish protein sequences (Figure 2). BETA2 is highly homologous to NeuroD-related factor (NDRF2), Nex1/Math2, and NGN1/NDF3 (Figure 3). Interestingly, these all have a very-well-conserved loop sequence (CYSKTQ) that could potentially define them as a subgroup (Figure 3 ).

Like most class B bHLHs, BETA2 has no DNA binding capacity by itself but is strongly stimulated by heterodimerization with class A bHLH proteins (e.g., E47). In the insulin promoter, the RIPE3 mini-enhancer is composed of binding sites for multiple proteins, including BETA2 and PDX1. Synergistic activation of this mini-enhancer is observed between BETA2 and PDX1. The molecular mechanism for this effect recently was reported and involved the direct interaction of BETA2 and PDX1 via the bHLH domain of BETA2 and the homeodomain of PDX1 (Glick et al., 2000; Ohneda et al., 2000). In addition, PDX1 is able to interact with other bHLH proteins, including the ubiquitous E47 (Ohneda et al., 2000; Poulin et al., 2000). This interaction does not result in cooperative DNA binding between bHLH proteins and PDX1 but rather in a clustering of multiple 
transcriptional activators, which enhance transcription by intcracting with coactivators and the basal transcriptional machinery.

Indeed, BETA2's transactivation potential is stimulated by the presence of the coactivator p300 or CREB binding protein (CBP) (Mutoh et al., 1998; Qiu et al., 1998; Sharma et al., 1999). Two activation domains (AD1 and AD2) were identified C-terminal of the bHLH domain (Figure 4). The AD1 located within amino acids 189 to 299 is more active than the AD2 (aa 300 to 355). The two ADs do not appear to synergize with each other; rather, they seem to act independently. The activity of both ADs correlates with their ability to interact with the coactivator $\mathrm{p} 300$. The bHLH domain of BETA2 also was reported to interact with $\mathrm{p} 300$, while no other region of BETA2 - including the C-terminal region — was able to interact with $\mathrm{p} 300$ in this study (Mutoh et al., 1998). It is interesting to point out that the myogenic bHLH MyoD is able to interact with p300 via its bHLH domain and its N-terminal activation domain (Puri et al., 1997; Sartorelli et al., 1999). Thus, it is possible that, depending on the system or context used, different interaction domains of BETA2 are important. Additionally, the transcriptional ability of BETA2 may be enhanced by its heterodimerization with a partner able to interact with the p300/CBP family of coactivators. Finally, most of the activation studies have been performed using transient transfection assays and artificially high amounts of bHLH or Gal4 fusion proteins and may not entirely recapitulate the in vivo environment. This is reflected by some lack of consistency in the reported data between various groups and further analysis is required to clarify the discrepancies.

\section{Physiological Functions of BETA2}

\section{A. BETA2 AND PANCREAS}

BETA2 is expressed in both the developing and adult pancreas. In mature islet, BETA2 is expressed in all endocrine cells, making the name BETA2 somewhat misleading. The exact function of BETA2 in the mature islet is unknown. BETA2 is involved in regulation of insulin and glucagon genes. However, this function does not appear to be a major one, since mutant mice lacking the BETA2 gene still are able to express insulin and glucagon in the pancreas (see below). In $\delta$ and PP cells, the role of BETA2 remains unknown. During embryonic development, the expression pattern of BETA2 is best visualized by X-gal staining as an assay for the enzymatic activity of $\beta$-galactosidase resulting from the replacement of the coding region of the murine BETA2 gene by the bacterial LacZ gene. When such an approach is used, the activity of the endogenous BETA2 gene can be monitored in heterozygous mice. To the best of our knowledge, the staining pattern detected by X-gal appears to reflect the endogenous BETA2 expression 
$\mathrm{MmBETA} 2$ $\mathrm{R} \cap \mathrm{BETA} 2$ C gBETA 2 H S B E T A 2 G g B T A 2 XIBEA 2 D $\triangle B E T A 2$ consensus

MmBETA 2 R n B T T 2 C gBETA 2 H $\triangle$ BETA 2 G gBET 2 $\mathrm{X} 1 \mathrm{BETA} 2$ D $~ B E T A 2$ consensus

M B B TA 2 R I B T A 2 C gBETA 2

HSBETA 2 G gBETA 2 X 1 B E T A 2

D $\triangle$ BETA 2

consensus

$\mathrm{MmBETA} 2$ RnB T A 2 C gBETA 2 H $\triangle B E T A 2$ G g B T T 2 $X 1$ BETA2 D $\triangle B E T A 2$ consensu
MTKSYSESGLMGEP QPQGPPSWTDECLSSQDEEHEADKK-GDELEAMN- - A EDSLK - - N MTKSYSESGLMGEP OPOGP P SWTDECLSSQDEEHEADKK-EDELEAMN- - A E EDS LR - - N MTKSYSESGLUGEPQPQGP SWTDECLSSQDEDHEADKK-EDELEAMN- - A E EDSLR- - N MTKSYSES CLMGBPQPQ GP PSWTDECZSSQDEEHEADKK-EDDLEAMN- - A EDSLR- -N MTKSYSESGPAGEP QAQAP PGWA A GCLSP PADGPEADKK-EEDLEALHGEAEEDALR- - N MTKSYGENGLILAETP-GCRGWVDECLSSQDEN-DLEKK-EGELMKED- - DEDSLNHHW

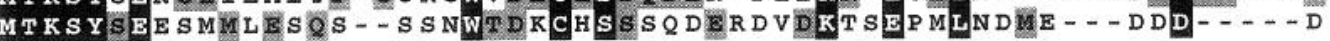

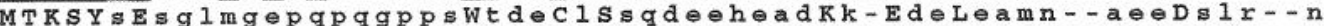

Basic

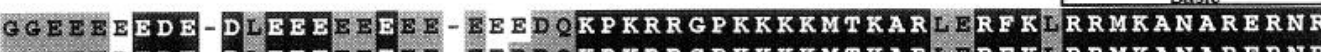
GGEEEDEDE-DLEEEEEEIE-EEDD OKPKRRGPKKKKMTKARLERFKLRRMKANARERNR C.CDEEDEDE-DLEEEDEEE E-B-DDQKPKRRGPKKKKMTKARLERFKLRRMKANARERNR GGEB BDEDE-DLEEEEEEE E- EDDDQKPKRRGPKKKKMTKARLERFKLRRMKANARERNR GEERDEEDELDEEEEEEEE E-EDDEQKPKRRGPKKKKMTKARLERFKLRRMKANARERNR GEENEEEDEGDEEEEDDEDDDEDDDQKPKRRGPKKKKMTKARVERFKVRRMKANARERNR A GLNRLEDE-DDEEEEEEEE-DGDDTKPKRRGPKKKKMTKARMQRFKMRRMKANARERNR

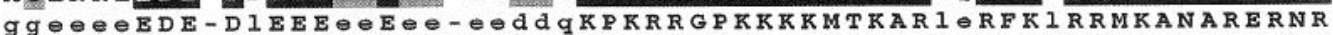
g gee e E DE-DIEEEe E Loop Helix

MHGLNAALDNLRKVVPCYSKTQKLSKIETLRLAKNYIWALSEILRSGKSPDLVSFVOTLC IH GLNAALDNLRKVVPCYSKTQKLSKIETLRLAKNYIVALSEILRSGKSPDLVSFVQTLC MH GLNAALDNLRKVVPCYSKTQKLSKIETLRLAKNYIWALSEILRSGKSPDLVSFVQTLC MH GLNAALDNLRKVVPCY STQRLSKIETLRLAKNYIWALSEILRSGKSPDLVSFVQTLC MH GLNAALDNLRKVVPCYSKTQKLSKIETLRLAKNYIWALSEILRSGKSPDLVSFVQTLC MH GLNDALDSLRKVVPCY K TQKLSKIETLRLAKNYIWALSEILRSGKSPDLVSFVQTLC MH GLNDALESLRKVVPCYSKTQKLSKIETLRLAKNY IWALSEILRSGKSPDLMSFVQALC MHGLN A L A LRKVVPCYSKTQKLSKIETLRLAKNYIWALSEILRSGKSPDLVSFVQTLC

KGLSQPTTNLVAGCLQLNP RTFLPEQNP DNP PHLPTASA SFPVH P SYQSPGLPSP PYGT $K G L S Q P T T N L V A G C L Q L N P R T F L P E Q N P D M P P H L P T A S A S F P V H P Y S Y Q S P G L P S P P Y G T$ KGLSQPTTNLVAGCLQLNPRTFLPEQNPDMP PHLP TASASFP VHPYSYQSPGLPSPPYGT $K G L S Q P T T N L V A G C L Q L N P R T F L P E Q N Q D M P P H L P T A S A S F P V H P Y S Y Q S P G L P S P P Y G T$ KGLSQPTTNLVAGCLQLNPRTFLPEQSADAARHLPPAGAPFAPPPFPYASPGLPSPPYGT KGLSQPTTNLVAGCLQLNPRTFLPEQSQDIQSHMQTASSSFELQGYPYQSPGLPSPPYGT KGLSOPTTNLVAGCLQLNPRTFLPEQSQEMP PHMQTASASESALPY SYQTPGLPSPPYGT KGLSQPTTNLVAGCLQLNPRTFLPEQn-dmpPHIPtAsasFPVhPYsYq 


\section{MmBETA 2 \\ R I B T A 2 \\ C g B ETA 2 \\ H $\triangle$ BETA 2 \\ G g B E T 2 \\ X1BETA 2 \\ D $\triangle B E T A 2$ \\ consensus}

M B B T A 2

R B BETA 2

C gBETA 2

H $\$$ B ETA 2

G $\mathrm{BETA} 2$

X 1 BETA 2

D $\triangle B E T A 2$

consensus

$\begin{array}{lll}\text { MMBETA2 } & 353 & \text { AIFHD- } \\ \text { RABETA2 } & 353 & \text { AIFHD- } \\ \text { CgBETA2 } & 351 & \text { AIFHD- } \\ \text { HSBETA2 } & 352 & \text { AIFHD- } \\ \text { GgBET2 } & 353 & \text { AIFHE- } \\ \text { XIBETA2 } & 348 & \text { AIFHD- } \\ \text { DIBETA2 } & 345 & \text { AIFHDS } \\ \text { COngensus } & 361 & \text { AIFHd - }\end{array}$
361 AIFHd -
234 MDSSHVFH VKPP PHAYSAALEPF FESP - ITDCTSPSFDGPLSP PLSTNGNFSFKHEPSAE 234 MDSSHVFHVKPP PHAYSAALEPFFBSP-LTDCPSPBFDGPLSPPLSTNGNFSFKHEPSTE 233 MDSSHVFQVKPE PHAYSATLEP F FE S - LTDCTSPSF DGPLSP PLSINGNFSFKHEPSAR 234 MDSSHVFHVKPP PHAYSAALEPFFESP-LTDCTSPSFDGPLSP L STNGNFSFKHEPSAE 237 MDSSHLFHLKPP-HAYGAALEPFFEGG-LPEGAGPAFDGPLSPPLSTYGNFSFRHEPAAD 235 MDSSHVFH VKP--HSYGA ALEPFFDSSTVTECTSPSFDGPLSP L SVNGNFTFKHEHS-E 229 MDSSHIFH VKP-- HAYGSALEPFFDTT-LTDCTSPSFDGPLSPPLSVNGNFSFKHEPSSE 241 MDSSHVFhvKPPPHaYsa a LPFFesp-1tdctsPsFDGPLSPPLSinGNFsFREP

293 FEKNYAFTHHYPAAIZA QPQSHGSIFSSGAA A PREIPIDNIMSFDSHSHHERVMSAQLN 293 FEKNYAFTMHYPAATLAQPQSHCSIFSSGAAAPRCEIPIDNIMSFDSHSH ERVMSAQLN 292 FEKNYAFTMHYPAATLA CPQSHCSIFS-CATAPRCEIPIDNIMSFDSHSHHERVMSAQLN 293 FEKNYAFTMHYPAATLACAQSHCSIFS- -TAAPRCEIPIDNIMSFDSHSH ERVMSAQLN 295 FDNSYAFTMHYPAG PLPA PAHAAVF S--GA AARCBLPADGLAPYEGHPH ERVLSAQLS 292 Y DKNYTFTMHYPAA I IS- - QGHGPLFS- - TGGPRCEIPIDTIMSYDGHSH ERVMSAQLN 286 FEKNYAFTMHYQAAGLAGAQGHAASLY - A G TQRCDIPMENIMSYDGHSHHERVMNAQLN 301 feknYaFTMHYpAatlag-qsHgsifs-gaapRCeipidnimsfds

FIG 2. Alignment of BETA2 protein sequences from various species. The bHLH domain is drawn on top of the sequence. The consensus line indicates sequence identity (upper case) and similarity (lower case). Mm, Mus musculus; $\mathrm{Rn}$, Rattus norvegicus; $\mathrm{Cg}$, Cricetinae gen. sp.; $\mathrm{Hs}$, Homo sapiens; $\mathrm{Gg}$, Gallus gallus; $\mathrm{Xl}$, Xenopus laevis; Dr, Danio rerio. Identical and conserved amino acids are boxed in black and light gray, respectively. 
B E T A 2

C gBETA 2

ND 2 / NDRF / NDF 2

MATH $2 / \mathrm{N} \in \mathrm{X} 1$

NGN 1 / NDF 3

MATH 3 / NeuroM

$N G N 3$

NGN 2 / MATH 4

DMATONAL

MATH 1

Beta 3

MIST 1

TWIST/DERMIS 1

PTF1/P48

MASH 1

My०D 1

E 2 A

HES 1

consensus

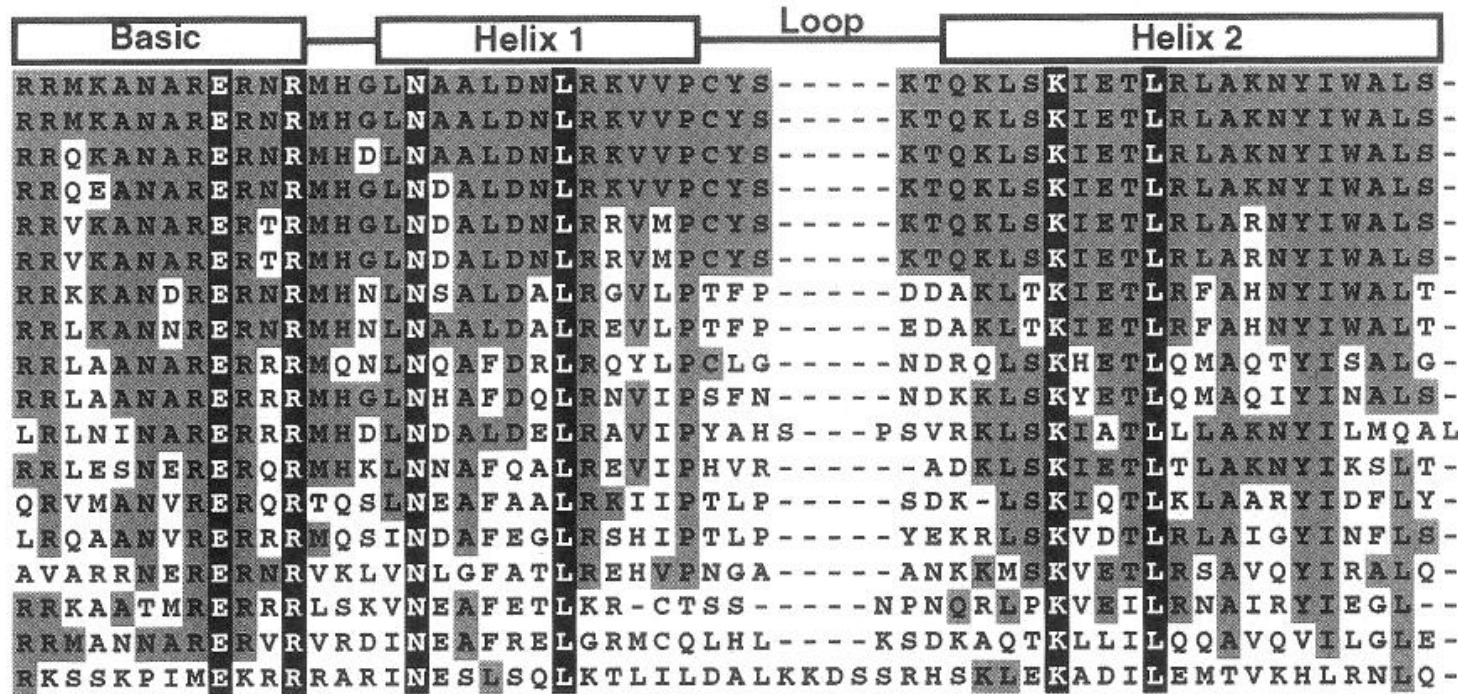

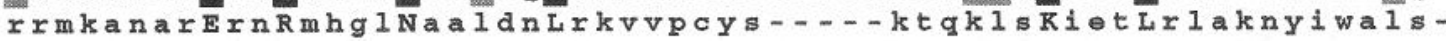

FIG. 3. Sequence comparison of the mouse BETA2 with other bHLH proteins. Unless stated otherwise, only murine sequence were used. The loop sequence of BETA2, ND2/NDRF/NDF2, MATH2/Nex1, and NGN1/NDRF3 is very well conserved, which suggests that they could form a subfamily of bHLH. Identical amino acids to mouse BETA2 are shown in gray, whereas fully conserved amino acids and conserved amino acids are boxed in light gray and black, respectively. Cg, Cricetinae gen. sp.; Dm, Drosophila melanogaster. 


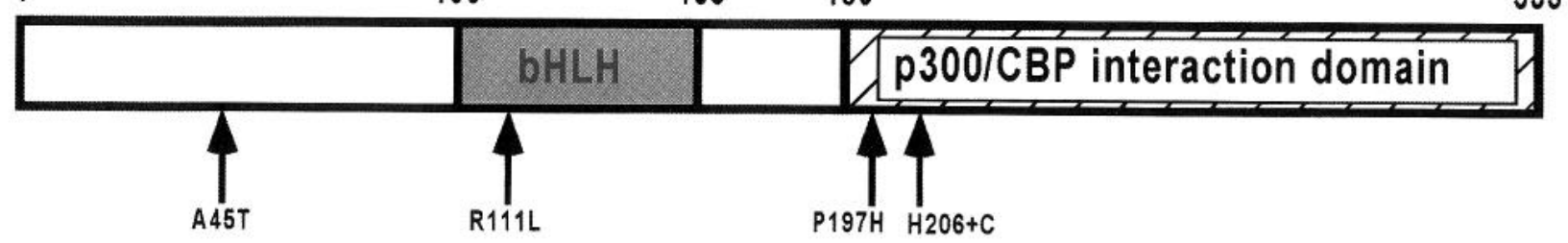

$\begin{array}{rr}189 & 299\end{array}$

$300 \quad$ AD 2

355

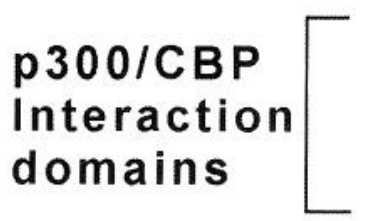

$$
100
$$

\section{5}

155 299 domain

FIG. 4. The BETA2 protein. Schematic representation of the functional domains of hamster BETA2. The bHLH is located between aa 100 and 155 . The p300/CBP-interacting domain is located C-terminal of the bHLH between aa 189 and 355. Two activation domains were identified in the C-terminal from aa 189 to 299 and 300 to 355 . Three p300/CBP interaction domains were characterized. bHLH itself is able to interact with p300; aa 155 to 299 , which overlaps with AD1; and aa 300 to 355 , which corresponds to AD2. Also shown are the four DNA polymorphisms identified and indicated by the arrows and their mutation. 
pattern. The BETA2 gene is expressed very early during pancreas ontogeny. It is detected at $\mathrm{e} 9.5$ in the pancreatic primordia in glucagon-expressing cells, suggesting that BETA2 is expressed in early precursor cells. At e12.5, BETA2 is found in both dorsal and ventral pancreatic buds. By e 14.5, BETA2 is expressed in ductal epithelial cells and in the small population of glucagon-producing cells that are budding from the duct. At e17.5, BETA2 mainly is expressed in small clusters of endocrine cells and rarely in ductal epithelial cells. Such expression pattern suggests a role for BETA2 in the terminal differentiation of endocrine cells, consistent with its proposed role in the terminal differentiation of neurons (see below).

\section{B. BETA2 KNOCKOUT MODEL}

In order to study the role of BETA2 in the pancreas, a mouse knockout model was generated (Naya et al., 1997). Homozygous BETA2 ${ }^{-/}$newborns appear to be malnourished and dehydrated and died within 5 days of birth. Mutant pups are hyperglycemic, when compared to age-matched wild-type or heterozygote littermates; mutants show ketonuria, suggesting severe diabetes. Close examination of homozygous BETA2 null mice indicates a striking reduction in the number of islet cells, especially $\beta$ cells ( 25 percent of the wild type). In addition, analysis of newborn pancreata of mutant mice reveals a drastic reduction of endocrine $\alpha$ cells as well as a failure of residual endocrine cells to aggregatc into mature islets (Naya et al., 1997), suggesting that BETA2 is essential for the morphogenesis of pancreatic islets.

Further examination of embryos indicates that the defect in BETA2 $2^{-/-}$animals occurs after e14.5. Prior to e14.5, the number of BETA2-expressing cells in the developing pancreas is similar between heterozygote and BETA2 ${ }^{-/-}$animals. E14.5 is an important threshold in pancreas development in mice. It marks the beginning of the second wave of endocrine cell differentiation, which is believed to generate the majority of endocrine cells. Thus, in the absence of BETA2, endocrine precursor cells do not massively differentiate and instead undergo programmed cell death. The pancreata of e17.5 null mutants have a profound decrease in endocrine cell numbers and fail to form islets. It is also important to point out that insulin is still expressed in the BETA2 $2^{-/}$pancreas, suggesting the lack of requirement of BETA2 for insulin gene expression. Alternatively, other bHLH proteins, known or novel, could compensate for the lack of BETA2 and play a role in transcription of the insulin gene. Furthermore, transcription factors such as PDXI (which is unaffected by the absence of BETA2) could be sufficient to regulate the insulin gene.

In the transgenic model of overexpression of BETA2 in the pancreas, a moderate increase in endocrine cells is observed in the developing pancreas (Schwitzgebel et al., 2000). This suggests a role of BETA2 in endocrine cell differentiation. A more-severe phenotype is observed when NGN3 is overex- 
pressed in the pancreas (Apelquist et al., 1999; Schwitzgebel et al., 2000). In this case, one observes a hypoplastic pancreas likely due to premature differentiation of endocrine precursor cells. On one hand, the more-severe phenotype of NGN3 overexpression, compared to BETA2 overexpression, suggests that NGN3 is an upstream regulator of BETA2. On the other hand, it indicates that NGN3 is likely to regulate genes other than BETA2. Overall, these results demonstrate that a tight regulation of bHLH expression is required for normal pancreas development.

\section{BETA2 AND DIABETES}

Several transcription factors important for the development and functioning of $\beta$ cells have been identified as the factors responsible for maturity-onset diabetes of the young (MODY). These include the HNF1 $\alpha,-1 \beta$, and $-4 \alpha$ for MODY 1, 3, and 5, respectively, and the homeobox protein PDX1 for MODY4 (Horikawa et al., 1997; Stoffers et al., 1997; Yamagata et al., 1996a,1996b). The BETA2 gene is located on chromosome $2 \mathrm{q} 32$ in humans, a region to which the insulin-dependent diabetes 7 (IDDM7) locus has been assigned (Owerbach et al., 1997). Different groups have identified five DNA polymorphisms at this locus (Table I). The most-common polymorphism is a mutation that results in an alanine-to-threonine conversion at position 45 (A45T) and is linked to type I diabetes in Japanese and Danish populations (Hansen et al., 2000; Iwata et al., 1999) but is not in American and French populations (Dupont et al., 1999; Malecki et al., 1999; Owerbach et al., 1997). Interestingly, two others mutations - arginine 111 to leucine (R111L) in the DNA binding domain and an insertion of a cytosine residue in a poly $\mathrm{C}$ tract at codon 206 (histidine), resulting in a frame shift premature termination at amino acid $242(\mathrm{H} 206+\mathrm{C})$ - have been linked to type 2 diabetes in American population in the heterozygous state (Malecki et al., 1999). The R111L mutation has no DNA binding activity, whereas the $\mathrm{H} 206+\mathrm{C}$ mutation retains its DNA binding ability but is unable to interact with the coactivator $\mathrm{p} 300$. The clinical profile indicates that the $\mathrm{H} 206+\mathrm{C}$ mutation is more severe than the R111L mutation in the heterozygous state. This suggests that the $\mathrm{H} 206+\mathrm{C}$ could be acting in a dominant-negative fashion by interacting with class A bHLH on DNA but is unable to activate transcription of target genes. In contrast, the R111L mutant is unable to interact with DNA but could heterodimerize with class A partner(s) or squelch important co-regulators of the remaining functional BETA2 protein, thus partially affecting the function of the wild-type BETA2.

\section{INTESTINE}

BETA2 also is expressed in all enteroendocrine cells of the diffuse neuroendocrine system of the gut (Rindi et al., 1999). However, in the absence of BETA2, only secretin- and CCK-expressing enteroendocrine cells are absent (Mutoh et al., 
TABLE I

DNA Polymorphisms Identified in the Human BETA2 Coding Region

\begin{tabular}{llll} 
& Polymorphism & Type I & Type II \\
\hline Owerbach et al., 1997 & A45T & No linkage & ND \\
Furuta et al., 1998 & A45T & ND & No linkage \\
Dupont et al., 1999 & A45T & No linkage & No linkage \\
Malecki et al., 1999 & A45T & ND & No linkage \\
& R111L & ND & Yes \\
P197H & ND & No linkage \\
Iwata et al., 1999 & H206+C* & ND & Yes \\
Hensen et al., 1999 & A45T & Yes & No linkage \\
& A45T & Yes & No linkage \\
& P197H & No linkage & No linkage \\
& S259S & No linkage & No linkage
\end{tabular}

The most-common one is an alanine to threonine at codon 45 (A45T), which is present in all the ethnic populations studied. This A45T mutation is linked to type I diabetes in Japanese and Danish populations but not in French or American populations. Two mutations in the BETA2 protein are linked to type II diabetes (R11L and $\mathrm{H} 206+\mathrm{C}$ ) in the heterozygous state (see text for more details). *This mutation results in an insertion of $\mathrm{a} C$ in a $\mathrm{C}$-stretch, which leads to a truncated protein. [See Figure 4 for more details.] ND, not determined.

1998; Naya et al., 1997). All other enteroendocrine hormones (i.e., gastrin inhibitory peptide, substance P, glucagon-like peptides, peptide YY, serotonin, somatostatin) are present. This suggests that BETA2 is a marker for enteroendocrine cells but is only required for the terminal differentiation of secretin- and CCKproducing cells and/or the expression of these genes in enteroendocrine cells. These results concur with the observation that secretin and CCK cells might arise from a common progenitor (Rindi et al., 1999). In addition, the absence of these two pancreatic secretagogues may explain the inability of pancreatic acinar exocrine cells to secrete zymogen granules in BETA2 mutant animals (Naya et al., 1997). Additional transcription factors likely are required for the differentiation of the enteroendocrine cell types observed in the gut epithelium. 


\section{E. BRAIN}

Although this review is not focused on the brain, understanding BETA2 function requires the discussion of its role in neuronal physiology. In general, BETA2 is expressed throughout neuronal development, mostly in terminally differentiating neurons (Lee et al., 1995,2000; Liu et al., 2000b; Naya et al., 1997).

During development, BETA2 is first detected around e8 in neuronal epithelium. At $\mathrm{e} 9.5$, high expression is observed in the developing trigeminal ganglia. At e1 1.5, strong expression is observed in the cranial ganglia ( $\mathrm{V}$ to $\mathrm{XI}$ ), the dorsal root ganglia, and spinal cord. BETA2 is not detected in the mitotically active ventricular zone in the cerebral cortex but rather adjacent to it. BETA2 is also detected in differentiating neurons of the nasal epithelium and the retina. At e14.5, the expression of BETA2 is turned off in cranial ganglia- and dorsal root gangliadifferentiated neurons. This suggests that BETA2 plays a role in the initiation of terminal differentiation of certain neurons. By e18.5, there is a general decrease of BETA2 expression throughout the brain, with the exception of the granule cells in the cerebellum and dentate gyrus, where an increase in expression is observed due to the differentiation of precursor cells into granule cells. In adult mice, BETA2 is expressed in the olfactory bulb, hippocampus, cerebellum, and pituitary gland. This expression is indicative of another function of BETA2 in fully differentiated neurons.

As mentioned previously, BETA2 null mutants are not viable in a mixed C57B6/129SvEv background but when they are crossed into a pure $129 \mathrm{SvJ}$ background, approximately 40-65 percent of the mutant animals survive (Liu et al., 2000a). This possibly is due to the presence of a modifier gene related to BETA2. The rescue of the BETA2 null mutant allowed our group to study the role of BETA2 in postnatal neuronal development, especially granule cells of the dentate gyrus and cerebellum (Liu et al., 2000a).

\section{F. DENTATE GYRUS}

In the hippocampus, BETA2 is expressed in the Ammon's horn and granule cells of the dentate gyrus in adult mice. Granule cells are generated in two phases during dentate gyrus development. The first phase occurs during embryogenesis, when granule cells appear at the ventricular surface of the neuroepithelium and migrate in conjunction with the precursor cells to the dentate anlage. The second wave of granule cells that will account for the majority of the hippocampus granule cells is generated postnatally from precursor cells that migrate from the ventricular zone to the dentate anlage. Interestingly, in addition to differentiated granule cells, BETA2 expression was observed in a few mitotic cells of the dentate gyrus as revealed by BrdU labeling (Lee et al., 2000; Liu et al., 2000a; Miyata et al., 1999). The presence of BETA2 in BrdU-positive cells could be explained by 
their recent exit from the cell cycle and an early onset of BETA2 expression. In the absence of BETA2, granule and progenitor cells are decreased such that the later events in development of the dentate gyrus is greatly affected. BETA2 may be required for the specification of an early subpopulation of granule cells that then organizes the dentate granule cell layer and regulates birth of additional granule cells, independent of continued BETA2 function. Thus, the role of BETA2 in the dentate gyrus appears complex, involving the maintenance or survival of precursor cells and the terminal differentiation of granule cells.

\section{G. CEREBELLUM}

As in the dentate gyrus, there is a defect in granule cells in the cerebellum. This is not surprising, since the granule cells of the cerebellum share similar developmental characteristics with those of the dentate gyrus (Altman, 1967). During development, BETA2 is expressed in the inner layer of the external granular layer (EGL), where postmitotic cells are located. Beginning at birth, granule cell precursors proliferate and differentiate into granule cells. As in the hippocampus, BETA2 is detected in both mitotic and postmitotic cells of the EGL in the postnatal cerebellum. Additionally, BETA2 is required for terminal differentiation of granule cells, since its absence leads to a decrease in granule cell number (M. Liu and M.J. Tsai, personal observations; Miyata et al., 1999). The effect is more severe in the posterior lobules ( $\mathrm{V}$ to XI) than in the anterior lobules of the cerebellum. The presence of surviving granule cells suggests a possible compensatory mechanism by other bHLHs, such as NeuroD2 or Nex 1/Math2, that also are expressed in the cerebellum. We are currently studying the mechanism of the differential depletion of granule cells that occurs between the anterior and posterior cerebellum.

\section{H. RETINA}

In adult mice, BETA2 expression is detected in the outer nuclear layer of the central retina and in all layers (i.e., outer and inner) of the more-peripheral retina (M. Liu and M.J. Tsai, personal observations; Morrow et al., 1999). In the retina, BETA2 likely plays a role in glial vs. neuronal cell fate determination. In the absence of BETA2, one observes an increase in Müller glial cells (Morrow et al., 1999). In a gain-of-function experiment, overexpression of BETA2 in postnatal retina resulted in a complete blockade of Müller glial cell development. In addition, BETA2 plays a role in interneuron development, determining the ratio between amacrine and bipolar neurons (Morrow et al., 1999). Finally, BETA2 is implicated in the survival of a subset of rod photoreceptor cells (Morrow et al., 1999). These recent findings broaden our view of BETA2 function and suggest that it can have an active role in cell fate determination. 


\section{IN VITRO}

In vitro, BETA2 can induce terminal differentiation of cell lines (P19, PC12, and F11) when transfected (Cho et al., 2000; Farah et al., 2000; Noma et al., 1999). When a mutant of BETA2 lacking the C-terminal activation domain is introduced in F11 neuroblastoma cells, differentiation induced by cAMP is inhibited (Cho et al., 2000). This implies that this truncated molecule harbors a dominant-negative function. BETA2 also can induce transdifferentiation of retinal pigment epithelium into cells that resembles young photoreceptors (Yan and Wang, 2000a,2000b). Furthermore, using retina explant cultures derived from rat or mouse, it was shown that BETA2 plays multiple functions in cell fate determination and differentiation as well as survival of various retinal cell types (Morrow et al., 1999). Overall, the role of BETA2 in neuronal development appears complex and dependent on the system being studied. These results also implicate BETA2 in cell fate determination, differentiation, and neuronal survival.

\section{Regulation of BETA2}

BETA2 expression pattern and timing indicate that it plays important functions in differentiation. NGN1 expression spatially overlaps and temporally precedes that of BETA2 during neuronal development (Fode et al., 1998; Ma et al, 1998,1999; Sommer et al., 1996). In addition, ectopic overexpression of NGN1 induces BETA2 gene expression and neurogenesis in Xenopus ectoderm (Ma et al., 1996; Perron et al., 1999). In order to analyze further the regulation of BETA2 expression, we have cloned the murine BETA2 promoter and shown its regulation by bHLH proteins in transient transfection assays (Huang et al., 2000). NGN3 was shown to stimulate the activity of the BETA2 promoter in HIT, $\beta$-TC, and Hela cells. NGN3 and BETA2 expression patterns also partially overlap in the developing pancreas, suggesting that the regulation observed in vitro might exist in vivo (I luang et al., 2000). Indeed, injection of NGN3 mRNA into Xenopus embryo causes ectopic expression of BETA2 in the trunk and an abnormal expansion of its expression in the head region (Huang et al., 2000). The neurogenin family of transcription factors generally is regarded as playing roles in neuronal determination. The NGN1 and NGN2 genes are important in the determination of selected neurons; their absence has revealed a block at the earliest stages of neurogenesis in complementary sets of cranial sensory ganglia (Fode et al., 1998,2000; Ma et al., 1998,1999).

Alignment of the mouse and human BETA2 promoters reveals strong conservation of the two promoters over about two kilobases. The first $400 \mathrm{bp}$ of the promoters are particularly well conserved. Within this region, three E-boxes and two $\mathrm{Spl}$ sites were identified as putative DNA response elements (Huang $e t$ al., 2000; Miyachi et al., 1999; Yoon et al., 1998). Promoter deletion analysis has 
revealed the presence of a proximal DNA element required for basal activity in BETA2-expressing cells such as $\beta$-TC, HIT, and $\beta$-HC3 (Huang et al., 2000; Miyachi et al., 1999; Yoon et al., 1998). Deletion of the three proximal E-boxes greatly affects basal promoter activity, suggesting a potential role for a $\mathrm{bHLH}$ protein in the regulation of BETA2 (Huang et al., 2000). In transient transfection assays, NGN3 and BETA2 upregulate a BETA2 promoler-luciferase construct (Huang et al., 2000). While the regulation of BETA2 by NGN3 observed in transient transfection assays is likely to be of physiological importance, the autoregulation of the BETA2 gene and its importance for developing and adult islets is less clear. This awaits the generation of a tissue-specific knockout of the BETA2 locus in islet cells. Finally, a more-detailed analysis of the BETA2 promoter is required for a full understanding of the regulation of the tissue-specific expression of the BETA2 gene.

\section{Summary}

The development of the pancreas is a very complex process. The discovery of transcription factors is a first step toward a better understanding of the genetic program responsible for the activation or repression of the genes necessary for the developmental process. In turn, the identification of the genes targeted by these transcription factors (e.g., other transcription factors, growth factors and their receptors, extracellular matrix components, modifying proteins, signaling molecules) will broaden our views of pancreatic development and diabetes. In this regard, we believe that the BETA2 knockout mouse is an attractive and interesting model for the identification of important regulators of endocrine cell development.

From the analysis of the BETA2 knockout mouse, it was shown that BETA2 is needed for the proper formation of pancreatic islets. Its expression pattern and functional analysis assigns to BETA2 a key role in the terminal differentiation of pancreatic endocrine cells. In its absence, endocrine cells are decreased in number due to increase in programmed cell death and a failure of these cells to aggregate into islets.

BETA2 also is expressed in various regions of the central nervous system. There, BETA2 is similarly involved in terminal differentiation of granule cells of the dentate gyrus and cerebellum. In addition to its established role in differentiation, BETA2 appears to play a role in maintenance and survival of granule cell progenitors in these structures and in cell fate determination in the retina. The expression of BETA2 in adult neuronal and pancreatic endocrine tissues suggests a role different from the differentiation function played during development. Interestingly, BETA2 expression decreases with age in the hippocampus but not in the cerebellum in adult mice (Uittenbogaard and Chiaramello, 2000). The significance of this age-related downregulation is unknown but may contribute to 
neurodegenerative diseases in the elderly. Finally, we have recent evidence for an additional role for BETA2 in ear development (Liu et al., 2000b). The observed phenotype has been attributed to defects in the delamination of the cochlear-vestibular ganglion neurons and in the differentiation and patterning of the sensory cochlear epithelium. Finally, it would be interesting to evaluate whether the BETA2 mutations that have been linked to diabetes in some populations (Hansen et al., 2000; Iwata et al., 1999; Malecki et al., 1999) affect other aspects of BETA2 physiology, including the development and functions of the hippocampus, cerebellum, retina, and ear, which might be associated with behavioral or perceptional deficits (Liu et al., 2000a,2000b; Miyata et al., 1999).

\section{ACKNOWLEDGMENTS}

The authors gratefully acknowledge helpful discussions with Dr. Debra Bramblett during the preparation of the manuscript. M.-.J.I. is supported by a grant from the National Institutes of Health (DK55325). E. N-.G. is a recipient of a postdoctoral fellowship from the Juvenile Diabetes Foundation International.

\section{REFERENCES}

Ahlgren, U., Jonsson, J., and Edlund, H. (1996). Development 122, 1409-1416.

Ahlgren, U., Pfaff, S.L., Jessell, T.M., Edlund, T., and Edlund, H. (1997). Nature 385, 257-260.

Ahlgren, U., Jonsson, J., Jonsson, L., Simu, K., and Edlund, H. (1998). Genes Dev. 12, 1763-1768.

Altman, J. (1967). In "The Neurosciences" (G.C. Quarton, T. Melnechuk, and F.O. Schmitt, eds.), pp. 723-743. The Rockefeller University Press, New York.

Ang, S.L., and Rossant, J. (1994). Cell 78, 561-574.

Apelqvist, A., Li, H., Sommer, L., Beatus, P., Anderson, D.J., Honjo, T., Hrabe de Angelis, M., Lendahl, U., and Edlund, H. (1999). Nature 400, 877-881.

Blackwell, T.K., and Weintraub, H. (1990). Science 250, 1104-1110.

Blackwell, T.K., Huang, J., Ma, A., Kretzner, L., Alt, F.W., Eisenman, R.N., and Weintraub, H. (1993). Mol. Cell. Biol. 13, 5216-5224.

Bramblett, D.E., Huang, H.P., and Tsai, M.J. (2000). Adv. Pharmacol. 47, 255-315.

Cho, J.-H., Kwon, I.-S., Ghil, S.-H., Tsai, M.-J., Kim, Y.-S., Lee, Y.-D., and Suh-Kin, H, submitted.

Crowe, D.T, and Tsai, M.J. (1989). Mol. Cell. Biol. 9, 1784-1789.

Crowe, D.T., Hwung, Y.P., Tsai, S.Y., and Tsai, M.J. (1988). Prog. Clin. Biol. Res. 284, 211-224.

Dupont, S., Dina, C., Hani, E.H., and Froguel, P. (1999). Diabetes Metab. 25, 516-517.

Edlund, H. (1998). Diabetes 47, 1817-1823.

Edlund, H. (1999). Curr. Opin. Cell Biol. 11, 663-668.

Ellenberger, T., Fass, D., Amaud, M., and Harrison, S.C. (1994). Genes Dev. 8, 970-980.

Farah, M.H., Olson, J.M., Sucic, H.B., Hume, R.I., Tapscott, S.J., and Turner, D.L. (2000). Development 127, 693-702.

Ferre-D'Amare, A.R., Prendergast, G.C., Ziff, E.B., and Burley, S.K. (1993). Nature 363, 38-45.

Ferre-D'Amare, A.R., Pognonec, P., Roeder, R.G., and Burley, S.K. (1994). EMBO J. 13, 180-189.

Fode, C., Gradwohl, G., Morin, X., Dierich, A., LeMeur, M., Goridis, C., and Guillemot, F. (1998). Neuron 20, 483-494.

Fode, C., Ma, Q., Casarosa, S., Ang, S.L., Anderson, D. J., and Guillemot, F. (2000). Genes Dev. 14, 67-80. 
Gannon, M., Ray, M.K., Van Zee, K., Rausa, F., Costa, R.H., and Wright, C.V. (2000). Development 127, 2883-2895.

Glick, E., Leshkowitz, D., and Walker, M.D. (2000). J. Biol. Chem. 275, 2199-2204.

Gradwohl, G., Dierich, A., LeMeur, M., and Guillemot, F. (2000). Proc. Natl. Acad Sici. U.S.A. 97, 1607-1611.

Grapin-Botton, A., and Melton, D.A. (2000). Trends Genet. 16, 124-130.

Guillemot, F. (1999). Exp. Cell Res. 253, 357-364.

Guz, Y., Montminy, M.R., Stein, R., Leonard, J., Gamer, L.W., Wright, C.V., and Teitelman, G. (1995) Development 121, 11-18.

Hansen, L., Jensen, J.N., Urioste, S., Petersen, H.V., Pociot, F., Eiberg, H., Kristiansen, O.P., Hansen, T., Serup, P., Nerup, J., and Pedersen, O. (2000). Diabetes 49, 876-878.

Harrison, K.A., Thaler, J., Pfaff, S.L., Gu, H., and Kehrl, J.H. (1999). Nature Genet. 23, 71-75.

Horikawa, Y., Iwasaki, N., Hara, M., Furuta, H., Hinokio, Y., Cockburn, B.N., Lindner, T., Yamagata, K., Ogata, M., Tomonaga, O., Kuroki, H., Kasahara, T., Iwamoto, Y., and Bell, G.I. (1997). Nature Genet. 17, 384-385.

Huang, H.P., Liu, M., El-Hodiri, H.M., Chu, K., Jamrich, M., and Tsai, M.J. (2000). Mol. Cell. Biol. 20, 3292-3307.

Hwung, Y.P., Gu, Y.Z., and Tsai, M.J. (1990). Mol. Cell. Biol. 10, 1784-1788.

Ishibashi, M., Ang, S.L., Shiota, K., Nakanishi, S., Kageyama, R., and Guillemot, F. (1995). Genes Dev. 9, 3136-3148.

Iwata, I., Nagafuchi, S., Nakashima, H., Kondo, S., Koga, T., Yokogawa, Y., Akashi, T., Shibuya, T., Umeno, Y., Okeda, T., Shibata, S., Kono, S., Yasunami, M., Ohkubo, H., and Niho, Y. (1999). Diabetes 48, 416-419.

Jacquemin, P., Durviaux, S.M., Jensen, J., Godfraind, C., Gradwohl, G., Guillemot, F., Madsen, O.D., Carmeliet, P., Dewerchin, M., Collen, D., Rousseau, G.G., and Lemaigre, F.P. (2000). Mol. Cell. Biol. 20, 4445-4454.

Jarriault, S., Brou, C., Logeat, F., Schroeter, E.H., Kopan, R., and Israel, A. (1995). Nature 377, 355-358.

Jarriault, S., Le Bail, O., Hirsinger, E., Pourquie, O., Logeat, F., Strong, C.F., Brou, C., Seidah, N.G., and Isra I, A. (1998). Mol. Cell. Biol. 18, 7423-7431.

Jensen, J., Heller, R.S., Funder-Nielsen, T., Pedersen, E.E., Lindsell, C., Weinmaster, G., Madsen, O.D., and Serup, P. (2000a). Diabetes 49, 163-176.

Jensen, J., Pedersen, E.E., Galante, P., Hald, J., Heller, R.S., Ishibashi, M., Kageyama, R., Guillemot, F., Serup, P., and Madsen, O.D. (2000b). Nature Genet. 24, 36-44.

Jonsson, J., Carlsson, L., Edlund, T., and Edlund, H. (1994). Nature 371, 606-609.

Jonsson, J., Ahlgren, U., Edlund, T., and Edlund, H. (1995). Intl. J. Dev. Biol. 39, 789-798.

Kaestner, K.H., Katz, J., Liu, Y., Drucker, D.J., and Schutz, G. (1999). Genes Dev. 13, 495-504.

Kim, S.K., Hebrok, M., and Melton, D.A. (1997). Cold Spring Harb. Symp. Quant. Biol. 62, 377-383.

Krapp, A., Knofler, M., Ledermann, B., Burki, K., Berney, C., Zoerkler, N., Hagenbuchle, O., and Wellauer, P.K. (1998). Genes Dev. 12, 3752-3763.

Lammert, E., Brown, J., and Melton, D.A. (2000). Mech. Dev. 94, 199-203.

Lee, J.E., Hollenberg, S.M., Snider, L., Turner, D.L., Lipnick, N., and Weintraub, H. (1995). Science 268, 836-844.

Lee, J.K., Cho, J.H., Hwang, W.S., Lee, Y.D., Reu, D.S., and Suh-Kim, H. (2000). Dev. Dynam. 217, 361-367.

Leonard, J., Peers, B., Johnson, T., Ferreri, K., Lee, S., and Montminy, M.R. (1993). Mol. Endocrinol. 7, 1275-1283.

Li, H., Arber, S., Jessell, T.M., and Edlund, H. (1999). Nature Genet. 23, 67-70.

Liu, M., Pleasure, S.J., Collins, A.E., Noebels, J.L., Naya, F.J., Tsai, M.J., and Lowenstein, D.H. (2000a). Proc. Natl. Acad. Sci. U.S.A. 97, 865-870. 
Liu, M., Pereira, F.A., Price, S.D., Chu, M.J., Shope, C., Himes, D., Eatock, R.A., Brownell, W.E., Lysakowski, A., and Tsai, M.-J. (2000b). Genes Dev. 14, 2839-2854.

Ma, P.C., Rould, M.A., Weintraub, H., and Pabo, C.O. (1994). Cell 77, 451-459.

Ma, Q., Kintner, C., and Anderson, D.J. (1996). Cell 87, 43-52.

Ma, Q., Chen, Z., del Barco Barrantes, I., de la Pompa, J.L., and Anderson, D.J. (1998). Neuron 20, 469-482.

Ma, Q., Fode, C., Guillemot, F., and Anderson, D.J. (1999). Genes Dev. 13, 1717-1728.

Malecki, M.T., Jhala, U.S., Antonellis, A., Fields, L., Doria, A., Orban, T., Saad, M., Warram, J.H., Montminy, M., and Krolewski, A.S. (1999). Nature Genet. 23, 323-328.

Miller, C.P., McGehee, R.E., Jr., and Habener, J.F. (1994). EMBO J. 13, 1145-1156.

Miyachi, T., Maruyama, H., Kitamura, T., Nakamura, S., and Kawakami, H. (1999). Brain Res. Mol. Brain Res. 69, 223-231.

Miyata, T., Maeda, T., and Lee, J.E. (1999). Genes Dev. 13, 1647-1652.

Morrow, E.M., Furukawa, T., Lee, J.E., and Cepko, C.L. (1999). Development 126, 23-36.

Murre, C., McCaw, P.S., and Baltimore, D. (1989a). Cell 56, 777-783.

Murre, C., McCaw, P.S., Vaessin, H., Caudy, M., Jan, L.Y., Jan, Y.N., Cabrera, C.V., Buskin, J.N., Hauschka, S.D., Lassar, A.B., et al. (1989b). Cell 58, 537-544.

Mutoh, H., Naya, F.J., Tsai, M.J., and Leiter, A.B. (1998). Genes Dev. 12, 820-830.

Naya, F.J., Stellirecht, C.M., and Tsai, M.J. (1995). Genes Dev. 9, 1009-1019.

Naya, F.J., Huang, H.P., Qiu, Y., Mutoh, H., DeMayo, F.J., Leiter, A.B., and Tsai, M.J. (1997). Genes Dev. 11, 2323-2334.

Noma, T., Yoon, Y.S., and Nakazawa, A. (1999). Brain Res. Mol. Brain Res. 67, 53-63.

Offield, M.F., Jetton, T.L., Labosky, P.A., Ray, M., Stein, R.W., Magnuson, M.A., Hogan, B.L., and Wright, C.V. (1996). Development 122, 983-995.

Ohneda, K., Mirmira, R.G., Wang, J., Johnson, J.D., and German, M.S. (2000). Mol. Cell. Biol. 20, 900-911

Owerbach, D., Naya, F.J., Tsai, M.J., Allander, S.V., Powell, D.R., and Gabbay, K.H. (1997). Diabetes 46, 1069-1074.

Perez, S.E., Rebelo, S., and Anderson, D.J. (1999). Development 126, 1715-1728.

Perron, M., Opdecamp, K., Butler, K., Harris, W.A., and Bellefroid, E.J. (1999). Proc. Natl. Acad. Sci. U.S.A. 96, 14996-15001.

Peshavaria, M., Gamer, L., Henderson, E., Teitelman, G., Wright, C.V., and Stein, R. (1994). Mol. Endocrinol. 8, 806-816.

Pictet, R.L., Clark, W.R., Williams, R.H., and Rutter, W.J. (1972). Dev. Biol. 29, 436-467.

Poulin, G., Lebel, M., Chamberland, M., Paradis, F.W., and Drouin, J. (2000). Mol. Cell. Biol. 20, 4826-4837.

Puri, P.L., Sartorelli, V., Yang, X.J., Hamamori, Y., Ogryzko, V.V., Howard, B.H., Kedes, L., Wang, J.Y., Graessmann, A., Nakatani, Y., and Levrero, M. (1997). Mol. Cell 1, 35-45.

Qiu, Y., Sharma, A., and Stein, R. (1998). Mol. Cell. Biol. 18, 2957-2964

Rindi, G., Ratineau, C., Ronco, A., Candusso, M.E., Tsai, M., and Leiter, A.B. (1999). Development 126, 4149-4156.

Robinson, G.L., Peshavaria, M., Henderson, E., Shieh, S.Y., Tsai, M.J., Teitelman, G., and Stein, R. (1994). J. Biol. Chem. 269, 2452-2460.

Sartorelli, V., Puri, P.L., Hamamori, Y., Ogryzko, V., Chung, G., Nakatani, Y., Wang, J. Y., and Kedes, L. (1999). Mol. Cell 4, 725-734.

Schwitzgebel, V.M., Scheel, D.W., Conners, J.R., Kalamaras, J., Lee, J.E., Anderson, D.J., Sussel, L., Johnson, J.D., and German, M.S. (2000). Development 127, 3533-3542.

Serup, P., Petersen, H.V., Pedersen, E.E., Edlund, H., Leonard, J., Petersen, J.S., Larsson, L.I., and Madsen, O.D. (1995). Biochem. J. 310, 997-1003. 
Sharma, A., Moore, M., Marcora, E., Lee, J.E., Qiu, Y., Samaras, S., and Stein, R. (1999). Mol. Cell. Biol. 19, 704-713.

Shieh, S.Y., and Tsai, M.J. (1991). J. Biol. Chem. 266, 16708-16714.

Slack, J.M. (1995). Development 121, 1569-1580.

Sommer, L., Ma, Q., and Anderson, D.J. (1996). Mol. Cell. Neurosci. 8, 221-241.

Sosa-Pineda, B., Chowdhury, K., Torres, M., Oliver, G., and Gruss, P. (1997). Nature 386, 399-402.

St-Onge, L., Sosa-Pineda, B., Chowdhury, K., Mansouri, A., and Gruss, P. (1997). Nature 387, 406-409.

Stoffers, D.A., Ferrer, J., Clarke, W.L., and Habener, J.F. (1997). Nature Genet. 17, 138-139.

Stoffers, D.A., Heller, R.S., Miller, C.P., and Habener, J.F. (1999). Endocrinology 140, 5374-5381.

Uittenbogaard, M., and Chiaramello, A. (2000). Neurosci. Lett. 280, 95-98.

Voronova, A., and Baltimore, D. (1990). Proc. Natl. Acad. Sci. U.S.A. 87, 4722-4726.

Weinstein, D.C., Ruiz i Altaba, A., Chen, W.S., Hoodless, P., Prezioso, V.R., Jessell, T.M., and Darnell, J.E., Jr. (1994). Cell 78, 575-588.

Wells, J.M., and Melton, D. A. (1999). Annu. Rev. Cell. Dev. Biol. 15, 393-410.

Yamagata, K., Furuta, H., Oda, N., Kaisaki, P.J., Menzel, S., Cox, N.J., Fajans, S.S., Signorini, S., Stoffel, M., and Bell, G.I. (1996a). Nature 384, 458-460.

Yamagata, K., Oda, N., Kaisaki, P.J., Menzel, S., Furuta, H., Vaxillaire, M., Southam, L., Cox, R.D., Lathrop, G.M., Boriraj, V.V., Chen, X., Cox, N.J., Oda, Y., Yano, H., Le Beau, M.M., Yamada, S., Nishigori, H., Takeda, J., Fajans, S.S., Hattersley, A.T., Iwasaki, N., Hansen, T., Pedersen, O., Polonsky, K.S., Bell, G.I., et al. (1996b). Nature 384, 455-458.

Yan, R.T., and Wang, S.Z. (2000a). Vis. Neurosci. 17, 157-164.

Yan, R.T., and Wang, S.Z. (2000b). Neurosci. Lett. 280, 83-86.

Yoon, Y.S., Noma, T., Yamashiro, Y., Ito, H., and Nakazawa, A. (1998). Neurosci. Lett. 251, 17-20. 


\title{
Of Mice and Men: $K_{A T P}$ Channels and Insulin Secretion
}

\author{
Lydia Aguilar-Bryan, ${ }^{*}$ Joseph Bryan, ${ }^{\dagger}$ and Mitsuhiro Nakazaki ${ }^{\dagger \ddagger}$ \\ *Departments of Medicine and ${ }^{\dagger}$ Molecular and Cellular Biology, Baylor College of Medicine, \\ Houston, Texas 77030; ${ }^{+}$The First Department of Internal Medicine, Faculty of Medicine, \\ Kagoshima University, Kagoshima, 890-8520, Japan
}

\begin{abstract}
$\mathrm{K}_{\mathrm{ATP}}$ channels are a unique, small family of potassium $\left(\mathrm{K}^{+}\right)$-selective ion channels assembled from four inward rectifier pore-forming subunits, $K_{I R} 6 . x$, paired with four sulfonylurea receptors (SURs), members of the adenosine triphosphate (ATP)-binding cassette superfamily. The activity of these channels can be regulated by metabolically driven changes in the ratio of adenosine diphosphate (ADP) to ATP, providing a means to couple membrane electrical activity with metabolism. In pancreatic $\beta$ cells in the islets of Langerhans, $K_{A T P}$ channels are part of an ionic mechanism that couples glucose metabolism to insulin secretion. This chapter 1) briefly describes the properties of $K_{\text {ATP }}$ channels; 2 ) discusses data on a genetically recessive form of persistent hyperinsulinemic hypoglycemia of infancy (PHHI), caused by loss of $\beta$-cell $\mathrm{K}_{\mathrm{AIP}}$ channel activity; and 3) compares the severe impairment of glucose homeostasis that characterizes the human phenotype with the near-normal phenotype observed in $\mathrm{K}_{\mathrm{ATP}}$ channel null mice.
\end{abstract}

\section{Introduction}

A wide range of factors or processes, often classified arbitrarily by their effects on insulin secretion vs. insulin action, are known to affect glucose homeostasis and produce either hypoglycemia or hyperglycemia. The hyperglycemia characteristic of type II or non-insulin dependent diabetes is associated with a decreased sensitivity of different tissues to the normal glucose-lowering action of insulin (insulin resistance) and a failure of pancreatic $\beta$ cells to produce sufficient insulin. The hyperglycemia present in type I or insulin-dependent diabetes is the result of reduced or absent insulin secretion secondary to the immunologic destruction of insulin-secreting $\beta$ cells in the islets of Langerhans within the pancreas. While the molecular mechanism(s) of insulin resistance and $\beta$-cell destruction are not completely understood, an enormous amount of work has been done in these areas for more than two decades.

Profound hypoglycemic states in adults, although rare, have been associated with hyperinsulinism resulting from insulinomas whose insulin secretion is inappropriately regulated. However, the factors leading to uncontrolled insulin secre- 
tion in nontumor states are less well understood. During the last half-dozen years, insight into the genetic basis of congenital hyperinsulinism, or persistent hyperinsulinemic hypoglycemia of infancy (HI or PHHI), has begun to provide deeper understanding of the normal regulation of insulin secretion and the types of metabolic defects that can produce this disorder. In overall outline, this work has largely confirmed the early ionic steps in a basic regulatory model developed by a number of workers over the past 20 years. In this model, the metabolism of glucose is coupled to the membrane electrical activity of $\beta$ cells, which controls the influx of calcium ions that initiate and potentiate insulin exocytosis. In the early steps of this model, glucose metabolism in $\beta$ cells, initiated by glucokinase (GCK), decreases the adenosine diphosphate/adenosine triphosphate (ADP/ATP) ratio. This, in turn, reduces the activity of ATP-sensitive $\mathrm{K}^{+}$channels $\left(\mathrm{K}_{\mathrm{ATP}}\right.$ channels). In low-glucose conditions, $\mathrm{K}_{\mathrm{ATP}}$ channels act to hyperpolarize the $\beta$-cell membrane. A decrease in their activity results in membrane depolarization and initiation of $\mathrm{Ca}^{2+}$-dependent action potentials. These action potentials involve both voltage-sensitive "L-type" $\mathrm{Ca}^{2+}$ channels, whose openings further depolarize the $\beta$-cell membrane, and other poorly characterized repolarizing currents. The increased $\mathrm{Ca}^{2+}$ influx through the L-type $\mathrm{Ca}^{2+}$ channels increases $\left[\mathrm{Ca}^{2+}\right]_{j}$. This stimulates insulin exocytosis in concert with other second messengers, including cyclic nucleotides, the hydrolytic products of phospholipases (A2, C, and D), and adenine and guanine nucleotides. Thus, $\mathrm{Ca}^{2+}$ facilitates exocytosis and $\mathrm{K}_{\mathrm{ATP}}$ channels regulate glucose-stimulated insulin secretion by controlling $\mathrm{Ca}^{2+}$ channel activity.

\section{Persistent Hyperinsulinemic Hypoglycemia of Infancy}

PHHI, also referred to as familial hyperinsulinism or pancreatic nesidioblastosis (Online Mendelian Inheritance in Man: OMIM 256450), is a metabolic disorder of neonates identified by inappropriate secretion of insulin in the presence of hypoglycemia (for recent reviews, see Aguilar-Bryan and Bryan, 1996,1999; Permutt et al., 1996; Glaser et al., 1999; Bryan and Aguilar-Bryan, 2000; Glaser, 2000; and Shepherd et al., 2000). Plasma insulin is often elevated, the key feature being an inappropriately high level for the degree of hypoglycemia. Infusion of large amounts of glucose, more than $12 \mathrm{mg} / \mathrm{kg} / \mathrm{min}$, is required to maintain euglycemia in newborns. In severe cases, patients are unresponsive to $\mathrm{K}_{\mathrm{ATP}}$ channel openers (e.g., diazoxide) or to treatment with somatostatin derivatives (e.g., octreotide). Partial or subtotal pancreatectomy is done to relieve the hypoglycemia (Aynsley-Green et al., 2000).

The diagnosis of hyperinsulinism - considered here as high insulin levels, low blood ketone levels, and low free fatty acid levels at the time of hypoglycemia - has been shown to result from three genetic defects. At the suggestion of Franz 
Matschinsky, we (Aguilar-Bryan and Bryan, 1999) have designated these subclasses of hyperinsulinism as "HI-," followed by the protein affected (e.g., HIGCK, HI-SUR). GCK, the type IV hexokinase found in $\beta$ cells, converts glucose to glucose-6-phosphate and is generally considered to be the $\beta$-cell "glucose sensor" (Matschinsky, 1996). GCK mutations have been identified as one cause of maturity onset diabetes of the young (MODY 2). In these cases, an increase in the $\mathrm{K}_{\mathrm{m}}$ of the enzyme is correlated with an increase in the glucose concentration needed for insulin release. Glaser et al. (1998) have described a "gain-of-function" mutation in GCK that lowers the $\mathrm{K}_{\mathrm{m}}$ and results in hypoglycemia. This mutation, val $\rightarrow$ met, V408M, causes a rare, mild, dominant form of PHHI, HI-GCK (OMIM 602485), that responds clinically to diazoxide. No homozygotes have been identified, suggesting two copies of the allele may be lethal. The result is consistent with the basic model, as the mutant enzyme should make $\beta$ cells more "efficient" by increasing the rate of glucose metabolism at lower glucose concentrations, thus reducing the glucose "setpoint."

Zammarchi et al. (1996) and Weinzimer et al. (1997) have described a different mild, dominant form of hyperinsulinism in which the affected individuals also present moderate asymptomatic hyperammonemia. Several mutations in the glutamate dehydrogenase (GLUD1) gene have been identified in isolated and familial cases, in patients that have normal weight at birth and a good response to diazoxide. This form of hyperinsulinism, HI-GLUD1 (OMIM 138130), appears to be the result of an increase in GLUDl activity that produces a subsequent increase in $\alpha$-ketoglutarate production and excessive ATP. Enzyme studies indicate that the glutamate dehydrogenase in two unrelated patients with this disorder was less sensitive to GTP, a negative allosteric effector as a result of mutations in the C-terminus of the enzyme (Stanley et al., 1997).

This review is focused on a third group of mutations, discovered prior to those described above, which are found in the two subunits that make up $\beta$ cell $\mathrm{K}_{\mathrm{ATP}}$ channels (Inagaki et al, 1995a). These mutations cause PHHI, termed HI-SUR1 (OMIM 600509) or HI-K $\mathrm{IR}_{\mathrm{R}} 6.2$ (OMIM 600937), depending on the affected subunit. This subclass of $\mathrm{HI}$ usually is considered to be recessive and can be either mild or severe, conditions usually distinguished by the clinical symptoms and the lack of response to diazoxide. Mutations in these two genes are inherited in a Mendelian way but loss of the maternal allele(s), in parallel with a heterozygous germline mutation in the paternal SURI allele, also has been described (Ryan et al., 1998; Verkarre et al., 1998). Mild forms have been associated with missense mutations in the regulatory subunit (SUR1), which produce channels that can be inhibited by ATP, are poorly stimulated by the addition of MgADP, but remain sensitive to diazoxide (Nichols et al., 1996; Shyng et al., 1998). Severe forms of the disease have been associated with splice site and nonsense mutations that truncate the regulatory subunit (Thomas et al., 1995; Dunne et al., 1997) and block 
expression of channels at the cell surface by inhibiting normal trafficking to the plasma membrane (Sharma et al., 1999). Similarly, a severely truncated form of $\mathrm{K}_{\mathrm{IR}} 6.2$ causes $\mathrm{PHHI}$ (Nestorowicz et al., 1997). These results were consistent with the basic model, which suggested that secondary to loss of $\mathrm{K}_{\mathrm{ATP}}$ channels, PHHI $\beta$ cells should display a predictable electrical phenotype characterized by persistent depolarization, the presence of $\mathrm{Ca}^{2+}$-dependent action potentials, and elevated $\left[\mathrm{Ca}^{2+}\right]_{\mathrm{i}}$. Analysis of human PHHI $\beta$ cells has largely confirmed this expectation (Dunne et al., 1997; Otonkoski et al., 1999), while observations on insulin release from cultured PHHI $\beta$ cells (Kaiser et al., 1990) and the increased insulin levels in PHHI patients suggested that elevated $\mathrm{Ca}^{2+}$ increased insulin exocytosis.

We have reviewed all aspects of this area over the past several years (AguilarBryan and Bryan, 1996,1999; Aguilar-Bryan et al.,1998; Bryan and AguilarBryan, 1997,1999,2000; Babenko et al., 1998). Our intention in this chapter is to minimize overlap with these previous publications. Rather, we review briefly recent studies aimed at understanding how particular $\mathrm{K}_{\mathrm{ATP}}$ channel mutations cause PHHI. We want to draw attention to the rather remarkable physiologic differences between human neonates missing $K_{\text {ATP }}$ channel activity and $K_{\text {ATP }}$ channel knockout mice. We have tried to provide the reader with the minimal background in $\mathrm{K}_{\mathrm{ATP}}$ channels needed to follow the story and have made frequent reference to earlier reviews where additional detail can be found.

\section{III. $K_{\mathrm{ATP}}$ Channels Are Assembled from SURs and $\mathrm{K}_{\mathrm{IR}} \mathrm{s}$}

$\mathrm{K}_{\mathrm{ATP}}$ channels initially were identified in cardiac myocytes (Noma, 1983; Trube and Hescheler, 1983,1984) and pancreatic $\beta$ cells (Cook and Hales, 1984). Their biophysical and pharmacologic properties have been studied intensively (Nelson and Quayle, 1995; Dukes and Philipson, 1996; Tucker et al., 1998). Our understanding of these channels increased markedly with the cloning of both their pore $\left(\mathrm{K}_{\mathrm{IR}} 6 . \mathrm{x}\right)$ (Inagaki et al., 1995a,1995b) and regulatory (SUR) (Aguilar-Bryan et al., 1995) subunits and with the reconstitution of the $\beta$ cell (Inagaki et al., 1995a), cardiac (Inagaki et al., 1996; Babenko et al., 1998b), and smooth muscle (Isomoto et al., 1996; Yamada et al., 1997) type channels. The cloning and reconstitution studies established that $\mathrm{K}_{\mathrm{ATP}}$ channels are a unique combination of two quite dissimilar subunits: 1) a large, regulatory subunit with homology to members of the transport ATPase superfamily, particularly the multidrug resistance (MDR) and multidrug resistance-associated proteins (MRP), and 2) a small, potassium ion channel subunit that forms the channel pore. While other transport ATPases couple ATP hydrolysis to movement of molecules through a membrane against their concentration gradient, SURs, the regulatory subunits of $\mathrm{K}_{\mathrm{ATP}}$ channels, control the flux of potassium ions through a pore driven by a concentration gradient or, more precisely, by the difference between the Nernst potential result- 
ing from a $\mathrm{K}^{+}$gradient and the membrane potential. Control is exerted in response to changes in the concentrations of cytosolic ADP/ATP that result from ATP synthesis and hydrolysis within the cell and presumably on the regulatory subunit itself. Thus, ATP hydrolysis is thought to occur as an integral part of the regulation of $\mathrm{K}_{\mathrm{ATP}}$ channels, although it does not power $\mathrm{K}^{+}$movement.

The regulatory subunits are termed SURs because they bind hypoglycemic sulfonylureas. The $\beta$-cell receptor, for example, binds glibenclamide with high affinity ( $\mathrm{K}_{\mathrm{D}}$ in the low $\mathrm{nM}$ range). The SURs consist of a small family, specified by the Sur 1 and Sur2 genes that encode the high-affinity SUR1 and low-affinity SUR2A and SUR2B receptors, respectively. The pore subunits are termed $\mathrm{K}_{\mathrm{IR}} \mathrm{S}$, or potassium inward rectifiers (see Doupnik et al., 1995, and Nichols and Lopatin, 1997, for reviews). Rectifiers are devices for converting alternating currents into direct currents - in short, devices able to conduct current more easily in one direction than another. $\mathrm{K}_{\mathrm{IR}} \mathrm{S}$ are able to pass potassium better into a cell than out when measured in excised patches and thus are termed "inward rectifiers." Under physiological conditions, the potassium concentration is greater inside the cell than out. Therefore, potassium leaves the cell through $\mathrm{K}_{\mathrm{ATP}}$ channels, thus lowering the membrane potential. SURs have been shown to assemble preferentially with two $\mathrm{K}_{\mathrm{IR}} \mathrm{s}, \mathrm{K}_{\mathrm{IR}} 6.1$ and $\mathrm{K}_{\mathrm{IR}} 6.2$, to form large octameric channels with a (SUR/K $\left.\mathrm{I}_{\mathrm{IR}} 6 . \mathrm{x}\right)_{4}$ stoichiometry (Clement et al., 1997; Shyng and Nichols, 1997; Inagaki et al., 1997; reviewed in Aguilar-Bryan et al., 1998, and Bryan and Aguilar-Bryan, 1999).

Several mechanisms have evolved to regulate the assembly and trafficking of the octameric channels, to insure that only those whose activity can be correctly regulated will reach the cell surface. Assembly occurs in the endoplasmic reticulum (ER); both subunits carry ER retrieval signals, an $-R K R-$ sequence motif, which cause incompletely assembled complexes to be retained in the ER (Zerangue et al., 1999). This ER retention mechanism accounts for the original observations that SUR 1 expressed alone was incompletely glycosylated (AguilarBryan et al., 1995; Clement et al., 1997) and that expression of $\mathrm{K}_{\mathrm{IR}} 6.2$ alone failed to produce channel activity (Inagaki et al., 1995a), while expression of truncated $\mathrm{K}_{\mathrm{IR}} 6.2$ missing the $-R K R$-motif produced aberrant channels (Tucker et al., 1997).

\section{Truncation of SUR1 Causes Severe PHHI}

The C-terminal 25 amino acids of SUR1 contain an additional peptide motif that is critical for the correct trafficking of $\mathrm{K}_{\mathrm{ATP}}$ channels. This motif consists of a dileucine pair and nearby phenylalanine (-XLLXXXXXXFX). Removal of this motif, or substitution of alanine residues, prevents $\mathrm{K}_{\mathrm{ATP}}$ channel expression at the cell surface (Sharma et al, 1999). In human SUR1, the specific sequence is -KLLSRKDSVFA. We have proposed that loss of this C-terminal signal explains 
the loss of $\mathrm{K}_{\mathrm{ATP}}$ channel activity that is observed in PHHI $\beta$ cells with mutations that truncate SUR1 (Sharma et al., 1999).

\section{Two Gene Clusters Encode the Isoforms of $K_{\mathrm{ATP}}$ Channels}

The size and chromosomal location of the human $\mathrm{K}_{\mathrm{ATP}}$ channel genes have been established. Two gene clusters on the short arms of human chromosomes 11 (11p15.1) and $12(12 \mathrm{p} 11.23)$ encode the SUR1 and $\mathrm{K}_{\mathrm{IR}} 6.2$ subunits and the SUR2 and $\mathrm{K}_{\mathrm{IR}} 6.1$ subunits, respectively (reviewed in Aguilar-Bryan and Bryan, 1999). The gene names within these clusters, using the updated nomenclature, are Sur 1 $=\mathrm{Abcc} 8+\mathrm{K}_{\mathrm{IR}} 6.2=\mathrm{KCNJ} 11$ and Sur2 $=\mathrm{Abcc} 9+\mathrm{K}_{\mathrm{IR}} 6.1=\mathrm{KCNJ} 8$, respectively. Differential splicing of the terminal exon in the Sur2 gene specifies the two major SUR2A and SUR2B splice variants (reviewed in Aguilar-Bryan et al., 1998; Aguilar-Bryan and Bryan, 1999). Additional minor splice variants have been described (Chutkow et al., 1996,1999). Based on extensive evidence (reviewed in Aguilar-Bryan et al., 1998; Aguilar-Bryan and Bryan, 1999; Babenko et al., $1998 \mathrm{a}$ ), the SUR $1 / \mathrm{K}_{\mathrm{IR}} 6.2$ pair is known to comprise the $\mathrm{K}_{\mathrm{ATP}}$ channels of pancreatic $\beta$ cells and also is found in neurons and neuroendocrine cells. The SUR2A/K $\mathrm{K}_{\mathrm{IR}} 6.2$ pair assembles the channels in cardiac ventricular myocytes and skeletal muscle (Inagaki et al., 1996; Babenko et al., 1998b). SUR2B pairs with $\mathrm{K}_{\mathrm{IR}} 6.1$ and $\mathrm{K}_{\mathrm{IR}} 6.2$ to form the $\mathrm{K}_{\mathrm{ATP}}$ channels found in vascular and nonvascular smooth muscle (Isomoto et al., 1996; Yamada et al., 1997). The SUR2-based channels are the targets for a variety of potassium channel openers of potential therapeutic importance (reviewed in Challinor-Rogers and McPherson, 1994; Quast et al., 1994; Quayle and Standon, 1994; and Edwards and Watson, 1995). The Sur $2 / \mathrm{K}_{\mathrm{IR}} 6.1$ gene cluster has not been connected with a disease at this time. Recent reports have suggested that mixed channels comprised of SUR1/SUR2/K $\mathrm{K}_{\mathrm{IR}} 6.1$ or SUR1/SUR2/ $\mathrm{K}_{\mathrm{IR}} 6.2$ subunits may exist (Miller et al., 1999; Liss et al., 1999; Gopalakrishnan et al., 1999; Yokoshiki et al., 1999). However, their pharmacologic and functional properties are not understood.

\section{Progress in Mapping Functionally Important Regions of $\mathbf{K}_{\mathrm{ATP}}$ Channel Subunits}

$\mathrm{K}_{\mathrm{ATP}}$ channels have a medically important pharmacology, the $\beta$-cell channels being the classical target for hypoglycemic drugs, specifically, the first- and second-generation sulfonylureas (e.g., tolbutamide, glibenclamide, glipizide) used to control hyperglycemia in non-insulin dependent diabetes mellitus (NIDDM). $\mathrm{K}_{\mathrm{ATP}}$ channels are also the target for potassium channel openers (KCOs), with diazoxide being used to control hyperinsulinemic states by hyperpolarization of $\beta$ cells via channel activation. The sulfonylureas and $\mathrm{KCO}$ both act through 
SURs. A substantial amount of progress has been made in understanding these interactions and how they act to block and stimulate channel activity (Dickinson et al., 1997; Schwanstecher et al., 1998; Loffler-Walz and Quast, 1998; Hambrock et al., 1998,1999; Gribble et al., 1999; Babenko et al., 1999a,1999b, 1999c,2000; Meyer et al., 1999; Uhde et al., 1999; D'Hahan et al., 1999; reviewed in Bryan and Aguilar-Bryan, 1999). In addition to their differential sensitivity to sulfonylureas and $\mathrm{KCOs}, \mathrm{K}_{\mathrm{ATP}}$ channel isoforms display differences in their kinetics and sensitivity to inhibitory ATP. Chimeric receptors, constructed from fragments of SUR1 and SUR2A, have been used to exploit these differences and to develop a map of the regions important in specifying 1) high-affinity sulfonylurea binding (Gribble et al., 1999; Babenko et al., 1999c; Meyer et al., 1999) 2) the action of diazoxide (Babenko et al., 2000) and other KCOs (Uhde et al., 1999; D'Hahan et al., 1999; Babenko et al., 2000); and 3) the segments that specify the differences in kinetics and sensitivity to ATP (Babenko et al., 2000). A similar chimeric strategy has been used to identify residucs in the pore subunit, in the extracellular linkers after $\mathrm{M} 1$ and before $M 2$, that specify the lower conductance of $\mathrm{K}_{\mathrm{IR}} 6.1 \mathrm{vs}$. $\mathrm{K}_{\mathrm{IR}} 6.2$ (Repunte et al., 1999). Figure 1 provides a schematic summary of this work that we have recently reviewed (Bryan and Aguilar-Bryan, 1999).

\section{Regulation of $\mathbf{K}_{\mathrm{ATP}}$ Channels}

Early studies on isolated, excised $\beta$-cell membrane patches demonstrated that ATP inhibited $\mathrm{K}_{\mathrm{ATP}}$ channels and that this inhibition was antagonized by $\mathrm{MgADP}$. This discovery suggested that the link between metabolism and channel activity was through changes in the ADP/ATP ratio (Dunne and Petersen, 1986; Misler et al., 1986; Hopkins et al., 1992; Detimary et al., 1994). Analysis of missense mutations that cause $\mathrm{PHHI}$ has provided strong genetic support for this mechanism in the form of channels whose inhibition is not affected by ATP but that display a reduced stimulatory response to MgADP (Nichols et al., 1996; Shyng et al., 1998).

A second, quite different line of evidence in support of this mechanism comes from studies on knockout mice missing the mitochondrial uncoupling protein, UCP2 (Zhang et al., 2000). UCP2 acts to reduce the mitochondrial membrane potential, thus reducing the efficiency of conversion of ADP to ATP. At a given rate of glucose metabolism, eliminating UCP2 should decrease the ADP/ATP ratio in $\beta$ cells, thus potentiating closure of $\mathrm{K}_{\mathrm{ATP}}$ channels and increasing insulin output. Interestingly, UCP- $2^{-/-}$and UCP- $2^{+/-}$mice in the fed state have significantly higher insulin and lower blood sugar levels than control animals. At the same time, biochemical measurements indicate that ADP/ATP ratios are lower in knockout islets. In support of the knockout data, the overexpression of UCP2 in rat islets has been shown to inhibit glucose-stimulated insulin secretion (Chan et al., 1999). 


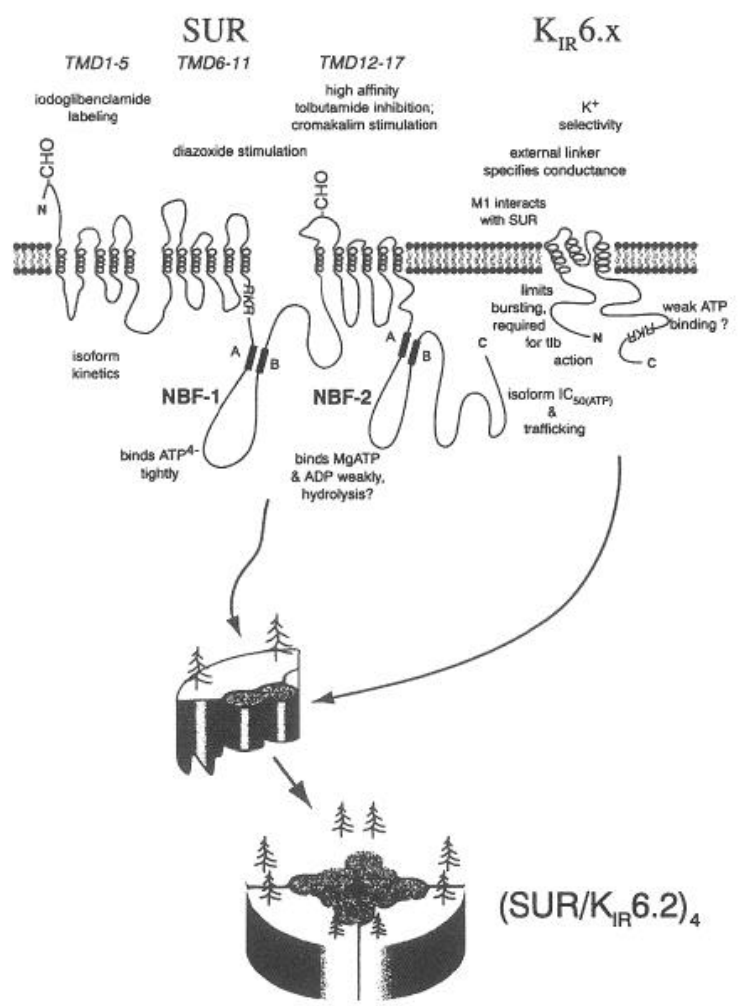

FIG. 1. Summary of the functional domains and stoichiometry of $\mathrm{K}_{\mathrm{ATP}}$ channels. The topology of SUR is based on the model proposed by Tusnady et al. (1997); the topology of the first five helices has been defined experimentally by Raab-Graham et al. (1999). Radio-iodinated glibenclamide has been shown to photolabel an N-terminal fragment of SUR1 (Aguilar-Bryan et al., 1995; Nelson et al., 1996). The evidence for the positioning of the domains required for high-affinity sulfonylurea (tolbutamide) binding and for the action of potassium channel openers (KCOs) is covered in the text. The importance of the TMD1-5 segment of SUR1 in determining the differences in kinetics of $\beta$ cell vs. cardiac $\mathrm{K}_{\mathrm{ATP}}$ channel isoforms and the C-terminus of SUR1 in specifying the isoform $\mathrm{IC}_{50(\mathrm{ATP})}$ is covered in Babenko et al. (1999c). The data supporting the existence of ER retention signals, the $-R K R-$ motifs, are in Zerangue et al. (1999). The data indicating there is a distinct trafficking signal in the C-terminus of SUR1 is in Sharma et al. (1999). The data on the differences in binding properties of the two nucleotide-binding folds (NBFs) are from Ueda et al. $(1997,1999)$. The importance of the $\mathrm{N}$-terminus of $\mathrm{K}_{\mathrm{IR}} 6.2$ in limiting bursting and for the action of tolbutamide is covered in Babenko et al. (1999a,1999b), and Koster et al. (1999). The importance of the Ml segment in SUR-K $\mathbb{R}_{\mathbb{R}} 6.2$ interactions is described in Schwappach et al. (2000). The data demonstrating that residues in the extracellular linkers specifies the differences in conductance of $\mathrm{K}_{\mathrm{IR}} 6.1 \mathrm{vs} . \mathrm{K}_{\mathrm{IR}} 6.2$ channels is given in Repunte et al. (1999). The evidence for a (SUR1/K $\left.\mathrm{K}_{\mathrm{IR}} 6.2\right)_{4}$ stoichiometry is in Clement et al. (1997), Shyng and Nichols (1997), and Inagaki et al. (1997). The location of the glycosylation sites is given in Aguilar-Bryan et al. $(1995,1998)$ and Raab-Graham et al. (1999). Direct evidence for ATP-binding to $\mathrm{K}_{\mathbb{R}} 6.2$ is given in Tanabe et al. (1999). The location of the binding site is not known. 
While the available data are consistent with changes in the ADP/ATP ratio controlling $\mathrm{K}_{\mathrm{ATP}}$ channel activity, other mechanisms have been proposed. Phosphatidyinositol phosphate $\left(\mathrm{PIP}_{2}\right)$ has been demonstrated to reduce the sensitivity of $K_{\text {ATP }}$ channels to inhibitory ATP when applied to excised patches at high concentrations (Fan and Makielski, 1997; Shyng and Nichols, 1998; Baukrowitz et al, 1998). This suggests a potential mechanism where, at a given concentration of ATP, a local increase in $\beta$-cell PIP ${ }_{2}$ would increase the number of $K_{A T P}$ channel openings. A local decrease in $\mathrm{PIP}_{2}$ would reduce channel activity, thus depolarizing the $\beta$-cell membrane initiating $\mathrm{Ca}^{2+}$ influx and potentiating insulin release. This mechanism would appear to require a $\mathrm{PIP}_{2}$ decrease before the rise in $\mathrm{Ca}^{2+}$ usually considered necessary to activate phospholipase C.

Additionally, several recent reports (Beguin et al., 1999; Lin et al., 2000) indicate that in vitro phosphorylation of $\mathrm{K}_{\mathrm{ATP}}$ subunits can modulate channel activity, although the physiological significance of these observations has not been established.

\section{PHHI vs. $K_{\text {ATP }}$ Channel Knockout Mice: Equivalent Electrophysiology, Remarkably Different Glucose Homeostasis}

Electrophysiological studies on isolated $\beta$ cells from three $\mathrm{K}_{\mathrm{ATP}}$ channel-deficient systems - the recessive form of PHHI in humans (Kane et al., 1996; Dunne et al., 1997; Otonkoski et al., 1999) and $\mathrm{K}_{\mathrm{IR}} 6.2^{-/-}$(Miki et al., 1998) and SUR $1^{-1}$ (Seghers et al., 2000) mice - have identified a common electrophysiological signature consistent with the basic model described above (see also Atwater et al., 1996; Cook and Taborsky, 1997; Aguilar-Bryan and Bryan, 1999; Mears and Atwater, 2000, for reviews). The features of this phenotype are 1) loss of $\mathrm{K}^{+}$currents activated by reduced intracellular nucleotides, as determined by whole-cell dialysis; 2) an elevated membrane potential (the cells are depolarized); 3 ) the presence of spontaneous, $\mathrm{Ca}^{2+}$-dependent action potentials under low glucose conditions; and 4) increased $\left[\mathrm{Ca}^{2+}\right]_{i}$ as a result of the increased $\mathrm{Ca}^{2+}$ influx. Figure 2 illustrates several aspects of this phenotype. In PHHI, the increase in $\beta$-cell $\left[\mathrm{Ca}^{2+}\right]_{i}$ appears to produce an inappropriately high rate of insulin release, producing hypoglycemia. Remarkably, and unexpectedly, the $\mathrm{K}_{\mathrm{ATP}}$ channel null mice display normal insulin and blood sugar levels when randomly feeding, despite the presence of elevated $\left[\mathrm{Ca}^{2+}\right]_{i}$ in isolated islets and $\beta$ cells. Table I compares the features of PHHI neonates vs. $\mathrm{K}_{\mathrm{ATP}}{ }^{-1-}$ mice.

Although the $\mathrm{K}_{\mathrm{ATP}}{ }^{-/-}$mice are normoglycemic when fed, their glucose homeostasis is impaired. Both $\mathrm{K}_{\mathrm{IR}} 6.2^{-/-}$(Miki et al., 1998) and Surl ${ }^{-/ \text {- }}$ (Seghers et al., 2000) pups display a transient hypoglycemia immediately after birth that persists for the first several days of life. The two mouse phenotypes are reported to differ subtly. The $\mathrm{K}_{\mathrm{IR}} 6.2^{-/-}$mice are not significantly hypoglycemic vs. controls 


\section{Whole Cell Mode}

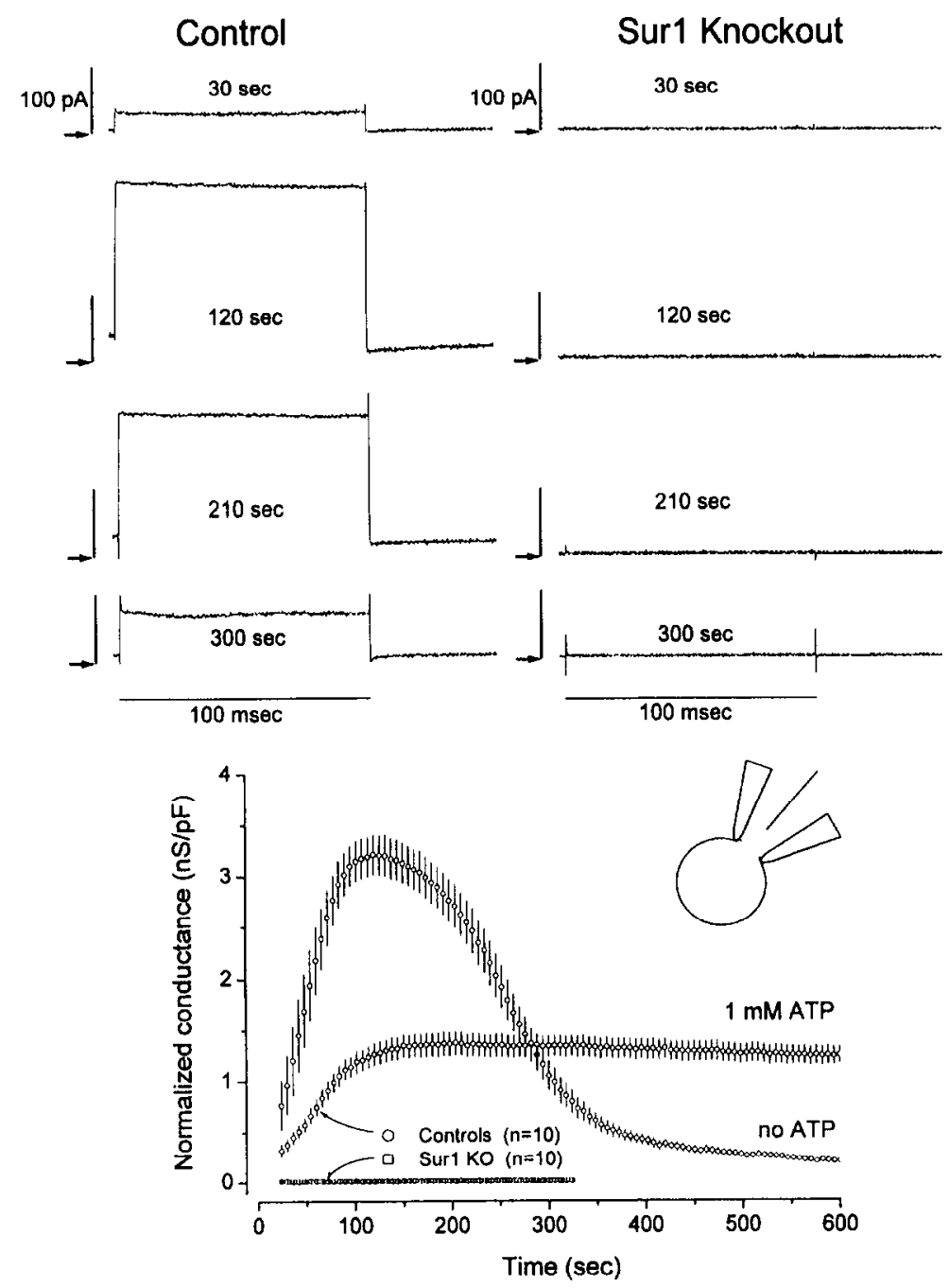

FIG. 2. (A) Whole-cell $\mathrm{K}^{+}$currents in control and Surl-knockout $\beta$ cells. The $\mathrm{K}^{+}$currents generated in response to a $100 \mathrm{msec}$ test pulse from -70 to $60 \mathrm{mV}$, every 3 seconds, at the indicated times after membrane rupture are shown for control and Sur $1-k n o c k o u t ~ \beta$ cells, respectively. As shown in the bottom plot of normalized conductance vs. time, the control currents peak at about 2 minutes, then proceed to rundown when there is no ATP in the pipette solution. No currents were detected in the Surl-knockout $\beta$ cells. Inclusion of $1 \mathrm{mM} \mathrm{ATP}$ in the pipette solution reduces the peak currents and reduces the rate of rundown significantly in the control cells. The error bars are \pm S.E. 


\section{Cell Attached Mode}

\section{Wildtype}
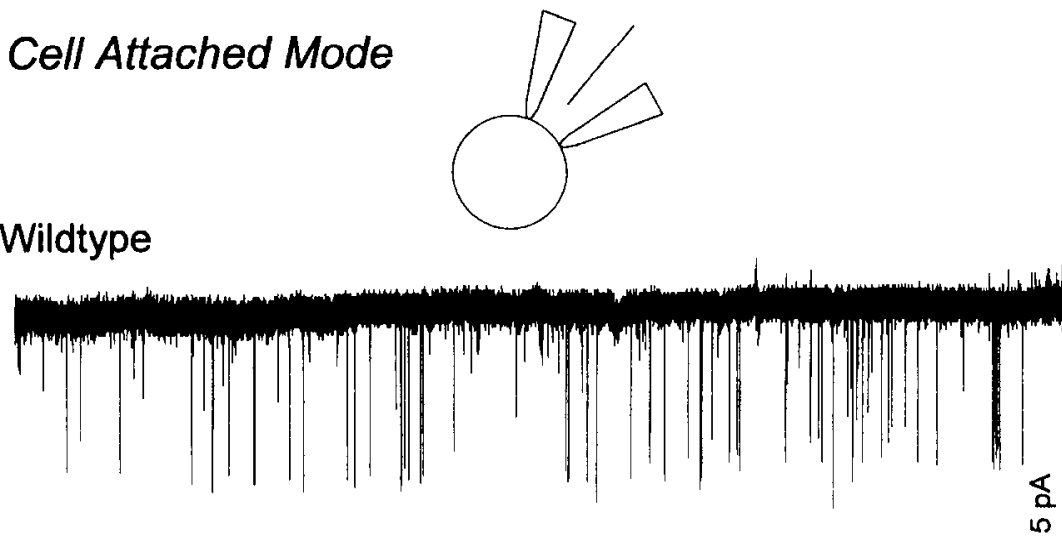

Sur1 Knockout

$300 \mu \mathrm{M}$ Diazoxide

$30 \mathrm{msec}$

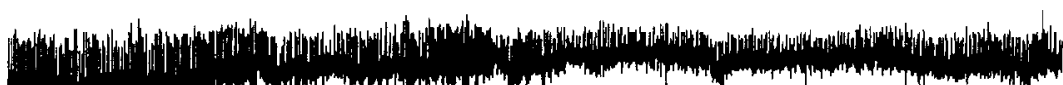

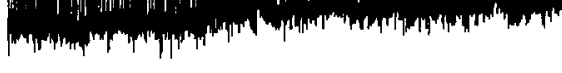
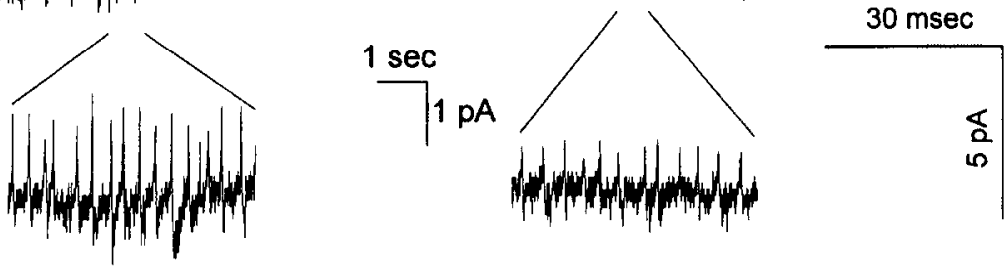

\section{$1 \mu \mathrm{M}$ Nifedipine}

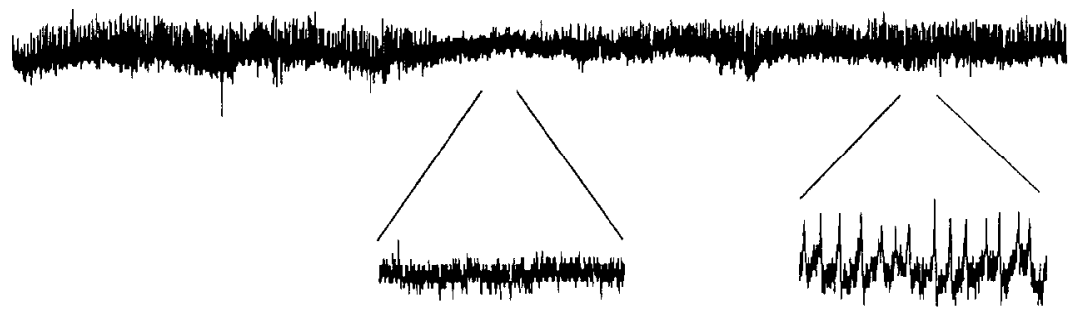

FIG. 2. (B) Sur 1-knockout $\beta$ cells exhibit spontaneous $\mathrm{Ca}^{2+}$-dependent action potentials. Records from cell-attached patches on control and Sur $1-$ knockout $\beta$ cells show spontaneous action potentials that are not inhibited by diazoxide but are suppressed by the L-type $\mathrm{Ca}^{21}$ channel blocker, nifedipine 
TABLE I

PHHI Patients vs. $K_{A T P}$ Channel Knockout Mice

Clinical

\begin{tabular}{|c|c|c|}
\hline & PHHI Patients & Surl $/ \mathrm{K}_{\mathrm{IR}} 6.2$ Knockout Mice \\
\hline Birth weight & $\begin{array}{l}\text { Large for gestational age, } \\
3.6-5.5 \mathrm{~kg}\end{array}$ & Normal, $1.47 \pm 0.18 \mathrm{~g}(1.47 \pm 0.20)$ \\
\hline Growth & $\begin{array}{l}\text { Normal, if diagnosed and } \\
\text { treated early }\end{array}$ & Normal \\
\hline Fertility & Normal & Normal \\
\hline \multicolumn{3}{|l|}{ Glucose } \\
\hline Newborn & $\begin{array}{l}\text { Persistent hypoglycemia } \\
<20 \mathrm{mg} / \mathrm{dl}(50 \pm 10)\end{array}$ & $\begin{array}{l}\text { Transient hypoglycemia } \\
\text { Day } 1,31 \pm 9 \mathrm{mg} / \mathrm{dl}(67 \pm 10) \\
\text { Day } 2,53 \pm 24 \mathrm{mg} / \mathrm{dl} \\
\text { Day } 5,149 \pm 31 \mathrm{mg} / \mathrm{dl}(112 \pm 9)\end{array}$ \\
\hline Adolescent & $\begin{array}{l}\text { Hyperglycemic; high } \\
\text { prevalence of IDDM }\end{array}$ & $\begin{array}{l}\text { Normoglycemic when random fed, } \\
121 \pm 18 \mathrm{mg} / \mathrm{dl}(113 \pm 17) \\
\text { Hypoglycemic upon fasting, } 48 \pm 13 \mathrm{mg} / \mathrm{dl} \\
(66 \pm 9) / \text { Normal } \\
\text { Glucose intolerant (IPGTT) }\end{array}$ \\
\hline \multicolumn{3}{|l|}{ Insulin } \\
\hline Newborn & $\begin{array}{l}\text { Inappropriate levels of insulin } \\
\text { for hypoglyccmia }\end{array}$ & $\begin{array}{l}\text { Transient hyperinsulinemia } \\
\text { Day } 1,86 \pm 53 \mathrm{mU} / \mathrm{l}(38 \pm 31) \\
\text { Day } 2,38 \pm 22 \mathrm{mU} / \mathrm{l} \\
\text { Day } 5,17 \pm 7 \mathrm{mU} / /(31 \pm 22)\end{array}$ \\
\hline \multirow[t]{2}{*}{ Adolescent } & $\begin{array}{l}\text { Normal for patients in } \\
\text { remission, absent in IDDM }\end{array}$ & $\begin{array}{l}\text { Normal insulin levels when random fed, } \\
25 \pm 13 \mathrm{mU} / \mathrm{l}(27 \pm 13) \\
\text { High insulin/blood glucose ration upon fasting, } \\
0.39 \pm 0.17(0.20 \pm 0.08) / \text { Normal }\end{array}$ \\
\hline & $\begin{array}{l}\text { Lack of acute insulin response } \\
\text { in patients with diffuse form } \\
\text { of PHHI }\end{array}$ & $\begin{array}{l}\text { Lack of acute insulin response and } \\
\text { attenuated insulin secretion (IPGTT) }\end{array}$ \\
\hline
\end{tabular}

(continued on next page) 


\begin{tabular}{|c|c|c|}
\hline \multicolumn{3}{|c|}{ Basic } \\
\hline & PHHI $\beta$ Cells & Sur $1 / \mathrm{K}_{\mathrm{IR}} 6.2$ Knockout $\beta$ Cells \\
\hline $\mathrm{K}_{\text {ATP }}$ channel activity & Absent & Absent \\
\hline Resting $\mathrm{Vm}$ & $\begin{array}{l}\text { Elevated with action } \\
\text { potentials }\end{array}$ & $\begin{array}{l}\text { Elevated with action potentials, } \\
-32.6+62 \mathrm{mV}(-624+12.31)\end{array}$ \\
\hline Resting $\left[\mathrm{Ca}^{2+}\right]_{i}$ & Elevated with oscillations & Elevated with oscillations \\
\hline \multicolumn{3}{|l|}{ Insulin secretion } \\
\hline Basal & Elevated & $\begin{array}{l}\text { Normal, } 52 \pm 14 \mu \mathrm{U} / \\
10 \text { islets/hr }(41 \pm 28)\end{array}$ \\
\hline \multicolumn{3}{|l|}{ Stimulation } \\
\hline Glucose & $\begin{array}{l}\text { Responds to } 6-11 \mathrm{nM} \\
\text { glucose }\end{array}$ & $\begin{array}{l}\text { Lack of first-phase and attenuated } \\
\text { second-phase ( } 16.7 \mathrm{mM} \text { glucose) } \\
\text { No response }\end{array}$ \\
\hline Tolbutamide & No response & No response \\
\hline $\operatorname{High~} \mathrm{K}^{+}$ & No response & Stimulated by $30 \mathrm{mM} \mathrm{KCl}$ \\
\hline
\end{tabular}

[Range in parentheses represents normal values.]

after a 16-hour fast, while the Surl $1^{-/-}$mice are. Both knockout animals exhibit a similar mild intolerance toward intraperitoneal injections of glucose, consistent with the observed loss of first-phase insulin release in isolated islets. The fasting plasma insulin values in the $\mathrm{K}_{\mathrm{IR}} 6.2^{-/-}$and Sur $1^{-/-}$animals are lower but not significantly different (at the $p<0.05$ level) from fasting control animals. In the case of the Sur $1^{-i_{-}}$animals, plasma insulin returns to control levels upon feeding, indicating insulin release is regulated in the absence of $\mathrm{K}_{\mathrm{ATP}}$ channels. Plasma insulin data have not been reported for fed $\mathrm{K}_{\mathrm{IR}} 6.2^{-/}$mice. In addition to being poorly able to regulate their insulin output when blood glucose falls during a fast, Sur $1^{-/}$pups do not properly regulate when blood glucose levels rise. This results in a peculiar plasma glucose profile, with the Surl ${ }^{-1}$ mice being more hyperglycemic when glucose loaded and more hypoglycemic when fasted, relative to control animals. The two $\mathrm{K}_{\mathrm{ATP}}{ }^{-1-}$ animals are reported to differ markedly in their sensitivity to insulin. The $\mathrm{K}_{\mathrm{IR}} 6.2^{-/-}$mice are reported to be insulin hypersensitive (Miki et al., 1998) secondary to loss of SUR $2 A / K_{\mathrm{IR}} 6.2$ channels in skeletal muscle, while we see no difference in the insulin sensitivity of the Surl ${ }^{-/-}$mice (Seghers et al., 2000). 
The secretion profiles of isolated $\mathrm{K}_{\mathrm{IR}} 6.2^{-/-}$and Sur ${ }^{-/-}$islets differ subtly. The Sur $1^{-/}$islets show essentially no first-phase insulin secretion in response to a shift from low (i.e., $2.8 \mathrm{mM}$ ) to high (i.e., $16.7 \mathrm{mM}$ ) glucose, while the $K_{\mathrm{IR}} 6.2^{-/-}$animals show a small first-phase release. By contrast, the $\mathrm{K}_{\mathrm{IR}} 6.2^{-/-}$islets exhibit no second-phase release, while the Sur $1^{-/}$islets show an attenuated, second-phase response, approximately 35 percent of the controls. Our hypothesis was that the $\mathrm{K}_{\mathrm{ATP}}$-independent pathway regulating secretion in the Sur1 $1^{-1-}$ animals had a slow response time. In extended perifusion experiments, Sur $1^{-/-}$islets displayed an increase in insulin output that paralleled that seen from control islets (Figure 3A). Extended static incubation experiments demonstrated that insulin output, from both control and Surl ${ }^{-1}$ islets, was glucose dependent and could be suppressed by the $\mathrm{Ca}^{2+}$ channel blocker, nifedipine (Figure $3 \mathrm{C}$ ). Furthermore, while reducing glucose in the medium resulted in a rapid return of wild-type islets to a basal secretory rate $\left(t_{1 / 2} \sim 5\right.$ minutes $)$, the Sur $1^{-/}$islets were unable to rapidly reduce their insulin output $\left(t_{1 / 2}-67\right.$ minutes), consistent with excess insulin during fasting (Figure 3B). We have correlated the lack of first-phase insulin release with the observed impaired ability of $\mathrm{K}_{\mathrm{ATP}}$ channel null mice to rapidly clear a glucose load (i.e., their mild glucose intolerance) and hypothesize that the greater hypoglycemia upon fasting is the result of their inability to shutdown insulin release when glucose levels fall. These arguments are summarized in a generalized model in Figure 4A.

Since the insulin secretory responses to both the rise and fall of glucose are blunted in the knockout animals, these results suggested that while the mean values of blood glucose may be equivalent, the variance $(\sigma)$ of measured values in randomly feeding animals should be greater. In short, wild-type animals should display a much-tighter control of blood glucose than the knockout animals. Figure $4 \mathrm{~B}$ illustrates a series of measurements consistent with the hypothesis in which wild-type and Surl-knockout animals were given constant access to food and glucose measurements were carried out over an extended period. We attempted to "stress" the animals by increasing the sugar content of their diet through supplementing the drinking water with dextrose. The data indicate that the mean values of the wild-type and knockout animals are the same - that increasing glucose in the drinking water had no clear effect and that the standard deviations (error bars) of the knockout values are significantly greater than for the wild type. As a measure of this increased variation, we averaged all of the control and knockout values. The values were $8.55 \pm 1.67 \mathrm{vs.} 8.41 \pm 3.19 \mathrm{mM}$ for the control $(\mathrm{n}=5)$ and Surl-knockout $(\mathrm{n}=6)$ animals, respectively. The values for the variance are 2.77 vs. 10.2 for the control and knockout animals, respectively. 


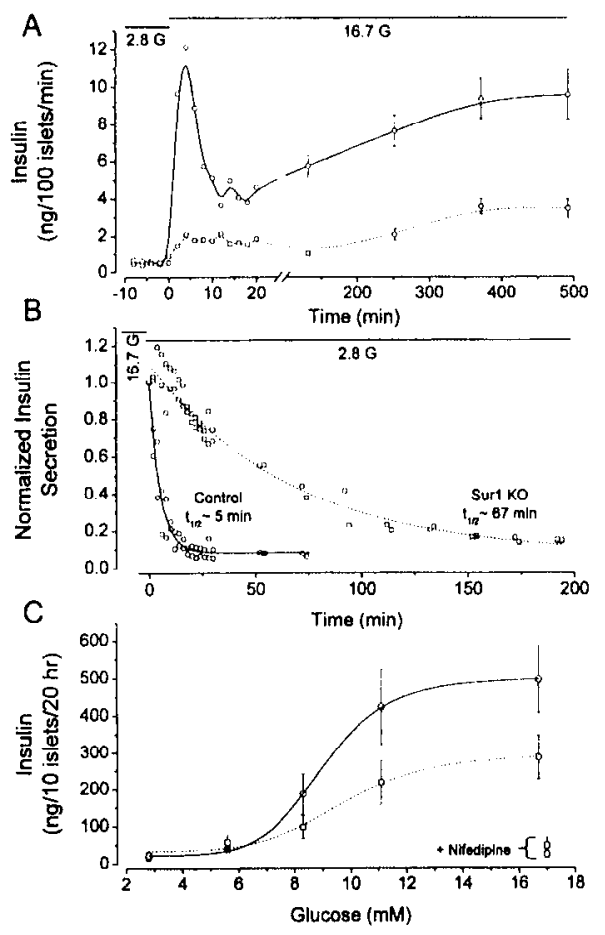

FIG. 3. Comparison of the insulin secretory response of control vs. Surl-knockuut islets. (A) Perifusion experiments that assess insulin output from isolated pancreatic islets as a function of time demonstrate that Surl-knockout islets lack first-phase insulin secretion and display an attenuated second-phase response to elevated glucose. Note the break in the time line at 25 minutes. The longer time values are the mean values of seven 1-minute time points flanking the given time, the error bars are \pm S.D. (B) At the end of the perifusion experiment shown in (A), the islets were switched to low glucose to assess their rates of return to basal secretory levels. For purposes of comparison, we have normalized the data to an initial value of 1 by dividing by the average value immediately before switching to $2.8 \mathrm{mM}$ glucose. Note that the absolute values of insulin output from control islets fall below that of the Sur $1^{-1}$ islets in approximately 5 minutes. The results from two separate experiments are plotted; the lines are the best fits of a monoexponential function to the combined data sets. The glucose concentrations in the perfusing medium in (A) and (B) are indicated by the bars. (C) Static incubation experiments demonstrate that the Surl islets display glucose-stimulated insulin secretion. Islets were cultured for 20 hours at the indicated glucose concentrations before assay of insulin released into the culture medium. At $16.7 \mathrm{mM}$ glucose, the values were $494.5 \pm 88.9 \mathrm{vs} .285 .1 \pm 62.0 \mathrm{ng} / \mathrm{ml} / 10$ islets $/ 20 \mathrm{hr}(\mathrm{n}=10$; mean \pm S.D.), respectively. The lines are best fits to a logistic equation; the half-maximal values are 9.0 and $9.8 \mathrm{mM}$ glucose for the control and SUR1 ${ }^{-t}$ groups, respectively. For comparative purposes, the mean values of blood glucose was $6.530 .99 \mathrm{mM}(\mathrm{n}=72)$ and did not differ significantly for randomly fed control vs. Sur ${ }^{1 /}$ animals. The + nifedipine samples $(1 \mu \mathrm{M})$ were incubated with the highest concentration of glucose tested $(16.7 \mathrm{mM})$. The error bars are \pm S.D. [Data from Seghers, V., Nakazaki, M., DeMayo, F., Aguilar-Bryan, L., and Bryan, J. J. Biol. Chem. 275, $9270-9277,2000$.] 
A
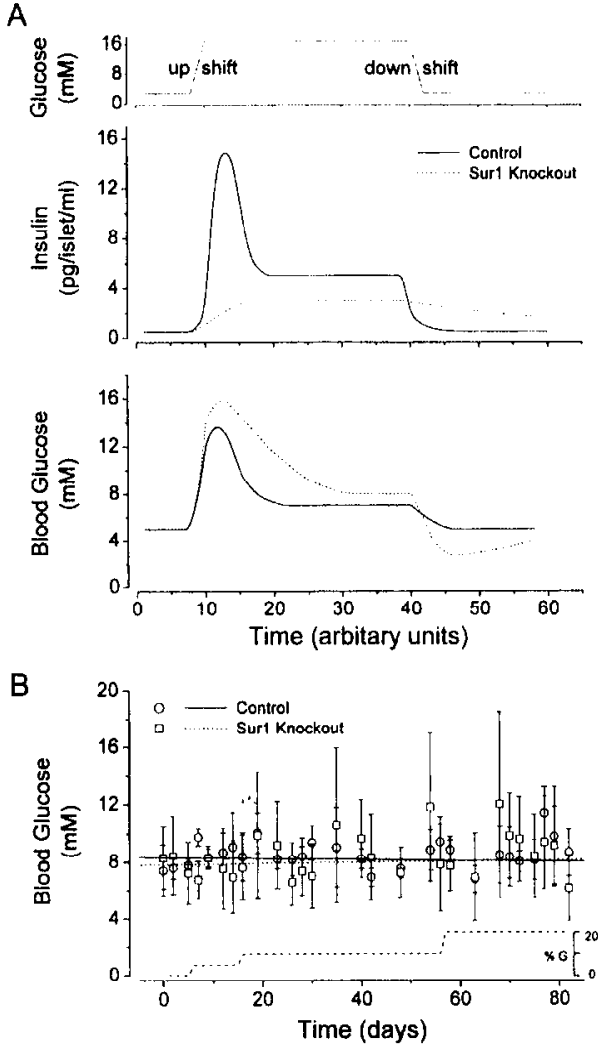

FIG. 4. Generalized insulin secretory responses and consequent blood glucose levels in Sur1knockout mice. The top panel in (A) illustrates hypothetical changes in glucose concentration as the result of glucose infusion into mice or through a change in the perifusion medium in isolated islet experiments. The middle panel illustrates the resulting insulin secretory response from control (solid line) or Sur 1-knockout islets (dotted line), in response to the shifts in glucose stimulation. The lower panel in (A) illustrates the proposed changes in blood glucose levels in response to insulin output. The loss of first-phase secretion in the Surl-knockout animals results in higher peak glucose levels and slower clearance of the glucose load. The reduced insulin output from the Surl-knockout islets would result in a higher steady-state glucose level. The impaired ability of the Sur 1-knockout islets to restrict insulin output during a fall in glucose concentration leads to more-pronounced hypoglycemia in the knockout animals, for example, during fasting. (B) The generalized results shown in (A) suggested that randomly feeding animals should display a wider variation in their blood glucose values. The results shown here are consistent with this hypothesis. Control (5) and knockout (6) animals were allowed free access to normal mouse chow; the drinking water was supplemented with glucose as indicated. Blood glucose values were taken at the indicated times. The mean values \pm S.D. are plotted for control and Surl-knockout animals. There was no significant effect of the additional glucose on the mean blood glucose levels but the knockout animals display an increased variation in their glucose levels vs controls 


\section{Compensation for Loss of $K_{A T P}$ Channels May Occur Shortly After Birth}

A comparison of the features of PHHI $\beta$ cells and neonates vs. mice missing $\mathrm{K}_{\mathrm{ATP}}$ channels indicates both have an equivalent $\beta$-cell signature. However, their insulin secretory responses and blood glucose values differ. Both the $K_{\mathrm{IR}} 6.2^{-/-}$and the Sur $1^{-/}$animals are hypoglycemic at birth but develop a compensatory mechanism to maintain normoglycemia. (We will restrict the specifics of this discussion to the SURI null animals, as Miki et al., 1998, have argued an alternative mechanism for the $\mathrm{K}_{\mathrm{IR}} 6.2$ null mice based on insulin hypersensitivity.) The Sur ${ }^{-1-}$ pups resolve their hypoglycemia within 2 to 3 days (the $\mathrm{K}_{\mathrm{IR}} 6.2^{-1-}$ pups display a similar pattern), while, for unknown reasons, PHHI neonates do not. Compensation requires that the knockout islets become refractory to elevated $\left[\mathrm{Ca}^{2+}\right]_{i}$ and "acquire" a $\mathrm{K}_{\mathrm{ATP}}$-independent mechanism for regulation of insulin release. It is not clear if the latter is always operative, perhaps during second-phase insulin release, or develops as a result of the loss of $\mathrm{K}_{\mathrm{ATP}}$ channels. We have pointed out that the pattern of insulin release in the Sur $1^{\%}$ mice is similar to the $\mathrm{K}_{\mathrm{ATP}}$-independent, $\mathrm{Ca}^{2+}$ - and glucose-dependent potentiation of insulin release observed from isolated islets by several groups (Panten et al., 1988; Best et al., 1992; Gembal et al., 1992; Straub et al., 1998a). However, it differs from the GTP-dependent, $\mathrm{Ca}^{2+}$-independent potentiation observed upon simultaneous activation of protein kinases A and C (Komatsu et al., 1995,1997a,1997b; Straub et al., 1998b) (see Aizawa et al., 1998, for a brief review of this area). We have begun to determine whether the Sur1 knockout animals retain sensitivity to other factors known to stimulate insulin release (Newgard and McGarry, 1995; Prenki, 1996; Sato and Henquin, 1998; Lang, 1999). Our preliminary comparison of the responsiveness of isolated islets to agents (i.e., IBMX, forskolin) that elevate cyclic AMP (cAMP) levels, thus activating cAMP-dependent protein kinases (PKA) and markedly stimulating glucose-induced insulin release from wild-type islets, shows the Surl knockout islets are poorly responsive to small changes (two- to five-fold) in cAMP but do respond to very high concentrations ( $>100$-fold increase). The Sur ${ }^{-1-}$ islets also display a reduced insulin secretory response to carbachol, although phorbol esters like trtradecanoyl phorbol acetate (TPA), known to activate protein kinase $\mathrm{C}$, can potentiate glucose-stimulated insulin secretion equally from both wild-type and Sur $1^{-/}$islets (Nakazaki et al., in preparation). The results are consistent with a reduced state of phosphorylation of protein(s) critical for insulin release. Alternatively, our results could be explained by an impairment in the cAMP-stimulated pathway that involves members of the novel Epac family of cAMP-dependent RapGEF's (Kawasaki et al., 1998; de Rooij et al., 1998). These proteins, Epac1 (cAMP-GEFI) and Epac2 (cAMP-GEFII), are closely related guanine-nucleotide exchange factors for the small GTPases Rap1 and Rap2 (de Rooij et al., 2000). Rapl has been implicated in insulin secretion (Leiser et al., 
1995; Kowluru et al., 1997). Clearly, myriad reasons could account for the impaired response of Sur $1^{-1-}$ islets to these various agents. With the available data, we cannot distinguish between possible mechanisms but hypothesize that the impaired responsiveness develops during the first few days of life in response to elevated cytosolic calcium and accounts for the resolution of the early hypoglycemia observed in the knockout animals.

\section{Summary}

The availability of cloned cDNAs encoding the subunits that comprise members of the $\mathrm{K}_{\mathrm{ATP}}$ channel family continues to provide insight into their structure and function. Studies over the past 5 years have illuminated the stoichiometry, biophysics, and pharmacology of $\mathrm{K}_{\mathrm{ATP}}$ channels and have identified functionally important regions of both subunits. Additionally, it is now clear that trafficking plays a major role in the corrcct asscmbly and surface expression of these channels. Endoplasmic reticulum retention/retrieval signals have been defined on both SUR 1 and $\mathrm{K}_{\mathrm{IR}} 6.2$ that insure that only completely assembled channels (SUR $\left.1 / \mathrm{K}_{\mathrm{IR}} 6.2\right)_{4}$, reach the cell surface. An additional motif has been identified in SUR1 that is required for surface expression and, when removed as a result of mutation, produces PHHI. The study of the functional properties of missense mutations that cause PHHI has provided insight into the mechanism by which the activity of these channels is coupled to glucose metabolism. Although PHHI neonates lacking $\mathrm{K}_{\mathrm{ATP}}$ channel activity are profoundly hypoglycemic, as a result of inappropriately high insulin secretion, recent work on knockout mice lacking these channels indicates that they are able to compensate using an as-yet-undefined, $\mathrm{K}_{\mathrm{ATP}}$-independent regulatory mechanism to maintain near-normal insulin and blood sugar levels. $\mathrm{K}_{\mathrm{ATP}}$-independent regulation in the knockout mice is glucose and $\mathrm{Ca}^{2+}$ dependent and is presumably equivalent to recently described

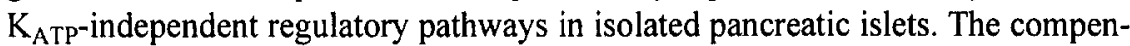
satory mechanism(s) are not known but preliminary results indicate that the loss of these channels and subsequent chronic increase in cytosolic calcium levels produce change beyond the simple loss of plasma membrane $\mathrm{K}^{+}$channels. Understanding these compensatory changes and learning how to activate them in PHHI neonates could provide a novel therapeutic approach for the treatment of this disorder.

\section{ACKNOWLEDGMENTS}

The authors thank Gabriela Gonzalez, Maria Janecki, Maria Shylapobersky, and Li-Zhen Song for their help and excellent technical assistance. 


\section{REFERENCES}

Aguilar-Bryan, L., and Bryan, J. (1996). Diabetes Rev. 4, 336-346.

Aguilar-Bryan, L., and Bryan, J. (1999). Fndocr. Rev. 20, 101-135

Aguilar-Bryan, L., Nichols, C.G., Wechsler, S.W., Clement, J.P. IV, Boyd, A.E. III, Gonzalez, G., Herrera-Sosa, H., Nguy, K., Bryan, J. and Nelson, D.A. (1995). Science 268, 423-426.

Aguilar-Bryan, L., Clement, J.P. IV, Gonzalez, G., Kunjilwar, K., Babenko, A., and Bryan, J. (1998). Physiol. Rev. 78, 227-245.

Aizawa, T., Komatsu, M., Asanuma, N., Sato, Y., and Sharp, G.W. (1998). TIPS 19, 496-499.

Atwater, I., Mears, D., and Rojas, E. (1996). In "Diabetes Mellitus" (D. LeRoith, S.I. Taylor, and J.M. Olefsky, eds.), pp. 78-102. Lippincott-Raven, Philadelphia.

Aynsley-Green, A., Hussain, K., Hall, J., Saudubray, J.M., Nihoul-Fekete, C., De Lonlay-Debeney, P., Brunelle, F., Otonkoski, T., Thornton, P., and Lindley, K.J. (2000). Arch. Dis. Child Fetal Neonatal. Ed. 82, F98-F107.

Babenko, A.P., Aguilar-Bryan, L., and Bryan, J. (1998a). Ann. Rev. Physiol. 60, 667-687.

Babenko, A.P., Gonzalez, G., Aguilar-Bryan, L., and Bryan, J. (1998b). Circ. Res. 83, 1132-1143.

Babenko, A.P., Gonzalez, G. and Bryan, J. (1999a). Biochem. Biophys. Res. Comm. 255, 231-238.

Babenko, A.P., Gonzalez, G., and Bryan, J. (1999b). FEBS Lett. 459, 367-376.

Babenko, A.P., Gonzalez, G., and Bryan, J. (1999c). J. Bicl. Chem. 274, 11587-11592.

Babenko, A.P., Gonzalez, G., and Bryan, J. (2000). J. Biol. Chem. 275, 717-720.

Baukrowitz, T., Schulte, U., Oliver, D., Herlitze, S., Krauter, T., Tucker, S.J., Ruppersberg, J.P., and Fakler, B. (1998). Science 282, 1141-1144.

Beguin, P., Nagashima, K., Nishimura, M., Gonoi, T., and Seino, S. (1999). EMBO J. 18, 4722-4732.

Best, L., Yates, A.P., and Tomlinson, S. (1992). Biochem. Pharmacol. 43, 2483-2485.

Bryan, J., and Aguilar-Bryan, L. (1997). Curr. Opin. Cell Biol. 9, 553-559.

Bryan, J., and Aguilar-Bryan, L. (1999). Biochim. Biophys. Acta 1461, 285-303.

Bryan, J., and Aguilar-Bryan (2000). In "Diabetes Mellitus" (D. LeRoith, S.I. Taylor, and J.M. Olefsky, eds.), edit. 2, pp. 61-78. Lippencott-Raven, Philadelphia.

Challinor-Rogers, J.L., and McPhorson, G.A. (1994). Clin. Expt. Pharmacol. Physiol. 21, 583-597.

Chan, C.B., MacDonald, P.E., Saleh, M.C., Johns, D.C., Marban, E., and Wheeler, M.B. (1999). Diabetes 48, 1482-1486.

Chutkow, W.A., Simon, M.C., Le Beau, M.M., and Burant, C.F. (1996). Diabetes 45, 1439-1445.

Chutkow, W.A., Makielski, J.C., Nelson, D.J., Burant, C.F., and Fan, Z. (1999). J. Biol Chem. 274, 13656-13665.

Clement, J.P. IV, Kunjilwar, K., Gonzalez, G., Schwanstecher, M., Panten, U., Aguilar-Bryan, L., and Bryan, J. (1997). Newron 18, 827-838.

Cook, D.L., and Hales, C.N. (1984). Nature 311, $271-273$.

Cook, D.L., and Taborsky, G.J. (1997). In "Elleberg and Rifkin's Diabetes Mellitus" (D. Porte and R.S. Sherwin, eds.), pp. 49-73. Appleton and Lange, Stamford, Conn.

de Rooij, J., Zwartkruis, F.J., Verheijen, M.H., Cool, R.H., Nijman, S.M., Wittinghofer, A., and Bos, J.L. (1998). Nature 396, 474-477.

de Rooij, J., Rehmann, H., van Triest, M., Cool, R.H., Wittinghofer, A., and Bos, J.L. (2000). J. Biol. Chem. 275, 20829-20836.

Detimary, P., Gilon, P., Nenquin, M., and Henquin, J.C. (1994). Biochem. J. 297, 455-461.

D'Hahan, N., Jacquet, H., Moreau, C., Catty, P., and Vivaudou, M. (1999). Mol. Pharmacol. 56, 308-315.

Dickinson, K.E., Bryson, C.C., Cohen, R.B., Rogers, L., Green, D.W., and Atwal, K.S. (1997), Mol. Pharmacol. 52, 473-481.

Doupnik, C.A., Davidson, N., and Lester, H.A. (1995). Curr. Opin. Neurobiol. 5, 268-277.

Dukes, I.D., and Philipson, L.H. (1996). Diabetes 45, 845-853. 
Dunne, M.J., and Petersen, O.H. (1986). FEBS Lett. 208, 59-62.

Dunne, M.J., Kane, C., Shepherd, R.M., Sanchez, J.A., James, R.F.L., Johnson, P.R.V., Aynsley-Green, A., Lu, S., Clement, J.P. IV, Lindley, K.J., Seino, S., and Aguilar-Bryan, L. (1997). N. Engl. J. Med. 336, 703-706.

Edwards, G., and Weston, A.H. (1995). Cardiovasc. Drugs Ther. 9, 185-193.

Fan, Z., and Makielski, J.C. (1997). J. Biol. Chem. 272, 5388-5395.

Gembal, M., Gilon, P., and Henquin, J.C. (1992). J. Clin. Invest. 89, 1288-1295.

Glaser, B. (2000). Semin. Perinatol. 24, 150-163.

Glaser, B., Kesavan, P., Heyman, M., Davis, E., Cuesta, A., Buchs, A., Stanley, C.A., Thornton, P.S., Permutt, M.A., Matschinsky, F.M., and Herold, K. (1998). N. Engl. J. Med. 338, 226-230.

Glaser, B., Landau, H., and Permutt, M.A. (1999). Trends Endocrinol. Metab. 10, 55-61.

Gopalakrishnan, M., Whiteaker, K.L., Molinari, E.J., Davis-Taber, R., Scott, V.E., Shieh, C.C., Buckner, S.A., Milicic, I., Cain, J.C., Postl, S., Sullivan, J.P., and Brioni, J.D. (1999). J. Pharmacol. Exp. Ther. 289, 551-558.

Gribble, F.M., Ashfield, R, and Ashcroft, F.M. (1999). Biophys. J. 76, Al4.

Hambrock, A., Loeffler-Walz, C., Kurachi, Y., and Quast, U. (1998). Br. J. Pharmacol. 125, 577-583.

Hambrock, A., Loffler-Walz, C., Kloor, D., Delabar, U., Horio, Y., Kurachi, Y., and Quast, U. (1999). Mol. Pharmacol. 55, 832-840.

Hopkins, W.F., Fatherazi, S., Peter-Riesch, B., Corkey, B.E., and Cook, D.L. (1992). J. Membr. Biol. 129, 287-295.

Inagaki, N., Gonoi, T., Clement, J.P. IV, Namba, N., Inazawa, J., Gonzalez, G., Aguilar-Bryan, L., Seino, S., and Bryan, J. (1995a). Science 270, 1166-1170.

Inagaki, N., Tsuura, Y., Namba, N., Masuda, K., Gonoi, T., Horie, M., Seino, Y., Mizuta, M. and Seino, S. (1995b). J. Biol. Chem. 270, 5691-5694.

Inagaki, N., Gonoi, T., Clcment, J.P., Wang, C.Z., Aguilar-Bryan, L., Bryan, J., and Seino, S. (1996). Neuron 16, 1011-1017.

Inagaki, N., Gonoi, T., and Seino, S. (1997). FEBS Lett. 409, 232-236.

Isomoto, S., Kondo, C., Yamada, M., Matsumoto, S., Higashiguchi, O., Horio, Y., Matsuzawa, Y., and Kurachi., Y. (1996). J. Biol. Chem. 271, 24321-24324.

Kaiser, N., Corcos, A.P., Tur, S.A., Ariav, Y., Glaser, B., Landau, H., and Cerasi, E. (1990). Diabetologia 33, 482-488.

Kane, C., Shepherd, R.M., Squires, P.E., Johnson, P.R., James, R.F., Milla, P.J., Nynsley-Green, A., Lindley, K.J., and Dunne, M.J. (1996). Nature Med. 2, 1344-1347.

Kawasaki, H., Springett, G.M., Mochizuki, N., Toki, S., Nakaya, M., Matsuda, M., Housman, D.E., and Graybiel, A.M. (1998). Science 282, 2275-2279.

Komatsu, M., Schermerhorn, T., Aizawa, T., and Sharp, G.W. (1995). Proc. Natl. Acad. Sci. U.S.A. 92 , 10728-10732.

Komatsu, M., Schermerhorn, T., Noda, M., Straub, S.G., Aizawa, T., and Sharp, G.W. (1997a). Diabetes 46, 1928-1938.

Komatsu, M., Sharp, G.W., Aizawa, T., and Hashizume, K. (1997b). Jpn. J. Physiol. 47, S22-S24.

Koster, J.C., Sha, Q., Shyng, S., and Nichols, C.G. (1999). J. Physiol. (Lond). 515, 19-30.

Kowluru, A., Li, G., Rabaglia, M.E., Segu, V.B., Hofmann, F., Aktories, K., and Metz, S.A. (1997) Biochem. Pharmacol. 54, 1097-1108.

Lang, J. (1999). Eur. J. Biochem. 259, 3-17.

Leiser, M., Efrat, S., and Fleischer, N. (1995). Endocrinology 136, 2521-2530.

Lin, Y.F., Jan, Y.N., and Jan, L.Y. (2000). EMBO J. 19, 942-955.

Liss, B., Bruns, R., and Roeper, J. (1999). EMBO J. 18, 833-846.

Loffler-Walz, C., and Quast, U. (1998). Br. J. Pharmacol. 123, 1395-1402.

Matschinsky, F.M. (1996). Diabetes 45, 223-241. 
Mears, D., and Atwater, I. (2000). In "Diabetes Mellitus" (D. LeRoith, S. I. Taylor and J. M. Olefsky, eds.), edit. 2, pp. 47-61. Lippincott Williams \& Wilkins, Philadelphia.

Meyer, M., Chudziak, F., Schwanstecher, C., Schwanstecher, M., and Panten, U. (1999). Br. J. Pharmacol. 128, 27-34.

Miki, T., Nagashima, K., Tashiro, F., Kotake, K., Yoshitomi, H., Tamamoto, A., Gonoi, T., Iwanaga, T., Miyazaki, J., and Seino, S. (1998). Proc. Natl. Acad. Sci. U.S.A. 95, 10402-10406.

Miller, T.R., Taber, R.D., Molinari, E.J., Whiteaker, K.L., Monteggia, L.M., Scott, V.E., Brioni, J.D., Sullivan, J.P., and Gopalakrishnan, M. (1999). Eur. J. Pharmacol. 370, 179-185.

Misler, S., Falke, L.C., Gillis, K., and McDaniel, M.L. (1986). Proc. Natl. Acad. Sci. U.S.A. 83, 7119-7123.

Nelson, D.A., Bryan, J., Wechsler, S., Clement, J.P. IV, and $\Lambda$ guilar-Bryan, L. (1996). Biochemistry 35, 14793-14799.

Nelson, M.T., and Quayle, J.M. (1995). Am. J. Phys. 268, C799-C822.

Nestorowicz, A., Inagaki, N., Gonoi, T., Schoor, K.P., Wilson, B.A., Glaser, B., Landau, H., Stanley, C.A., Thornton, T.S., Seino, S., and Permutt, M.A. (1997). Diabetes 46, 1743-1748.

Newgard, C.B., and McGarry, J.D. (1995). Ann. Rev. Biochem. 64, 689-719.

Nichols, C.G., and Lopatin, A.N. (1997). Ann. Rev. Physiol. 59, 171-191.

Nichols, C.G., Shyng, S.L., Nestorowicz, A., Glaser, B., Clement, J.P. IV, Gonzalez, G., Aguilar-Bryan, L., Permutt, M.A., and Bryan, J. (1996). Science 272, 1785-1787.

Noma, A. (1983). Nature 305, 147-148.

Otonkoski, T., Ammala, C., Huopio, H., Cote, G.J., Chapman, J., Cosgrove, K., Ashfield, R., Huang, E., Komulainen, J., Ashcroft, F.M., Dunne, M.J., Kere, J., and Thomas, P.M. (1999). Diabetes 48, 408-415.

Panten, U., Schwanstecher, M., Wallasch, A., and Lenzen, S. (1988). Naunyn Schmiedebergs Arch. Pharmacol. 338, 459-462.

Permutt, M.A., Nestorowicz, A., and Glaser, B. (1996). Diabetes Rev. 4, 347-355.

Prenki, M. (1996). Eur. J. Endocrinol. 134, 272-286.

Quast, U., Guillon, J.M., and Cavero, I. (1994). Cardiovasc. Res. 28, 805-810.

Quayle. J.M., and Standen, N.B. (1994). Cardiovasc. Res. 28, 797-804.

Raab-uraham, K.F., Cirilo, L.J., Boettcher, A.A., Radeke, C.M., and Vandenberg, C.A. (1999). J. Biol. Chem. 274, 29122-29129.

Repunte, V.P., Nakamura, H., Fujita, A., Horin, Y., Findlay, I., Pott, L., and Kurachi, Y. (1999). EMBO J. 18, 3317-3324.

Ryan, F., Devaney, D., Joyce, C., Nestorowicz, A., Permutt, M.A., Glaser, B., Barton, D.E., and Thornton, P.S. (1998). Arch. Dis. Child. 79, 445-447.

Sato, Y., and Henquin, J.C. (1998). Diabetes 47, 1713-1721.

Schwanstecher, M., Sieverding, C., Dorschner, H., Gross, I., Aguilar-Bryan, L., Schwanstecher, C., and Bryan, J. (1998). EMBO J. 17, 5529-5535.

Schwappach, B., Zerangue, N., Jan, Y.N., and Jan, L.Y. (2000). Neuron 26, 155-167.

Seghers, V., Nakazaki, M., DeMayo, F., Aguilar-Bryan, L., and Bryan, J. (2000). J. Biol. Chem. 275, 9270-9277.

Sharma, N., Crane, A., Clement, J.P. IV, Gonzalez, G., Babenko, A.P., Bryan, J., and Aguilar-Bryan, L. (1999). J. Biol. Chem. 274, 20628-20632.

Shepherd, R.M., Cosgrove, K.E., O'Brien, R.E., Barnes, P.D., Ammala, C., and Dunne, M.J. (2000) Arch. Dis. Child. Fetal. Neonatal. Ed. 82, F87-F97.

Shyng, S., and Nichols, C.G. (1997). J. Gen. Physiol. 110, 655-664.

Shyng, S.L., and Nichols, C.G. (1998). Science 282, 1138-1141.

Shyng, S.L., Ferrigni, T., Shepard, J.B., Nestorowicz, A., Glaser, B., Permutt, M.A., and Nichols, C.G (1998). Diabetes 47, 1145-1151.

Stanley, C.A., Lieu, Y., Hsu, B., and Poncz, M. (1997). Diabetes 46, 217A. 
Straub, S.G., James, R.F., Dunne, M.J., and Sharp, G.W. (1998a). Diabetes 47, 758-763.

Straub, S.G., James, R.F., Dunne, M.J., and Sharp, G.W. (1998b). Diabetes 47, 1053-1057.

Tanabe, K., Tucker, S.J., Matsuo, M., Proks, P., Ashcroft, F.M., Seino, S., Amachi, T., and Ueda, K. (1999). J. Biol Chem. 274, 3931 -3933.

Thomas, P.M., Cote, G.J., Wohllk, N., Haddad, B., Mathew, P.M., Rabl, W., Aguilar-Bryan, L., Gagel, R.F., and Bryan, J. (1995). Science 268, 426-429.

Trube, G., and Hescheler, J. (1983). Naunyn-Schmiedeberg 's Arch. Pharmacol. 322, R64.

Trube, $\mathrm{G}_{\text {. }}$ and Hescheler, J. (1984). Pflugers Arch. 401, 178-184.

Tucker, S.J., Gribble, F.M., Zhao, C., Trapp, S., and Ashcroft, F.M. (1997). Nature 387, 179-183

Tucker, S.J., Gribble, F.M., Proks, P., Trapp, S., Ryder, T.J., Haug, T., Reimann, F., and Ashcroft, F.M. (1998). EMBO J. 17, 3290-3296.

Tusnady, G.E., Bakos, E., Varadi, A., and Sarkadi, B. (1997). FEBS Lett. 402, 1-3.

Ueda, K., Inagaki, N., and Seino, S. (1997). J. Biol. Chem. 272, 22983-22986.

Ueda, K., Komine, J., Matsuo, M., Seino, S., and Amachi, T. (1999). Proc. Natl. Acad. Sci. U.S.A. 96, 1268-1272.

Uhde, I., Toman, A., Gross, I., Schwanstecher, C., and Schwanstecher, M. (1999). J. Biol. Chem. 274, 28079-28082.

Verkarre, V., Fournet, J.C., de Lonlay, P., Gross-Morand, M.S., Devillers, M., Rahier, J., Brunelle, F., Robert, J.J., Nihoul-Fekete, C., Saudubray, J.M., and Junien, C. (1998). J. Clin. Invest. 102, 1286-1291.

Weinzimer, S.A., Stanley, C.A., Berry, G.T., Yudkoff, M., Tuchman, M., and Thornton, P.S. (1997). J. Pediatr. 130, 661-664.

Yamada, M., Isomoto, S., Matsumoto, S., Kondo, C., Shindo, T., Horie, Y., and Kurachi, Y. (1997). J. Physiol. 499, 715-720.

Yokoshiki, H., Sunagawa, M., Seki, T., and Sperelakis, N. (1999). Pflugers Arch. 437, 400-408.

Zammarchi, E., Filippi, L., Novembre, E., and Donati, M.A. (1996). Metabolism 45, 957-960.

Zerangue, N., Schwappach, B., Jan, Y.N., and Jan, L.Y. (1999). Neuron 22, 537-548.

Zhang, C.Y., De-Leo, D., Joseph, J., Boss, O., Hagen, T,, Grujic, D., Vidal-Puig, A.J., Chan, C.B., Wheeler, M., and Lowell, B.B. (2000). In "Program \& Abstracts of the 82nd Annual Meeting of The Endocrine Society," June 2000, Toronto, Canada, abstract OP274, p. 75. 


\title{
Genetics of Type 1A Diabetes
}

\author{
Maria J. Redondo, Pamela R. Fain, and George S. Eisenbarth \\ Barbara Davis Center for Childhood Diabetes, University of Colorado Health Sciences Center, \\ 4200 East 9 th Avenue, Denver, Colorado 80262
}

\begin{abstract}
Type $1 \mathrm{~A}$ diabetes is an autoimmune disease with genetic and environmental factors contributing to its etiology. Twin studies, family studies, and animal models have helped to elucidate the genetics of autoimmune diabetes. Most of the genetic susceptibility is accounted for by human leukocyte antigen (HLA) alleles. The most-common susceptibility haplotypes are DQA $1 * 0301-\mathrm{DQB} 1 * 0302$ and DQA 1*0501-DQB1*0201. Less-common haplotypes such as DQA1*0401-DQB1*0402 and DQA I*0101-DQBI*0501 are associated with high risk for diabetes; however, large study populations are needed to analyze their effect. The DQA1*0102-DQB1*0602 haplotype is associated with diabetes resistance. DR molecules, such as DRBI*1401, confer protection from diabetes. Monozygotic twins of patients with type $1 \mathrm{~A}$ diabetes have a diabetes risk higher than that for HLA-identical ordinary siblings, suggesting that non-HLA genes contribute to diabetes risk. Polymorphisms in the regulatory region of the insulin gene (designated IDDM2), polymorphisms in cytotoxic T lymphocyte antigen-4 (CTLA-4) gene (IDDM12), and other genes are likely to contribute to diabetes risk and susceptibility in some individuals. In selected families, major diabetogenes (e.g., IDDM17, autoimmune regulator gene (AIRE)) are likely to be of importance. Other factors - either noninherited genes (i.e., somatic mutations and T-cell receptor or inmunoglobulin rearrangements) or environment - may have a rolc in progression to diabetes. This is suggested by the finding that the risk for monozygotic twins of patients with type $1 \mathrm{~A}$ diabetes is not 100 percent. Studying the genetics of type $1 \mathrm{~A}$ diabetes will allow us to better define this disease, to improve our ability to identify individuals at risk, and to predict the risk of associated disorders.
\end{abstract}

\section{Introduction}

Type 1A diabetes, formerly known as juvenile-onset diabetes or insulin-dependent diabetes mellitus (IDDM), is defined as immune-mediated diabetes (American Diabetes Association, 1997). Serum anti-islet autoantibodies, a marker of immune diabetes, are found in more than 90 percent of European patients with childhood-onset diabetes. Some cases of autoantibody-negative diabetes are clinically similar to those with positive autoantibodies (e.g., appear in lean, young individuals who need insulin therapy to avoid ketosis). At least some of these cases are likely to have immune-mediated diabetes in which current techniques cannot detect serum autoantibodies. The remaining cases of autoantibody-negative diabetes in children correspond to diabetes that is distinguishable from type 
1A diabetes: type 1B diabetes, type 2 diabetes, maturity onset diabetes of the young (MODY), cystic fibrosis-related diabetes, or other defined forms of diabetes. Type $1 \Lambda$ diabetes has been described among adults. Probably as many adults as children develop type $1 \mathrm{~A}$ diabetes, although type 2 diabetes is far more prevalent in adults than type $1 \mathrm{~A}$.

Knowlcdge of the genetics of type 1A diabetes allows better disease definition and improved ability to identify individuals at risk of diabetes and its associated disorders.

\section{Twin Studies}

Twin sludies help define the role of genetic and environmental factors in the etiology of disease (Martin et al., 1997; Phillips, 1993; Smith, 1974). In a primarily genetic disorder, concordance (i.e., both twins affected) is higher in monozygotic (identical) than dizygotic (fraternal) twins. Differences between monozygotic twins must be due to environmental factors, to genetic factors not coded in the germ line (e.g., somatic mutations), or to genes that undergo random rearrangement (e.g., immunoglobulin, T-cell receptor genes). Limitations of twin studies are small sample sizes, assignment of zygosity, and a patient-referral bias leading to over-ascertainment of concordant twin pairs (Hawkes, 1997; Phillips, 1993; Rowe and Leslie, 1995).

The problem of over-ascertainment has been addressed by analyzing twins identified through national registries (Petersen et al., 1997; Kyvik et al., 1995) and prospective studies of twins discordant for diabetes when recruited (Srikanta et al., 1983; Verge et al., 1995; Hawa et al., 1997). Concordance rates for type 1A diabetes have been reported among dizygotic twins of between zero percent (Hawa et al., 1997) and 13 percent (Kumar et al., 1993; Lorenzen et al., 1994; Redondo et al., 1999a). The concordance rate among monozygotic twins has been reported between 21 percent and 70 percent (Verge et al., 1995; Hawa et al., 1997; Srikanta et al., 1983; Kyvik et al., 1995; Redondo et al., 1999b). We have observed that monozygotic twins may become concordant many years after diagnosis in the index twin (the first twin to be diagnosed). Studies with long-term follow-up and lifetable projections have the highest concordance in monozygotic twins (Petersen et al., 1997; Verge et al., 1995). Nondiabetic monozygotic twins of patients with type $1 \mathrm{~A}$ diabetes show a high prevalence of anti-islet autoantibodies, between 42 and 76 percent (Verge et al., 1995; Hawa et al., 1997; Petersen et al., 1997). The appearance of multiple autoantibodies is followed in most instances by progression to diabetes (Verge et al., 1995; Hawa et al., 1997).

We recently assembled a series of nondiabetic dizygotic twins of patients with type 1A diabetes to compare with our series of monozygotic twins. Anti-islet autoantibody expression was analyzed in nondiabetic monozygotic twins $(n=53)$, dizygotic twins $(n=30)$, and siblings $(n=149)$ of patients with type 1 A diabetes, 
plus 101 controls (Redondo et al., 1999a). To reduce a potential patient-referral bias, we included only those pairs that were discordant at referral (i.e., one twin was diabetic and the other was not). Monozygotic twins had a much-higher risk of progressing to diabetes and expressing autoantibodies than did dizygotic twins. This finding is consistent with previous studies (Kaprio et al., 1992; Kumar et al., 1993; Hawa et al., 1997; Petersen et al., 1997) and supports the hypothesis of an important role for genetic factors in the determination of anti-islet autoimmunity. The risk for dizygotic twins and siblings was similar. This latter observation suggests that environmental factors, shared by dizygotic twins more than by ordinary siblings, does not increase progression to diabetes. There was also a higher penetrance of anti-islet autoimmunity in monozygotic twins who were heterozygous for the two high-risk human leukocyte antigen (HLA) haplotypes, DQ2/DQ8. Johnston et al. (1983) found a higher concordance for monozygotic twins with HLA DR3/DR4, the alleles most-frequently found on the same haplotype with DQ2/DQ8. This finding further supports the hypothesis that islet-cell autoimmunity may be predominantly genetically determined.

The observation that the concordance rate in monozygotic twins is not 100 percent indicates that nongermline (i.e., noninherited) genetic or environmental factors play a role. We recently combined two series, from Britain and the United States, to provide a database with 187 monozygotic twins of patients with type 1 A diabetes, with many twin pairs followed for up to 40 years (Redondo et al., 1999b). Progression to diabetes was similar in both series of monozygotic twins from the two countries. The hazard rate of progression to diabetes was higher within 10 years from diagnosis in the index twin, in line with observations by Olmos and coworkers (1998). However, progression to diabetes may happen many years after diagnosis in the index twin. In the combined series from Britain and the United States, 20 percent of the twins who progressed did so after more than 14 years of discordance, consistent with our previous studies (Verge et al., 1995). In addition we observed a higher progression rate to diabetes for twins whose index twin developed diabetes at a younger age.

Studies of identical twins of patients with type 1A diabetes provided the first evidence that the disorder developed chronically with progressive loss of firstphase insulin secretion (Srikanta, 1983). The observation that linear loss of firstphase insulin secretion preceded diabetes has been confirmed by Colman and coworkers (2000) in studies of first-degree relatives.

\section{Family Studies}

The observation of familial clustering of type $1 \mathrm{~A}$ diabetes is a strong indication that genetic factors are involved in the etiology of type 1A diabetes. Eightyfive percent of newly diagnosed cases are sporadic; that is, the patients do not have a first-degree relative with type $1 \mathrm{~A}$ diabetes. In spite of this, first-degree 
relatives of patients with type $1 \mathrm{~A}$ diabetes are at increased risk of developing positive autoantibodies and diabetes, compared to the general population. The familial clustering $\left(\lambda_{\mathrm{s}}\right)$ for a given disease can be calculated as the ratio of the risk for siblings divided by the prevalence in the general population (Risch et al., 1993; Todd and Farrall, 1996). The risk of type 1A diabetes in the general population is 0.4 percent, while the risk for siblings of affected individuals is approximately 6 percent. Thus, $\lambda_{\mathrm{s}}$ for type $1 \mathrm{~A}$ diabetes is approximately 15 times as great. The offspring of a mother with type 1A diabetes has a 1.3-4 percent risk of developing type $1 \mathrm{~A}$ diabetes. 'The risk for the offspring of a father with type $1 \mathrm{~A}$ diabetes is approximately six to nine percent (Warram et al., 1984; Tillil and Kobberling, 1987; Bleich et al., 1993). This parent-of-origin difference in transmission of diabetes is observed also for expression of anti-islet autoantibodies. The frequency and levels of expression of islet autoantibodies (glutamic acid decarboxylase (GAD)65, insulin antibodies (IAA), and islet cell antibodies (ICA)) were increased in 148 offspring of 103 fathers with diabetes, compared to 144 offspring of 102 mothers with diabetes (Yu et al., 1995).

The gender bias in transmission rate of diabetes has not been fully explained. Environmental hypotheses include maternal sex hormones modulating susceptibility in utero, immunologic tolerance to islet autoantigens, or selective fetal loss of potentially diabetic offspring (Warram et al., 1988). We did not find evidence for an effect of duration of diabetes in mothers before pregnancy nor the presence vs. the absence of diabetes at the time of the pregnancy (Yu et al., 1995). Genetic hypotheses for this parent-of-origin effect include genomic imprinting. Imprinting has been reported for a $19-\mathrm{kb}$ region in the insulin-like growth factor- 2 associated with diabetes risk (Baker et al., 1995). We found evidence for maternal imprinting of the insulin gene, with the B FOK allele of the insulin gene associated with protection only upon paternal transmission (Schwartz, 1990). It is not known how this observation correlates with the contradictory finding of a higher risk of diabetes transmission to offspring from diabetic fathers, compared to diabetic mothers.

\section{Genes Underlying Diabetes Susceptibility}

\section{A. MAJOR HISTOCOMPATIBILITY COMPLEX (MHC): DESCRIPTION AND NOMENCLATURE}

Peptides bound to MHC class I molecules are recognized by CD8 $\mathrm{T}$ cells. Antigens bound to MHC class II molecules are recognized by CD4 T cells. Nearly all nucleated cells express MHC class I molecules. MHC class II molecules are expressed by $\mathrm{B}$ lymphocytes, macrophages, and other antigen-presenting cells, and, in humans, by activated $\mathrm{T}$ lymphocytes. In addition, most epithelial cells can 
be induced to express class II molecules. The MHC molecules (in humans, also called HLA, human leukocyte antigen) are highly polymorphic; multiple different peptides can be presented to $T$ cells. MHC polymorphism results from different genes encoding families of MHC molecules, with each of these genes having multiple, germline-encoded alleles (Figure 1). Both MHC class I and class II moleculcs consist of two chains, alpha and beta. The alpha chain of MHC class I and both chains of MHC class II are encoded by genes within the MHC region on chromosome 6. The beta chain of MHC class I (beta-2-microglobulin), which is not polymorphic, is encoded by a genc on chromosome 15 . The MHC region in humans contains more than 100 genes, including HLA genes, extending over $4 \times 10^{6}$ base pairs of DNA. The alpha chains of three major MHC class I molecules are encoded by three different genes, HLA-A, HLA-B, and HLA-C. Each allele is identified by a unique number corresponding to a unique nucleotide sequence of the alpha chain (e.g., HLA-B0801).

The class II molecules, also known as immune response genes, are termed DR, DP, and DQ. Only the beta chain in DR molecules is polymorphic. Thus, different $D R$ molecules are identified by one number denoting the allele encoding the beta chain (e.g., DRB ${ }^{*} 0401$ ). Both beta and alpha chains in DQ molecules are polymorphic. The resulting DQ molecule is therefore identified by two numbers (e.g., DQA 1*0301, DQB1*0302). Additional genes within the MHC encode other molecules involved in the immune response, such as transporters associated

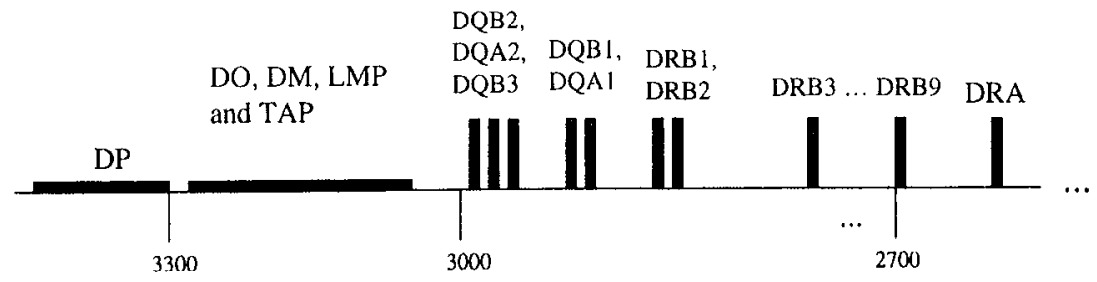

Class II

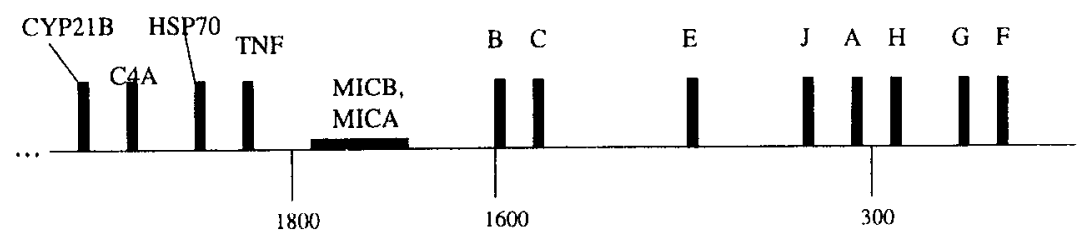

Class III

Class I

FIG. 1. Outline of the major histocompatibility complex region on chromosome 6 . 
with antigen processing (TAP-1 and TAP-2), the DM molecule, and proteosome subunits. The MHC class III region encodes complement and other genes.

\section{B. HLA AND TYPE 1A DIABETES}

\section{HLA Molecules Associated with Diabetes Susceptibility or Resistance}

The association of HLA alleles and type 1A diabetes was recognized more than 20 years ago by Nerup and co-workers (Nerup et al., 1974). Polyglandular failure syndrome type 2 , a constellation of autoimmune disorders associated with type $1 \mathrm{~A}$ diabetes, was also associated with HLA class I alleles, specifically, HLA-B8 (Eisenbarth et al., 1978). HLA class II alleles were found to be moresignificantly associated with type $1 \mathrm{~A}$ diabetes than class I alleles. It is currently believed that approximately half of the familial aggregation of type 1 diabetes is determined by HLA genes.

HLA DR 4 and DR3 are strongly associated with type 1A diabetes. However, this association is likely in part secondary to linkage disequilibrium (nonrandom association of alleles) between DR and DQ (Park et al., 1998). A haplotype is defined as a series of alleles of different genes on a chromosomal segment. HLA DRB1*0401, ${ }^{*} 0402$, and ${ }^{*} 0405$ alleles with DQA $1{ }^{*} 0301-\mathrm{DQB} 1{ }^{*} 0302$ (also called DQ8) are associated with high risk for diabetes. HLA DRB1*0301DQA 1*0501-DQB1*0201 (also called DQ2) is another high-risk haplotype. Ninety percent of individuals with type 1A diabetes have at least one of these two high-risk HLA haplotypes, compared to 20 percent of the general population. Approximately 35 percent of U.S. patients with type 1A diabetes are "DR3/DR4" heterozygotes, vs. only 2.4 percent of the general population. Overall, siblings of a patient with type $1 \mathrm{~A}$ diabetes have a risk of diabetes of approximately 6 percent. However, if a sibling is HLA identical to a sibling with type $1 \mathrm{~A}$ diabetes, the estimated risk increases to 20 percent. If the nonaffected sibling of a patient with diabetes carries both high-risk haplotypes DR4-DQA 1*0301-DQB1*0302 and HLA DR3-DQA $1 * 0501-\mathrm{DQB} 1 * 0201$, the risk of diabetes is approximately 25 to 40 percent. The risk conferred by the DQ8 molecule is modified by the DRB1 allele present on the same haplotype. The DRB $1 * 0402,{ }^{*} 0401$, and ${ }^{*} 0405$ alleles are associated with high susceptibility, the DRB ${ }^{*} 0404$ with moderate susceptibility, and the DRB1*0403 with "protection" against type 1A diabetes (Park et al., 1998). The presence of the HLA-DR4-DQ8 haplotype in first-degree relatives of patients with type 1A diabetes is associated with anti-insulin and ICA512 autoantibodies, both in individuals who developed diabetes on follow-up and in those who did not (Ziegler et al., 1991). In another study where 72 ICA-positive, first-degree relatives and 126 autoantibody-negative, first-degree relatives were studied, the frequency of the high-risk haplotypes was higher among ICA-positive relatives, compared to autoantibody-negative relatives (Pugliese et al., 1995). In 
addition, first-degree relatives with the high-risk allele DQB1*0302 progressed to diabetes at a younger age than relatives without this allele. The relatives with the high-risk allele DQB1*0201 without DQB 1*0302 progressed at an older age, compared to those relatives without it.

Uncommon HLA alleles also significantly affect diabetes risk of protection. However, large study populations are needed to identify their influence. The Human Biological Data Interchange (HBDI) type 1 repository has made it possible to analyze transmission of DQ haplotypes to affected and unaffected offspring from 276 families. The HBDI created a repository for DNA and cell lines in part for the purpose of mapping non-HLA genes associated with type 1A diabetes by linkage analysis. Over 95 percent of the HBDI families were multiplex; that is, ascertained with two or more affected siblings. Spurious association can result from artifacts of population structure, such as admixture or stratification. The transmission disequilibrium test (TDT) has been used to help distinguish between association due to linkage and spurious association (Spielman and Ewens, 1996). Under the null hypothesis of no linkage, a haplotype is expected to be transmitted 50 percent of the time from a heterozygous parent to an offspring. The TDT compares the observed transmission frequency to diabetic offspring against the expected 50 percent. A significant TDT result could be due to meiotic segregation distortion. If so, both affected and unaffected offspring would have this distortion. To rule out this possibility, the haplotype transmission frequencies to affected and unaffected offspring are compared by a $2 \times 2$ contingency chi-square test. We used the TDT analysis to assess the transmission of DQ haplotypes from heterozygous parents to children with diabetes and to their nondiabetic siblings. The rank order of transmission to affected offspring was DQA 1*0301-DQB1*0302 (associated with the highest risk), DQA1*0501-DQB 1*0201, DQA1*0401-DQB 1*0402, and DQA 1*0101-DQB $1 * 0501$. The DQ haplotype that was least-often transmitted to affected offspring was the well-known protective DQA 1*0102-DQB1*0602, followed by DQA 1*0103-DQB1*0603.

HLA alleles also confer protection from diabetes (Pugliese et al., 1995; Baisch et al., 1990). The best-known "protective" allele is DQB 1*0602, usually found on "DR2" (DRB1*1501-DQA1*0102-DQB1*0602) haplotypes. Approximately 20 percent of Americans and Europeans have DQA ${ }^{*} 0102-D Q B 1 * 0602$, while less than one percent of children with type $1 \mathrm{~A}$ diabetes carry these alleles. Table I illustrates the risk or protection associated with different HI.A alleles. The protection conferred by the DQA 1*0102-DQB1*0602 haplotype appears to be dominant, since it is protective even in the presence of a high-risk haplotype in the same individual. Of note, the frequency of the protective allele $\mathrm{DQB} 1 * 0602$ was similar among 72 ICA-positive, first-degree relatives and 126 first-degree relatives who were autoantibody negative (Pugliese et al., 1995). Progression to diabetes is, however, rare in 0602-positive, first-degree relatives, even in the 
TABLE 1

Risk of Type IA Diabetes Associated with DR and DQ Alleles

\begin{tabular}{|c|c|c|}
\hline DQAl & DQB1 & DRB \\
\hline \multicolumn{3}{|c|}{ High Risk } \\
\hline 0301 & 0302 & 0401 \\
\hline 0301 & 0302 & 0402 \\
\hline 0301 & 0302 & 0405 \\
\hline 0501 & 0201 & 0301 \\
\hline \multicolumn{3}{|c|}{ Moderate Risk } \\
\hline 0401 & 0402 & 0801 \\
\hline 0101 & 0501 & 0101 \\
\hline 0301 & 0303 & 0901 \\
\hline \multicolumn{3}{|c|}{ Weak or Moderate Protection } \\
\hline 0301 & 0302 & 0403 \\
\hline 0201 & 0201 & 0701 \\
\hline 0501 & 0301 & 1101 \\
\hline \multicolumn{3}{|c|}{ Strong Protection } \\
\hline 0102 & 0602 & 1501 \\
\hline 0101 & 0503 & 1401 \\
\hline 0201 & 0303 & 0701 \\
\hline
\end{tabular}

presence of positive autoantibodies. Interestingly, autoantibody-positive, 0602positive patients who did not develop diabetes had a restricted pattern of ICA staining, associated with high titers of GAD65 autoantibodies (Verge et al., 1996). Among ICA-positive, 0602-positive relatives, there is a more-limited response to islet antigens, often directed to GAD, compared to ICA-positive relatives without the 0602 allele (Gianani et al., 1992,1996).

A progressive decrease in first-phase insulin release (FPIR) on intravenous glucose tolerance testing (IVGTT) predicts progression to diabetes. DQB ${ }^{*} 0602$ positive, ICA-positive relatives were more likely to have a normal insulin secretion on IVGTT. In another study among individuals identified through the Dia- 
betes Prevention Trial, 0602-positive individuals were less likely to have positive insulin autoantibodies or low FPIR than relatives without DQB 1*0602 (Greenbaum et al., 2000). However, 29 percent of the ICA-positivc, 0602-positive relatives did have insulin autoantibodies or low FPIR, defined as below the 10th percentile. These findings suggest that the protective effect of the DQB $1 * 0602$ allele is likely to occur after the autoimmune destruction of the pancreas has begun. The presence of $\mathrm{DQB} 1 * 0602$ or sequence-related alleles protects from diabetes also in patients with stiff-man syndrome, who are otherwise at increased risk for type $1 \mathrm{~A}$ diabetes. Of note, this syndrome is associated with extremely high values of GAD autoantibodies.

It was reported that four patients with type $1 \mathrm{~A}$ diabetes who were initially found to have the conventional DQB1*0602 with standard sequence-specific oligonucleotide (SSO) typing techniques actually had novel variants of this allele with sequencing (Hoover and Marta, 1997). This finding would suggest that the conventional DQA $1{ }^{*} 0102-\mathrm{DQB} 1{ }^{*} 0602$ haplotype is never found in patients with type 1A diabetes and that this haplotype confers absolute protection from the disease. However, we have documented eight nondiabetic, ICA-positive, first-degree relatives and six patients with type 1 diabetes who carry the DQA ${ }^{*} 0102-$ DQB1*0602 haplotype. These individuals were first typed with standard SSO techniques, then confirmed by direct sequencing of the second exon of $\mathrm{DQB} 1^{*} 0602$ and DQA ${ }^{*} 0102$ alleles. All subjects carried conventional DQA 1*0102-DQB1*0602 exon 2 sequences (Pugliese et al., 1999). Type 1A diabetes can develop in individuals carrying the $\mathrm{DQB} 1{ }^{*} 0602$ allele, indicating that the protective effect associated with this allele is not absolute. The protective effect of DQB1*0602 may differ among ethnic groups. African American, ICApositive relatives were more likely to carry the $\mathrm{DQA} 1 * 0102-\mathrm{DQB} 1 * 0602$ haplotype than other ethnic groups. Hispanic, ICA-positive relatives with DQB $1 * 0602$ were more likely to have positive insulin autoantibodies or to have low FPIR than other racial groups (Greenbaum et al., 2000).

The DQB1*0602 allele is usually found on DR2-DRB1*1501-DQA 1*0102$\mathrm{DQB} 1{ }^{*} 0602$ haplotypes. However, protection from diabetes by this haplotype seems to be an effect of the DQB molecule. We described three siblings with type 1A diabetes who carried DR2 (Erlich et al., 1991). Utilizing sequence analysis, an unusual DQB1 allele was found on their DR2 haplotype, while the DRB1 alleles on these DR2 haplotypes was the conventional allele $\left({ }^{*} 1501\right)$. Further evidence was found in a recent study of 1371 subjects from the HBDI type 1 diabetes repository and 2441 subjects from the Norwegian Type 1 Diabetes Simplex Families (NODIAB) study (Redondo et al., 2000). DQA 1*0102$\mathrm{DQB} 1 * 0602$ haplotypes were infrequently transmitted to diabetic offspring $(2 / 313)$, while rare $\mathrm{DRB} 1 * 1501$ haplotypes without DQA $1 * 0102-\mathrm{DQB} 1 * 0602$ were transmitted to $5 / 11$ affected offspring. This finding again supports the hypothesis that the protection associated with the DQA1*0102-DQB1 ${ }^{*} 0602$ haplo- 
type is an effect of the DQ alleles rather than the DRB1*1501 in linkage disequilibrium.

$\mathrm{DR}$ alleles also may be protective against type $1 \mathrm{~A}$ diabetes. In the non-obesc diabetic (NOD) mouse, both I-E and I-A (DR and DQ molecules) protect from autoimmune diabetes (Yamane et al., 1996). Combining families from NODIAB and the HBDI, 728 patients with type 1A diabetes, and 110 healthy controls from the Barbara Davis Center provided us with a database to analyze the risk or protection associated with uncommon alleles. The HBDI study has been described above. The NODIAB study included 526 Norwegian families with one child diagnosed with diabetes before age 15. Less than three percent of families in this study had two affected children. We used the TDT analysis to analyze transmission to affected and unaffected offspring within fannilies in the HBDI and NODIAB studies. DQA1*0101-DQB ${ }^{*} 0503$ is a rare haplotype, present only in 1.6 percent of haplotypes in North America (Gjertson and Terasaki, 1998). This haplotype was transmitted from heterozygous parents to affected offspring only $2 / 42$ times. In the analysis of patients and controls, we also found that this allele is significantly less frequent among cases than controls. These findings are in agreement with previous reports (Ronningen et al., 1991). Interestingly, the only two affected offspring who inherited this haplotype in our combined family study did not have the DRB1*1401 allele usually found on the same haplotype with DQB I*0503, while all nonaffected children who inherited DQA ${ }^{*} 0101-$ DQB 1*0503 had DRB $1 * 1401$. Among the three patients with type 1A diabetes from the Barbara Davis Center who had DQB 1*0503, only one had DRB1*1401.

Polymorphisms in the heat shock protein genes located within the MHC are associated with type $1 \mathrm{~A}$ diabetes. However, this association is believed to be due to linkage disequilibrium of alleles on extended haplotypes associated with diabetes (Pugliese et al., 1992). Similarly, tumor necrosis factor (TNF) microsatellite haplotype associations with type I diabetes and multiple sclerosis were attributable to the known extended haplotype associations of these diseases (Garcia-Merino, et al., 1996).

\section{Mechanisms of Diabetogenicity of HLA Molecules}

The diabetes susceptibility or protection associated with HLA molecules may be related to their ability to present peptides of relevance to diabetogenic $T$ cells. Individuals with HLA molecules that are not able to effectively present specific peptides to naïve $T$ cells in the thymus might fail to engender tolerance. Alternatively, specific HLA alleles may selectively present an islet peptide to mature $T$ lymphocytes that have escaped negative selection. The two mechanisms - lack of central tolerance affecting the T-cell repertoire in the thymus or abnormal tolerance in the periphery - are not mutually exclusive and could coexist. The ability to present antigens may depend on conformational properties of the HLA 
molecule, with some alleles binding to peptides in such a fashion that the antigen would never be effectively presented to the $T$ cells. The DQA $1 * 0301$ allele frequently found on DR4 haplotypes, as well as *0101, *0102, *0103, and *0201 (lineage 1-3 DQA1 alleles), are associated with high serum levels of insulin autoantibodies (Pugliese et al., 1994b).

The hypothesis of conformational structure influencing diabetogenicity of some HLA alleles or haplotypes is further supported by inter-ethnic group studies. For instance, among the Japanese, the $\mathrm{DQB} 1{ }^{*} 0401$ allele is usually found on DR4-DQA 1*0301-DQB1*0401 haplotypes and is associated with diabetes risk (Aparicio et al., 1988). The DQB $1 * 0401$ and $\mathrm{DQB} 1 * 0402$ alleles differ only by one amino acid. Among Norwegians, the heterozygous DQA ${ }^{*} 0301$ DQB 1*0302/DQA 1*0401-DQB 1*0402 genotype appears to be associated with type 1 A diabetes. A very similar molecule could be formed among Japanese in cis (DR4-DQA 1*0301-DQB1*0401) and among Norwegians in trans (DQA 1*0301- DQB1*0402).

Animal models of type $1 \mathrm{~A}$ diabetes, such as the NOD mouse or transgenic mice, are helping in the identification of self antigens and MHC molecules associated with anti-islet autoimmunity. Sherwin and coworkers have demonstrated that transgenic mice expressing human DQ8 with beta-cell expression of the co-stimulatory molecule B7-1 develop type 1A diabetes (Wen et al., 2000). The crystal structure of the diabetogenic I- $\mathrm{A}^{\mathrm{g} 7}$ molecule, the only class II allele expressed by the NOD mouse, has been elucidated. The molecule has a "normal" class II binding cleft with a large pocket and the ability to bind multiple peptides (Corper et al., 2000). Santamaria and colleagues have defined the structure of a peptide mimotope that elicits the preferential expansion of beta-reactive $T$ cells in NOD mice (Anderson et al., 1999). Finally, a mouse with a transgene encoding a "diabetogenic" T-cell receptor is protected from diabetes by multiple "deleting" class II alleles (Schmidt et al., 1999).

The protective DQ haplotype, DQA 1*0102-DQB $1 * 0602$, has an aspartic acid at position 57 of the DQ beta chain. On the other hand, the two DQ haplotypes that determine the highest risk for diabetes, DQA 1*0301-DQB1*0302 and DQA $1{ }^{*} 0501-\mathrm{DQB} 1 * 0201$, do not have an aspartic acid residue at that position but rather alanine. It was hypothesized that the presence of aspartic acid at position 57 of the DQ-beta chain determines protection from diabetes (Morel et al., 1988). Further reports suggested that an arginine at position 52 of the DQ-alpha chain determined susceptibility to type $1 \mathrm{~A}$ diabetes, as it is found on the DQA1*0301DQB 1*0302 and DQA 1*0501-DQB1*0201 haplotypes (Gutierrez-Lopez, 1992; Khalil et al., 1990,1992). However, the DQA1*0401-DQB1*0402 haplotype is also associated with diabetes risk. Although it has arginine at position 52 of the DQ-alpha chain, it has aspartic acid at position 57 of the DQ-beta chain, as do DQB $1 * 0301$ and DQB $1 * 0303$ alleles associated with moderate risk. 


\section{Disorders Associated with Type 1A Diabetes}

Individuals with type $1 \mathrm{~A}$ diabetes experience other diseases at an increased frequency, compared with the general population. In most cases, that association is with autoimmune disorders. Most dramatic are the autoimmune polyendocrine syndromes (APS) type 1 and type 2. APS type 1 is a rare constellation of autoimmune disorders often diagnosed in infancy, with hypoparathyroidism, mucocutaneous candidiasis, Addison's disease, and hepatitis (Maes and Eisenbarth, 1999). This syndrome is associated with mutations of the autoimmune regulator gene (AIRE) on chromosome 21(Aaltonen et al., 1997; Nagamine et al., 1997) and has no association with HLA alleles. The basis for the association of the different disorders that constitute this syndrome is not yet clear. Therefore, APS-1 is an interesting case of HLA-independent autoimmune diabetes, although the diabetes is clinically similar to HLA-dependent type 1A diabetes and is associated with anti-islet autoantibodies such as GAD. However, the presence of GAD anti-islet autoantibodies in individuals with this syndrome does not seem to predict progression to diabetes, as it does in sporadic cases of type 1A diabetes (Husebye et al., 1997), while individuals with ICA512 (IA-2) autoantibodies frequently progress.

The disorders commonly associated in APS type 2 are Addison's disease, type 1A diabetes, autoimmune thyroid disease, gonadal failure, celiac disease, pernicious anemia, and vitiligo (Redondo and Eisenbarth, 1997). This syndrome is associated with HLA DR and DQ alleles. Table II illustrates the HLA association of disorders found in APS type 2.

Stiff-man syndrome (SMS) is a rare neurologic disorder characterized by rigidity of the body musculature, with spasms triggered by sensory or emotional stimuli with a characteristic electromyographic pattern. Autoantibodies against GAD are found in the serum and cerebroespinal fluid of 60 percent of patients with SMS. SMS is associated with DQB1*0201 (Pugliese et al., 1993).

Patients with type $1 \mathrm{~A}$ diabetes have an increased risk of celiac disease. This is a common autoimmune disorder associated with characteristic intestinal lesions that occur upon the ingestion of gliadin, a glutamine-rich protein present in wheat, rye, and barley. Typically, the lesions disappcar rapidly when this protein is removed from the diet. Patients may present with gastrointestinal complaints and poor growth. In rare instances, intestinal lymphoma may occur. Extra-intestinal lesions include skin and enamel lesions. Intestinal biopsy is the standard procedure for the diagnosis of this condition. The major autoantigen in celiac disease is transglutaminase, accounting for the presence of serum antiendomysial autoantibodies. We developed a radioassay for autoantibodies to tissue transglutaminase and found that transglutaminase autoantibodies are highly predictive of celiac disease. All patients (13/13) with an index greater than 0.7 had a positive intestinal biopsy (Bao et al., 1999b). Patients with transglutaminase autoantibodies at a titer 
TABLE II

Major HLA Associations of Disorders Found in Autoimmune Polyendocrine Syndrome Type 2

\begin{tabular}{lll} 
Disease & DRB Association & DQADQB Association \\
\hline Addison's disease & 0404 & $0301 / 0302$ \\
& 0301 & $0501 / 0201$ \\
& DR5 & $0501 / 0301$ \\
Graves' disease & 0301 & $0501 / 0201$ \\
Type 1A diabetes & $0401,0402,0404$ & $0301 / 0302$ \\
& 0301 & $0501 / 0201$ \\
Pernicious anemia & DR5 & \\
Celiac disease & 0301 & $0501 / 0201$ in "cis" \\
& DR5/DR7 & $0501 / 0201$ in "trans" \\
Myasthenia gravis & 0301 & $0501 / 0201$ \\
Sjogren syndrome & 0301 & $0501 / 0201$ \\
\hline
\end{tabular}

lower than 0.3 had a negative biopsy. Patients (49/49) with the conventional antiendomysial autoantibodies had positive transglutaminase antibodies. Susceptibility to celiac disease is mainly associated with the DQA 1*0501-DQB1*0201 haplotype. Interestingly, this heterodimer may be encoded either in cis, usually with a DR3 haplotype, or in trans, with DR7-DQA $1 * 0201-D Q B 1 * 0201$ on one chromosome and DR5-DQA1*0501-DQB1*0301 on the other (Bao et al., 1999a). The expression of transglutaminase antibodies was greatly increased (approximately one third) among patients with type $1 \mathrm{~A}$ diabetes homozygous for DQA $1 * 0501-D Q B 1 * 0201$.

Addison's disease is an uncommon condition associated with autoimmune failure of the adrenal gland that leads to fatigue, hyperpigmentation of the skin, abdominal pain, dehydration, low blood pressure, cardiovascular shock, and, if untreated, death. Adrenal cortex autoantibodies (ACA) are found in 43 to 84 percent (Soderbergh et al., 1996; Falorni et al., 1997) of patients with idiopathic adrenal failure. The major target of ACA is the steroidogenic enzyme P450c21 (21-hydroxylase). Autoantibodies against 21-hydroxylase have been documented in over 90 percent of patients with Addison's disease associated to APS-1 or APS-2 (Tanaka et al., 1997) and have not been found in nonautoimmune adrenal insufficiency and other conditions. We did not find positive 21-hydroxylase autoantibodies in any of 240 healthy controls. Approximately 1.5 percent of 
patients with type $1 \mathrm{~A}$ diabetes are positive for these autoantibodies. Five percent (10/208) of patients with type 1 diabetes heterozygous for DQ8 and DQ2 expressed 21-hydroxylase antibodies, compared to less than 0.5 percent in patients with type 1A diabetes who have neither DQ8 nor DQ2. Three of the 21-hydroxylase diabetic patients were found to have Addison's disease. The genotype DRB 1*0404-DQA 1*0301-DQB 1*0302/DRB1*0301-DQA 1*0501-DQB1*0201 was present in 14/21 patients with Addison's disease (8/12 with diabetes and 6/9 without diabetes or anti-islet antibodies), vs. 0.7 percent of the general population $(109 / 15,547)$ and 11 percent of patients with type $1 \mathrm{~A}$ diabetes without Addison's disease (62/578). Among patients with diabetes with DQA $1^{*} 0301-\mathrm{DQB} 1^{*} 0302$, Addison's disease was strongly associated with DRB $1 * 0404$. Eighty percent (12/15) of patients carrying DRB $1{ }^{*} 0404-\mathrm{DQA} 1{ }^{*} 0301-\mathrm{DQB} 1{ }^{*} 0302$ and positive for 21-hydroxylase autoantibody had Addison's disease, in contrast to 10 percent (1/10) 21-hydroxylase autoantibody-positive patients who had other DRB1 alleles (DRB1*0401 or DRB $1 * 0402)$ (Yu, 1999).

\section{Insulin Gene (IDDM2)}

Most of the genetic susceptibility for type 1A diabetes is determined by HLA genes. However, polymorphisms in a noncoding region of the insulin gene, on chromosome $11 \mathrm{pl}$, have long been known to be associated with diabetes risk (Bennett et al., 1995,1996; Bell et al., 1984). This locus has been termed IDDM2 and includes a variable nucleotide tandem repeat (VNTR) minisatellite located at the $5^{\prime}$ end of the insulin gene. There are three main VNTR classes defined by VNTR size: class I (26-63 repeats), class II (approximately 80 repeats), and class III (140-200 repeats). Each of these classes may be further divided. Homozygosity for class I VNTR determines high risk for diabetes, while class III VNTRs confer dominant protection. The VNTR class III allele is associated with higher expression of messenger RNA for insulin within the thymus (Pugliese et al,, 1997). One hypothesis is that expression of insulin within the thymus leads to negative selection (deletion) of autoreactive $T$ cells and thus to development of tolerance. VNTR class I alleles, associated with low levels of proinsulin and insulin in the thymus during fetal life and childhood, may fail to shape the T-cell repertoire and lead to the presence of anti-insulin autoreactive $T$ cells. A parent-of-origin effect has been described for IDDM2, with protective alleles protecting when paternally inherited (Pugliese et al., 1994a; Sospedra et al., 1998)

\section{IDDM3-17}

At least 15 more loci have been associated with type $1 \mathrm{~A}$ diabetes, in addition to HI.A (IDDM1) and the insulin VNTR (IDDM2) (Table III). For most associa- 
TABLE III

Defined and Putative IDDM loci

\begin{tabular}{|c|c|c|}
\hline Locus & Chromosome & Marker \\
\hline IDDMl & $6 \mathrm{p} 21.31$ & HLA \\
\hline IDDM2 & $11 \mathrm{lp} 15.5$ & $5^{\prime}$ insulin VNTR \\
\hline IDDM3 & $15 q 26$ & D15S107 \\
\hline IDDM4 & $11 q 13$ & Fibroblast growth factor-3 (FGF3) \\
\hline IDDM5 & $6 \mathrm{q} 24-27$ & D65476-D65448 \\
\hline IDDM6 & $18 \mathrm{q} 21$ & D18S64 \\
\hline IDDM7 & $2 q 31-33$ & D2S 152 \\
\hline IDDM8 & $6 q 27$ & D6S 1590 \\
\hline IDDM9 & $3 q 21-q 25$ & D3S1303 \\
\hline IDDM 10 & $10 p 11-q 11$ & D10S193 \\
\hline IDDMII & $14 q 24.3-14 q 31$ & DD14567 \\
\hline IDDM12 & $2 q 33$ & CTLA-4 \\
\hline IDDM13 & $2 q 34$ & D2S164 \\
\hline IDDM15 & $6 q 21$ & D65283 \\
\hline IDDM17 & $10 \mathrm{q} 25.1$ & D10S1681 \\
\hline No "IDDM" & $16 q$ & D1653098 \\
\hline No "IDDM" & lq & D15617 \\
\hline
\end{tabular}

tions, the responsible gene has not been identified. Of note, there are three more loci in addition to HLA located on chromosome 6, three loci on the short arm or chromosome 2 , and three loci on chromosome 10 . The polymorphic, cytotoxic, T-lymphocyte-associated antigen-4 (CTLA-4) gene on chromosome 2q33 (designated IDDM12) encodes a receptor expressed by activated T cells. CTLA-4 binds B7 molecules and limits the proliferative response of activated T cells. An A/G polymorphism in the first exon in CTLA4 results in an amino acid change (Thr/Ala). The presence of an alanine at codon 17 of CTLA4 has been associated with susceptibility to type $1 \mathrm{~A}$ diabetes, autoimmune thyroid disease, and primary biliary cirrhosis (Agarwal et al., 2000). The effect of IDDM12 is independent of other genetic markers of type 1A diabetes, such as HLA alleles or haplotypes, or 
the INS VNTR I/I risk genotype (van der Auwera et al., 1997). Ethnic heterogenity has been reported with strong effect in three Mediterranean-European populations (Italian, Spanish, and French), the Mexican American population, and the Korean population. There is a weak transmission deviation in the Caucasian American and no deviation in the British, Sardinian, and Chinese populations (Marron et al., 1997). Another study in the Danish population did not support IDDM12 as a type 1 diabetes susceptibility locus (Larsen et al., 1999).

In nonautoimmune BALB/c mice, CTLA-4 (CD152) is also a downregulator of T-cell activation responses. CD152 blockade unmasked islet cell-specific, autoreactive T cells. Conversely, CD152 blockade in NOD mice failed to regulate islet-specific, auto-reactive $T$-cell responses but enhanced the T-cell response to the exogenous, foreign antigen $\mathrm{KLH}$ in both nonautoimmune $\mathrm{BALB} / \mathrm{c}$ and autoimmune NOD mice. These results suggest that there is not a global defect in CD152-mediated regulation of peripheral T-cell immune responses in NOD autoimmune mice. Rather, there is a defect specific to $T$ cells recognizing self antigen (Piganelli et al., 2000). In the NOD mouse, macrophages, dendritic cells, and $T$ cells, but not $B$ cells, express lower basal levels of CD86. It has been proposed that this low level of CD86 expression contributes to a defective regulation of autoreactive $\mathrm{T}$ cells by preventing the full activation of $\mathrm{T}$ cells and therefore the upregulation of CTLA-4 (Dahlen et al., 2000). Protection against diabetes in the NOD mouse has been associated with increased CD40L and CTLA-4-expressing Th cells and with the generation of a CD40- $\mathrm{IgG}+\mathrm{B}$ cells. This study associated the induction by mycobacterial infection of regulatory CD45RBlo CD38 + Th cells with the ability to trigger deletion or anergy of peripheral, self-reactive lymphocytes and shutting down IgG+ B-cell responses (Martins and Aguas, 1999). Interestingly, in spontaneously diabetic BB rats, ex vivo, adenovirus-mediated CTLA4Ig gene transfer induced local immunomodulation, blocking autoimmune recurrence and rejection of pancreaticoduodenal grafts. The transferred CTLA4Ig gene was strongly expressed in both endocrine and exocrine tissues. These results indicate the potential utility of local CD28-B7 co-stimulatory blockade for prevention of alloimmune and autoimmune destruction of pancreatic grafts in type 1 diabetic hosts (Uchikoshi et al., 1999).

An alternative hypothesis to polygenic inheritance is that type $1 \mathrm{~A}$ diabetes is an oligogenic, heterogeneous disease. In this hypothesis, HLA is a major diabetogenic locus with relatively few non-HLA genes determining diabetes susceptibility (i.e., different genes causing disease in different families). We have studied a Bedouin Arab family with 21 members with type 1A diabetes. Based on a search for linkage, we identified a locus (termed IDDM17) contributing to type 1 diabetes mapping to the long arm of chromosome $10(10 \mathrm{q} 25$; nonparametric linkage $=$ $4.99, p=0.00004)$. In this family, HLA DR3 or DR4 plus IDDM17 is associated with a diabetes risk of 40 percent (Verge et al., 1998). 


\section{Summary}

Type 1A diabetes is an autoimmune disease with genetic and environmental factors contributing to its etiology. Most of the genetic susceptibility is accounted for by HLA alleles. The most-common susceptibility haplotypes are DQA ${ }^{*} 0301$ DQB 1*0302 and DQA 1*0501-DQB 1*0201. Other haplotypes (e.g., DQA $1 * 0401-\mathrm{DQB} 1 * 0402$ and DQA $1 * 0101-\mathrm{DQB} 1 * 0501$ ) are diabetogenic; strongly protective alleles (e.g., DQB1*0602) exist. Differential expression of insulin or proinsulin in the thymus may contribute to diabetes susceptibility. This autoimmune process also can result from mutations of a transcription factor (AIRE gene), independent of HLA alleles. It is likely that an abnormal presentation of antigens, potentially insulin or proinsulin, to $T$ cells either within the thymus or in the periphery, or both, results in an abnormal $\mathrm{T}$-cell repertoire or an abnormal immune response. Interestingly, protective HLA alleles continue to have an effect after the autoimmune process has started. The observation that the diabetes risk for monozygotic twins of patients with type 1A diabetes is higher than the diabetes risk for HLA-identical ordinary siblings supports the hypothesis of non-HLA genes contributing to diabetes risk. The non-HI A genetic susceptibility is probably explained by multiple genes, different in different families. In addition, the

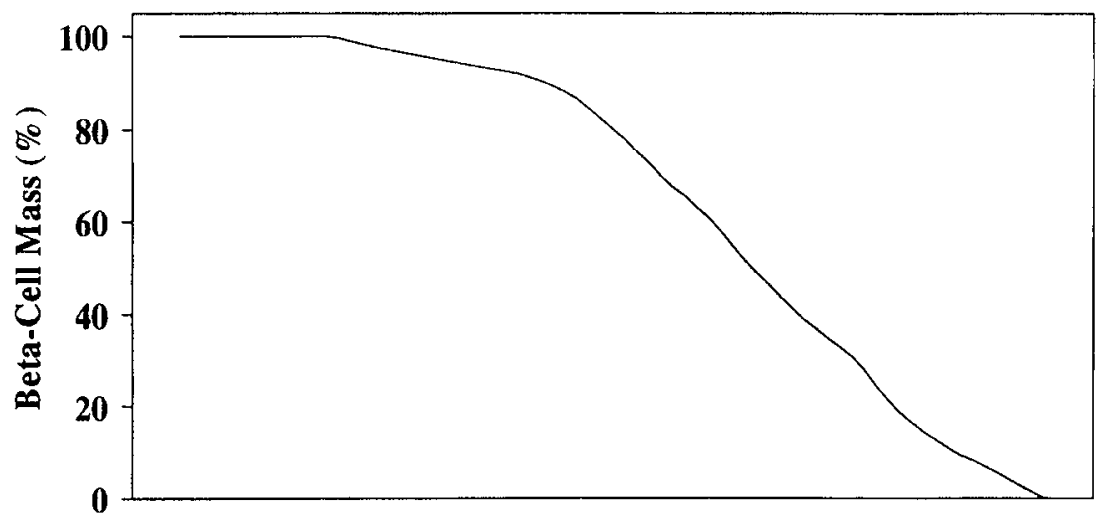

Time

Genetic Susceptibility

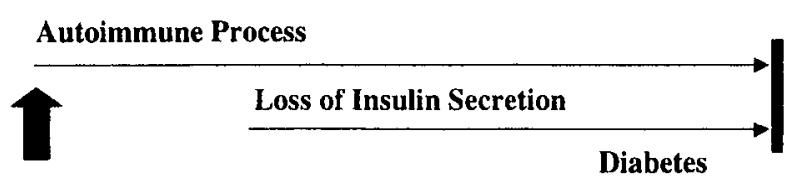

Triggering Factor(s)

FIG. 2. Hypothetical stages of the development of type 1A diabetes. 
observation that the risk for monozygotic twins of patients with type $1 \mathrm{~A}$ diabetes is not 100 percent indicates that there are other factors - either nongermline (noninherited) genes or environment - that have a role in progression to diabetes. Type $1 \mathrm{~A}$ diabetes is predictable, based on genetic, immunologic, and metabolic factors (Figure 2), such that trials of diabetes prevention are underway (e.g., Diabetes Prevention Trial 1 (DPT-1)). Several studies, including DAISY (Diabetes Autoimmunity Study of the Young) in the United States, suggest that diabetes will be predictable for individuals in the general population as well as first-degree relatives of patients. This should facilitate future preventive trials.

\section{REFERENCES}

Aaltonen, J., Bjorses, P., Perheentupa, J., Horelli-Kuitunen, N., Palotie, A., Peltonen, L., et al (1997). Nature Genet. 17, 399-403.

Agarwal, K., Jones, D.E., Daly, A.K., James, O.F., Vaidya, B., Pearce, S., et al. (2000). J. Hepatol. 32, 538-541.

American Diabetes Association. (1997). Diabetes Care 20, 1183-1197.

Anderson, B., Park, B.J., Verdaguer, J., Amrani, A., and Santamaria, P. (1999). Proc. Natl. Acad. Sci. U.S.A. 96, 9311-9316.

Aparicio, J.M.R., Wakisaka, A., Takada, A., Matsuura, N., and Aizawa, M. (1988). Immunogenetics 28, 240-246.

Baisch, J.M., Weeks, T., Giles, R., Hoover, M., Stastny, P., and Capra, J.D. (1990). N. Engl. J. Med. 322, 1836-1841.

Baker, B.S., Garioch, J.J., Bokth, S., Leonard, J., and Fry, L.J. (1995). J. Autoimmunity 8, 75-82.

Bao, F., Rewers, M., Scott, F., and Eisenbarth, G.S. (1999a). In "Endocrine and Organ Specific Autoimmunity" (G.S. Eisenbarth, ed.), pp. 85-96. R.G. Landes, Austin, Tex.

Bao, F., Yu, L., Babu, S., Wang, T., Hoffenberg, E.J., Rewers, M., et al. (1999b). J. Autoimmunity 13, 143-148.

Bell, G.I., Horita, S., and Karam, J.H. (1984). Diabetes 33, 176-183.

Bennett, S.T., Lucassen, A.M., Gough, S.C.L., Powell, E.E., Undlien, D.E., Pritchard, L.E., et al. (1995). Nature Genet. 9, 284-292.

Bennett, S.T., Wilson, A.J., Cucca, F., Nerup, J., Pociot, F., McKinney, P.A., et al. (1996). J. Autoimmunity 9, 415-421

Bleich, D., Polak, M., Eisenbarth, G.S., and Jackson, R.A. (1993). Diabetes 42, 1433-1439.

Colman, P.G., McNair, P., Steele, C., Gellert, S., Tait, B., Honeyman, M., et al. (2000). Diabetes 49, A36-A36 (abstract)

Corper, A.L., Stratmann, T., Apostolopoulos, V., Scott, C.A., Garcia, K.C., Kang, A.S., et al. (2000). Science 288, 505-511.

Dahlen, E., Hedlund, G., and Dawe, K. (2000). J. Immunol. 164, 2444-2456.

Eisenbarth, G.S., Wilson, P., Ward, F., and Lebovitz, H.E. (1978). N. Fngl I. Med. 298, 92-94.

Erlich, H.A., Griffith, R.L., Bugawan, T.L., Ziegler, R., Alper, C., and Eisenbarth, G.S. (1991). Diabetes 40, 478-481.

Falorni, A., Laureti, S., Nikoshkov, A., Picchio, M.L., Hallengren, B., Vandewalles, C.L., et al. (1997). Clin. Exp. Immunol. 107, 341-346.

Garcia-Merino, A., Alper, C.A., Usuku, K., Marcus-Bagley, D., Lincoln, R., Awdeh, Z., et al. (1996). Hum. Immunol. 50, 11-21.

Gianani, R., Pugliese, A., Bonner-Weir, S., Shiffrin, A.J., Soeldner, J.S., Erlich, H., et al. (1992). Diabetes 41, 347-353. 
Gianani, R., Verge, C.F., Moromisato-Gianani, R.I., Yu, L., Zhang, Y.J., Pugliese, A., et al. (1996). J. Autoimmunity 9, 423-425.

Gjertson, D.W., and Terasaki, P.I. (eds.) (1998). "HLA." American Society of Immunocompatibility and Genetics, Lenexa, Tex.

Greenbaum, C.J., Cuthbertson, D., Eisenbarth, G.S., Schatz, D.A., Zeidler, A., and Krischer, J.P. (2000). J. Clin. Endocrinol. Metab. 85, 1255-1260.

Gutierrez-Lopez, M.D., Bertera, S., Chantres, M.T., Vavassori, C., Dorman, J.S., Trucco, M., et al. (1992). Diabetologia 35, 583.588.

Hawa, M., Rowe, R., Lan, M.S., Notkins, A.L., Pozzilli, P., Christie, M.R., et al. (1997). Diabetes 46, $1270-1275$.

Hawkes, C.H. (1997). Diabetic Med. 17, 347-352.

Hoover, M.L., and Marta, R.T. (1997). Scand. J. Immunol. 45, 193-202.

Husebye, E.S., Gebre-Medhin, G., Tuomi, T., Perheentupa, J., Landin-Olsson, M., Gustafsson, J., et al . (1997), J. Clin. Endocrinol. Metab. 82, 147-150.

Johnston, C., Pyke, D.A., Cudworth, A.G., and Wolf, E. (1983). Br. Med. J. 286, 253-255.

Kaprio, J., Tuomilehto, J., Koskenvuo, M., Romanov, K., Reunanen, A., Eriksson, J., et al. (1992). Diabetologia 35, 1060-1067.

Khalil, I., D'Auriol, L., Gobet, M., Morin, L., Lepage, V., Deschamps, I., et al. (1990). J. Clin. Invest. 85, 1315-1319

Khalil, I., Deschamps, I., Lepage, V., Al-Daccak, R., Degos, L., and Hors, J. (1992). Diabetes 41, 378-384.

Kumar, D., Gemayel, N.S., Deapen, D., Kapadia, D., Yamashita, P.H., Lee, M., et al. (1993). Diabetes 42, 1351-1363

Kyvik, K.O., Green, A., and Beck-Nielsen, H. (1995), Br. Med. J. 311, 913-917.

Larsen, Z.M., Kristiansen, O.P., Mato, E., Johannesen, J., Puig-Domingo, M., de Leiva, A., et al. (1999). Autoimmunity 31, 35-42.

Lorenzen, T., Pociot, F., Hougaard, P., and Nerup, J. (1994). Diabetologia 37, 321-327.

Maes, M, and Eisenbarth, G.S. (1999). In "Contemporary Endocrinology on Autoimmune Endocrinopathies" (R. Volpé, ed.), pp. 349-363. Humana Press, Inc., Totowa, N.J.

Marron, M.P., Raffel, L.J., Garchon, H.J., Jacob, C.O., Serrano-Rios, M., Martinez, L.M., et al. (1997). Hum. Mol. Genet. 6, 1275-1282.

Martin, N., Boomsma, D., and Machin, G. (1997). Nature Genet. 17, 387-392.

Martins, T.C., and Aguas, A.P. (1999). Immunology 96, 600-605.

Morel, P.A., Dorman, J.S., Todd, J.A., McDevitt, H.O., and Trucco, M. (1988). Proc. Natl. Acad. Sci. U.S.A. 85, $8111-8115$.

Nagamine, K., Peterson, P., Scott, H.S., Kudoh, J., Minoshima, S., Heino, M., et al. (1997). Nature Genet. 17, 393-398.

Nerup, J., Platz, P., Anderson, O.O., Christy, M., Lyngsoe, J., Poulsen, J.E., et al. (1974). Lancet ii, 864-866.

Olmos, P., A'Hearn, R., Heaton, D.A., Millward, B.A., Risley, D., Pyke, D.A., et al. (1988). Diabetologia 31, 747-750.

Park, Y.S., Wang, C.Y., Ko, K.W., Yang, S.W., Park, M., Yang, M.C., et al. (1998). Hum. Immunol. 59, 794-801.

Petersen, J.S., Kyvik, K.O., Bingley, P.J., Gale, E.A.M., Green, A., Dyrberg, T., et al. (1997). Br. Med. J. 314, 1575-1579.

Phillips, D.I.W. (1993). Lancet 341, 1008-1009

Piganelli, J.D., Poulin, M., Martin, T., Allison, J.P., and Haskins, K. (2000). J. Autoimmunity 14, 123-131.

Pugliese, A., Awdeh, Z., Galluzzo, A., Yunis, E.J., Alper, C.A., and Eisenbarth, G.S. (1992). Diabetes 41, 788-791. 
Pugliese, A., Solimena, M., Awdeh, Z.L., Alper, C.A., Bugawan, T., Erlich, H.A., et al. (1993). J. Clin. Endocrinol. Metab. 77, 1550-1553.

Pugliese, A., Awdeh, Z.L., Alper, C.A., Jackson, R.A., and Eisenbarth, G.S. (1994a). J. Autoimmunity 7, 687-694.

Pugliese, A., Bugawan, T., Moromisato, R., Awdeh, Z.L., Alper, C.A., Jackson, R.A., et al. (1994b). J. Clin. Invest. 93, 2447-2452.

Pugliese, A., Gianani, R., Moromisato, R., Awdeh, Z.L., Alper, C.A., Erlich, H.A., et al. (1995). Diabetes 44, 608-613.

Pugliese, A., Zeller, M., Fernandez, A., Zalcberg, L.J., Bartlett, R.J., Ricordi, C., et al. (1997). Nature Genet. 15, 293-297.

Pugliese, A., Kawasaki, E., Zeller, M., Yu, L., Babu, S., Solimena, M., et al. (1999). J. Clin. Endocrinol. Metab. 84, 1722-1728

Redondo, M.J., and Eisenbarth, G.S. (1997). In "Endocrine and Organ Specific Autoimmunity" (G.S. Eisenbarth, ed.), pp. 41-61. R.G. Landes Company, Austin, Tex.

Redondo, M.J., Kawasaki, E., Mulgrew, C.L., Noble, J., Erlich, H., Freed, J., et al. (2000). J. Clin. Endocrinol. Metab. 85, 3793-3797.

Redondo, M.J., Rewers, M., Yu, L., Garg, S., Pilcher, C.C., Elliott, R.B., et al. (1999a). Br. Med. J. 318, 698-702.

Redondo, M.J., Yu, L., Hawa, M., Leslie, D.G., and Eisenbarth, G.S. (1999b). Diabetes 48, 780-780 (abstract).

Risch, N., Ghosh, S., and Todd, J.A. (1993). Am. J. Hum. Genet. 53, 702-714.

Ronningen, K.S., Spurkland, A., Iwe, T., Vartdal, F., and Thorsby, E. (1991). Tissue Antigens 37, 105-111.

Rowe, R.E., and Leslie, R.D.G. (1995). Diabetes Metab. Rev. 11, 121-136.

Schmidt, D., Amrani, A., Verdaguer, J., Bou, S., and Santamaria, P. (1999). J. Immunol, 162, 4627-4636.

Schwartz, R. (1990). N. Engl. J. Med. 323, 340-342.

Smith, C. (1974). Am. J. Hum. Genet. 26, 454-466.

Soderbergh, A., Winqvist, O., Norheim, I., Rorsman, F., Husebye, E.S., Dolva, O., et al. (1996). Clin. Endocrinol. (Oxf.) 45, 453-460.

Sospedra, M., Ferrer-Francesch, X., Dominguez, O., Juan, M., Foz-Sala, M., and Pujol-Borrell, R. (1998). J. Immunol. 161, 5918-5929.

Spielman, R.S., and Ewens, W.J. (1996). Am. J. Hum. Genet. 59, 983-989.

Srikanta, S., Ganda, O.P., Eisenbarth, G.S., and Soeldner, J.S. (1983). N. Engl. J. Med. 308, 322-325.

Tanaka, H., Perez, M.S., Powell, M., Sanders, J.F., Sawicka, J., Chen, S., et al. (1997). J. Clin. Endocrinol. Metab. 82, 1440-1446.

Tillil, H., and Kobberling, J. (1987). Diabetes 36, 93-99.

Todd, J.A., and Farrall, M. (1996). Hum. Mol. Genet. 5, 1443-1448.

Uchikoshi, F., Yang, Z.D., Rostami, S., Yokoi, Y., Capocci, P., Barker, C.F., et al. (1999). Diabetes 48, 652-657.

van der Auwera, B.J., Vandewalle, C.L., Schuit, F.C., Winnock, F., De Leeuw, I.H., Van Imschoot, S., et al. (1997). Clin. Exp. Immunol. 110, 98-103.

Verge, C.F., Gianani, R., Yu, L., Pietropaolo, M., Smith, T., Jackson, R.A., et al. (1995). Diabetes 44, 1176-1179.

Verge, C.F., Gianani, R., Kawasaki, E., Yu, L., Pietropaolo, M., Jackson, R.A., et al. (1996). Diabetes 45, 926-933.

Verge, C.F., Vardi, P., Babu, S., Bao, F., Erlich, H.A., Bugawan, T., et al. (1998). J. Clin. Invest. 102, 1569-1575

Warram, J.H., Krolewski, A.S., Gottlieb, M.S., and Kahn, C.R. (1984). N. Engl. J. Med. 311, 149-152.

Warram, J.H., Kroleski, A.S., and Kahn, C.R. (1988). Diabetes 37, 1328-1334.

Wen, L., Wong, F.S., Tang, J., Chen, N.Y., Altieri, M., David, C., et al. (2000). J. Exp. Med. 191, 97-104. 
Yamane, K., Yamamoto, K., Yoshikawa, Y., and Sasazuki, T. (1996). Clin. Exp. Immunol. 103, 141-148. Yu, L., Chase, H.P., Falorni, A., Rewers, M., Lernmark, $\AA$., and Eisenbarth, G.S. (1995). Diabetologia 38, 1353-1357.

Yu, L., Brewer, K.W., Gates, S., Wang, T., Babu, S., Gottlieb, P.A., et al. (1999). J. Clin. Endocrinol. Metab. 84, 328-335.

Ziegler, R., Alper, C.A., Awdeh, Z.L., Castano, L., Brink, S.J., Soeldner, J.S., et al. (1991). Diabetes 40, 709-714. 



\title{
Genetic Determinants of Type 2 Diabetes
}

\author{
Philippe froguel ${ }^{*}$ and Gilberto Velho ${ }^{\dagger}$ \\ *Institute of Biology-CNRS 8090, Institut Pasteur de Lille, France; \\ $\dagger_{\text {INSERM U342, Ilopital Saint Vincent de Paul, Paris, France }}$
}

\begin{abstract}
Hyperglycemia of type 2 diabetes mellitus (T2DM) results from a complex interplay of genetic and environmental factors that influence a number of intermediate traits (e.g., $\beta$-cell mass, insulin secretion, insulin action, fat distribution, obesity). The primary biochemical events leading to diabetes are still unknown in most cases. Although several monogenic forms of diabetes have been identified, T2DM seems to be a polygenic disorder in the majority of cases. T2DM is probably also multigenic, meaning that many different combinations of gene defects may exist among diabetic patients. Significant results were obtained in the identification of the genetic determinants of monogenic forms of diabetes with young age of onset. However, despite the evidence for a strong genetic background, litle of the genetic risk factors for the more-common forms of polygenic T2DM are known to date. The goal of this chapter is to summarize and discuss the significant results of recent literature on the genetics of both the monogenic and polygenic forms of T2DM.
\end{abstract}

\section{Introduction}

Type 2 diabetes mellitus (T2DM) is a heterogeneous syndrome resulting from defects of both insulin secretion and action (DeFronzo, 1997). The precise molecular mechanisms leading to chronic hyperglycemia are largely unknown (Ferrannini, 1998). It is generally accepted that T2DM results from a complex interplay of genetic and environmental factors that influence a number of intermediate traits of relevance to the diabetic phenotype (e.g., $\beta$-cell mass, insulin secretion, insulin action, fat distribution, obesity). In fact, T2DM appears to be composed of subtypes whereby genetic susceptibility is strongly associated with environmental factors at one end of the spectrum and highly genetic forms at the other end. Although several monogenic forms of diabetes have been identified - such as maturity onset diabetes of the young (MODY) and maternally inherited diabetes and deafness (MIDD) (Froguel and Velho, 1999; van den Ouweland et al., 1994) - T2DM seems to be a polygenic disorder in the majority of cases. T2DM shows clear familial aggregation but does not segregate in classical Mendelian fashion. T2DM seems to result either from several combined gene defects or from the simultaneous action of several susceptibility alleles or else from combinations of 
frequent variants at several loci that may have deleterious effects when predisposing environmental factors are present. Type 2 diabetes is probably also multigenic, meaning that many different combinations of genc defects may exist among diabetic patients.

The primary biochemical events leading to diabetes are still unknown in most cases. Genetic and environmental factors may affect both insulin secretion and insulin action (DeFronzo, 1997). A variety of environmental factors can be implicated in the clinical expression of T2DM, such as the degree and type of obesity, sedentarity, malnutrition in fetal and perinatal periods, lifestyle, and different kinds of drugs (e.g, steroids, diuretics, antihypertensive agents). It is noteworthy that obesity, which is one of the so-called environmental determinants of T2DM, is also clearly under genetic control (Hager et al., 1998). Both disorders are frequently associated and share many metabolic abnormalities, which suggests that they might also share susceptibility genes (Carmelli et al., 1994). Moreover, retrospective studies showed that low birth weight was associated with insulin resistance and T2DM in adulthood (Hales et al., 1991; Lithell et al., 1996). It has been proposed that this association results from a metabolic adaptation to poor fetal nutrition (Barker, 1995). However, the identification of gene variants that contribute both to variation in fetal growth and to the susceptibility to T2DM suggests that this metabolic "programming" could be partly genetically determined (Hattersley and Tooke, 1999).

These complex interactions between genes and environment complicate the task of identifying any single genetic susceptibility factor to T2DM. Three general approaches have been adopted to search for genes underlying complex traits such as T2DM. The first approach is to focus on candidate genes - that is, genes selected as having a plausible role in the control of glucose homeostasis - on the basis of their known or presumed biological functions. Although this approach led to the identification of several susceptibility genes with small effects (see below), no genes with moderate or major effect on the polygenic forms of diabetes have been identified. Possible explanations for this failure to identify genes with a major effect include the possibility that they do not exist. It is also possible that our ignorance of the pathophysiological mechanisms of T2DM (and the genes that control them) has misled our choice of candidates. The second approach is to perform genome-wide scans for linkage in collections of nuclear families or sib-pairs with T2DM. This strategy requires no presumptions as to the function of the susceptibility loci. Although a large number of regions of presumed linkage have been mapped (Elbein et al., 1999; Hanis et al., 1996; Mahtani et al., 1996; Pratley et al., 1998), identification of the susceptibility genes is proceeding at very slow pace. The third approach to identify diabetes genes is to study spontaneous (Naggert et al., 1995), bred (Gauguier et al., 1996), or transgenic (Ahlgren et al., 1998; Jonsson et al., 1994; Kulkarni et al., 1999; Naya et al., 1997; Pontoglio et al., 1998; Withers et al., 1998) animal models of T2DM. The genes responsible 
for diabetes in these models may not necessarily be major players in typical T2DM in humans. However, such studies provide the most-direct way to improve overall understanding of the molecular circuitry that maintains glucose homeostasis. Nevertheless, despite the evidence for a strong genetic background in T2DM, very few of the genetic risk factors for T2DM are known. Most of the available results were obtained by studying the highly familial and monogenic forms of diabetes with young age of onset.

\section{Genetics of MODY}

The well-defined mode of inheritance with high penetrance and the early age of onset of diabetes allows the collection of multigenerational pedigrees, making MODY an attractive model for genetic studies. MODY is a familial form of non-insulin dependent diabetes (NIDDM) with autosomal dominant inheritance. MODY usually develops at childhood, adolescence, or young adulthood and presents primary insulin-secretion defects (Froguel and Velho, 1999; Velho and Froguel, 1998). MODY is not a single entity but presents genetic, metabolic, and clinical heterogeneity. Mutations in six genes cause most of the MODY cases. These genes encode the enzyme glucokinase (MODY2/GCK) (Froguel et al., 1992,1993; Velho et al., 1997) and the transcription factors hepatocyte nuclear factor 4 alpha (HNF-4/MODY1) (Bell et al., 1991; Yamagata et al., 1996a), HNF 1 alpha (HNF-1/MODY3) (Vaxillaire et al, 1995,1997; Yamagata et al., 1996b), insulin promoter factor 1 (IPF-1/MODY4) (Stoffers et al., 1997a,1997b), HNF 1 beta (HNF-1/MODY5) (Horikawa et al., 1997), and NeuroD1/Beta2 (Malecki et al., 1999). Moreover, additional MODY genes probably exist, since there are families in which MODY does not co-segregate with markers tightly linked to the known MODY loci (Chèvre et al., 1998).

Although the prevalence of MODY is unknown, it has been estimated that 2 to 5 percent of patients with T2DM may, in fact, have MODY (Ledermann, 1995). The relative prevalence of the different subtypes of MODY has been shown to vary greatly in studies of British, French, German, and Spanish families (Chèvre et al., 1998; Costa et al., 2000; Frayling et al., 1997; Lindner et al., 1999). MODY2 represents from 8 to 63 percent of cases (the most-prevalent form in French families) and MODY 3 from 21 to 64 percent of cases (the most-prevalent form in British families). The other MODY subtypes are rare disorders in all these populations, having been described only in a few families. Additional unknown MODY locus/loci (MODY-X) represent 16 to 45 percent of the cases of MODY (the most-prevalent form in German and Spanish families). These contrasting results may be due to differences in the genetic background of these populations or else may reflect, at least partially, ascertainment bias in the recruitment of families. 


\section{A. GLUCOKINASE MUTATIONS AND MODY2}

Glucokinase (GCK) phosphorylates glucose to glucose-6-phosphate in pancreatic $\beta$ cells and hepatocytes and plays a major role in the regulation and integration of glucose metabolism (Matschinsky, 1996). More than 80 different GCK mutations have been observed (Blanché et al., 1997; Velho et al., 1997). Expression studies have shown that the enzymatic activity of the mutant proteins was impaired (Gidh-Jain et al., 1993), resulting in decreased glycolytic flux in pancreatic $\beta$ cells (Sturis et al., 1994). This defect translates in vivo as a glucosesensing defect, leading to an increase in the blood glucose threshold that triggers insulin secretion (Velho et al., 1992) and a right shift in the dose-response curve of glucose-induced insulin secretion (Byrne et al., 1994). Decreased net accumulation of hepatic glycogen and augmented hepatic gluconeogenesis following meals were observed in GCK-deficient subjects and contribute to the post-prandial hyperglycemia of MODY2 (Velho et al., 1996b). Despite these multiple defects in the pancreas and the liver, the hyperglycemia associated with GCK mutations is often mild, with fewer than 50 percent of subjects presenting overt diabetes (Velho et al., 1997). There is a lower prevalence of diabetes microvascular complications (i.e., retinopathy and proteinuria) in MODY2 than in other subtypes of MODY and late-onset T2DM (Velho et al., 1996c,1997).

\section{B. MUTATIONS IN TRANSCRIPTION FACTOR GENES}

Positional cloning of MODY loci and studies in candidate genes have led to the identification of mutations in six transcription factors: HNF-1 $\alpha$, HNF-1 $\beta$, HNF-4a, IPF1, and NeuroD1/Beta2 (Horikawa et al., 1997; Malecki et al., 1999; Stoffers et al., 1997a; Yamagata et al., 1996a, 1996b). Gene targeting experiments in animals recently has demonstrated that many of these islet-expressed genes have a key role in fetal development, $\beta$-cell differentiation, proliferation, and neogenesis (Ahlgren et al., 1998; Jonsson et al., 1994; Naya et al., 1997). Mutations in HNF-1 $\alpha$ account for most of the mutations associated with MODY identified in nuclear factors. More than 80 different mutations located in the coding regions or in the promoter were found in various populations (Boutin et al., 1997; Chèvre et al., 1997; Frayling et al., 1997; Glucksmann et al., 1997; Hansen et al., 1997; Kaisaki et al., 1997; Vaxillaire et al., 1997; Yamagata et al., 1996b). An insulin secretory defect in the absence of insulin resistance was observed in diabetic and nondiabetic carriers of MODY3 mutations (Byrne et al., 1996; Vaxillaire et al., 1999b), suggesting that HNF-1 $\alpha$ is, indeed, implicated in pancreatic $\beta$-cell function. In contrast to the usually mild hyperglycemia due to glucokinase deficiency, MODY3 is a severe form of diabetes, often evolving to insulin requirement. Microvascular complications of diabetes are observed as frequently in MODY3 as in late age of onset T2DM subjects (Isomaa et al., 1998; 
Velho et al., 1996c). HNF-1 $\alpha$ also is expressed in the kidney; a defect in the renal resorption of glucose often is associated with the pancreatic $\beta$-cell defect in MODY3 subjects (Menzel et al., 1998; Velho et al., 1998). Heterozygous knockout mice lacking one copy of HNF-1 $\alpha$ have a normal phenotype, while MODY3 subjects are all heterozygous for their mutations and fully express the diabetes phenotype (Pontoglio et al., 1996,1998). This observation suggests that these mutations might have a dominant-negative effect. However, experimental data show that only the mutations located in the transactivation domain of HNF-1 $\alpha$ have a dominant-negative effect on HNF- $1 \alpha$ transactivation potential (Vaxillaire et al., 1999a). Mutations located elsewhere in the protein do not interfere with the activity of the normal allele. The target genes associated with the $\beta$-cell defect of MODY3 remain unknown. Contrasting results were observed in studies of knockout mice (Dukes et al., 1998; Okita et al., 1999; Pontoglio et al., 1998; Wang et al., 1998), notably regarding the role of the insulin gene.

MODY1 is much less prevalent than MODY2 and MODY3. Only a few kindred other than the large American RW family were found to carry an HNF-4 $\alpha$ mutation (Bulman et al., 1997; Furuta et al., 1997; Lindner et al., 1997; Yamagata et al., 1996a). HNF- $4 \alpha$ is a member of the steroid/thyroid hormone receptor superfamily and upstream regulator of $\mathrm{HNF}-1 \alpha$ expression. Interestingly, it was demonstrated that long-chain fatty acids directly modulate the transcriptional activity of HNF- $4 \alpha$ by binding as acyl-CoA thioesters to the ligand binding domain of HNF-4 $\alpha$ (Hertz et al., 1998). This binding results in the activation or the inhibition of HNF- $4 \alpha$ transcriptional activity as a function of chain length and the degree of saturation of the fatty acyl-CoA ligand (Hertz et al., 1998). This observation contributes important data to the understanding of the role of dietary fats in the control of insulin secretion. Here, again, the target genes of HNF-4 $\alpha$ associated with $\beta$-cell defect are not clearly determined (Stoffel and Duncan, 1997).

Mutations in HNF-1 $1 \beta$ recently were described in a few families with familial diabetes with early onset consistent with MODY (Horikawa et al., 1997; Nishigori et al., 1998). In these pedigrees, HNF-1 $\beta$ mutations were associated with diabetes and severe kidney disease that may appear before the impairment of glucose tolerance. Polycystic renal disease and/or particular histological abnormalities showing meganephrons were present in some subjects, suggesting that this gene could play a major role in kidney development and nephron differentiation. It is noteworthy that HNF-1 $\beta$ and HNF- $1 \alpha$ can form heterodimers to bind DNA (Tronche and Yaniv, 1992).

All of these genetic defects in transcription factors lead to abnormalities of glucose homeostasis, thereby promoting the development of chronic hyperglycemia through alterations in insulin secretion and possibly in the development of 
the pancreatic islets. In this regard, a deletion in the homeodomain transcription factor insulin promoter factor-1 (IPF-1 or IDX-1, STF-1, PDX-1) was found to co-segregate with MODY in a large kindred presenting a consanguineous link (Stoffers et al., 1997a). This mutation results in a premature stop codon and a protein lacking a domain that is crucial for DNA binding. The phenotype of the subjects who are heterozygous for the mutation ranges from normal to impaired glucose tolerance to overt NIDDM. One child who is homozygous for the mutation was born with pancreatic agenesis and suffers from diabetes as well as exocrine insufficiency (Stoffers et al., 1997b). IPF-1 is critically required for the embryonic development of the pancreatic islets as well as for transcriptional regulation of endocrine pancreatic tissue-specific genes in adults, such as the insulin, glucose transporter-2 (GLUT2), and glucokinase genes in $\beta$ cells and the somatostatin gene in $\delta$-cells. IPF-1 normally is expressed in all cells of the pancreatic bud. Its absence in mice arrests development at the bud stage, leading to pancreatic agenesis (Jonsson et al., 1994).

The transcription factor NeuroD1 (also known as Beta 2) is involved in the regulation of endocrine pancreas development. In mice homozygous for a targeted disruption of NeuroD1, pancreatic islet morphogenesis is abnormal and hyperglycemia develops, due in part to inadequate expression of the insulin gene (Naya et al., 1997). Recently, mutations in NeuroDl were shown to co-segregate with T2DM of early age of onset and autosomal dominant-like transmission in two Caucasian kindreds (Malecki et al., 1999). This observation suggests that NeuroD1 might play an important role in endocrine pancreas development and/or insulin gene expression in humans.

\section{Mitochondrial Diabetes and Wolfram Syndrome}

Mitochondria contain their own genetic information in the form of a circular DNA molecule of 16,569 base pairs that encodes 13 subunits of the oxidative phosphorylation complex, two ribosomal RNAs, and 22 transfer RNAs (tRNA) needed for mitochondrial protein synthesis. Several mitochondrial cytopathies and syndromes caused by point mutations, deletions, or duplications of mitochondrial DNA (mtDNA) and characterized by decreased oxidative phosphorylation are associated with diabetes (Gerbitz et al., 1995; Luft and Landau, 1995). Moreover, about 40 point mutations of mtDNA have been identified in subjects and families having maternally inherited diabetes as the main phenotypic trait (Mathews and Berdanier, 1998). Only one of these mutations, an A-to-G transition in the mito-

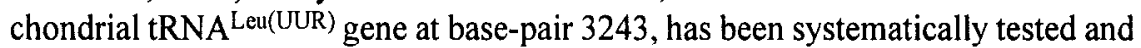
phenotypically characterized in several populations (Maassen and Kadowaki, 1996; Massin et al., 1995; Van den Ouweland et al., 1992,1994; Vialettes et al., 1995,1997; Vionnet et al., 1993). It co-segregates in families with diabetes and 
sensorineural deafness of maternal transmission, a syndrome known as maternally inherited diabetes and deafness (MIDD). In some populations, MIDD might represent 1 to 3 percent of all cases of T2DM. The same mutation was observed in patients with MELAS - a syndrome of mitochondrial myopathy, encephalopathy, lactic acidosis, and stroke-like episodes - that is often accompanied by diabetes and deafness (Ciafaloni et al., 1992). The mechanisms underlying the different phenotypic expression (MIDD or MELAS) are unknown but might be related to the variable degree of heteroplasmy in different tissues.

Subjects with the 3243 mutation may present with variable clinical features, ranging from normal glucose tolerance to insulin-requiring diabetes. However, abnormalities in insulin secretion were found in all MIDD subjects that were tested, including those with normal glucose tolerance (Velho et al., 1996a). The pathophysiological mechanisms leading to hyperglycemia and often to insulin-requiring diabetes in this syndrome are probably complex and multifactorial and might include defects in insulin production or glucose toxicity as well as insulin resistance. However, a defect of glucose-regulated insulin secretion is an early, possible primary abnormality in carriers of the mutation (Velho et al., 1996a). This defect probably results from the progressive reduction of oxidative phosphorylation in $\beta$ cells caused by the accumulation of mutant mitochondrial DNA (Luft and Landau, 1995; Maassen and Kadowaki, 1996).

Wolfram syndrome (or the acronym DIDMOAD) describes patients with diabetes insipidus, diabetes mellitus, optical atrophy, and deafness. Other endocrine and neurological abnormalities often are associated in this genetically and clinically heterogeneous syndrome. Wolfram syndrome is frequently transmitted as an autosomal recessive disorder by a locus mapped to the short arm of chromosome 4. This gene, named WFS1, recently was identified (Inoue et al., 1998; Strom et al., 1998). It encodes wolframin, a protein showing no perceptible homology to known DNA or protein sequences (Gerbitz, 1999). The physiological function of wolframin and its link to diabetes remain unclear. In contrast to this autosomal recessive transmission, a few cases of Wolfram syndrome were found to be associated with mitochondrial DNA mutations (Pilz et al., 1994; Rotig et al., 1993).

\section{Candidate Genes and Polygenic Forms of T2DM}

The majority of the genes found to play a role in the common forms of T2DM have been identified by testing candidate genes, the most-used approach, up to now, to tackle the genetic determinants of T2DM. Reasons for candidacy are numerous: 1) known or presumed biological function in glucose homeostasis or energy balance in humans; 2) gene implicated in subtypes of diabetes (e.g., MODY); 3) gene associaled with diabetes or associated traits in animal models; 
4) gene responsible for an inherited disease that includes diabetes (e.g., mitochondrial cytopathies, Wolfram syndrome); or 5) product differentially expressed in diabetic and normal tissues.

For obvious reasons, the insulin gene was among the first genes to be studied. Mutations in the coding regions of the insulin gene (chromosome 11p) have been reported in less than 10 families but are not consistently associated with T2DM (Haneda et al., 1984). However, mutations in the promoter region could affect the regulation of the insulin gene, leading to a decrease of transcription and absolute or relative hypoinsulinemia. A variant allele of the promoter was observed in about 5 percent of African Americans with T2DM and shown to be associated with decreased transcriptional activity (Olansky et al., 1992). More recently, an association between T2DM and paternally transmitted class III alleles of the variable region upstream of the insulin gene (INS-VNTR) was observed in British families (Huxtable et al., 2000). Interestingly, class III alleles also were found to be associated with increased length and weight at birth (Dunger et al., 1998) and with a dominant protection against type 1 diabetes (Bennett et al., 1995), as compared with type I alleles.

The role of the MODY genes and of other transcription factors in the development of the more-common forms of late-onset T2DM is still under investigation. Regarding the MODY genes, most studies have excluded a major role in the genetic determinants of T2DM. However, mutations in HNF-1 $\alpha$ were identified in African-American and Japanese subjects with atypical non-autoimmune diabetes of acute onset (Boutin et al., 1999; Iwasaki et al., 1997). A common polymorphism in HNF-1 $\alpha$ was found to be associated with mild insulin secretion defects

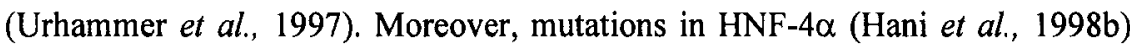
and IPF1 (Hani et al., 1999; Macfarlane et al., 1999) were identified in a few families with late-onset T2DM. Several other transcription factors have been studied: a mutation in islet brain 1 (IB1) was found to be associated with diabetes in one family (Waeber et al., 2000). IBl is a homologue of the c-jun amino-terminal kinase interacting protein 1 (JIP-1), which plays a role in the modulation of apoptosis. IB1 is also a transactivator of the islet glucose transporter, GLUT2. The mutant IBI was found to be unable to prevent apoptosis in vitro. It is thus possible that the abnormal function of this mutant IB1 may render $\beta$ cells more susceptible to apoptotic stimuli, thus decreasing $\beta$-cell mass. As glucotoxicity and lipotoxicity are known to induce both apoptosis and transcription factor downregulation in pancreatic $\beta$ cells, inherited or acquired limitations in IB1 activity could have deleterious effects in $\beta$-cell function.

On the other hand, mutations in peroxisome proliferator-activated receptor gamma (PPAR $\gamma$ ) that severely decrease the transactivation potential were found to co-segregate with extreme insulin resistance, diabetes, and hypertension in two families with autosomal dominant inheritance (Barroso et al., 1999). Interestingly, 
given the proposed role of PPAR $\gamma$ in adipogenesis, all affected family members had no evidence of lipoatrophy or abnormal fat distribution. All together, these data suggest that mutations in transcription factors may contribute to the genelic risk to T2DM through various mechanisms: 1) dysregulation of target genes involved in glucose or lipid metabolism (i.e., HNFs, PPAR $\gamma$, IPF1, IB1); 2) abnormal $\beta$-cell development and differentiation (i.e., IPF1, NeuroD1/Beta2); or 3 ) dysregulation of $\beta$-cell apoptosis (IBI). Deleterious mutations that significantly impair the transactivation activity of these transcription factors can be responsible in some families for monogenic-like forms of diabetes with late age of onset, which may represent an intermediary phenotype between MODY and the mostcommon forms of T2DM.

Other genes encoding key components of insulin secretion pathways were tested as potential candidates for a role in the genetic susceptibility of T2DM. The pancreatic $\beta$-cell ATP-sensitive potassium channel (IKATP) plays a central role in glucose-induced insulin secretion by linking signals derived from glucose metabolism to cell membrane depolarization and insulin exocytosis (Dukes and Philipson, 1996). IKATP is composed of two distinct subunits: an inwardly rectifying ion channel forming the pore (Kir6.2) and a regulatory subunit, a sulfonylurea receptor (SUR1) belonging to the ATP binding cassette (ABC) superfamily (Inagaki et al., 1995). The genes encoding these two subunits are located $4.5 \mathrm{~kb}$ apart on human chromosome $11 \mathrm{p} 15.1$. Mutations in each of these genes may result in familial persistent hyperinsulinemic hypoglycemia of infancy, demonstrating their role in the regulation of insulin secretion (Ashcroft and Gribble, 1999). Studies in various populations with different ethnic backgrounds provided evidence for associations of single nucleotide polymorphisms (SNPs) in these genes with T2DM ('t Hart et al., 1999; Hani et al., 1997,1998a; Hansen et al., 1998; Inoue et al., 1996; Ohta et al., 1998). However, sib-pair analyses in several populations indicated that the SUR 1/Kir6.2 region is not a major diabetogenic locus (Hani et al., 1997; Iwasaki et al., 1996; Stirling et al., 1995).

Key components of the insulin-signaling pathways also were tested. They were at first thought to be important players in the context of the insulin resistance of T2DM. Several of these genes are expressed in pancreatic $\beta$ cells. Recent results in knockout animals demonstrated that they also play an important role in the mechanisms of insulin secretion (Kulkarni et al., 1999; Withers et al., 1998). More than 50 different mutations have been found in the coding regions of the insulin receptor gene on chromosome 19p (Taylor, 1992). However, patients with these mutations seldom present with the common form of T2DM (Kan et al., 1995) but rather with syndromes of severe insulin resistance associated with leprechaunism or with acanthosis nigricans, hirsutism, or major hyperinsulinemia (Flier, 1992). Missense variants in the coding regions of the gene encoding the first substrate for the insulin receptor kinase (IRS-1) on chromosome $2 q$ have been detected in 
several populations (Almind et al., 1993; Hager et al., 1993; Hitman et al., 1995; Ura et al., 1996). However, association of these variants with diabetes was not observed in all these studies. Similarly, an association between polymorphisms of the muscle glycogen synthase gene (GSY1) on chromosome 19q and T2DM was observed in Finnish (Groop et al., 1993) and in Japanese (Kuroyama et al., 1994) subjects but not in French ones (Zouali et al., 1993). Taken together, these results suggest that IRS-1 and GSYl genes might act in some populations as minor susceptibility genes, which are neither necessary nor sufficient for disease expression but may nevertheless modulate the phenotype of patients.

Other genes were shown to be implicated in the genetic susceptibility to insulin resistance. Although they do not seem to be directly linked to or associated with T2DM, they could modulate the expression of diabetes. A common and widespread polymorphism at codon 905 of the gene encoding the glycogen-associated regulatory subunit of protein phosphatase- 1 of the skeletal muscle was shown to be associated with insulin resistance and hypersecretion of insulin in Danish T2DM subjects (Hansen et al., 1995). A missense mutation in the intestinal fatty acid binding protein 2 (FABP2) gene on chromosome $4 \mathrm{q}$ was found to be associated with increased fatty acid binding, increased fat oxidation, and insulin resistance in the Pima Indians of Arizona (Baier et al., 1995), an ethnic group with the highest reported prevalence of T2DM and insulin resistance in the world. A point mutation in the gene encoding the beta- 3 adrenergic receptor was found to be associated with an increased capacity to gain weight in a population of morbidly obese subjects (Clément et al., 1995). This same mutation was associated with reduced metabolic rate and early onset of diabetes (Walston et al., 1995) and with the development of upper-body obesity and insulin resistance (Widen et al., 1995) in two T2DM populations.

\section{Positional Cloning of T2DM Genes}

The candidate gene approach presents limitations, as it is now clear that at least some T2DM susceptibility genes are likely to code for proteins of unknown function or a function not obviously implicated in glucose metabolism. The genome-wide linkage approach attempts to locate these unknown genes by a systematic search throughout the genome. This consists of genotyping the entire genome of affected sib-pairs or families with panels of 250-300 anonymous polymorphic markers to identify regions showing excess of allele sharing with the disease. This total genome approach has been successful in other multifactorial diseases such as type 1 diabetes (Davies et al., 1994) and obesity (Hager et al., 1998). More than 20 genome scans for T2DM are currently underway, involving thousands of pedigrees from different populations and ethnic groups. One of the limitations of the genome-scan approach is the relatively low power of the method, (i.e., inabilty to detect weak linkage signal), which is due to the low relative risk 
for diabetes in siblings (about a 3- to 5-fold increase, compared to the general population). Working on large family collections, in homogenous ethnic groups, or in large pedigrees using quantitative traits instead of the dichotomous diabetes status could improve the efficiency to detect linkage. Moreover, because of the large number of markers that are tested, false-positive results are likely to occur. Thus, stringent criteria for linkage $\left(\mathrm{p}<10^{-5}\right)$ need to be used to minimize the bias due to multiple testing.

Results of several genome scans already have been published. A locus for T2DM on chromosome 2q (NIDDMl) was localized in Mexican Americans (Hanis et al., 1996). It showed that an interaction of this locus with a locus on chromosome 15 further increases the susceptibility to diabetes in this population (Cox et al., 1999). Linkage was found at a locus near MODY3 on chromosome 12q in Finnish T2DM families characterized by a predominant insulin secretion defect (Mahtani et al., 1996). Evidence for an obesity-diabetes locus on chromosomc 11q23-q25 (Norman et al., 1998) and linkage of several chromosomal regions with pre-diabetic traits (Pratley et al., 1998) were observed in Pima Indians, an ethnic group with a high prevalence of diabetes and obesity. A strong linkage between diabetes and chromosome 1q21-1q23 was reported in multigenerational families of northern European ancestry from Utah (Elbein et al., 1999). Linkages with diabetes and with the age at onset of diabetes were found in a region on chromosome 10q in Mexican American families from San Antonio (Duggirala et al., 1999). Evidence for the presence of one or more diabetes loci on chromosome 20 was found in different populations (Ji et al., 1997; Zouali et al., 1997). In these and other studies, many loci showing only suggestive or weak indication of linkage with diabetes-related traits have been reported, several of which fall in overlapping regions. Although many of these loci may represent false-positive results, some may harbor true diabetes-susceptibility genes. Comparisons of linkage result in different populations or family collections and/or meta-analysis of the data may help to guide positional cloning efforts. New statistical methods exploiting multiloci effects or analyzing quantitative traits should permit researchers to squeeze more power from genome-scan data.

These genome scans have mapped loci within large chromosomal regions containing 10-20 million nucleotides. Now, the challenge is to identify the diabetes-related genes within this interval. The classical approach that consisted of building a physical map of the region through contiguous artificial chromosomes spanning the entire region of linkage, followed by the cloning of the gene, is limited by size of the regions of linkage. An integrated genomic approach might be needed. It would combine linkage disequilibrium mapping, to define more-precise gene locations, with techniques to pick out the genes of these smaller regions (e.g., microarrays for the identification of genes differentially expressed in diabetic and nondiabetic subjects). These investigations will benefit from recent technological developments in SNP identification and genotyping. Moreover, the 
results from the Human Genome Project - which include genomic DNA sequences, expressed sequences, and expression profile databanks - will certainly make easier the identification of T2DM-susceptibility genes by positional cloning. The recent identification by Graeme Bell and coworkers of NIDDMl as the gene encoding calpain 10 (cAPN10), a nonlysosomal cysteine protease, demonstrates the feasibility of positional cloning of polygenic T2DM genes. Currently, it is believed that less than 15 percent of the genetic determinants of T2DM have been unveiled. It is likely that other genes contributing to the genetic risk of T2DM will be shortly discovered.

\section{Conclusions and Perspective}

The identification of T2DM genes will improve our understanding of the molecular mechanisms that maintain glucose homeostasis and of the precise molecular defects leading to chronic hyperglycemia. A nosological classification of T2DM, based on primary pathophysiological mechanisms, will then be possible. This could lead to the development of more-specifically targeted antidiabetic drugs or even gene-based therapies. Moreover, pharmacogenetic testing might then be used to predict for each patient the therapeutic response to different classes of drugs. The identification of T2DM genes also will provide the tools for the timely identification of high-risk individuals who might benefit from early behavioral or medical intervention to prevent the development of diabetes. An important reduction in diabetes-related morbidity and mortality could be then expected, along with a reduction in the costs of treatment of diabetes and its complications.

\section{RFFERENCES}

Ahlgren, U., et al. (1998). Gene Devel. 12, 1763-1768.

Almind, K., et al. (1993). Lancet 342, 828-832.

Ashicroft, F.M., and Gribble, F.M. (1999). Diabetologia 42, 903-919.

Baier, L.J., et al. (1995). J. Clin. Invest. 95, 1281-1287.

Barker, D.J. (1995). Mol. Med. Today 1, 418-423.

Barroso, I., et al. (1999). Nature 402, 880-883.

Bell, G.I., et al. (1991). Proc. Natl. Acad. Sci. U.S.A. 88, 1484-1488.

Bennett, S.T., el al. (1995). Nature Genet. 9, 284-292.

Blanché, H, et al (1997), Diab. Metab. 23(suppl.1), 29.

Boutin, P., et al. (1997). Diabetes 46, 2108-2109.

Boutin, P., et al. (1999). Diabetologia 42, 380-381.

Bulman, M.P., et al. (1997), Diabetologia 40, 859-862.

Byrne, M.M., et al. (1994). J. Clin. Invest. 93, 1120-1130.

Byrne, M.M., et al. (1996). Diabetes 45, 1503-1510.

Carmelli, D., et al. (1994). Am. J. Hum. Genet. 55, 566-573.

Chèvre, J.C., et al. (1997). Diabetologia 40(suppl.1), A157.

Chèvre, J.C., et al. (1998). Diabetologia 41, 1017-1023.

Ciafaloni, E., et al. (1992). Ann. Neurol. 31, 391-398. 
Clément, K., et al. (1995). N. Engl. J. Med. 333, 352-354.

Costa, A., et al. (2000). Eur. J. Endocrinol. 142, 380-386.

Cox, N.J., et al. (1999). Nature Genet. 21, 213-215.

Davies, J.L., et al. (1994). Nature 371, 130-136.

DeFronzo, R.A. (1997). Diabetes Rev 5, 177-269.

Duggirala, R., et al. (1999). Am. J. Hum. Genet. 64, 1127-1140.

Dukes, I.D., and Philipson, L.H. (1996). Diabetes 45, 845-853.

Dukes, I.D, et al. (1998). J. Biol. Chem. 273, 24457-24464.

Dunger, D.B., et al. (1998). Nature Genet. 19, 98-100.

Elbcin, S.C., et al. (1999). Diabetes 48, 1175-1182.

Ferrannini, E. (1998). Endocr. Rev. 19, 477-490.

Flier, J.S. (1992). Diabetes 41, 1207-1219.

Frayling, T.M., et al. (1997). Diabetes 46, 720-725.

Froguel, P., and Velho, G. (1999). Trends Endocrinol. Metab. 10, 142-146.

Froguel, P., et al. (1992). Nature 356, 162-164.

Froguel, P., et al. (1993). N. Engl. J. Med. 328, 697-702.

Furuta, H., et al. (1997). Diabetes 46, 1652-1657.

Gauguier, D., et al. (1996). Nature Genet. 12, 38-43.

Gerbitz, K.D. (1999). Diabetologia 42, 627-630.

Gerbitz, K.D., et al. (1995). B.B.A. - Mol. Basis Dis. 1271, 253-260.

Gidh-Jain, M., et al. (1993). Proc. Natl. Acad. Sci. U.S.A. 90, 1932-1936

Glucksmann, M.A., et al. (1997). Diabetes 46, 1081-1086.

Groop, L.C., et al. (1993). N. Engl. J. Med. 328, 10-14.

Hager, J., et al. (1993). Lancet 342, 1430.

Hager, J., et al. (1998). Nature Genet. 20, 304-308.

Hales, C.N., et al. (1991). Br. Med. J. 303, 1019-1022.

Haneda, M., et al. (1984). N. Engl. J. Med 310, 1288-1294.

Hani, E.H., et al. (1997). Diabetes 46, 688-694.

Hani, E.H., et al. (1998a). Diabetologia 41, 1511-1515.

Hani, E.H., et al. (1998b). J. Clin. Invest. 101, 521-526.

Hani, E.H., et al. (1999). J. Clin. Invest. 104, R41-R48.

Hanis, C.L., et al. (1996). Nature Genet. 13, 161-166.

Hansen, L., et al. (1995). Hum. Mol. Genet. 4, 1313-1320.

Hansen, T, et al. (1997). Diabetes 46, 726-730.

Hansen, T., et al. (1998). Diabetes 47, 598-605.

Hattersley, A.T., and Tooke, J.E. (1999). Lancet 353, 1789-1792.

Hertz, R., et al. (1998). Nature 392, 512-516.

Hitman, G.A., et al. (1995). Diabetologia 38, 481-486.

Horikawa, Y, et al. (1997). Nature Genet. 17, 384-385

Huxtable, S.J., et al. (2000). Diabetes 49, 126-130.

Inagaki, N., et al. (1995). Science 270, 1166-1170.

Inoue, H., et al. (1996). Diabetes 45, 825-831.

Inoue, H., et al. (1998). Nature Genet. 20, 143-148.

Isomaa, B., et al. (1998). Diabetologia 41, 467-473.

Iwasaki, N., et al. (1996). Diabetes 45, 267-269.

Iwasaki, N., et al. (1997). Diabetes 46, 1504-1508.

Ji, L.N., et al. (1997). Diabetes 46, 876-881.

Jonsson, J., et al. (1994). Nature 371, 606-609.

Kaisaki, P.J., et al. (1997). Diabetes 46, 528-535.

Kan, M., et al. (1995). Diabetes 44, 1081-1086. 
Kulkarni, R.N., et al. (1999). Cell 96, 329-339.

Kuroyama, H., et al. (1994). Diabetologia 37, 536-539.

Ledermann, H.M. (1995). Lancet 345, 648.

Lindner, T., et al. (1997). J. Clin. Invest. 100, 1400-1405.

Lindner, T.H., et al. (1999). Diabetologia 42, 121-123.

Lithell, H.O., et al. (1996). Br. Med. J. 312, 406-410.

Luft, R., and Landau, B.R. (1995). J. Intern. Med. 238, 405-421.

Maassen, J.A., and Kadowaki, T. (1996). Diabetologia 39, 375-382.

Macfarlane, W.M., et al. (1999). J. Clin. Invest. 104, R33-R39.

Mahtani, M.M., et al. (1996). Nature Genet. 14,90-94.

Malecki, M.T., et al. (1999). Nature Genet. 23, 323-328.

Massin, P., et al. (1995). Am. J. Ophtalmol. 120, 247-248.

Mathews, C.E., and Berdanier, C.D. (1998). Proc. Soc. Exp. Biol. Med. 219, 97-108.

Matschinsky, F.M. (1996). Diabetes 45, 223-241.

Menzel, R., et al. (1998). Diabet. Med. 15, 816-820.

Naggert, J.K., et al. (1995). Nature Genet. 10, 135-142.

Naya, F.J., et al. (1997). Gene Devel. 11, 2323-2334.

Nishigori, H., et al. (1998). Diabetes 47, 1354-1355.

Norman, R., et al. (1998). Am. J. Hum. Genet. 62, 659-668.

Ohta, Y., et al. (1998). Diabetes 47, 476-481.

Okita, K, et al. (1999). Biochem. Biophys. Res. Commun. 263, 566-569.

Olansky, L., et al. (1992). J. Clin. Invest. 89, 1596-1602.

Pilz, D., et al. (1994). J. Med. Genet. 31, 328-330.

Pontoglio, M., et al. (1996). Cell 84, 572-585.

Pontoglio, M., et al. (1998). J. Clin. Invest. 101, 2215-2222.

Pratley, R.E., et al. (1998). J. Clin. Invest. 101, 1757-1764.

Rotig, A., et al. (1993). J. Clin. Invest. 91, 1095-1098.

Stirling, B., et al. (1995). Diabetologia 38, 1479-1481.

Stoffel, M., and Duncan, S.A. (1997). Proc. Natl. Acad. Sci. U.S.A. 94, 13209-13214.

Stoffers, D.A., et al. (1997a). Nature Genet. 17, 138-139.

Stoffers, D.A., et al. (1997b). Nature Genet. 15, 106-110.

Strom, T.M., et al. (1998). Hum. Mol. Genet. 7, 2021-2028.

Sturis, J., et al. (1994). Diabetes 43, 718-723.

Taylor, S.I. (1992). Diabetes 41, 1473-1490.

't Hart, L.M. et al. (1999). Diabetologia 42, 617-620.

Tronche, F., and Yaniv, M. (1992). Bioessays 14, 579-587.

Ura, S., et al. (1996). Diabetologia 39, 600-608.

Urhammer, S.A., et al. (1997). Diabetologia 40, 473-475.

van den Ouweland, J.M.W., et al. (1992). Nature Genet. 1, 368-371.

van den Ouweland, J.M.W., et al. (1994). Diabetes 43, 746-751.

Vaxillaire, M., et al. (1995). Nature Genet. 9, 418-423.

Vaxillaire, M., et al. (1997). Hum. Mol. Genet. 6, 583-586.

Vaxillaire, M., et al. (1999a). J. Biol. Chem. 274, 35639-35646.

Vaxillaire, M., et al. (1999b). Eur. J. Endocrinol. 141, 609-618.

Velho, G., and Froguel, P. (1998). Eur. J. Endocrinol. 138, 233-239.

Velho, G., et al. (1992). Lancet 340, 444-448.

Velho, G., et al. (1996a). Diabetes 45, 478-487.

Velho, G., et al. (1996b). J. Clin. Invest. 98, 1755-1761.

Velho, G., et al. (1996c). Diabetes Care 19, 915-919.

Velho, G., et al. (1997). Diabetologia 40, 217-224. 
Velho, G., et al. (1998). Diabetologia 41(suppl. 1), A108.

Vialettes, B., et al. (1995). Diabetes Care 18, 1023-1028.

Vialettes, B., et al. (1997). Diabetes Metah. 23, 52-56.

Vionnet, N., et al. (1993). Lancet 342, 1429-1430.

Waeber, G., et al. (2000). Nature Genet. 24, 291-295.

Walston, J., et al. (1995). N. Engl. J. Med. 333, 343-347.

Wang, H., et al. (1998). EMBO J. 17, 6701-6713.

Widen, E., et al. (1995). N. Engl. J. Med. 333, 348-351.

Withers, D.J., et al. (1998). Nature 391, 900-904.

Yamagata, K., et al. (1996a). Nature 384, 458-460.

Yamagata, K., et al. (1996b). Nature 384, 455-458.

Zouali, H., et al. (1993). N. Engl. J. Med. 328, 1568

Zouali, H., et al. (1997). Hum. Mol. Genet. 6, 1401-1408. 



\title{
Discovery of a Small Molecule Insulin Receptor Activator
}

\author{
Gino M. Salituro, ${ }^{*}$ Fernando Pelaez, ${ }^{\dagger}$ and Bei B. Zhang ${ }^{\ddagger}$ \\ *Department of Natural Product Drug Discovery, ${ }^{\dagger}$ Department of Molecular Endocrinology, \\ R80W250, Merck Research Laboratories, Rahway, New Jersey 07065; ${ }^{t}$ Centro de Investigacion \\ Basica, Merck. Sharp \& Dohme de Espana, S.A. Josefa Valcarcel 38, Madrid 28027, Spain
}

\begin{abstract}
Insulin elicits diverse biological responses in many tissues and cell types by binding to its specific receptor. The insulin receptor (IR) is a tetramer consisting of two extracellular $\alpha$ subunits and two membrane-spanning $\beta$ subunits. The binding of insulin to the receptor causes conformational changes that lead to autophosphorylation and activation of the tyrosine kinase intrinsic to the $\beta$ subunits. Insulin receptor transphosphorylates several immediate substrates, resulting in modulation of a cascade of downstrearn signal transduction molecules. In order to discover small molecules that activate the human insulin receptor tyrosine kinase (IRTK), a cell-based assay was established and utilized to screen a collection of synthetic chemicals and natural product extracts. This effort led to the identification of a nonpeptidyl, small molecule, insulin-mimetic compound (demethylasterriquinone B-1, DMAQ-B1) that was isolated from a mixture of metabolites produced by a tropical endophytic fungus, Pseudomassaria sp. This compound induced human IRTK activation and increased tyrosine phosphorylation of IR $\beta$ subunit. It mediated insulin-like effects, including insulin receptor substrate-1 (IRS-1) phosphorylation and activation of phosphotidylinositide 3-kinase and Akt kinase. DMAQ-BI also exhibited an insulin-like effect on glucose uptake in adipocytes and skeletal muscle tissue. Furthermore, the compound was relatively selective for IR vs. insulin-like growth factor-I (IGF-I) receptor and other homologous receptor tyrosine kinases. In addition, it activated partially purified native IR or recombinant IR kinase, demonstrating the direct interaction of the small molecule with the IR. Oral administration of DMAQ-B1 resulted in significant glucose lowering in two mouse models of diabetes. Thus, DMAQ-B1 represents the first orally active insulin-mimetic agent. Pharmaceutical intervention aimed at augmenting IR function ultimately may prove beneficial as a novel therapeutic option in patients with diabetes.
\end{abstract}

\section{Introduction}

Diabetes is a chronic metabolic disorder affecting approximately five percent of the population of industrialized nations. Type 1 diabetes results from a ccllmediated autoimmune destruction of the $\beta$ cells of the pancreas, leading to absolute insulin deficiency. The more-prevalent form, type 2 diabetes, accounts for more than 90 percent of cases. The pathogenesis of type 2 diabetes is complex, involving progressive development of insulin resistance and a relative deficiency in insulin secretion, leading to overt hyperglycemia. 
Insulin elicits a diverse array of biological responses by binding to its specific receptor (White and Kahn, 1994). The insulin receptor (IR) is a heterotetrameric protein consisting of two extracellular $\alpha$ subunits and two transmembrane $\beta$ subunits. The binding of the ligand to the $\alpha$ subunit of IR not only concentrates insulin at its site of action but also induces conformational changes in the receptor. These changes, in turn, stimulate the tyrosine kinase activity intrinsic to the $\beta$ subunit of the IR. Extensive studies have indicated that the ability of the receptor to autophosphorylate and phosphorylate intracellular substrates is essential for its mediation of the complex cellular responses of insulin (Ellis et al., 1987; Kasuga et al., 1982; Rosen et al., 1983; Yu and Czech, 1984). Insulin receptors trans phosphorylate several immediate substrates (on Tyr residues), including insulin receptor substrate (IRS) proteins 1-4, Shc, and Gab 1, each of which provide specific docking sites for other signaling proteins containing Src homology 2 (SH2) domains (White and Yenush, 1998). These events lead to the activation of downstream signaling molecules, including phosphatidylinositol-3-kinase (PI-3kinase). Numerous studies have adduced that PI-3-kinase is required for the metabolic effects of insulin. Although the discrete pathways that couple PI-3-kinase to glucose regulation remain poorly defined, Akt (or protein kinase B), a Ser/Thr kinase known to be PI-3-kinase dependent, appears to be involved in insulin-mediated activation of glucose transport (Kohn et al., 1996) and glycogen synthesis (Alessi and Cohen, 1998).

Insulin is essential for maintaining glucose homeostasis and regulating carbohydrate, lipid, and protein metabolism (Rosen, 1989). The central role of the IR in metabolic and growth control has been validated in IR null mouse models (Accili et al., 1996; Joshi et al., 1996). Alterations of IR in specific tissues and/or insulin receptor substrate (IRS) 1 and 2 function via genetic manipulation have been shown to produce insulin resistance and diabetes in mice (Bruning et al., 1997a,1997b,1998; Kido et al., 2000; Kulkarni et al., 1999a,1999b; Lauro et al., 1998; Taylor, 1999). Decreased cellular responses to insulin or perturbation of the insulin signaling pathways are associated with a number of pathological states. Mutations in the IR gene - which lead to alterations of receptor synthesis, degradation, and function - have been described in patients with several uncommon syndromes associated with severe insulin resistance (Taylor, 1992). The molecular basis for insulin resistance that precedes, or is associated with, common forms of type 2 diabetes remains poorly understood. However, several studies have shown modest decreases in IR numbers attributed to downregulation in response to hyperinsulinemia in tissues or cells from type 2 diabetic patients (Caro et al., 1986; Olefsky, 1976). Substantial decreases in insulin-stimulated receptor tyrosine kinase (IRTK) activity and an even-more-substantial defect in receptormediated IRS phosphorylation or PI-3-kinase activation have been described using samples of tissue (e.g., muscle or fat) from rodents or human subjects with 
type 2 diabetes (Caro et al., 1987; Goodyear et al., 1995; Kerouz et al., 1997). Although controversial, diminished insulin-stimulated Akt activation was documented in skeletal muscle from type 2 diabetic patients (Kim et al., 1999; Krook et al., 1998). Thus, in patients with type 2 diabetes, clear defects exist that involve the IR and proximal steps in insulin signaling.

Discovery of oral insulin mimetic agents has been a long-standing yet elusive goal of pharmaceutical research. Given that most type 2 patients respond to insulinotropic agents or exogenously administered insulin, pharmaceutical intervention aimed at mimicking insulin's effect and augmenting IR function may prove beneficial. As patients with type I (insulin-dependent) diabetes are dependent upon parenteral, exogenous insulin injections for metabolic control, the discovery of orally active small molecules that might serve to mimic insulin's effects could lead to alternative therapies for this disorder.

\section{Discovery of DMAQ-B1 Through Screening of Natural Products}

\section{A. ASSAY}

A cell-based, 96-well screening assay was designed to identify small molecule IR activators. This assay utilizes Chinese hamster ovary cells (CHO.IR) that overexpress human IRs (Zhang et al., 1994). Following incubation of intact cells with insulin or test compounds, IRs are immunopurified using a monoclonal antibody specific for IR. Activation of insulin receptor is determined by measuring the IRTK activity towards an exogenous substrate (polyGlu:Tyr 4:1). This assay was employed to screen synthetic chemicals and natural product extracts to identify novel compounds capable of activating IR. Such an approach has advantages over a receptor-binding assay in that small molecules that compete with insulin binding are likely to be antagonists for the receptor. Furthermore, the assay was designed to identify compounds that work in intact cells, either by interacting directly with the receptor or by indirectly stimulating receptor activation (e.g., a specific phosphatase inhibitor).

\section{B. SOURCES OF NATURAL PRODUCTS}

Natural products historically have been a prolific source of therapeutically useful drugs. Approximately 160 marketed drugs utilized in a variety of disease states have been derived from natural sources. These include antibiotics (e.g., erythromycin, vancomycin, cephamycin, thienamycin, penicillins); antifungals (e.g., amphotericin B); immunosuppressants (e.g., FK 506, cyclosporin); antitumor agents (e.g., taxol, bleomycin, doxorubicin); antiparasitic agents (e.g., avermectin, milbemycin), and the cholesterol-lowering agent lovastatin. 
Several drugs derived from natural sources have been approved recently, including the anti-obesity compound tetrahydrolipstatin and the antibiotic streptogramin. Moreover, the antifungal lipopeptide known as caspofungin acetate (MK0991, CANCIDAS ${ }^{\mathrm{TM}}$ ), a semisynthetic derivative of the fungal metabolite pneumocandin $\mathrm{B}_{0}$, is now in phase III clinical trials. In the particular case of diabetes, a number of plant-derived secondary metabolites have been described to possess antidiabetic activity in a variety of model systems (Cignarella et al., 1996; Perez et al., 1997; Carney et al., 1999; Inman et aL, 1999).

In our screening program, over 50,000 samples of natural extracts were tested for their ability to mimic insulin activity using the assay described above. These extracts were obtained from more than 5000 microbial isolates recovered from diverse ecological niches (Figure 1). Substrates for microbial isolation were collected from a broad geographical range, covering both tropical and temperate regions. Fungal extracts were the predominant source of samples tested. The rationale behind focusing the screening on fungi is founded on the proven success of these microorganisms as a source of secondary metabolites (i.e., about 20,000 fungal metabolites are known to science, with 25 percent of them possessing biological activity) and the large sample pool from which to draw. Over 1.5 million species of fungi are thought to exist, with only 70,000 having been described (Hawksworth, 1991).

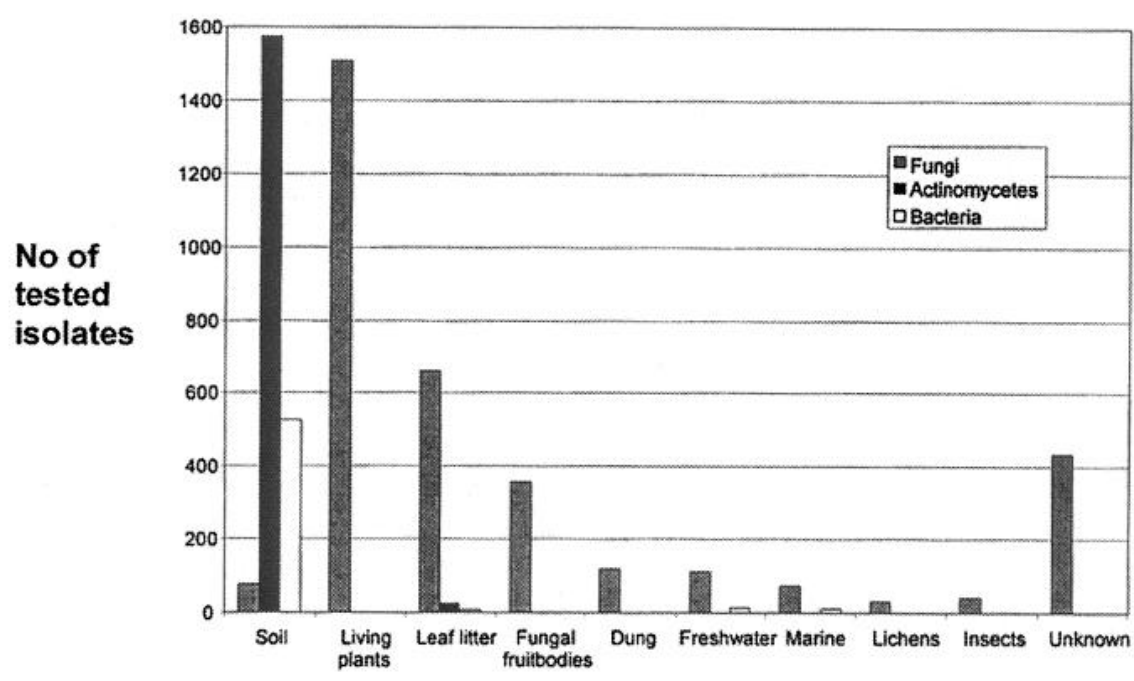

Substrate type

FIG. 1. Ecological distribution of microbial isolates tested in IRTK assay. 


\section{LEAD IDENTIFICATION AND ISOLATION}

In the course of screening microbial extracts in the cell-based IRTK activation assay, a sample obtained from the fermentation of a fungus isolated from unidentified living leaves collected near Kinshasa, Democratic Republic of Congo, was found to be moderately active. The fungus was recovered from the leaves using a standard surface sterilization technique for the isolation of endophytic fungi (Collado et al., 1996). This isolate (American Type Culture Collection 74411) was later tentatively identified as an undetermined species of the genus Pseudomassaria (Hyponectriaceae, incertae sedis, Ascomycota), based upon examination of an ascoma that was obtained by forcing the culture to grow on sterilized wood strips (G. Bills, unpublished results). Partial sequencing of the ribosomal DNA region, including the $18 \mathrm{~S}$ and 5.8S rRNA genes and the two internally transcribed spacers ITS1 and 1TS2, failed to provide a more-accurate identification for this strain. Pseudomassaria species typically are found growing on plant materials and are distributed in both tropical and temperate regions (Barr, 1964; Hyde et al., 1998). They had been never reported as producers of bioactive metabolites.

Seed cultures of Pseudomassaria sp. ATCC 74411 were prepared from slants grown on Difco Potato Dextrose Agar (PDA) at $25^{\circ} \mathrm{C}$ and 80 percent relative humidity (rh) for 14-2l days. The top third of one slant was aseptically transferred to a 250 -mL unbaffled flask containing $50 \mathrm{~mL}$ of a complex seed medium, then incubated on a shaker $(220 \mathrm{rpm})$ at $25^{\circ} \mathrm{C}$ and 80 percent rh for 4 days or until good growth was achieved. Two $\mathrm{mL}$ of a seed culture were used to inoculate 50 $\mathrm{mL}$ of production medium in $250-\mathrm{mL}$ Erlenmeyer flasks, which were incubated at $25^{\circ} \mathrm{C}, 220 \mathrm{rpm}, 80$ percent rh for 21 days (Zhang et al., 1999).

Interestingly, production of the IRTK-stimulating compound by the fungus appeared to be highly dependent on the culture conditions. Although the isolate was grown in as many as five different production media containing diverse nutritional components, the activity was recovered only in the medium described above.

For the isolation of the active compound present in the fermentation of Pseudomassaria sp. ATCC 74411, a 25-mL sample of whole broth was extracted with methylethyl ketone. A portion of the extract $(10 \mathrm{~mL})$ was dried $(250 \mathrm{mg})$ and purified by size exclusion chromatography on Sephadex LH-20 in methanol followed by semipreparative reverse-phase high-performance liquid chromotography (HPLC). The active compound crystallized as dark purple needles from ethyl acetate when stored in the cold $\left(4^{\circ} \mathrm{C}\right)$. Spectroscopic data: ${ }^{1} \mathrm{H},{ }^{13} \mathrm{C}$, correlation spectroscopy (COSY), heteronuclear multiple-quantum correlation (HMQC), heteronuclear mutiple-bond correlation (HMBC), and nuclear Overhauser effect spectroscopy (NOSEY) nuclear magnetic resonance (NMR) data and high-resolution fast atom bombardment mass spectroscopy (HRFABMS) were collected on 
$2.4 \mathrm{mg}$ of the major compound (Figure 2). Following data analysis, the compound was identified as demethylasterriquinone B-1, DMAQ-B1 (Figure 3), a member of the family of the asterriquinones (Yamamoto et al., 1976). The methylated analog asterriquinone B-1 had previously been isolated from Aspergillus terreus (Arai et al., 1981a). Ultimately, the structure of DMAQ-B1 was confirmed by a total synthesis of the compound (Liu et al., 1999).

The asterriquinones are widely known fungal bis-indolyl-quinones, structurally highly related to cochliodinoles (Jerram et al., 1975) and ochrindoles (De Guzman et al., 1994). These compounds have been found in many diverse, phylogenetically unrelated ascomycetes, including Aspergillus spp. (and the teleomorph Petromyces muricatus), Chaetomium spp., Chrysosporium merdarium, Humicola spp., and Botryotrichum spp. (De Guzman et al., 1994; Fredenhagen et al., 1997; Mocek et al., 1996; Ooike et al., 1997; Sekita, 1983). The asterriquinones and related bis-indolyl-quinones have been studied as potential antitumor agents because of their DNA-intercalating properties (Kaji et al., 1998; Yamamoto et al., 1976). Some of them are also HIV-1 protease inhibitors (Fredenhagen et al., 1997), antibacterial, and toxic to insects (De Guzman et al., 1994). Other biological activities reported for these molecules include inhibition of epidermal growth factor receptor (EGF-R) tyrosine kinase (Fredenhagen et al., 1997) and inhibition of the binding of the Grb-2 adapter to phosphorylated EGFR tyrosine kinase (Alvi et al., 1999). The compound found in our screening previously had been obtained by chemical treatment of asterriquinone B1 (Arai $e t$

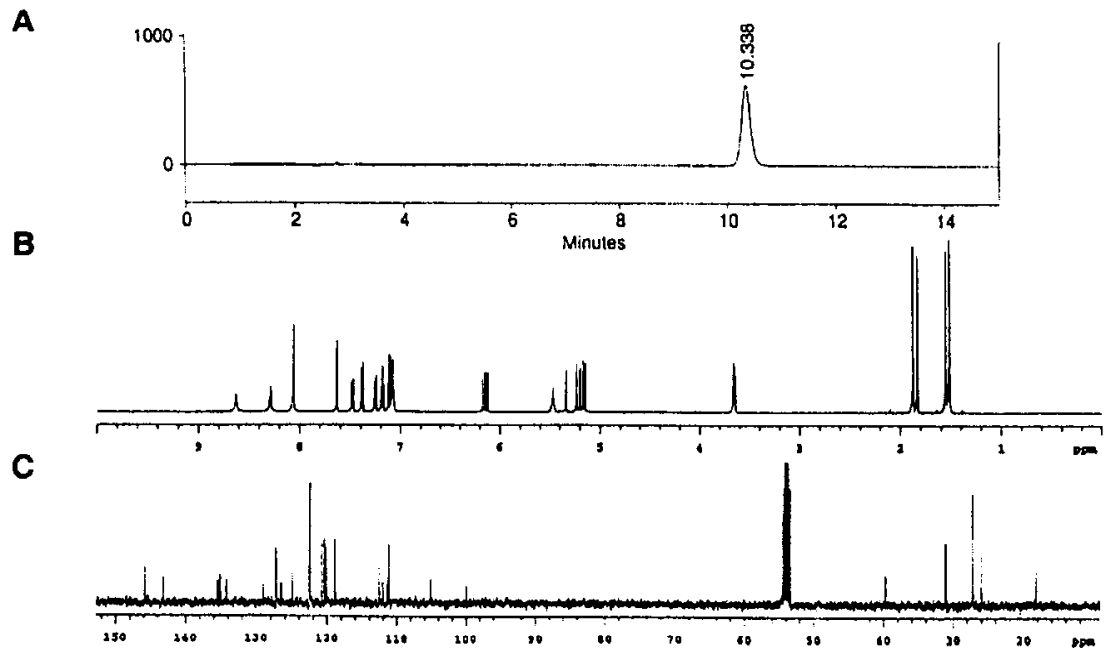

FIG. 2. Spectroscopic data on pure DMAQ-B1. (A) RP HPLC (Zorbax Rx C- $94.6 \times 250 \mathrm{~mm}$, $55: 45$ acetonitrile: $0.1 \%$ trifluroacetic acid $1 \mathrm{~mL} / \mathrm{min}$., $220 \mathrm{~nm}$ ). (B) $500 \mathrm{MHz}{ }^{1} \mathrm{H}_{\mathrm{NMR}}$ in $\mathrm{CD}_{2} \mathrm{Cl}_{2}$. (C) $125 \mathrm{MHz}{ }^{13} \mathrm{C} \mathrm{NMR}$ in $\mathrm{CD}_{2} \mathrm{Cl}_{2}$. 


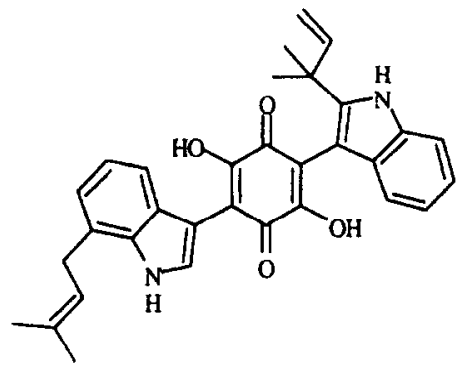

Demethylasterriquinone B1

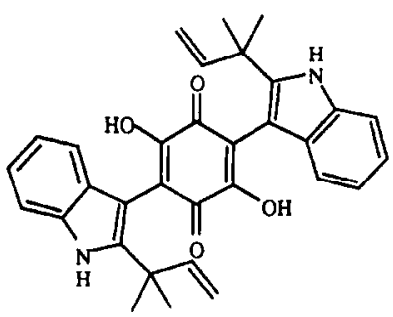

Hinnuliquinone

FIG. 3. Structures of demethylasterriquinone B-1 and hinnuliquinone.

al., 1981b) but its insulin-mimetic properties were unknown. Although asterriquinones seem to be widely distributed within the fungal kingdom, DMAQ-B1 has been found only once through several thousand fungal isolates screened. This suggests that, although the general metabolic pathway for the biosynthesis of asterriquinones may be present in many fungi, some of the specific steps involved in the synthesis of DMAQ-B1 could be much more restricted in nature.

In order to provide sufficient material for further biological testing, largescale fermentation and isolation methods were developed. A 120-liter batch of culture ATCC 74411 that contained approximated $630 \mathrm{mg} / \mathrm{L}$ of DMAQ-B1 was processed using a streamlined isolation procedure employing an adsorption-elution step, followed by liquid-liquid partitioning, and a single chromatography column (Figure 4). This yielded approximately $18 \mathrm{~g}$ of pure DMAQ-B1.

\section{ANALOGS (NATURAL PRODUCTS)}

This large-scale fermentation and isolation process led to the detection of a number of related minor components in the fermentation extracts. These components were purified by reverse-phase HPLC and their structures were elucidated using the same spectroscopic methods employed for the elucidation of DMAQ-B1 (Figure 5). Components 2 and 3 are most likely derived from alternate biosynthetic sequences, as they differ only in the functionality or number of isoprenyl sidechains on the indole rings. Components 4 and $\mathrm{S}$ can be envisioned as arising from oxidative transformations of DMAQ-B1. A major decomposition product also was observed in some aged solutions of DMAQ-B1. The appearance of this compound could be artificially accelerated under basic conditions $(\mathrm{pH}>10)$. 


\section{L WBE whole broth of Pseudomassaria}

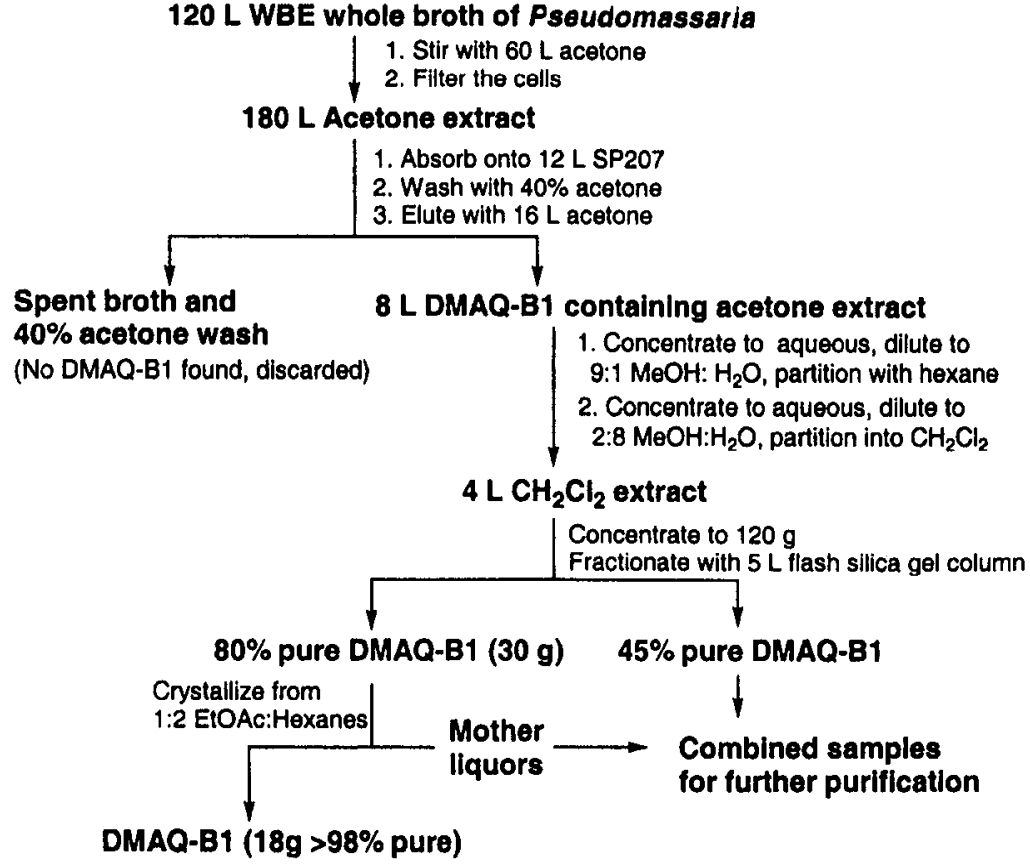

FIG. 4. Large-scale purification of DMAQ-B1 from Pseudomassaria fermentation.

\section{Biological Characterization of DMAQ-B1}

\section{A. ACTIVATION OF IR AND THE INSULIN SIGNALING PATHWAY}

In the cell-based IR activation assay, DMAQ-B1 was able to stimulate IRTK activity with 50 percent and 100 percent of the maximal activity achieved at 3-6 $\mathrm{uM}$ and 10-20 uM of compound concentrations, respectively (Figure 6A). The maximal IRTK activity induced by the compound was comparable to that stimulated by insulin. Hinulliquinone, the analog that is closely related in structure to DMAQ-B1, was about 100 times less potent in activating IRTK, suggesting that the effect of DMAQ-B1 is unlikely due to nonspecific effects exerted on cells by this class of compounds. When insulin was titrated in the assay in the presence of low concentrations of DMAQ-B1 (0.6 to $2 \mathrm{uM})$, there was a leftward shift of the insulin dose-response curve. This observation was consistent with the notion that DMAQ-B1 could function as an insulin sensitizer under the experimental conditions. 

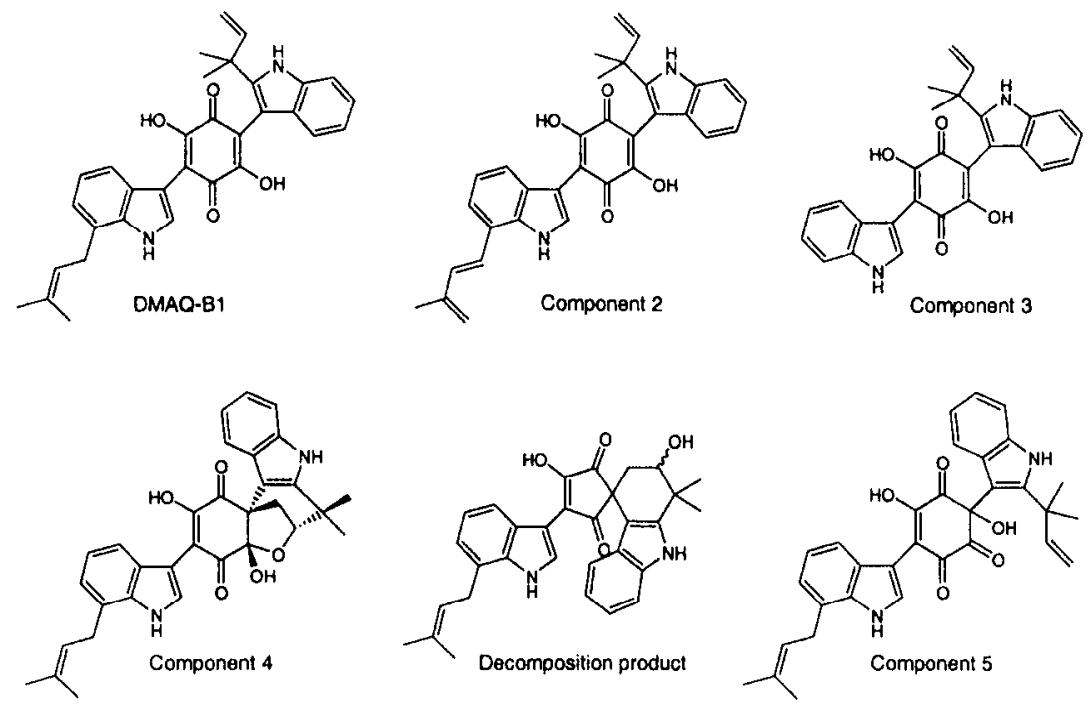
products.

FIG. 5. DMAQ-B1 and analogs isolated as minor fermentation components or degradation

DMAQ-B1 induced tyrosine phosphorylation of the IR $\beta$ subunit and IRS-1 in CHO.IR cells, as evidenced by antiphosphotyrosine immunoblotting (Figure $6 \mathrm{~B})$. Activation of these proximal insulin-signaling molecules was coupled to stimulation of other components of the signaling cascade. Thus, DMAQ-B1 stimulated PI-3-kinase activity (Endemann et al., 1990; Levy-Toledano et al., 1994) and phosphorylation of Akt kinase on the activating Ser-473 residue (Franke et al., 1995) in CHO.IR cells (Figure 7A and B). Moreover, DMAQ-B1 induced a classical insulin-like effect by acutely stimulating glucose uptake in rat primary adipocytes (263 percent of basal level at $10 \mu \mathrm{M}$ ) (Figure 7C) and in isolated soleus muscle from lean mice ( 237 percent of basal level at $2 \mu \mathrm{M}$ ) (Figure 7D). These results indicated that the small molecule, insulin-mimetic compound was capable of modulating multiple steps of the insulin signal transduction pathway.

\section{B. SELECTIVITY FOR IR}

Insulin receptor belongs to a superfamily of receptor tyrosine kinases with a high degree of sequence homology in the tyrosine kinase domain (Ullrich and Schlessinger, 1990). This family includes the IR and the receptors for growth factors such as insulin-like growth factor-I (IGF-I), EGF, and platelet-derived growth factor (PDGF). Activation of receptor tyrosine kinases leads to a wide 


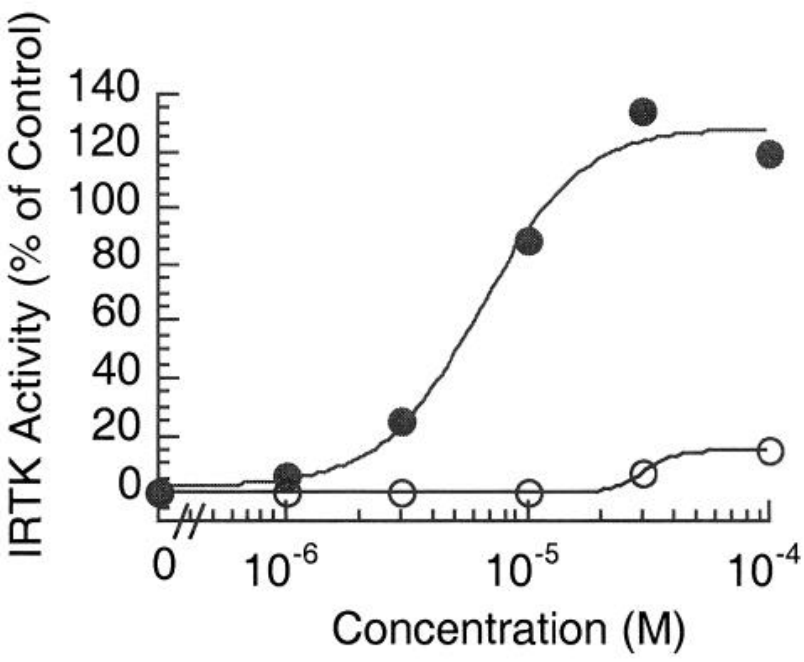

B

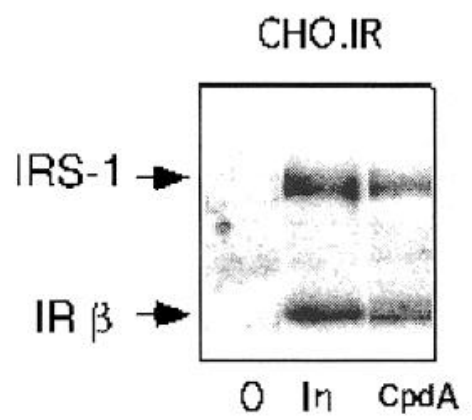

FIG. 6. Activation of IRTK in CHO.IR cells. (A) Dose- response curves are shown for DMAQB1 (filled circles) and hinulliquinone (open circles). (B) Stimulation of tyrosine phosphorylation of IRS- 1 and $\beta$ subunits of IR in CHO.IR cells by DMAQ-B1 (cpd A). [Reprinted with permission from Zhang, B., Salituro, G., Szalkowski, D., Li, Z., Zhang, Y., Royo, I., Vilella, D., Pelaez, F., Ruby, C., Kendall, R., Mao, X., Griffin, P., Calaycay, J., Heck, J.V., Smith, R.G., and Moller, D. Science 284, 974-977, 1999. Copyright 1999 American Association for the Advancement of Science.] 
A

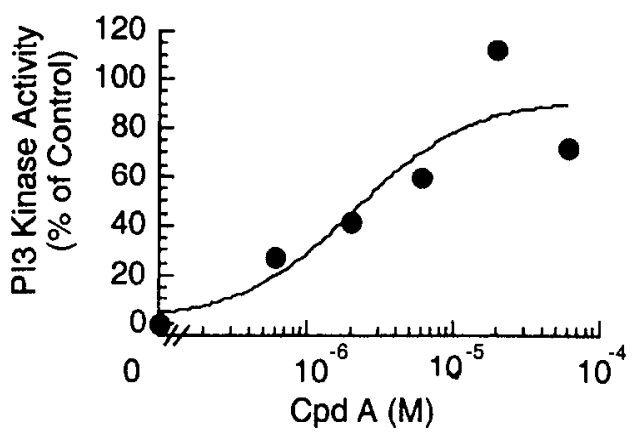

B

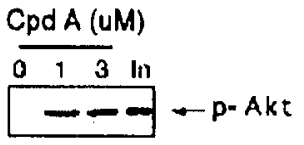

C
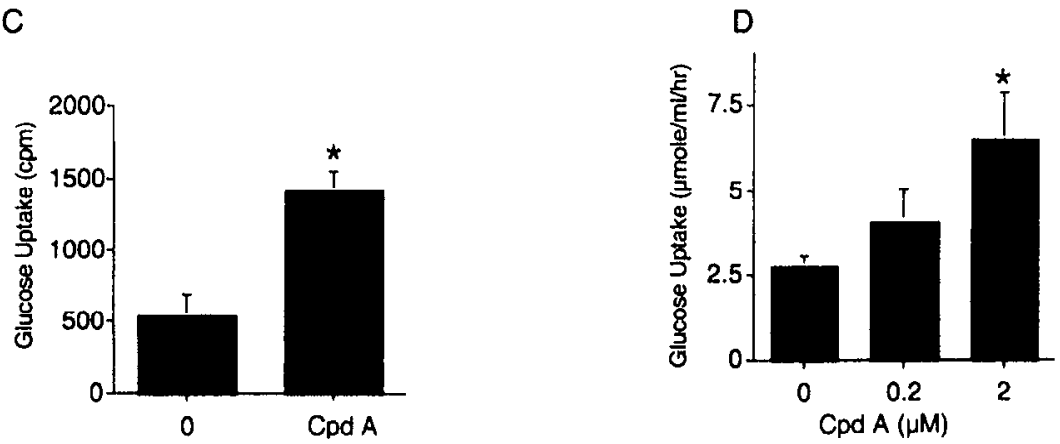

FIG. 7. Activation of insulin signaling pathway in cells treated with DMAQ-B1. (A) Activation of PI-3-kinase in CHO.IR cells. (B) Stimulation of phosphorylation of Akt in CHO.IR cells. (C) Glucose uptake in rat primary adipocytes. (D) Glucose uptake in isolated soleus muscles. ${ }^{*}, p<0.02$ by student $t$ test. [Reprinted with permission from Zhang, B., Salituro, G., Szalkowski, D., Li, Z., Zhang, Y., Royo, I., Vilella, D., Pelaez, F., Ruby, C., Kendall, R., Mao, X., Griffin, P., Calaycay, J., Heck, J.V., Smith, R.G., and Moller, D. Science 284, 974-977, 1999. Copyright 1999 American Association for the Advancement of Science.] 
variety of beneficial biological effects, ranging from metabolic regulation to embryonic development to tissue regeneration. These receptor tyrosine kinases also play pivotal roles in pathological conditions, including diabetic retinopathy, atherosclerosis, and deleterious neoplastic transformation. Thus, a critically important aspect in the search for small molecules capable of activating IRTK is to determine the selectivity of such compounds. Although these tyrosine kinases are highly homologous, small variations in primary sequences have provided the basis for identification of potent and exquisitely selective inhibitors (Fry and Bridges, 1995; Fry et al., 1994; Levitzki and Gazit, 1995; Mohammadi et al., 1996, 1997,1998).

In order to determine the specificity of test compounds for IR vs. other selected homologous receptors, parallel, cell-based assays were established and used to counterscreen IR activators against IGF-I-R, EGF-R, and PDFG-R. In CHO cells overexpressing IGF-I-R (CHO.IGFIR), DMAQ-B1 (at $10 \mu \mathrm{M}$ ) did not stimulate IGF-I-R or IRS-1 tyrosyl phosphorylation. No other compound-mediated tyrosyl protein phosphorylation was evident, suggesting that the compound is selective for IR vs. IGF-I-R activation. In subsequent studies using CHO.IGFIR cells, EGF-R-overexpressing cells (CHO.EGFR), and quantitative tyrosine kinase (IGF-I-R) or anti-phosphotyrosine ELISA (EGF-R) assays, DMAQ-BI was

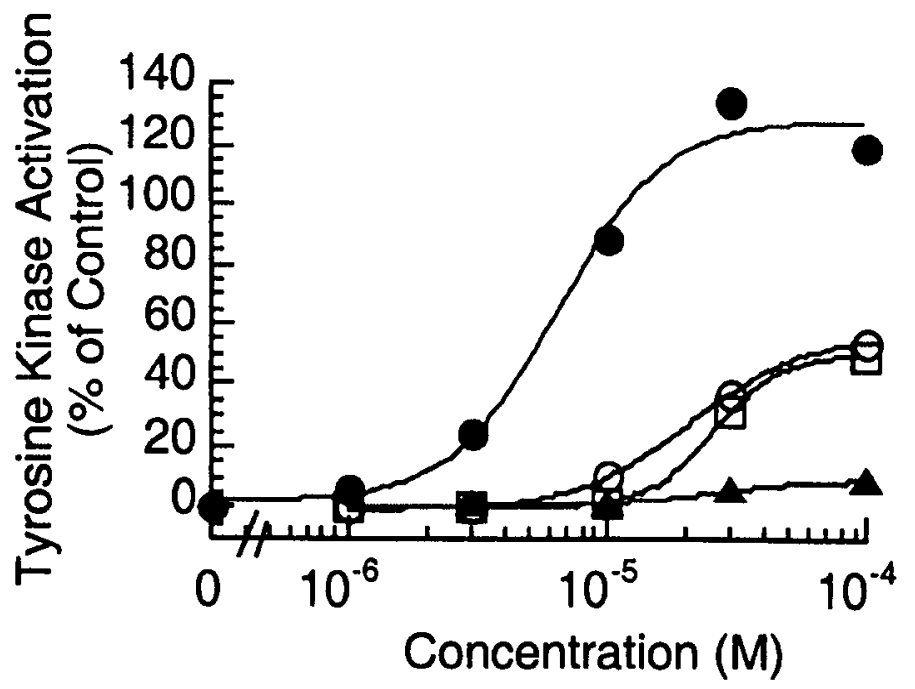

FIG. 8. Selectivity of DMAQ-B1. Activation of IRTKs by DMAQ-B1 in CHO.IR (filled circles), CHO.IGFIR (open circles), CHO.EGFR (open squares), or CHO.PDGFR (filled triangles) cells. [Reprinted with permission from Zhang, B., Salituro, G., Szalkowski, D., Li, Z., Zhang, Y., Royo, I., Vilella, D., Pelaez, F., Ruby, C., Kendall, R., Mao, X., Griffin, P., Calaycay, J., Heck, J.V., Smith, R.G., and Moller, D. Science 284, 974-977, 1999. Copyright 1999 American Association for the Advancement of Science.] 
shown to induce weak IGF-I-R and EGF-R activation; $100 \mu \mathrm{M}$ concentrations were required to achieve 50 percent efficacy of either IGF-I or EGF (Figure 8). In addition, DMAQ-Bl did not induce EGF-R activation (up to $60 \mu \mathrm{M}$ ) in cells (A431) that express high levels of endogenous EGF-Rs (not shown). The compound (up to $100 \mu \mathrm{M}$ ) also failed to induce detectable activation of PDGF-R in overexpressing $\mathrm{CHO}$ cells (Figure 8) or fetal human fibroblasts, which express high endogenous PDGF-R levels (not shown). Based on these data, DMAQ-B1 has greater than 10 -fold selectivity for IR and may provide a scaffold for building in greater selectivity.

\section{DIRECT INTERACTION WITH IR}

To establish that the effect of DMAQ-BI observed in the cell-based assays is due to direct activation of IR, experiments were conducted to study the compound's mechanism of action. Several lines of evidence suggested that DMAQ-B1 acted directly to activate the IR intracellular $\beta$ subunit (tyrosine kinase domain). First, transfected $\mathrm{CHO}$ cells (CHO.IRR/IR) that overexpress chimeric receptors composed of the IR intracellular domain fused to the nonhomologous insulin receptor-related receptor (IRR) extracellular domain (Zhang and Roth, 1992) were used to show that DMAQ-B1 (but not insulin) could activate receptor tyrosine kinase activity in intact cells (Figure 9A). Second, DMAQ-BI did not displace radiolabeled insulin binding to IRs expressed in intact CHO.IR cells, nor was the affinity of insulin for the receptor affected (Figure 9B). Third, direct, in vitro incubation with DMAQ-B1, but not compound $\mathrm{B}$, was able to increase tyrosine kinase activity of recombinant IRTK protein (Figure 9C). Furthermore, IR was partially purified from CHO.IR cells using wheat germ agglutanin (WGA) affinity chromatography. When the partially purified IR was incubated in the presence of $\gamma^{32}$ P-ATP and a 12-mer IR peptide substrate, DMAQ-B1 stimulated IR kinase activity in a dose-dependent manner, as measured by increased incorporation of radiolabel into the substrate peptide (Figure 9D).

The mechanism for activation of IRTK has been a subject of intensive investigation. High-resolution structural information has been obtained through crystallographic studies of the IR kinase domain (Hubbard et al., 1994; Hubbard, 1997). Based on crystal structures of the unphosphorylated, low-activity form as well as the phosphorylated, active form of IR - a model of cis-inhibition and trans-activation of the receptor was proposed. The unliganded receptors exist in the autoinhibitory conformation that prevents access of ATP and substrate to the active site. Upon autophosphorylation of Tyr1158, Tyr1162, and Tyr1163 in the activation loop, the IR kinase undergoes a major conformational change, resulting in unrestricted access of adenosine triphosphate (ATP) and substrate to the active site and full activation of the kinase. More recently, the three-dimensional (3D) structure of IR bound to insulin was determined by electron cryomicroscopy (Luo 


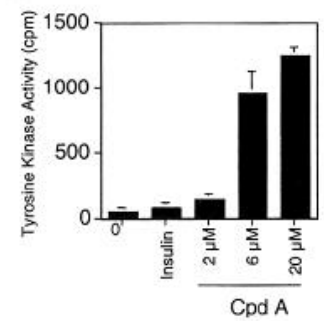

B

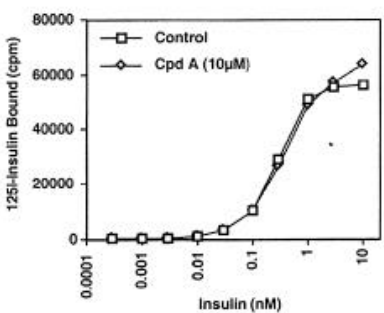

Histone $\mathrm{H} 2 \mathrm{~B}$
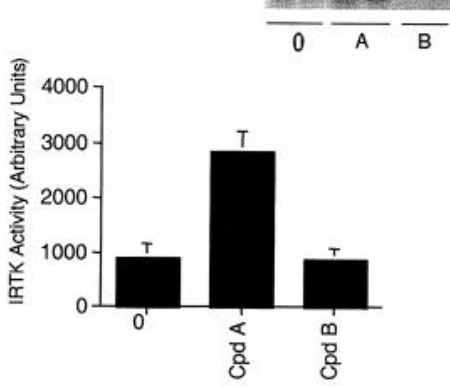

D

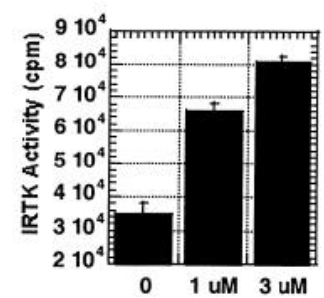

FIG. 9. Interaction of DMAQ-B1 with IR. (A) Activation of tyrosine kinase in CHO cells expressing IRR/IR chimeric receptor. (B) Lack of effect of DMAQ-B1 on insulin binding to the receptor. (C) In vitro activation of recombinant IRTK. (D) Activation of partially purified insulin receptor by DMAQ-B1. [Modified with permission from Zhang, B., Salituro, G., Szalkowski, D., Li, Z., Zhang, Y., Royo, I., Vilella, D., Pelaez, F., Ruby, C., Kendall, R., Mao, X., Griffin, P., Calaycay, J., Heck, J.V., Smith, R.G., and Moller, D. Science 284, 974-977, 1999. Copyright 1999 American Association for the Advancement of Science.] 
et al., 1999). The 3D reconstruction of the quaternary structure reveals that both $\alpha$ subunits are involved in insulin binding and that the two $\beta$ subunits are poised for trans-autophosphorylation. These structural studies have provided the molecular basis of IR activation.

In order to assess whether interaction of DMAQ-B1 with IRTK domain could alter conformation of the protein, recombinant IR intracellular domain protein (48 $\mathrm{kDa}$ ) was incubated with DMAQ-B1, followed by partial protease digestion. This approach revealed that the compound induced a change in protease sensitivity of IRTK, as illustrated by altered proteolytic pattern of the protein (Figure 10). A different pattern of altered protease sensitivity was observed following incubation of protein with an ATP analog (ATP $\gamma$-S), which is known to affect IR kinase conformation (Hubbard, 1997; Hubbard et al., 1994). A third protease digestion pattern was observed following combined incubation with DMAQ-B1 and ATP $\gamma-$ $\mathrm{S}$. Of particular interest was the presence of a $30-\mathrm{kDa}$ band when the $48-\mathrm{kDa}$ protein was incubated with DMAQ-B1 followed by partial trypsin digestion

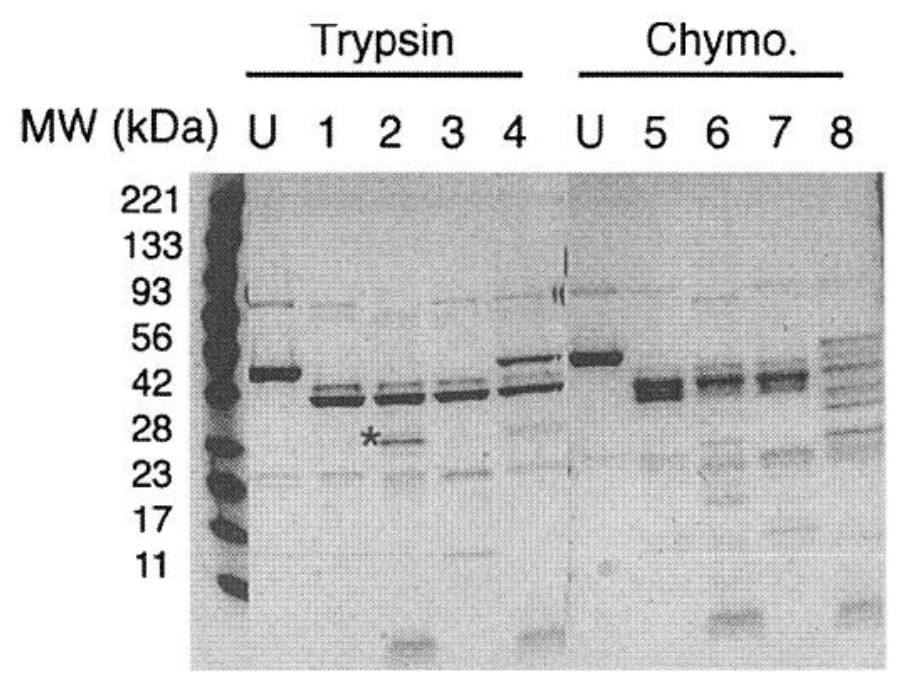

FIG. 10. DMAQ-B1 alters protease sensitivity of recombinant IR intracellular domain protein. The 48-kDa IRTK protein was subject to limited trypsin or chymotrypsin (chymo.) digestion in the presence or absence of compounds. The digestion mixtures were separated with electrophoresis and the gels were stained with Commassie Blue. $\mathrm{U}=$ undigested protein; lanes $1,5=\mathrm{DMSO}$ control; lanes 2, 6 = DMAQ-B1; lanes 3, 7 = ATP $\gamma$-S; lanes 4, 8 = DMAQ-B1 plus ATP $\gamma$-S. [Reprinted with permission from Zhang, B., Salituro, G., Szalkowski, D., Li, Z., Zhang, Y., Royo, I., Vilella, D., Pelaez, F., Ruby, C., Kendall, R., Mao, X., Griffin, P., Calaycay, J., Heck, J.V., Smith, R.G., and Moller, D. Science 284, 974-977, 1999. Copyright 1999 American Association for the Advancement of Science.] 
(denoted by $*$ in lane 2). Further experiments with titration of the protease showed that 10 to $50 \times$ decreased concentrations of trypsin could produce the approximately $30-\mathrm{kDa}$ digestion product in the presence of DMAQ-B1. The trypsin digestion products were separated and visualized in a Coomassie-blue stained $4 \%-20 \%$ SDS polyacrylamide gel. Following electrotransfer onto a polyvinylidene difluoride (PVDF) membrane, the 30-kDa band was excised and subjected to automated Edman sequence analysis to reveal the $\mathrm{N}$-terminal peptide sequence - $\mathrm{T}^{1031}$ VNESASL. This peptide is immediately adjacent to $\mathrm{K}^{1030}$, which has been shown to be involved in ATP binding to the active site of the IRTK domain (Chou et al., 1987; Ebina et al., 1985,1987; McClain et al., 1987). Thus, interaction of DMAQ-B1 with the IRTK domain apparently altered the conformation of the protein in the region encompassing the ATP binding site. This could conceivably lead to activation of the kinase by partially relieving the cis-inhibition of the enzyme. Structural biology studies will be necessary to further elucidate the mechanism of action.

\section{ANTIDIABETIC ACTIVITY IN MURINE MODELS OF DIABETES}

The results of in vitro experiments demonstrated that DMAQ-B1 is an insulin mimetic with selectivity for IR in cell-based assays, suggesting that it might exhibit substantial glucose-lowering effects in vivo. This hypothesis was tested using $\mathrm{db} / \mathrm{db}$ and ob/ob mice, obese animal models of type 2 diabetes characterized by severe insulin resistance and hyperglycemia. The purpose of the in vivo studies was to determine the potential ability of DMAQ-B1 to correct the diabetic state in the mouse models without resulting in undesirable side effects.

Oral administration of DMAQ-B1 to diabetic $\mathrm{db} / \mathrm{db}$ mice was shown to be efficacious for lowering the elevated blood glucose. Single-dose, oral administration of DMAQ-B1 resulted in dose-dependent glucose lowering (Figure 11A), with over 50 percent transient correction of hyperglycemia achieved at 25 $\mathrm{mg} / \mathrm{kg} /$ day (over 3-6 hours; food withheld). In addition, chronic, daily, oral administration of DMAQ-B1 (at 5 or $20 \mathrm{mg} / \mathrm{kg} /$ day) for 7 days was shown to result in significant correction of hyperglycemia in ad libitum-fed $\mathrm{db} / \mathrm{db}$ mice (Figure 11B). The in vivo effects of DMAQ-B1 on blood glucose levels in $\mathrm{db} / \mathrm{db}$ mice also were shown to be independent of potential effects on circulating insulin or glucagon (not shown) and were clearly evident in food-withheld mice where changes in feed intake were not a factor. In addition, when DMAQ-B1 was administered to ob/ob mice with extreme hyperinsulinemia and mild hyperglycemia, followed by a glucose tolerance test, significant and dose-dependent reductions in glucose excursion were observed (Figure 11C). Single, oral doses of DMAQ-B1 also acted to suppress elevated plasma insulin levels in ob/ob mice (Figure 11D). Chronic treatment of mice (up to 15 days) with therapeutic doses of DMAQ-B1 did not affect food intake, body weight, organ weights, or blood 
A
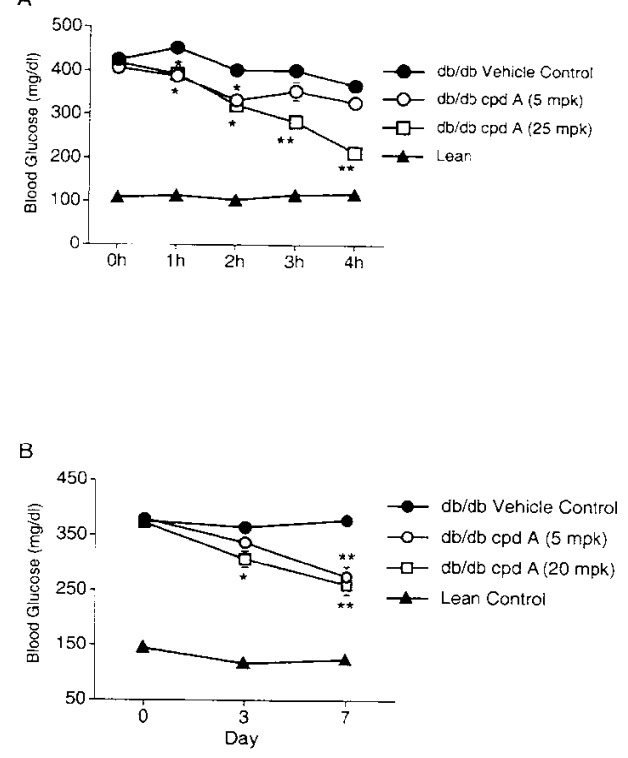

$\mathrm{C}$
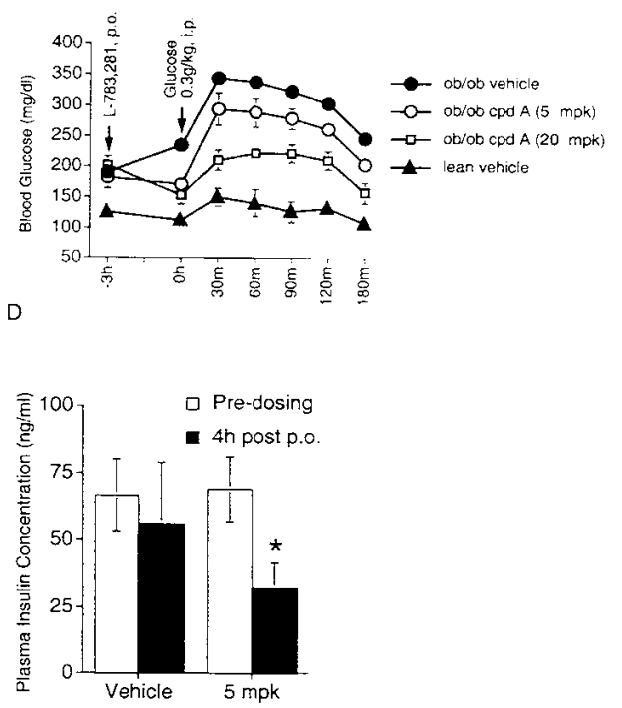
in $\mathrm{db} / \mathrm{db}$ mice following chronic dosing. (C) Glucose tolerance test in ob/ob mice. (D) Plasma insulin level in 12-week-old ob/ob mice at pre- and 4-hour post single oral dosing of DMAQ-B1. Shown are mean \pm S.E.M. values for each data point ( $\mathrm{n}=7-9$ for each group). In some cases, S.E.M. values are within the area of the symbols. ${ }^{*}, \mathrm{p}<0.05 ;{ }^{* *} \mathrm{p}<0.002$ by student $t$ test. All animals were fed ad libitum prior to the study and animal care was in accordance with institutional guidelines. [Modified with permission from Zhang, B., Salituro, G., Szalkowski, D., Li, Z., Zhang, Y., Royo, I., Vilella, D., Pelaez, F., Ruby, C., Kendall, R., Mao, X., Griffin, P., Calaycay, J., Heck, J.V., Smith, R.G., and Moller, D. Science 284, 974-977, 1999. Copyright 1999 American Association for the Advancement of Science.] 
chemistry values. These data indicate that DMAQ-B1 functions as an efficacious antidiabetic agent in murine NIDDM models.

Vanadate is another orally active compound that can function as an insulin mimetic agent (Goldfine et al., 1995). Unlike the IR activator exemplified by DMAQ-B1, vanadate inhibits protein tyrosine phosphatases (PTP) and augments tyrosyl phosphorylation of a wide variety of cellular proteins, including the IR (Huyer et al., 1997). Recent studies indicate that PTP1B plays an important role in modulating insulin signaling, since augmented insulin sensitivity was observed in PTP1B knockout mice (Elchebly et al., 1999). Interestingly, PTP1B null mice also were protected from obesity. Therefore, specific inhibitors of PTP1B represent a potential treatment for diabetes or other metabolic disorders by augmenting activity of the IRTK.

\section{Summary}

The discovery of DMAQ-B1 demonstrated that a small, nonpeptidyl molecule is capable of mimicking the in vitro and in vivo function of a protein hormone by interacting with and activating its receptor. It is worth noting that small molecule agonists have been identified for other peptidyl hormone receptors such as erythropoeitin and granulocyte colony-stimulating factor receptors (Johnson $e t$ al., 1998; Livnah et al., 1996; Tian et al., 1998). These agonists interact with the extracellular ligand-binding domain and induce dimerization of the monomeric receptor subunits, leading to activation of the receptors. Selective IR activators, as exemplified by DMAQ-B1, may lead to the development of a novel class of antidiabetic agents.

\section{REFERENCES}

Accili, D., Drago, J., Lee, E.J., Johnson, M.D., Cool, M.H., Salvatore, P., Asico, L.D., Jose, P.A., Taylor, S.I., and Westphal, H. (1996). Nature Genet. 12, 106-109.

Alessi, D.R., and Cohen, P. (1998). Curr. Opin. Genet. Devel. 8, 55-62.

Alvi, K.A., Pu, H., Luche, M., Rice, A., App, H., McMahon, G., Dare, H., and Margolis, B. (1999). J. Antibiotics 52, 215-223.

Arai, K., Shimizu, S., Taguchi, Y., and Yamamoto, Y. (1981a). Chem. Pharmacol. Bull. 29, 991-999.

Arai, K., Masuda, K., Kiriyama, N., Nitta, K., Yamamoto, Y., and Shimizu, S. (198lb). Chem. Pharmacol. Bull. 29, 961-969.

Barr, M.E. (1964). Mycologia 56, 841-862.

Bruning, J.C., Winnay, J., Bonner-Weir, S., Taylor, S.I., Accili, D., and Kahn, C.R. (1997a). Cell 88, $561-572$.

Bruning, J.C., Winnay, J., Cheatham, B., and Kahn, C.R. (1997b). Mol. Cell Biol. 17, 1513-1521.

Bruning, J.C., Michael, M.D., Winnay, J.N., Hayashi, T., Horsch, D., Accili, D., Goodyear, L.J., and Kahn, C.R. (1998). Mol. Cell 2, 559-569.

Carney, J.R., Krenisky, J.M., Williamson, R.T., Luo, J., Carlson, T.J., Hsu, V.L., and Moswa, J.L. (1999). J. Nat. Products 62, 345-347. 
Caro, J.F., Itoop, O., Pories, W.J., Meelheim, D., Flickinger, E.G., Thomas, F., Jenquin, M., Silverman, J.F., Khazanie, P.G., and Sinha, M.K. (1986). J. Clin. Invest. 78, 249-258.

Caro, J.F., Sinha, M.K., Raju, S.M., Itoop, O., Pories, W.J., Flickinger, E.G., Meelheim, D., and Dohm, G.L. (1987). J. Clin. Invest. 79, 1330-1337.

Chou, C.K., Dull, T.J., Russell, D.S., Gherzi, R., Lebwohl, D., Ullrich, A., and Rosen, O.R. (1987). J. Biol. Chem. 262, 14663-14667.

Cignarella, A., Nastasi, M., Cavalli, E., and Puglisi, L. (1996). Thrombasis Res. 84, $311-322$

Collado, J., Platas, G., and Pelaez, F. (1996). Nova Hedwigia 63, 347-360.

De Guzman, F.S., Bruss, D.R., Rippentrop, J.M., Gloer, K.B., Gloer, J.B., Wicklow, D.T., and Dowd, P.F. (1994). J. Nat. Products 57, 634-639.

Ebina, Y., Ellis, L., Jarnagin, K., Edery, M., Graf, L., Clauser, E., Ou, J.H., Masiarz, F., Kan, Y.W., Goldfine, I.D., Roth, R.A., and Rutter, W.J. (1985). Cell 40, 747-758.

Ebina, Y., Araki, E., Taira, M., Shimada, F., Mori, M., Craik, C.S., Siddle, K., Pierce, S.B., Roth, R.A. and Rutter, W.J. (1987). Proc. Natl. Acad. Sci. U.S.A. 84, 704-708.

Elchebly, M., Payette, P., Michaliszyn, E., Cromlish, W., Collins, S., Loy, A.L., Normandin, D., Cheng, A., Himms-Hagen, J., Chan, C.C., Ramachandran, C., Gresser, M.J., Tremblay, M.L., and Kennedy, B.P. (1999). Science 283, 1544-1548.

Ellis, L., Morgan, D.O., Clauser, E., Roth, R.A., and Rutter, W.J. (1987). Mol. Endocrinol. 1, 15-24.

Endemann, G., Yonezawa, K., and Roth, R.A. (1990). J. Biol. Chem. 265, 396-400.

Franke, T.F., Yang, S.I., Chan, T.O., Datta, K., Kazlauskas, A., Morrison, D.K., Kaplan, D.R., and Tsichlis, P.N. (1995). Cell 81, 727-736.

Fredenhagen, A., Petersen, F., Tintelnot-Blomley, M., Rosel, J., Mett, H., and Hug, P. (1997). J. Antibiotics 59, 395-401.

Fry, D.W., and Bridges, A.J. (1995). Curr. Opin. Biotechnol. 6, 662-667.

Fry, D.W., Kraker, A.J., McMichael, A., Ambroso, L.A., Nelson, J.M., Leopold, W.R., Connors, R.W., and Bridges, A.J. (1994). Science 265, 1093-1095.

Goldfine, A.B., Simonson, D.C., Folli, F., Patti, M.E., and Kahn, C.R. (1995). Mol. Cell Biochem. 153, $217-23 !$.

Goodyear, L.J., Giorgino, F., Sherman, L.A., Carey, J., Smith, R.J., and Dohm, G.L. (1995). J. Clin. Invest. 95, 2195-2204.

Hawksworth, D.L. (1991). Mycol. Res. 95, 641-655.

Hubbard, S.R. (1997). EMBO J. 16, 5572-5581

Hubbard, S.R., Wei, L., Ellis, L., and Hendrickson, W.A. (1994). Nature 372, 746-754.

Huyer, G., Liu, S., Kelly, J., Moffat, J., Payette, P., Kennedy, B., Tsaprailis, G., Gresser, M.J., and Ramachandran, C. (1997). J. Biol. Chem. 272, 843-851.

Hyde, K.D., Frohlich, J. and Taylor, J.E. (1998). Sydowia 50, 21-80.

Inman, W.D., Luo, J., Jolad, S.D., King, S.R., and Cooper, R. (1999). J. Nat. Products 62, 1088-1092.

Jerram, W.A., Mclnnes, A.G., Maass, W.S.G., Smith, D.G., Taylor, A., and Walter, J.A. (1975). Can J. Chem. 53, 727-737.

Johnson, D.L., Farrell, F.X., Barbone, F.P., McMahon, F.J., Tullai, J., Hoey, K., Livnah, O., Wrighton, N.C., Middleton, S.A., Loughney, D.A., Stura, E.A., Dower, W.J., Mulcahy, L.S., Wilson, I.A., and Jolliffe, L.K. (1998). Biochemistry 37, 3699-3710

Joshi, R.L., Lamothe, B., Cordonnier, N., Mesbah, K., Monthioux, E., Jami, J., and Bucchini, D. (1996). EMBO J. 15, 1542-1547.

Kaji, A., Iwata, T., and Kiriyama, N. (1998). J. Antibiotics 51, 235-238.

Kasuga, M., Karlsson, F.A., and Kahn, C.R. (1982). Science 215, 185-187.

Kerouz, N.J., Horsch, D., Pons, S., and Kahn, C.R. (1997). J. Clin. Invest. 100, 3164-3172.

Kido, Y., Burks, D.J., Withers, D., Bruning, J.C., Kahn, C.R., White, M.F., and Accili, D. (2000). J. Clin. Invest. 105, 199-205. 
Kim, Y.B., Zhu, J.S., Zierath, J.R., Shen, H.Q., Baron, A.D., and Kahn, B.B. (1999). Diabetes 48, 310-320.

Kohn, A.D., Summers, S.A., Birnbaum, M.J., and Roth, R.A. (1996). J. Biol. Chem. 271, 31372-31378.

Krook, A., Roth, R.A., Jiang, X.J., Zierath, J.R., and Wallberg-Henriksson, H. (1998). Diabetes 47, 1281-1286.

Kulkarni, R.N., Bruning, J.C., Winnay, J.N., Postic, C., Magnuson, M.A., and Kahn, C.R. (1999a). Cell 96, 329-339.

Kulkarni, R.N., Winnay, J.N., Daniels, M., Bruning, J.C., Flier, S.N., Hanahan, D., and Kahn, C.R. (1999b). J. Clin. Invest. 104, R69-R75.

Lauro, D., Kido, Y., Castle, A.L., Zarnowski, M.J., Hayashi, H., Ebina, Y., and Accili, D. (1998). Nature Genet. 20, 294-298.

Levitzki, A., and Gazit, A. (1995). Science 267, 1782-1788.

Levy-Toledano, R., Taouis, M., Blaettler, D.H., Gorden, P., and Taylor, S.I. (1994). J. Biol. Chem. 269, 31178-31182

Liu, K., Wood, H.B., and Jones, A.B. (1999). Tetrahedron Lett. 40, $5119-5122$.

Livnah, O., Stura, E.A., Johnson, D.L., Middleton, S.A., Mulcahy, L.S., Wrighton, N.C., Dower, W.J., Jolliffe, L.K., and Wilson, I.A. (1996). Science 273, 464-471.

Luo, R.Z., Beniac, D.R., Fernandes, A., Yip, C.C., and Ottensmeyer, F.P. (1999). Science 285, 1077-1080.

McClain, D.A., Maegawa, H., Lee, J., Dull, T.J., Ullrich, A., and Olefsky, J.M. (1987). J. Biol. Chem. 262, 14663-14671.

Mocek, U., Schultz, L., Buchan, T., Baek, C., Fretto, L., Nzerem, J., Sehl, L., and Sinha, U. (1996). J. Antibiotics 49, 854-859.

Mohammadi, M., Schlessinger, J., and Hubbard, S.R. (1996). Cell 86, 577-587.

Mohammadi, M., McMahon, G., Sun, L., Tang, C., Hirth, P., Yeh, B.K., Hubbard, S.R., and Schlessinger, J. (1997). Science 276, 955-960.

Mohammadi, M., Froum, S., Hamby, J.M., Schroeder, M.C., Panek, R.L., Lu, G.H., Eliseenkova, A.V., Green, D., Schlessinger, J., and Hubbard, S.R. (1998). EMBOJ. 17, 5896-5904.

Olefsky, J.M. (1976). J. Clin. Invest. 57, 1165-1172.

Ooike, M., Nozawa, K., Udagawa, S., and Kawai, K. (1997). Chem. Pharm. Bull. 45, 1694-1696.

Perez, S.G., Perez, R.M.G., Perez, C.G., Zavala, M.A.S., and Vargas, R.S. (1997). Pharm. Acta Helv. 72, 105-111

Rosen, O.M. (1989). Diabetes 38, 1508-1511.

Rosen, O.M., Herrera, R., Olowe, Y., Petruzzelli, M., and Cobb, M.H. (1983). Proc. Natl. Acad. Sci. U.S.A. 80, 3237-3240.

Sekita, S. (1983). Chem. Pharm. Bull. 31, 2998-3001.

Taylor, S.I. (1992). Diabetes 41, 1473-1490.

Taylor, S.I. (1999). Cell 97, 9-12.

Tian, S.S., Lamb, P., King, A.G., Miller, S.G., Kessler, L., Luengo, J.I., Avcrill, L., Johnson, R.K., Gleason, J.G., Pelus, L.M., Dillon, S.B., and Rosen, J. (1998). Science 281, 257-259.

Ullrich, A., and Schlessinger, J. (1990). Cell 61, 203-212.

White, M.F., and Kahn, C.R. (1994). J. Biol. Chem. 269, 1-4.

White, M.F, and Yenush, L. (1998). Curr. Top. Microbiol. Immunol, 228, 179-208.

Yamamoto, Y., Nishimura, K., and Kiriyama, N. (1976). Chem. Pharm. Bull. 24, 1853-1859.

Yu, K.T., and Czech, M.P. (1984). J. Biol. Chem. 259, 5277-5286.

Zhang, B., and Roth, R.A. (1992). J. Biol. Chem. 267, 18320-18328.

Zhang, B., Szalkowski, D., Diaz, E., Hayes, N., Smith, R., and Berger, J. (1994). J. Biol. Chem. 269, 25735-25741. 


\title{
Ras Activation of the Raf Kinase: Tyrosine Kinase Recruitment of the MAP Kinase Cascade
}

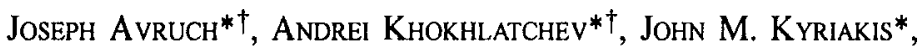 \\ ZhiJun Luo $\ddagger$, Guri Tzivion ${ }^{\dagger}$, Demetrios Vavvas $\$$, and Xian-Feng Zhang* \\ *Diabetes Unit and Medical Services and the tDepartment of Molecular Biology, \\ Massachusetts General Hospital, and the Department of Medicine, Harvard Medical School, \\ Boston, Massachusetts 02114; 'Boston Medical Center, Boston, Massachusetts 02118;

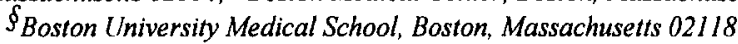

\begin{abstract}
A continuing focus of our work has been an effort to understand the signal transduction pathways through which insulin achieves its cellular actions. In the mid-1970s, we and others observed that insulin promoted an increase in Ser/Thr phosphorylation of a subset of cellular proteins. This finding was unanticipated, inasmuch as nearly all of the actions of insulin then known appeared to result from protein dephosphorylation. In fact, nearly 15 years elapsed before any physiologic response to insulin attributable to stimulated (Ser/Thr) phosphorylation was established. Nevertheless, based on the hypothesis that insulin-stimulated Ser/Thr phosphorylation reflected the activation of protein (Ser/Thr) kinases downstream of the insulin receptor, we sought to detect and purify these putative, insulin-responsive protein (Ser/Thr) kinases. Our effort was based on the presumption that an understanding of the mechanism for their activation would provide an entry into the biochemical reactions through which the insulin receptor activated its downstream effectors. To a degree that, in retrospect, is surprising, this goal was accomplished, much in the way originally envisioned. It is now well known that receptor tyrosine kinases (RTKs) recruit a large network of protein (Ser/Thr) kinases to execute their cellular programs. The first of these insulin-activated protein kinase networks to be fully elucidated was the Ras-Raf-mitogen-activated protein kinase (MAPK) cascade. This pathway is a central effector of cellular differentiation in development; moreover, its inappropriate and continuous activation provides a potent promitogenic force and is a very common occurrence in human cancers. Conversely, this pathway contributes minimally, if at all, to insulin's program of metabolic regulation. Nevertheless, the importance of the Ras-MAPK pathway in metazoan biology and human malignancies has impelled us to an ongoing analysis of the functions and regulation of Ras and Raf. This chapter will summarize briefly the way in which work from this and other laboratories on insulin signaling led to the discovery of the mammalian MAP kinase cascade and, in turn, to the identification of unique role of the Raf kinases in RTK activation of this protein (Ser/Thr) kinase cascade. We will then review in more detail current understanding of the biochemical mechanism through which the Ras proto-oncogene, in collaboration with the 14-3-3 protein and other protein kinases, initiates activation of the Raf kinase.
\end{abstract}




\section{Insulin and Growth Factors Promote Widespread Protein (Ser/Thr) Phosphorylation}

The discovery of cyclic AMP (cAMP) in the mid-1950s provided the first paradigm for signal transduction by polypeptide hormones. In this model, the hormone interacts with its receptor at the cell surface, leading to the generation within a cell of a chemically distinct, intracellular molecule responsible for conveying all the information necessary to carry out the complete program of hormone action (Robison et al., 1971). The nature of the biochemical reactions through which the "second messenger" reoriented cell function remained obscure until the discovery and elucidation of the cAMP-dependent protein kinase, protein kinase A (PKA), by Krebs and coworkers in 1968. A substantial body of evidence accumulated rapidly thereafter that demonstrated that PKA-catalyzed protein phosphorylation explains most of the actions of cAMP (Krebs, 1972). Although other cAMP effectors - such as cAMP-gated ion channels (Zagotta and Siegelbaum, 1996) and guanyl nucleotide exchangers for the small GTPase, Rap1 (DeRooij et al.,1998) - have been identified more recently, PKA nevertheless remains the dominant cAMP effector.

Shortly after the discovery of cAMP, Larner and coworkers showed that insulin, like glucagon or beta-adrenergic catecholeamines, can induce stable alterations in the activity of the intracellular enzyme, glycogen synthase, which can be measured in the homogenate or after purification, if cells are disrupted under appropriate conditions (Larner, 1988). This property usually reflects the occurrence of a stable, post-translational modification (e.g., phosphorylation, dephosphorylation), suggesting a parallel between insulin and cAMP action. As the effects of insulin on the activity of enzymes such as glycogen synthase, pyruvate dehydrogenase, and hormone-sensitive triglyceride lipase are opposite to those caused by cAMP/PKA, it was anticipated that insulin would execute much of its cellular program by promoting the dephosphorylation of target proteins. This would be accomplished either by opposing cAMP generation, or by activating protein phosphatases to reverse cAMP/PKA action, or both. Direct assessment of the effect of insulin on overall protein phosphorylation in target cells readily demonstrated insulin inhibition of cAMP-directed phosphorylation, if cAMP levels were elevated by catecholamines or glucagon. Unexpectedly, however, the dominant response to insulin as the sole agonist was not dephosphorylation but rather an increase in ${ }^{32} \mathrm{P}$ incorporation into (Ser/Thr) residues on a variety of polypeptides, through a mechanism entirely independent of the cAMP/PKA system (Benjamin and Singer, 1975; Avruch et al., 1976; Forn and Greengard, 1976). The only purpose known for agonist-stimulated Ser/Thr phosphorylation is regulation of protein target function. However, no examples of enzyme regulation by insulin-stimulated protein phosphorylation were then known. Nevertheless, reasoning that these novel, insulin-stimulated phosphorylations reflected intermedi- 
ate steps in insulin signal transduction, efforts were undertaken to identify the targets and the responsible insulin-regulated protein kinases/protein phosphatases. Among the initial set of insulin-stimulated phosphoproteins identified were several metabolic enzymes (ATP-citrate lyase, Alexander et al., 1979; acetyl CoA carboxylase, Witters, 1981). However, only the 40S ribosomal subunit protein S6 (Haselbacher et al., 1979; Smith et al., 1979) provided a substrate useful for the reliable detection of the insulin-regulated interconverting enzymes (Avruch et al., 1985). Thus, once suitable extraction conditions had been defined (Novak-Hofer and Thomas, 1984), a kinase activity capable of phosphorylating $40 \mathrm{~S}$ subunits exclusively on $\$ 6$ could be reliably detected in extracts of cells treated with insulin or mitogens (Tabarini et al., 1985; Erikson and Maller, 1985; Nemenoff et al., 1986; Pelech et al., 1986). An in vivo counterpart of this response is the tenfold increase in S6 kinase activity in the regenerating liver remnant that occurs within 2 hours after partial hepatectomy in the rat (Nemenoff et al., 1988). Similarly, the induction of germinal vesicle breakdown in stage VI Xenopus oocytes by progesterone or insulin is accompanied by a dramatic increase in 40S-S6 kinase activity (Maller et al., 1986).

\section{Insulin and Growth Factors Activate Multiple (Ser/Thr) Kinases: Discovery of the Mammalian MAPKinase Cascade}

The Xenopus S6 kinase activity was first to be purified, yielding two 85- to 90-kDa kinase polypeptide isoforms (Erikson and Maller, 1985). cDNAs corresponding to these enzymes were cloned from Xenopus (Jones et al., 1988) and murine (Alcorta et al., 1989) sources and named ribosomal $\underline{\text { S6 }}$ protein kinase (Rsk) 1 and 2. Xenopus Rsk was shown to undergo extensive (Ser/Thr) phosphorylation in vivo concomitant with activation. It could be deactivated in vitro by treatment with protein phosphatase (Erikson and Maller, 1989). Rsk thus appeared to be activated by protein (Ser/Thr) phosphorylation, suggesting that this S6 kinase itself was the target of an insulin/mitogen-activated protein (Ser/Thr) kinase.

A second insulin-stimulated protein kinase was discovered shortly thereafter by Sturgill and coworkers. In the course of examining whether insulin activated a protein kinase activity toward the protein phosphatase modulatory protein called inhibitor-2, they observed the insulin-stimulated phosphorylation of a high $\mathrm{Mr}$ polypeptide contaminant, identified subsequently as the microtubule-associated protein, MAP-2 (Ray and Sturgill, 1987). Partial purification of this insulin-stimulated MAP-2 kinase from $3 \mathrm{~T} 3 \mathrm{~L} 1$ adipocytes indicated that it co-purified with a 42-kDa polypeptide, whose phosphorylation on Thr and Tyr residues was strongly stimulated by insulin (Ray and Sturgill, 1988). Remarkably, the partially purified, insulin-activated p42 MAP-2 kinase was found to phosphorylate and partially reactivate the dephosphorylated Xenopus Rsk (S6 kinase II), suggesting that the 
MAP-2 kinase and Rsk, both insulin-activated kinases, might represent sequential elements in an insulin-regulated protein kinase cascade (Sturgill et al., 1988). Independently, Ahn and Krebs (1990), fractionating protein kinases in NIH3T3 cell extracts active toward a synthetic S6 peptide, garnered evidence for the existence of an analogous mitogen-activated S6-peptide kinase and "kinase-kinase" module.

Protein kinase cascades were first described by Krebs and coworkers, who discovered PKA as the immediate upstream activator of phosphorylase $b$ kinase (Walsh et al., 1968). Gibson's laboratory later provided evidence for the operation of a protein kinase cascade in the regulation of hydroxymethyglutaryl (HMG) CoA reductase (Ingebritsen et al., 1981). However, the components of this kinase cascade, which include the AMP-activated protein kinase, were not identified at a molecular level until much later, while the AMP kinase-kinase remains unidentified. Thus, little consideration was given to the significance of protein kinase cascades, until interest was rekindled by the elucidation of the MAPK-Rsk relationship. A forceful demonstration of ubiquity of this architectural motif in signal transduction was provided by the elucidation of the molecular structure of the $44-\mathrm{kDa}$ isoform of the MAP kinase. This polypeptide, named erk1 (Boulton et al., 1990), is approximately 45 percent identical in the amino acid sequence of its catalytic domain to a pair of $S$. cerevisiae protein kinases, FUS3 and KSS1, that had been identified by genetic analysis as indispensable elements in the signal transduction pathway mediating the yeast response to the mating pheromones (Elion et al., 1990; Courchesne et al., 1989). In fact, among the genes then known to be encoding components of the pheromone response pathway, in addition to the two MAPK homologs FUS3/KSS, were three other kinases - namely, Ste20, Ste 11, and Ste7. An epistatic analysis indicated that these three kinases acted in a sequential manner upstream of KSS1/FUS3 (Errede and Levin, 1993). However, no biochemical evidence was available as to whether any of these five kinases acted directly on another. The possibility remained that as-yet-unidentified gene products were interposed. Biochemical analysis of mammalian MAPK clearly showed that its activation required both Thr and Tyr phosphorylation of the MAPK polypeptide (Anderson et al., 1990). Mammalian cell extracts contained a potent MAPK activator that appeared to be a protein kinase with such dual specificity (Ahn et al., 1991; Gomez and Cohen, 1991). This entity was purified and molecularly cloned by several laboratories in rapid succession and proved to be a MAP kinase kinase (MAPKK, usually called MEK1) highly homologous to the yeast Ste7, the gene product immediately upstream of the yeast MAPKs, FUS3/KSS1 (Crews et al., 1992; Seger et al., 1992; Ashworth et al., 1992; Wu et al., 1993). This remarkable conservation of both the architectural design and individual structures made clear the ubiquitous and fundamental importance of the MAP kinase cascade as a signaling module. 
Our primary effort at this time was focused on the elucidation of the mammalian $\mathrm{S} 6$ kinase. We purified this enzyme from rat liver as a 70-kDa polypeptide (Price et al., 1989) and found by molecular cloning that it had a structure quite distinct from the Xenopus/mammalian Rsks (Banerjee et al., 1990). Moreover, although the p70 S6 kinase, like Rsk, is activated through insulin-stimulated Ser/Thr phosphorylation (Price et al., 1990), the p70 S6 kinase is poorly phosphorylated in vitro (and not at all reactivated) by the p42/44 MAPK (Price et al., 1990; Mukhopadhyay et al., 1992). This provided the first indication that the $\mathrm{p} 70$ S6 kinase and Rsk are on separate limbs of the signal transduction outflow downstream of the insulin receptor. This conclusion was strongly supported by our finding that the immunosuppressant drug rapamycin causes a potent inhibition of p70 S6kinase in intact cells, with no effect on Rsk activity (Price et al., 1992). Once convinced that Rsk and p70 were on separate insulin-directed signaling pathways, we attempted to identify the components of each signaling module. We have reviewed elsewhere current information concerning the RTK-PI-3 kinase$\mathrm{mTOR}$ and cell cycle-regulated pathways that impinge on p70 S6 kinase (Avruch et al., in press). The remainder of this discussion will focus on the control of the $\mathrm{MAPK} /$ pathway by insulin and growth factors.

\section{RTKs Activate the MAPK Cascade Through Raf Kinases}

One candidate RTK effector we selected for study was the cRaf-1 protein kinase. The cRaf-1 protein kinase is the cellular homolog of vRaf, one of the very few oncogenes then known to encode a protein (Ser/Thr) kinase (Rapp et al., 1983; Moelling et al., 1984). On inspecting the cRaf-1 amino acid sequence, a number of canonical MAPK phosphorylation sites (e.g., PXSP, XXSP) were seen to reside in a segment whose deletion had been shown to activate Raf-transforming activity, suggesting that Raf, like Rsk, might be activated by MAPK. Our efforts to examine this idea, however, were impeded by the lack of a suitable assay for Raf kinase activity. We observed that, although insulin or mitogen treatment increased the ability of immunoprecipitated cRafl to catalyze an autophosphorylation in vitro, we were not able to detect insulin/mitogen-stimulated protein kinase activity toward exogenous protein substrates previously reported (Siegel $e t$ al., 1990) (primarily various histones), that could survive stringent washing of the cRaf1 immunoprecipitate. Moreover, the recombinant cRaf-1 ATP site mutant, presumably catalytically inactive, exhibited autophosphorylating and kinase activity quite similar to the recombinant wild-type cRaf-1, indicating that the bulk of these activities were due to (randomly and/or specifically) adsorbed kinases.

A fortunate clue came from the characterization of a line of v-Raf-transformed NIH $3 \mathrm{~T} 3$ cells. We found that the endogenous p42/44 MAPKs in these cells appeared to be constitutively activated and unresponsive to further stimulation by serum or mitogens. Moreover, the activity of MAPKK in the v-Raf-trans- 
formed cells was markedly greater than that in the parental NIH 3T3 cells. After partial purification, this active MAPKK could be completely deactivated by PP2A in vitro (Kyriakis et al., 1992). The sensitivity of active MAPKK to PP2A was not unexpected, as the architecture of the $S$. cerevisiae mating pathway indicated that the yeast MAPKK Ste7 was situated downstream of another protein (Ser/Thr) kinase, Ste 11 (Errede and Levin, 1993). Although the highly conserved nature of the MAPK pathway components then known suggested that a mammalian homolog of Stel1 would serve as the immediate activator (MAPKKK) upstream of MAPKK, the constitutive, phosphorylation-dependent activity of MAPKK in the $v$-Raf-transformed cells raised the simple possibility that the v-Raf kinase itself acted as the MAPKKK. The activation of MAPKK by Raf proved to be readily demonstrable; an aminoterminally truncated, constitutively active Raf mutant, immunoprecipitated from NIH $3 \mathrm{~T} 3$ cells, catalyzed the phosphorylation and complete reactivation of a partially purified, PP2A-treated MAPKK (Kyriakis et al., 1992). These findings were rapidly confirmed (Dent et al., 1992; Howe et al., 1992). Having established MAPKK as a reliable substrate for Raf kinase in vitro, we next showed that insulin and polypeptide growth factor treatment of a wide variety of cultured cells induced, within 2 minutes, a robust increase in the MAPKKK activity immunoprecipitated by anti-cRaf-1, with a slightly slower activation of endogenous MAPKK (Kyriakis et al., 1993). These results established cRaf $I$ as an insulin/mitogen-activated (SerThr) kinase and defined MAPKK as one of its likely physiologic substrates. All three Raf isoforms (cRaf, B-Raf, and A-Raf) are capable of direct activation of the MAPKKs, MEK1 and MEK2. Conversely, Raf is specific for these two MAPKK isoforms and does not appear to participate in other MAPK pathways. Several MAPKKKs in addition to the Raf subfamily are now known to be capable of activating the MAPKKs, MEK1/2 in vitro, including cMos, MEKK1, MEKK2, MEKK3, and cCot/Tpl2 (Kyriakis and Avruch, in press). Nevertheless, Raf loss-of-function mutations invariably interrupt RTK-driven, MAPK-dependent cellular differentiation in Drosophila (Dickson et al. 1992) and C. elegans (Han et al., 1993), indicating that Rafs are the only MEK 1/2-specific MAPKKKs recruited by receptor tyrosine kinases in metazoans.

\section{Ras-GTP Recruits Raf to the Membrane to Initiate Raf Activation and Mitogenesis}

We next sought to understand the mechanism by which RTKs recruit cRafl into an active state. Our approach was influenced most strongly by earlier work of Rapp and colleagues, who first identified the v-Raf oncogene (Rapp et al., 1983) and its cellular homologs (Moelling et al., 1984). The 648 amino acid (74-kDa) cRaf1 polypeptide is composed of a carboxyterminal catalytic domain (AA335 to 627) and an aminoterminal noncatalytic segment that contains a zinc 
finger structure (AA139-184) homologous to those found in the PKCs (Nishizuka, 1992) (Figure 1). Deletion of the aminoterminal segment to AA303 abruptly activates the Raf-transforming activity. Most v-Raf oncogenes exhibit aminoterminal truncation, generally between AA250-300 (Stanton et al., 1989; Heidecker et al., 1990). The aminoterminal segment is thus an inhibitor of the catalytic domain. In addition, however, the ability of insulin and growth factors to activate cRafl (Kovacina et al., 1990; Blackshear et al., 1991) implied that the aminoterminal segment may serve as the receptor for the upstream activating signal. Strong evidence for this idea is the demonstration by Rapp and colleagues that the ability of catalytically inactive, full-length cRafl to inhibit RTK-induced mitogenesis (Kolch et al., 1991) and transcriptional activation resides in the cRafl aminoterminal segment (AA 1-257). A point mutation (Cys165 Ser) in the cRaf zinc finger largely eliminates the inhibitory potency of the cRaf (1-257) segment (Bruder $e t$

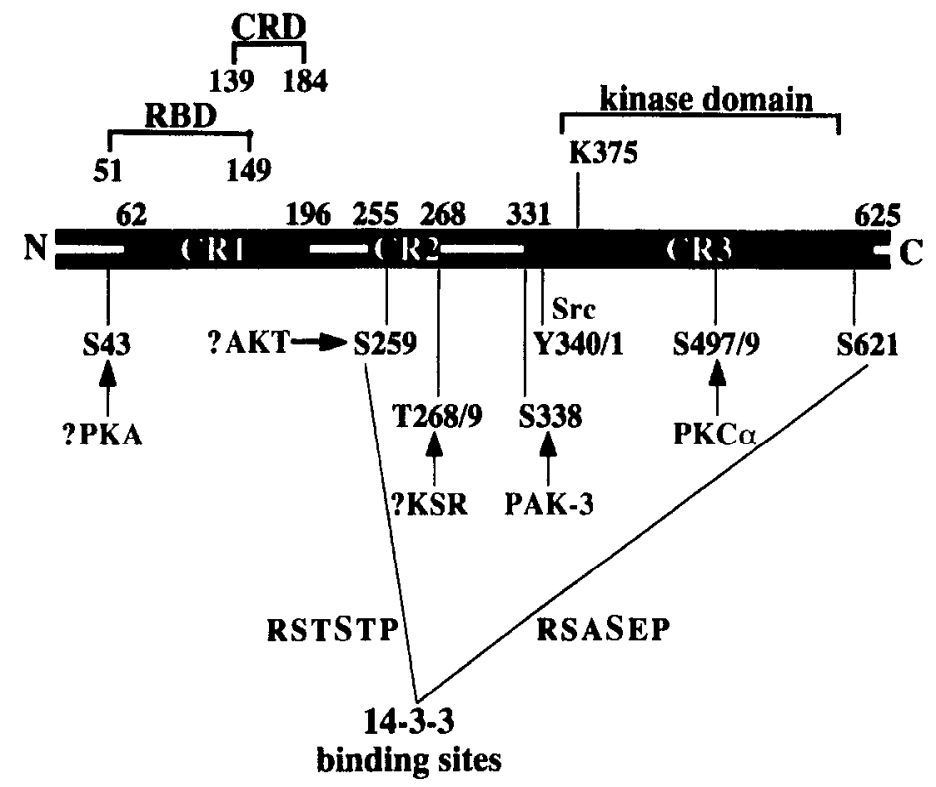

RBD: Ras-binding domain

CRD: cysteine-rich domain

FIG. 1. cRafl: Domain structure and relevant, known phosphorylation sites. $\mathrm{CR}=$ conserved regions in $\mathrm{C}^{-}, \mathrm{A}-$, and $\mathrm{B}-\mathrm{Raf}$. 
al., 1992). As to the nature of this upstream activating signal, the cRaflzinc finger binds zinc (two moles) and phosphatidylserine (Ghosh et al., 1994) but does not bind diacylyccrol (DAG). The structural homology between the Raf- and PKCzinc fingers, however, suggested that Raf activation might be initiated by the binding of some other signal-dependent membrane lipid. Nevertheless, to cover the possibility that the upstream activator required the participation of, or was itself a cellular polypeptide, we employed the yeast two-hybrid expression system to inquire whether the Raf-1 aminoterminal segment (AA1-257) interacted with cellular polypeptides in a manner that was dependent on an intact zinc finger. One of the first cDNAs found to interact with the Raf aminoterminus in this manner encoded the small GTPase, Rap- 1 b.

Rap 1 had been discovered in 1988 independently by three groups (Kawata $e t$ al., 1988; Kitayama et al., 1989; Pizon et al., 1988), one of which isolated Rap 1 through selection of cDNAs capable of causing reversion of the transformed phenotype induced by v-Ras (Kitayama et al., 1989). Rap 1 is 50 percent identical to Ras in overall amino acid sequence; however, the two small GTPases share complete identity in the region corresponding to the Ras effector domain, amino acids $32-40$ (Figure 2). This segment had been identified through a comprehensive examination of the effects of site-specific mutation on the transforming activity of the GTPase-deficient, constitutively active V12 Ras. Mutations in Ras amino acids 32-40 were found to severely inhibit V12 Ras-transforming activity without

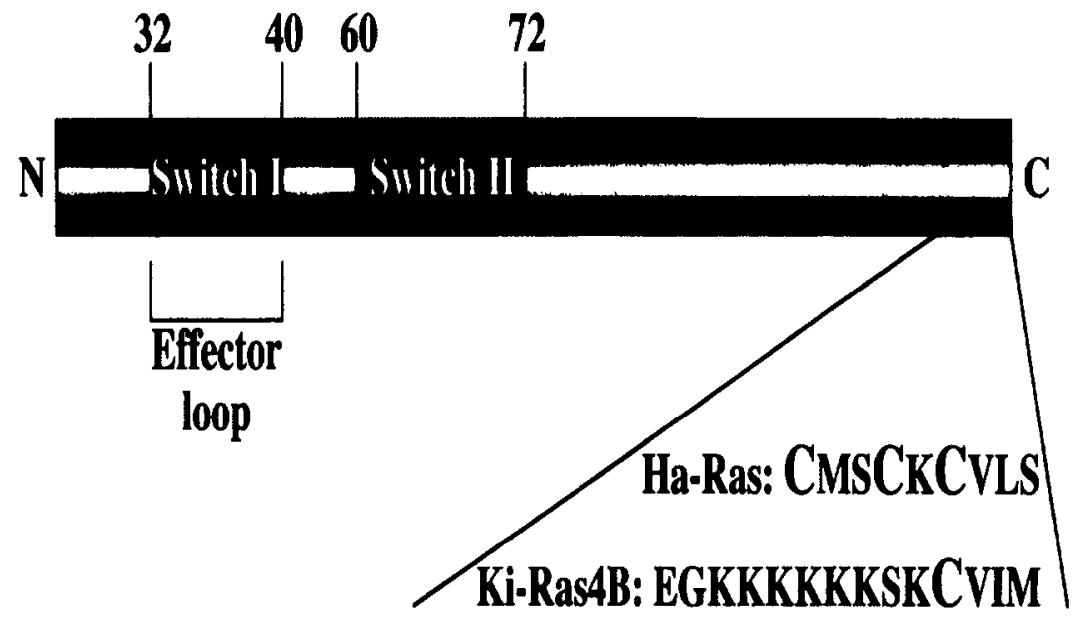

FIG. 2. Ras: Domains relevant to activity. Switch = segments whose configuration is altered by guanyl nucleotides. The cysteine residues subject to prenylation (CAAX) or palmitoylation (Ha-Ras only) are enlarged. 
affecting Ras cellular localization or the ability of cRas to bind, hydrolyze, or exchange guanosine triphosphate (GTP) (Marshall, 1993). The ability of mutations in this segment to abrogate transformation without altering the biochemical activities of Ras known to be critical to transforming activity led to the proposal that the structure of this segment was important to the ability of Ras to recruit its mitogenic "effectors." This view was reinforced when comparison of the crystal structure of Ras liganded with either guanosine diphosphate (GDP) or GTP showed that the loop encoded by Ras AA31-39 (i.e., the "effector" loop identified by mitogenesis) was one of only two segments that differed in configuration (switch 1 and 2) between inactive and active Ras (Figure 2). The inhibitory action of Rapl on Ras-induced transformation could be rationalized with this hypothesis if their identical "effector" loops enabled Rap 1 to interact with Ras "effectors" but in a manner that was nonproductive for the activation of mitogenesis (Marshall, 1993). Our finding that the proto-oncogene Raf interacted directly with the anti-oncogene Rap was consistent with this formulation and implied that the Raf activator was likely to be Ras rather than Rap. In fact, expression of cRafl with V12 Ras gave strong activation of Raf kinase activity, whereas V12 Rapl was without effect. Moreover, we readily demonstrated that Ras interacted strongly with Raf 1-257 in the yeast expression system (Zhang et al., 1993). To determine whether this interaction was truly direct, baculoviral-recombinant, full-length V12 Ha-Ras polypeptide was purified from Sf9 cells and examined for its ability to bind directly to a purified, immobilized prokaryotic recombinant GST-Raf (1-257) fusion protein. Ras charged with GTP $\gamma$ S bound avidly to GST-Raf, whereas Ras charged with GDP $\beta S$ was only slightly retained; GST bound neither form of Ras. Mutation of the Raf zinc finger (GST-Raf 1-257 Cl68S) reduced the binding of V12 Ras GTP $\gamma S$ and abolished binding to V12 Ras GDP $\beta$ S. Fully processed, membrane-bound V12 Ha-Ras and cytoplasmic (farnesylated but incompletely palmitoylated) V12 Ha-Ras polypeptides behaved in a manner similar to each other, binding preferentially to wild-type Raf, as compared to the Raf C168S mutant. Prokaryotic (unprocessed) recombinant Ras liganded with GTP also bound to GST Raf (1-257) but bound equally well to GST Raf (1-257, C168S). Thus, the initial binding studies established that Ras bound to the aminoterminal regulatory domain of Raf in a GTP-dependent manner. Ras carboxyterminal processing, although not required for high-affinity, GTP-dependent Ras-Raf interaction in vitro, did appear to influence the interaction. Reciprocally, an intact Raf zinc finger, while not necessary for the GTP-dependent Ras-Raf binding in vitro, somehow influenced avidity, at least for processed Ras. An indirect estimate of the affinity of this interaction was enabled by the ability of GST-Raf (1-257) to inhibit $\left(\mathrm{IC}_{50}, 0.13 \mu \mathrm{M}\right)$ the stimulation of Ras-GTPase activity caused by the addition of p120 Ras-GAP (Zhang et al., 1993). 
The direct, specific binding of the aminoterminal regulatory domain of the cRafl proto-oncogene to GTP-liganded, "active" Ras established Raf as a strong candidate to serve as a mitogenic effector of Ras. Because the interaction of such an effector with Ras would be expected to occur through the Ras "effector" loop, we examined a panel of Ras effector loop mutants that had been characterized quantitatively for their transforming ability relative to V12 Ras, measured as focus formation in NIH 3T3 cells. The ability of V12 Ras and the various effector Ras loop mutants to bind to Raf (1-257), as assessed quantitatively in the yeast two-hybrid system, paralleled almost completely the relative transforming efficiency of these Ras variants. This close correlation provided additional strong evidence in support of Raf as a direct effector of Ras's mitogenic program (Zhang et al., 1993). Contemporaneously, several other laboratories provided evidence for an association of Ras-GTP and Raf, either in the two-hybrid system, or in vitro using purified polypeptides, or both (reviewed in Avruch et al., 1994).

The identification of Raf as a Ras effector, the concurrent discovery of the role of Grb2 and son of sevenless (SOS) in coupling RTKs to Ras GTP charging, and the earlier discovery of Raf as the conduit for RTK signals into the MAPK pathway together enabled the first description of an RTK signaling pathway from ligand binding to an ultimate intracellular target (e.g., transcription factors such as ternary complex factor (TCF) are regulated directly by MAPK-catalyzed phosphorylation). The ubiquity of the Ras-MAPK pathway in cellular differentiation during development attests to the biologic importance of this pathway. Moreover, the Ras-Raf interaction established in concrete terms a paradigm for the biochemical action of a small GTPase. This has proven applicable to the entire superfamily (i.e., GTP induced reconfiguration of a binding surface on the G protein, enabling a high-affinity interaction with target proteins, which are thereby recruited to the G protein, resulting in either target activation and/or target apposition to critical substrates). The central role of protein kinases as effectors of the small GTPases has been repeatedly demonstrated with the subsequent discovery of the p21-activated kinases (PAKs) and (probably) mixed-lineage kinases (MLKs) as effectors of the Racl and Cdc 42 GTPases, the rho-activated kinases (ROKs) and proteinkinase C-related kinases (PRKs) as effectors of the Rho A GTPases, germinal center (GC) kinase as a probable Rab8 effector, and so forth. Finally, mutant active forms of Ras are present in an estimated 30 percent of human cancers (Bos, 1989). Considerable evidence indicates that that transforming activity of all tyrosine kinase oncogenes and most nonnuclear oncogenes requires activation of endogenous Ras. Thus, the biochemical mechanism of Ras signaling is crucial to the understanding of oncogenesis and to the development of rationally targeted anticancer therapies

In collaboration with Mark Marshall, we undertook a more-refined analysis of the sites of interaction between Raf and Ras-GTP. Using the competitive inhibition of p120 Ras-GAP, a Raf fragment (AA 51-149) was found to bind 
prokaryotic recombinant Ras-GTP with an affinity tenfold higher $\left(\mathrm{IC}_{50}, 12 \mathrm{nM}\right)$ than the full Raf aminoterminus (AA 1-257). Further deletion, especially from the aminoterminus, reduced affinity for Ras-GTP significantly (Chuang et al., 1994). Subsequent mutagenesis of this Raf segment by substitution of single or multiple amino acids revealed especially strong inhibitory effects of substituting $A$ for the basic residues $\mathrm{R} 67, \mathrm{~K} 84, \mathrm{~K} 87$, and especially $\mathrm{R} 89$, as well as $\mathrm{L} 86$ (Barnard et al., 1995). R89 had been identified independently as a loss-of-function mutation in Drosophila Raf (Fabian et al., 1994). Conversely, substitution of acidic residues D33, E37, D38 on the Ras effector loop by uncharged amino acids reduced affinity for Raf by 20 - to 50-fold or more. This analysis suggested that the GTP-dependent binding between Raf and Ras involved multiple basic residues in the Raf aminoterminal segment, interacting with acidic and/or hydrophilic residues on the Ras effector loop. The subsequent visualization of a co-crystal between prokaryotic recombinant Rap1 GTP and the Raf fragment AA 51-131 strongly reinforced this view (Nassar et al., 1995).

\section{Once at the Membrane, the Raf Zinc Finger Binds to a Second Site on Ras}

The relatively clearcut picture of the structural basis of the high-affinity, GTP-dependent binding of Raf to Ras did not actually clarify the question of how the Ras-Raf interaction functions to promote Raf activation. In fact, discrepancies between the requirements for Raf-Ras binding as presented above and those for Raf activation became evident as these data accumulated. Thus, several mutations in Ras just outside of the effector loop (e.g., Ras N26 H27 to GI; Ras V45E) result in dramatic inhibition of Ras-transforming activity, with essentially no inhibition of the binding in vitro of Ras-GTP to Raf (1-257) (Chuang et al., 1994; Barnard et al., 1995). The discrepancy we found most striking was that the Raf fragment (51-149) showing the highest affinity for prokaryotic recombinant Ras-GTP in a direct, in vitro binding assay (Chuang et al., 1994) lacked nearly all of the zinc finger structure. However, an intact Raf zinc finger appeared to be important to the Ras-Raf interaction, as assessed both in the very-sensitive yeast two-hybrid system and in vitro using baculoviral, recombinant Ras polypeptides (Zhang et al., 1993). In addition, the zinc finger was clearly important to Ras-dependent Raf activation. Mutation of the Raf zinc finger (C168S), although causing a small increase in Raf transactivating function in serum-deprived cells, completely abolished the ability of V12 Ras to further augment Raf activity (Bruder et al., 1992).

If the Raf zinc finger is not critical for the high-affinity, GTP-dependent binding of Raf to Ras, why does zinc finger mutation disturb Raf activation so severely? Two mutually exclusive explanations for these apparently conflicting data seemed possible. First, the Raf Cys-rich domain could make an important direct contribution to Raf activation and/or the Ras-Raf interaction that was poorly 
reflected in the in vitro binding assay because of unappreciated technical issues. Second, the zinc finger mutation might simply cause a propagated disturbance in the structure of the contiguous Ras-GTP-binding domain, thereby interfering with Ras binding in vivo. Attempting to distinguish these alternatives, we sought to avoid the loss-of-function phenotype engendered by the point mutations by replacing the Raf zinc finger with the homologous, diacylglycerol (DAG)-binding zinc finger structure from $\mathrm{PKC} \gamma$ (Luo et al., 1997). We then examined whether the newly introduced PKC zinc finger was itself functionally intact and could effectively substitute for the endogenous Raf zinc finger. The results were quite clearcut: the Raf/PKC $\gamma$ fusion proved to be a fully activatable protein kinase, which had acquired the ability to bind active phorbol ester (TPA) in vivo and in vitro. The Raf $1 / \mathrm{PKC} \gamma$ fusion was activated by TPA in intact cells but not in vitro, indicating that TPA activated $\mathrm{Raf} 1 / \mathrm{PKC} \gamma$ by recruiting the fusion protein to the membrane, rather than by an allosteric mechanism. Conversely, in sharp contrast to wild-type Raf 1, the Raf/PKC $\gamma$ fusion, like the $\operatorname{Raf}(\mathrm{Cl} 165,168 \mathrm{~S})$ zinc finger mutant, showed virtually no activation in response to epidermal growth factor (EGF). Thus, the presence of a functional, DAG-binding zinc finger in place of the native Raf zinc finger did not interfere with the ability of the catalytic domain to be activated; however, it did not enable Ras-dependent activation of the Raf kinase domain any better than a mutant Raf zinc finger. Seeking an explanation for this outcome, we compared the association of wild-type and variant Raf polypeptides with V12 Ras. Surprisingly, in spite of earlier studies pointing to the dispensability of the zinc finger for high-affinity Ras-Raf binding in vitro, we found that both the Raf $(\mathrm{C165}, 168 \mathrm{~S})$ and $\mathrm{Raf} / \mathrm{PKC} \gamma$ polypeptides were markedly impaired in their ability to associate with V12 Ras during coexpression in COS cells and in vitro, to an extent comparable to that caused by mutation of the critical basic residues (K84 ALK87 to A) in Raf's primary Ras-binding domain. This indicated that the Raf zinc finger contributed in a significant way to the creation of a stable Ras-Raf interaction. The difference between prokaryotic (unprocessed) recombinant Ras and mammalian recombinant Ras in their ability to bind wildtype Raf vs. Raf with mutant or variant zinc fingers proved to be attributable to Ras prenylation. Abolition of Ras farnesylation by conversion of Ras C 186 to S does not impair high-affinity, GTP-dependent binding of Ras to Raf in vitro. However, it does abolish the ability of mammalian recombinant Ras-GTP to distinguish between wild-type Raf and $\operatorname{Raf}(\mathrm{C} 165,168 \mathrm{~S})$ zinc finger mutant, as was observed for prokaryotic (unprocessed) recombinant Ras. Thus, the Raf zinc finger binds to an Ras epitope, distinct from the effector loop, that is fully expressed only on prenylated Ras (Luo et al., 1997).

The first direct demonstration of a second binding site between Ras and Raf was provided by Hu et al. (1995). This group showed that a recombinant Raf fragment (AA 132-206) encompassing the Cys-rich zinc finger bound directly to 
Ras in vitro. In contrast to the GTP-dependent, high-affinity (nM) binding of the Raf aminoterminal segment (e.g., AA 48-148) to Ras, the binding of the Raf zinc finger to Ras exhibits a much-lower affinity that is entirely GTP independent and abolished by the Raf (C168S) mutation. Our estimates indicate that Raf (130-220) has a binding affinity for prenylated Ras in the low micromolar range (Figure 3 ). Campbell and coworkers (Williams et al., 2000), using a sensitive fluoresence assay, demonstrated that the enzymatic farnesylation of prokaryotic recombinant Ras in vitro greatly increases its affinity for the Ras zinc finger (to a $\mathrm{Kd}$ near 20-30 $\mu \mathrm{M}$ ). This low-affinity, GTP-independent binding becomes physiologically relevant only through the proximity induced by the primary, GTP-dependent Ras-Raf interaction. Hu and colleagues (1995) further showed that the Ras mutations N26G and V45E, which greatly impair V12 Ras-dependent Raf activation without significantly interfering with the GTP-dependent binding of Ras to Raf in vitro, greatly inhibit the GTP-independent binding of Ras to the Raf zinc finger

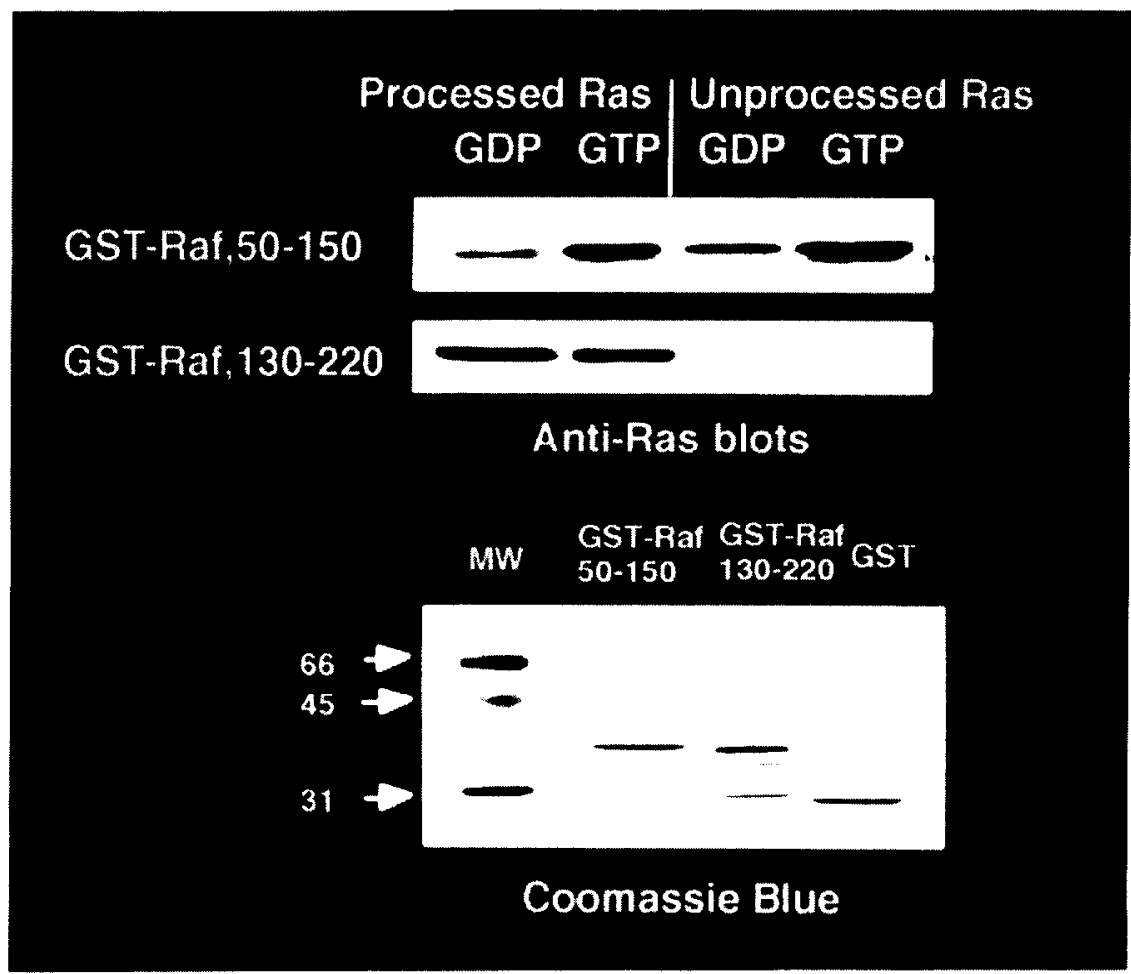

FIG. 3. Binding in vitro of processed (prenylated) or unprocessed Ras to two overlapping domains in the cRafl aminoterminal segment. 
domain (AA132-206). How Ras prenylation reconfigures this epitope (sometimes called the Ras "activation" domain) to increase its affinity for the Raf zinc finger remains to be established.

\section{The Interaction of the Raf Zinc Finger with Ras Is Critical for Raf Activation}

The idea that Ras was dispensable once Raf had been recruited to the membrane was inferred from the observation that fusion of the carboxyterminal membrane targeting sequence from Ki-Ras (Figure 2) onto cRafl to create Raf CAAX enables Raf to promote transformation of NIH $3 \mathrm{~T} 3$ cells in a manner resistant to inhibition by N17 Ras (Leevers et al., 1994; Stokoe et al., 1994). Subsequent work has shown this interpretation to be incorrect; the resistance of Raf CAAX to N17Ras only indicates that the GTP-dependent function of Ras has been bypassed. A continued interaction between Ras and Raf is necessary to enable Raf activation. The Raf zinc finger is critical for this function.

An extensive, surface-scanning mutagenesis (Daub et al., 1998) of the Raf zinc finger region identified S177, T182, and M183 as well as L149 and F151 (Williams et al., 2000) as residues critical to Ras-Raf binding energy. Mutation of Raf K144, R164, and L160 has little affect on Ras binding but interferes strongly with Raf activation, even in the context of Raf CAAX. An elegant, although indirect, demonstration of the importance of the second site, GTP-independent Ras-Raf interaction to Raf activation, was provided by the Ras E37G effector loop mutant, which is unable to bind Raf in a GTP-dependent manner. White and colleagues (1995) used the yeast two-hybrid system to select a Raf mutant (S257L) that is capable of binding to Ras E37G. The Raf (Ser257 Leu) mutation does not restore high-affinity, GTP-dependent binding to Ras E37G but causes a slightly higher level of basal kinase activity that is further augmented by both wild-type Ras and Ras E37G (Jaitner et al., 1997). Raf (S257L) CAAX, like wild-type Raf CAAX, is transforming. Coexpression with Ras E37G substantially increases Raf (S257L) CAAX-dependent focus formation, kinase activity, and MAPK activation. However, Ras E37G has no effect on wild-type Raf CAAX (Mineo et al., 1997). The Raf S257L mutation thus facilitates the second site Ras-Raf interaction that is crucial to Raf activation.

How does the zinc finger participate in Raf activation? The ability of Raf microinjected into Xenopus oocytes to activate germinal vesicle breakdown (GVBD) provides an exquisitely sensitive assay for Raf activity. Raf wild-type and the Raf (R89L) mutant do not activate GVBD. However, various mutations in the zinc finger - C165, 168S, F163I, P181L, as well as R143E or W and K144E - enable significant GVBD, even in the Raf (R89L) background (Cutler and Morrison, 1997; Cutler et al., 1998; Winkler et al., 1998). These results suggest that the Raf zinc finger, in addition to its ability to bind Ras, is the element 
in the Raf aminoterminal domain that participates directly in the inhibition of the Raf catalytic domain. The binding of Ras to the Raf zinc finger may therefore displace the zinc finger from the catalytic domain and promote the disinhibition of the catalytic domain. Support for this view is provided by the ability of Raf 1-330 to inhibit the ability of the catalytically active Raf kinase domain (Raf 306-648) to cause GVBD. Raf 1-330 (R89L) also inhibits but the Raf zinc finger mutants indicated above as allowing GVBD in full-length Raf abolish the ability of Raf 1-330 to inhibit Raf 306-648 (Cutler et al., 1998). Reciprocally, introduction of Y340/341D mutations into Raf 306-648 abrogates inhibition by Raf 1-330, suggesting that the zinc finger inhibits catalytic function by an interaction near Y340 and 341 (i.e., the aminoterminal region of the catalytic domain) (Cutler et al., 1998; Roy et al., 1997).

Interestingly, Rap 1, which binds both cRafl and B-Raf in a GTP-dependent manner, is incapable of activating cRafl in vivo but activates B-Raf both in vivo and on direct addition in vitro (Ohtsuka et al., 1996). This response to Rap 1-GTP depends on the respective Raf zinc finger structures. Swapping the zinc fingers between B-Raf and cRafl swaps the susceptibility to activation by Rapl (Okada et al., 1999). The cRaf zinc fingers actually bind Rapl more tightly than Ras (Hu et al., 1997). Rapl inhibition of Ras-dependent Raf activation therefore may occur through Rapl sequestion of the Raf zinc finger rather than through the more-aminoterminal, GIP-dependent interaction sites. 'The differential affinity of Ras and Rap 1 for the cRaf zinc finger is attributable to residue 31 , which is $\mathrm{K}$ in $\operatorname{Rap} 1$ and $\mathrm{E}$ in Ras; Ras E31K behaves like Rap 1. Conversely, the B-Raf zinc finger binds comparably to both small GTPases. A B-Raf zinc finger mutant (K252E M278T) with selectively enhanced binding to Rap 1 is no longer activated by Rap1 but continues to be activated by Ras (Okada et al., 1999). This behavior is consistent with the idea that the Raf zinc finger-Ras interaction must be strong enough to enable displacement of the zinc finger from the catalytic domain. An excessively strong interaction between the small GTPase and the Raf zinc finger, however, will block activation.

\section{Conversion of cRaf-1 to a Stably Active State Requires Raf Phosphorylation}

The discovery of the Ras/Raf interaction provided an instant insight into both the function of Ras and the regulation of Raf. The subsequent, more-torturous elaboration of the complexities of this interaction, partially described above, uncovered the dual role of Ras: first, to recruit Raf to the membrane through a GTP-dependent interaction between the Ras effector loop and Raf 51-149 (the Ras-binding domain, RBD) and then to initiate the disinhibition of the catalytic domain by a GTP-independent interaction between a second site on Ras with the Raf zinc finger. Altogether, however, these steps do not fully account for the 
mechanism of Ras-dependent cRafl activation in vivo. Ras-dependent activation in vivo converts Raf to a stably activated form in the cytosol, no longer bound to Ras. In our hands, addition of purified, fully processed Ras failed to alter the activity of purified, baculoviral recombinant cRafl (Zhang et al., 1993). Stokoe and McCormick (1997) subsequently reported evidence for such direct activation. Ilowever, the extent of activation appeared to be very slight, compared to that achieved in vivo, and also appeared to require the continued presence of Ras-GTP. We infer that this reflects an intermediate step in the conversion of Raf to a stably activated, Ras-free form, a view supported by other work (Mizutani et al., 1998). As indicated earlier, such stable activation most often reflects the introduction of a post-translational modification, usually phosphorylation. In fact, $\mathrm{cRaf1}$ is extensively phosphorylated in vivo prior to activation and its phosphorylation increases several-fold after RTK activation. Moreover, coexpression of $\mathrm{cRaf} 1$ with a variety

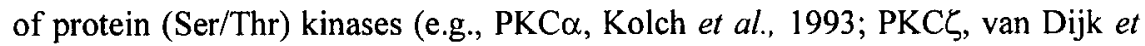
al., 1997) or tyrosine kinases (e.g., Src, Fabian et al., 1993; Marais et al., 1995; Jelinek et al., 1996; Mason et al., 1999; Xia et al., 1999) or JAK2 (Xia et al., 1996), either in mammalian or Sf9 cells, results in the phosphorylation and activation of cRafl. Reciprocally, dephosphorylation of active cRafl with protein (Ser/Thr) phosphatases, alone or together with protein tyrosine phosphatases (Dent et al., 1995) as well as the dual-specificity phosphatase Cdc25A (Mason et al., 1999), have all been reported to deactivate cRafl. Nevertheless, the manner in which altered phosphorylation promotes the Ras-dependent activation of cRafl remains elusive.

A very definite conclusion is that phosphorylation of one or more residues on the cRafl activation loop, the most-common mechanism for phosphorylationdependent kinase activation, is not contributory to Ras-dependent Raf activation (Barnard et al., 1998). Much attention has been devoted to the role of phosphorylation at $\mathrm{Y} 340 / 341$, inasmuch as conversion of these residues to A greatly impedes cRafl activation. However, conversion to $D$ (which is the amino acid found at these two sites in B-Raf) results in increased basal activity (Fabian et al., 1993; Marais et al., 1995; Jelinek et al., 1996; Mason et al., 1999). This site is phosphorylated in vivo in response to overexpression of Src, in a manner augmented by V12Ras. We and others, however, have been unable to detect significant cRafl tyrosine phosphorylation during activation by insulin, EGF, or platelet-derived growth factor (PDGF). Thus, while phosphorylation of Y340/41 is a plausible and attractive mechanism for activation, especially in view of the role of this region as the probable site of negative regulation of Raf kinase activation by the Raf zinc finger, the evidence that Y340/41 phosphorylation participates in RTK-initiated, Ras-dependent activation of $\mathrm{cRaf}$ is unpersuasive.

A more-compelling body of evidence points to an important role for cRaf Ser338 phosphorylation in facilitating Raf activation. As first shown by Marshall 
and coworkers, conversion of S338/339 to A blocks Raf activation by V12 Ras or vSrc and abrogates the transforming activity of Raf CAAX. Conversion of S338/339 to D is not itself activating but restores responsiveness to V12Ras and vSrc as well as the transforming activity of Raf CAAX (Diaz et al., 1997). These workers subsequently identified PAK 3 as a kinase capable of phosphorylating in vitro a peptide whose sequence is corresponds to that surrounding cRafl Ser338/339 (King et al., 1998). Substantial evidence was presented for the participation of PAK3 in S338 phosphorylation in vivo, acting downstream of Cdc42 and/or Rac1, which can be recruited either by V12Ras or by RTKs, through the recruitment and activation of PI-3 kinase (Sun et al., 2000). PAK3 may not be the only kinase active on the Raf S338 site; the identity of the S338 kinase is likely to vary, depending on the initiating stimulus (e.g., tumor necrosis factor receptors (TNFRs) vs. RTKs). Moreover, it is plausible that some stimuli (e.g., hematopoeitic cytokine receptors) use Y341 phosphorylation rather than S338 phosphorylation for this "priming" or facilitative function. Nevertheless, phosphorylation at S338, while necessary, is not sufficient to activate Raf in vivo or in vitro.

\section{Raf Binds Chaperones and 14-3-3 Proteins}

Our inability to activate cRafl by direct addition in vitro of purified, fully processed Ras-GTP and Mg-ATP led us to the view that additional components are necessary to complete the process of Raf activation. We therefore again used two-hybrid expression cloning to seek proteins that interacted with cRafl and immediately recovered cDNAs encoding the 14-3-3 $\zeta$ polypeptides (Luo et al., 1995). Concurrently, purification of baculoviral recombinant cRafl yielded a complex of proteins consisting of the 74-kDa Raf polypeptide, heat shock protein (hsp) 90, hsp 50 (also known as cdc37), and the 14-3-3 proteins (Luo et al., 1995). The association of these heat shock proteins with other protein kinases, particularly Src, had been previously described (Pratt, 1998). While these chaperones are undoubtedly critical for ensuring the proper conformation of the kinase, we considered it unlikely that they participated actively in the regulation of kinase activity. We therefore examined the role of the 14-3-3 proteins in Raf regulation. The 14-3-3s are a family of 28- to $30-\mathrm{kDa}$ polypeptides known to assemble as homo- or heterodimers and repeatedly rediscovered as binding partners for a diverse array of polypeptides (Aitken, 1996). As first shown by Muslin et al. (1996), the 14-3-3s bind to motifs that contain phosphoserine (and probably phosphothreonine) residues, situated in a specific sequence. This context was defined as RSXS(P)XP or RXXXS(P)XP using synthetic peptides (Yaffe et al., 1997). However, it is clear that a considerably broader specificity is accommodated. This tolerance is explained in part by the dimeric nature of the 14-3-3 assembly. The stable binding of a peptide containing a single phosphoserine motif 
to 14-3-3 requires a high-affinity $(\mathrm{nM})$ interaction. A polypeptide with multiple phosphoserines, even though these are encompassed in lower-affinity motifs, may achieve stable association with 14-3-3 due to the approximation of a low-affinity motif enabled by association of the first phosphoserine site with a 14-3-3 half dimer.

We carried out a deletion analysis of the 245 amino acid 14-3-3 $\zeta$ polypeptide. This analysis demonstrated that the 14-3-3 dimerizeration interface involved an extensive part of the aminoterminal half of the $14-3-3 \zeta$ polypeptide, whereas the Raf binding function resided primarily in the carboxyterminal half of the 14-3-3 polypeptide (Luo et al., 1995). Structures of 14-3-3 $\zeta$ crystals confirmed this arrangement, revealing each 14-3-3 $\zeta$ polypeptide as an L-shaped set of antiparallel helices, with the $\mathrm{N}$-terminal four helices involved in dimerization and the carboxyterminal helices free, with helices 3,5,7, and 9 forming the inner walls of a cavity within the dimer that accommodates its binding partners (Liu et al., 1995). Modeling the phosphoserine peptide into the 14-3-3 structure indicates that the two facing binding sites within the cavity are arranged in an antiparallel fashion. The most-striking result of our analysis was the finding that, although cRafl bound quite well in vivo to a GST-14-3-3 (139-245) fusion protein, the cRaf 1 polypeptide recovered with this monomeric form of 14-3-3 is completely devoid of catalytic activity, either before or after EGF stimulation (Luo et al., 1995). This strongly suggested that a dimeric 14-3-3 was critical to the process of Raf activation.

\section{Raf Dimerization Promotes Raf Activation}

The ability of monomeric GST 14-3-3 to bind cRafl suggested the possibility that the native 14-3-3 dimer might enable cRafl to be dimerized, either with itself or with another kinase that could catalyze Raf phosphorylation and activation. Coexpression of variously epitope-tagged Raf polypeptides demonstrated that a small fraction of recombinant Raf is recovered as homodimers and that the abundance of these homodimers is unaffected by EGF stimulation (Luo et al., 1996). We next inquired whether forced dimerization of Raf polypeptides affected Raf activity or susceptibility to activation. A set of three tandem FKBP-12 polypeptides was fused to the Raf aminoterminus, each conferring a single binding sitc for the macrolide drug FK506 or its dimeric congener, FK1012. Addition of FK1012 to cells expressing FK506-binding protein (FKBP)-Raf promoted the oligomerization of FKBP-Raf in a manner that was inhibited competitively by the monomeric FK506. The oligomerization of Myc-Raf was unaffected by FK1012. Similarly, FK 1012 produced a time- and concentration-dependent activation of FKBP-Raf but not wild-type Raf that was about half the magnitude seen with EGF. Strikingly, however, FK1012 and EGF together produced a synergistic 
activation of FKBP-Raf. Activation of FKBP-Raf by both EGF and FK1012 was inhibited by coexpression with $\mathrm{N} 17$ Ras and abolished completely by mutation in either FKBP-Raf Ras-binding domain (RBD) (K84 ALK-A84AA) or zinc finger (C165,168S) (Luo et al., 1996). Thus, Raf dimerization strongly promotes Rasdependent activation in vivo. Whether Raf dimerization is a necessary precondition for Raf activation in vivo and whether dimerization is regulated are not yet known. Nevertheless, it is striking that Inouye et al. (2000) recently observed that the ability of Ras-GTP to partially activate cRafl in vitro is entirely dependent on Ras dimerization. Ras dimerization apparently occurs spontaneously when Ras is inserted into a phospholipid membrane in vitro and perhaps within caveoli in vivo. Ras and Raf dimerizations appear to be significant elements in the Raf activation process in a manner somewhat analogous to the ligand-induced dimerization of the RTKs themselves. In contrast to the RTKs, however, it is unclear whether Raf dimerization is initiated by dimeric Ras, as Raf homodimers are recovered from cells free of Ras. Nevertheless, further analysis of the structural basis for Raf homodimerization revealed that the $\operatorname{Raf}(1-257)$ aminoterminal fragment can homodimerize, whereas the constitutively active carboxyterminal Raf fragment known as BXB-Raf (1-25/306-648) does not. Inasmuch as BXB Raf binds 14-3-3 avidly, while Raf (1-257) binds Ras but exhibits no binding to 14-3-3 in the two-hybrid system, it seems clear that Raf homodimerization is not mediated by 14-3-3. However, a role for Ras in initiating Raf dimerization remains tenable (J. Avruch et al., unpublished observations).

\section{$X$. Binding of Raf to Dimeric 14-3-3 Is Necessary for the Initiation and Maintenance of Kinase Activity}

Previous work had provided evidence for the binding of 14-3-3 to the Raf zinc finger (Michaud et al., 1995; Clark et al., 1997) as well as to the phosphoserines at 259 and 621 (Muslin et al., 1996; Michaud et al., 1995; Rommel et al., 1996). We mutated S259 and S621 to A and examined the effects on the interaction of Raf with 14-3-3 in the two-hybrid assay and on the activation of Raf kinase in COS cells in response to EGF (Tzivion et al, 1998). Removal of either S259 or S621 singly did not eliminate interaction with 14-3-3. However, the double mutation S259/621A eliminated interaction with 14-3-3 completely without affecting the interaction of Raf with Ras or MEK. Thus, each Raf polypeptide contains two phosphoserine-based 14-3-3-binding sides. The S259A (single) mutant exhibits a modest increase in basal kinase activity and two- to three-fold greater activation in response to EGF than is seen with wild-type Raf. Thus, the S259 phosphorylation is inhibitory and contributes to the maintenance of unstimulated Raf in a low-activity state. Conversely, the S621A mutant (and the S259/621A double mutant) is completely inactive. To ascertain whether the inactive state of the S621A mutant is due to the loss of 14-3-3 binding from that site, 
we displaced 14-3-3 from the recombinant Raf in vitro by addition of a synthetic phosphopeptide whose sequence corresponds to the motif surrounding cRaf1 S621. The displacement of 14-3-3 from Raf resulted in the complete deactivation of the Raf kinase, both wild type and Raf (S259A). This deactivation is not due to interference with Raf oligomerization, which was unaltered by displacement of 14-3-3. Moreover, the activity of FKBP-Raf induced by the dimeric ligand FK1012 was inhibited by displacement of 14-3-3. Thus, the binding of 14-3-3 to Raf is necessary both for initiation and maintenance of the active state. The deactivation of Raf caused by displacement of 14-3-3 can be reversed simply by addition in vitro of prokaryotic, recombinant 14-3-3. However, Raf must have been previously activated in vivo to be (re)activated by 14-3-3 in vitro. The ability of 14-3-3 to reactivate Raf in vitro is dependent on Raf serine phosphorylation, as treatment of Raf with protein phosphatase $1 \gamma$ after 14-3-3 displacement prevents reactivation by 14-3-3. Moreover, reactivation in vitro, just like activation in vivo, depends on the integrity of the 14-3-3 dimer. We constructed a mutant, monomeric 14-3-3 polypeptide by introducing a series of mutations into the dimer interface. Monomeric, full-length 14-3-3 bound Raf avidly in vivo but, as with the monomeric GST 14-3-3 (139-245), the Raf bound to monomeric, full-length 14-3-3 was devoid of activity. Moreover, in contrast to wild-type, dimeric 14-3-3, prokaryotic, recombinant monomeric 14-3-3 is completely unable to support the reactivation of wild-type Raf or Raf (S259A) in vitro after displacement of endogenous 14-3-3. Thus, Raf (S259A), which contains only one known 14-3-3binding motif (at S621) nevertheless requires a dimeric 14-3-3 to sustain activity in vivo and to restore activity in vitro. The explanation for this requirement is not known. Our working hypothesis is simply that a 14-3-3 dimer is required because a second, yet to be identified, phospho (Ser/Thr)-dependent 14-3-3 binding site exists on active cRafl (S259A). In fact, we believe that the introduction of this phosphorylation underlies the stable activation of Raf (i.e., the active state requires the simultaneous binding of both S621P and this putative new phosphorylation site on a single Raf polypeptide by a single 14-3-3 dimer) (Tzivion et al., 1998 ). We recently have identified a set of novel phosphorylation sites in the Raf catalytic domain and are examining the role of those sites in the initiation and maintenance of Raf activation.

\section{Mechanism of Ras-dependent cRafl Activation}

Our current hypothesis (Figure 4) for the mechanism of RTK-induced cRaf activation is the following:

1) cRafl resides in the cytosol in an inactive state, bound in a complex with hsp90, hsp50, and the 14-3-3 protein. The configuration of inactive Raf involves the occlusion of the catalytic domain by the Raf zinc finger, which obstructs 
access to the ATP-binding site. The inactive configuration is stabilized by the binding of a 14-3-3 dimer to Ptd Ser259 and Ptd Ser621, which acts like a clothespin, folding the Raf polypeptide into a closed configuration. Mutation of the zinc finger or the S259A mutation each relaxes slightly the inhibited configuration. A portion of Raf polypeptides exist as dimers, an interaction mediated, directly or indirectly, through a segment in the aminoterminal (1-257) region, yet to be fully defined.

2) Ligand activation of RTK promotes Ras-GTP charging, which creates a high-affinity binding site for cRafl residues (51-149), the primary Ras-binding domain. This enables recruitment of a Raf dimer to Ras-GTP dimer at the membrane.

3) Once bound to the Ras effector loop at the membrane, Raf then interacts through its zinc finger with a second epitope on Ras that includes Ras N26, H27, and E45. Its configuration is unaffected by GTP-GDP but is fully developed only on prenylated Ras.

\section{Inactive Raf in resting cells}

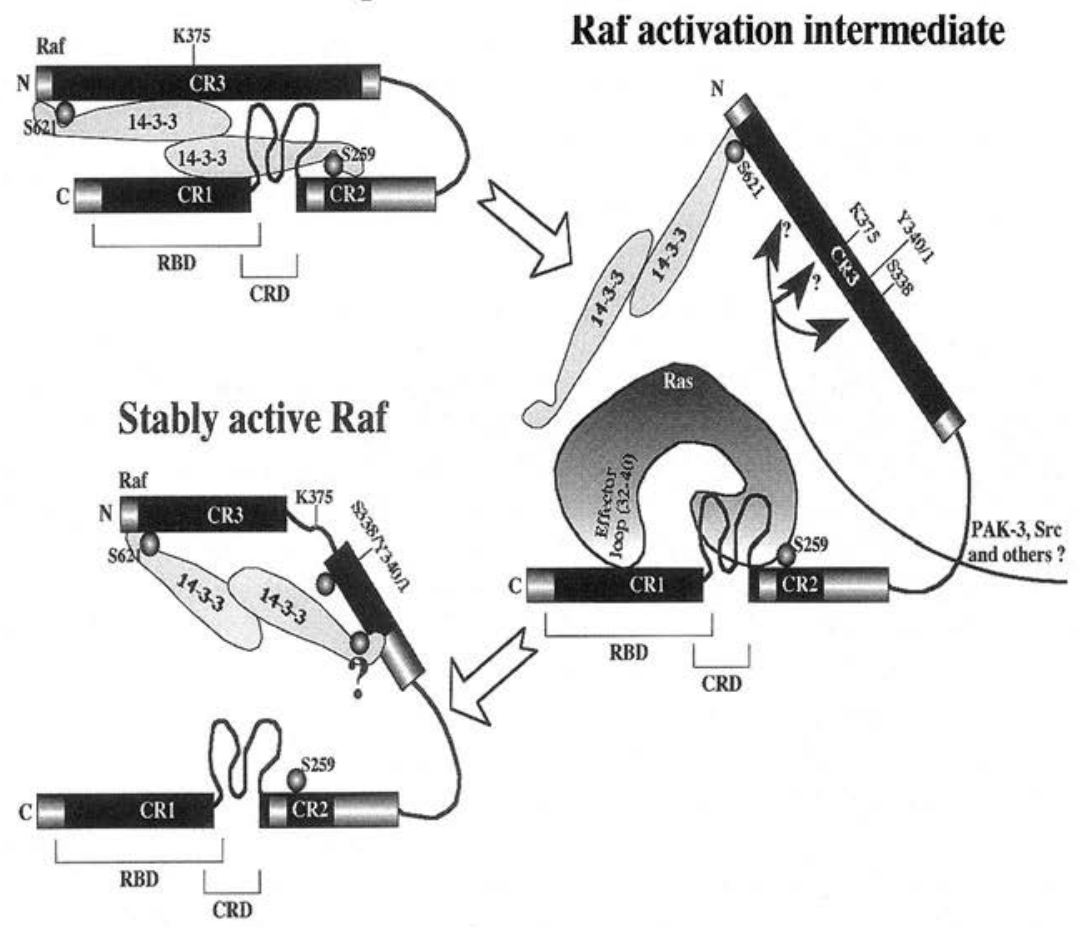

FIG. 4. A model for Ras-dependent cRaf1 activation. 
4) Once at the membrane, the Raf catalytic domain also may bind to phosphatidic acid (PA) through a segment between cRafl residues 389-423 (Ghosh et al., 1996). Although PA does not alter Raf activity per se, it may contribute to the activation process in vivo (Ghosh et al., 1996; Rizzo et al., 2000).

5) The second site Ras-Raf interaction, perhaps aided by phosphatidylserine binding to the zinc finger, promotes the displacement of the 14-3-3 half-dimer from the P-Ser259 site (Rommel et al., 1996). This enables a partial activation of Raf kinase, which remains dependent on the continued presence of Ras. Ras binding to the Raf zinc finger also interferes with the interaction of the zinc finger with the region around Raf S338-Y341, further relaxing the inhibited configuration.

6) PAK3-catalyzed phosphorylation of S338 impedes the reassociation of the Raf zinc finger with this segment, limiting autoinhibition.

7) cRafl undergoes phosphorylation at an unidentified site in the catalytic domain, distinct from the $\$ 338-Y 340$ region, which creates a new binding site for the 14-3-3 half-dimer just displaced from Ptd Ser259. The binding of 14-3-3 to this putative site stabilizes an open, active configuration of cRafl, enabling its release from Ras-GTP into the cytosol in a stably active state. Deactivation is initiated by protein (Ser/Thr) phosphatase action.

Steps 1-6 in this model are well supported by available data; step 7 is speculative and under active investigation. Other models, which differ in significant details regarding the mechanism of activation, have been proposed (Roy et al., 1998; Thorson et al., 1998; McPherson et al., 1999).

\section{Ras Effectors Other Than Raf}

Although the Raf kinases are the most-securely established, direct mitogenic effectors of Ras, other Ras effectors crucial to mitogenesis have emerged (Shields et al., 2000). The existence of such elements was predictable from the early observation that although both v-Raf and v-Ras are able to transform fully NIH $3 \mathrm{~T} 3$ cells and other cells of fibroblastic origin, most human Ras oncogenes are found in cancers of epithelial origin (e.g., pancreas, colon, nonsmall cell lung cancers). In such cell backgrounds, $v$-Ras is itself transforming but v-Raf is not. Inhibitors of MAPK activation uniformly inhibit the growth of Ras-transformed epithelial cell lines in cell culture. This indicates that Ras activation of the RafMAPK pathway is necessary to transformation; however, mitogenic pathway(s) in addition to Raf-MAPK clearly are required. Moreover, a variety of Ras effector loop mutations have been shown to attenuate or eliminate Ras-transforming activity while retaining the ability to elicit components of the vRas biologic response and to complement each other to enable mitogenesis (White et al., 1995; Joneson et al., 1996). These mutants have been very useful in parsing the outflows from 
V12 Ras (Miller et al., 1998). At this time, two families of Ras-regulated signaling molecules in addition to the Rafs have been clearly identified.

Rodriquez-Viciana et al. (1994) first demonstrated that constitutively active Ras can promote the accumulation of $3^{\prime} \mathrm{OH}$ phosphorylated Ptd Ins lipids. Subsequent to the molecular cloning of the p1 10 catalytic subunits of the type 1a PI-3 kinases, this group showed that a region near the pl10 aminoterminus binds directly to Ras-GTP in preference to Ras-GDP. A point mutation (K227E) that abrogates this interaction increases basal p110 activity (about four-fold) but abolishes the response to V12 Ras (Rodriquez-Viciana et al., 1996). Moreover, the ability of V12 Ras to cause membrane ruffling, a Rac-regulated response, is abolished by the PI-3 kinase inhibitor wortmannin (Rodriquez-Viciana et al., 1997). Thus, type 1a PI-3 kinases appear to be among the mitogenic effectors recruited by V12 Ras. The caveat, however, relates to the role of Ras in the activation of PI-3 kinase in response to RTK activation. Unlike Raf, whose recruitment to the membrane and subsequent activation in response to RTKs is entirely dependent on its $\mathrm{nM}$ affinity for and recruitment by Ras-GTP, the affinity of Ras-GTP for p1 10 is substantially lower than for Raf (Rodriquez-Viciana $e t$ al., 1994,1996). In response to RTK activation, type 1a PI-3 kinases are not recruited to the membrane through their low-affinity interaction with Ras-GTP but rather by the avidity of the SH2 domains of the p85/55 adaptor subunits of the type IA PI-3 kinases for the tyrosine-phosphorylated RTKs and docking protein (e.g., insulin receptor substrate (IRS), FGF receptor substrate (FRS)). Thus, in the context of ligand activation of the RTK, the impact of Ras-GTP on PI-3 kinase activity is secondary and collateral, serving to augment the activation engendered by direct recruitment of the p85/p110 PI-3 kinase heterodimer by $\mathrm{RTK}$ and/or docking proteins. Conversely, the idea that the constitutively active V12 Ras oncogene is able to promote a direct activation of PI-3 kinase, sufficient to recruit some Ptd Ins 3,4,5 $\mathrm{P}_{3}\left(\mathrm{PIP}_{3}\right)$ downstream effectors in achieving cellular transformation, is quite plausible. Such effectors include the $\mathrm{PKB} / \mathrm{cAkt}$ protein kinases and members of the Rho subfamily of GTPases (e.g., Rac1), which are activated by $\mathrm{PIP}_{3}$-induced recruitment of Dbl-family guanylnucleotide exchange factors (Shields et al., 2000).

A second, well-established group of Ras-GTP binding partners is a family of guanynucleotide exchange proteins (GNEFs) for the RalA GTPases (Shields $e t$ al., 2000). These GNEFs - including Ral-GDS, RGL, and RIf - were identified as candidate Ras effectors through two-hybrid screens. Each is capable of binding directly to Ras GTP, in preference to Ras GDP in vitro. Dominant inhibitors of RalA can interfere with Ras-induced mitogenesis. However, the role of RalA in Ras signaling and the specific biochemical effectors of the RalA GTPase are poorly understood. Ral GDS also binds to Rap 1 GTP in vitro, with an affinity that apparently is higher than for Ras-GTP. This situation is reversed from cRafl, 
which clearly prefers Ras GTP over Rap 1 GTP. Thus, the question of whether the Ral GDS are effectors for Ras, Rap1, or both is currently unresolved. This exemplifies as well the current uncertainty over the actual biologic functions of the Rapl GTPases (Zwartkruis and Bos, 1999). Based on work in Drosophila, it appears probable that Rapl has functions entirely distinct from its ability to interfere with Ras signaling (Li et al., 1997; Asha et al., 1999). The latter phenotype, in fact, may occur only when Rap1 is overexpressed and may not reflect a physiologic function of Rapl (Zwartkruis et al., 1998). Whereas Rapl opposes Ras-induced activation of $\mathrm{cRafl}$, both Ras and Rap 1 are capable of promoting by direct addition the activation in vitro of B Raf (Yamamori et al., 1995; Ohtsuka et al., 1996), the isoform predominant in neural cells in vivo (Vossler et al., 1997). Interestingly, constitutively active Rap 1 is transforming in some cell backgrounds (e.g., Swiss 3T3 cells, but not NIH 3T3 cells) (Altshuler and Rheiro-Neto, 1998), possibly as a function of its ability to support the activation of B-Raf.

Our own efforts to identify additional Ras effectors have primarily employed two-hybrid screens using constitutively active forms of Ras and Rapl. Although we never encountered p1 10 PI3 kinase, we have recovered cDNAs corresponding to all three Ral-GDS isoforms from several cDNA libraries. In addition, we have retrieved an array of noncatalytic proteins, some of which (e.g., AF6) have been characterized as candidate Ras-GTP effectors by others. We are presently attempting to elucidate the biologic function of NOREl, a 46-kDa noncatalytic polypeptide that binds to Ras in vivo in response to EGF (Vavvas, 1998). We presume NORE 1 to be an adaptor protein and have recently found it to be constitutively associated with a protein kinase of the Ste 20 subfamily. The significance of this association to the biologic programs controlled by NOREl acting downstream of Ras remains to be defined.

\section{Conclusion}

The Ras-Raf-MAPK pathway is arguably the most-extensively characterized and thoroughly studied signal transduction pathway in metazoans, certainly over the last decade. This scrutiny reflects the central role of this pathway in development, cellular differentiation, and, especially, mitogenesis. Continuous overactivity of this pathway is necessary to the growth of a substantial fraction of human malignancies. The pathway components therefore are attractive targets for antiproliferative therapies. This chapter has focused selectively on Ras and cRaf1, components that we have investigated directly. The understanding of the interaction between these elements and their operation during the process of Raf activation has expanded greatly but remains incomplete. Moreover, the outflows from Ras other than Raf, and the targets of Raf other than the MAPPKs (MEK 1 and 2), are areas where significant discoveries remain to be accomplished. Finally, we have not discussed a number of elements that appear to be important modulators 
of the activity of either Ras or Raf or of the interaction of Ras and Raf with each other. Such molecules include the Ras-GAPs, p120 and NF1/2, and Sprouty (Casci et al., 1999), an inhibitory element acting on Ras by an unknown mechanism. By contrast, SUR8 (Steward et al., 1999), connector enhancer of kinase suppressor of Ras (CNK) (Therrien et al., 1999), and kinase suppressor of Ras (KSR) (Li et $a l ., 2000)$ are positive modulators of Ras-Raf signaling that interact directly with Ras, Raf, or both. Other modulators are Raf kinase inhibitor protein (RKIP), a putative Raf inhibitor (Yeung et al., 1999), and Bag-1, a Bcl2-like, putative Raf activator (Wang et al., 1996). Understanding the mechanism of action and biologic role of these novel elements will keep this field vibrant for many years to come.

\section{ACKNOWLEDGMENTS}

The work described herein from our laboratory was supported by grants from the National Institutes of Health, the American Cancer Society, and Eli Lilly, Inc. We thank our outstanding collaborators, Ulf Rapp and Mark Marshall.

\section{REFERENCES}

Ahn, N.G., and Krebs, E.G. (1990). J. Biol. Chem. 265, 11487-11494.

Ahn, N.G., Seger, R., Bratlien, R.L., Diltz, C.D., Tonks, N.K., and Krebs, E.G. (1991). J. Biol. Chem. 266, 4220-4227.

Aitken, A. (1996). Trends Cell Biol. 6, 341-347.

Alcorta, D.A., Crews, C.M., Sweet, L.J., Bankston, L., Jones, S.W., and Erikson, R.L. (1989). Mol. Cell. Biol. 9, 3850-3859.

Alexander, M.C., Kowaloff, E.M., Witters, L.A., Dennihy, D., and Avruch, J. (1979). J. Biol. Chem. 254, 8052-8056.

Altshuler, D.L., and Ribeiro-Neto, F. (1998). Proc. Natl. Acad. Sci. U.S.A. 95, 7475-7479.

Anderson, N.G., Maller, J.L., Tonks, N.K., and Sturgill, T.W. (1990). Nature 343, 651-653.

Asha, H., deRuiter, N.D., Wang, M.G., and Hariharan, I.K. (1999). EMBO J. 18, 605-615.

Ashworth, A., Nakielny, S., Cohen, P., and Marshall, C. (1992). Oncogene 7, 2555-2556.

Avruch, J., Leone, G.R., and Martin, D.B. (1976). J. Biol. Biochem. 251, 151 1-1515

Avruch, J., Nemenoff, R.A., Pierce, M., Kwok, Y.C., and Blackshear, P.J. (1985). In "Insulin Action" (M.P. Czech, ed.), pp. 263-296. Plenum Press, New York.

Avruch, J., Zhang, X.F, and Kyriakis, J.M. (1994). Trends Biochem. Sci. 19, 279-283.

Avruch, J.A., Belham, C., and Weng, Q-P. (2001). Prog. Mol. Subcell. Biol., in press.

Banerjee, P., Ahmad, M.F., Grove, J.R., Kozlosky, C., Price, D.J., and Avruch, J. (1990). Proc. Natl. Acad. Sci. U.S.4. 87, 8550-8554

Barnard, D., Diaz, B., Hettich, L., Chuang, E., Zhang, X-f., Avruch, J., and Marshall, M.S. (1995). Oncogene 10, 1283-1290.

Barnard, D., Diaz, B., Clawson, D., and Marshall, M. (1998). Oncogene 17, 1539-1547

Benjamin, W.B., and Singer, 1. (1975). Biochemistry 14, 3301-3309

Blackshear, P.J., Haupt, D.M., App, H., and Rapp, U.R. (1991). J. Biol. Chem. 265, 12131-12134.

Bos, J.L. (1989). Cancer Res. 49, 4682-4689.

Boulton, T.G., Yancopoulos, G.D., Gregory, J.S., Slaughter, C., Moomaw, C., Hsu, J., and Cobb, M.H. (1990). Science 249, 64-67. 
Bruder, J.T., Heidecker, G., and Rapp, U.R. (1992). Genes Devel. 6, 545-556.

Casci, T., Vinos, J., and Freeman, M. (1999). Cell 96, 655-665.

Chuang, E., Barnard, D., Hettich, L., Zhang, X.-f., Avruch, J., and Marshall, M.S. (1994). Mol. Cell Biol. 14, 5318-5325.

Clark, G.J., Drugan, J.K., Rossman, K.L., Carpenter, J.W., Rogers-Graham, K., Fu, H., Der, C.J., and Campbell, S.L. (1997). J. Biol. Chem. 272, 20990-20993.

Courchesne,W.E., Kunisawa, R., and Thorner, J. (1989). Cell 58, 1107-1119.

Crews, C.M., Alessandrini, A., and Erikson, R.L. (1992). Science 258, 478-480.

Cutler, R.E. Jr., and Morrison, D.K. (1997). EMBO J. 16, 1953-1960.

Cutler, R.E. Jr., Stephens, R.M., Saracino, M.R., and Morrison, D.K. (1998). Proc. Natl. Acad. Sci. U.S.A. 95, 9214-9219.

Daub, M., Jockel, J., Quack, T., Weber, C.K., Schmitz, F., Rapp, U.R., Wittinghofer, A., and Block, C. (1998). Mol. Cell. Biol. 18, 6698-6710.

Dent, P., Haser, W., Haystead, T.A., Vincent, L.A., Roberts, T.M., and Sturgill, T.W. (1992). Science 257, 1404-1407.

Dent, P., Jelinek, T., Morrison, D.K., Weber, M.J., and Sturgill, T.W. (1995). Science 268, 1902-1906.

DeRooij, J., Zwartkruis F.J.T., Verheijen M.H.G., Cool, R.H., Nijman, S.M.B., Wittinghofer, A., and Bos, J.L. (1998). Nature 396, 474-477.

Diaz, R., Barnard, D., Filson, A., Macdonald, S., King, Alastair, and Marshall, M. (1997). Mol. Cell Biol. 17, 4509-4515.

Dickson, B., Sprenger, F., Morrison, D., and Hafen, E. (1992). Nature (Lond.) 360, 600-603.

Elion, E.A., Grisafi, P.L., and Fink, G.R. (1990). Cell 60, 649-664.

Erikson, E., and Maller, J.L. (1985). Proc. Natl. Acad. Sci. U.S.A. 82, 742-746.

Erikson, E., and Maller, J.L. (1989). J. Biol. Chem. 264, 13711-12717.

Errede, B., and Levin, D.E. (1993). Curr. Opin. Cell Biol. 5, 254-260.

Fabian, J.R., Daar, I.O., and Morrison, D.K. (1993). Mol. Cell. Biol. 13, 7170-7179.

Fabian, J.R., Vojtek, A.B. Cooper, J.A., and Morrison, D.K. (1994). Proc. Natl. Acad. Sci. U.S.A. 91, 5982-5986.

Forn, J., and Greengard, P. (1976). Arch. Biochem. Biophys. 176, 721-733

Ghosh, S., Xie, W-Q,., Quest, A.F.G., Mabrouk, G.M., Strum, J.C., and Bell, R.M. (1994). J. Biol. Chem. 269, 1000-10007.

Ghosh, S., Strum, J.C., Sciorra, V.A., Daniel, L., and Bell, R.M. (1996). J. Biol. Chem. 271, 8472-8480.

Gomez, N., and Cohen, P. (1991). Nature 353, 170-173.

Han, M.,, Golden, A., Han, Y., and Sternberg, P.W. (1993). Nature (Lond.) 363, 133-140.

Haselbacher, G.K., Humbel, R.E., and Thomas, G. (1979). FEBS Lett. 100, 185-189.

Heidecker, G., Huleihel, M., Cleveland, L., Kolch, W., Beck, T.W., Lloyd, P., Pawson, T., and Rapp, U.R. (1990). Mol. Cell. Biol. 10, 2503-2512.

Howe, L.R., Leevers, S.J., Gomez, N., Nakielny, S., Cohen, P., and Marshall, C.J. (1992). Cell 71, 335-342.

Hu, C-D., Kariya, Ki., Tamada, M., Akasaka, K., Shirouzu, M., Yokoyama, S., and Kataoka, T. (1995). J. Biol. Chem. 270, 30274-30277.

Hu, C-D., Kariya, K-i., Kotani, G., Shirouzu, M., Yokoyama, S., and Kataoka, T. (1997). J. Biol. Chem. 272, 11702-11706.

Ingebritsen, T.S., Parker, R.A., and Gibson, D.M. (1981). J. Biol. Chem. 256, 1138-1144.

Inouye, K., Mizutani, S., Koide, H., and Kaziro, Y. (2000). J. Biol. Chem. 275, 3737-3740.

Jaitner, B.K., Becker, J., Linnemann, T., Herrmann, C., Wittinghofer, A., and Block, C. (1997). J. Biol. Chem. 272, 29927-29933.

Jelinek, T., Dent, P., Sturgill, T.W., and Weber, M.J. (1996). Mol. Cell. Biol. 16, 1027-1034.

Jones, S.W. Erikson, E., Blenis, J., Maller, J.L., and Erikson, R.L. (1988). Proc. Nat. Acad. Sci. U.S.A 85, 3377-3381 
Joneson, T., White, M.A., Wigler, M.H., and Bar-Sagi, D. (1996). Science 271, 810-812.

Kawata, M., Matsui, Y., Kondo, J., Hishida, T., Teranishi, Y., and Takai Y. (1988). J. Biol. Chem. 263, 18965-18971.

King, A.J., Sun, H., Diaz, B., Barnard, D., Miao, W., Bagrodia, S., and Marshall, M.S. (1998). Nature 396, 180-183.

Kitayama, H., Sugimoto, Y., Matsuzaki, T., Ikawa, Y., and Noda, M. (1989). Cell 56, 77-84.

Kolch, W., Heidecker, G., Lloyd P., and Rapp, U.R. (1991). Nature 349, 426-428.

Kolch, W., Heldecker, G., Kochs, G., Hummel, R., Vahidl, H., Mischak, H., Finkenzeller, G., Marme, D., and Rapp, U.R. (1993). Nature 364, 249-252.

Kovacina, K.S., Yonezawa, K., Brautigan, D.L., Tonks, N.K., Rapp, U.R., and Roth, R. (1990). J. Biol Chem. 265,12115-12118.

Krebs, E.G. (1972). Curr. Topics Cell Reg. 5, 99-133.

Kyriakis, J., and Avruch, J. (2001). Physiol. Rev, in press.

Kyriakis, J.M., App, H., Zhang, X-F., Banerjee, P., Brautigan, D.L., Rapp, U.R., and Avruch, J. (1992). Nature 358, 417-421.

Kyriakis, J.M., Force, T.L., Rapp, U.R., Bonventre, J.V., and Avruch, J. (1993). J. Biol. Chem. 268, 16909-16919.

Larner, J. (1988). Diabetes 37, 262-275.

Leevers, S.J., Paterson, H.F., and Marshall, C.J. (1994). Nature 369, 411 -414.

Li, Q., Hariharan, I.K., Chen, F., Huang, Y., and Fischer, J.A. (1997). Proc. Natl. Acad. Sci. U.S.A. 94, 12515-12520.

Li, W., Han, M., and Guan, K-L. (2000). Genes Devel. 14, 895-900.

Liu, D., Bienkowska, J., Petosa, C., Collier, R.J,, Fu, H., and Liddington, R. (1995). Nature 376, 191-194.

Luo, Z., Zhang, X-f., Rapp, U., and Avruch, J. (1995). J. Biol. Chem. 270, 23681-2387.

Luo, Z., Tzivion, G., Belshaw, P.J., Vavvas, D., Marshall, M., and Avruch, J. (1996). Nature 383, 181-185.

Luo, Z., Diaz, B., Marshall, M.S., and Avruch, J. (1997). Mol. Cell. Biol. 17, 46-53.

Maller, J.L., Pike, L.J., Freidenberg, G.R., Cordera, R.. Stith, B.J., Olefsky, J.M., and Krebs, E.G. (1986). Nature 320, 459-461.

Marais, R., Light, Y., Paterson, H.F., and Marshall, C.J. (1995). EMBO J. 14, 3136-3145.

Marshall, M.S. (1993). Trends Biochem. Sci. 18, 250-254.

Mason, C.S., Springer, C.J., Cooper, R.G., Superti-Furga, G., Marshall, C.J., and Marias, R. (1999). EMBO J. 18, 2137-2148.

McPherson, R.A., Harding, A., Roy, S., Lane, A., and Hancock, J.F. (1999). Oncogene 18, 3862-3869.

Michaud, N.R., Fabian, J.R., Mathes, K.D., and Morrison, D.K. (1995). Mol. Cell. Biol. 15, 3390-3397.

Miller, M.J., Rioux, L., Prendergast,G.V., Cannon, S., White, M.A., and Meinkoth, J.L. (1998). Mol. Cell. Biol. 18, 3718-3726.

Mineo, C., Anderson, R.G.W., and White, M.A. (1997). J. Biol. Chem. 272, 10345-10348.

Mizutani, S., Koide, H., and Kaziro, Y. (1998). Oncogene 16, 2781-2786.

Moelling, K., Heimann, B., Beimling, P., Rapp, U.R., and Sander, T. (1984). Nature 312, 558-561.

Mukhopadhyay, N.K., Price, D.J., Kyriakis, J.M., Pelech, S.J., Sanghera, J., and Avruch, J. (1992). J. Biol. Chem. 267, 3325-3335.

Muslin, A.J., Tanner, J.W., Allen, P.M., and Shaw, A.S. (1996). Cell 84, 889-897.

Nassar N., Horn G., Herrmann, C., Scherer, A., McCormick, F., and Wittinghofer, A. (1995). Nature $375,554-560$.

Nemenoff, R.A., Gunsalas, R., and Avruch, J. (1986). Arch. Biochem. Biophys. 245, 196-203.

Nemenoff, R.A., Price, D.J., Carter, E.A., and Avruch, J. (1988). J. Biol. Chem. 263, 19455-19460.

Nishizuka, Y. (1992). Science 258, 607-614

Novak-Hofer, I, and Thomas, G. (1984). J. Biol. Chem. 259, 5991-6000. 
Ohtsuka, T., Shimizu, K., Yamamori, B., Kuroda, S., and Takai, Y. (1996). J. Biol. Chem. 271, 1258-1261.

Okada, T., Hu, C-D., Jin, T-G., Kariya, K-I., Yamawaki-Kataoka, Y., and Kataoka, T. (1999). Mol. Cell. Biol. 19, 6057-6064.

Pelech, S.L., Olwin, B.B., and Krebs, E.G. (1986). Proc. Natl. Acad. Sci. U.S.A. 83, 5968-5972.

Pizon, V., Chardin, P., Lerosey, I., Olofsson, B., and Tavitian, A. (1988). Oncogene 3, $201-204$.

Pratt, W.B. (1998). Proc. Soc. Exp. Biol. Med. 217, 420-434.

Price, D.J., Nemenoff, R.A., and Avruch, J. (1989). J. Biol. Chem. 264, 13825-13833.

Price, D.J., Gunsalus, J.R., and Avruch, J. (1990). Proc. Natl. Acad. Sci. U.S.A. 87, 7944-7948.

Price, D.J., Grove, J.R., Calvo, V., Avruch, J., and Bierer, B.E. (1992). Science 257, 973-977.

Rapp, U.R., Goldsborough, M.D., Mark, G.E., Bonner, T.I., Groffen, J., Reynolds, F.H. Jr., et al. (1983).

Proc. Natl. Acad. Sci. U.S.A. 80, 4218-4222.

Ray, L.B., and Sturgill, T.W. (1987). Proc. Natl. Acad. Sci. U.S.A. 84, 1502-1506.

Ray, L.B., and Sturgill, T.W. (1988). Proc. Natl. Acad. Sci. U.S.A. 85, 3753-3757.

Rizzo, M.A., Shome, K., Watkins, S.M., and Romero, G. (2000) J. Biol. Chem. 275, 23911-23918.

Robison, G.A., Butcher, R.W., and Sutherland, E.W. (1971). "Cyclic AMP." Academic Press, New York, pp. 1-23.

Rodriquez-Viciana, P., Warne, P.H., Dhand, R., Vanhaesebroeck, B., Gout, I., Fry, M.J., Waterfield, M.D., and Downward, J. (1994). Nature 370, 527-532.

Rodriquez-Viciana, P., Warne, P.H., Vanhaesebroeck, B., M.J., Waterfield, M.D., and Downward, J. (1996). EMBO J. 15, 2442-2451

Rodriquez-Viciana, P., Warne, P.H., Khwaja, A., Marte, B.M., Pappin, D., Das, P., Waterfield, M.D., Ridley, A., and Downward, J. (1997). Cell 89, 457-467.

Rommel, C., Radziwill, G., Lovric, J., Noeldeke, J., Heinicke, T., Jones, D., Aitken, A., and Moelling, K. (1996). Oncogene 12, 609-619.

Roy, S., Lane, A., Yan, J., McPherson, R., and Hancock, J.F. (1997). J. Biol. Chem. 272, 20139-20145.

Roy, S., McPherson. A., Apolloni, A., Yan, J., Lane, A., Clyde-Smith, J., and Hancock, J.F. (1998). Mol. Cell Biol. 18, 3947-3955.

Seger, R., Seger, D., Lozeman, F.J., Ahn, N.G., Graves, L.M., Campbell, J.S., Ericsson, L., Harrylock, M., Jensen, A.M., and Krebs, E.G. (1992). J. Biol. Chem. 267, 25628-15631.

Shields, J.M., Pruitt, K., McFall, A., Shaub, A., and Der, C.J. (2000). Trends Cell Biol. 10, 147-154.

Siegel, J.N., Klausner, R.D., Rapp, U.R., and Samuelson, L.E. (1990). J. Biol. Chem. 265, 18472-18480.

Smith, C.J., Wejkshora, P.J., Warner, J.R., Rubin, C.S., and Rosen, O.M. (1979). Proc. Natl. Acad. Sci. U.S.A. 76, 2725-2729.

Stanton, V.P. Jr., Nichols, D.W., Laudano, A.P., and Cooper, G.M. (1989). Mol. Cell. Biol. 9, 636-647.

Steward, S., Sundaram, M., Zhang, Y, Lee, J., and Guan, K-L. (1999). Mol. Cell. Biol. 19, 5523-5534.

Stokoe, D., and McCormick, F. (1997). EMBO J. 16, 2384-2396.

Stokoe, D., Macdonald, S.G., Cadwallader, K., Symons, M., and Hancock, J.F. (1994). Science 264, 1463-1467.

Sturgill, T.W., Ray, L.B., Erikson, E., and Maller, J.L. (1988). Nature 334, 715-718.

Sun, H., King, A.J., Diaz, H.B., and Marshall, M.S. (2000). Curr. Biol. 10, 281-284.

Tabarini, D., Heinrich, J., and Rosen, O.M. (1985). Proc. Natl. Acad. Sci. U.S.A, 82, 4369-4373.

Therrien, M., Wong, A.M., Kwan, E., and Rubin, G.M. (1999). Proc. Natl. Acad. Sci. U.S.A. 96,1325913263.

Thorson, J.A., Yu, L.W.K., Hsu, A.L., Shih, N., Graves, P.R., Tanner, J.W., Allen, P.M., PiwnicaWorms, H., and Shaw, A.S. (1998). Mol. Cell. Biol. 18, 5229-5238.

Tzivion, G., Luo, Z., and Avruch, J. (1998). Nature 394, 88-92.

van Dijk, M.C.M., Hilkmann, H., and van Blitterswijk, W.J. (1997). Biochem J. 325, 303-307.

Vavvas, D., Li., Y., Avruch, J., and Zhang, X.-F. (1998). J. Biol. Chem. 273, 5439-5442.

Vossler, M.R., Yao, H., York, R.D., Pan, MG., Rim, C.S., and Stork, P.J.S. (1997). Cell 89, 73-82. 
Walsh, D.A., Perkins, J.P., and Krebs, E.G. (1968). J. Biol. Chem. 243, 3763-3765.

Wang, H.-G., Takayama, S., Rapp, U.R., and Reed, J.C. (1996). Proc. Natl. Acad. Sci. U.S.A. 93, 7063-7068.

White, M.A., Nicolette, C., Minden., Polverino, A., Aelst, L.V., Karin, M., and Wigler, M.H. (1995). Cell 80, 533-541.

Williams, J.G., Drugan J.K., Yi, G-S., Clark, G.J., Der, C.J., and Campbell, S.L. (2000). J. Biol. Chem. 275, 22172-22179.

Winkler, D.G., Cutler, R.E. Jr.,Drugan, J.K., Campbell, S., Morrison, D.K., and Cooper, J.A. (1998). J. Biol. Chem.273, 21578-21584.

Witters, L.A. (1981). Biochem. Biophys. Res. Comm. 100, 872-875

Wu, J., Harrison, J.K., Vincent, L.A., Haystead, C., Haystead, T.A.J., Michel, H., Hunt, D.F., Lynch, K.R., and Sturgill, T.W. (1993). Proc. Natl. Acad. Sci. U.S.A. 90, 173-177.

Xia, K., Mukhopadhyay, N.K., Inhorn, R.C., Barber, D.L., Rose, P.E., Lee, R.S., Narsimhan, R.P., D'Andrea, A.D., Griffin, J.D., and Roberts, T.M. (1996). Proc. Natl. Acad Sci. U.S.A. 93, 11681-11686.

Xia, K., Lee, R.S., Narshimhan, R.P., Mukhopadhyay, N.K., Neel, B.G., and Roberts, 'T.M. (1999). Mol. Cell. Biol. 19, 4819-4824.

Yaffe, M.B., Rittinger, K., Volinia, S., Caron, P.R., Aitken, A., Leffers, H., Gamblin, S.J., Smerdon, S.J., and Cantley, L.C. (1997). Cell 91, 961-971.

Yamamori, B., Kuroda, S., Shimizu, K., Fukui, K., Ohtsuka, T., and Takai, Y. (1995). J. Biol. Chem. 270, $11723-11726$.

Yeung, K., Seitz, T., Li, S., Janosch, P., McFerran, Kaiser, C., Fee, F., Katsanakis, K.D., Rose, D.W., Mischak, H., Sedivy, J.M., and Kolch, W. (1999). Nature 401, 173-177.

Zagotta, W.N, and Siegelbaum, S.A. (1996). Annu. Rev. Neurosci. 19, 235-263.

Zhang, X.F., Settleman, J., Kyriakis, J.M., Takeuchi-Suzuki, E., Elledge, S.J., Marshall, M.S., Bruder, J.T., Rapp, U.R., and Avruch, J. (1993). Nature 364, 308-313.

Zwartkruis, F.J.T., Wolthuis, R.M.F., Nabben, N.M.J.M., Franke, B., and Bos, J.L. (1998). EMBO J. $17,5905-5912$.

Zwartkruis, F.J.T., and Bos, J.L. (1999). Exp. Cell Res. 253, 157-165. 



\title{
The Role of Protein Phosphatase-1 in Insulin Action
}

\author{
Matthew J. Brady and Alan R. Saltiel \\ Department of Cell Biology, Parke-Davis Pharmaceutical Research Division, \\ 2800 Plymouth Road, Ann Arbor, Michigan 48105
}

\begin{abstract}
Insulin is the most-potent physiological anabolic agent known, promoting the synthesis and storage of carbohydrates and lipids and inhibiting their degradation and release into the circulation. This action of the hormone is due in part to the acute regulation of metabolic enzymes through changes in their phosphorylation state. In fat, liver, and muscle, insulin stimulates the dephosphorylation of a number of enzymes involved in glycogen and lipid metabolism via activation of protein phosphatases. Numerous studies have indicated that protein phosphatase-1 (PP1) is the primary phosphatase involved in insulin action. Although PP1 is a cytosolic protein, the phosphatase is compartmentalized in cells by discrete targeting subunits. These proteins confer substrate specificity to PP1 and mediate the specific regulation of intracellular pools of PP1 by a variety of extracellular signals. Four proteins have been described that target the phosphatase to the glycogen particle. $G_{M}$ and $G_{L}$ are expressed exclusively in striated muscle and liver, while protein targeting to glycogen (PTG) and R6 are more widely expressed. Despite a common targeting function, these four proteins are not highly conserved, suggesting profound differences in the mechanisms by which they contribute to the hormonal regulation of PPI activity. Overexpression studies in cell lines or animals have revealed major differences among these proteins regarding basal glycogen levels and hormonal responsiveness. Furthermore, alterations in the expression or function of PP1 glycogen-targeting subunits may contribute to the onset of insulin resistance and type 2 diabetes.
\end{abstract}

\section{Introduction}

Insulin is the principal anabolic hormone that regulates glucose and lipid metabolism. However, the precise molecular mechanisms by which this important factor exerts its effects on target cells are still unclear. Although insulin stimulates protein phosphorylation by the activation of several well-characterized kinase cascades, many of the metabolic effects of this hormone are mediated through protein dephosphorylation. Most of these dephosphorylation reactions are thought to be catalyzed by protein phosphatase-1 (PP1), which has been implicated in the regulation of several rate-limiting enzymes in both glucose and lipid metabolism.

The activation of PP1 by insulin is complex and paradoxical. A number of pharmacological studies have revealed that the insulin-dependent dephosphorylation of metabolic enzymes such as glycogen synthase, hormone-sensitive lipase, 
and acetyl CoA-carboxylase is catalyzed by PP1 (Haystead et al., 1989; Hess et al., 1991). However, PP1 is involved in the regulation of a wide variety of cellular processes that are not influenced by insulin, such as cell division, vesicle fusion, and ion channel function (Berndt, 1999; Kwon et al., 1997; Peters et al., 1999; Westphal et al., 1999). Moreover, in a single cell type, insulin can stimulate the phosphorylation of some proteins, while simultaneously reducing the phosphorylation of others (Saltiel, 1996). To further complicate matters, insulin produces the dephosphorylation of only a small subset of proteins at discrete locations, whereas PP1 is ubiquitously expressed and found in virtually all cellular compartments. These findings suggest that mechanisms must exist for the targeted regulation of PP1 in insulin-responsive cells. Furthermore, these mechanisms must selectively permit activation of the enzyme only at these sites.

One clue in understanding the compartmentalized regulation of PPI activity has emerged from the interaction of the protein with "targeting" subunits. A number of studies have shown that PPI is localized to the nucleus, plasma membrane, neuronal dendrites, myosin filaments, and glycogen particles through binding to a growing family of PP1-targeting proteins (Alessi et al., 1992; Allen et al., 1998; Hsieh-Wilson et al., 1999; Hubbard and Cohen, 1993; Schillace and Scott, 1999; Terry-Lorenzo et al, 2000). These interactions allow for the compartmentalized activation of the phosphatase by a variety of extracellular signals, resulting in the specific dephosphorylation of discrete pools of proteins within the cell. This chapter will focus on the role of PPl glycogen-targeting subunits in the regulation of glycogen metabolism by insulin.

\section{Regulation of Glycogen-metabolizing Enzymes}

\section{A. GLYCOGEN SYNTHASE IS REGULATED BY ALLOSTERIC AND COVALENT MODIFICATION}

Glycogen is an important form of energy storage in mammals. As such, its synthesis and degradation are subject to multiple levels of hormonal and allosteric regulation via control of the enzymes glycogen synthase (GS) and phosphorylase. Although glucose and its metabolites can modulate enzymatic activity and localization, GS and phosphorylase are principally regulated by phosphorylation. Glycogen synthase, the rate-limiting enzyme in glycogen synthesis, is phosphorylated on up to nine regulatory serine residues by several kinases, resulting in progressive inactivation (Lawrence and Roach, 1997). These kinases - which include cAMPdependent kinase (PKA), calmodulin-dependent kinases, glycogen synthase kinase-3 (GSK-3), and adenosine monophosphate (AMP) kinase - respond to a variety of catabolic stimuli to reduce glycogen synthesis. In contrast, phosphorylase, which catalyzes glycogen breakdown, is activated by phosphorylation of a single serine by phosphorylase kinase. The opposing activities of GS and phos- 
phorylase arise from a summation of allosteric regulators and covalent modifications that allow glycogen levels to fluctuate in parallel to the energy needs of the cell.

In peripheral tissues, insulin modulates glycogen accumulation through a coordinate increase in glucose transport and regulation of glycogen-metabolizing enzymes (Azpiazu et al., 2000; Brady et al., 1999b) (Figure 1). Insulin increases glycogen synthesis via the activation of GS by promoting the net dephosphorylation of the protein. Insulin both attenuates the activity of kinases such as PKA or GSK-3 (Cross et al., 1995; Welsh and Proud, 1993) and activates serine/threonine phosphatases, particularly PP1 (Brady et al., 1997a; Dent et al., 1990; Ragolia and Begum, 1998; Wang et al., 1995). Additionally, insulin stimulates the PP1mediated dephosphorylation of both phosphorylase and phosphorylase kinase.

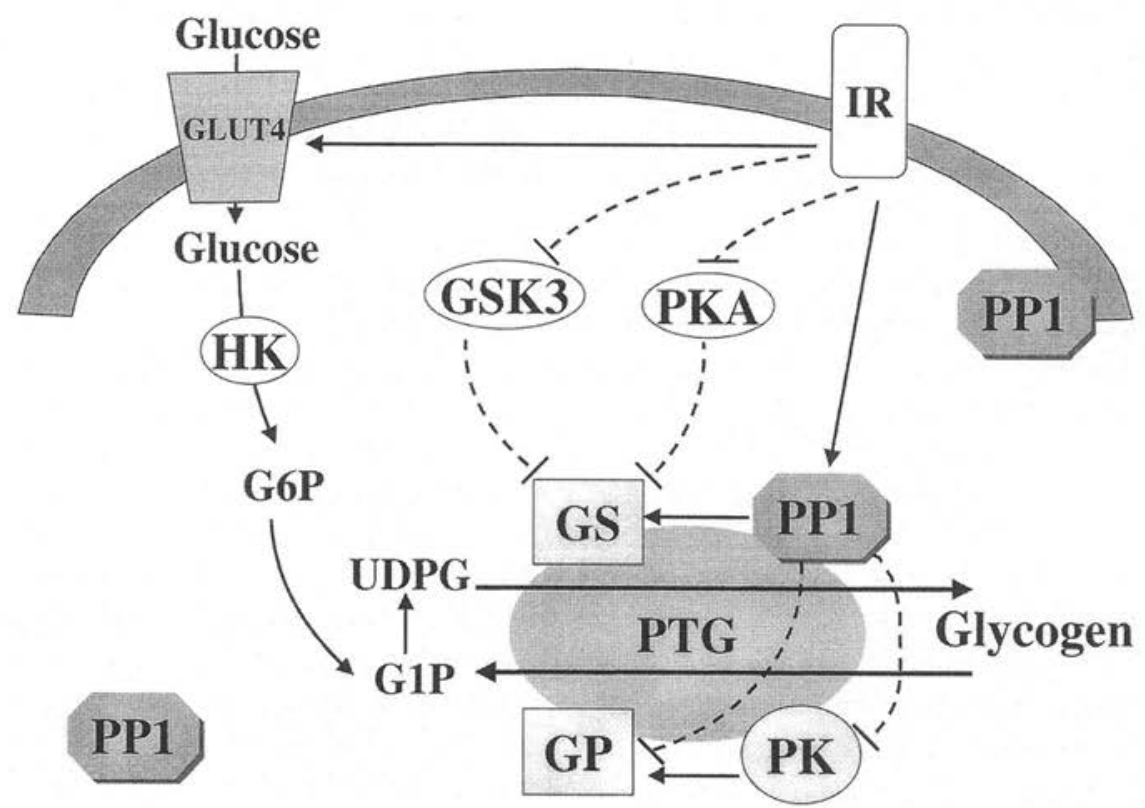

FIG. 1. Insulin regulates glycogen metabolism by promoting protein dephosphorylation. Insulin binds to its receptor and stimulates glycogen synthesis by increasing glucose transport via GLUT4 and modulating enzymatic activities. Insulin induces the dephosphorylation and activation of glycogen synthase (GS) through activation of PP1 and inactivation of upstream kinases (e.g., PKA, GSK-3). Additionally, insulin inhibits glycogenolysis by promoting the PP1-mediated dephosphorylation and inactivation of phosphorylase and phosphorylase kinase. Thus, modulation of glycogen-metabolizing enzymatic activities by insulin requires both phosphatase activation and kinase inactivation. Stimulatory pathways are denoted with solid lines; inhibitory pathways are denoted with dashed lines. Abbreviations: IR = insulin receptor; GSK3 = glycogen synthase kinase-3; PKA = cAMP-dependent kinase; $\mathrm{PP1}=$ protein phosphatase $-1 ; \mathrm{GS}=$ glycogen synthase $; \mathrm{GP}=$ glycogen phosphorylase; $\mathrm{PK}=$ phosphorylase kinase; HK = hexokinase; G6P = glucose-6-phosphate; G1P = glucose-1-phosphate; UDPG $=$ UDP-glucose. 
resulting in enzymatic inactivation and a decrease in glycogenolysis (Figure 1). Thus, the insulin-mediated dephosphorylation of GS and phosphorylase involves both phosphatase activation and kinase inactivation. Insulin-stimulated glucose transport also plays a critical role in regulating GS, since removal of extracellular glucose markedly reduces the activation of GS by insulin (Brady et al., 1999a; Lawrence and James, 1984; Lawrence and Larner, 1978). Increased intracellular levels of glucose-6-phosphate (G6P) and uridine diphosphate (UDP)-glucose induce the translocation of cytosolic GS to glycogen-containing fractions in primary hepatocytes and 3T3-L1 adipocytes (Brady et al., 1999a; Fernandez-Novell et al., 1992a,1992b). Additionally, G6P binding to GS allosterically activates glycogen synthase and increases its susceptibility to dephosphorylation (Lawrence and Roach, 1997; Villar-Palasi and Guinovart, 1997). Therefore, insulin modulates GS activity through covalent modification, translocation, and allosteric regulation.

\section{B. STIMULATION OF GLYCOGEN SYNTHASE ACTIVITY BY INSULIN INVOLVES KINASE INHIBITION AND PHOSPHATASE ACTIVATION}

The molecular mechanisms by which insulin regulates glycogen metabolism are complex and may vary among cell types. Recently, a kinase cascade has been described that may mediate the activation of GS through inhibition of GSK-3 (Cohen et al., 1997). Stimulation of phosphatidylinositol 3-kinase (PI 3-K) by extracellular signals results in an increase in membrane phosphatidyl inositol $(3,4,5)$-trisphosphate $\left(\mathrm{PIP}_{3}\right)$ levels and the sequential translocation, phosphorylation, and activation of Akt-kinase by another membrane-bound, PIP $_{3}$-stimulated kinase, PDK. Akt subsequently phosphorylates and inactivates GSK-3 kinase, decreasing the rate of phosphorylation of GS, resulting in enzymatic activation. Insulin inhibits GSK-3 activity in a variety of cell types (Cross et al., 1995,1997; Welsh and Proud, 1993), concomitant with dephosphorylation of GS. Overexpression of wild-type and mutant GSK-3 constructs diminished basal and insulinstimulated GS activity (Eldar-Finkelman et al., 1996; Summers et al., 1999). Use of pharmacological agents has suggested a role for GSK-3 inactivation in insulin metabolic signaling, since the PI 3-K inhibitor wortmannin blocks both GSK-3 inactivation and GS activation (Hurel et al., 1996; Moule et al., 1995; Shepherd et al., 1995). Additionally, the GSK-3 inhibitor lithium stimulates GS activity in cell lines and primary muscle (Furnsinn et al., 1997; Orena el al., 2000; Summers et al., 1999).

Despite this evidence, the precise role for GSK-3 inactivation in the regulation of GS activity is unclear. Inhibition of GSK-3 is not sufficient to mediate GS activation, since GSK-3 does not phosphorylate several of the residues on GS that are dephosphorylated by insulin (Lawrence and Roach, 1997; Skurat et al., 1994). Furthermore, wortmannin inhibits the activation of PP1 (Brady et al., 1998; De 
Luca et al., 1999) and GLUT4 translocation by insulin (reviewed in Czech and Corvera, 1999), both of which contribute to GS activation. Additionally, GSK-3 inactivation by other factors does not result in GS activation (Brady et al., 1998; Moule et al., 1997). Thus, stimulation of GS activity by insulin requires increased glucose transport, kinase inactivation, and phosphatase activation, although the relative contribution of each signaling component is unclear. Furthermore, insulin promotes the dephosphorylation of phosphorylase (Zhang et al., 1989) and inhibition of glycogenolysis. Conversely, glycogenolytic agents may act, in part, by promoting the dissociation of PP1 from the glycogen particle (Hiraga and Cohen, 1986; Hubbard and Cohen, 1993), thus further enhancing the phosphorylation of GS and phosphorylase. Together, these findings indicate that PP1 plays a central role in the hormonal control of glycogen metabolism.

\section{Structure/Function Analysis of PP1 Glycogen-targeting Subunits}

As mentioned earlier, insulin does not globally activate PP1 but rather specifically targets discrete pools of phosphatase activity. Insulin has been reported to activate a cytosolic pool of PPI (Villa-Moruzzi, 1989) via a mitogen-activated protein kinase (MAPK)-dependent pathway (Wang et al., 1995). However, since inhibition of MAPK had no effect on metabolic signaling by insulin (Lazar et al., 1995), the significance of these results is unclear. Insulin primarily increases PP1 activity localized at the glycogen particle. Thus, glycogen-targeting subunits were initially proposed to mediate the compartmentalized activation of PP1 by insulin (Hubbard and Cohen, 1993). However, recent data indicate that these targeting proteins actually serve as "molecular scaffolds," bringing together the enzyme directly with its substrates in a macromolecular complex. In the process, they exert profound effects on PP1 activity in a substrate-specific manner.

\section{A. PP1 IS LOCALIZED TO GLYCOGEN BY A FAMILY OF TARGETING SUBUNITS}

Four different proteins have been reported to target PP1 to the glycogen particle. $G_{M} / R 3$ and $G_{L} / R 4$ are primarily expressed in striated muscle and liver, respectively, while PTG/R5 and R6 are more ubiquitously distributed (Armstrong et al., 1997; Doherty et al., 1996; Printen et al., 1997). $\mathrm{G}_{\mathrm{M}}$ is a 124-kDa protein that is significantly larger than the other three approximately $35-\mathrm{kDa}$ family members. The nonhomologous portion of $\mathrm{G}_{\mathrm{M}}$ mediates localization at the sarcoplasmic reticulum (Hubbard and Cohen, 1993; Tang et al., 1991). Despite a proposed common function, no two targeting subunits share more than 50 percent sequence homology, which is largely confined to the PP1- and putative glycogenbinding regions (Figure 2A). Additionally, northern blot analysis revealed that three subunits are expressed in both liver and skeletal muscle (Armstrong et al., 
1997; Doherty et al., 1995; Printen et al., 1997; Tang et al., 1991). These observations suggest that each targeting subunit may possess unique regulatory properties in regard to PP1 activity and hormonal responsiveness.

Sequence analysis predicted that PP1 binds to the N-terminal region of the glycogen-targeting subunits through a consensus VXF binding motif that is conserved in other PP1 binding proteins (Zhao and Lee, 1997) (Figure 2B). This supposition was confirmed for $\mathrm{G}_{\mathrm{M}}, \mathrm{G}_{\mathrm{L}}$, and PTG through peptide competition, deletion analysis, and crystallization studies (Armstrong et al., 1998; Egloff $e t$ al., 1997; Fong et al., in press; Johnson et al., 1996). Furthermore, mutagenesis of the invariant $\underline{\mathrm{V}}$ and $\underline{\mathrm{F}}$ residues in PTG completely abrogates PP1 binding (Fong et al., in press). Although the importance of these conserved residues explains the mutually exclusive binding of PP1 to glycogen-targeting subunits, substitution of the single amino acid in the $\underline{X}$ position on $G_{M}$ with the corresponding residue from PTG also disrupted PP1 binding (Liu et al., 2000). This result indicates that the immediate consensus motif is not necessarily interchangeable between targeting proteins. Additionally, a series of point mutations in PP1 differentially affected interaction with the glycogen-targeting subunits in a two-hybrid array (N.M. Fong, M.J. Brady, and A.R. Saltiel, unpublished observation). These results suggest that multiple contact points mediate the interaction of PP1 with each glycogen-targeting subunit.

\section{B. DIFFERENTIATAL MODULATION OF PP1 ACTIVITY BY BINDING TO GLYCOGEN-TARGETING SUBUNITS}

The glycogen-targeting proteins confer substrate specificity to PP1 by localizing the phosphatase to glycogen particles. The conserved region on the targeting subunits that binds to glycogen has been loosely defined (Figure 2A) through deletion analysis, mutagenesis, and sequence comparison (Armstrong et al., 1997; Doherty et al., 1996; Fong et al., in press; Wu et al., 1998). However, these subunits also have differential effects on phosphatase activity against glycogenlocalized substrates. PTG stimulates the PP1-catalyzed dephosphorylation of phosphorylase in vitro (Brady et al., 1997b), while $\mathrm{G}_{\mathrm{M}}$ increases PP1 activity against GS, phosphorylase, and phosphorylase kinase (Hubbard and Cohen, 1989b). In contrast, the binding of PPl to $G_{L}$ inhibits phosphatase activity against phosphorylase, while increasing the dephosphorylation of GS (Doherty et al., 1995). These effects on phosphatase activity can be reversed by interfering with PP1 interaction with PTG or $G_{L}$, either through mutagenesis or addition of a peptide corresponding to the PP1 binding site on $\mathrm{G}_{\mathrm{M}}$ (Fong et al., in press; Johnson et al., 1996). Thus, the unique PP1-binding pockets of the various glycogen-targeting proteins may differentially modulate PP1 activity against the same glycogen-metabolizing enzymes. 
A

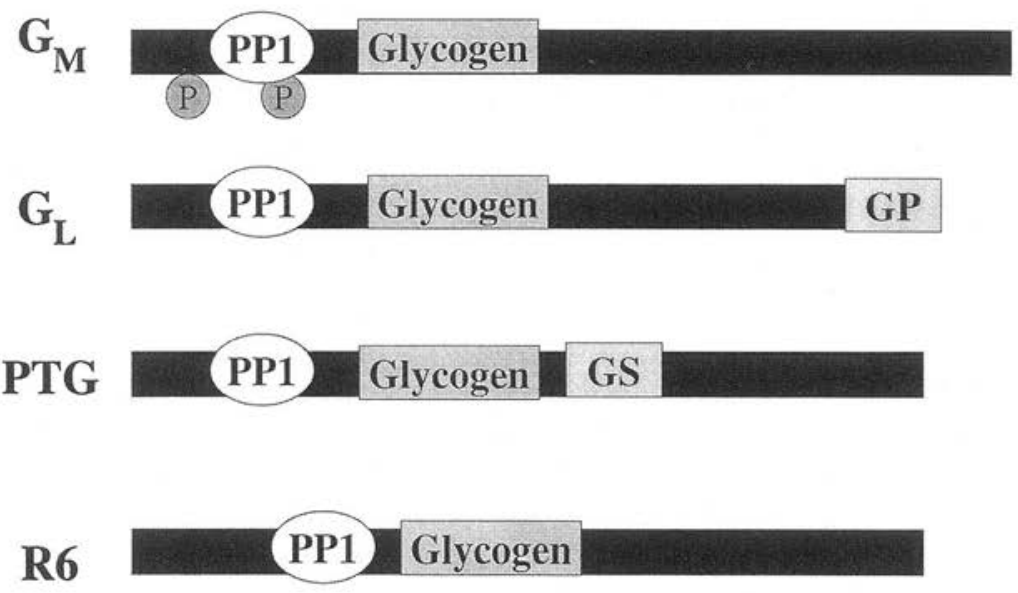

B

\begin{tabular}{cc} 
PP1-GTS & AAA \\
\cline { 1 - 1 } GM & $62-76$ \\
GL & $58-72$ \\
PTG & $58-72$ \\
R6 & $99-113$
\end{tabular}

Sequence

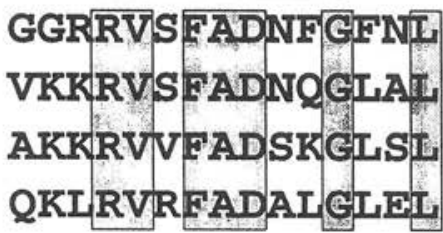

FIG. 2. (A) Schematic diagram of the functional domains of PP1 glycogen-targeting subunits. Abbreviations: GS = glycogen synthase; GP = glycogen phosphorylase. The PP1-, glycogen-, and GS-binding domains of the glycogen-targeted subunits are indicated by ovals and squares. $G_{M}$ is a $124-\mathrm{kDa}$ protein, while the other three targeting subunits are around $35 \mathrm{kDa}$. Only the homologous $\mathrm{N}$-terminal portion of $\mathrm{G}_{\mathrm{M}}$ is shown. The Ser- 48 and Ser- 67 residues on $\mathrm{G}_{\mathrm{M}}$ that may be phosphorylated in response to hormones are indicated by shaded Ps. PP1 binds to the $\mathrm{N}$-terminal region of all four targeting subunits. Glycogen phosphorylase binds to the extreme C-terminus of $\mathrm{G}_{\mathrm{L}}$, while mutation of two residues in the GS-binding region of PTG completely abrogates interaction with the enzyme. (B) Alignment of the PP1-binding domains from glycogen-targeting subunits. The VXF motif in the PP1-binding domains is highly conserved with other PP1-binding proteins. However, substitution of Ser-67 on $\mathrm{G}_{\mathrm{M}}$ with Val-63 from PTG completely abrogated PP1 binding, indicating that other residues must be involved in the binding of PP1 by glycogen-targeting subunits. Abbreviations: GTS = glycogen-targeting subunit; $\mathrm{AA}=$ amino acid residue; invariant residues are shaded gray . 
Glycogen-targeting subunits also form specific complexes with PP1 substrates that regulate glycogen synthesis. PTG directly binds to GS, phosphorylase, and phosphorylase kinase (Printen et al., 1997). Deletion analysis revealed that the glycogen regulatory enzymes bind to a single domain located in the C-terminal region of PTG (Fong et al., in press). Mutagenesis of two acidic residues in this region completely blocked the binding of these proteins to PTG, while having no effect on PPI binding. Conversely, mutagenesis of the two highly conserved residues in the PP1-binding domain of PTG abrogated PP1 interaction without affecting complex formation with the glycogen-metabolizing enzymes (Fong $e t$ al., submitted). Overexpression of wild-type PTG in Chinese hamster ovary cells overexpressing insulin receptor (CHO-IR) cells induces a redistribution of PP1 and GS from the cytosol to glycogen particles and a commensurate marked increase in glycogen synthesis rate (Printen et al., 1997). Interestingly, disruption of either PP1 or GS binding to PTG mutants completely blocked glycogen accumulation (Fong et al., in press). These results demonstrate that targeting PP1 to glycogen alone is not sufficient to increase glycogen storage and implicate PP1 substrate binding as a critical function of PTG.

$\mathrm{G}_{\mathrm{L}}$ does not bind to GS but does bind to phosphorylase with an approximately 1000-fold higher affinity than PTG (Armstrong et al., 1998). The extreme C-terminus of $\mathrm{G}_{\mathrm{L}}$ contains a phosphorylase-binding site that is not conserved in the other glycogen-targeting subunits (Armstrong et al., 1998). PP1 binding to $\mathrm{G}_{\mathrm{L}}$ potently inhibits phosphatase activity against phosphorylase. Furthermore, the $\mathrm{G}_{\mathrm{L}}$-PP1 complex is subject to allosteric inhibition by phosphorylase, which decreases PP1 activity against GS in vitro (Doherty et al., 1995). Removal of the last 17 amino acids from $\mathrm{G}_{\mathrm{L}}$ completely abrogated phosphorylase binding (Armstrong et al., 1998). However, the effects of this deletion on PP1 activity or glycogen metabolism were not reported. Interestingly, recent data suggest that $\mathrm{G}_{\mathrm{L}}$ preferentially regulates glycogenolysis, while PTG primarily mediates insulinstimulated glycogen synthesis (Gasa et al., 2000). Thus, multiple glycogen-targeting subunits may be required for the differential modulation of PP1 substrate specificity through intracellular localization, distinct mechanisms of binding to PPl, and direct interaction with phosphatase substrates.

\section{Tissue-specific Regulation of Glycogen-targeted PP1}

Insulin-stimulated glycogen synthesis depends on increased glucose uptake, which coordinately regulates glycogen-metabolizing enzymes. In skeletal muscle and adipose tissue, insulin-stimulated glucose uptake is mediated by the translocation of the glucose transporter GLUT4 to the cell surface. In contrast, hepatic glucose transport is primarily mediated by GLUT2, which enables the rate of glucose uptake to change in parallel with plasma glucose levels, independently of insulin. PP1-targeting subunits also display tissue-specific expression patterns. In 
skeletal muscle, PPl is primarily localized to glycogen through binding of $\mathrm{G}_{M}$ (Tang et al., 1991), although PTG and R6 are expressed at lower levels (Armstrong et al., 1997; Printen et al., 1997). In adipose tissue, PTG is the principal PP1 glycogen-targeting subunit (Printen et al., 1997). However, in liver, PTG and $\mathrm{G}_{\mathrm{L}}$ are expressed at equivalent levels (O'Doherty et al., 2000), which may facilitate the utilization of hepatic glycogen for both glucose storage and hepatic glucose output. Thus, the tissue-specific hormonal regulation of glycogen metabolism partially arises from differential expression of facilitative glucose transporters in combination with PP1 glycogen-targeting subunits.

\section{A. HORMONAL REGULATION OF \\ GLYCOGEN-TARGETED PP1 IN STRIATED MUSCLE MAY INVOLVE PHOSPHORYLATION OF $\mathrm{G}_{M}$}

In rabbit skeletal muscle, $G_{M}$ was reported to be phosphorylated on Ser-48 and Ser-67 in response to both insulin and adenylyl cyclase activators (Dent $e t$ al., 1990; Hubbard and Cohen, 1993). Phosphorylation of Ser-48 in response to insulin increased PP1 activity against phosphorylase kinase and GS but not phosphorylase (Dent $e t$ al., 1990). This is a further indication that glycogen-targeting subunits may preferentially regulate PP1 activity against specific glycogen-localized substrates. The proposed involvement of the MAPK cascade in the insulinstimulated phosphorylation of $\mathrm{G}_{\mathrm{M}}$ (Dent et al., 1990) has been disproven (Azpiazu et al., 1996; Lazar et al., 1995). The putative insulin-activated kinase that phosphorylates Ser- 48 has not been identified. However, mutagenesis of Ser-48 to alanine has no effect on GS activation by insulin, indicating that other regulatory mechanisms are involved (Liu and Brautigan, 2000). Additionally, conflicting reports exist regarding whether the insulin-stimulated phosphorylation of $G_{M}$ occurs in primary tissue and cell lines (Begum, 1995; Dent et al., 1990; Liu and Brautigan, 2000; Walker et al., 2000).

$\mathrm{G}_{\mathrm{M}}$ is also phosphorylated on Ser-48 and Ser-67 in response to elevation of intracellular cAMP levels (Hubbard and Cohen, 1989a; Walker et al., 2000). Ser-67 is located in the middle of the PP1-binding region. Phosphorylation of this site results in dissociation of the phosphatase from $\mathrm{G}_{\mathrm{M}}$ and glycogen (Hubbard and Cohen, 1989a; Liu et al., 2000) and a corresponding reduction in PP1 activity against glycogen-bound substrates (Hubbard and Cohen, 1989b) (Figure 3). Thus, disruption of PP1 binding to $G_{M}$ would further enhance the stimulation of glycogenolysis induced by cAMP elevation. Substitution of Ser-67 with a threonine residue blocks PKA-mediated phosphorylation as well as the release of PP1 from $\mathrm{G}_{\mathrm{M}}$ (Liu et al., 2000). Thus, while the hormonal regulation of glycogen metabolism in skeletal muscle may involve the phosphorylation of $\mathrm{G}_{\mathrm{M}}$, the putative regulatory phosphorylation sites are not conserved in the other glycogen-targeting 


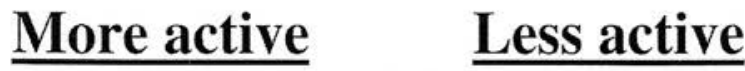
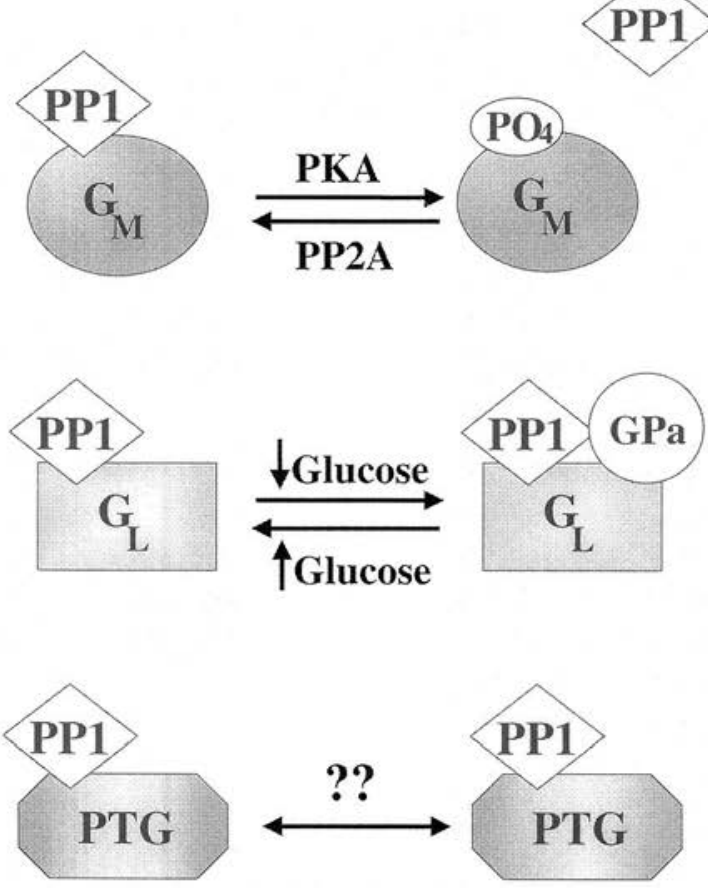

FIG. 3. Regulation of glycogen-targeted PP1 activity. The PKA-mediated phosphorylation of Ser- 67 on $G_{M}$ disrupts PP1 binding to the targeting subunit. The disassociation of PP1 from glycogen further enhances glycogen breakdown by glycogenolytic agents. In liver, reduction of intracellular glucose levels results in binding of activated phosphorylase to $G_{L}$ and allosteric inhibition of PP1. Conversely, increased intracellular glucose levels removes phosphorylase from $G_{L}$, causing PP1 activation. The mechanisms of hormonal regulation of the PP1-PTG complex are unknown. Abbreviations: $\mathrm{PKA}=\mathrm{cAMP}$-dependent protein kinase; $\mathrm{PP} 2 \mathrm{~A}=$ protein phosphatase $2 \mathrm{~A} ; \mathrm{GPa}$, glycogen phosphorylase a.

subunits, indicating that additional mechanisms must be utilized by insulin to activate glycogen-targeted PP1.

\section{B. HORMONAL REGULATION OF HEPATIC GLYCOGEN METABOLISM}

Glycogen metabolism in liver is responsive to changes in both extracellular glucose and hormone levels (Villar-Palasi and Guinovart, 1997). Since GLUT2 acts as a glucose sensor, hepatic glucose levels change in parallel to plasma 
glucose concentrations. During hyperglycemic conditions, glucose is stored as glycogen, while, conversely, hypoglycemia induces glycogenolysis, which contributes to the elevation of hepatic glucose output. The regulation of the $G_{L}-P P 1$ complex by phosphorylase binding may mediate these effects. During hyperglycemic conditions, glucose binds to phosphorylase, relieving the allosteric inhibition of glycogen-targeted PP1 (Figure 3). Additionally, elevation of intracellular G6P levels increases PP1 activity against GS. Conversely, during hypoglycemic conditions, when hepatic glycogen stores are mobilized to increase hepatic glucose output, phosphorylase allosterically inhibits the $\mathrm{G}_{\mathrm{L}}-\mathrm{PP} 1$ complex, reducing GS activity (Figure 3 ). Insulin and glycogenolytic agents can further regulate these enzymes by modulating kinase activities, thus establishing a delicate balance between hepatic glycogen synthesis and the energy needs of peripheral tissues. However, since $G_{L}$ and PTG are expressed at equal levels in the liver (O'Doherty et al., 2000), multiple, undefined, second messenger cascades most likely are also involved in the hormonal regulation of hepatic glycogen metabolism.

\section{INSULIN ACTIVATES THE PTG-PPI COMPLEX}

The mechanisms of hormonal regulation of PP1 bound to PTG are unclear. Insulin stimulates both PP1 and GS activity in 3T3-L1 adipocytes (Brady et al., 1997a), which contain high levels of PTG (Printen et al., 1997). However, treatment of adipocytes with either insulin or adenylyl cyclase activators does not cause phosphorylation of PTG or any detectable movement of PPI between cellular fractions (Brady et al., 1997b). The potential regulation of the PTG-PP1 complex by DARPP-32 in 3T3-Ll adipocytes has been suggested (Brady et al., 1997a). Following phosphorylation of a single site by PKA, DARPP-32 specifically and potently inhibits PP1 (Hemmings et al., 1984). Increased phosphorylation of DARPP-32 might mediate inhibition of PP1 by cAMP elevation, while disruption of the PTG-PP1-DARPP-32 complex by insulin would allow for the specific activation of glycogen-targeted PP1. However, recent data indicate that PTG and DARPP-32 share overlapping binding sites on PP1, precluding trimeric formation (Zhao and Lee, 1997). Additionally, mouse models lacking PP1 inhibitor proteins displayed no perturbations in the stimulation of GS by insulin (Scrimgeour et al., 1999). However, novel PP1-inhibitor proteins recently were described that differentially inhibited PP1 bound to myosin- and glycogen-targeting subunits (Eto et al., 1999). The potential interaction of the PTG-PPI complex with other regulatory proteins is currently under investigation. 


\section{Modulation of PP1 Glycogen-targeting Subunit Levels Markedly Alters Glycogen Metabolism in Vivo}

\section{A. CELLULAR OVEREXPRESSION OF GLYCOGEN-TARGETING SUBUNITS}

PTG overexpression in CHO-IR cells results in a dramatic increase in glycogen accumulation. Although there was no change in cellular GS or PP1 protein levels, PTG overexpression resulted in a redistribution of both enzymes to glycogen particles and a corresponding marked increase in GS activity and glycogen synthesis (Printen et al., 1997). Similarly, basal and insulin-stimulated GS activity was elevated in muscle cell lines overexpressing $G_{M}$ (Liu and Brautigan, 2000; Ragolia and Begum, 1997). These results indicate a critical role for PPl glycogen-targeting subunits in the control of key regulatory enzymes involved in glycogen metabolism.

Recently, Newgard and colleagues have expanded these initial findings through use of adenoviral-mediated gene transfer into primary cell cultures and animals. Transfection of primary hepatocytes with PTG resulted in a dramatic increase in basal cellular glycogen levels, even when the extracellular medium was supplemented only with amino acids (Berman et al., 1998). Furthermore, glycogen stores in the cultures infected with PTG were refractory to breakdown by agents that raise intracellular cAMP levels. These results suggested that PTG overexpression locks the cell into a glycogenic mode by pulling carbon chains into glycogen through various metabolic pathways, desensitizing the cell to glycogenolytic stimuli (Berman et al., 1998). Similar findings were reported when rats were inoculated with the PTG adenoviral construct, which results in preferential protein expression in liver (O'Doherty et al., 2000). Hepatic glycogen stores were markedly elevated and did not substantially decrease during fasting conditions. Interestingly, despite increased glucose deposition into glycogen, the transgenic animals displayed no alterations in plasma glucose, insulin, free fatty acid, or triglyceride levels (O'Doherty et al., 2000). Recently, the same group has reported that transfection of primary hepatocytes with $\mathrm{G}_{\mathrm{L}}$ or PTG results in similarly large incrcases in glycogen levels, while $\mathrm{G}_{M}$ overexpression has moremodest effects (Gasa et al., 2000). Cells containing $G_{L}$ were no longer responsive to insulin-stimulated glycogen synthesis, while PTG-overexpressing cells were refractory to glycogenolytic stimuli. These results suggest that PTG may play a greater role in mediating insulin-stimulated glycogen synthesis, while $G_{L}$ may preferentially transduce cAMP elevation into glycogen breakdown. 


\section{B. PP1-GLYCOGEN-TARGETING SUBUNIT KNOCKOUT MOUSE MODELS}

Two transgenic mouse strains have been made that lack either $\mathrm{G}_{\mathrm{M}}$ or PTG. The $G_{M}$ knockout mice exhibited no apparent developmental defects but showed reduced GS activity and enhanced phosphorylase activity. The mice produced a 90 percent reduction in skeletal muscle glycogen levels (Depaoli-Roach et al., 1999). Despite the dramatic disruption of peripheral glucose storage, fasting blood glucose and rate of clearance of an injected glucose challenge were normal. In contrast, PTG knockout mice are not viable, although the cause and precise timing of the embryonic lethality is not yet known (M.J. Brady, S.M. Crosson, P.A. Hansen, J.E. Pessin, and A.R. Saltiel, unpublished observation). Due to the redundancy of expression, disruption of an individual PP1 glycogen-targeting subunit may not be sufficient to produce fasting hyperglycemia. Interbreeding of the glycogen-targeting knockout lines and crossbreeding with other diabetic rodent models may be needed before perturbations in glucose homeostasis are realized.

\section{POTENTIAL ROLE FOR ALTERATIONS IN PPI GLYCOGEN-TARGETING SUBUNIT FUNCTION IN TYPE 2 DIABETES}

Insulin-stimulated glucose transport and storage is inhibited in all experimental models of non-insulin dependent or type 2 diabetes. Additionally, reduced glucose clearance by peripheral tissues is one of the first defects detected in insulin-resistant patients, which may be the primary lesion leading to the development of diabetes. Shulman and colleagues (Cline et al., 1999) recently have pinpointed reduced glucose transport as the primary defect causing insulin resistance. However, the activation of GS and PP1 by insulin is also diminished (Freymond et al., 1988). Overexpression of PP1 glycogen-targeting subunits has been reported to markedly alter glycogen synthesis rates without affecting protein levels of key regulatory enzymes (Berman et al., 1998; Gasa et al., 2000; Printen et al., 1997; Ragolia and Begum, 1997). Thus, defects in the expression or function of PP1 glycogen-targeting subunits might impact on the metabolic actions of insulin in vivo, resulting in insulin resistance. However, a detailed, comprehensive characterization of PP1 glycogen-targeting subunit expression in normal, obese, and diabetic patients has not been reported.

Genetic linkage analyses have suggested possible alterations in $\mathrm{G}_{\mathrm{M}}$ function and/or expression in the onset of insulin resistance and type 2 diabetes. A widespread amino acid mutation in $G_{M}$ was associated with altered rates of glucose metabolism in Danish subjects in vivo (Hansen et al., 1995) and increased sensitivity to glycogenolytic agents in vitro (Ragolia et al., 1999). However, despite the widespread penetrance of this allele in the Japanese population, no linkage could be established between the mutation and insulin resistance (Shen et al., 
1998). A second mutation in the $3^{\prime}$ noncoding region of $G_{M}$ has been reported to result in mRNA instability (Xia et al., 1998,1999) and reduced $G_{M}$ protein levels and may be linked to development of insulin resistance in Native American populations (Hegele et al., 1998; Xia et al., 1998). Clearly, more analysis will be required to address the potential link between aberrant PP1-glycogen-targeting subunit function and the development of insulin resistance and type 2 diabetes.

\section{Summary}

Significant progress has been made in identifying many of the signaling components involved in insulin action and the development of insulin resistance (Cline et al., 1999; Virkamaki et al., 1999). However, relatively little is known concerning the precise molecular mechanisms by which insulin regulates glycogen metabolism. Insulin modulates the activities of the two rate-limiting enzymes of glycogen synthesis - GS and phosphorylase - through allosteric activation, intracellular translocation, and covalent modification. Increased glucose uptake and metabolism mediate the allosteric regulation and localization of GS and phosphorylase. Insulin also promotes the dephosphorylation of both enzymes, resulting in activation of GS and inactivation of phosphorylase. Although insulin inhibits upstream kinases for both enzymes, the activation of PP1 plays a crucial role in the control of glycogen levels. PP1 is present in nearly every cellular compartment through the action of targeting subunits. However, insulin promotes the dephosphorylation of a limited number of proteins that are mainly involved in glycogen metabolism. These results suggest that mechanisms must exist whereby PP1 is targeted to the glycogen particle and insulin can specifically activate this pool of phosphatase, while not affecting PP1 activity in other cellular compartments.

Recently, four proteins have been described that bind to PP1 and glycogen, thus targeting the phosphatase to the glycogen particle. These proteins are not highly homologous but display overlapping tissue expression, suggesting distinct functions. The mechanisms by which insulin activates PP1 activity bound to each targeting subunit are still poorly understood. The continued construction of chimeric targeting subunits, through point mutagenesis and swapping of putative domains, will aid in the elucidation of tissue-specific mechanisms for the regulation of glycogen synthesis. Additionally, these studies should help to elucidate why so many relatively nonconserved proteins are required to target PP1 to the glycogen particle. Finally, comparison of this family of glycogen-targeting proteins to other PP1-targeting subunits will establish the importance of intracellular localization in the specific regulation of protein dephosphorylation by a variety of hormones and extracellular signals in different cell types. 


\section{REFERENCES}

Alessi, D., MacDougall, L.K., Sola, M.M., Ikebe, M., and Cohen, P. (1992). Eur. J. Biochem. 210, 1023-1035

Allen, P.B., Kwon, Y.G., Nairn, A.C., and Greengard, P. (1998). J. Biol. Chem. 273, 4089-4095.

Armstrong, C.G., Browne, G.J., Cohen, P., and Cohen, P.T. (1997). FEBS Lett. 418, $210-214$.

Armstrong, C.G., Doherty, M.J., and Cohen, P.'T. (1998). Biochem. J. 336, 699-704.

Azpiazu, I., Saltiel, A.R., DePaoli-Roach, A.A., and Lawrence, J.C. (1996). J. Biol. Chem. 271, 5033-5039

Azpiazu, I., Manchester, J., Skurat, A.V., Roach, P.J., and Lawrence, J.C., Jr. (2000). Am. J. Physiol. Endocrinol. Metab. 278, E234-E243

Begum, N. (1995). J. Biol. Chem. 270, 709-714.

Berman, H.K., O'Doherty, R.M., Anderson, P., and Newgard, C.B. (1998). J. Biol. Chem. 273, 26421-26425.

Berndt, N. (1999). Front. Biosci. 4, D22-D42.

Brady, M.J., Nairn, A.C., and Saltiel, A.R. (1997a). J. Biol. Chem. 272, 29698-29703.

Brady, M.J., Printen, J.A., Mastick, C.C., and Saltiel, A.R. (1997b). J. Biol. Chem. 272, 20198-20204.

Brady, M.J., Bourbunais, F.J., and Saltiel, A.R. (1998). J. Biol. Chem. 273, 14063-14066.

Brady, M.J., Kartha, P.M., Aysola, A.A., and Saltiel, A.R. (1999a). J. Biol. Chem. 274, 27497-27504

Brady, M.J., Pessin, J.E., and Saltiel, A.R. (1999b). Trends Endocrinol. Metab. 10, 408-4l3.

Cline, G.W., Petersen, K.F., Krssak, M., Shen, J., Hundal, R.S., Trajanoski, Z., Inzucchi, S., Dresner, A., Rothman, D.L., and Shulman, G.I. (1999). N. Engl. J. Med. 341, 240-246.

Cohen, P., Alessi, D.R., and Cross, D.A. (1997). FEBS Lett. 410, 3-10.

Cross, D.A., Alessi, D.R., Cohen, P., Andjelkovich, M., and Hemmings, B.A. (1995). Nature 378, 785-789.

Cross, D.A., Watt, P.W., Shaw, M., van der Kaay, J., Downes, C.P., Holder, J.C., and Cohen, P. (1997). FEBS Lett. 406, 211-215.

Czech, M.P., and Corvera, S. (1999). J. Biol. Chem. 274, 1865-1868.

De Luca, J.P., Garnache, A.K., Rulfs, J., and Miller, T.B., Jr. (1999). Am. J. Physiol. 276, H1520-H1526.

Dent, P., Lavoinne, A., Nakielny, S., Caudwell, F.B., Watt, P., and Cohen, P. (1990). Nature 348, 302-308.

Depaoli-Roach, A.A., Suzuki, Y., Lanner, C., Zhang, H., Yang, J., Scrimgeour, A., and Lawrence, J.C. (1999) Diabetes 48(suppl. 1), A25

Doherty, M.J., Moorhead, G., Morrice, N., Cohen, P., and Cohen, P.T. (1995). FEBS Lett. 375, 294-298.

Doherty, M.J., Young, P.R., and Cohen, P.T. (1996). FEBS Lett. 399, 339-343.

Egloff, M.P., Johnson, D.F., Moorhead, G., Cohen, P.T., Cohen, P., and Barford, D. (1997). EMBO J. 16, 1876-1887.

Eldar-Finkelman, H., Argast, G.M., Foord, O., Fischer, E.H., and Krebs, E.G. (1996). Proc. Natl. Acad. Sci. U.S.A. 93, 10228-10233.

Eto, M., Karginov, A., and Brautigan, D.L. (1999). Biochemistry 38, 16952-16957.

Fernandez-Novell, J.M., Arino, J., Vilaro, S., Bellido, D., and Guinovart, J.J. (1992a). Biochem. J. 288, 497-501.

Fernandez-Novell, J.M., Arino, J., Vilaro, S., and Guinovart, J.J. (1992b). Biochem. J. 281, 443-448.

Fong, N.M., Jensen, T.C., Shah, A.M., Parekh, N., Saltiel, A.R., and Brady, M.J. (2000). J. Biol. Chem. 275, 35034-35039.

Freymond, D., Bogardus, C., Okubo, M., Stone, K., and Mott, D. (1988). J. Clin Invest. 82, 1503-1509.

Furnsinn, C., Noe, C., Herdlicka, R., Roden, M., Nowotny, P., Leighton, B., and Waldhausl, W. (1997). Am. J. Physiol. 273, E514-E520.

Gasa, R., Jensen, P.B., Berman, H.K., Brady, M.J., Depaoli-Roach, A.A., and Newgard, C.B. (2000). J. Biol. Chem. 275, 26396-26403. 
Hansen, L., Hansen, T., Vestergaard, H., Bjorbaek, C., Echwald, S.M., Clausen, J.O., Chen, Y.H., Chen, M.X., Cohen, P.T., and Pedersen, O. (1995). Hum. Mol. Genet. 4, 1313-1320.

Haystead, T.A., Sim, A.T, Carling, D., Honnor, R.C., Tsukitani, Y., Cohen, P., and Hardie, D.G. (1989) Nature 337, 78-81.

Hegele, R.A., Harris, S.B., Zinman, B., Wang, J., Cao, H., Hanley, A.J., Tsui, L.C., and Scherer, S.W. (1998). J. Clin. Endocrinol. Metab. 83, 3980-3983.

Hemmings, H.C., Jr., Greengard, P., Tung, H.Y., and Cohen, P. (1984). Nature 310, 503-505.

Hess, S.L., Suchin, C.R., and Saltiel, A.R. (1991). J. Cell Biochem. 45, 374-380.

Hiraga, A., and Cohen, P. (1986). Eur. J. Biochem. 161, 763-769.

Hsieh-Wilson, L.C., Allen, P.B., Watanabe, T., Nairn, A.C., and Greengard, P. (1999). Biochemistry $38,4365-4373$.

Hubbard, M.J., and Cohen, P. (1989a). Eur. J. Biochem. 186, 701-709.

Hubbard, M.J., and Cohen, P. (1989b). Eur. J. Biochem. 186, $711-716$.

Hubbard, M.J., and Cohen, P. (1993). Trends Biochem. Sci 18, 172-177.

Hurel, S.J., Rochford, J.J., Borthwick, A.C., Wells, A.M., Vandenheede, J.R., Turnbull, D.M., and Yeaman, S.J. (1996). Biochem. J. 320, 871-877.

Johnson, D.F., Moorhead, G., Caudwell, F.B., Cohen, P., Chen, Y.H., Chen, M.X., and Cohen, P.T. (1996). Eur. J. Biochem. 239, 317-325

Kwon, Y.G., Lee, S.Y., Choi, Y., Greengard, P., and Nairn, A.C. (1997). Proc, Natl. Acad. Sci. U.S.A. 94, 2168-2173.

Lawrence, J.C., Jr., and James, C. (1984). J. Biol. Chem. 259, 7975-7982.

Lawrence, J.C., Jr., and Larner, J. (1978). J. Biol. Chem. 253, 2104-2113.

Lawrence, J.C., Jr., and Roach, P.J. (1997). Diabetes 46, 541-547.

Lazar, D.F., Wiese, R.J., Brady, M.J., Mastick, C.C., Waters, S.B., Yamauchi, K., Pessin, J.E., Cuatrecasas, P., and Saltiel, A.R. (1995). J. Biol. Chem. 270, 20801-20807.

Liu, J., and Brautigan, D.L. (2000). J. Biol. Chem. 275, 15940-15947.

Liu, J., Wu, J., Oliver, C., Shenolikar, S., and Brautigan, D.L. (2000). Biochem. J. 346, 77-82.

Moule, S.K., Edgell, N.J., Welsh, G.I., Diggle, T.A., Foulstone, E.J., Heesom, K.J., Proud, C.G., and Denton, R.M. (1995). Biochem. J. 311, 595-601.

Moule, S.K., Welsh, G.I., Edgell, N.J., Foulstone, E.J., Proud, C.G., and Denton, R.M. (1997). J. Biol. Chem. 272, 7713-7719.

O’Doherty, R.M., Jensen, P.B., Anderson, P., Jones, J.G., Berman, H.K., Kearney, D., and Newgard, C.B. (2000). J. Clin. Invest. 105, 479-488.

Orena, S.J., Torchia, A.J., and Garofalo, R.S. (2000). J. Biol. Chem. 275, 15765-15772.

Peters, C., Andrews, P.D., Stark, M.J., Cesaro-Tadic, S., Glatz, A., Podtelejnikov, A., Mann, M., and Mayer, A. (1999). Science 285, 1084-1087.

Printen, J.A., Brady, M.J., and Saltiel, A.R. (1997). Science 275, 1475-1478.

Ragolia, L., and Begum, N. (1997). Endocrinology 138, 2398-2404.

Ragolia, L., and Begum, N. (1998). Mol. Cell Biochem. 182, 49-58.

Ragolia, L., Duddy, N., and Begum, N. (1999). Mol. Endocrinol. 13, 1773-1783.

Saltiel, A.R. (1996). Am. J. Physiol. 270, E375-E385.

Schillace, R.V., and Scott, J.D. (1999). Curr. Biol. 9, 321-324.

Scrimgeour, A.G., Allen, P.B., Fienberg, A.A., Greengard, P., and Lawrence, J.C., Jr. (1999). J. Biol. Chem. 274, 20949-20952.

Shen, G.Q., Ikegami, H., Kawaguchi, Y., Fujisawa, T., Hamada, Y., Ueda, H., Shintani, M., Nojima, K., Kawabata, Y., Yamada, K., Babaya, N., and Ogihara, T. (1998). Diabetes Care 21, 1086-1089.

Shepherd, P.R., Nave, B.T., and Siddle, K. (1995). Biochem. J. 305, 25-28.

Skurat, A.V., Wang, Y., and Roach, P.J. (1994). J. Biol. Chem. 269, 25534-25542. 
Summers, S.A., Kao, A.W., Kohn, A.D., Backus, G.S., Roth, R.A., Pessin, J.E., and Birnbaum, M.J. (1999). J. Biol. Chem. 274, 17934-17940.

Tang, P.M., Bondor, J.A., Swiderek, K.M., and DePaoli-Roach, A.A. (1991). J. Biol. Chem. 266, 15782-15789.

Terry-Lorenzo, R.T., Inoue, M., Connor, J.H., Haystead, T.A., Armbruster, B.N., Gupta, R.P., Oliver, C.J., and Shenolikar, S. (2000). J. Biol. Chem. 275, 2439-2446.

Villa-Moruzzi, E. (1989). FEBS Lett. 258, 208-210.

Villar-Palasi, C., and Guinovart, J.J. (1997). FASEB J. 11, 544-558.

Virkamaki, A., Ueki, K., and Kahn, C.R. (1999). J. Clin. Invest. 103, 931-943.

Walker, K.S., Watt, P.W., and Cohen, P. (2000). FEBS Lett. 466, 121-124.

Wang, Q.M., Guan, K.L., Roach, P.J., and DePaoli-Roach, A.A. (1995). J. Biol. Chem. 270, 1835218358.

Welsh, G.I., and Proud, C.G. (1993). Biochem. J. 294, 625-629.

Westphal, R.S., Tavalin, S.J., Lin, J.W., Alto, N.M., Fraser, I.D., Langeberg, L.K., Sheng, M., and Scott, J.D. (1999). Science 285, 93-96.

Wu, J., Liu, J., Thompson, I., Oliver, C.J., Shenolikar, S., and Brautigan, D.L. (1998). FEBS Lett. 439 , 185-191.

Xia, J., Scherer, S.W., Cohen, P.T., Majer, M., Xi, T., Norman, R.A., Knowler, W.C., Bogardus, C., and Prochazka, M. (1998). Diabetes 47, 1519-1524.

Xia, J., Bogardus, C., and Prochazka, M. (1999). Mol. Genet. Metab. 68, 48-55.

7hang, J.N., Hiken, I, Davis, A. .., and l awrence, I.C., Ir (1989). I. Riol Chem. 264, 17513-17523.

Zhao, S., and Lee, E.Y. (1997). J. Biol. Chem. 272, 28368-28372. 



\title{
Intracellular Organization of Insulin Signaling and GLUT4 Translocation
}

\author{
Robert T. Watson and Jeffrey E. Pessin \\ Department of Physiology \& Biophysics, 51 Newton Road, \\ The University of Iowa, Iowa City, Iowa 52242
}

\begin{abstract}
Glucose is cleared from the bloodstream by a family of facilitative transporters (GLUTs), which catalyze the transport of glucose down its concentration gradient and into cells of target tissues, primarily striated muscle and adipose. Currently, there are five established functional facilitative glucose transporter isoforms (GLUT1-4 and GLUTXI), with GLUT5 being a fructose transporter. GLUT1 is ubiquitously expressed with particularly high levels in human erythrocytes and in the endothelial cells lining the blood vessels of the brain. GLUT3 is expressed primarily in neurons and, together, GLUT1 and GLUT3 allow glucose to cross the blood-brain barrier and enter neurons. GLUT2 is a low-affinity (high $\mathrm{Km}$ ) glucose transporter present in liver, intestine, kidney, and pancreatic $\beta$ cells. This transporter functions as part of the glucose sensor system in $\beta$ cells and in the basolateral transport of intestinal epithelial cells that absorb glucose from the diet. A new facilitative glucose transporter protein, GLUTX1, has been identified and appears to be important in early blastocyst development. The GLUT4 isoform is the major insulin-responsive transporter that is predominantly restricted to striated muscle and adipose tissue. In contrast to the other GLUT isoforms, which are primarily localized to the cell surface membrane, GLUT4 transporter proteins are sequestered into specialized storage vesicles that remain within the cell's interior under basal conditions. As postprandial glucose levels rise, the subsequent increase in circulating insulin activates intracellular signaling cascades that ultimately result in the translocation of the GLUT4 storage compartments to the plasma membrane. Importantly, this process is readily reversible such that when circulating insulin levels decline, GLUT4 transporters are removed from the plasma membrane by endocytosis and are recycled back to their intracellular storagc compartments. Therefore, by establishing an internal membrane compartment as the default localization for the GLUT4 transporters, insulin-responsive tissues are poised to respond rapidly and efficiently to fluctuations in circulating insulin levels. Unfortunately, the complexity of these regulatory processes provides numerous potential targets that may be defective and eventually result in peripheral tissue insulin resistance and possibly diabetes. As such, understanding the molecular details of GLUT4 expression, GLUT4 vesicle compartment biogenesis, GLUT4 sequestration, vesicle trafficking, and fusion with the plasma membrane has become a major focus for many laboratories. This chapter will focus on recently elucidated insulin signal transduction pathways and GLUT4 vesicle trafficking components that are necessary for insulin-stimulated glucose uptake and GLUT4 translocation in adipoctyes.
\end{abstract}




\section{Proximal Insulin Receptor Signaling}

The mature cell surface insulin receptor is composed of two extracellular $\alpha$ and two transmembrane $\beta$ subunits disulfide-linked into an $\alpha_{2} \beta_{2}$ heterotetrameric structure (Czech and Corvera, 1999; Lee and Pilch, 1994). Following insulin binding to the extracellular subunits, a transmembrane conformational change is generated that activates the intracellular subunit tyrosine kinase domain. Subsequently, the $\beta$ subunits undergo a series of intramolecular transautophosphorylation reactions, resulting in tyrosine autophosphorylation at multiple sites. For example, tyrosine phosphorylation at the juxtamembrane Y960 residue is necessary for appropriate substrate recognition, whereas tyrosine phosphorylation at residues $\mathrm{Y} 1146, \mathrm{Y} 1150$, and $\mathrm{Y} 1151$ in the kinase activation domain relieves pseudosubstrate inhibition and results in the constitutive activation of insulin receptor substrate protein tyrosine kinase activity.

However, in contrast to many other receptor tyrosine kinases that directly recruit effector molecules to the phosphorylated receptor, the insulin receptor phosphorylates several proximal intracellular targets that serve as docking sites for effector proteins. These include the four members of the insulin receptor substrate family (i.e., IRSI, 2, 3, and 4), Gabl, Shc, signal-regulated proteins (SIRPs), Cbl, and the newly identified APS (adapter protein with pleckstrin homology and src homology domains) protein. Tyrosine phosphorylation of these proteins creates recognition sites for both src homology ( $\mathrm{SH} 2$ ) binding and phosphotyrosine binding (PTB) domains of several downstream effector proteins. For example, tyrosine phosphorylation of the IRS proteins provides docking sites for $\mathrm{p} 85$, the regulatory subunit of the type 1A phosphatidylinositol (PI) 3-kinase, the protein tyrosine phosphatase SHP2, the Src family member kinase Fyn, and the small adapter proteins Grb2 and Nck.

Although the precise signaling function of each of these adapters/effectors remain poorly defined, each apparently serves distinct yet overlapping biological functions. For example, Grb2 appears to primarily function in the insulin regulation of Ras activation through the appropriate engagement of the Ras guanylnucleotide exchange factor, SOS. This pathway directly leads to extracellular signal-regulated kinase (ERK) activation (via Raf and MEK) and is an important cascade regulating several transcription events and eventually mitogenesis. On the other hand, there is well-documented evidence that Ras can interact with the catalytic subunit of the PI 3-kinase and modulate its activity (Avruch, 1998). The PI 3-kinase has been implicated in numerous biological responses, including the stimulation of transcription, mitogenesis, anti-apoptosis, protein synthesis, glycogen synthesis, and glucose transport (Shepherd et al., 1997). In addition, PI 3-kinase is involved in the regulation of the actin cytoskeleton, which is under the control of Rac and Rho family members. Thus, understanding the interrelationship between all these possible initial signaling events and the in vivo specificity 
required for a defined biological response remains a daunting but critical problem in cell biology and insulin action.

\section{Insulin-stimulated GLUT4 Vesicle Translocation}

One critical aspect of insulin action is the regulation of whole-body glucose homeostasis and peripheral tissue glucose uptake. This primarily results from the translocation of intracellular stored GLUT4 protein to the plasma membrane (Pessin et al., 1999; Rea and James, 1997). In the basal state, GLUT4 cycles slowly between the plasma membrane and one or more intracellular compartments, with the vast majority of the transporter residing within the cell interior. Activation of the insulin receptor triggers a large increase in the rate of GLUT4 vesicle exocytosis and a smaller decrease in the rate of internalization by endocytosis. The insulin-mediated increase in exocytosis is probably the major step for GLUT4 translocation, since a complete inhibition of GLUT4 endocytosis results only in a partial increase in plasma membrane-associated GLUT4 protein without affecting the extent of insulin-stimulated GLUT4 translocation. The overall insulin-dependent shift in the cellular dynamics of GLUT4 vesicle trafficking results in a net increase of GLUT4 protein levels on the cell surface, thereby increasing the rate of glucose uptake.

Substantial data have been accumulated demonstrating that the activation of the type IA PI 3-kinase and subsequent generation of $\mathrm{PI}(3,4,5) \mathrm{P} 3$ are essential for the insulin stimulation of GLUT4 translocation. For example, multiple studies using various pharmacological inhibitors, dominant-interfering mutants, expression of a phosphatidylinositol 5 ' phosphatase, and expression of a constitutively active catalytic subunit all are consistent with a necessary PI 3-kinase activity for insulin-stimulated GLUT4 translocation (reviewed in Shepherd et al., 1998). The formation of $3^{\prime}$ phosphoinositides activates the phosphoinositide-dependent protein kinase (PDK1). This phosphorylates another serine/threonine kinase Akt, also known as protein kinase $\mathrm{B}$ (PKB) or RAC-PK, on threonine 308. In addition, binding of PI-3,4,5-P3 to the amino-terminal pleckstrin homology $(\mathrm{PH})$ domain of Akt releases an inhibitory constraint, making it a more-efficient substrate for PDK1. Full activation of Akt also requires phosphorylation on serine 473 that may result from the activation of another putative kinase, PDK2, or perhaps through an alteration in substrate recognition of PDK 1 following threonine phoshorylation of Akt and/or via autophosphorylation (Balendran et al., 1999; Toker and Newton, 2000).

In any case, several studies have observed a direct correlation between Akt function and insulin-stimulated GLUT4 translocation (reviewed in Czech and Corvera, 1999). For example, stable expression of a constitutively active, membrane-bound form of Akt in 3T3L1 adipocytes results in increased glucose trans- 
port and persistent localization of GLUT4 to the plasma membrane. In addition, co-expression of an epitope-tagged GLUT4 with a dominant-interfering Akt mutant was reported to inhibit insulin-stimulated GLUT4 translocation. However, this issue appears to be substantially more complicated, as protein kinase $C \zeta$ (PKC $\zeta)$ also is activated by insulin through the formation of polyphosphoinositides in a wortmannin-sensitive (PI 3-kinase-dependent) manner. In addition, expression of $\mathrm{PKC} \zeta$ or PKC $\lambda$ (both atypical PKC family members) induces GLUT4 translocation (Kotani et al., 1998; Standaert et al., 1997). Expression of a dominant-interfering Akt mutant was found to inhibit insulin-stimulated protein synthesis without any significant effect on PKC $\lambda$ activation or GLUT4 translocation. Although expression of a dominant-interfering PKC $\lambda$ mutant had no effect on insulin-stimulated Akt activation, GLUT4 translocation was inhibited. It should also be noted that, in all these studies, the dominant-interfering PKC or Akt mutants resulted in only an approximate 50 percent reduction in insulinstimulated GLUT4 translocation. Thus, at present, there is no clear consensus for either Akt, PKC $\zeta$, and/or PKC $\lambda$ as essential downstream targets of PI 3-kinase activation mediating insulin-stimulated GLUT4 translocation (Figure 1).

Despite the absolute requirement for PI 3-kinase activation, it is also clear that insulin must generate additional signals that function in conjunction with the PI 3-kinase to stimulate GLUT4 translocation. For example, activation of PI 3-kinase by platelet-derived growth factor (PDGF), interleukin-4 (IL-4), or through engagement of the integrin receptors does not induce GLUT4 translocation in adipocytes (Guilherme and Czech, 1998; Isakoff et al., 1995; Staubs et al., 1998). In addition, two insulin receptor mutants have been identified that effectively undergo insulin-stimulated activation of the PI 3-kinase but are unable to induce GLUT4 translocation (Krook et al., 1997). These observations are not to be confused with studies in which expression of high levels of a constitutively active PI 3-kinase catalytic subunit alone induces GLUT4 translocation. In this situation, the PI 3-kinase is highly promiscuous, causing massive increases in various polyphosphoinositides, serine/threonine protein phosphorylation, and marked changes in cellular morphology. Therefore, it may activate GLUT4 translocation through a stress response or by circumventing the normal insulin regulatory pathways. In any case, the most-compelling evidence for a required additional PI 3-kinase-independent pathway was the use of a cell-permeable analog of $\mathrm{PI}(3,4,5) \mathrm{P} 3$ (Jiang et al., 1998). In these experiments, addition of the $\mathrm{PI}(3,4,5) \mathrm{P} 3$ analog had no effect on glucose uptake in the absence of insulin. As expected, treatment of cells with wortmannin (a PI 3-kinase inhibitor) prevented insulin-stimulated glucose transport, consistent with a requirement for the activation of the PI 3-kinase. However, treatment of adipocytes with wortmannin, insulin, plus the PI $(3,4,5) \mathrm{P} 3$ analog resulted in enhanced glucose uptake. Together, these data provide compelling evidence that, although the PI 3-kinase 


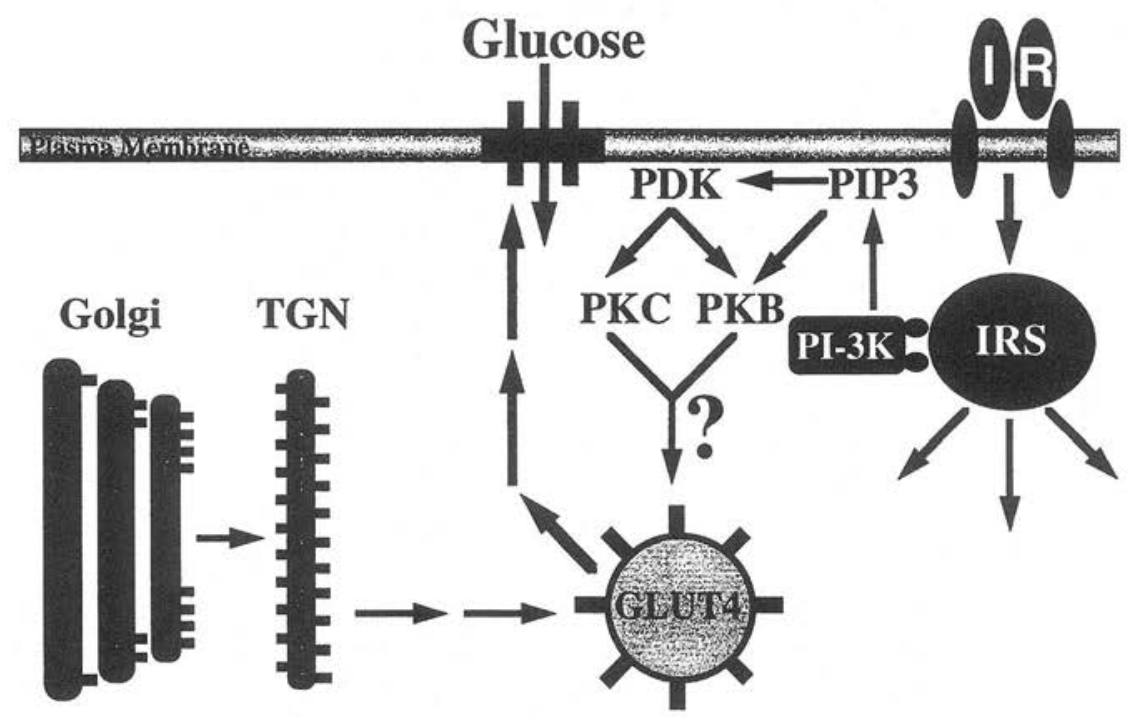

FIG. 1. Schematic model for the role of PI 3-kinase in insulin-stimulated GLUT4 translocation. Following GLUT4 protein synthesis in the endoplasmic reticulum and processing through the Golgi apparatus and the trans-Golgi network (TGN), the GLUT4 protein is localized to both tubulovesicular bodies and small cytosolic vesicles scattered throughout the cytoplasm. Activation of the insulin receptor induces the tyrosine phosphorylation of the IRS family of protein substrates that, in turn, engages the $\mathrm{SH} 2$ domains of the p85 PI 3-kinase regulator subunit. The activation and/or localization of the PI 3-kinase generates the formation of phosphatidylinositol $(3,4,5)$ trisphosphate (PIP3). PIP3 stimulates the kinase activity of phosphoinositide-dependent protein kinase (PDK) and interacts with protein kinase $\mathrm{B}(\mathrm{PKB})$ to make it a more-efficient substrate. In addition, PDK can phosphorylate and activate the atypical protein kinase $\mathrm{C}$ (PKC) family members, zeta and lambda.

pathway is necessary, there is at least one additional insulin receptor-signaling pathway that functions independently of the PI 3-kinase.

What is the nature of this potential second signaling pathway? Several possibilities have been suggested, based upon the study of insulinomimetic agents that can stimulate glucose uptake and GLUT4 translocation without activating the insulin receptor. For example, exercise, hypoxia, and/or contraction in skeletal muscle induce GLUT4 translocation independently of PI 3-kinase activation (Goodyear and Kahn, 1998). It has been suggested that this results from activation of the adenosine monophosphate (AMP)-dependent protein kinase. In adipocytes, introduction of the nonhydrolyzable guanosine triphosphate(GTP) analogue $5^{\prime}-[\gamma$ thio]triphosphate (GTP $\gamma$ S) rapidly induces GLUT4 translocation by a PI 3-kinaseindependent mechanism (Elmendorf et al., 1998). More recently, several studies have suggested that the trimeric GTP-binding protein $(\mathrm{Gq} / \mathrm{G} 11) \alpha$ subunit may be 
required for insulin-stimulated GLUT4 translocation (Imamura et al., 1999; Kanzaki et al., 2000). Although controversial, the Gq/G11 stimulation of GLUT4 translocation was reported to function either downstream or in parallel with the PI 3-kinase. Thus, it is intriguing to speculate that the stimulatory action of GTP $\gamma \mathrm{S}$ may occur through activation of the Gq and/or G11 $\alpha$ subunits. However, since all these events appear to function independently of the insulin receptor, it is unlikely that they can account for the putative second pathway directly initiated by insulin.

A clue to this elusive PI 3-kinase-independent signal has emerged from a comparison of the proximal insulin receptor tyrosine phosphorylated substrates. As previously described, insulin stimulation results in the tyrosine phosphorylation of the IRS proteins, causing association and activation of the PI 3-kinase. Although many receptor and nonreceptor tyrosine kinases can phosphorylate $\mathrm{Cbl}$ in various cell types, only insulin is capable of tyrosine phosphorylating $\mathrm{Cbl}$ in adipocytes (Ribon and Saltiel, 1997). This appears to result from the presence of the $\mathrm{Cbl}$-associated adaptor protein (CAP) in adipocytes, which is required for insulin-stimulated $\mathrm{Cbl}$ tyrosine phosphorylation (Ribon et al., 1998). The CAP protein contains three adjacent carboxyl-terminal SH3 domains, with the last SH3 domain responsible for its association with the proline-rich domain of $\mathrm{Cbl}$. More recently, the amino-terminal domain of CAP was found to specifically interact with the caveolar protein flotillin. The insulin-stimulated, tyrosine-phosphorylated $\mathrm{Cbl}$ protein is localized to the lipid raft subdomains of the plasma membrane (Baumann et al., 2000). Importantly, expression of a dominant-interfering CAP mutant prevented the recruitment of tyrosine-phosphorylated $\mathrm{Cbl}$ to the lipid raft subdomains and inhibited insulin-stimulated glucose uptake and GLUT4 translocation. This occurred without any significant effect on the insulin stimulation of the PI 3-kinase or MAP kinase pathways. Since several studies have suggested that at least a portion of the insulin receptor is localized in caveolae, this may account for the unique ability of the insulin receptor to tyrosine phosphorylate $\mathrm{Cbl}$ in adipocytes via a CAP-dependent recruitment to the lipid raft subdomains (Figure 2). Obviously, additional studies are needed to connect the signaling events that occur downstream of $\mathrm{Cbl}$ and their relationship to the PI 3-kinase signaling pathway.

\section{GLUT4 Storage Compartments}

Irrespective of the specific signal transduction pathways required, they must necessarily impinge upon the intracellular GLUT4 storage compartments to induce their trafficking, docking, and fusion with the plasma membrane. Immunoelectron microscopy and subcellular fractionation studies have localized GLUT4 to several compartments of the recycling pathway, including the trans- 


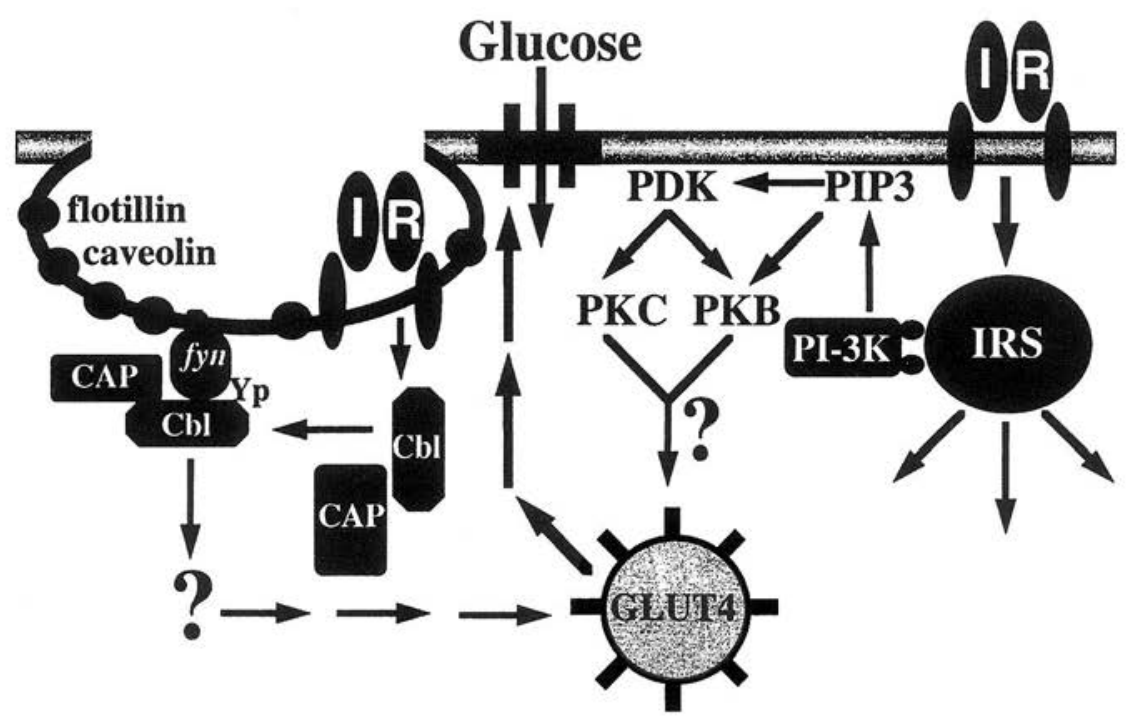

FIG. 2. Schematic model depicting the hypothesized role of the CAP/Cbl complex functioning in concert with the PI 3-kinase pathway in mediating insulin-stimulated GLUT4 translocation. In addition to the activation of the PI 3-kinase pathway, a portion of the cell surface insulin receptor is thought to reside within the flotillin/caveolin-enriched lipid raft domains. The insulin receptor can then tyrosine phoshorylate $\mathrm{Cbl}$ due to its localization (recruitment) to the lipid raft domains through the adaptor protein CAP.

Golgi network (TGN), clathrin-coated vesicles, and endosomes (Rea and James, 1997). However, the majority of GLUT4 appears to reside in tubulovesicular elements in the cytoplasm that lie beneath the plasma membrane and may represent specialized GLUT4 storage compartments. Several lines of evidence support this model. First, GLUT4 is targeted differently from GLUT1, which shows a markedly different steady-state distribution and localizes strongly to the plasma membrane under basal conditions. Second, compartment ablation studies using a horseradish peroxidase-transferrin receptor conjugate have shown that GLUT4 partitions into vesicular compartments that are largely distinct from those occupied by vesicle-associated membrane protein 3 (VAMP3) and transferrin receptor. Third, vesicle immunoabsorption studies have shown that, although some vesicle populations are enriched for both GLUT4 and endosomal markers, other vesicles appear to harbor a preponderance of GLUT4 and seem to exclude general endosomal markers.

These data support a model in which GLUT4 is partitioned into specialized, insulin-responsive storage compartments that represent the primary site of insulin action. One corollary of this model is that the GLUT4 protein must contain 
information that directs its localization to these specific compartments. Several groups have attempted to identify GLUT4 targeting motifs by expression of various mutant and chimeric GLUT4/GLUTI or GLUT4/transferrin receptor reporter constructs. A number of these early studies suggested that either the amino terminal FQQI motif and/or the carboxyl dileucine SLL motif was responsible for appropriate intracellular GLUT4 sequestration. Ilowever, subsequent analysis has demonstrated that these domains appear to function in the endocytosis of GLUT4. Thus, disruption of their function resulted in a default accumulation at the plasma membrane (Corvera et al., 1994; Garippa et al., 1996). Although a defined, intracellular sequestration sequence has not yet been identified, introduction of the GLUT4 carboxyl-terminal domain into adipocytes resulted in the spontaneous translocation of GLUT4 to the cell surface (Lee and Jung, 1997). Similarly, the insulin-responsive aminopeptidase (IRAP) co-localizes with GLUT4 and undergoes an insulin-stimulated plasma membrane translocation (Kandror and Pilch, 1994; Keller and Lienhard, 1994). Expression of the IRAP carboxyl terminus, which shares some sequence similarity with the GLUT4 carboxyl terminus, also caused GLUT4 translocation (Waters et al., 1997). Furthermore, insulin stimulation appears to unmask a cryptic antibody epitope at the carboxyl terminus (Smith et al., 1991; Wang et al., 1996). These data suggest that GLUT4 is sequestered away from the recycling endosome systems through the association of its carboxyl-terminal domain with a retention receptor. Competition with a related peptide releases the GLUT4-containing vesicles that can then enter the recycling endosome system and subsequently traffick to the plasma membrane. However, the identity of such a putative retention receptor has not been established and remains speculative. The lack of progress in this area leaves open the strong possibility that the elusive GLUT4-targeting motif may be scattered over noncontiguous residues that assemble into a discrete localization domain in the folded tertiary protein structure.

\section{Role of SNARE Proteins in GLUT4 Translocation}

Early studies examining intracellular transport processes suggested that regulated interactions between cognate receptor proteins displayed on the cytoplasmic faces of vesicle and target membranes mediate bilayer fusion events. The ability to reconstitute intercisternal Golgi transport in vitro provided the experimental means to identify the protein components of the vesicle fusion machinery. In this manner, two cytosolic proteins - the $\mathrm{N}$-ethylmaleimide (NEM)-sensitive fusion protein (NSF) and the soluble NSF attachment proteins (SNAPs) - were isolated (Calakos and Scheller, 1996). With the soluble components in hand, it became possible to search for their membrane-bound partners, termed SNARES for SNAP receptors. These studies, in combination with the characterization of highly purified synaptic vesicles, led to the identification of the target membrane SNAP 
receptors (t-SNAREs) as members of the syntaxin family and the vesicle membrane SNAP receptors (v-SNAREs) as members of the VAMP family. Subsequently, another target membrane SNARE, termed SNAP25 for synaptosomeassociated protein of $25 \mathrm{kDa}$, was found to form a stable complex with syntaxin. The fundamental importance of $\mathrm{v}$ - and t-SNAREs for synaptic vesicle exocytosis was demonstrated in neurons through the use of the tetanus and botulinum clostridial neurotoxins, which irreversibly block neurotransmitter release by selectively cleaving VAMP2, syntaxin 1, or SNAP-25 (Nieman et al., 1994). Subsequent studies using v- and t-SNARE partners reconstituted into separate liposome populations showed that interactions between cognate SNARE proteins form a bridge that brings vesicle pairs into sufficiently close apposition to cause bilayer mixing (Weber et al., 1998). Indeed, v- and t-SNARE partners may represent the minimal machinery for membrane fusion. Consistent with this hypothesis, structural studies have demonstrated that cognate $\mathrm{v}$ - and t-SNAREs form a parallel, four-helix bundle through coiled-coil domain interactions that may be sufficient to overcome the energetic barrier to bilayer fusion (Hanson et al., 1997; Sutton et al., 1998). In this model, the v-/t-SNARE complex forms first, allowing the subsequent binding of SNAPs, followed by NSF. The energy derived from ATP hydrolysis by NSF may serve to disassemble the very stable SNARE complex, freeing the SNAREs for another round of fusion (Figure 3).

Although in vitro binding studies have observed that $v$ - and t-SNAREs have the potential to interact promiscuously, under in vivo conditions, specific pairing of SNARE partners most likely provides one layer of specificity for membrane fusion events (Calakos et al., 1994; Scales et al., 2000). Consistent with this notion, both $\mathrm{v}$ and $\mathrm{t}$-SNAREs comprise large families of proteins that localize to discrete membrane compartments within the cell. SNAREs may thus help to demarcate membrane compartments with the potential to participate in the fusion process. Because their distribution within the cell may be critical for maintaining membrane compartment identity, several studies have investigated the mechanism by which SNAREs localize to specific membrane compartments. In the case of VAMP2, a specific signal within an $\alpha$-helical domain was found to specify localization to synaptic vesicles (Grote et al., 1995). More recently, two independent signals that cooperate to maintain syntaxin 6 in the TGN were identified: an $\alpha$-helical retention motif that traps syntaxin 6 in the TGN and a retrieval signal that returns wayward syntaxin 6 molecules back to the TGN (Watson and Pessin, 2000 ). These results are consistent with a model whereby the cell actively maintains the specific membrane compartment localization of SNARE proteins. Indeed, the fidelity of bilayer fusion events may be ensured at least in part by sequestering the fusogenic SNARE proteins in spatially segregated compartments. Maintaining compartment identity and fusion specificity is particularly important for GLUT4 and other protcins that navigate through multiple membrane compart- 


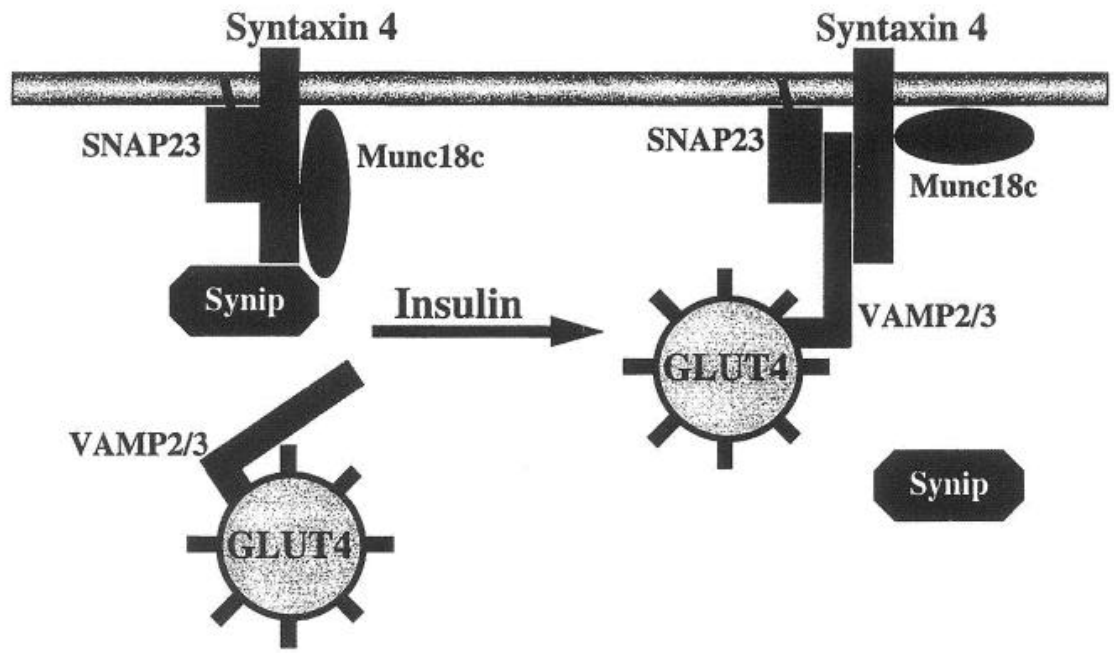

FIG. 3. SNARE model of GLUT4 vesicle docking with the plasma membrane. The intracellular GLUT4 vesicles also contain the v-SNARE proteins VAMP2 and/or VAMP3. The plasma membrane t-SNARE complex is composed of the transmembrane syntaxin 4 protein associated with the peripheral membrane protein, SNAP23. Moreover, the t-SNARE complex interacts with two additional proteins, Munc18c and Synip. Following insulin stimulation, the GLUT4 vesicles traffick to the plasma membrane, where a ternary complex involving VAMP2/3 and syntaxin 4/SNAP23 forms. Complex formation occurs concomitantly with the dissociation of the Synip protein, unmasking the syntaxin 4 coiled-coil domains, and through a conformation change in Munc18c.

ments during their biogenesis, intracellular storage, exocytosis, and retrieval from the plasma membrane. Although SNAREs play key roles during membrane fusion, several additional proteins help regulate the fusion process and may contribute to bilayer fusion specificity (Mayer, 1999; Pfeffer, 1999).

In the case of insulin-stimulated glucose transport in adipocytes, only syntaxin 4 has been implicated in GLUT4 vesicle trafficking. Inhibition of endogenous syntaxin 4 function by a variety of approaches - including overexpression of the cytosolic domain of syntaxin 4 or introduction of inhibitory syntaxin 4 antibodies - prevented insulin-stimulated GLUT4 translocation (reviewed in Pessin et al., 1999). Since the majority of syntaxin 4 is localized to the plasma membrane, GLUT4 vesicles presumably are prevented from docking or fusing with the cell surface when syntaxin 4 binding function is disrupted. Similarly, SNAP23 (a SNAP25 homolog in adipocytes) has been identified as a functional component of the t-SNARE complex and may contribute to GLUT4 vesicle fusion with the plasma membrane (Rea et al., 1998).

Two potential GLUT4 vesicle v-SNAREs, VAMP2 and VAMP3 (also known as cellubrevin), are expressed in adipocytes. Both of these isoforms share several 
characteristics expected of a GLUT4 v-SNARE protein. For example, both proteins partially co-localize in intracellular membranes with GLUT4 and translocate to the plasma membrane in response to insulin. Moreover, these two proteins can form stable complexes with syntaxin 4. Expression of either the VAMP2 or VAMP3 cytosolic domains inhibits GLUT4 translocation. Although these two proteins differ in their cytoplasmic amino-terminal regions, they differ by only one amino acid in their central t-SNARE-binding, coiled-coil domains. Based upon these close structural and physical properties between VAMP2 and VAMP3, a series of elegant cell and molecular approaches have been employed to distinguish the functional differences between these two isoforms. Initially, studies using relatively VAMP-specific proteases suggested that specific cleavage of VAMP2, but not VAMP3, reduced insulin-stimulated GLUT4 translocation (Cheatham et al., 1996). Consistent with a specific role for VAMP2, introduction of a short peptide corresponding to the unique amino-terminal extension of VAMP2 had a small effect on the appearance of GLUT4 at the plasma membrane (Rea et al., 1998). More importantly, in a series of specific compartment ablation studies, VAMP3 was found to be predominantly localized to the recycling endosome compartments defined by the transferrin receptor (Martin et al., 1996,1998; Millar et al., 1999b). These compartments contained approximately 50 percent of the GLUT4 protein but only a small fraction of the VAMP2-containing population. Ablation of the VAMP3/transferrin receptor endosomes had only a small effect on insulin-stimulated GLUT4 translocation but effectively inhibited GLUT4 translocation stimulated by GTPyS. These results support the notion that VAMP2 is the primary v-SNARE for insulin-stimulated translocation of GLUT4 vesicles to the plasma membrane. In contrast, VAMP3 appears to function in a different subpopulation of GLUT4 vesicles that are responsive to alternative signaling pathways.

\section{SNARE-associated Proteins in GLUT4 Translocation}

In addition to NSF, SNAPs, and SNAREs, several other proteins participate in the lipid bilayer fusion process. One critically important syntaxin-binding protein originally was identified in yeast as secl and subsquently as uncl8 in $\mathrm{C}$. elegans, ROP in D. melanogaster, and $\mathrm{nSecl}$ or Munc18a in mammals. The cytosolic $\mathrm{nSecl}$ protein binds with high affinity to syntaxin 1 and, at least in vitro, competes with the syntaxin 1 binding of VAMP2 and SNAP25, thereby preventing the formation of the syntaxin 1-VAMP2-SNAP25 ternary complex (Fujita $e t$ al., 1996; Pevsner et al., 1994). Consistent with a negative regulatory role for $\mathrm{nSec} 1$, increased ROP expression inhibited neurotransmitter release in vivo (Schulze et al., 1994). However, null or temperature-sensitive mutants of Secl homologues in S. cerevisiae, C. elegans, and D. melanogaster blocked secretion 
at specific steps, suggesting a positive role for Secl proteins during membrane fusion (Harrison et al., 1994; Hosono et al., 1992; Novick and Schekman, 1979). Recently, structural analysis of the nSecl-syntaxin 1 complex has been revealed and suggests that the nSecl maintains syntaxin 1 in a closed, inactive, conformational state, thereby preventing promiscuous membrane fusion (Misura et al., 2000 ). It is postulated that, upon activation, $\mathrm{nSec} 1$ could undergo a conformation change that leads to the open syntaxin 1 conformational state, thereby allowing membrane fusion. In this model, the function of $\mathrm{nSecl}$ would be to regulate the transition state between the open and closed syntaxin 1 conformations.

Since the original characterization of $\mathrm{nSec}$ 1/Munc18a as a neuronal-specific isoform, two additional ubiquitously expressed isoforms, termed Munc $18 \mathrm{~b}$ and Munc 18c, have been identified. Since only Munc $18 \mathrm{c}$ binds to syntaxin 4 with high affinity, substantial attention has focused on this isoform for its potential role in GLUT4 vesicle trafficking. We and others have shown that overexpression of Munc $18 \mathrm{c}$ inhibits GLUT4 vesicle translocation, perhaps by binding to syntaxin 4 and preventing its interaction with VAMP2 (Tellam et al., 1997; Thurmond et al., 1998,2000). Consistent with this view, Munc18c blocked the association of VAMP2 with syntaxin 4 in the yeast two-hybrid system and in in vitro binding experiments. However, although overexpression studies have yielded valuable information concerning the possible endogenous function of Munc18c, it is also important to examine Munc18c under physiological conditions, especially given the conflicting data and possible dual roles played by other Secl family members. To address this issue, we have used short peptides corresponding to conserved regions of Munc 18 isoforms to investigate the functional role of the syntaxin 4-Munc18c association in 3T3L1 adipocytes (Thurmond et al., 2000). In these studies, a short peptide corresponding to amino acids 459-483 of Munc18c blocked the ability of GLUT4 vesicles to integrate into the plasma membrane but did not block the movement of GLUT4 vesicles to the cell surface. Instead, GLUT4 vesicles were seen to accumulate beneath the plasma membrane, suggesting that an early stage of the fusion process was blocked. Since the Munc18c peptide disrupts the endogenous Munc18c-syntaxin 4 complex, these results suggest that native Munc18c plays a positive role during the fusion of GLUT4 vesicles with the plasma membrane, perhaps by maintaining syntaxin 4 in an optimal conformation for interactions with VAMP2. According to this model, Munc $18 \mathrm{c}$ binds to syntaxin 4 and inhibits GLUT4 vesicle fusion in the basal state, perhaps by keeping syntaxin 4 in the inactive, closed conformation. Upon insulin stimulation, Munc $18 \mathrm{c}$ may undergo a conformation change such that interactions between syntaxin 4 and VAMP2 are facilitated. When overexpressed, superphysiological doses of Munc18c may shift the equilibrium such that syntaxin 4 is maintained in a closed conformation. This leads to the observed inhibition of insulin-stimulated GLUT4 translocation under these conditions. Although this is an appealing model, there are currently no structural data regarding Munc18c/syn- 
taxin 4 binding or evidence that insulin can modulate these interactions in a timeframe consistent with GLUT4 translocation.

In ongoing efforts to identify additional regulatory components in GLUT4 translocation, we have isolated a novel syntaxin 4-binding protein termed synip (syntaxin 4-interacting protein). Synip was isolated using the yeast two-hybrid system with syntaxin 4 as "bait" and a 3T3L1 adipocyte cDNA expression library as a source for potential "prey" molecules (Min et al., 1999). Synip is predicted to contain several domains, including PDZ and EF hand domains at the amino terminus as well as two tandem coiled-coil domains and a WW motif at the carboxyl terminus. Synip interacts preferentially with syntaxin 4 (probably through the coiled-coil domains) and is expressed at high levels in adipose and skeletal muscle. Overexpression of wild-type synip had no effect on insulin-stimulated GLUT4 vesicle translocation. However, the carboxyl-terminal half of synip (which includes the coiled-coil and WW domains) strongly inhibited the ability of insulin to stimulate GLUT4 translocation when overexpressed. In addition, insulin stimulation resulted in the dissociation of full-length synip from syntaxin 4. These results suggest that the carboxyl-terminal region binds to syntaxin 4 and that the amino-terminal region provides the insulin regulatory sites required for the dissociation of the synip-syntaxin 4 complex. Thus, synip may function as a fusion control switch at the plasma membrane. In the basal state, the binding of synip effectively masks the syntaxin 4 molecule and prevents nonspecific vesicle fusion. In this model, insulin stimulation would result in the release of synip, thereby exposing the syntaxin 4 coiled-coil domains, making them available for interactions with VAMP2 (Figure 3). Obviously, further experimentation is needed to test this model. We currently are investigating the upstream signaling mechanism used by insulin to cause the dissociation of the synip-syntaxin 4 complex.

\section{Role of Small GTP Binding Proteins in GLUT4 Vesicle Translocation}

The large family of small GTP binding proteins play essential roles in many cell biological processes, including receptor tyrosine kinase signal transduction and intracellular vesicle trafficking. Rab proteins comprise the largest branch of the Ras superfamily of small GTPases, with over 50 mammalian isoforms known. In contrast to the SNARE proteins that are central in catalyzing the bilayer fusion event, Rab GTPases appear to function in upstream processes such as the initial tethering or docking of vesicles with their target membranes (Brennwald, 2000). Consistent with this proposed function, Rabs localize to the cytoplasmic faces of all organelles involved in membrane transport. This process of membrane delivery requires the cycling of the Rab proteins between the guanosine diphosphate (GDP)- and GTP-bound states. For example, the delivery of cargo vesicles requires GTP-bound Rab followed by GTP hydrolysis. Subsequently, the GDP- 
bound Rab is extracted from the target membrane by guanylnucleotide dissociation inhibitor (GDI) proteins and recycled back to the donor membrane for another round of vesicle transport.

Although insulin-responsive cells express many Rab isoforms, Rab4 has been specifically implicated in the insulin regulation of GLUT4 exocytosis (Cormont et al., 1993,1996; Mora et al., 1997; Shibata et al., 1996). Initially, Rab4 was found to co-localize with the GLUT4-enriched, low-density microsome fraction of adipocytes. In addition, insulin stimulation resulted in the redistribution of Rab4 from the microsome fraction to the cytosolic fraction, in parallel with the movement of GLUT4 to the plasma membrane. Moreover, introduction of a Rab4 carboxyl-terminal peptide or expression of a carboxyl-terminal Rab4 truncation mutant inhibited insulin-stimulated GLUT4 translocation (Knight et al., 2000; Shibata et al., 1996). Finally, overexpression of wild-type Rab4 inhibited GLUT4 translocation, presumably by preventing correct cycling of the GTPase (Mora et al., 1997). Together, these data implicate Rab4 as a possible mediator of fusion events related to GLUT4 trafficking to the cell surface. Although these data provide suggestive evidence for an important functional role of Rab4, there is no data indicating whether or not insulin modulates Rab4 GDP/GTP cycling or the specific Rab4 effectors required for GLUT4 vesicle translocation.

Another important Ras subfamily of small, GTP binding proteins involved in vesicle trafficking is the ADP-ribosylation factors, ARFs (Chavrier and Goud, 1999). ARFs are thought to function during coat protein recruitment in the early stages of vesicle budding. In the GDP-bound state, ARFs are cytosolic; however, upon GTP loading, they associate with membranes and recruit cytosolic coat components to the donor membrane compartment. The coat proteins then deform the donor membrane into a bud and bind cargo molecules. Given their widespread roles in membrane-trafficking processes, it seems likely that one or more ARF isoforms participate in various stages of GLUT4 vesicle trafficking. ARF6 has been implicated in GLUT4 vesicle trafficking. Introduction of myristoylated peptides corresponding to the amino terminus of ARF6 was reported to inhibit GLUT4 vesicle translocation by about 50 percent in permeabilized adipocytes (Millar et al., 1999a). In contrast, myristoylated peptides from other ARF isoforms did not affect GLUT4 translocation. ARF6 is intriguing because experiments in fibroblasts have suggested that ARF6 links membrane trafficking with the organization of the actin cytoskeleton. Agents that disrupt actin organization also inhibit GLUT4 translocation (Omata et al., 2000; Radhakrishna et al., 1999). However, other studies using a dominant-interfering ARF6 mutant found no significant effect on basal or insulin-stimulated glucose transport (Yang and Mueckler, 1999). Thus, although ARF isoforms are likely to participate in GLUT4 vesicle trafficking, additional work is needed to refine our understanding of the potential role played by ARF6 and perhaps other ARF isoforms. 


\section{GLUT4 Endocytosis}

Although it is well documented that the major effect of insulin is to enhance the rate of GLUT4 exocytosis, insulin also decreases the rate of GLUT4 endocytosis approximately two to three fold (Czech, 1995; Holman and Cushman, 1994; Kandror and Pilch, 1996). Indeed, a complete understanding of the molecular mechanism underlying GLUT4 endocytosis could afford opportunities to develop novel drug therapies aimed at slowing the rate of GLUT4 internalization, thus enhancing glucose uptake under diabetic conditions. Currently, we only have a vague understanding of the molecular mechanism responsible for GLUT4 internalization. Several lines of evidence suggest that GLUT4 endocytosis primarily occurs through clathrin-coated pits. For example, GLUT4 has been localized to clathrin-coated pits by immunofluorescence and electron microscopy studies. Maneuvers that inhibit clathrin-mediated endocytosis prevent GLUT4 internalization (Garippa et al., 1996; Nishimura et al., 1993; Robinson et al., 1992; Slot et al., 1991a,1991b).

The mechanism by which clathrin-coated pits invaginate and form free vesicles is unclear; however, the cytosolic GTPase dynamin appears to play a key role in this process. Dynamin is a $100-\mathrm{kDa}$ protein with an amino-terminal GTPase domain, a central pleckstrin homology domain, and a carboxyl-terminal proline-rich region. Dynamin wraps around the necks of invaginating vesicles as a spiral. This process has been visualized in seminal experiments in Drosophila, where temperature-sensitive mutants of the dynamin homologue shibire yield a paralytic phenotype at the nonpermissive temperature. Electron microscopy analysis of the presynaptic termini of these flies revealed an absence of synaptic vesicles and an abundance of clathrin-coated pits with electron-dense collars around their necks, suggesting that a late stage of endocytosis was blocked (Kosaka and Ikeda, 1983). The shibire temperature-sensitive mutations occur near the GTPase domain and, together with experiments using GTP $\gamma \mathrm{S}$, it was proposed that GTPase activity of dynamin was necessary for the scission process. In this model, the energy derived from GTP hydrolysis induces a conformational change in dynamin such that the necks of invaginating vesicles are constricted to the point where scission occurs (McNiven, 1998). However, this model recently has been challenged. GTP hydrolysis may instead cause the elongation of dynamin spirals, resulting in stretching and eventual scission of the vesicle neck (McNiven et al., 2000). Alternatively, recent work suggests that dynamin may function as a classical GTPase molecular switch that recruits other effector molecules (e.g., endophilin), which then participate in the formation of coated vesicles (Ringstad et al., 1999).

Although the specific details of its molecular mechanism remain controversial, it is clear the dynamin plays an essential role in GLUT4 endocytosis. Multiple studies expressing dominant-interfering dynamin mutants or specific peptides to disrupt dynamin binding to other essential effectors resulted in a near-complete 
inhibition of GLUT4 endocytosis (Al-Hasani et al., 1998; Kao et al., 1998; Volchuk et al., 1998). However, a potential mechanism by which insulin might regulate dynamin function has not been forthcoming. One possibility is that the proline-rich domain of dynamin is well known to associate with various effector SH3 domains that, in turn, enhance the dynamin GTPase activity (Muhlberg et al., 1997). In this manner, Grb2 has been reported to associate with dynamin and induce the binding of the dynamin-Grb2 complex to tyrosine phosphorylated Shc and IRS1 (Ando et al., 1994). These results suggest that the formation of this higher-order complex either inhibits dynamin activity and/or results in the sequestration of dynamin away from the GLUT4-containing, clathrin-coated pits. Alternatively, a recent study reported that insulin induces the tyrosine phosphorylation of dynamin (Baron et al., 1998). In either case, the functional role of these events remains to be determined, as there is currently no evidence that insulin modulates dynamin localization, GTPase activity, or pinchase function in vivo.

\section{Summary and Future Prospects}

Since the discovery of insulin in the 1920 s, it took another 60 years to make the seminal observation that insulin stimulates glucose uptake by inducing the translocation of glucose transporter proteins from intracellular storage sites to the plasma membrane. During the next 20 years, researchers have identified multiple family members of the facilitative glucose transporter and demonstrated that the GLUT4 isoform is the predominant insulin-responsive transporter in striated muscle and adipose tissue. This protein is localized to at least two distinct intracellular compartments, one that is apparently similar to the general recycling endosome system and a second, more-specialized insulin-responsive compartment. The overall translocation process is similar to that utilized during synaptic transmission. It requires the specific pairing of GLUT4 vesicle v-SNAREs with plasma membrane t-SNAREs. Future studies are needed to identify the specific events and regulatory steps involved in the trafficking, docking, and fusion of these intracellular GLUT4 compartments with the plasma membrane.

In addition to the important role of intracellular GLUT4 localization, it is becoming increasingly apparent that insulin signaling is compartmentalized. We now know that insulin activation of PI 3-kinase and generation of phosphatidylinositol-3,4,5-trisphosphate is necessary for insulin-stimulated glucose uptake and GLUT4 translocation. More-recent studies indicate that this pathway is not sufficient and that a second insulin-stimulated pathway functions in concert with the PI 3-kinase. This pathway is also compartmentalized and utilizes the insulinstimulated recruitment of tyrosine-phosphorylated $\mathrm{CAP} / \mathrm{Cbl}$ complex to the flotillin-enriched lipid raft plasma membrane subdomains. Thus, understanding the subsequent downstream events from both the PI 3-kinase and the CAP/Cbl complex are critical issues that require substantial investigation. Moreover, the 
integration of these signals and their specific roles in modulating t-SNARE and v-SNARE interactions will be a fruitful area to pursue. With an improved molecular understanding of these regulatory events, the prospects for the rational development of specifically targeted antidiabetic drugs will become a viable possibility.

\section{REFERENCES}

Al-Hasani, H., Hinck, C.S., and Cushman, S.W. (1998). J. Biol. Chem. 273, 17504-17510.

Ando, A., Yonezawa, K., Gout, I., Nakata, T., Ueda, H., Hara, K., Kitamura, Y., Noda, Y., Takenawa, T., Hirokawa, N. (1994). EMBO J. 13, 3033-3038.

Avruch, J. (1998). Mol. Cell. Biochem. 182, 31-48.

Balendran, A., Casamayor, A., Deak, M., Paterson, A., Gaffney, P., Currie, R., Downes, C.P., and Alessi, D.R. (1999). Curr. Biol. 22, 393-404

Baron, V., Alengrin, F., and Van Obberghen, E. (1998). Endocrinology 139, 3034-3037.

Baumann, C.A., Ribon, V., Kanzaki, K., Thurmond, D.C., Mora, S., Shigematsu, S., Bickel, P.E., Pessin, J.E., and Saltiel, A.R. (2000). Nature 407, 202-207.

Brennwald, P. (2000). J. Cell. Biol. 149, 1-4.

Calakos, N., and Scheller, R.H. (1996). Physiol. Rev. 76, 1-29.

Calakos, N., Bennett, M.K., and Peterson, K.E. (1994). Science 263, 1146-1149.

Chavrier, P., and Goud, B. (1999). Curr. Opin. Cell Biol. 11, 466-475.

Cheatham, B., Volchuk, A., Kahn, C.R., Wang, L., Rhodes, C.J., and Klip, A. (1996). Proc. Natl. Acad. Sci. U.S.A. 93, 15169.15173.

Cormont, M., Tanti, J.F., Zahraoui, A., Vanobberghen, E., Tavitian, A., and Le Marchand-Brustel, Y. (1993). J. Biol. Chem. 268, $19491-19497$.

Cormont, M., Bortoluzzi, M. N., Gautier, N., Mari, M., Van Obberghen, E., and Le Marchand-Brustel, Y. (1996). Mol. Cell. Biol. 16, 6879-6886.

Corvera, S., Chawla, A., Chakrabarti, R., Joly, M., Buxton, J., and Czech, M.P. (1994). J. Cell. Biol. 126, 979-989

Czech, M.P. (1995). Annu. Rev. Nutrit. 15, 441-471.

Czech, M.P., and Corvera, S. (1999). J. Biol. Chem. 274, 1865-1868.

Elmendorf, J.S., Chen, D., and Pessin, J.E. (1998). J. Biol. Chem. 273, 13289-13296.

Fujita, Y., Sasaki, T., Fukui, K., Kotani, H., Kimura, T., Hata, Y., Sudhof, T.C., Scheller, R.H., and Takai, Y. (1996). J. Biol. Chem. 271, 7265-7268.

Garippa, R.J., Johnson, A., Park, J., Petrush, R.L., and McGraw, T.E. (1996). J. Biol. Chem. 271, 20660-20668.

Goodyear, L.J., and Kahn, B.B. (1998). Annu. Rev. Med. 49, 235-261.

Grote, E., Hao, J.C., Bennett, M.K., and Kelly, R.B. (1995). Cell 81, 581-589.

Guilherme, A., and Czech, M.P. (1998). J. Biol. Chem. 273, 33119-33122.

Hanson, P.I., Roth, R., Morisaki, H., Jahn, R, and Heuser, J.E. (1997). Cell 90, 523-535.

Harrison, S.D., Broadie, K., van de Goor, J., and Rubiı, G.M. (1994). Neurun 13, 555-566.

Holman, G.D., and Cushman, S.W. (1994). Bioessays 16, 753-759.

Hosono, R., Hekimi, S., Kamuya, Y., Sassa, T., Murakami, S., Nishiwaki, K., Miwa, J., Taketo, A., and Kodaira, K.I. (1992). J. Neurochem. 58, 1517-1525.

Imamura, T., Vollenweider, P., Egawa, K., Clodi, M., Ishibashi, K., Nakashima, N., Ugi, S., Adams, J.W., Brown, J.H., and Olefsky, J.M. (1999). Mol. Biol. Cell 19, 6765-6774.

Isakoff, S.J., Taha, C., Rose, E., Marcusohn, J., Klip, A., and Skolnik, E.Y. (1995). Proc. Natl. Acad. Sci. U.S.A. 92, 10247-10251. 
Jiang, T., Sweeney, G., Rudolf, M.T., Klip, A., Traynor-Kaplan, A., and Tsien, R.Y. (1998). J. Biol. Chem. 273, 11017-11024.

Kandror, K., and Pilch, P.F. (1994). J. Biol. Chem. 269, 138-142.

Kandror, K.V., and Pilch, P.F. (1996). Am. J. Physiol. 271, E1-E14.

Kanzaki, M., Watson, R.T., Artemyev, N.O., and Pessin, J.E. (2000). J. Biol. Chem. 275, 7167-7175.

Kao, A.W., Ceresa, B.P., Santeler, S.R., and Pessin, J.E. (1998). J. Biol. Chem. 273, 25450-25457.

Keller, S.R., and Lienhard, G.E. (1994). Trends Cell Biol. 4, 115-119.

Knight, J.B., Cao, K.T., Gibson, G.V., and Olson, A.L. (2000). Endocrinology 141, 208-218.

Kosaka, T., and Ikeda, K. (1983). J. Neurobiology 14, 207-225.

Kotani, K., Ogawa, W., Matsumoto, M., Kitamura, T., Sakaue, H., Hino, Y., Miyake, K., Sano, W., Akimoto, K., Ohno, S., and Kasuga, M. (1998). Mol. Cell. Biol. 18, 6971-6982.

Krook, A., Whitehead, J.P., Dobson, S.P., Griffiths, M.R., Ouwens, M., Baker, C., Hayward, A.C., Sen, S.K., Maassen, J.A., Siddle, K., et al. (1997). J. Biol. Chem. 272, 30208-30214.

Lee, J.S., and Pilch, P.F. (1994). Am. J. Physiol. 266, C319-C334.

Lee, W., and Jung, C.Y. (1997). J. Biol. Chem. 272, 21427-21431.

Martin, L.B., Shewan, A., Millar, C.A., Gould, G.W., and James, D.E. (1998). J. Biol. Chem. 273, 1444-1452.

Martin, S., Tellam, J., Livingstone, C., Slot, J.W., Gould, G.W., and James, D.E. (1996). J. Cell. Biol. $134,625-635$.

Mayer, A. (1999). Curr. Opin. Cell Biol. 11, 447-452.

McNiven, M.A. (1998). Cell 94, 151-154

McNiven, M.A., Cao, H., Pitts, K.R., and Yoon, Y. (2000). Trends Biochem. Sci. 25, 115-120.

Millar, C.A., Powell, K.A., Hickson, G.R., Bader, M.F., and Gould, G.W. (1999a). J. Biol. Chem. 274, 17619-17625.

Millar, C.A., Shewan, A., Hickson, G.R., James, D.E., and Gould, G.W. (1999b). Mol. Biol. Cell 10, 3675-3688.

Min, J., Okada, S., Coker, K., Ceresa, B.P., Elmendorf, J.S., Syu, L.-J., Noda, Y., Saltiel, A.R., and Pessin, J.E. (1999). Mol. Cell 3, 751-760.

Misura, K.M., Scheller, R.H., and Weis, W.I. (2000). Nature 404, 355-362.

Mora, S., Monden, I., Zorzano, A., and Keller, K. (1997). Biochem. J. 324, 455-459.

Muhlberg, A.B., Warnock, D.E., and Schmid, S.L. (1997). EMBO J. 16, 6676-6683.

Nieman, H., Blasi, J., and Jahn, R. (1994). Trends. Cell Biol. 4, 179-185.

Nishimura, H., Zarnowski, M.J., and Simpson, I.A. (1993). J. Biol. Chem. 268, 19246-19253.

Novick, P., and Schekman, R. (1979). Proc. Natl. Acad. Sci. U.S.A. 76, 1858-1862.

Omata, W., Shibata, H., Li, L., Takata, K., and Kojima, I. (2000). Biochem. J. 346(pt. 2), 321-328.

Pessin, J., Thurmond, D., Elmendorf, J., Coker, K., and Okada, S. (1999). J. Biol. Chem. 274, 2593-2596.

Pevsner, J., Hsu, S.-C., and Scheller, R.H. (1994). Proc. Natl. Acad. Sci. U.S.A. 91, 1445-1449.

Pfeffer, S.R. (1999). Nature Cell Biol. 1, E17-E22.

Radhakrishna, H., Al-Awar, O., Khachikian, Z., and Donaldson, J.G. (1999). J. Cell. Sci. 112, 855-866.

Rea, S., and James, D. (1997). Diabetes 46, 1667-1677.

Rea, S., Martin, L.B., McIntosh, S., Macaulay, S.L., Ramsdale, T., Baldini, G., and James, D.E. (1998). J. Biol. Chem. 273, 18784-18792.

Ribon, V., and Saltiel, A.R. (1997). Biochem. J. 324, 839.

Ribon, V., Printen, J.A., Hoffman, N.G., Kay, B.K., and Saltiel, A.R. (1998). Mol. Cell. Biol. 18, 872-879.

Ringstad, N., Gad, II., Low, P., Di Paolo, G., Brodin, L., Shupliakov, O., and De Camilli, P. (1999). Neuron 24, 143-154.

Robinson, L.J., Pang, S., Harris, D.S., Heuser, J., and James, D.E. (1992). J. Cell. Biol. 117, 1181-1196.

Scales, S.J., Chen, Y.A., Yoo, B.Y., Patel, S.M., Doung, Y.-C., and Scheller, R.H. (2000). Neuron 26, 457-464. 
Schulze, K.L., Littleton, J.T., Salzberg, A., Halachmi, N., Stern, M., Lev, Z., and Bellen, H.J. (1994). Neuron 13, 1099-1108.

Shepherd, P.R., Siddle, K., and Nave, B.T. (1997). Biochem. Soc. Trans. 25, 978-981.

Shepherd, P.R., Withers, D.J, and Siddle, K. (1998). Biochem. J. 333, 471-490.

Shibata, H., Omata, W., Suzuki, Y., Tanaka, S., and Kojima, I. (1996). J. Biol. Chem. 271, 9704-9709.

Slot, J.W., Geuze, H.J., Gigengack, S., James, D.E., and Lienhard, G.E. (1991a). Proc. Natl. Acad. Sci. U.S.A. 88, 7815-7819.

Slot, J.W., Geuze, H.J., Gigengack, S., Lienhard, G.E., and James, D.E. (1991b). J. Cell. Biot. 113, 123-135.

Smith, R.M., Charron, M.J., Shah, N., Lodish, H.F., and Jarett, L. (1991). Proc. Natl. Acad. Sci. U.S.A. 88, 6893-6897.

Standaert, M.L., Galloway, L., Karnam, P., Bandyopadhyay, G., Moscat, J., and Farese, R.V. (1997). J. Biol. Chem. 272, 30075-30082.

Staubs, P.A., Nelson, J.G., Reichart, D.R., and Olefsky, I.M. (1998). J. Biol. Chem. 273, 25139-25147.

Sutton, R., Fasshauer, D., Jahn, R, and Brunger, A. (1998). Nature 395, 347-353.

Tellam, J.T., Macaulay, S.L., Mcintosh, S., Hewish, D.R., Ward, C.W., and James, D.E. (1997). J. Biol. Chem. 272, 6179-6186.

Thurmond, D.C., Ceresa, B.P., Okada, S., Elmendorf, J.S., Coker, K., and Pessin, J.E. (1998). J. Biol. Chem. 273, 33876-33883.

Thurmond, D.C., Kanzaki, M., Khan, A.H., and Pessin, J.E. (2000). Mol. Cell. Biol. 20, 379-388.

Toker, A, and Newton, A.C. (2000). .J. Biol. Chem. 275, 8271-8274.

Volchuk, A., Narine, S., Foster, L.J., Grabs, D., De Camilli, P., and Klip, A. (1998). J. Biol. Chem. 273, $8169-8176$.

Wang, W., Hansen, P.A., Marshall, B.A., Holloszy, J.O., and Mueckler, M. (1996). J. Cell. Biol. 135, 415-430.

Waters, S. B., D'Auria, M., Martin, S.S., Nguyen, C., Kozma, L.M., and Luskey, K.L. (1997). J. Biol. Chem. 272, 23323-23327.

Watson, R.T., and Pessin, J.E. (2000), J. Biol. Chem. 275, 1261-1268.

Weber, T., Zemelman, B. V., McNew, J.A., Westermann, B., Gmachl, M., Parlati, F., Sollner, T.H., and Rothman, J.E. (1998). Cell 92, 759-772.

Yang, C.Z., and Mueckler, M. (1999). J. Biol. Chem. 274, 25297-25300. 



\title{
Cell-specific Roles of Glucokinase in Glucose Homeostasis
}

\author{
Catherine Postic, Masakazu Shiota, and Mark A. Magnuson \\ Department of Molecular Physiology and Biophysics, \\ Vanderbilt University School of Medicine. Nashville, Tennessee 37232
}

\begin{abstract}
Mutations in the glucokinase (GK) gene cause two different diseases of blood glucose regulation: maturity onset diabetes of the young, type 2 (MODY-2) and persistent hyperinsulinemic hypoglycemia of infancy (PHHI). To gain further understanding of the pathophysiology of these disorders, we have used both transgenic and gene-targeting strategies to explore the relationship between GK gene expression in specific tissues and the blood glucose concentration. These studies, which have included the use of a Cre/loxP gene-targeting strategy to perform both pancreatic $\beta$-cell-and hepatocyte-specific knockouts of GK, clearly demonstrate multiple, cell-specific roles for this hexokinase that, together, contribute to the maintainance of euglycemia. In the pancreatic $\beta$ cell, GK functions as the glucose sensor, determining the threshold for insulin secretion. Mice lacking GK in the pancreatic $\beta$ cell die within 3 days of birth of profound hyperglycemia. In the liver, GK facilitates hepatic glucose uptake during hyperglycemia and is essential for the appropriate regulation of a network of glucose-responsive genes. While mice lacking hepatic GK are viable, and are only mildly hyperglycemic when fasted, they also have impaired insulin secretion in response to hyperglycemia. The mechanisms that enable hepatic GK to affect $\beta$-cell function are not yet understood. Thus, the hyperglycemia that occurs in MODY- 2 is due to impaired GK function in both the liver and pancreatic $\beta$ cell, although the defect in $\beta$-cell function is clearly more dominant. Whether defects in GK gene expression also impair glucose sensing by neurons in the brain or enteroendocrine cells in gut, two other sites known to express GK, remains to be determined. Moreover, whether the pathophysiology of PHHI also involves multitissue dysfunction remains to be explored.
\end{abstract}

\section{Kinetic Behavior and Tissue-specific Functions of Glucokinase}

The first step in glycolysis in mammals is catalyzed by four different hexokinases. Hexokinases I-III are cach about $100 \mathrm{kDa}$ in size, have relatively high affinities for several different hexose substrates, and are inhibited by physiological concentrations of glucose-6-phosphate (G6P) (Wilson, 1995). In contrast, hexokinase IV, more commonly known as glucokinase (GK), is about half the molecular mass, has a $\mathrm{K}_{\mathrm{cat}} \mathrm{S}_{0.5}$ that is similar to the blood glucose concentration, displays sigmoidal kinetics, and is not significantly affected by physiological concentrations of G6P. These unique kinetic features of GK have long been thought to be of key functional significance in the liver and pancreatic $\beta$ cell, two sites where 
the enzyme has been known for years to be expressed (Weinhouse, 1976; Meglasson and Matschinsky, 1983). The predicted functional importance of this enzyme in these cell types has led to intensive investigation, both in humans and in animal model systems, to understand the role of GK in determining the setpoint for the blood glucose concentration. These studies have led to the discovery that mutations in GK cause two different glycemic disorders in humans, clearly demonstrating the pivotal role of this enzyme in maintaining euglycemia.

In the pancreatic $\beta$ cell, GK is a key component of the glucose-sensing machinery (Matschinsky et al., 1998). The phosphorylation of glucose within $\beta$ cells is tightly coupled to insulin secretion. The unique kinetics of GK underlie the ability of these cells to sense and respond to fluctuations in the plasma glucose concentration. Because of this, GK is considered the "glucose sensor" of the $\beta$ cell, a term coined by Matschinsky (Meglasson and Matschinsky, 1984). Modeling of changes in GK activity, as well as studies of the effects of graded increases in the enzyme, have established that glucose phosphorylation is the key point of control for glycolytic flux in the $\beta$ cell (Liang et al., 1992; Wang and Iynedjian, 1997). Consequently, even small changes in GK activity can be physiologically significant, since they directly affect the threshold for glucose-stimulated insulin secretion.

The now-well-established functional principals of how GK contributes to a biochemical sensing mechanism in $\beta$ cells have enabled these cells to be considered a prototype for exploring the mechanisms of glucose sensing in other cell types that exhibit functional changes in response to alterations in the glucose concentration to which they are exposed. For instance, it has long been known that certain cells in both the hypothalamus and gut are also glucose responsive, although the biochemical mechanisms involved remain unknown. In this regard, the identification of GK gene expression in these sites led us to suggest that GK also functions as a glucose sensor in some or all of these cells (Jetton et al., 1994) as it does in the $\beta$ cell. While direct data in support of this notion are only beginning to be generated (Roncero et al., 2000), this remains a plausible hypothesis.

In liver, GK has long been thought to be essential for the unique metabolic functions of this tissue. Indeed, GK was first discovered in the liver over 40 years ago (Grossbard and Schimke, 1966). Studies over the past few years in humans, animal models, and isolated hepatocytes have established that hepatic GK exerts a very strong influence on glucose utilization and glycogen synthesis. Even small changes in the expression of GK in transgenic mice lead to a measurable impact on the blood glucose concentration (Ferre et al., 1996; Hariharan et al., 1997; Niswender et al., 1997b). Moreover, complementary studies in primary hepatocytes have shown that GK overexpression elevates intracellular G6P (Seoane $e t$ 
al., 1996), which triggers an increase in both glycolysis and glycogen synthesis (Aiston et al., 1999).

Metabolic flux in the liver reflects the net activitics of several major pathways. Enzymes in these pathways are regulated largely at the transcriptional level by the metabolism of glucose (Girard, 1997). Genes regulated by glucose - such as L-type pyruvate kinase (L-PK) (Vaulont and Kahn, 1994), $\mathrm{S}_{14}$ (Shih and Towle, 1994), fatty acid synthase (Foufelle et al., 1996), and GLUT2 (Rencurel et al., 1996) - have all been found to contain carbohydrate response elements. While the mechanisms whereby glucose is able to affect transcription of these genes is not very well understood, the essential role that GK plays in enabling glucose to regulate the expression of a network of glucose-responsive genes is evident. Thus, GK is widely thought to play a key role in regulating hepalic glucose metabolism. Indeed, GK gene expression, by determining the regulation of genes involved in fatty acid metabolism, plays an important role in hepatic lipogenesis (Girard, 1997).

\section{Transcriptional and Post-translational Regulation of GK}

Given the vital role of this hexokinase in determining insulin secretion by the cell, glucose uptake by the liver, and in modulating expression of an entire network of hepatic glucose-responsive genes, it is of little surprise that the regulation of GK gene expression is complex. However, two aspects of GK regulation are particularly intriguing and merit discussion. First, the GK gene contains two widely separated and functionally distinct promoters that regulate expression at the transcriptional level. These alternate promoters lead to the production of tissue-specific $\mathrm{GK}$ isoforms that differ in their $\mathrm{N}$-terminal amino acids sequences (Magnuson, 1990). Second, hepatic GK, in addition to being regulated at the level of gene transcription, is regulated at a post-translational level by binding to the GK regulatory protein (GKRP) (Van Schaftingen, 1989).

Cloning and characterization of an $83-\mathrm{kb} \mathrm{P} 1$ clone that contains both promoter regions and all coding sequences of the mouse GK preceded genetic manipulations in mice (Postic et al., 1995). This effort revealed that the 11 exons of the gene span $49 \mathrm{~kb}$, most of which is accounted for by $35 \mathrm{~kb}$ of DNA between exons $1 \beta$ and $1 \mathrm{~L}$. More importantly, the availability of a single, contiguous DNA fragment containing the entire mouse GK gene allowed the generation of transgenic mice with increased gene copy number, as will be described in detail. Sequencing of the mouse GK gene revealed that murine GK is more than 94 percent identical to rat and human GK. Moreover, DNA sequence alignment of the upstream and downstream promoter regions of the mouse, rat, and human genes has revealed many different, evolutionarily conserved regions whose functions remain to be determined. 
The upstream GK promoter initially was thought to be expressed only in pancreatic $\beta$ cells. However, studies have demonstrated GK expression from the upstream promoter in pituitary corticotropes as well as in certain neural/neuroendocrine (NE) cells of the brain and the gut (Hughes et al., 1991; Jetton et al., 1994,1998). All of the regulatory elements necessary for expression in these sites, including $\beta$ cells, appear to be contained within a 294-bp fragment of DNA (Shelton et al., 1992; Jetton et al., 1994,1998). Indeed, a detailed mutational analysis of the upstream promoter has revealed several different classes of regulatory elements within this region (Shelton et al., 1992; Moates, et al., 1996), at least one of which, termed the Pal elements, appears to be unique. Expression of the upstream promoter has, until recently, been thought to be largely constitutive. However, retinoic acid was shown to increase pancreatic GK expression in cultured islets (Cabrera-Valladares et al., 1999). Since the physiological role of retinoic acid in $\beta$-cell function has not been determined, further studies are required to pinpoint the regulatory elements and signal transduction pathways involved. While there have been several efforts to examine the transcriptional regulation of the upstream promoter, the changes observed have been small and thus may have been dismissed as not being significant. However, now that we know that even small changes in GK activity can affect the threshold for insulin secretion, a re-examination of the effects of various hormones and other agents on the expression of GK in the islet has begun. Moreover, given that GK gene expression has been found to occur in a phenotypically diverse set of neu$\mathrm{ral} /$ neuroendocrine cells, further studies of the elements and factors involved in the cell-specific expression of this enzyme likely will provide additional insights.

In liver, GK gene transcription is stimulated by insulin and repressed by glucagon acting via cAMP (lynedjian et al., 1989; Magnuson et al., 1989). Unfortunately, despite extensive efforts using both classical transfection experiments and transgenic mice (Niswender et al., 1997a; Iynedjian, 1998), we continue to have very little knowledge about the cis-acting elements in the hepatic (downstream) GK promoter that are responsible for its cell-specific expression and regulation. Preliminary results of fusion gene studies in transgenic mice suggest that the conserved regions near the transcription start site in hepatocytes are themselves not sufficient for either position-independent expression in liver or regulation by insulin (Postic et al., data not shown). Indeed, given the results of DNAse I hypersensitive site-mapping studies - which have revealed no less than 11 liver-specific, hypersensitive sites in an approximately $50-\mathrm{kb}$ region of the mouse gene - the downstream GK promoter appears to be more complicated in its regulation than many other hepatic genes studied to date (Postic et al., 1995; Moates et al., 1997).

Although mapping regulatory elements in the hepatic GK promoter via a fusion gene approach has been hindered by the apparent size of the regulatory unit 
and the lack of hepatoma cell lines that express this gene, recent work by Foretz et al. (1999) has implicated sterol regulatory element-binding protein-1c (SREBP$1 c)$ as an activator for transcription of hepatic GK in cultured hepatocytes. In this study, SREBP-1c, which belongs to a family of transcription factors involved in cholesterol and fatty acid metabolism (Brown and Golstein, 1997), was suggested to mediate the effect of insulin on hepatic GK gene transcription. However, this notion contrasts with the observation that overexpression of SREBP-1c does not affect the levels of GK mRNA in liver of transgenic animals (Sebastian et al., 2000). Although the reason for this discrepancy is not readily evident, further studies of the role of SREBP-1c in determining hepatic GK expression may help us to understand how the downstream GK promoter is regulated by insulin and other hormones.

The interaction between GK and GKRP in liver affects both activity and subcellular location of the enzyme (Van Schaftingen, 1989; Vandercarmmen and Van Schaftingen, 1990; de la Iglesia et al., 1999; Shiota et al., 1999). By reversibly binding to and inhibiting GK, GKRP is able to affect hepatic glucose usage and probably also the responsiveness and sensitivity of the hepatocytes to changes in glucose concentrations (de la Iglesia et al., 2000). Interestingly, GKRP knockout mice have impaired glucose tolerance and less GK than normal animals with this protein (Farrelly et al., 1999; Grimsby et al., 2000). Thus, binding of GK to GKRP may protect it from degradation, providing a reservoir of protein that can be quickly mobilized after feeding. While further studies are necessary to understand the role that GKRP plays in regulating hepatic GK, we and others have obtained data indicating that GKRP is necessary for the translocation of GK into the nucleus (Shiota et al., 1999; Bosco et al., 2000). Moreover, our studies have shown that GK contains a nuclear export signal sequence that enables GK to return to the cytoplasm after metabolic cues lead to its dissociation from GKRP in the nucleus (Shiota et al., 1999). These studies have led us to suggest the existence of a novel nuclear translocation cycle for GK. However, it remains unclear how the binding of GK to GKRP actually stimulates movement of the dimerized complex into the nucleus.

\section{GK Gene Mutations in Humans}

In humans, mutations in the GK gene can cause two distinct diseases: maturity onset diabetes of the young, type 2 (MODY-2) and persistent hyperinsulinemic hypoglycemia of infancy (PHHI). Most mutations in the GK gene - in fact, over 100 different ones - have been identified that cause hyperglycemia in people (Froguel et al., 1992; Vionnet et al., 1992; Velho et al., 1997). The identification of GK as a diabetes-susceptibility gene has provided a major impulse for the reassessment of the physiological role of GK as a glucose sensor and the understanding of the pathophysiogical importance of this key enzyme in glucose ho- 
meostasis. At a biochemical level, missense mutations of GK impair either or both the $\mathrm{V}_{\max }$ and $\mathrm{K}_{\mathrm{cat}} \mathrm{S}_{0.5}$ of the enzyme, although some also appear to shorten the half-life of the protein (Pilkis et al., 1994; Liang et al., 1995; Guazzini et al., 1998; Miller et al., 1999). In addition to the effects of mutations on the $\mathrm{K}_{\mathrm{cat}} \mathrm{S}_{0.5}$ and $V_{\max }$, a decrease in the ATP-binding affinity or stability of the mutated enzyme may contribute to a reduction of GK activity in some individuals. Other mutations impair GK gene expression by affecting splice site utilization or by leading to premature termination of protein translation. In all cases, whether by activity or gene expression, the net effect is a reduction in the amount of GK activity within cells.

Congenital hyperinsulinism is a clinically and genetically heterogeneous disease. While most cases that have been described are caused by mutations in the sulfonylurea receptor-type 1 , a component of the ATP-sensitive potassium channel in $\beta$ cells (Thomas et al., 1995), mutations in other genes have been identified in patients with this clinical syndrome. Indeed, in one instance, the disease is due to a mutation in GK (V455M) that has the unusual effect of increasing the catalytic activity of the enzyme (Glaser et al., 1998). Given this important observation, as well as the fortuitous finding during experimental analysis of a second mutation capable of enhancing the activity of GK (Davis et al., 1999), it is possible that additional mutations that cause hypoglycemia will be identified.

\section{Rationale for Studies in Mice}

Given that GK is expressed in the liver, pancreatic islet, brain, and gut tissues that play central and interacting roles in controlling the blood glucose concentration - and that the regulation of GK activity is complex, a full understanding of how mutations in this enzyme cause either hyperglycemia or hypoglycemia can be achieved only in animal model systems. Indeed, given the complex nature of the regulation of both the islet and hepatic GK isoforms, as well as the fact that they arise from the same gene, it is unlikely that we will ever accurately predict the in vivo ramifications of altered GK gene expression by simply extrapolating from in vitro experimental model systems. Due to these various issues, controversy has persisted about the relative contributions of the liver and the islet in perturbations of glucose homeostasis observed in humans with GK gene mutations.

Part of the problem in determining the role of GK in glucose homeostasis is that both the liver and islet, key sites of GK gene expression, participate in a glucose-insulin feedback loop (Meglasson and Matschinsky, 1986; Magnuson, 1990). For instance, the secretion of both insulin and glucagon occurs in response to changes in the plasma glucose concentration, which is, in turn, affected by the metabolic status of the liver. Similarly, in the islet, glucose modulates islet GK 
content, probably by directly affecting the half-life of the enzyme (Liang et al., 1990,1992). The translocation of GK in hepatocytes between the cytoplasm and the nucleus is another good example of the complexity of the mechanisms involved. Indeed, the blood glucose concentration is a physiological endpoint that is determined by a myriad of different biochemical and physiological interactions. Thus, we thought it was necessary to generate several animal models with specific differences in GK gene expression and to carefully determine their phenotypes, in order to obtain unambiguous experimental information about tissue-specific roles of GK in regulating the blood glucose concentration.

\section{Effects of Overexpressing GK in Mice}

The effects of increased GK gene expression on blood glucose homeostasis were first examined by generating transgenic mice that contained additional copies of the entire mouse GK gene locus (Niswender et al., 1997a). These studies made use of the 83-kb fragment of mouse DNA, described earlier, that contained both the upstream and downstream promoters, all coding exons, as well as a large amount of tlanking DNA sequence (Postic et al., 1995). Indeed, the latter fact was an important consideration since, prior to these studies, a DNA fragment capable of regulated expression of GK in liver had not been demonstrated. By pronuclear DNA microinjection experiments using this DNA fragment, a line of transgenics that contained a single extra copy of the entire GK gene locus was generated. This was fortuitous, since it allowed the effects of increased GK gene copy number on glucose homeostasis to be studied.

In the GK gene locus transgenic mice, GK mRNA concentration was increased by approximately 1.5 fold in heterozygous transgenic animals and by about twofold in homozygous animals (Niswender et al., 1997a). Mice with a single extra copy of the GK gene locus had a plasma glucose concentration 25 percent lower than littermates with two normal alleles. Animals with a total of four functional copies of the GK gene had a plasma glucose concentration that was reduced even further (Figure 1). The effect of increased GK gene number on whole-body homeostasis was assessed further by performing both basal and hyperglycemic clamp studies (Niswender et al., 1997b). This involved implanting two catheters that enabled the precise determination of rates of hepatic glucose utilization and of insulin secretion under both basal and hyperglycemic conditions. During the hyperglycemic clamp study, a variable infusion of glucose was used to raise blood glucose levels to about $300 \mathrm{mg} / \mathrm{dl}$. Glucose turnover and glucose clearance rates as well as net hepatic and muscle glycogen are measured by incorporating the use of $\left[3-{ }^{3} \mathrm{H}\right]$ glucose as a metabolic tracer. The glucose infusion rate required to maintain the plasma glucose at this level provides an index of glucose tolerance. 


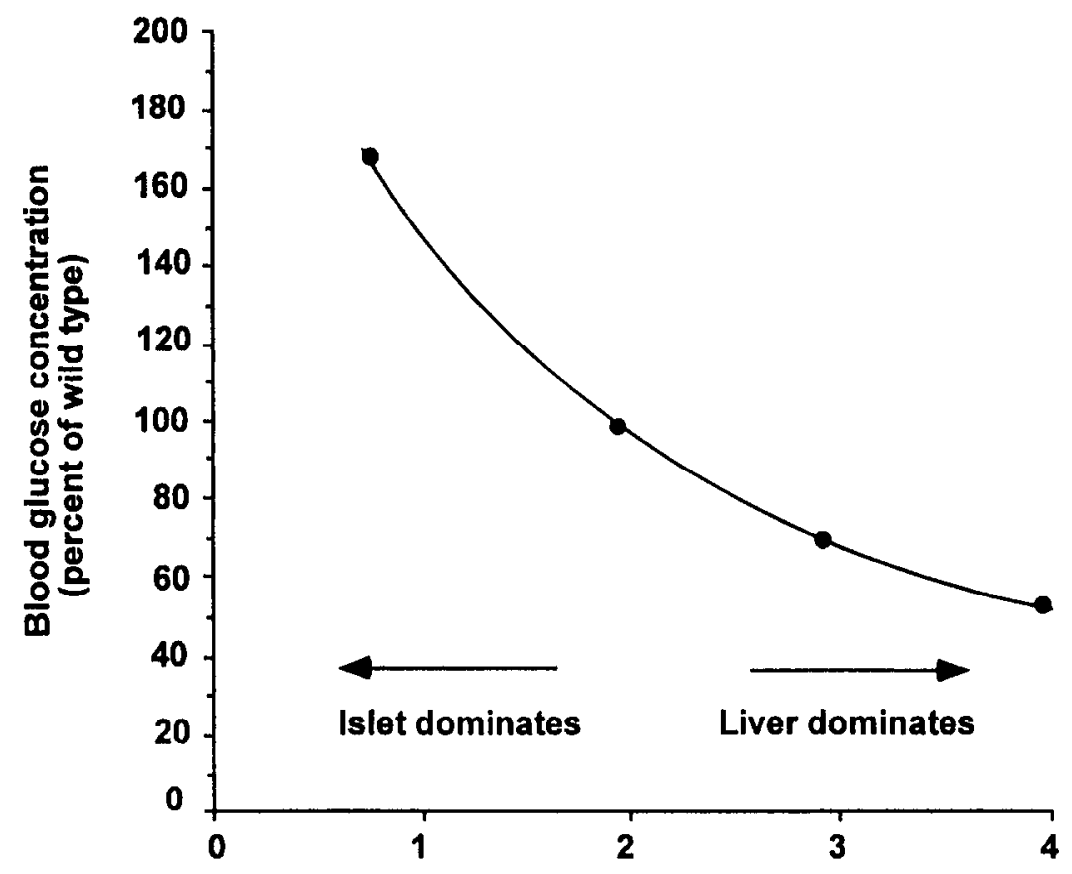

GK gene copy number

FIG. 1. Relationship between GK gene copy number and the blood glucose concentration. This figure shows the effect on the blood glucose concentration of changes in the GK gene copy number, obtained from GK-overexpressing transgenic mice and heterozygous global GK knockout mice. Mice that totally lack GK have blood glucose concentration greater than $500 \mathrm{mg} / \mathrm{dl}$ and do not survive the neonatal period, as described previously. [Adapted with permission from Postic, C., and Magnuson, M.A. Front. Diabetes 15, 293-298, 2000. Copyright 2000 by Karger, Basel.]

Increased GK gene expression from the presence of the GK gene locus transgene resulted in a 21 percent increase in glucose clearance rate under basal conditions, in the absence of any difference in basal insulin concentrations (Niswender, et al., 1997b). This result clearly demonstrated a role for GK in determining the basal rate of glucose clearance. Under hyperglycemic conditions, GK transgenic mice had glucose turnover and clearance rates similar to controls but secreted about 50 percent less insulin (Table I). Interestingly, despite diminished insulin secretion, hepatic glycogen content was markedly increased after the 2-hour glucose infusion (Niswender, et al., 1997b).

Transgenic mice that overexpress GK in the liver have been generated by other groups (Ferre et al., 1996; Hariharan et al., 1997). The results of these studies, which are similar to ours, have confirmed a direct role for hepatic GK in 
TABLE I

Summary of Metabolic Parameters from Basal and Hyperglycemic Clamp Experiments

\begin{tabular}{|c|c|c|c|c|c|c|c|c|}
\hline \multirow[b]{2}{*}{ Mouse Model } & \multicolumn{4}{|c|}{ Basal } & \multicolumn{4}{|c|}{ Hyperglycemic Clamp } \\
\hline & $g k^{3}$ & $g k^{\mathrm{del} / \mathrm{w}}$ & $\begin{array}{l}g k^{\mathrm{lox} / \mathrm{w}}+ \\
\text { Rip-Cre }\end{array}$ & $\begin{array}{l}\boldsymbol{g h}^{\text {lox/ox }}+ \\
\text { Alb-Cre }\end{array}$ & $g k^{3}$ & $g k^{\mathrm{del} / \mathrm{w}}$ & $\begin{array}{l}\text { gk } k^{10 x / \mathrm{w}}+ \\
\text { Rip-Cre }\end{array}$ & $\begin{array}{l}g k^{l o x} / 0 x+ \\
\text { Alb-Cre }\end{array}$ \\
\hline Blood glucose & $-12 \%$ & $+38 \%$ & $+23 \% *$ & $+40 \% *$ & $-40 \% *$ & \multicolumn{3}{|c|}{ Raised to $\sim 300 \mathrm{mg} / \mathrm{dl}$} \\
\hline Plasma insulin & nd & nd & nd & nd & $-40 \% *$ & $-70 \% * *$ & $-70 \% * *$ & $-70 \% * *$ \\
\hline Glucose turnover & nd & $-50 \% *$ & nd & nd & nd & $-67 \% *$ & $-56 \% *$ & $-60 \% *$ \\
\hline Glycogen synthesis & - & - & - & - & $+360 \% * *$ & $-90 \% * *$ & $-55 \% *$ & $-88 \%$ ** \\
\hline
\end{tabular}

The following mouse models were used: $\boldsymbol{g} \boldsymbol{k}^{3}$ : transgenic mice over expressing the entire GK gene locus (three functional genes in all tissues); $\boldsymbol{g}^{\text {del/w: }}$ heterozygoL GK knockuul mice (une functional gene in all tissues); $g k^{\text {tox } / w}+$ Rip-cre: heterozygous $\beta$-cell-specific GK knockout mice (a single functional gene in the $\beta$ cell, tw elsewhere); $g^{\text {loxhox }}+\boldsymbol{A l b}$-cre: liver-specific GK knockout mice (no hepatic GK, two functional genes elsewhere). For the hyperglycemic clamp studies, blood glucos concentrations were raised to $-300 \mathrm{mg} / \mathrm{dl}$. Each group of mice was comparcd to its appropriate control and differences between parameters are expressed as percer of controls. Only significant values are indicated in the table. nd: not statistically different. ${ }^{*} p<0.05 ; * \mathrm{p}<0.001$. 
maintaining basal plasma glucose concentration. Together, they clearly demonstrate that increased hepatic GK, by itself, is able to increase the rate of glucose clearance by the liver without increasing insulin secretion. Additionally, these studies show the pivotal role that hepatic GK plays in controlling glycogen synthesis in liver (Table I). Since we used a transgene containing promoters for the islet and hepatic isoforms of GK, it is interesting that islet GK expression was not increased, as might have been expected. Instead, these animals had a diminished amount of GK in islets, probably due to development of mild hypoglycemia. This hypoglycemia probably led to a diminished amount or stability of $\beta$-cell GK (Niswender et al., 1997a). In addition, elegant studies that have made use of recombinant adenoviruses to overexpress GK in hepatocytes (Agius et al., 1996; O'Doherty et al., 1996), as well as in the liver of rats (O'Doherty et al., 1999), confirm a direct role for hepatic GK in determining hepatic glucose uptake and, consequently, in maintaining basal plasma glucose concentration. Therefore, the role of liver in determining the blood glucose concentration appears to dominate in the situation of increased GK gene expression, as occurs in the gene locus transgenic mice (Niswender et al., 1997a). These results suggest that, normally, there is a fine balance between the function of the liver and the islet in the usual situation of two functional GK gene copies. Any perturbation in GK gene expression would cause a change in the blood glucose concentration by shifting the glucose setpoint (Niswender et al., 1997b).

Overexpression of GK, besides reducing the blood glucose concentration, appears to prevent the development of type 2 diabetes. Male GK locus transgenic mice that were fed a high-fat diet were protected against the development of hyperinsulinemia and hyperglycemia, even though these animals were as obese as their nontransgenic littermates (Shiota $e t$ al., in press). This suggests that if GK activity could somehow be enhanced, it might be an effective strategy of treating diabetes.

\section{Conventional GK Gene Knockout Mice}

Information complementary to the GK gain-of-function studies has been obtained by three groups who used gene-targeting studies to totally eliminate GK gene expression or to knock out expression only of the islet GK isoform (Bali et al., 1995; Grupe et al., 1995; Terauchi et al., 1995). In all cases, heterozygous GK knockout mice were hyperglycemic, thereby indicating that studies in the mouse provide a relevant model for MODY-2. Furthermore, all of the heterozygous GK null mouse models showed defective insulin secretion in response to glucose, indicating that the haploinsufficiency of GK impairs insulin secretion. This finding is consistent with the proposed role of GK as glucose sensor in the cell (Bali et al., 1995; Grupe et al., 1995; Terauchi et al., 1995). However, the 
fact that the global lack of GK is lethal either at midgestation (Bali, et al., 1995) or a few days after birth (Grupe et al., 1995) has prevented a detailed physiological analysis in these animal models. Additionally, none of these studies allowed the function of GK in the liver to be directly examined.

\section{Tissue-specific GK Gene Knockouts Using the Cre-loxP Approach}

To overcome the limitations of conventional gene-targeting approaches, we made use of a conditional targeting strategy in the site-specific DNA recombinase Cre to investigate the cell-specific roles of GK in glucose homeostasis. The Cre/lox $P$ recombinase system has been found to function efficiently both in vitro and in vivo (Sauer and Henderson, 1988; Sauer, 1993). Cre catalyzes recombination between two 34-bp recognition elements, termed loxP sites, thereby causing excision of intervening sequences. A virtually 100 percent excision efficiency can be achieved by transient expression of the Cre recombinase in embryonic stem (ES) cells or by pronuclear injections in zygotes (Araki et al., 1995). Transgenic mouse lines that express Cre under tissue-specific promoters can be crossed with mice harboring loxP sites to achieve gene excision in specific cell types. However, the difficulty of the technique resides in the need to obtain reliable Cre transgenic lines in which this recombinase is expressed in a cell-specific manner. While this method provides many advantages over a conventional gene knockout, the strategy is more complex because two components are necessary. First, a conditional gene locus is made by inserting two or more lox $P$ sites in nonessential portions of the gene, usually introns, by gene targeting in ES cells. This results in a gene locus that remains functional until exposed to Cre, which then causes excision of intervening sequences. For our studies, lox $P$ sites were inserted into the GK gene between exons 8 and 9 and downstream of exon 10, generating the loxed GK allele $\left(g k^{\text {lox }}\right)$ shown in Figure 2. The second essential component is a transgenic mouse that expresses Cre under the control of one or another different, cell type-specific promoters. For our studies, we generated transgenic mice that expresses $C r e$ under the control of either the insulin or the albumin promoters. These mice expressed this site-specific recombinase in pancreatic $\beta$ cells and hepatocytes, respectively (Figure 3 ). By crossing the $g k^{\text {tox }}$ allele into mice bearing one or another of the Cre transgenes, we were able to specifically eliminate GK in either $\beta$ cells or in the liver. In addition, by microinjecting a Cre expression plasmid into single-cell mouse embryos, we were able to generate a null allele $\left(g k^{\mathrm{del}}\right)$, thereby generating mice that confirm the effects of a global GK gene knockout (Postic et al., 1999). 


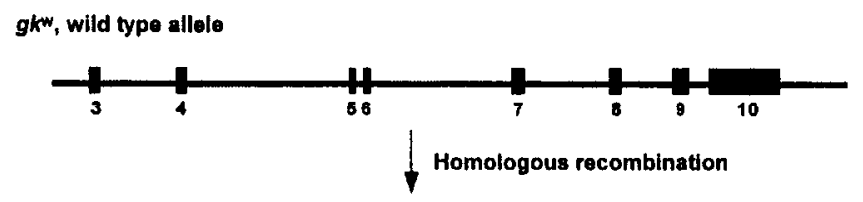

gklox+neo

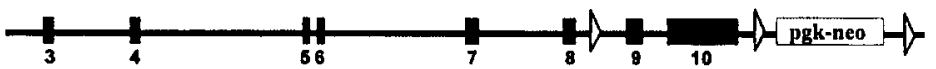

Cre expression in cells or embryos

gklox, conditional allele

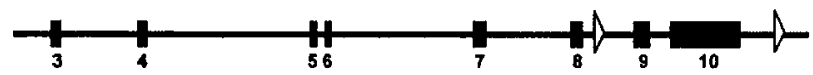

Cell-specific recombination using Cre transgenic mice

gkdel, deleted allele (null)

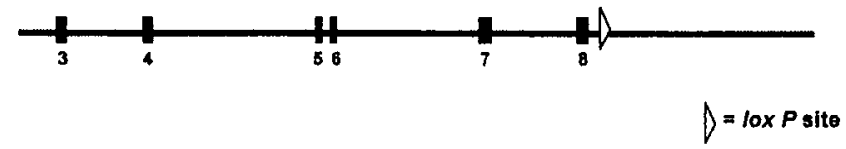

FIG. 2. Generation of mice with conditional $g k$ gene alleles. Exons are indicated as solid rectangles. A partial map of the wild-type allele $\left(g k^{\mathrm{w}}\right)$ is shown. The conditional allele was obtained by homologous recombination in ES cells and partial Cre recombination $\left(g k^{\text {lox }}\right)$. The deleted allele ( $g k^{\text {del }) ~ l a c k s ~ e x o n s ~} 9$ and 10 and was obtained by microinjection of a Cre expression plasmid into single-cell mouse embryos or by cell-specific expression of Cre in transgenic mice.

\section{A. GLOBAL GK NULL MICE}

To determine the effect of a total deletion of GK, we converted the $g k^{\text {lox+neo }}$ allele into a deleted allele $\left(g k^{\mathrm{del}}\right)$ by microinjecting different concentrations of a CMV-Cre expression plasmid into single-cell $g k^{\mathrm{lox}+\text { neo/w }}$ mouse embryos (Araki $e t$ $a l ., 1995)$. At concentrations of $0.1 \mathrm{ng} / \mu \mathrm{l}$ or greater, Cre efficiently excised both loxP-flanked DNA fragments, thereby creating several mice that were heterozygous for the $g k^{\mathrm{del}}$ allele (e.g., $g k^{\mathrm{del} / \mathrm{w}}$ ). Intercrossing of $g k^{\mathrm{del} / \mathrm{w}}$ resulted in mice that were homozygous null for GK $\left(g k^{\mathrm{del} / \mathrm{del}}\right)$. These mice appeared normal at birth but died within 4 days of profound hyperglycemia. The metabolic alterations observed in $g k^{\text {del/del }}$ pups, particularly hepatic steatosis and decreased glycogen content, were consistent with insufficient insulin secretion due the inability of $\beta$ cells in the GK null mice to respond to an elevated plasma glucose concentration. Indeed, mice that have induced mutations of both insulin genes have nearly an identical phenotype (Duvillie et al., 1997). This phenotype closely resembled that of Grupe 


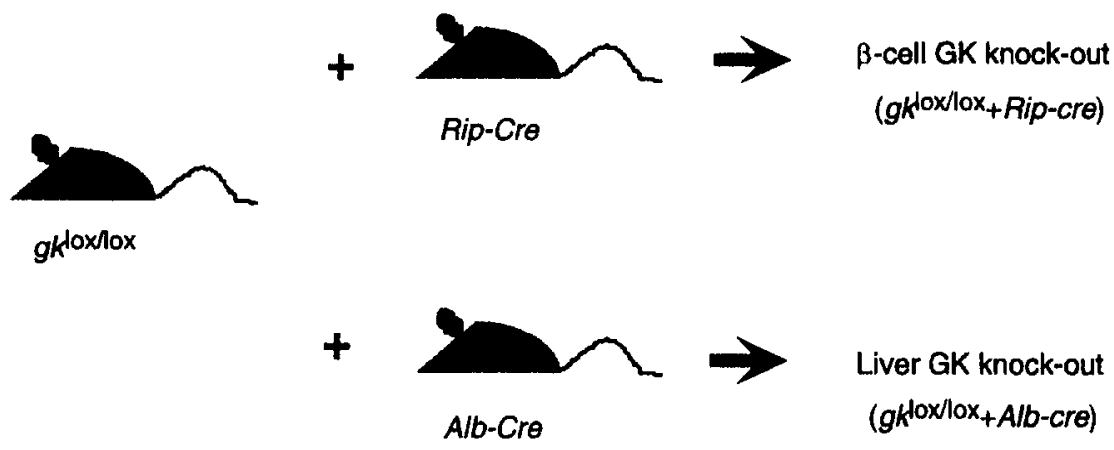

FIG. 3. Strategy for $\beta$-cell- and liver-specific deletion of the $g k^{\text {tox }}$ allele in mice. Two lines of Cre transgenic mice were created. Rip-cre transgenic mice express $C r e$ in the pancreatic $\beta$ cells under the control of the rat insulin promoter. Alb-cre transgenic mice express Cre in liver under the control of the promoter/enhancer albumin gene. Intercrossing Cre transgenic mice with $g k^{\text {lox } \lambda_{0 x}}$ mice produces both $\beta$-cell and liver-specific GK gene knockout animals.

et al. (1995) but was substantially different from mice generated by Bali et al. (1995), which died in utero at embryonic day 9.5. We think this difference is due to the random insertion of a second copy of the targeting vector in the genome of the mice made by Bali et al. (1995), as indicated by the presence of an unexpected band after hybridization of DNA from these mice with a probe containing sequences from the neomycin resistance cassette (Niswender et al., 1997a). This may have led to the inadvertent knockout of a second gene whose function is necessary during early embryogenesis.

\section{B. $\beta$-CELL-SPECIFIC KNOCKOUT OF GK}

To unambiguously determine the role of GK in pancreatic $\beta$ cells, mice with the conditional GK allele $\left(g k^{\mathrm{lox}}\right)$ were interbred with animals that express Cre under the control of a 668-bp fragment of the rat insulin 2 promoter (Rip-Cre) (Figure 3). The Rip-Cre transgene contained the entire human growth hormone gene placed downstream of the Cre coding sequences that had been modified to contain a nuclear localization signal sequence and an optimized translation start site. All of these features were included to ensure high-fidelity expression of this recombinase. Moreover, since Cre-mediated conditional gene inactivation requires precise knowledge of sites of recombination, we also used ROSA26 LacZ reporter mice (Soriano, 1999) to determine sites of Rip-Cre expression, both during mouse development and in the adult animal (Gannon et al., 2000). Rip-Cre mediated recombination was first detected in early developmental (i.e., 11.5 days postcoitum) pancreatic endocrine cells (Gannon et al., 2000). In adult mice, 
recombination was first detected within pancreatic $\beta$ cells and in the ventral cerebral cortex and some neurons (Gannon et al., 2000). Beta-cell-specific GK knockout mice (e.g., $g k^{\text {lox/lox }+R i p-C r e)}$ are phenotypically similar to animals with a global GK knockout, showing signs of steatosis and a depletion of hepatic glycogen content due to approximately a 70 percent decrease in plasma insulin concentrations. Furthermore, the $g k^{\mathrm{lox} / \mathrm{ox}}+R i p-C r e$ die within a few days of birth as a result of severe diabetes. Both basal and hyperglycemic clamp studies of the heterozygous $\beta$-cell-specific GK knockout mice $\left(g k^{\text {lox/w }}+R i p-C r e\right)$ showed that they had fasting hyperglycemia $(+25 \%)$ without measurable difference in basal insulin levels (Table I). During the hyperglycemic clamp, glucose turnover rates and insulin secretion were reduced by about $70 \%$. Moreover, as a consequence of diminished insulin secretion, net glycogen synthesis in liver was reduced by nearly 50 percent.

\section{LIVER-SPECIFIC KNOCKOUT OF GK}

To determine the role of hepatic GK in glucose homeostasis, we next generated liver-specific GK knockout mice (e.g., $g k^{\mathrm{lox} / \mathrm{ox}}+A l b-C r e$ ) by interbreeding albumin-Cre transgenic mice with animals bearing the loxed GK allele (Figure 3). Cre expression in hepatocytes was obtained using a $2.35-\mathrm{kb}$ rat albumin enhancer/promoter fragment (Pinkcrt et al., 1987). Otherwise, the transgenic design was similar to that used for expression of $\mathrm{Cre}$ in the $\beta$ cell. To examine the efficiency of recombination as a function of age, we performed Southern blot analysis using liver genomic DNA from $g k^{\mathrm{lox} / \mathrm{lox}}+A l b$-Cre mice isolated at ages ranging from 1 day to 12 weeks. The timing of recombination in liver of $g k^{\mathrm{lox} / \mathrm{lox}}+A l b$-Cre mice, while age dependent, appeared to be complete by 6 weeks of age (Postic and Magnuson, 2000a). Moreover, when the Alb-Cre transgene was crossed with the ROSA26A Cre-inducible reporter mice, more than 99 percent of hepatocytes showed positive $\beta$-galactosidase staining at 12 days of age. The lack of complete recombination at birth in $g k^{\text {lox/lox }}+A l b$-Cre mice and progressive recombination thereafter is likely due to expression of Cre below the threshold level necessary to cause rapid recombination (Postic and Magnuson, 2000a). Nonetheless, by 6 to 8 weeks of age, recombination in liver was complete and there was no evidence for Cre-mediated deletion of the loxed GK gene locus in other tissues.

The complete loss of hepatic GK was not lethal and had only a relatively small effect on the plasma glucose concentration (Postic et al., 1999). Indeed, mice totally lacking hepatic GK had fed blood glucose values that were only about 10 percent higher that their $g k^{\text {lox/lox }}$ littermates. Again, to explore the consequences of the total loss of hepatic GK on both insulin secretion and hepatic fluxes, basal and hyperglycemic clamp studies were performed (Table I). After an 8-hour fast, no differences were detected in either plasma insulin concentration or glucose 
turnover rate, even though the blood glucose concentrations were higher $(+40$ percent) than the controls. During the hyperglycemic clamp phase of the study, glucose turnover rate was markedly reduced in the liver-specific GK knockout mice (Table I). Surprisingly, the liver-specific GK knockout mice secreted 70 percent less insulin in response to the glucose stimulus, compared to controls (Table I; Figure 4A), a finding that will be discussed in more detail. Net glycogen synthesis was reduced by approximately 90 percent in liver (Figure 4B). In addition, several glucose-responsive genes, including L-pyruvate kinase and phosphoenolpyruvate kinase, were found to be inappropriately regulated (Postic $e t a l$., unpublished data). Together, these results clearly indicate that hepatic GK plays an important role in glucose homeostasis, largely by a direct effect on hepatic glucose utilization but possibly also by an indirect effect on insulin secretion.

\section{Importance of GK in Glucose Homeostasis and Glucose Sensing}

\section{A. ROLE OF $\beta$-CELL GK}

An extensive body of evidence indicates that GK plays a critical role in the control of insulin secretion in the $\beta$ cells. Insulin secretion depends upon glucose metabolism and GK exerts nearly total control on the rate of glycolysis in these cells (Meglasson and Matschinsky, 1986; Matschinsky et al., 1993). Indeed, the control strength of GK for metabolic flux is close to unity (Sweet et al., 1996), implying that even small changes in total $\beta$-cell GK activity have an impact on the rate of glucose metabolism and, hence, the rate of glucose-induced insulin release.

In our mouse models, as well as those of other investigators, the reduced level of GK activity in $\beta$ cells appears to be responsible for much of the hyperglycemia that developes as a result of diminished GK gene expression, as occurs in MODY2 (Bali et al., 1995; Grupe et al., 1995; Terauchi et al., 1995, Postic et al., 1999). Interestingly, despite the close similarities between mice and humans in the mechanisms used to maintain glucose homeostasis, the loss of one functional GK allele in mice may result in a greater degree of hyperglycemia than is observed in humans. In several subjects with GK gene mutations that were studied by Sturis et al. (1994), insulin secretion was found to be reduced compared to controls but less than predicted from the calculated decrease in GK flux, suggesting the existence of compensatory changes. These changes include a potential glucoseinduced overexpression of the normal allele or a decrease in the inhibitory effect of the regulatory protein (Sturis et al., 1994). Using islets isolated from a linc of heterozygous GK knockout mice, Sreenan and colleagues have shown that these islets may be protected against the toxic effect of high glucose through enhanced expression of GK protein and activity, resulting from the remaining normal allele 
A

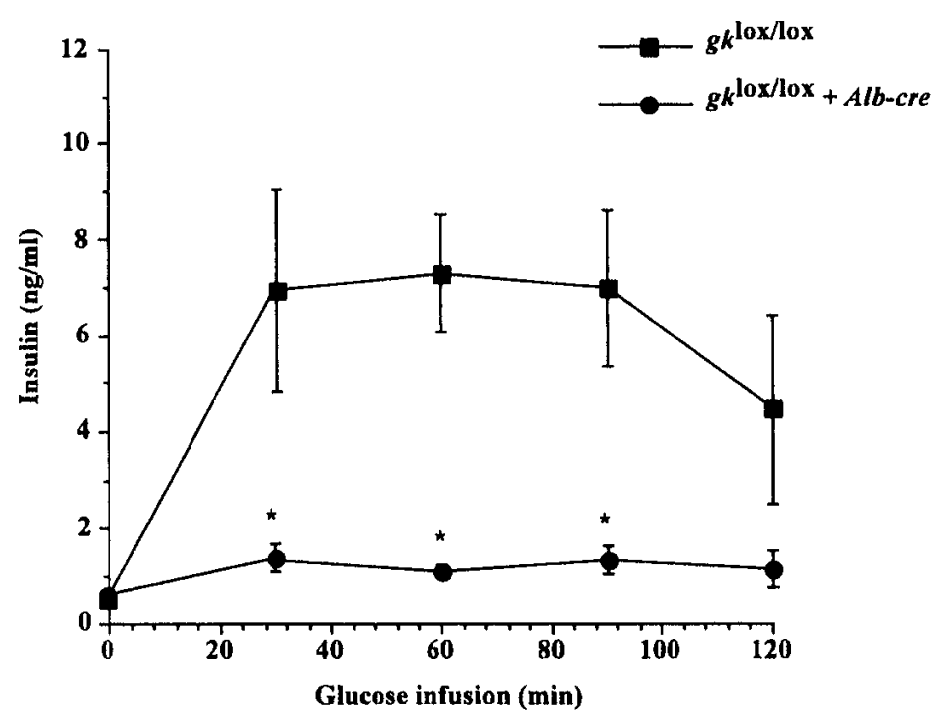

B

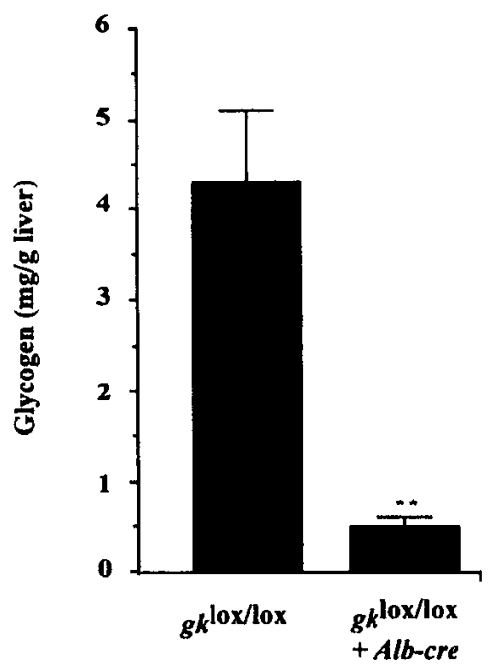

FIG. 4. Impaired insulin secretion and reduced glycogen content in liver of $g k^{\text {lox/lox }}+A l b-c r e$ mice. (A) $g k^{\mathrm{lox} / 0 \mathrm{x}}+A l b$-cre mice secreted 70 percent less insulin in response to the glucose stimulus, compared to controls. (B) Net hepatic glycogen synthesis, measured at the end of the 2-hour hyperglycemic clamp, was reduced by about 90 percent in liver of $g k^{\text {lox } \lambda \text { ox }}+A l b$-cre mice, compared to their controls. 
(Sreenan et al., 1998). These studies suggest that there is compensation by the functional GK allele in the heterozygous GK null mice to hyperglycemia, perhaps explaining why MODY-2 in humans is characterized by mild hyperglycemia that generally does not worsen over time.

While physiological studies of the $\beta$-cell-specific GK knockout mice have provided compelling data indicating a role for GK in determining insulin secretion, additional evidence for GK functioning as the $\beta$-cell glucose sensor was obtained by Piston et al. (1999). In this study, islets from $g k^{\text {lox/lox }}$ mice were isolated and treated with a recombinant adenovirus that expresses Cre (Anton and Graham, 1995). This caused the elimination of GK expression from some $\beta$ cells, while retaining it in others. Two-photon excitation microscopy of these islets was then used to show that individual $\beta$ cells that lack GK do not have a normal rise in NADP(H) autofluorescence, in contrast to cells that contain GK (Piston et al., 1999). Interestingly, while confirming the key role of GK in the metabolism of glucose by $\beta$ cells, these studies have suggested that one or another of the three known lower $K_{\mathrm{m}}$ hexokinases may play a small role in determining insulin secretion but probably only at low blood glucose concentrations. GK clearly plays the dominant role in determining glucose-induced insulin secretion (Piston et al., 1999). In any case, these studies illustrate the experimental flexibility of Cre-loxP technology and suggest that recombinant Cre-expressing adenoviruses will be generally useful for deleting other loxed alleles in isolated islets.

\section{B. ROLE OF HEPATIC GK}

While it has been widely assumed for years that GK is necessary for hepatic glucose metabolism, the complete loss of hepatic GK is not lethal. In fact, it has only a relatively small effect on the plasma glucose concentration. In addition, both glycogen concentrations and a number of other plasma and hepatic metabolites in these animals are normal at the basal state. Surprisingly, GLUT-2 knockout mice show a more-severe hepatic phenotype, including dysregulation of both glycogen metabolism and abnormal expression of some glucose-responsive genes (Burcelin et al., 2000). The fact that basal glucose turnover rates in mice totally lacking GK are similar to control animals suggests that a near-normal flux can be maintained at the basal state through one or another of the low $K_{\mathrm{m}}$ hexokinases. Indeed, the effect of a deficiency of hepatic GK becomes apparent only under hyperglycemic conditions, when an impairment in glucose turnover, glucose clearance, hepatic glycogen synthesis, and insulin secretion become apparent (Table I; Figure 4). During hyperglycemia, the phosphorylating capacities of all of the low $K_{\mathrm{m}}$ hexokinases likely become saturated, thereby leading to functional deficits that have been observed. Whether increased activity from one or another of low $K_{\mathrm{m}}$ hexokinase compensates for the lack of hepatic GK in $g k^{\text {lox/lox }}+A l b-C r e$ mice remains to be determined. It also appears that the lack of hepatic GK impairs 
the ability of certain hepatic genes to respond to changes in the glucose concentration, which is consistent with hepatic GK functioning as a glucose sensor in the liver, much as it does in the $\beta$ cell.

The finding of a blunted insulin secretory response to hyperglycemia was unexpected and unexplained. It seems unlikely that this is due to glucose toxicity, since these mice have only a small elevation in basal plasma glucose concentrations. Moreover, we have found no evidence for recombination in $\beta$ cells of liver-specific GK knockout mice (C. Postic, data not shown). It is possible that hepatic GK is somehow necessary for the function of a neural regulatory loop or for the secretion of a factor whose function is to modulate insulin secretion. However, it could also simply reflect a lower insulin clearance rate. Thus, this intriguing observation merits further study. In any case, these findings indicate that alteration in GK expression in the liver, in addition to the $\beta$ cell, probably contributes to the pathogenesis of MODY-2. Thus, while the total loss of hepatic GK has less of an impact on the animal than that of $\beta$-cell GK, these studies provide clear evidence that hepatic GK plays an important role in determining hepatic glucose utilization during hyperglycemia. Hepatic GK also enables the induction of other key hepatic genes in response to glucose.

The finding that deficiencies of both hepatic and $\beta$-cell GK cause hyperglycemia in mice is consistent with findings by other investigators who have studied individuals with MODY-2. Indeed, these studies have shown that GK gene mutations are associated with abnormal regulation of hepatic glucose output and glycogen synthesis (Velho et al., 1996; Tappy et al., 1997). Furthermore, the high control coefficient exerted by GK on glucose metabolism (Agius et al., 1996) is consistent with the dominant inheritance pattern of MODY-2. Abnormal suppression of hepatic glucose production has been demonstrated in heterozygous GK knockout mice (Rossetti et al., 1997). Indeed, the inability of hyperglycemia to inhibit hepatic glucose production is fully consistent with GK functioning as a hepatic glucose sensor for regulating gene expression by glucose. Taken together, both the gain-of-function and loss-of-function studies that have been performed indicate that hepatic GK is a major determinant of glucose homeostasis.

\section{Toward Further Understanding of Type 2 Diabetes Mellitus Using Cre-loxP}

Type 2 diabetes is now understood to be a complex metabolic disorder caused by two main physiological defects, resistance to the action of insulin combined with a deficiency in insulin secretion (De Fronzo, 1997). Because the molecular defects that cause type 2 diabetes are largely unknown, a large number of animal studies have been undertaken by different investigators to determine how different gene defects contribute or cause a diabetic phenotype. Further application of the 
Cre/loxP strategy is likely to yield novel insights into the role of specific tissues in the development of insulin resistance and type 2 diabetes. While our studies have been focused on understanding how a haploinsufficiency of GK is able to cause hyperglycemia, there are other forms of MODY caused by mutations in genes encoding several different transcription factors. Specifically, mutations in HNF-4 $\alpha$ (Yamagata et al., 1996a), HNF-1 $\alpha$ (Yamagata et al., 1996b), PDX-1 (Stoffers et al., 1997a,1997b), and HNF-1 $\beta$ (Horikawa et al., 1997) cause MODY$1,-3,-4$, and -5 , respectively. These transcription factors are expressed in pancreatic $\beta$ cells. The mutations are believed to cause insulin deficiency by impairing $\beta$-cell function. However, because HNFs are also expressed in hepatocytes, these mutations may cause abnormalities in hepatic metabolism. While the generation of HNF- $1 \alpha$ null mice has shed some light on the molecular basis of MODY-1 (Lee et al., 1998; Pontoglio et al., 1998), analysis of mice with a tissue-specific defect in HNF-1 $\alpha$ expression would clearly resolve the contribution of the liver and pancreatic islets in the pathogenesis of the disease.

Recently, Cre/lox $P$ methodology has been used to begin to sort out the physiological effects of insulin resistance in various tissues. Although mice with a global insulin receptor defect die of uncontrolled diabetes (Accili et al., 1996; Joshi et al., 1996), mice with tissue-specific knockouts of insulin receptor are viable (Bruning et al., 1998; Kulkarni et al., 1999; Michael et al., 2000). Analysis of mice with a liver-specific insulin receptor knockout (LIRKO) has revealed the critical role that hepatic insulin signaling plays in the regulation of glucose homcostasis and maintenance of normal hepatic function (Michael et al., 2000). Indeed, LIRKO mice exhibit dramatic insulin resistance, severe glucose intolerance, and a failure of insulin to suppress hepatic glucose production and to regulate hepatic gene expression (Michael et al., 2000). In the liver of LIRKO mice, genes encoding a variety of different hepatic enzymes, known to be regulated both by insulin and glucose, are either induced or repressed (Michael et al., 2000). Whether this effect is caused by the virtual absence of hepatic GK in liver of LIRKO mice or by a direct loss of hepatic insulin signaling remains to be determined. Given that mice with a muscle-specific insulin receptor knockout (MIRKO) have normal fasting glucose levels and normal glucose tolerance (Bruning et al., 1998), the liver may play a more-important role in the control of glucose homeostasis than has been previously thought. Moreover, studies of mice with a selective defect in pancreatic $\beta$-cell insulin receptor (BIRKO) suggest that insulin resistance in $\beta$ cells may contribute to the inability of these cells to sustain sufficient insulin secretion (Kulkarni et al., 1999). How this occurs is not known; however, because there is a selective loss of glucose-stimulated insulin secretion, it is possible that expression of islet GK is impaired in the BIRKO mice. Whether or not this is the case, further studies of how insulin signaling affects $\beta$-cell 
function is clearly needed and may lead to dramatic changes in our understanding of how this cell contributes to the pathogenesis of type 2 diabetes.

\section{Concluding Comments}

In conclusion, our studies have illustrated how various mousc models, some of which used Cre-loxP methodologies, have been valuable for determining the pathophysiological basis of MODY-2. Since type 2 diabetes is even a more-complex physiological disorder, we expect that various site-specific recombinases, particularly Cre, are likely to see additional use in determining the cell-specific functions of genes in intact animals. Indeed, by enabling specific genes to be activated or deleted in virtually any tissue, Cre/loxP is certain to be a very important tool in mouse functional genomics, as suggested by various recent reviews (Nagy, 2000; Le and Sauer, 2000; Hardouin and Nagy, 2000).

\section{ACKNOWLEDGMENTS}

These studies were supported by grants from the National Institutes of Health (NIH) (DK42612 and DK 42502). We thank K.D. Niswender, Y. Chen, J.M. Moates, K.D. Shelton, J. Lindner, S. Knobel, D. Piston, A.D. Cherrington, M. Gannon, and C. Wright for their participation in the primary studies that are summarized here.

\section{REFERENCES}

Accili, D., Drago, J., lee, E., Johnson, M., Cool, M., Salvatore, P., Asico, L., Jose, P., Taylor, S., and Westphal, H. (1996). Nature Genet. 12, 106-109.

Agius, L., Peak, M., Newgard, C.B., Gomez-Foix, A.M., and Guinovart, J.J. (1996), J. Biol. Chem. 271, 30479-30486.

Aiston, S., Trinh, K.Y., Lange, A.J., Newgard, C.B., and Agius, L. (1999). J. Biol. Chem. 274, 24559-24566.

Anton, M., and Graham, F.L. (1995). J. Virol. 69, 4600-4606.

Araki, K., Araki, M., Miyazaki, J., and Vassalli, P. (1995). Proc. Natl. Acad. Sci. U.S.A. 92, 160-164.

Bali, D., Svetlanov, A., Lee, H.-W., Fusco-DeMane, D., Leiser, M., Li, B., Barzilai, N., Surana, M., Hou, H., Fleisher, N., DePinho, R., Rossetti, L., and Efrat, S. (1995). J. Biol. Chem. 270, 21464-21467.

Bosco, D., Meda, P., and lynedjian, P.B. (2000). Biochem. J. 15, 215-222.

Brown, M.S., and Golstein, J.L. (1997). Cell 89, 331-340.

Bruning, J.C., Michael, M., Winney, J., Hayashi, T., Horsch, D., Accili, D., Goodyear, L., and Kahn, C.R. (1998). Mol. Cell. 2, 559-569.

Burcelin, R., Munoz, M.C., Guillam, M.-T., and Thorens, B. (2000). J. Biol. Chem. 275, 10930-10936.

Cabrera-Valladares, G., German, M.S., Matschinsky, F.M., Wang, J., and Fernandez-Meja, C. (1999). Endocrinology 140, 3091-3096.

Davis, E.A., Cuesta-Munoz, A., Raoul, M., Buettger, C., Sweet, I., Moates, M., Magnuson, M.A., and Matschskiny, F.M. (1999). Diabetologia 42, 1175-1186.

De Fronzo, R. (1997). Diabetes Rev. 5, 177-269. 
de la Iglesia, N., Veiga-da-Cunha, M., Van Shaftingen, E., Giunovart, J.J., and Ferrer, J.C. (1999). FEBS Lett. 456, 332-338.

de la Iglesia, N., Mukhtar, M., Seone, J., Guinovart, J.J., and Agius, L. (2000). J. Biol. Chem. 275, 10597-10603.

Duvillie, B., Cordonnier, N., Deltour, L., Dandoy-Dron, F., Itier, J.-M., Monthioux, E., Jami, J., Joshi, R.L., and Bucchini, D. (1997). Proc. Natl. Acad. Sci. U.S.A. 94, 5137-5140.

Farrelly, D., Brown, K.S., Tieman, A., Ren, J., Lira, S.A., Hagan, D., Gregg, R., Mookhtiar, K.A., and Hariharan, N. (1999). Proc. Natl. Acad. Sci. U.S.A. 96, 14511-14516.

Ferre, T., Riu, E., Bosch, F., and Valera, A. (1996). FASEB J. 10, 1213-1218.

Foretz, M., Giuchard, c., Ferré, P., and Foufelle, F. (1999). Proc. Natl. Acad. Sci. U.S.A. 96, 12737 12742 .

Foufelle, F., Girard, J., and Ferre, P. (1996). Adv. Enzyme Regul. 36, 199-226.

Froguel, P., Vaxillaire, M., Sun, F., Velho, G., Zouali, H., Butel, M.O., Lesage, S., Vionnet, N., Clément, K., Fougerousse, F., Tanizawa, Y., Weissenbach, J., Beckman, S.J., Lathrop, J.M., Passa, P., Permutt, M.A., and Cohen, D. (1992). Nature 356, 162-164.

Gannon, M., Shiota, C., Postic, C., Wright, C.V.E., and Magnuson, M.A. (2000). Genesis 26, 139-141. Girard, J. (1997). Annu. Rev. Nutrit. 17, 325-352.

Glaser, B., Kesavan, P., Heyman, M., Davis, E., Cuesta, A., Busch, A., Stanley, C.A., Thornton, P.S., Permutt, M.A., Matschinsky, F.M., and Herold, K.C. (1998). N. Engl. J. Med. 338, 226-230.

Grimsby, J., Coffey, J.W., Dvorozniak, M.T. Shiota, Magnuson, M.A., and Grippo, J. (2000). J. Biol. Chem. 275, 7826-7831.

Grossbard, L, and Schimke, R.T. (1966). J. Biol. Chem. 241, 3546-3560.

Grupe, A., Hultgren, B., Ryan, S., Ma, Y.H., Bauer, M., and Stewart, T.A. (1995). Cell 83, 69-78.

Guazzini, B., Gaffi, D., Mainieri, D., Multari, G., Cordera, R., Bertolini, S., Meschi, F., and Barbetti, F. (1998). Hum. Mutat. 12, 136.

Hardouin, N., and Nagy, A. (2000). Genesis 26, 245-252.

Hariharan, N., Farrelly, D., Hagan, D., Hillyer, D., Arbeeny, C., Sabrah, T., Treloar, A., Brown, K., Kalinowski, S., and Mookhtiar, K. (1997). Diabetes 46, 11-16.

Horikawa, Y., Iwasaki, N., Hara, M., Furuta, H., Hinokio, Y., Cockburn, B.N., Lindner, T., Yamagata, K., Ogata, M., Tomonaga, O., Kuroki, H., Kasahara, T., Iwamoto, Y., and Bell, G. (1997). Nature Genet. 17, 384-385.

Hughes, S.D., Quaade, C., Milburn, J.L., Cassidy, L., and Newgard, C.B. (1991), J. Biol. Chem. 266, $4521-4530$.

Iynedjian, P.B. (1998). Biochem. J. 333, 705-712.

Iynedjian, P.B., Jotterand, D., Nouspikel, T., Asfari, M., and Pilot, P.R. (1989). J. Biol. Chem. 264, 21824-21829.

Jetton, T., Liang, Y., Petterpher, C.C., Zimmerman, E.C., Cox, G.F., Horvath, K., Matchinsky, F.M., and Magnuson, M. (1994). J. Biol. Chem. 269, 3641-3654.

Jetton, T.L., Moates, J.M., Lindner, J., Wright, C.V.E., and Magnuson, M.A. (1998). Proc. Natl. Acad. Sci. U.S.A. 95, 8654-8659.

Joshi, R., Lamothe, B., Cordonnier, N., Mesbah, K., Monthioux, E., Jami, J., and Bucchini, D. (1996). EMBO J. 15, 1542-1547.

Kulkarni, R.N., Bruning, J.C., Winnay, J.N., Postic, C., Magnuson, M.A., and Kahn, C.R. (1999). Cell 96, 329-339.

Le, Y., and Sauer, B. (2000). Methods Mol. Biol. 136, 477-485

Lee, Y.H., Sauer, B., and Gonzalez, F.J. (1998). Mol. Cell Biol. 18, 3059-3068.

Liang, Y., Najafi, H., and Matschinsky, F.M. (1990). J. Biol. Chem. 265, 16863-16866.

Liang, Y., Najafi, H., Smith, R.M., Zimmerman, E.C., Magnuson, M.A., Tal, M., and Matchinsky, F.M. (1992). Diabetes 41, 792-806. 
Liang, Y., Kesavan, P., Niswender, K., Tanizawa, Y., Permutt, M.A., Magnuson, M.A., and Matchinsky, F.M. (1995). Biochem. J. 309, 167-173.

Magnuson, M.A. (1990). Diabetes 39, 523-527.

Magnuson, M.A., Andreone, T.L., Printz, R.L., Koch, S., and Granner, D.K. (1989). Proc. Natl. Acad. Sci. U.S.A. 86, 4838-4842.

Matschinsky, F.M., Glaser, B., and Magnuson, M.A. (1998). Diabetes 47, 307-315.

Meglasson, M.D., and Matschinsky, F.M. (1983). J. Biol. Chem. 258, 6705-6708.

Meglasson, M.D., and Matschinsky, F.M. (1984). Am. J. Physiol. 246, E1-E13.

Meglasson, M.D., and Matschinsky, F.M. (1986). Diabetes Metab. Rev. 2, 163-214.

Michael, M.D., Postic, C., Previs, S.F., Kulkarni, R.N., Shulman, G.I., Magnuson, M.A., and Kahn, C.R. (2000). Mol. Cell. 6, 87-97.

Miller, S.P., Anand, G.R., Karschnia, E.J., Bell, G.I., LaPorte, D.C., and Lange, A.J. (1999). Diabetes 48, 1645-1651.

Moates, J.M., Shelton, K.D., and Magnuson, M.A. (1996). Mol. Endocrinol. 10, 723-731.

Moates, J.M., Postic, C., Decaux, J.-F., Girard, J., and Magnuson, M.A. (1997). Genomics 45, 185-193.

Nagy, A. (2000). Genesis 26, 99-109.

Niswender, K.D., Postic, C., Jetton, T.L., Bennet, B., Piston, D., Efrat, S., and Magnuson, M.A. (1997a). J. Biol. Chem. 272, 22564-22569.

Niswender, K.D., Shiota, M., Postic, C., Cherrington, A.D., and Magnuson, M.A. (1997b). J. Biol. Chem. 272, 22570-22572.

O'Doherty, R.M., Lehman, D.L., Seoane, J., Gomez-Foix, A.M., Guinovart, J.J., and Newgard, C.B. (1996). J. Biol. Chem. 271, 20524-20530.

O'Doherty, R.M., Lehman, D.L., Telemaque-Potts, S., and Newgard, C.B. (1999). Diabetes 48, 2022-2027.

Pilkis, S.J., Weber, I.T., Harrison, R.W., and Bell, G.I. (1994). J. Biol. Chem. 69, 1925-1928.

Pinkert, C.A., Ornitz, D.M., Brinster, R.L., and Palmiter, R.D. (1987). Genes Devel. 3, 268-276.

Piston, D.W., Knobel, S.M., Postic, C., Shelton, K.D., and Magnuson, M.A. (1999). J. Biol. Chem. 274, 1000-1004.

Pontoglio, M., Sreenan, S., Roe, M., Pugh, W., Ostrega, D., Doyen, A., Pick, A.J., Baldwin, A., Velho, G., Froguel, P., Levisetti, M., Bonnerweir, S., Bell, G.I., Yaniv, M., and Polonsky, KS. (1998). J. Clin. Invest. 101, 2215-2222.

Postic, C., and Magnuson, M.A. (2000a). Genesis 26, 149-150.

Postic, C., and Magnuson, M.A. (2000b). In "Molecular Pathogenesis of MODYs" (F.M. Matschinsky and M.A. Magnuson, eds.), Frontiers in Diabetes, vol. 15, pp 293-298. Karger, Basel, Switzerland.

Postic, C., and Magnuson, M.A. (2001). In "Molecular Basis of Endocrine Pancreas Development and Function"' (M.A. Hussain and J.F. Habener, eds.). Kluwer Academic Publishers, Norwell, Mass., in press.

Postic, C., Niswender, K.D., Decaux, J.-F., Parsa, R., Shelton, K.D., Gouhot, B., Pettepher, C.C., Granner, D.K., Girard, J., and Magnuson, M.A. (1995). Genomics 29, 740-750.

Postic, C., Shiota, M., Niswender, K.D., Jetton, T.L., Chen, Y., Moates, J.M., Shelton, K.D., Lindner, J., Cherrington, A.D., and Magnuson, M.A. (1999). J. Biol. Chem. 274, 305-315.

Rencurel, F., Waeber, G., Antoine, B., Rocchiccioli, F., Maulard, P., Girard, J., and Leturque, A. (1996). Biochem. J. 14, 903-909.

Roncero, I., Alvarez, E., Vazquez, P., and Blazquez, E. (2000). J. Neurochem. 74, 1848-1856.

Rossetti, L., Chen, W., Hu, M.Z., Hawkins, M., Barzilai, N., and Efrat, S. (1997). Am. J. Physiol. 36, E743-E750.

Sauer, B. (1993). Meth. Enzymol. 225, 890-900.

Sauer, B., and Henderson, N. (1988). Proc. Natl. Acad. Sci. U.S.A. 85, 5166-5170.

Sebastian, S., Horton, J.D., and Wilson, J. (2000). Biochem. Biophys. Res. Comm. 270, 886-891. 
Seoane, J., Gomez-Foix, A.M., O’Doherty, R.M., Gomez-Ara, C., Newgard, C.B., and Guinovart, J.J. (1996). J. Biol. Chem. 271, 23756-23760.

Shelton, K.D., Franklin, A., Khoor, A., Beechem, J., and Magnuson, M.A. (1992). Mol. Cell Biol. 12, 4578-4589.

Shih, H., and Towle, H.C. (1994). J. Biol. Chem. 269, 9380-9387

Shiota, C., Coffey, J., Grimsby, J., Grippo, J.F., and Magnuson, M.A. (1999). J. Biol. Chem. 274, 37125-37130.

Shiota, M., Postic, C., Fujimoto, Y., Jetton, T.L., Dixon, K., Pan, D., Grimsby, J., Grippo, J.F., Magnuson, M.A., and Cherrington, A.D. (2001). Diabetes, in press.

Soriano, P. (1999). Nature Genet. 21, 70-71.

Sreenan, S.K., Cockburn, B.N., Baldwin, A.C., Ostrega, D.M., Levisetti, M., Grupe, A., Bell, G.I., Stewart, T.A., Roe, M.W., and Polonsky, K.S. (1998). Diabetes 47, 1881-1888.

Stoffers, D.A., Ferrer, J., Clarke, W.L., and Habener, J.F. (1997a). Nature Genet. 17, 138-139.

Stoffers, D.A., Zinkin, N.T., Stanojevic, V., Clarke, W.L., and Habener, J.F. (1997b). Nature Genet. 15, 106-110.

Sturis, J., Kurland, I.J., Byrne, M.M., Mosekilde, E., Froguel, P., Pilkis, S.J., Bell, G.I., and Polonsky, K.S. (1994). Diabetes 43, 718-723.

Sweet, I.R., Li, G., Najafi, H., Berner, D., and Matschskiny, F.M. (1996). Am. J. Physiol. 271, E606-E625.

Tappy, L., Dussoix, P., Iynedjian, P., Henry, S., Schneiter, P., Zahnd, G., Jequier, E., and Philippe, J. (1997). Diabetes 46, 204-208.

Terauchi, Y., Sakura, H., Yasuda, K., Iwamoto, K., Takahashi, N., Ito, K., Kasai, H., Susuki, H., Ueda, O., Kamada, N., Jishage, K., Komeda, K., Noda, M., Kanazawa, N., Tanigushi, S., Miwa, I, Akanuma, Y., Kodama, T., Yasaki, Y., and Kadowaki, T. (1995). J. Biol. Chem. 270, 3025330256.

Thomas, P.M., Cote, G.J., Wohllk, N., Haddad, B., Matthew, P.M., Rabl, W., Aguilar-Bryan, L., Gagel, R.F., and Bryan, J. (1995). Science 268, 426-429.

Vandercarmmen, A., and Van Schaftingen, E. (1990). Eur. J. Biochem. 191, 483-489.

Van Schaftingen, E. (1989). Eur. J. Biochem. 179, 179-184.

Vaulont, S., and Kahn, A. (1994). FASEB J. 8, 28-35.

Velho, G., Petersen, K.F., Perseghin, G., Hwang, J.-H., Rothman, D.L., Pueyo, M.E, Cline, G.W., Froguel, P., and Shulman, G.I. (1996). J. Clin. Invest. 98, 1755-1761.

Velho, G., Blanche, H., Vaxillaire, M., Bellanne-Chantelot, C., Pardini, V.C., Timsit, J., Passa, P., Deschamps, I., Robert, J.-J., Weber, I.T., Marotta, D., Pilkis, S.J., Lipkind, G.M., Bell, G.I., and Froguel, P. (1997). Diabetologia 40, 217-224.

Vionnet, N., Stoffel, M., Takeda, J., Yasuda, K., Bell, G.I., Zouali, H., Sesage, S., Lesage, S., Velho, G., Iris, F., Passa, P., Froguel, P., and Cohen, D. (1992). Nature 356, 721-722.

Wang, H., and Iynedjian, P.B. (1997). Proc. Natl. Acad. Sci. U.S.A. 94, 4372-4377.

Weinhouse, S. (1976). Curr. Top. Cell Reg. 11, 1-50.

Wilson, J.E. (1995). Rev. Physiol. Biochem. Pharmacol. 126, 65-198.

Yamagata, K., Furuta, H., Oda, N., Kaisaki, P.J., Menzel, S., Cox, N.J., Fajans, S.S., Signorini, S., Stoffel, M., and Bell, G.I. (1996a). Nature 384, 458-460.

Yamagata, K., Oda, N., Kaisaki, P.J., Menzel, S., Furuta, H., Vaxillaire, M., Southam, L., Cox, R.D., Lathrop, G.M., Boriraj, V.V., Chen, X., Cox, N.J., Y., O., Yano, H., Le Beau, M.M., Yamada, S., Nishigori, H., Takeda, J., Fajans, S.S., Hattersley, A.T., Iwasaki, N., Hansen, T., Pedersen, O., Polonsky, K.S., Bell, G.I., et al. (1996b). Nature 384, 455-458. 



\author{
Nuclear Magnetic Resonance Studies of \\ Hepatic Glucose Metabolism in Humans \\ Michael Roden, ${ }^{*}$ Kitt Falk Petersen, ${ }^{\dagger}$ and Gerald I. Shulman ${ }^{\dagger}$ \\ "Division of Endocrinology and Metabolism, Department of Internal Medicine III, \\ University of Vienna Medical School, Vienna, A-I090, Austria;

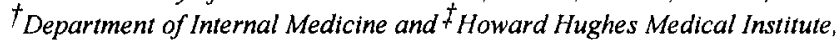 \\ Yale University, New Haven, Connecticut 06520
}

\begin{abstract}
Nuclear magnetic resonance (NMR) spectroscopy has made noninvasive and repetitive measurements of human hepatic glycogen concentrations possible. Monitoring of liver glycogen in real-time mode has demonstrated that glycogen concentrations decrease linearly and that net hepatic glycogenolysis contributes only about 50 percent to glucose production during the early period of a fast. Following a mixed meal, hepatic glycogen represents approximately 20 percent of the ingested carbohydrates, while only about 10 percent of an intravenous glucose load is retained by the liver as glycogen. During mixed-meal ingestion, poorly controlled type 1 diabetic patients synthesize only about 30 percent of the glycogen stored in livers of nondiabetic humans studied under similar conditions. Reduced net glycogen synthesis can be improved but not normalized by short-term, intensified insulin treatment. A decreased increment in liver glycogen content following meals was also found in patients with maturity-onset diabetes of the young due to glucokinase mutations (MODY2). In patients with poorly controlled type 2 diabetes, fasting hyperglycemia can be attributed mainly to increased rates of endogenous glucose production, which was found by ${ }^{13} \mathrm{C} \mathrm{NMR}$ to be due to increased rates of gluconeogenesis. Metformin treatment improved fasting hyperglycemia in these patients through a reduction in hepalic glucose production, which could be attributed to a decrease in gluconeogenesis. In conclusion, NMR spectroscopy has provided new insights into the pathogenesis of hyperglycemia in type 1 , type 2 , and MODY diabetes and offers the potential of providing new insights into the mechanism of action of novel antidabetic therapies.
\end{abstract}

\title{
I. Background
}

The pivotal role of the liver for maintaining normal glucose homeostasis resides in its ability to both deposit large amounts of glucose in the form of glycogen and to rapidly release glucose from its glycogen stores into the circulation by glycogenolysis. In addition, the liver - and, to a minor extent, the kidney - take up lactate, glycerol, and amino acids and convert these substrates to glucose by gluconeogenesis, which also contributes to glucose production. While hepatic glucose metabolism has been extensively investigated in vitro, many of its features remain unclear in humans. 
Prior to the introduction of in vivo NMR spectroscopy (Rothman et al., 1991a), studies in humans to estimate hepatic glucose metabolism were limited to either invasive or indirect approaches. Liver biopsies allow direct quantification of glycogen concentration but are invasive and cannot easily be repeated within one experiment (Behringer and Thaler, 1964; Nilsson et al., 1973,1974). Hepatic venous catheterization has been used to measure net splanchnic uptake of gluconeogenic substrates, thereby providing an estimate of hepatic gluconeogenesis (Wahren et al., 1972). Because this method does not account for intrahepatic gluconeogenic substrates or uptake of lactate and amino acids formed within the splanchnic bed (Björkman et al., 1990), it will underestimate hepatic gluconeogenesis. In addition, incorporation of labeled carbons into glucose using isotope dilution techniques (Consoli et al., 1987) does not permit quantification of gluconeogenesis (Landau, 1999; Landau et al., 1995,1996). Finally, mass isotopomer analysis following $\left[\mathrm{U}^{-13} \mathrm{C}_{6}\right]$ glucose (Tayek and Katz, 1997) or ${ }^{13} \mathrm{C}$ glycerol administration (Hellerstein et al., 1997) will underestimate gluconeogenesis, as was reviewed recently (Kelleher, 1999; Radziuk and Lee, 1999; Landau, 1999; Previs et al., 1999).

Carbon-13 nuclear magnetic resonance $\left({ }^{13} \mathrm{C}\right.$ NMK) spectroscopy has made noninvasive and repetitive measurements of hepatic glycogen concentrations in real-time mode possible, permitting the calculation of rates of net hepatic glycogen synthesis and glycogenolysis in vivo. Furthermore, using ${ }^{13} \mathrm{C}$-labeled glucose pulse- ${ }^{12} \mathrm{C}$ glucose chase experiments, rates of hepatic glycogen turnover can be assessed. Previous reviews have discussed other aspects of this topic (Alger et al., 1981; Shulman et al., 1984; Rothman et al., 1991b; Taylor and Shulman, 1994; Beckmann, 1995; Roden and Shulman, 1999).

\section{Basic Aspects of in Vivo NMR Spectroscopy}

The basic principles of in vivo NMR spectroscopy have been described previously in detail (Gadian et al., 1982; Taylor and Shulman, 1994; Roden and Shulman, 1999). Briefly, some nuclei possess magnetic properties - the magnetic moment or "spin." Within a strong, static magnetic field ( $B_{0}$ field) of a NMR spectrometer, the nuclei spin around their own axis with a characteristic frequency in order to align with or against the magnetic field. Stimulation of nuclei by an additional oscillating magnetic field $\left(B_{1}\right.$ field) at their frequency of processing causes these nuclei to swing out of alignment. Relaxation of the nuclei to the low-energetic state causes emission of radiowaves that are detected by a receiver coil. Under experimental conditions, resonant waves from various nuclei are superimposed, giving the picture of oscillating amplitudes in a display of intensities vs. time (free induction decay, FID). Fourier transformation translates the FID into a display of signal intensities vs. frequencies. The peak frequency is characteristic for a certain nucleus/compound; the area under that peak corresponds to 
its tissue concentration. That concentration can be converted into molar terms by comparison with the area under the peak of a phantom containing a defined concentration of that nucleus/compound (i.e., external standard) or by comparison with the resonance of an intrinsic compound with known concentration (i.e., internal standard). Several measures have been developed to improve the signalto-noise ratio of NMR spectroscopy: 1) using special ' $\mathrm{H}$ (decoupling) coils to eliminate the interaction between ${ }^{13} \mathrm{C}$ and its surrounding protons; 2) shimming procedures to increase the homogeneity of the static magnetic field by changing currents; 3 ) increasing the field strength of whole-body NMR spectrometers; 4) adding spectra prior to Fourier transformation; 5) placing surface coils tightly over the region of interest, to ensure homogenous tissue filling; and 6) selecting the pulse angle to suppress signals from other tissues such as the subcutaneous fat layer.

Despite a low natural abundance of $1.1 \%,{ }^{13} \mathrm{C}$ can be used to measure hepatic glycogen (Rothman et al., 1991a) and triglyceride content (Petersen et al., 1996a), which typically occur in concentrations over $50 \mathrm{mmol} / \mathrm{l}$. Since the resonance of ${ }^{13} \mathrm{C}$ in the $\mathrm{C} 1$ position of glycogen is clearly resolved at $100.5 \mathrm{ppm}$ and all ${ }^{13} \mathrm{C}$ signals from glycogen are detected by ${ }^{13} \mathrm{C}$ NMR spectroscopy (Shulman et al., 1988; Gruetter et al., 1994), the peak integral of $\mathrm{C} 1$ glycogen corresponds to a defined glycogen concentration (Figure 1). Validation of ${ }^{13} \mathrm{C}$ NMR spectroscopic measurements revealed an excellent agreement $(r=0.95)$ with classical chemical determination of glycogen over a broad range of glycogen concentrations (Shulman et al., 1988; Borgs et al., 1993), demonstrating that liver (Sillerud and Shulman, 1983; Shalwitz et al., 1987; Gruetter et al., 1994) and muscle (Gruetter et al., 1991; Taylor et al., 1992; Roussel et al., 1997) glycogen is essentially 100 percent visible by ${ }^{13} \mathrm{C}$ NMR. NMR spectrossopy is even more precise than the biopsy measurements. Coefficients of variation between multiple measurements in the same subject are 7 percent in liver (Rothman et al., 1991a).

In addition to quantification of natural abundance ${ }^{13} \mathrm{C}$ glycogen, ${ }^{13} \mathrm{C}$ NMR spectroscopy can be applied to trace ${ }^{13} \mathrm{C}$ incorporation into glycogen during infusion or ingestion of $\left[1-^{13} \mathrm{C}\right]$-enriched glucose (10-99 percent), which increases the sensitivity of the method by up to 100 -fold. Sequential infusions of ${ }^{13} \mathrm{C}$-enriched and unlabeled glucose $\left({ }^{13} \mathrm{C}\right.$ pulse- ${ }^{12} \mathrm{C}$ chase experiments) (Shulman et al., 1988) allow assessment of rates of glycogen synthesis and simultaneous glycogenolysis in humans (Magnusson et al., 1994; Roden et al., 1996; Petersen et al., 1998). In these experiments, the increment in total hepatic glycogen over time during infusion of $\left[1-{ }^{13} \mathrm{C}\right]$ glucose gives the flux through glycogen synthase. To obtain an estimate of glycogenolysis, the change of the $\left[1-{ }^{13} \mathrm{C}\right] \mathrm{glycogen}$ concentration is compared with the predicted increment, assuming constant flux through glycogen synthase and no glycogen breakdown. From the difference between predicted and observed glycogen concentrations, one can assess a minimum estimate of simultaneous glycogenolysis (i.e., only the glycogen that is broken down 


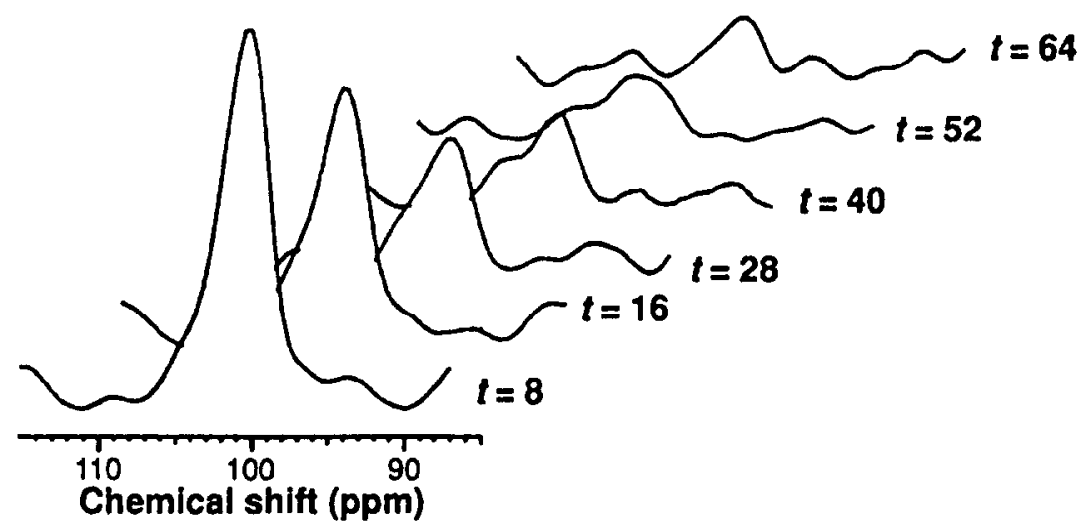

FIG. 1. Staggered plot of ${ }^{13} \mathrm{C}$ NMR spectra of the $\mathrm{Cl}$ position of natural abundance hepatic glycogen in one subject at indicated hours of fasting. [Reprinted with permission from Rothman, D.L., Magnusson, I., Katz, L.D., Shulman, R.G., and Shulman, G.I. Science 254, 573-576, 1991. Copyright 1991 American Association for the Advancement of Science.]

and escapes the hexose-1-phosphate pool). The ratio of glycogen breakdown to glycogen synthesis yields relative rates of glycogen turnover (Villar-Palasi and Larner, 1960).

The liver offers specific anatomical advantages to study glycogen metabolism by NMR spectroscopy. Its volume $\left(\sim 1500 \mathrm{~cm}^{3}\right)$ and proximity to the body surface allow homogenous tissue filling under the surface coil. Hepatocytes, which primarily account for glycogen metabolism, represent approximately 80 percent of parenchymal volume of the liver. In addition, glucose metabolism - and, in particular, the relative contribution of the pathways of glycogen synthesis - is similar across the liver acinus (Cline and Shulman, 1991,1995), although hepatocytes from periportal and perivenous zones of the liver acinus may differ in their enzyme pattern and subcellular structures (Jungermann and Katz, 1989). 


\title{
III. Studies on Liver Glycogen Metabolism in Humans
}

\author{
A. PHYSIOLOGIC REGULATION OF \\ HEPATIC GLYCOGEN METABOLISM
}

\section{The Postabsorptive State}

In the postabsorptive (i.e., fasting) state, both glycogenolysis and gluconeogenesis are required to maintain normal rates of endogenous glucose production. However, prior to NMR techniques, the relative contributions of gluconeogenesis and glycogenolysis to glucose production in humans were unknown.

Employing ${ }^{13} \mathrm{C}$ NMR spectroscopy in combination with magnetic resonance imaging (MRI) of liver volume and infusion of $\left[3-{ }^{3} \mathrm{H}\right]$ glucose to determine glucose production, the time course of hepatic glycogen concentrations was followed over 68 hours of fasting (Rothman et al., 1991a) (Figure 1). During the first 22 hours of fasting, liver glycogen decreased nearly linearly, from $396 \pm 29 \mathrm{mmol} / \mathrm{l}$ at 4 hours after a modest-sized meal of $650 \mathrm{kcal}$ to $251 \pm 30 \mathrm{mmol} / \mathrm{l}$ at 15 hours. This fasting concentration agreed with that of approximately $270 \mathrm{mmol} / \mathrm{l}$ obtained by needle biopsy (Nilsson and Hultman, 1973) and was later confirmed by another group employing ${ }^{13} \mathrm{C}$ NMR spectroscopy (Fried et al., 1996). Net rates of glycogenolysis were calculated to be $4.3 \pm 0.6 \mu \mathrm{mol} . \mathrm{kg}^{-1} \cdot \mathrm{min}^{-1}(0.20 \pm 0.02 \mu \mathrm{mol}$. $\left.\mathrm{l}^{-1} \cdot \mathrm{min}^{-1}\right)$, which surprisingly accounted for only 36 percent of whole-body glucose production (Figure 2). Thereafter, net glycogenolysis decreased to $1.7 \pm 0.5$ $\mu \mathrm{mol} . \mathrm{kg}^{-1} \cdot \mathrm{min}^{-1}$ between 22 hours and 46 hours and to $0.3 \pm 0.6 \mu \mathrm{mol} . \mathrm{kg}^{-1} \cdot \mathrm{min}^{-1}$ between 46 hours and 64 hours. After 64 hours, liver glycogen content and volume had decreased by 83 and 23 percent, respectively, and gluconeogenesis was almost exclusively (i.e., 96 percent) responsible for endogenous glucose production. The relatively large contribution of gluconeogenesis ( $64 \pm 5$ percent) to glucose production, even after 22 hours of fasting, is in contrast to earlier studies reporting less than 38 percent after a fast of 12-14 hours (Nilsson and Hultman, 1973; Wahren et al., 1972; Consoli et al., 1987). In order to estimate the contribution of glycogenolysis to glucose production during the earliest hours of an overnight fast, net glycogenolysis was followed 5-12 hours after a large, 1000-kcal meal (Petersen et al., 1996b). Whole-body glucose production was determined using $\left[6,6{ }^{2} \mathrm{H}\right] \mathrm{glucose}$, which yields a minimum estimate of glucose production (Miyoshi et al., 1988). Even under these conditions, net hepatic glycogenolysis (5.8 $\left.\pm 0.8 \mu \mathrm{mol} . \mathrm{kg}^{-1} \cdot \mathrm{min}{ }^{-1}\right)$ contributed only $45 \pm 6$ percent to whole-body glucose production. This result implies that gluconeogenesis typically contributes about 50 percent to the rate of whole-body glucose metabolism during the early hours following a mixed meal (Figure 2) (Petersen et al., 1996b). This provocative results was recently confirmed using a novel $\mathrm{D}_{2} \mathrm{O}$ method to directly assess gluconeogenesis (Landau et al., 1996). 


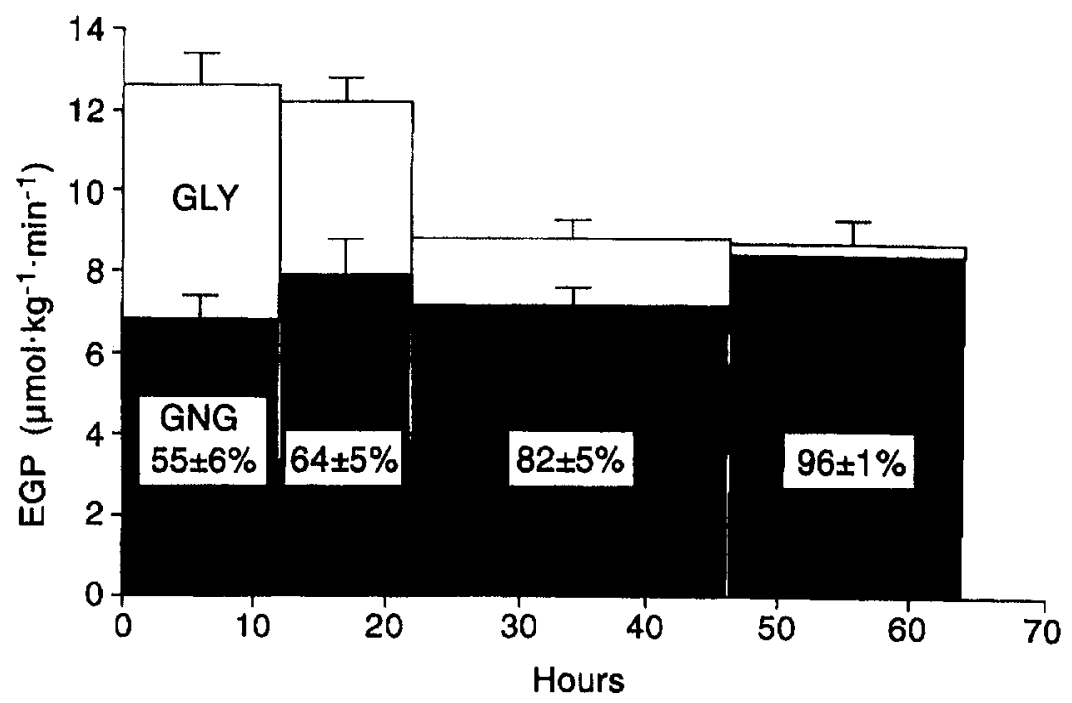

FIG. 2. Percent contribution of gluconeogenesis (GNG) and glycogenolysis (GLY) to glucose production during fasting. [Data from Rothman, D.L., Magnusson, I., Katz, L.D., Shulman, R.G., and Shulman, G.I. Science 254, 573-576, 1991; and Petersen, K.F., Price, T., Cline, G.W., Rothman, D.L., and Shulman, G.I. Am. J. Physiol. 270, E186-E191, 1996.].

\section{The Postprandial State}

Following an oral glucose load, hepatic glucose production is suppressed within 30 minutes (Taylor et al., 1996) and the liver takes up glucose to replenish hepatic glycogen stores. Typically, studies on the regulation of hepatic glucose removal were limited to measuring splanchnic glucose uptake by isotopic methods or hepatic vein catheterization (Davies et al., 1994; DeFronzo, 1997) but could not determine the fate of glucose within the liver.

When liver glycogen concentrations were monitored with ${ }^{13} \mathrm{C}$ NMR following a mixed meal, liver glycogen increased from $207 \pm 22 \mathrm{mmol} / \mathrm{l}$ after an overnight fast, to peak values of $316 \pm 19 \mathrm{mmol} / \mathrm{l}$ at 5 hours, giving a net rate of glycogen synthesis of $0.34 \mu \mathrm{mol} . \mathrm{I}^{-1} \cdot \mathrm{min}^{-1}$ (Taylor et al., 1996) (Figure 3). From these data, Taylor and coworkers (1996) calculated that $28.3 \pm 3.7 \mathrm{~g}$ glucose were deposited as glycogen in the liver, which represents approximately 19 percent of the carbohydrate contents in the meal. The data are consistent with estimates of about 25 percent obtained after an oral 150 -g glucose load by double tracer studies (Radziuk et al., 1978), splanchnic balance techniques (Katz et al., 1983), and a different NMR study (Beckmann et al., 1993). After the peak, liver glycogen concentrations decreased linearly, at a rate of $0.26 \mathrm{mmol}_{1 . \mathrm{l}^{-1}} \mathrm{~min}^{-1}$, in parallel with the fall of the insulin/glucagon ratio. 

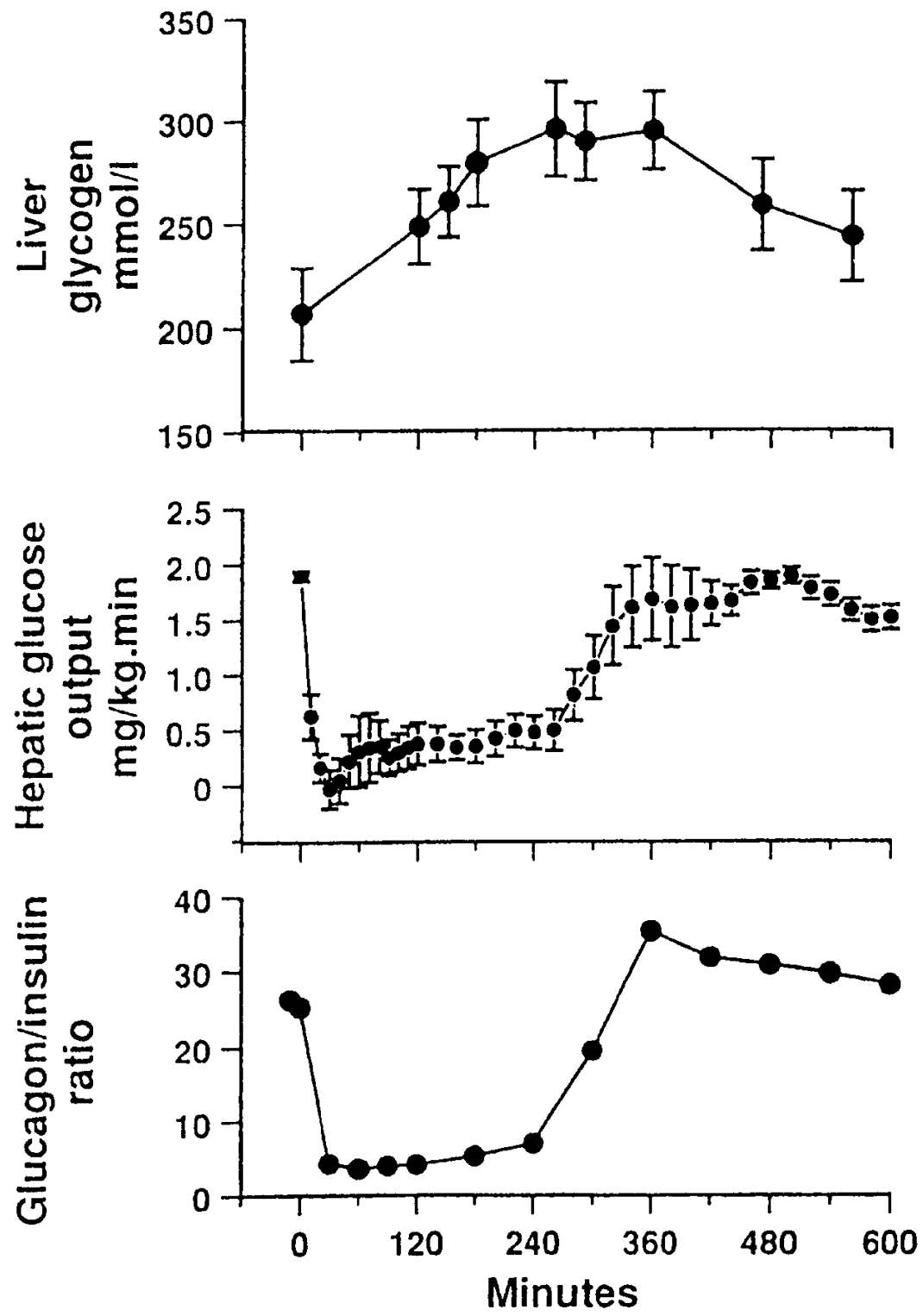

FIG. 3. Changes in liver glycogen concentration, endogenous glucose production, and plasma glucagon/insulin ratio after a mixed meal ingested at zero time. [Reprinted with permission from Taylor, R., Magnusson, I., Rothman, D.L., Cline, G.W., Caumo, A., Cobelli, C., and Shulman, G.I. J. Clin. Invest. 97, 126-132, 1996.] 
During an intravenous glucose load enriched with $\left[1-{ }^{13} \mathrm{C}\right]$ glucose, glycogen synthetic rate was $0.49 \pm 0.10 \mu \mathrm{mol}^{1-1} \cdot \mathrm{min}^{-1}$ (Cline et al., 1994). Net hepatic glycogen synthesis $\left(\sim 0.34 \mu \mathrm{mol}^{-1} \mathrm{I}^{-1} \mathrm{~min}^{-1}\right)$ accounted for around 12 percent of whole-body nonoxidative glucose metabolism. Using ${ }^{13} \mathrm{C}$ NMR spectroscopy, another group estimated that glucose uptake by the liver could account for up to 10 percent of the infused glucose under similar conditions of hyperglycemia $(\sim 9$ $\mathrm{mmol} / \mathrm{l} \mathrm{vs} .10 .5 \mathrm{mmol} / \mathrm{l})$ but lower plasma concentrations of insulin $(\sim 120 \mathrm{pmol} / 1$ vs. $400 \mathrm{pmol} / \mathrm{l})$ (Beckmann et al., 1993).

In order to examine changes in hepatic glycogen content under conditions of normal eating behavior, nondiabetic subjects ingested three mixed meals over the course of a day, with liver glycogen levels monitored following each meal (Hwang et al., 1995). Liver glycogen stores increased from $274 \pm 11 \mathrm{mmol} / \mathrm{l}$, reached a peak just before the next meal, and were maximal at 4 hours after dinner (420 \pm $19 \mathrm{mmol} / \mathrm{l})$. This indicates negligible net hepatic glycogenolysis, when meals are ingested 5 hours apart during the course of a day. Thus, glucose absorption from meals and gluconeogenesis account for the major part of whole-body glucose appearance during daytime, while net hepatic glycogenolysis contributes to whole-body glucose production only during the night.

\section{Regulation of Hepatic Glycogen Turnover by Nutritional State}

Studies in animals have provided evidence that hepatic glycogen phosphorylase may be active during periods of glycogen synthesis (Newman and Armstrong, 1978; van de Werve and Jeanrenaud, 1987). Simultaneous active glycogenolysis and synthesis is termed glycogen turnover or glycogen cycling (Villar-Palasi and Larner, 1960). Some authors reported that glycogen cycling is negligible in rat (Katz et al., 1979) or ranges between 2 and 12 percent in healthy and diabetic humans employing $\left[2-{ }^{3} \mathrm{H},{ }^{14} \mathrm{C}\right.$-]galactose infusions (Wajngot et al., 1991).

Combining ${ }^{13} \mathrm{C}$ NMR spectroscopy with the ${ }^{13} \mathrm{C}$ glucose pulse- ${ }^{12} \mathrm{C}$ glucose chase technique demonstrated that there is a substantial amount of glycogen turnover occuring, both in rat liver (Shulman et al., 1988; David et al., 1990) and in human liver (Magnusson et al., 1994; Roden et al., 1996; Petersen et al., 1998). Magnusson and colleagues (1994) studied healthy male subjects during hyperglycemia $(\sim 9.4 \mathrm{mmol} / \mathrm{l})$ maintained by sequential infusion of ${ }^{13} \mathrm{C}$-labeled and unlabeled glucose in the fed and fasted state. Fluxes through glycogen synthase were $0.43 \pm 0.09$ and $0.53 \pm 0.12 \mu \mathrm{mol}^{1 \mathrm{l}^{-1}} \cdot \mathrm{min}^{-1}$ and through glycogen phosphorylate were $0.15 \pm 0.06$ and $0.30 \pm 0.06 \mu \mathrm{mol} . \mathrm{l}^{-1} \cdot \mathrm{min}^{-1}$ in the fed and in the fasted states, respectively. Calculating the ratio of glucose phosphorylase flux to glycogen synthase flux yielded minimum estimates of relative glycogen turnover rate of 31 \pm 8 percent in the fed state and of $57 \pm 3$ percent in the fasted state. Considering the linear correlation between hepatic glycogen concentration and glycogenolysis, it is conceivable that the higher liver glycogen content in the fed state may itself 
stimulate glycogen breakdown by hepatic autoregulation (Clore et al., 1991; Cherrington et al., 1998).

\section{Regulation of Hepatic Glycogen Turnover by Glucose, Insulin, and Glucagon}

Hormones such as insulin and glucagon are known to affect the enzymes involved in glycogen metabolism. The rate of hepatic glycogen synthesis depends on portal vein insulin, requiring concentrations in the $130-170 \mathrm{pmol} / \mathrm{l}$ range for half-maximal stimulation of glycogen synthesis (Roden et al., 1996) (Figure 4). This agrees well with the concentration for suppression of glucose production by insulin (DeFronzo et al., 1983; Kolterman et al., 1980; Rizza et al., 1981). Although the overall flux through glycogen synthase might plateau at high insulin levels, substantial differences in net glycogen synthesis and glycogen turnover occur.

The ${ }^{13} \mathrm{C}$-labeled glucose- ${ }^{12} \mathrm{C}$ glucose chase technique was used to assess flux rates through glycogen phosphorylase in healthy humans during hypoglucagonemic $(\sim 30 \mathrm{ng} / \mathrm{l})$ conditions of 1$)$ hyperglycemic $(\sim 10 \mathrm{mmol} / \mathrm{l})$-hypoinsulinemia $(\sim 40 \mathrm{pmol} / \mathrm{l}) ; 2)$ euglycemic $(\sim 5 \mathrm{mmol} / \mathrm{l})$-hyperinsulinemia $(\sim 400$ pmol/l); and 3) hyperglycemic $(\sim 10 \mathrm{mmol} / \mathrm{l})$-hyperinsulinemia $(\sim 400 \mathrm{pmol} / \mathrm{l})$ (Petersen et al., 1998) (Figure 5). Hyperglycemia per se inhibited net hepatic glycogenolysis, primarily through inhibition of glycogen phosphorylase flux,

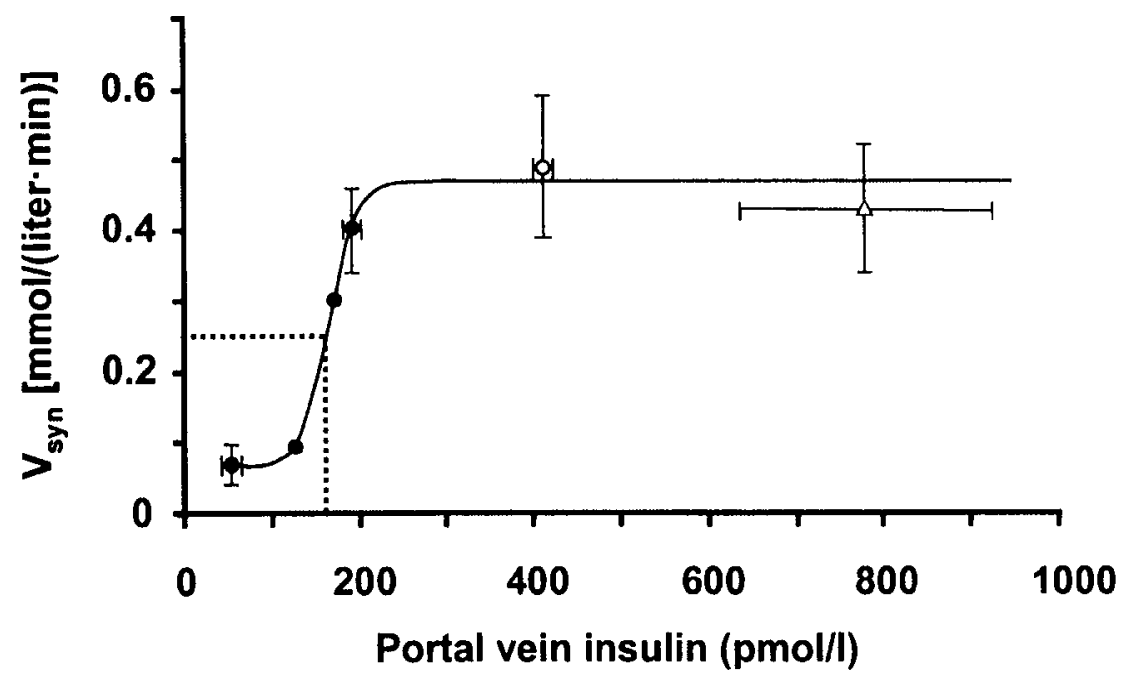

FIG. 4. Concentration dependence of mean glycogen synthetic rate at varying (portal vein) insulin concentrations under conditions of intravenous glucose infusion and suppressed glucagon secretion. [Reprinted with permission from Roden, M., Perseghin, G., Petersen, K.F., Hwang, J.H., Cline, G.W., Gerow, K., Rothman, D.L., and Shulman, G.I. J. Clin. Invest. 97, 642-648, 1996.] 


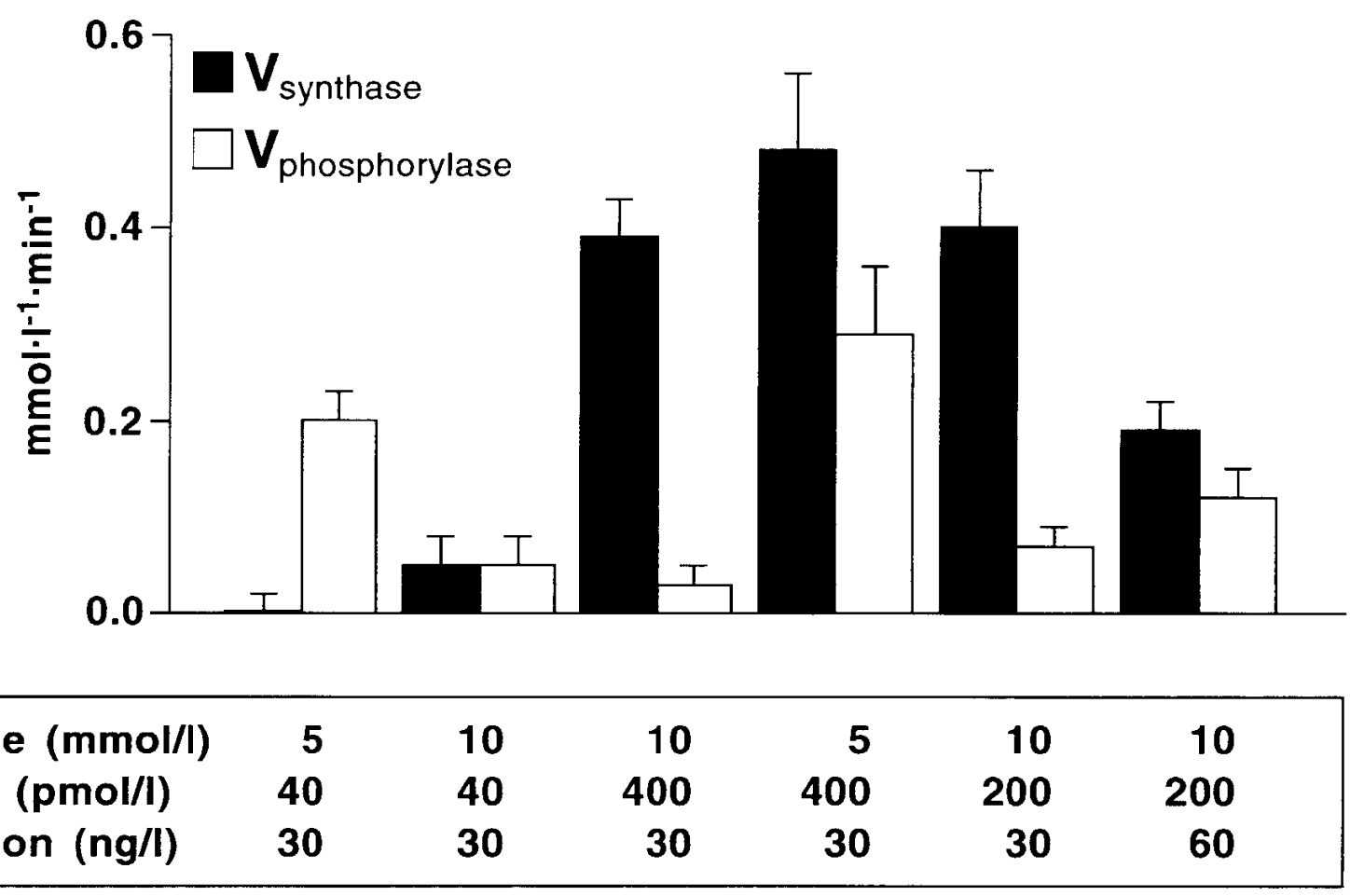

\begin{tabular}{|lrrrrrr|}
\hline Glucose (mmol/l) & 5 & 10 & 10 & 5 & 10 & 10 \\
Insulin (pmol/l) & 40 & 40 & 400 & 400 & 200 & 200 \\
Glucagon (ng/l) & 30 & 30 & 30 & 30 & 30 & 60 \\
\hline
\end{tabular}

FIG. 5. Hepatic flux rates through glycogen synthase $\left(V_{\text {synthase }}\right)$ and glycogen phosphorylase $\left(V_{\text {phosphorylase }}\right)$ in healthy humans. [Data from Roden, $M$., Perseghin, G. Petersen, K.F., Hwang, J.H., Cline, G.W., Gerow, K., Rothman, D.L., and Shulman, G.I. J. Clin. Invest. 97,642-648, 1996; Petersen, K.F., Krssak, M., Navarro, V., Chandramouli, V., Hundal, R., Schumann, W.C., Landau, B.R., and Shulman, G.I. Am. J. Physiol. 276, E529-E535, 1998.] 
while hyperinsulinemia per se inhibited net hepatic glycogenolysis, primarily through stimulation of glycogen synthase flux. When hyperglycemia was combined with hyperinsulinemia, phosphorylase flux was inhibited and glycogen synthesis was activated, resulting in the highest rates of net hepatic glycogen synthesis (Figure 5).

To determine the role of glucagon on hepatic glycogen synthesis and cycling, hyperglycemic $(\sim 10 \mathrm{mmol} / \mathrm{l})$-somatostatin clamps were performed under conditions of 1) fasting portal vein insulinemia $(\sim 200 \mathrm{pmol} / \mathrm{l})$-hypoglucagonemia $(\sim$ $30 \mathrm{ng} / \mathrm{l}), 2)$ fasting portal vein insulinemia $(\sim 200 \mathrm{pmol} / \mathrm{l})$ and glucagonemia $(\sim$ $60 \mathrm{ng} / \mathrm{l})$, and 3) fasting peripheral insulinemia ( $\sim 50 \mathrm{pmol} / \mathrm{l})$-hypoglucagonemia $(\sim 60 \mathrm{ng} / \mathrm{l})$ (Roden et al., 1996) (Figure 5). Suppression of glucagon secretion doubled the hepatic glycogen synthase flux and decreased glycogen turnover by approximately 73 percent, so that net glycogen synthesis was markedly increased, compared with conditions of fasting plasma glucagon concentrations.

\section{B. HEPATIC GLYCOGEN METABOLISM IN DISEASE}

\section{Type I Diabetes Mellitus (DM)}

In order to examine the effect of type 1 diabetes on hepatic glycogen synthesis, patients with poorly controlled (i.e., glycosylated hemoglobin, $10.2+0.3$ percent) type 1 diabetes were studied during a normal-life day during which they ingested three mixed meals. Compared to healthy control subjects, these patients had similar fasting hepatic glycogen stores but, at the end of the day, they had synthesized only around 30 percent of the glycogen stored in the livers of the nondiabetic subjects studied under similar conditions (Hwang et al., 1995) (Figure 6). In addition, the contribution of the gluconeogenic relative to the direct pathway of glycogen synthesis was higher in the type 1 diabetic patients over the 5 hours after breakfast ( $59 \pm 4$ percent vs. $35 \pm 4$ percent of control).

To examine the effect of intensified insulin treatment on hepatic glycogen metabolism, type 1 diabetic patients with moderate metabolic control (i.e., mean glycosylated hemoglobin $A_{1 c}$ of $8.8 \pm 0.3$ percent) were examined before and during variable insulin infusion, resulting in near-normal glycemia for 24 hours (Bischof et al., 1999). After dinner, rates of net glycogen synthesis in the type 1 diabetic patients were approximately 25 percent of that in healthy volunteers and doubled during intensified insulin treatment. Nevertheless, the rate was still markedly lower, compared with the control group $(0.11 \pm 0.02$ vs. $0.23 \pm 0.04 \mu \mathrm{mol}$. $\left.\mathrm{I}^{-1} \cdot \mathrm{min}^{-1}\right)$. During overnight fasting, net hepatic glycogenolysis was reduced by about 50 percent in the type 1 diabetic patients and increased by around 40 percent by intensified insulin treatment but was still lower than in the control group $(0.14$ 


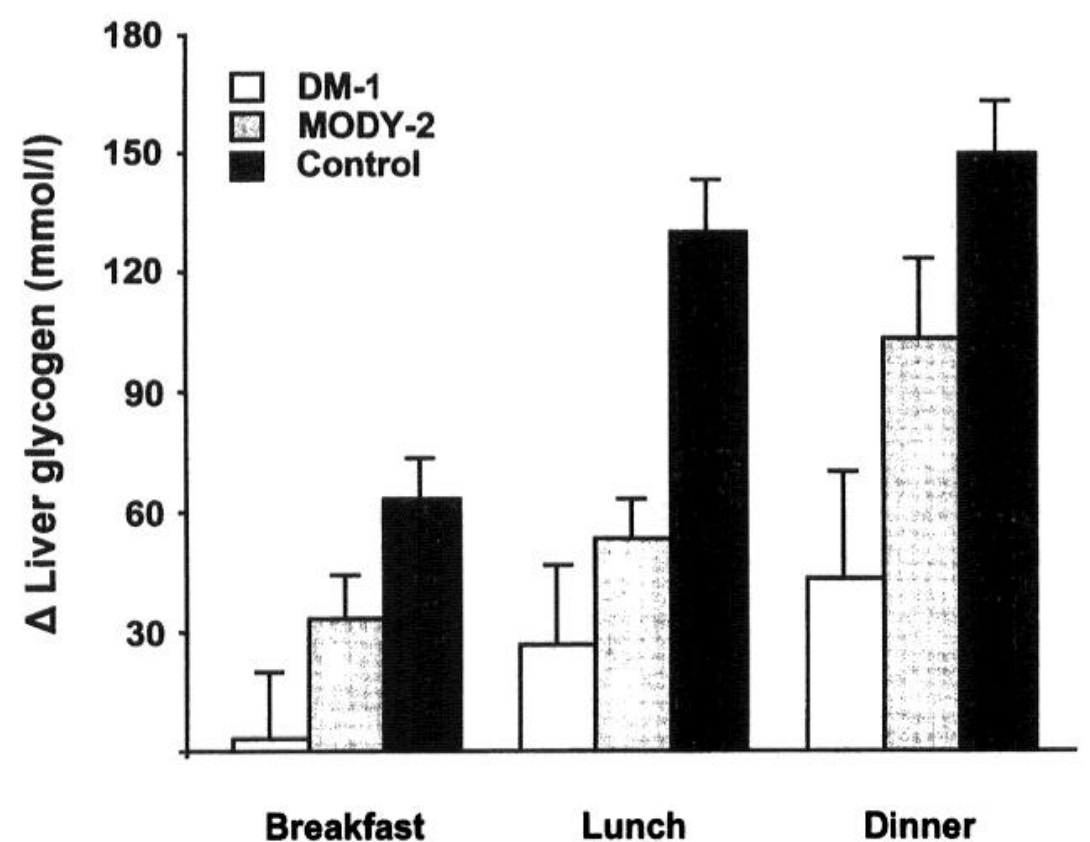

FIG. 6. Increments in hepatic glycogen concentration in type 1 diabetic (DM-1), maturity-onset diabetic (MODY-2), and healthy subjects (CONTROL) during a day in which three isocaloric mixed meals were ingested 5 hours apart. [Reprinted with permission from Hwang, J.H., Perseghin, G., Rothman, D.L., Cline, G.W., Magnusson, I., Petersen, K.F., and Shulman, G.I. J. Clin. Invest. 95, 783-787, 1995; and Velho, G., Petersen, K.F., Perseghin, G., Hwang, J.H., Rothman, D.L., Pueyo, M.E., Cline, G.W., Froguel, P., and Shulman, G.I. J. Clin. Invest. 98, 1755-1761, 1996.]

\pm 0.01 vs. $\left.0.19 \pm 0.01 \mu \mathrm{mol}^{1} \mathrm{l}^{-1} \cdot \mathrm{min}^{-1}\right)$. Thus, short-term improvement of metabolic control resulting in near-normoglycemia and peripheral hyperinsulinemia was not sufficient to completely reverse the abnormalities of hepatic glycogen metabolism in type 1 diabetes.

On the other hand, in the presence of both hyperglycemia and hyperinsulinemia, rates of hepatic glycogen synthesis did not differ between poorly controlled (mean glycosylated hemoglobin, $14.1 \pm 1.7$ percent) type 1 diabetic and nondiabetic subjects $\left(0.49 \pm 0.10\right.$ vs. $\left.0.46 \pm 0.07 \mu \mathrm{mol}^{1-1} \cdot \mathrm{min}^{-1}\right)$ (Cline et al., 1994). These data suggest that glycogen synthesis can be normalized under highglucose, high-insulin, but low-glucagon conditions in type 1 diabetic patients. However, the percent contribution of the direct pathway to glycogen synthesis 
remained lower in type 1 diabetic than in nondiabetic subjects, despite the presence of hyperinsulinemia ( 35 vs. 60 percent). This agrees with the results obtained after meal ingestion (Hwang et al., 1995), reflecting higher gluconeogenic rates that are not acutely normalized by hyperinsulinemia (Cline et al., 1994).

\section{Type $2 D M$}

It is well established that fasting hyperglycemia in patients with type 2 diabetes is strongly related to increased rates of endogenous glucose production (Fery, 1994; Bogardus et al., 1984; DeFronzo, 1997). These increased rates of glucose production could be secondary to increased rates of gluconeogenesis, hepatic glycogenolysis, or both. In order to address this question, rates of net hepatic glycogenolysis were measured in patients with poorly controlled type 2 diabetes (mean hemoglobin $A_{1 c}, 12 \pm 1$ percent) and gluconeogenesis was calculated as the difference between rates of glycogenolysis and whole-body glucose production (Magnusson et al., 1992) (Figure 7). Despite pair feeding, liver glycogen concentration was lower in type 2 diabetic than in healthy control subjects at 4 hours after a meal ( $131 \pm 20$ vs. $282 \pm 60 \mathrm{mmol} / \mathrm{l}$ liver $)$, consistent with a defect in hepatic glycogen synthesis in type 2 diabetes (Magnusson et al., 1992). The postprandial rise in the rate of whole-body glucose production (around 25 percent), estimated by using $\left[6{ }^{3} \mathrm{H}\right] \mathrm{glucose}$, was accompanied by decreased rates of net hepatic glycogenolysis (around 50 percent). Thus, increased gluconeogenesis could completely account for the higher rates of glucose production obtained in patients with poorly controlled type 2 diabetes.

The effect of the oral hypoglycemic drug, metformin, which decreases fasting plasma glucose concentrations by reducing hepatic glucose production (Cusi and DeFronzo, 1997; Inzucchi et al., 1998), was examined in poorly controlled (i.e., fasting plasma glucose, $\sim 15.5 \mathrm{mmol} / \mathrm{l}$ ) type 2 diabetic patients by employing two independent methods: 1) ${ }^{13} \mathrm{C}$ NMR spcctroscopy of the liver, combined with determination of glucose production with $\left[6,6^{2} \mathrm{H}\right]$ glucose, gives the rate of net hepatic glycogenolysis to glucose production; and 2) the ratio of ${ }^{2} \mathrm{H}$ enrichments in carbon 5 over carbon 2 in blood glucose upon administration of ${ }^{2} \mathrm{H}_{2} \mathrm{O}$ gives the contribution of gluconeogenesis to glucose production (Landau et al., 1995,1996). Rates of glucose production and gluconeogenesis were two- and three-fold higher in diabetic than in healthy subjects (Hundal et al., 2000). Treatment with metformin for 3 months reduced fasting plasma glucose concentration by approximately 30 percent, glucose production by approximately 24 percent, and gluconeogenesis by approximately 36 percent. Employing the ${ }^{2} \mathrm{H}_{2} \mathrm{O}$ method, gluconeogenesis was two-fold higher in the diabetic patients and was reduced by 


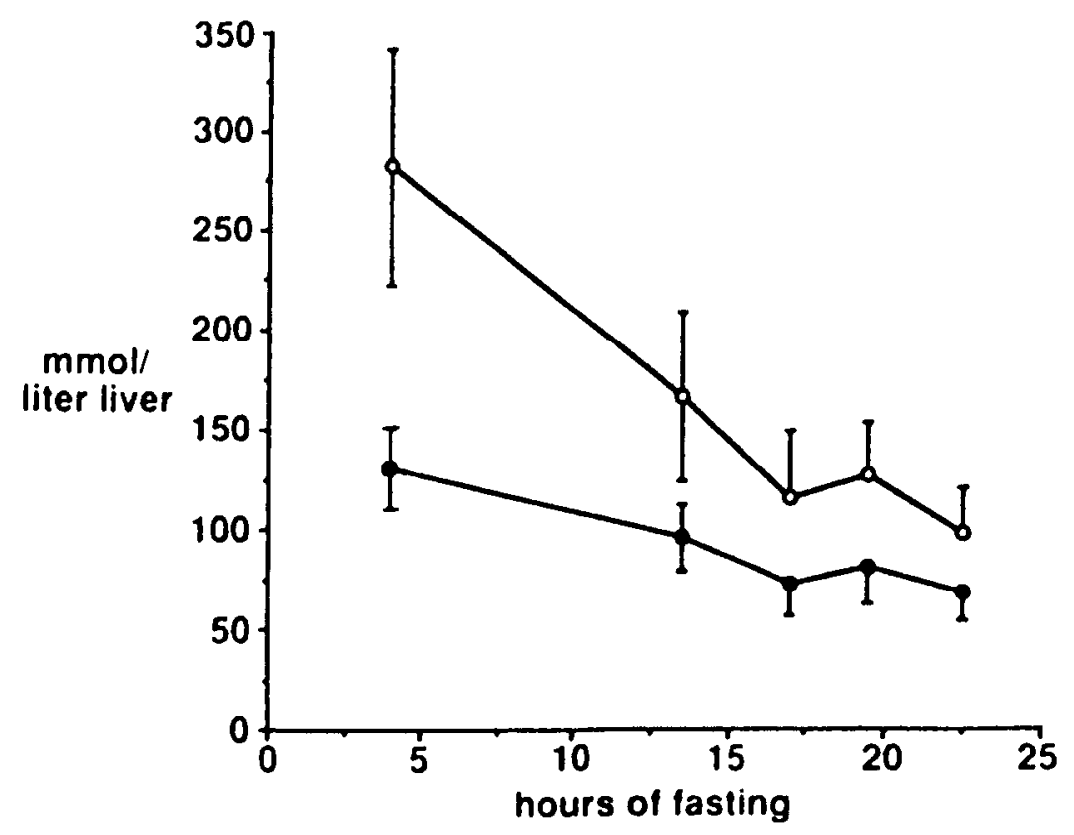

FIG. 7. Time course of mean liver glycogen concentration in type 2 diabetic and healthy subjects. [Reprinted with permission from Magnusson, I., Rothman, D.L., Katz, L.D., and Shulman, G.I. $J$. Clin. Invest. 90, 1323-1327, 1992.]

around 33 percent by metformin treatment. Net hepatic glycogenolysis (by ${ }^{13} \mathrm{C}$ NMR) was lower before $\left(0.11 \pm 0.02 \mathrm{mmol} \cdot \mathrm{m}^{-2} \cdot \mathrm{min}^{-1}\right)$ but almost normalized after metformin therapy $\left(0.15 \pm 0.02 \mathrm{mmol} \cdot \mathrm{m}^{-2} \cdot \mathrm{min}^{-1}\right.$ vs. control of $0.18 \pm 0.03$ mmol. $\left.\mathrm{m}^{-2} \cdot \mathrm{min}^{-1}\right)$. Since ${ }^{13} \mathrm{C}$ NMR spectroscopy measures net hepatic glycogenolysis, while the ${ }^{2} \mathrm{H}_{2} \mathrm{O}$ method indirectly measures total glycogenolysis (glucose production minus gluconeogenesis), the difference between total and net glycogenolysis can be used to assess hepatic glycogen cycling. Glycogen cycling was negligible in healthy subjects but contributed as much as 25 percent of glucose production in type 2 diabetic patients. The reduction of gluconeogenesis by metformin also may be due in part to the reduction of plasma free fatty acids, which have been shown to increase gluconeogenesis (Chen et al., 1999; Roden et al., 2000; Stingl et al., in press). 


\section{Maturity Onset Diabetes of Young 2(MODY2)}

Studies in a familial subgroup of diabetes, MODY 2, have shed some light on the potential intrahepatic defects occurring in type 2 DM. MODY 2 subjects present with moderate (postprandial) hyperglycemia due to autosomal dominant mutations in the coding regions of the hepatic and pancreatic isoforms of the glucokinase gene (Stoffel et al., 1992; Froguel et al., 1993). During a study employing three mixed meals over 1 day, the net increase in liver glycogen was 30-60 percent lower in MODY 2 than in control subjects. At the end of the day, glucokinase-deficient subjects had only about 70 percent of the increment in glycogen observed in control subjects (Figure 6) (Velho et al., 1996). In addition, the relative contribution of the gluconeogenic pathway to glycogen synthesis was higher in these subjects during the 4 hours after breakfast ( $50 \pm 2$ percent vs. 34 \pm 5 percent of control). Since relative hypoinsulinemia was present in the MODY 2 patients only during the first 2 hours following breakfast, the decreased insulin/glucagon ratio cannot entirely explain the reduction of liver glycogen synthesis following lunch and dinner and suggests that decreased activity of hepatic glucokinase is responsible for the impaired glycogen storage and postprandial hyperglycemia during these periods. From these results, one might speculate that the similar defects of liver glucose metabolism observed in patients with poorly controlled type 1 and type 2 DM might be related at least in part to an acquired abnormality of hepatic glucokinase.

\section{Liver Cirrhosis}

Hepatic glucose metabolism is generally thought to be altered in liver cirrhosis, although rates of glucose production were found to be decreased only in some (Owen et al., 1981; Merli et al., 1986) but not all studies (Shmueli et al., 1993; Petrides et al., 1991). To examine hepatic glycogen metabolism, Petersen and coworkers (1999) studied patients with biopsy-confirmed liver cirrhosis by combining ${ }^{13} \mathrm{C}$ NMR spectroscopy with $\left[6,6-{ }^{2} \mathrm{H}\right]$ glucose infusion and the ${ }^{2} \mathrm{H}_{2} \mathrm{O}$ method described above. Despite comparable rates of glucose production, net hepatic glycogenolysis was about 3.5 -fold lower in cirrhotic patients $(1.1 \pm 0.4$ $\mu \mathrm{mol} . \mathrm{kg}^{-1} \cdot \mathrm{min}^{-1}$ vs $3.9 \pm 0.9 \mu \mathrm{mol} . \mathrm{kg}^{-1} \cdot \mathrm{min}^{-1}$ ) and the relative contribution of gluconeogenesis was markedly increased. Using the ${ }^{2} \mathrm{H}_{2} \mathrm{O}$ method, the contribution of gluconeogenesis to glucose production was also found to be markedly higher in the cirrhotic patients compared to the healthy subjects $(68 \pm 3$ percent vs. $54 \pm 2$ percent). From these data, rates of total glycogenolysis can be calculated to be approximately $3.2 \mu \mathrm{mol} . \mathrm{kg}^{-1} . \mathrm{min}^{-1}$ and $4.6 \mu \mathrm{mol} . \mathrm{kg}^{-1} . \mathrm{min}^{-1}$, respectively. Glycogen cycling, as assessed from the difference between total and net glyco- 
genolysis, contributed to about 23 percent and 7 percent of glucose production in cirrhotic and healthy subjects, respectively. Increased glycogen cycling in liver cirrhosis may result from the approximately 2.5 -fold higher plasma concentrations of insulin and glucagon, both of which stimulate glycogen turnover (Magnusson et al., 1994; Petersen et al., 1998; Roden et al., 1996). These patients also had increased plasma concentrations of free fatty acids, which stimulate glycogen cycling by increasing gluconeogenesis (Chen et al., 1999; Roden et al., 2000) and decreasing net hepatic glycogenolysis (Stingl et al., in press). Finally, the lower insulin-like growth factor-I (IGF-I) concentrations may favor skeletal muscle proteolysis with subsequent stimulation of gluconeogenesis by amino acids in patients with liver cirrhosis.

\section{Glycogen Storage Diseases}

${ }^{13} \mathrm{C}$ NMR spectroscopy also may be useful to identify patients with glycogen storage disease. Elevated hepatic glycogen concentrations were reported in one patient with type IIIA glycogenosis (Beckmann et al., 1990), which is characterized by inactivation of the amylo-1,6-glucosidase debrancher enzyme, and in one patient with type IA glycogenosis, which is due to deficiency of glucose-6phosphatase (Roser et al., 1996).

\section{Summary and Conclusions}

Application of NMR spectroscopy offers the unique ability to noninvasively and continuously monitor intracellular substrates in humans. With this method, the time course of hepatic glycogen concentrations can be followed both in the fasted and in the postprandial state in vivo. Using this technique, substrate fluxes through metabolic pathways such as glycogen synthase and glycogen phosphorylase can be examined. Moreover, NMR spectroscopy has made it feasible to detect defects of hepatic glycogen accumulation in poorly controlled type 1 , type 2 , and MODY-2 diabetic patients. NMR spectroscopy identified increased gluconeogenesis as the major cause of elevated glucose production in patients with poorly controlled type 2 diabetes. These studies demonstrate that NMR spectroscopy can be applied effectively in humans and that it has provided new insights into the pathogenesis of type 1, type 2 , and MODY-2 diabetes.

\section{REFERENCES}

Alger, J.R., Sillerud, L.O., Behar, K.J., Gillies, R.J., Shulman, R.G., Gordon, R.E., Shaw, D., and Hanley, P.E. (1981). Science 214, 660-662.

Beckmann, N. (1995). In "Carbon-13 NMR Spectroscopy of Biological Systems" (N. Beckmann, ed.), chap. 6, pp. 269-322. Academic Press. San Diego, Calif. 
Beckmann, N., Seelig, J., and Wick, H. (1990). Magnet. Reson. Med. 16, 150-160.

Beckmann. N., Fried, R., Turkalj, I., Seelig, J., Keller, U., and Stalder, G. (1993). Magnet. Reson. Med. 29, 583-590.

Behringer, A., and Thaler, H. (1964). Wien. Klin. Wschr. 76, 627-630.

Bischof, M.G., Krssak, M., Krebs, M., Stingl, H., Hofer, A., Fürnsinn, C., Waldhausl, W., and Roden, M. (1999). Diabetes 48(suppl. 1), A29l (abstract).

Björkman, O., Eriksson, L.S., Nyberg, B., and Wahren, J. (1990). Diabetes 39, 747-751.

Bogardus, C., Lillioja, S., Howard, B.V., Reaven, G., and Mott, D. (1984). J. Clin. Invest. 74, 1238-1246. Borgs, M., van Hecke, P., Overloop, K., Decaniere, C., van Huffel, S., Stalmans, W., and Vanstapel, F. (1993). NMR Biomed. 6, 371-376.

Chen, X., Iqbal, N., and Boden, G. (1999). J. Clin. Invest. 103, 365-372

Cherrington, A.D., Edgerton, D., and Sindelar, D.K. (1998). Diabetologia 41, 987-996.

Cline, G.W., and Shulman, G.I. (1991). J. Biol. Chem. 266, 4094-4098.

Cline, G.W., and Shulman, G.I. (1995). J. Biol. Chem. 270, 28062-28067.

Cline, G.W., Rothman, D.L., Magnusson, I., Katz, L.D., and Shulman, G.I. (1994). J. Clin. Invest. 94, 2369-2376.

Clore, J.N., Glickman, P.S., Nestler, J.E., and Blackard, W.G. (1991). Am. J. Physiol. 261, E425-E429.

Consoli, A. (1992). Diabetes Care 15, 430-441.

Consoli, A., Kennedy, F., Miles, J., and Gerich, J. (1987). J. Clin. Invest. 80, 1303-1310

Cusi, K., and DeFronzo, R.A. (1998). Diabetes Rev. 6, 89-131.

David, M., Petit, W.A., Laughlin, M.R., Shulman, R.G., King, J.E., and Barrett, E.J. (1990). J. Clin. Invest. 86, 612-617.

Davies, S.N., Pagliassotti, M., and Cherrington, A.D. (1994). In "The Diabetes Annual" (S.M. Marschall and P.D. Home, eds.), vol. 8, pp. 119-133.

DeFronzo, R.A. (1997). Diabetes Rev. 5, 177-269.

DeFronzo, R.A., Ferrannini, E., Hendler, R., Felig, P., and Wahren, J. (1983). Diabetes 32, 35-45

Fery, F. (1994). J. Clin. Endocrinol. Metab. 78, 536-542.

Fried, R., Beckmann, N., Keller, U., Ninnis, R., Stalder, G., and Seelig, J. (1996). Am. J. Physiol. 270, G14-G19.

Froguel, P., Zouali, H., Vionnet, N., Velho, G., Vaxillaire, M., Sun, F., Lesage, S., Stoffel, M., Takeda, J., Passa, P., et al. (1993). N. Engl. J. Med. 328, 697-702.

Gadian, D.G. (ed.) (1982). "Nuclear Magnetic Resonance and Its Applications to Living Systems." Clarendon Press, Clarendon, III.

Gruetter, R., Prolla, T.A., and Shulman, R.G. (1991). Magnet. Reson. Med. 20, 327-332.

Gruetter, R., Magnusson, I., Rothman, D.L., Avison, D.L., Shulman, R.G., and Shulman, G.I. (1994). Magnet. Reson. Med. 31, 583-588.

Hellerstein, M.K., Neese, R.A., Linfoot, P., Christiansen, M., Turner, S., and Letscher, A. (1997). J. Clin. Invest. 100, 1305-1319.

Hundal, R., Krssak, M., Dufour, S., Laurent, D., Lebon, V., Chandramouli, V., Inzucchi, S., Schumann, W.C., Petersen, K.F., Landau, B.R., and Shulman, G.I. (2000). Diabetes 59, 2063-2069.

Hwang, J.H., Perseghin, G., Rothman, D.L., Cline, G.W., Magnusson, I., Petersen, K.F., and Shulman, G.I. (1995). J. Clin. Invest. 95, 783-787.

Inzucchi, S.E., Maggs, D.G., Spollett, G.R., Page, S.L., Rife, F.S., Walton, V, and Shulman, G.I. (1998). N. Engl. J. Med. 338, 867-872.

Jungermann, K., and Katz, N. (1989). Physiol. Rev. 69, 708-764.

Katz, J., Goiden, S., and Wals, P.A. (1979). Biochem. J. 180, 389-402.

Katz, L.D., Glickman, M.G., Rapoport, S., Ferrannini, E., and DeFronzo, R.A. (1983). Diabetes 32, 675-679. 
Kelleher, J.K. (1999). Am. J. Physiol. 277, E395-E400.

Kolterman, O.G., Insel, J., Saekow, M., and Olefsky, J.M. (1980). J. Clin. Invest. 65, 1272-1284.

Landau, B.R. (1999). Proc. Nutrit. Soc. 58, 963-972.

Landau, B.R., Wahren, J., Chandramouli, V., Schumann, W.C., Ekberg, K., and Kalhan, S.C. (1995). J. Clin. Invest. 95, 172-178.

Landau, B.R., Wahren, J., Chandramouli, V., Schumann, W.C., Ekberg, K., and Kalhan, S.C. (1996). J. Clin. Invest. 98, 378-385.

Magnusson, I., Rothman, D.L., Katz, L.D., and Shulman, G.I. (1992). J. Clin. Invest. 90, 1323-1327.

Magnusson, I., Rothman, D.L., Jucker, B., Cline, G.W., Shulman, R.G., and Shulman, G.I. (1994). Am. J. Physiol. 266, E796-E803.

Merli, M., Eriksson, L.S., Hagenfeldt, L., and Wahren, J. (1986). J. Hepatol. 3, 348-355.

Miyoshi, H., Shulman, G.I., Peters, E.J., Wolfe, M.H., Elahi, D., and Wolfe, R.R. (1988). J. Clin. Invest. 81, 1545-1555.

Newman, J.D., and Armstrong, I.M. (1978). Biochim. Biophys. Acta 544, 225-233.

Nilsson, L.H., and Hultman, E. (1973). Scand. J. Clin. Lab. Invest. 32, 325-330.

Nilsson, L.H., and Hultman, E. (1974). Scand. J. Clin. Lab. Invest. 33, 5-10.

Olefsky, J.M., Kolterman, O.G., and Scarlett, J.A. (1982). Am. J. Physiol. 243, E15-E30.

Owen, D.E., Reichle, F.A., Mozzoli, M.A., Kreulen, T., Patel, M.S., Elfenbein, I.B., Golsorkhi, M., Chang, K.H., Rao, N.S., Sue, H.S., and Boden, G. (1981). J. Clin. Invest. 68, 240-252.

Petersen, K.F., West, A.B., Reuben, A., Rothman, D.L., and Shulman, G.I. (1996a). Hepatology 24, 114-117.

Petersen, K.F., Price, T., Cline, G.W., Rothman, D.L., and Shulman, G.I. (1996b). Am. J. Physiol. 270, E186-E191.

Petersen, K.F., Laurent, D., Rothman, D.L., Cline, G.W., and Shulman, G.I. (1998). J. Clin. Invest. 101, 1203-1209.

Petersen, K.F., Krssak, M., Navarro, V., Chandramouli, V., Hundal, R., Schumann, W.C., Landau, B.R., and Shulman, G.I. (1999). Am. J. Physiol. 276, E529-E535.

Petrides, A.S., Groop, L.C., Riely, C.A., and DeFronzo, R.A. (1991). J. Clin. Invest. 88, 561-570.

Previs, S.F., Cline, G.W., and Shulman, G.I. (1999). Am. J. Physiol. 277, E154-E160.

Radziuk, J., and Lee, W.N.P. (1999). Am. J. Physiol. 277, E199-E207.

Radziuk, J., McDonald, T.J., Rubenstein, D., and Dupre, J. (1978). Metabolism 27, 657-669.

Rizza, R.A., Mandarino, L.J., and Gerich, J.E. (1981). Am. J. Physiol. 240, E630-E639.

Roden, M., and Shulman, G.I. (1999). Annu. Rev. Med. 50, 277-290.

Roden, M., Perseghin, G., Petersen, K.F., Hwang, J.H., Cline, G.W., Gerow, K., Rothman, D.L., and Shulman, G.I. (1996). J. Clin. Invest. 97, 642-648.

Roden, M., Stingl, H., Chandramouli, V., Schumann, W.C., Hofer, A, Landau, B.R., Nowotny, P., Waldhäusl, W., and Shulman, G.I. (2000). Diabetes 49, 701-707.

Roser, W., Beckmann, N., Wiesmann, U., and Seelig, J. (1996). Magnet. Reson. Med. 14, 1217-1220.

Rothman, D.L., Magnusson, I., Katz, L.D., Shulman, R.G., and Shulman, G.I. (1991a). Science 254, 573-576.

Rothman, D.L., Shulman, R.G., and Shulman, G.I. (1991b). Biochem. Soc. Trans, 19, 992-994.

Roussel, R., Carlier, P.G., Wary, C., Velho, G., and Bloch, G. (1997). Magnet. Reson. Med. 37, 821-824.

Shalwitz, R.A., Reo, N.V., Becker, N.N., and Ackerman, J.J. (1987). Magnet. Reson. Med. 5, 462-465.

Shmueli, E., Walker, M., Alberti, G., and Record, C.D. (1993). Hepatology 18, 86-95.

Shulman, G.I., Alger, J.R., Prichard, J.W., and Shulman, R.G. (1984). J. Clin. Invest. 74, 1127-1131.

Shulman, G.I., Rothman, D.L., Chung, Y., Rossetti, L., Petit, W.A. Jr., Barrett, E.J., and Shulman, R.G (1988). J. Biol. Chem. 263, 5027-5029

Sillerud, L.O., and Shulman, R.G. (1983). Biochemistry 22, 1087-1094. 
Stingl, H., Krssak, M., Bischof, M., Krebs, M., Fürnsinn, C., Nowonty, P., Waldhausl, W., Shulman, G.I., and Roden, M. (2001). Diabetologia, in press.

Stoffel, M., Froguel, P., Takeda, J., Zouali, H., Vionnet, N., Nishi, S., Weber, I.T., Harrison, R.W., Pilkis, S.J., Lesage, S., et al. (1992). Proc. Natl. Acad. Sci. U.S.A. 89, 7698-7702.

Tayek, J.A., and Katz, J. (1997). Am. J. Physiol. 272, E476-E484.

Taylor, R., and Shulman, G.I. (1994). In "The Diabetes Annual" (S.M. Marschall and P.D. Home, eds.), vol. 8, pp. 157-175.

Taylor, R., Price, T.B., Rothman, D.L., Shulman, R.G., and Shulman, G.I. (1992). Magnet. Reson. Med. 27, 13-20.

Taylor, R., Magnusson, I., Rothman, D.L., Cline, G.W., Caumo, A., Cobelli, C., and Shulman, G.I. (1996). J. Clin. Invest. 97, 126-132.

van de Werve, G., and Jeanrenaud, B. (1987). Diabetologia 30, 169-174.

Vetho, G., Petersen, K.F., Perseghin, G., Hwang, J.H., Rothman, D.L., Pueyo, M.E., Cline, G.W., Froguel, P., and Shulman, G.I. (1996). J. Clin. Invest. 98, 1755-1761.

Villar-Palasi, C., and Larner, J. (1960). Arch. Biochem. Biophys. 86, 270-273.

Wahren, J., Felig, P., Cerasi, E., and Luft, R. (1972). J. Clin. Invest. 51, 1870-1878.

Wajngot, A., Chandramouli, V., Schumann, W.C., Efendic, S., and Landau, B.R. (1991). Metabolism 40, 877-881. 



\title{
Peroxisome Proliferator-Activated Receptors: From Genes to Physiology
}

\author{
Steven A. Kliewer, H. ERIC Xu, \\ Millard H. Lambert, and Timothy M. Willson \\ Glaxo Wellcome, Inc., 5 Moore Drive, Research Triangle Park, North Carolina 27709
}

\begin{abstract}
The peroxisome proliferator-activated receptors (PPAR $\alpha, \gamma, \delta)$ are members of the nuclear receptor superfamily of ligand-activated transcription factors that have central roles in the storage and catabolism of fatty acids. Although the three PPAR subtypes are closely related and bind to similar DNA response elements as heterodimers with the 9-cis retinoic acid receptor RXR, each subserves a distinct physiology. PPAR $\alpha$ (NR1C1) is the receptor for the fibrate drugs, which are widely used to lower triglycerides and raise high-density lipoprotein cholesterol levels in the treatment and prevention of coronary artery disease. In rodents, PPAR $\alpha$ agonists induce hepatomegaly and stimulate a dramatic proliferation of peroxisomes as part of a coordinated physiological response to lipid overload. PPAR $\gamma$ (NR1C3) plays a critical role in adipocyte differentiation and serves as the receptor for the glitazone class of insulin-sensitizing drugs used in the treatment of type 2 diabetes. In contrast to PPAR $\alpha$ and PPARy, relatively little is known about the biology of PPAR $\delta$ (NR1C2), although recent findings suggest that this subtype also has a role in lipid homeostasis. All three PPARs are activated by naturally occurring fatty acids and fatty acid metabolites, indicating that they function as the body's fatty acid sensors. Three-dimensional crystal structures reveal that the ligand-binding pockets of the PPARs are much larger and more accessible than those of other nuclear receptors, providing a molecular basis for the promiscuous ligand-binding properties of these receptors. Given the fundamental roles that the PPARs play in energy balance, drugs that modulate PPAR activity are likely to be useful for treating a wide range of metabolic disorders, including atherosclerosis, dyslipidemia, obesity, and type 2 diabetes.
\end{abstract}

\section{Introduction}

Peroxisomes are organelles that are involved in the $\beta$ oxidation of long-chain chain fatty acids and the catabolism of cholesterol to bile acids (Vamecq and Draye, 1989). In rodents, the number and size of peroxisomes are dramatically increased in the liver and, to a lesser extent, the heart and kidney in response to treatment with a variety of different amphipathic acids, including hypolipidemic drugs and plasticizers, which are collectively referred to as "peroxisome proliferators." Peroxisome proliferation in rodents is accompanied by a marked hepatomegaly and increases in the transcription of genes involved in peroxisomal and 
microsomal oxidation of fatty acids as well as by alterations in lipid metabolism, including reductions in serum triglyceride and cholesterol levels. Under normal physiological conditions, peroxisomal $\beta$ oxidation represents only a minor pathway for fatty acid catabolism relative to the mitochondrial system. However, the peroxisomal pathway is engaged in rodents during periods of lipid overload (e.g., precipitated by high-fat diets or metabolic disturbances). Interestingly, the phenomenon of peroxisome proliferation does not occur in humans, although peroxisomes are essential organelles (Vamecq and Draye, 1989). The molecular basis for this difference between species is not yet clear.

In 1990, Issemann and Green reported the cloning and initial characterization of a novel murine orphan nuclear receptor that was activated by a range of established peroxisome proliferators (Issemann and Green, 1990). This new receptor originally was named the peroxisome proliferator-activated receptor (PPAR) but it is now referred to as PPAR $\alpha$. Like other members of the nuclear receptor superfamily, PPAR $\alpha$ contains an approximately 70 amino acid DNAbinding domain comprised of two highly conserved zinc fingers and a C-terminal ligand-binding domain (LBD) of approximately 250 amino acids. In addition to its ligand-binding capabilities, the LBD contains dimerization and transcriptional activation domains, including the well-characterized transcriptional activation function 2 (AF-2), which is embedded in the extreme C-terminal portion of the receptor. The $\mathrm{N}$-terminal domain of PPAR $\alpha$ is less well characterized but appears to encode an additional transcriptional activation function.

In 1992, the Wahli laboratory reported the cloning of the Xenopus laevis ortholog of PPAR $\alpha$ (Dreyer et al., 1992). Like the mouse receptor, the Xenopus PPAR $\alpha$ was activated by micromolar concentrations of peroxisome proliferators. The Wahli group also reported the cloning of two closely related orphan receptors encoded by distinct genes, which they named PPAR $\beta$ and PPAR $\gamma$. Several groups subsequently reported the cloning of mammalian orthologs of PPAR $\beta$ and PPAR $\gamma$. Although PPAR $\alpha$ and PPAR $\gamma$ are quite highly conserved across species, the third mammalian PPAR subtype diverged considerably from Xenopus to mammals, leaving in question whether these orphan receptors were, in fact, orthologs or paralogs (Figure 1A). The murine clone was named PPAR $\delta$ because of this divergence (Kliewer et al., 1994). However, the recent cloning and characterization of the chicken PPAR $\beta$ has made it possible to construct an evolutionary tree from which it is apparent that the Xenopus PPAR $\beta$ and the mammalian PPAR $\delta$ are, in fact, divergent orthologs (Takada et al., 2000). We will refer to this subtype as PPAR $\delta$ in the rest of this chapter. The PPAR nomenclature for PPAR $\delta$ and PPAR $\gamma$ is a misnomer, since neither of these PPAR subtypes has been associated with peroxisome proliferation. 
A

$\operatorname{PPAR} \alpha$

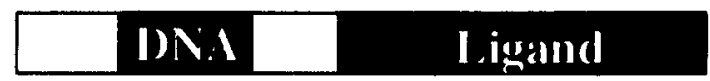

PPAR $\gamma$

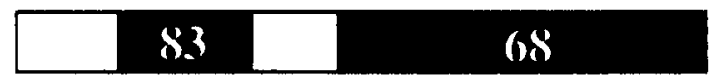

PPAR $\delta$

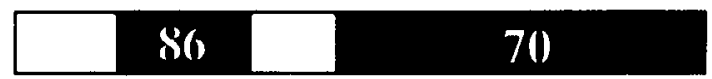

B

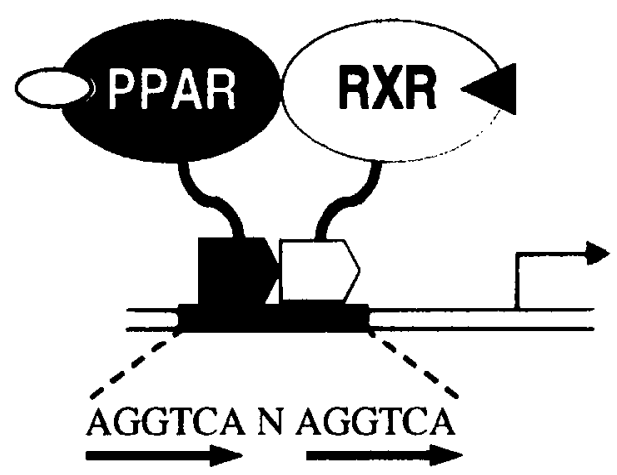

FIG. 1. The PPAR family and its DNA-binding properties. (A) The murine PPAR subfamily. The DNA and ligand-binding domains are indicated. Numbers represent percent amino acid identity. (B) The PPARs bind to DR-1-type DNA response elements as heterodimers with RXR. The PPAR/RXR heterodimer can be activated by ligands for either PPAR or RXR.

Each of the three PPAR subtypes is expressed in a distinct, tissue-specific pattern. PPAR $\alpha$ is highly expressed in liver, heart, kidney, skeletal muscle, and brown adipose, tissues that are metabolically very active. PPAR $\gamma$ is most-highly expressed in white and brown adipose tissue, large intestine, and spleen. In contrast to PPAR $\alpha$ and PPAR $\gamma$, which are abundantly expressed in just a few tissues, PPAR $\delta$ is expressed in virtually all tissues at comparable levels. Like several other members of the nuclear receptor superfamily, the PPARs bind to DNA as obligate heterodimers with the 9-cis retinoic acid receptors (RXRs) (Figure 1B). The PPAR/RXR heterodimers bind to two half sites of the consensus 
sequence AGGTCA, organized as a direct repeat with a single nucleotide spacer, a so-called DR-1 motif. Peroxisome proliferator response elements (PPREs) have been identified in the transcriptional regulatory regions of numerous genes involved in carbohydrate and lipid metabolism. There is emerging evidence that optimal binding sites differ slightly for each PPAR subtype. These subtle differences in binding site preference, together with the differences in tissue expression patterns, undoubtedly contribute to the different biologies of the three PPAR subtypes.

Shortly after their cloning, it was noted that the PPARs are unusual in their ligand activation properties in two important respects. First, they are relatively promiscuous, compared to most other nuclear receptors (e.g., the steroid hormone and retinoid receptors). The PPARs are activated by structurally diverse amphipathic acids, including both natural and synthetic compounds. Interestingly, this promiscuity has since been observed with several other orphan nuclear receptors, including the pregnane $\mathrm{X}$ receptor $(\mathrm{PXR})$ and the constitutive androstane receptor (CAR) (Moore et al., 2000). Second, the PPAR/RXR heterodimers are "permissive" for activation by 9-cis retinoic acid and synthetic RXR ligands through the RXR subunit of the heterodimer (Kliewer et al., 1992). Although the PPAR $\alpha / R X R$ complex was the first permissive heterodimer to be characterized, we now know that heterodimers formed between RXR and several other nuclear receptors - including all three PPAR subtypes, the liver $\mathrm{X}$ oxysterol receptors (LXRs), and the farnesoid X bile acid receptor (FXR) - are permissive for activation by RXR ligands (Mangelsdorf and Evans, 1995).

During the past 7 years, our nuclear receptor group at Glaxo Wellcome has focused much of its effort on the identification of PPAR subtype-selective agonists and antagonists and the use of these chemical tools to uncover the physiological roles of the PPARs and the therapeutic utility of PPAR ligands. We refer to this approach of using small-molecule ligands to unravel the biology of orphan nuclear receptors as "reverse endocrinology" (Kliewer et al., 1999; Willson et al., 2000a). In order to understand how ligands modulate PPAR activity at the molecular level, we have determined the three-dimensional structures of PPARs in the presence of agonists, partial agonists, and antagonists by $x$-ray crystallography. This review will focus on the insights into the structure and function of the PPARs provided by reverse endocrinology.

\section{PPAR $\alpha$ : The Fibrate Receptor}

The discovery that PPAR $\alpha$ is activated by a range of peroxisome proliferators, together with its high expression in tissues in which peroxisome proliferation occurs, first suggested that PPAR $\alpha$ might mediate this biological phenomenon (Isseman and Green, 1990). This hypothesis was confirmed in dramatic fashion 
by the generation of mice lacking functional PPAR $\alpha$ (Lee et al., 1995). PPAR $\alpha-$ null mice were viable and normal in appearance but did not display any evidence of characteristic morphological or biochemical changes when treated with efficacious peroxisome proliferators such as clofibrate and Wy14,643. Subsequent studies revealed that PPAR $\alpha$-null mice have increased basal plasma levels of cholesterol and become obese with age (Peters et al., 1997; Costet et al., 1998). These data provided strong evidence that PPAR $\alpha$ mediates the hypolipidemic effects of fibrates and other peroxisome proliferators.

The hypolipidemic fibrates - including gemfibrozil, fenofibrate, and bezafibrate - are a class of drugs widely prescribed in western countries for reducing triglyceride levels, a risk factor for cardiovascular disease. Fibrates have the added benefit of raising levels of high-density lipoprotein (HDL), or "good," cholesterol and reducing levels of low-density lipoprotein (LDL), or "bad," cholesterol in certain subpopulations of dyslipidemic individuals. The fibrates originally were developed as cardiovascular drugs through optimization of their in vivo activity in rodent models of hyperlipidemia prior to the discovery of the PPARs. Although these drugs activate PPAR $\alpha$ in vitro, they do so only at concentrations in the high micromolar range, which may explain why large doses (i.e., 200-1200 mg/day) are required for clinical activity. Moreover, the fibrates in clinical use are only weakly selective for PPAR $\alpha$ over the other two PPAR subtypes (Brown et al., 1999).

A search for more-potent fibrates by scientists at Burroughs Wellcome led to synthesis of a series of urea-substituted (ureido) fibrate analogs that were active at greater than 100-fold lower doses in rodent models of hyperlipidemia (Hawke et al., 1997). In our hands, these same compounds were very potent in cell-based PPAR $\alpha$ activation assays, activating the receptor with half-maximal effective concentrations in the low nanomolar range (Kliewer et al., 1997; Brown et al., 1999). The strong correlation between the in vivo and in vitro potencies of these compounds provided additional evidence that PPAR $\alpha$ is the molecular target for the hypolipidemic effects of fibrates (Brown et al., 1999).

The lack of potency of the fibrates in clinical use had raised the question whether they exerted their effects on PPAR $\alpha$ activity by binding directly to the receptor or by an indirect mechanism. The discovery of potent PPAR $\alpha$ activators provided us with the reagents necessary to address this question. Standard binding assays performed with a tritiated derivative of the potent ureido fibrate GW 2331 (Figure 2) showed that this compound bound directly to recombinant PPAR $\alpha$ LBD with a $K_{d}$ of $\sim 10 \mathrm{nM}$ (Kliewer et al., 1997). In competition binding assays, a series of established hypolipidemic agents and peroxisome proliferators efficiently displaced $\left[{ }^{3} \mathrm{H}\right] \mathrm{GW} 2331$ from the PPAR $\alpha$ LBD. These data provide strong evidence that the fibrates and other hypolipidemic agents mediate their therapeutic 


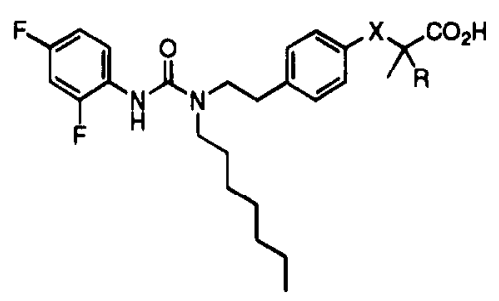

GW 2331, $X=0, R=E t$

GW 9578, $X=S, R=M e$<smiles>CN(CCOc1ccc(CC2SC(=O)NC2=O)cc1)c1ccccn1</smiles>

rosiglitazone

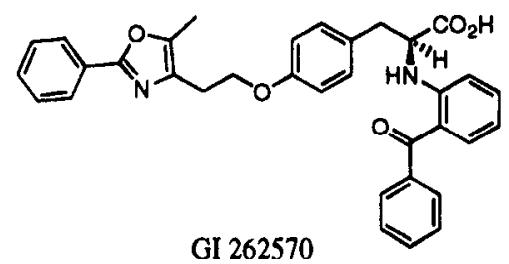

GI 262570<smiles>CCCCCCCC[C@H]1S[C@@H](CC(=O)N(Cc2ccccc2)Cc2ccccc2)C(=O)N1CCCCc1ccc(C(=O)O)cc1</smiles>

GW 0072

GW 2433

FIG. 2. Chemical structures of synthetic PPAR ligands.

effects by direct binding to PPAR $\alpha$. Ligand-sensing assays that detect ligand-induced conformational changes in PPAR $\alpha$ also have been used to show that fibrates and other peroxisome proliferators bind directly to this nuclear receptor (Dowell et al., 1997; Forman et al., 1997; Krey et al., 1997).

The availability of potent, selective PPAR $\alpha$ ligands as chemical tools has provided new, unexpected insights into the biology of this receptor. In addition to dramatically reducing serum triglyceride levels, the potent rodent PPAR $\alpha$-selective ureido fibrate GW 9578 (Figure 2) recently was shown to prevent hyperinsulinemia in rodent models of insulin resistance (Guerre-Millo et al., 2000). Since hyperlipidemia and insulin resistance are independent risk factors for coronary heart disease in diabetes patients, the development of potent human PPAR $\alpha$ - 
selective agonists may lead to more-effective drugs for the prevention of cardiovascular mortality.

\section{PPAR $\gamma$ : The Glitazone Receptor}

Type 2 diabetes is characterized by a resistance of peripheral tissues including skeletal muscle, liver, and adipose - to the actions of insulin. The development of insulin resistance is an early event in the progression of type 2 diabetes. In the early 1980s, scientists at the Japanese pharmaceutical company Takeda reported the first of a promising new class of thiazolidinedione-based antidiabetic compounds, termed glitazones (Sohda et al., 1982). These molecules lowered glucose levels in rodent models of insulin resistance without increasing insulin secretion from the pancreas, hence, their designation of insulin sensitizers. The glitazones originally were derived from the fibrate clofibrate, which was noted to have weak antihyperglycemic activity in rodents. Optimization of the glucose-lowering activity of clofibrate through a combination of traditional medicinal chemistry and in vivo pharmacology yielded the prototypical glitazone, ciglitazone. In addition to its antihyperglycemic effects, ciglitazone reduced levels of insulin and lipids, including both triglycerides and nonesterified fatty acids. Subsequent optimization of these glitazone activities in animal models led to more-potent members of this chemical class, including troglitazone, pioglitazone, and rosiglitazone (Figure 2) (Spiegelman, 1998). Rosiglitazone and pioglitazone are currently approved for use for type 2 diabetes under the trade names Avandia $^{\mathrm{TM}}$ and Actos ${ }^{\mathrm{TM}}$, respectively. Troglitazone (Rezulin ${ }^{\mathrm{TM}}$ ), the first of the glitazones approved by the U.S. Food and Drug Administration, was recently removed from the market due to idiosyncratic hepatotoxicity, which in extreme cases caused liver failure.

In 1995, we reported that PPAR $\gamma$ was the receptor for the glitazones (Lehmann et al., 1995). Two seemingly disparate bodies of literature led us to test whether glitazones might modulate PPAR $\gamma$ activity. First, PPAR $\gamma$ was shown to be highly expressed in adipocytes (Chawla et al., 1994; Tontonoz et al., 1994a) and to serve as a critical regulator of fat cell differentiation in vitro. Ectopic expression of PPAR $\gamma$ in fibroblasts in the presence of weak PPAR $\gamma$ activators resulted in their efficient differentiation into mature adipocytes (Tontonoz et al., 1994b). Second, the glitazones had been reported to dramatically enhance insulin-dependent differentiation of various preadipocyte and stem cell lines to fat cells (Hiragun et al., 1988; Sparks et al., 1991). Among the genes regulated by glitazones in fat cells was the adipocyte fatty acid-binding protein aP2, a gene that is regulated directly by PPAR $\gamma$ (Kletzein et al., 1992; Harris and Kletzien, 1994; Tontonoz et al., 1994a). We initially demonstrated that glitazones were selective activators of PPAR $\gamma$ in transfection assays and bound directly to the receptor 
(Lehmann et al., 1995). We subsequently showed that the potencies of glitazones in binding and activating PPARy in vitro correlated closely with their glucoselowering activities in vivo (Willson et al., 1996). Taken together, these findings provided compelling evidence that PPAR $\gamma$ was the molecular target for the antidiabetic actions of the glitazones. At first glance, it appeared counterintuitive that a key regulator of fat cell differentiation would be the molecular target for these antidiabetic drugs. However, work from a number of laboratories using a variety of experimental approaches has confirmed that PPAR $\gamma$ is, indeed, the molecular target for the antidiabetic actions of the glitazones (Spiegelman, 1998).

The glitazones were developed in rodent models of insulin resistance in the absence of any knowledge about their molecular target. Moreover, the glitazones contain a chiral center at carbon 5 of the thiazolidinedione headgroup but have been developed as a mixture of isomers (racemates), since they undergo racemization in vivo. Having the knowledge of their molecular target, we quickly established that only the (S)-enantiomers of the glitazones bind with high affinity to PPAR $\gamma$ (Nolte et al., 1998; Parks et al., 1998; Gampe et al., 2000; Willson et al., $2000 \mathrm{~b}$ ). Thus, only 50 percent of the drug substance in the currently approved glitazones is likely to be active. We also found that one of the glitazones, troglitazone, has significant activity on the nuclear receptor PXR, a key transcriptional regulator of CYP3A expression in the liver and intestine (Jones et al., 2000). Consistent with this observation, troglitazone was reported to interact with drugs metabolized by CYP3A (e.g., oral contraceptives). Over the last 5 years, our group has focused much of its effort on the identification of antidiabetic drugs optimized for their activity on PPAR $\gamma$. Recently, we reported a series of tyrosine-based PPAR $\gamma$ agonists - including GI 262570, GW 1929, and GW 7845 - that fulfill these criteria (Figure 2) (Cobb et al., 1998; Collins et al., 1998; Henke et al., 1998; Brown et al., 1999). These compounds are single enantiomers synthesized from the amino acid L-tyrosine and are extremely potent PPAR $\gamma$ agonists, binding to the receptor at low nanomolar concentrations. Moreover, these PPAR $\gamma$ agonists do not activate PXR. Importantly, GW 1929 was shown to lower glucose levels in Zucker diabetic fatty (ZDF) rats at serum plasma concentrations more than 100-fold lower than troglitazone, which mirrors the differences in the potencies of these two compounds at PPAR $\gamma$ in vitro (Brown et al., 1999). One of these compounds, GI 262570, has subnanomolar activity on human PPAR $\gamma$ and submicromolar activity on PPAR $\alpha$. GI 262570, currently in phase III of clinical trials for the treatment of type 2 diabetes, represents the first PPAR agonist optimized against the human receptor to be progressed into the clinic. Early clinical data indicate that GI 262570 has potent glucose-lowering effects and also lowers triglycerides and raises HDL cholesterol in diabetes patients (Fiedorek et al., 2000; Wilson et al., 2000). 
Although it is clearly established that PPAR $\gamma$ modulates insulin sensitivity, a paradox remains: how does activation of a nuclear receptor that is highly expressed in fat sensitize skeletal muscle and liver to the actions of insulin? We now believe that several plausible explanations exist. First, PPAR $\gamma$ may regulate signaling molecules that are secreted from adipose and affect glucose utilization and/or production in other tissues. Examples of signaling molecules that are secreted by adipocytes and affect energy balance are tumor necrosis factor $\alpha$ $(\mathrm{TNF} \alpha)$ and leptin. Expression of both these genes is repressed by PPAR $\gamma$ agonists (Hofmann et al., 1994; De Vos et al., 1996; Kallen and Lazar, 1996). A second possibility is that the effects of PPAR $\gamma$ agonists on glucose utilization are secondary to those on fatty acids. High circulating levels of fatty acids are known to interfere with glucose utilization in skeletal muscle through the Randle cycle and to promote glucose production in the liver (Randle, 1998). PPAR $\gamma$ agonists reduce serum concentrations of both esterified and nonesterified fatty acids, presumably by promoting their uptake and storage in adipocytes. Finally, PPAR $\gamma$ is expressed, albeit at low levels, in both skeletal muscle and liver. Thus, PPAR $\gamma$ agonists may have direct effects on glucose utilization or production in both of these tissues.

In order to better understand the mechanisms underlying the antidiabetic actions of PPAR $\gamma$ agonists, we recently used a comprehensive mRNA-profiling technique to identify PPAR $\gamma$ target genes in insulin-sensitive tissues of an animal model of type 2 diabetes. ZDF rats, a standard animal model of type 2 diabetes, were treated for 7 days with either the potent, selective PPAR $\gamma$ agonist GW 1929 or with vehicle alone. Genes that were regulated in response to PPAR $\gamma$ agonist in epididymal white adipose tissue (WAT), interscapular brown adipose tissue (BAT), liver, and soleus skeletal muscle were identified using CuraGen Corporation's GeneCalling TM technology (Shimkets et al., 1999). We observed the coordinated upregulation of a number of genes involved in fatty acid transport, storage, and oxidation in both WAT and BAT from PPARy agonist-treated rats. By contrast, a subset of these genes was coordinately downregulated in skeletal muscle upon treatment. Notably, we observed a marked decrease in pyruvate dehydrogenase kinase 4 (PDK4) expression in muscle from GW 1929-treated rats. PDK4 phosphorylates pyruvate dehydrogenase (PDH) and inhibits the activity of this mitochondrial enzymatic complex, which catalyzes the first irreversible step in glucose oxidation (Randle, 1998). Interestingly, decreased PDH activity and increased PDK activity are associated with insulin resistance and type 2 diabetes (Majer et al., 1998; Sreenan et al., 1999). Thus, the decreases that we observe in PDK4 activity may be an important component of the insulin-sensitizing activity of PPAR $\gamma$ agonists in skeletal muscle. In liver, PPAR $\gamma$ agonist treatment resulted in decreased expression of phosphoenolpyruvate carboxykinase, which encodes the rate-limiting step in hepatic gluconeogenesis. These findings are consistent 
with reports that glitazones reduce gluconeogenesis in patients with type 2 diabetes. Overall, the gene-expression patterns suggest that PPAR $\gamma$ agonists promote a flux of fatty acids into adipose tissue and away from skeletal muscle and liver. Decreased fatty acid metabolism in muscle is likely to account in large measure for increased glucose utilization via the Randle cycle (Randle, 1998). At the same time, decreases in fatty acid levels in the liver may account for reduced glucose production (Randle, 1998). Our data provide evidence that the antidiabetic actions of PPAR $\gamma$ agonists are the consequence of coordinate regulation of gene expression in multiple insulin-sensitive tissues (Figure 3). However, it remains to be determined whether the effects of PPAR $\gamma$ agonists in skeletal muscle and liver are mediated directly through activation of PPAR $\gamma$ in these tissues or are the indirect consequence of PPAR $\gamma$ activation in adipocytes.

\section{PPAR $\delta$}

Much less is known about the biology of PPAR $\delta$ than either of the other two PPAR subtypes. It seems likely that PPAR $\delta$ is involved in lipid metabolism, since fatty acids activate the receptor (see below). Fibroblasts transfected with a PPAR $\delta$

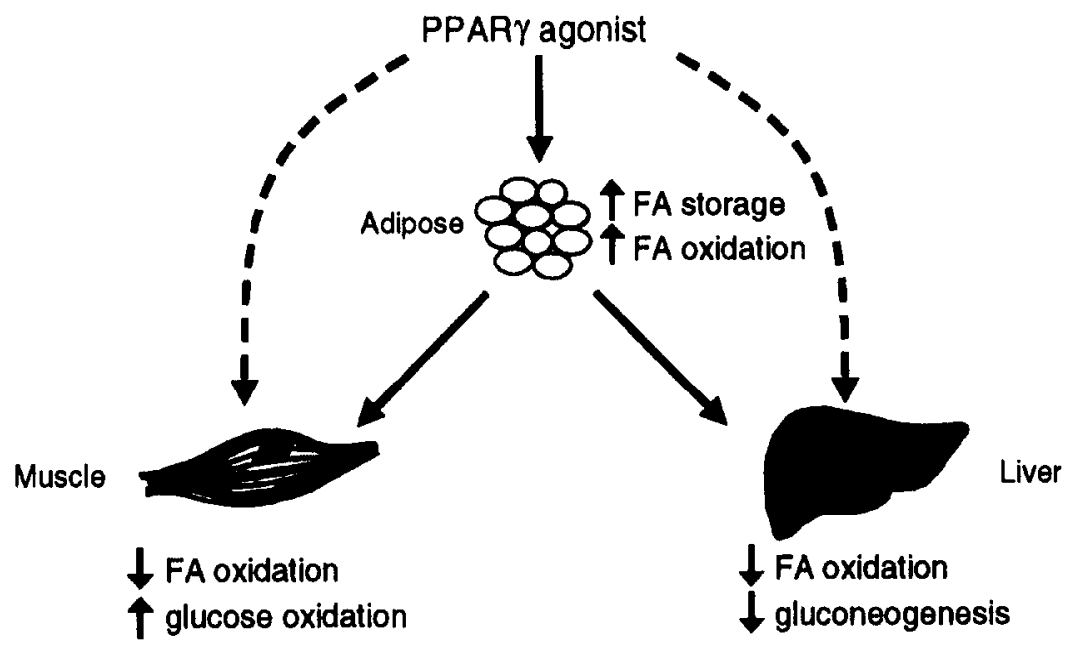

FIG. 3. Model for the insulin-sensitizing actions of PPAR $y$ agonists. PPARy agonists regulate genes in fat cells, resulting in a flux of fatty acids into adipocytes, where they are stored or metabolized, and away from skeletal muscle and liver (solid arrows). The net effect of this fatty acid repartitioning is an increase in glucose oxidation in muscle and a decrease in glucose production by the liver. PPAR $y$ agonists also may have direct effects on gene expression in muscle and liver (dotted arrows). 
expression vector become responsive to fatty acids, as measured by the induction of early genes in the adipocyte differentiation cascade (Bastie et al., 1999). In support of this idea, the Gonzalez laboratory recently reported that mice lacking functional PPAR $\delta$ have reduced adipose tissue stores compared to control animals (Peters et al., 2000). The PPAR $\delta$-null mice are smaller than control animals and display myelination defects in the corpus callosum and enhanced hyperplasia of the epidermis in response to the phorbol ester O-tetradecanoylphorbol-13-acetate (TPA). These findings establish roles for PPAR $\delta$ in development and in brain and skin function. The spectrum of defects observed in the PPAR $\delta$-null mice is consistent with the broad expression pattern of this PPAR subtype.

Unlike PPAR $\alpha$ and PPAR $\gamma$, no drugs have been identified that work through PPAR $\delta$. Moreover, the synthetic PPAR $\delta$ ligands that have been reported to date lack either potency or selectivity or both. Thus, a major challenge in unraveling the biology of PPAR $\delta$ is to identify the appropriate chemical tools. Through solid-phase synthesis of a combinatorial library of fibrate analogs, we identified several compounds that activated human PPAR (Brown et al., 1997). GW 2433 (Figure 2) was the most-potent activator of PPAR $\delta$ in this series, although it showed only modest selectivity over PPAR $\alpha .\left[{ }^{3} \mathrm{H}\right] \mathrm{GW} 2433$ bound to PPAR $\delta$ with a $\mathrm{K}_{\mathrm{i}}$ of $40 \mathrm{nM}$ and has been employed as a radioligand to aid in the development of selective PPAR $\delta$ agonists. Interestingly, scientists at Merck demonstrated that the leukotriene antagonist L-16504l activated PPAR $\delta$ and raised serum cholesterol levels in $\mathrm{db} / \mathrm{db}$ mice (Berger et al., 1999). Although this compound is not selective for murine PPAR $\delta$ over murine PPAR $\gamma$, the pharmacological effect was attributed to activation of PPAR $\delta$, since neither serum glucose nor triglycerides were lowered at the same dose. These data suggest that PPAR $\delta$, like the other two PPAR subtypes, may have important roles in lipid metabolism.

\section{Natural PPAR Ligands}

Although synthetic ligands, including the fibrates and glitazones, have provided a great deal of insight into PPARs, a comprehensive understanding of PPAR physiology requires the identification of their natural ligands. The Gustafsson laboratory was the first to show that PPAR $\alpha$ is activated by micromolar concentrations of a surprisingly diverse collection of fatty acids that vary in both chain length and degree of saturation (Gottlicher et al., 1992). A search for natural PPAR $\alpha$ ligands in fractionated human serum identified palmitic acid, oleic acid, linoleic acid, and arachidonic acid as naturally occurring activators of this orphan receptor (Figure 4) (Banner et al., 1993). Subsequent work showed that fatty acids also activate PPAR $\gamma$ and PPAR $\delta$. Given their important role in energy balance, the idea that the PPARs might serve as fatty acid receptors was attractive. How- 

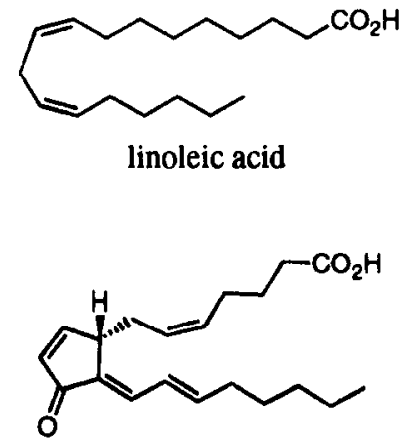

15-deoxy- $\Delta^{12,14}-\mathrm{PGJ}_{2}$

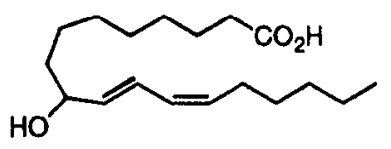

9-HODE

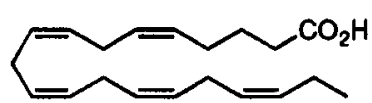

eicosapentaenoic acid

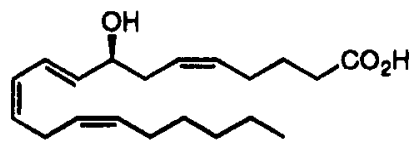

8(S)-HETE

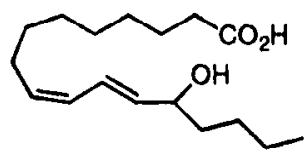

13-HODE

FIG. 4. Chemical structures of natural PPAR ligands.

ever, the relative lack of potency and biophysical properties of fatty acids had made them unsuitable for use in ligand-binding assays. Thus, it had remained unclear whether the fatty acids regulate the activity of PPARs by binding directly to them or via an indirect mechanism.

The availability of high-affinity, synthetic radioligands for all three of the PPAR subtypes provided us with the opportunity to address whether fatty acids bind directly to the PPARs. We tested a large number of saturated and unsaturated fatty acids for their ability to displace radioligands in competition binding assays. We found that many of these structurally diverse fatty acids interact directly with the PPARs at concentrations in the low micromolar range (Xu et al., 1999). Although these concentrations are higher than those typically required for ligands to bind to their cognate nuclear receptors, they are consistent with the levels of nonesterified fatty acids found in human serum. Interestingly, the three PPAR subtypes have different fatty acid-binding profiles. PPAR $\alpha$ is the most-promiscuous subtype, interacting efficiently with both saturated and unsaturated fatty acids. By contrast, PPAR $\gamma$ is the most-selective subtype, interacting efficiently with only a subset of the polyunsaturated fatty acids, including eicosapentaenoic (EPA) and arachidonic acid. PPAR $\delta$ binds to both unsaturated and saturated fatty acids but 
with slightly lower affinity than PPAR $\alpha$. Thus, the PPARs are capable of interacting with multiple fatty acids in vitro.

Certain oxidized fatty acid metabolites have been shown to function as PPAR ligands in vitro. Two of these eicosanoids, 15-deoxy $-\Delta^{12,14}$-prostaglandin $\mathrm{J}_{2}(15 \mathrm{~d}$ $\mathrm{PGJ}_{2}$ ) and $8(\mathrm{~S})$-hydroxyeicosatetraenoic acid (8(S)-HETE) (Figure 4), were found to be slightly more-potent activators of the PPARs than their polyunsaturated fatty acids precursors (Forman et al., 1995; Kliewer et al., 1995; Yu et al., 1995). These results were surprising, given that eicosanoids are known to mediate many of their biological effects through interactions with $\mathrm{G}$ protein-coupled cell-surface receptors. Interestingly, $15 \mathrm{~d}-\mathrm{PGJ}_{2}$ and $8(\mathrm{~S})$-HETE were subtype-selective in their interactions with the PPARs. Whereas $15 \mathrm{~d}-\mathrm{PGJ}_{2}$ was selective for PPAR $\gamma, 8(\mathrm{~S})$ HETE interacted preferentially with $\operatorname{PPAR} \alpha$. More recently, the lipoxygenase products 9-hydroxyoctadecadienoic acid (HODE), 13-HODE, and 15-HETE (Figure 4), which are components of oxidized LDL, were shown to bind and activate both PPAR $\alpha$ and PPAR $\gamma$ (Nagy et al., 1998; Huang et al., 1999; Delerive et al., 2000 ). There is also evidence to suggest that prostacyclin is a natural ligand for PPAR $\delta$. Mice lacking cyclooxygenase 2 (COX2), which is involved in prostacyclin biosynthesis, are impaired for blastocyst implantation in the uterus during pregnancy (Lim et al., 1999). Expression of both PPAR $\delta$ and prostacyclin synthase is induced in the stroma surrounding the blastocyst during implantation. Notably, PPAR $\delta$ ligands, including the stable prostacyclin analog carbaprostacyclin, restore implantation in the COX2-deficient mice (Lim et al., 1999). Although prostacyclin is too unstable to test in standard PPAR $\delta$-binding assays, these data raise the possibility that this prostanoid is a PPAR $\delta$ ligand in vivo. Thus, the regulated conversion of polyunsaturated fatty acids to eicosanoids through either the cyclooxygenase or lipoxygenase pathways may provide a mechanism for modulating the activities of one or more of the PPAR subtypes.

The discovery that the PPARs are capable of binding to a variety of fatty acids and their metabolites suggests that their activation in vivo may not determined through interactions with a single, high-affinity ligand like the classical steroid hormone and retinoid receptors, but rather through interactions with a number of fatty acids and fatty acid metabolites. Thus, the PPARs may function as generalized sensors of fatty acid levels, coupling fluxes in the levels of these fatty acids to the transcriptional regulation of genes involved in lipid and glucose homeostasis. In this regard, it is interesting that several other orphan nuclear receptors, including FXR and the LXRs, also bind to their natural oxysterol and bile acid ligands at low micromolar concentrations (Repa and Mangelsdorf, 1999). Thus, many of the orphan nuclear receptors may function as metabolic sensors. However, this idea remains controversial and the search for high-affinity PPAR ligands continues. 


\section{A Structural Basis for PPAR Promiscuity and Permissiveness}

As receptors for various fatty acids and fatty acid metabolites, the PPARs are much more promiscuous in terms of their interactions with ligands than other members of the nuclear receptor family. Recent x-ray crystal structures of the PPAR $\gamma$ and PPAR $\delta$ LBDs in either the absence or presence of ligand have provided tremendous insight into the molecular basis for the unusual ligand-binding properties of the PPARs. The structures of the PPAR LBDs are composed of $13 \alpha$ helices and a small, four-stranded $\beta$ sheet, which fold to form a "helical sandwich" (Figure 5A) (Nolte et al., 1998; Uppenberg et al., 1998; Xu et al., 1999; Gampe et al., 2000). The overall fold of the PPAR LBDs is similar to that of other nuclear receptors from helix 3 to the C-terminus. However, the PPARs are unique in having an additional helix, termed helix $2^{\prime}$, present between helices 2 and 3. A striking feature of the PPAR LBDs is the very large volume of their hydrophobic, ligand-binding cavities (Figure 5A). The solvent-accessible cavities of both the apo PPAR $\gamma$ and PPAR $\delta$ LBDs are over $1000 \AA^{3}$ in volume, which is approximately three times larger than those of other nuclear receptors for which the structure has been determined. This large volume is due in large part to the presence of helix $2^{\prime}$ and the position of helix 2 , which effectively wedge open the ligand-binding pocket. The positioning of these helices also results in a $100 \AA^{2}$ channel through which ligands can enter the pocket. The presence of a very-flexible loop between helices $2^{\prime}$ and 3 suggests that the channel opening has the potential to be even bigger. This mode of ligand entry appears to be unique to the PPARs. The structures of the other nuclear receptors determined to date including steroid, retinoid, and thyroid hormone receptors - suggest that their ligands enter the binding cavity on the other side of the protein through a channel formed by helices 3,4 , and 10 and capped by the AF-2 helix in the ligand-bound conformation.

The structure of the PPAR $\gamma$ LBD was solved more recently as a six-component complex with the LBD of RXR $\alpha$, an antidiabetic drug, the RXR $\alpha$ ligand, 9-cis retinoic acid, and two peptide fragments of steroid receptor coactivator-1 (SRC-1) (Gampe et al., 2000). Overall, the complex is shaped like a butterfly, with both PPAR $\gamma$ and RXR $\alpha$ adopting the helical sandwich fold that is conserved among nuclear receptors. The PPAR $\gamma / \mathrm{RXR} \alpha$ dimerization interface is comprised of a complex network of hydrophobic and polar interactions mediated by helices 7,9 , and 10 and the loop between helices 8 and 9 of each receptor. The residues of PPAR $\gamma$ that are involved in these interactions are highly conserved in other nuclear receptors that heterodimerize with RXR $\alpha$. Notably, the PPAR $\gamma / R X R \alpha$ dimerization interface is asymmetric, with each LBD tilted approximately $10^{\circ}$ from the $\mathrm{C} 2$ axis of symmetry. This asymmetry results in additional, unexpected interactions between $\operatorname{PPAR} \gamma$ and $\operatorname{RXR} \alpha$, including the packing of a negatively 
A

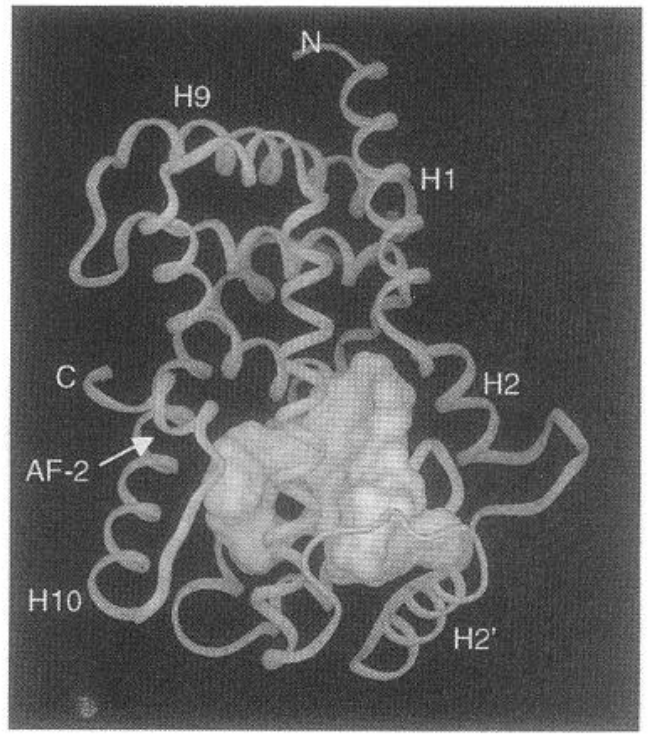

B

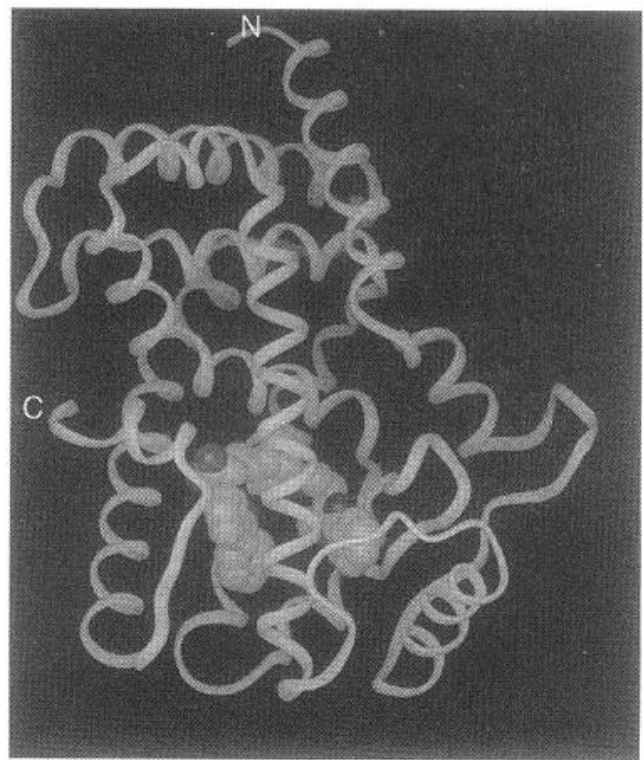

FIG. 5. PPAR $\gamma$ LBD crystal structures. (A) PPAR $\gamma$ LBD crystal structure with the solvent-accessible, ligand-binding cavity highlighted. The carboxy $(\mathrm{C})$ and amino $(\mathrm{N})$ termini and selected helices are indicated. (B) Crystal structure of the antidiabetic drug GI 262570 bound to the PPAR $\gamma$ LBD. 
charged surface of PPAR $\gamma$ composed of the end of the AF-2 helix and the loop between helices 8 and 9 against a positively charged region of RXR $\alpha$ helix 7 . These additional interactions increase the total area of the dimerization interface from roughly $500 \AA^{2}$ to $550 \AA^{2}$, which accounts in large measure for the preference of PPAR $\gamma$ and $R X R \alpha$ to form heterodimers rather than their respective homodimers.

The antidiabetic, insulin-sensitizing drugs GI 262570 or rosiglitazone bind into the large hydrophobic pocket of the PPAR $\gamma$ subunit of the heterodimer in a U-shaped conformation around helix 3 . There, they are stabilized by numerous hydrophobic interactions between the lipophilic portion of the molecule and the ligand-binding pocket (Figure 5B). Moreover, the carboxylic acid of GI 262570 and the thiazolidinedione headgroup of rosiglitazone make essentially the same network of hydrogen bonds with residues in PPAR $\gamma$, including Y473 in the AF-2 helix and $\mathrm{H} 323$ and $\mathrm{H} 449$ in helices 5 and 10, respectively. This pattern of hydrogen bonding effectively stabilizes the AF-2 domain of PPAR $\gamma$ in a conformation that is permissive for interaction with SRC-1. The peptide fragments of SRC-1 each contain an LXXLL motif, which adopts an $\alpha$-helical conformation when bound to the PPAR $\gamma$ LBD. The leucine residues of the LXXLL motif pack into a hydrophobic groove formed by helices 3, 4, and AF-2 of the PPAR $\gamma$ LBD, with the ends of the helix stabilized by salt bridges with E471 in the AF-2 helix and $\mathrm{K} 301$ in helix 3. The residues that comprise this groove as well as E471 and $\mathrm{K} 301$ are highly conserved among members of the nuclear receptor superfamily, suggesting a common mechanism underlying ligand-mediated recruitment of coactivator proteins.

Although many similarities exist in the binding of GI 262570 and rosiglitazone to PPAR $\gamma$, there are also important differences. Whereas GI 262570 occupies about 40 percent of the ligand-binding cavity, rosiglitazone occupies only about 25 percent of the pocket. The benzophenone group attached to the tyrosine nitrogen of GI 262570 reaches approximately $7 \AA$ into a hydrophobic cavity formed by helices 3,7 , and 10 . These hydrophobic interactions made by the benzophenone group of GI 262570 are not available to rosiglitazone or other glitazones. At its other end, the phenyloxazole tail of GI 262570 is inserted approximatley $2.3 \AA$ deeper into a cleft formed by helices 6 and 7 in the ligandbinding pocket than is the corresponding hydrophobic tail of rosiglitazone. The additional hydrophobic interactions between GI 262570 and both ends of the PPAR $\gamma$ ligand-binding pocket provide a molecular basis for the nearly 50-fold increase in potency of this antidiabetic agent, relative to rosiglitazone.

The PPAR $\gamma / \mathrm{RXR} \alpha$ complex is activated efficiently by ligands for both the PPAR $\gamma$ and RXR $\alpha$ subunits of the heterodimer. As a consequence, RXR ligands have many of the same biological actions as PPAR $\gamma$ ligands, including the glucose 
and lipid-lowering effects in rodent models of type 2 diabetes (Mukherjee et al., 1997). The asymmetric interactions between the AF-2 helix of PPAR $\gamma$ and helices 7 and 10 of $\operatorname{RXR} \alpha$ in the heterodimer suggest an unexpected basis for the permissive activation of this complex by RXR ligands. The net effect of these interactions may be the stabilization of the PPAR $\gamma$ AF-2 helix in a "quasi-active" position that is permissive for interactions with coactivator proteins, even in the absence of a PPAR $\gamma$ agonist. Assuming that two coactivator interfaces are required for stable receptor-coactivator interactions and transcriptional activation, the binding of an agonist to the RXR $\alpha$ component of the heterodimer may be sufficient to form the second interface and activate the complex. The addition of a PPAR $\gamma$ agonist would be expected to further increase the stability of this activated complex. Thus, the asymmetric nature of the PPAR $\gamma / \mathrm{RXR} \alpha$ dimerization interface also may explain the additive or synergistic effects that have been reported between PPAR $\gamma$ and RXR agonists.

Comparing the PPAR $\gamma$ and $R X R \alpha$ complexes within the heterodimer with their respective apo structures reveals important differences in the mechanism of activation of these receptors. The apo RXR $\alpha$ LBD and the ligand-bound RAR $\gamma$ LBD were the first nuclear receptor LBDs to be solved by $x$-ray crystallography (Bourguet et al., 1995; Renaud et al., 1995). Comparison of these structures led to the dogma that all nuclear receptors undergo a large "mouse-trap" conformational change upon ligand binding. With the apo and ligand-bound LBD structures available for both RXR $\alpha$ and PPAR $\gamma$, we were able to re-examine this hypothesis. Our structures show that $\mathrm{RXR} \alpha$ does, indeed, undergo a large conformational change upon binding of 9-cis-retinoic acid but that it may be unique among the nuclear receptors in this respect. The apo RXR $\alpha$ structure is forced to break helix 10 in order to accommodate three consecutive phenylalanine residues. This break in helix 10 drives the C-terminal AF-2 helix away from the body of the receptor but allows two of the phenylalanine residues to bury themselves in the hydrophobic core of the receptor. The binding of the RXR ligand displaces these residues from this position, which triggers the conformational change that folds the AF-2 helix against the body of the receptor.

Analysis of the PPAR $\gamma$ structures indicates a fundamentally different mechanism of activation. PPAR $\gamma$, like most nuclear receptors, does not contain three consecutive phenylalanines in helix 10. The apo PPAR $\gamma$ structure shows that there is no drive to break helix 10 . The C-terminal AF-2 helix already is folded against the body of the receptor in the unliganded state. The PPAR $\gamma / \operatorname{RXR} \alpha$ heterodimer suggests that, even in the unliganded state, the AF-2 helix will be stabilized in this "quasi-active" position by its interactions with RXR $\alpha$. In PPAR $\gamma$, the ligandbound structures reveal that the ligand plays an important role in stabilizing the AF-2 helix in the active position through a direct $\mathrm{H}$-bond between the acidic 
ligand and Tyr473 on the AF-2 helix. This tyrosine residue is conserved in PPAR $\alpha$ and PPARS. Interestingly, the other permissive nuclear receptors, FXR and LXR, have a tryptophan at this position, which might be capable of making a similar hydrogen bond with their respective natural ligands. Thus, within the PPAR $\gamma / \operatorname{RXR} \alpha$ complex, we now have evidence that there are two distinct mechanisms for activation of nuclear receptors by their ligands.

How do the PPARs interact with such a structurally diverse collection of natural fatty acids? We recently reported that the structures of PPAR $\delta$ bound to either the polyunsaturated fatty acid EPA or a fibrate, GW 2433, which binds to the receptor with high affinity $\left(K_{d}=40 \mathrm{nM}\right)(X u$ et al., 1999). The acid moieties of EPA and GW 2433 made the same intricate series of hydrogen bonds in PPAR $\delta$ that GI 262570 makes in PPAR $\gamma$, stabilizing the AF-2 helix in a conformation permissive for coactivator interactions. The conservation of this hydrogen bond network further confirms that it must be crucial for ligand-mediated activation of the PPARs. Remarkably, the PPAR $\delta$ ligand-binding cavity is sufficiently large to allow the long lipophilic tail of EPA to bind in two completely different conformations, which we designated "tail up" and "tail down" (Figure 6). In the tail-up conformation, the hydrophobic tail of EPA was bent up into the upper portion of the ligand-binding pocket. EPA was completely buried within the pocket in this conformation. In the second binding mode, the tail of EPA was bent down into the lower region of the ligand-binding cavity. EPA was partially exposed to solvent via the open channel in the tail-down conformation. In each docking mode, EPA occupied approximately $300 \AA^{3}$, which is only about 30 percent of the total accessible volume of the hydrophobic pocket. Notably, the fibrate GW 2433 bound to PPAR $\delta$ in a manner such that it occupied essentially the same space as EPA bound in both of its binding modes. The large number of hydrophobic interactions between GW 2433 and PPAR are likely to account for the increased potency of this ligand relative to EPA.

The $\mathrm{x}$-ray crystal structures provide a molecular explanation for the ability of the PPARs to interact with a structurally diverse set of amphipathic acids. First, the acid moieties of PPAR ligands, including thiazolidinediones and carboxylic acids, make an intricate series of hydrogen bonds with the receptor that includes the conserved tyrosine in the AF-2 helix. These hydrogen bonds stabilize the PPARs in a conformation that is permissive for coactivator interactions. This mode of ligand binding is different from that of the retinoic acid and thyroid hormone receptors, in which the carboxylic acids of the ligands are positioned in the interior of the hydrophobic cavity, away from the AF-2 helix. Second, the hydrophobic pockets of the PPARs are sufficiently large to allow the hydrophobic tails of their ligands to bind in multiple conformations, providing a basis for the promiscuous binding properties of the PPARs. The PPAR $\delta$-EPA structure explains why the PPARs bind only to fatty acids of certain chain lengths. Short fatty 


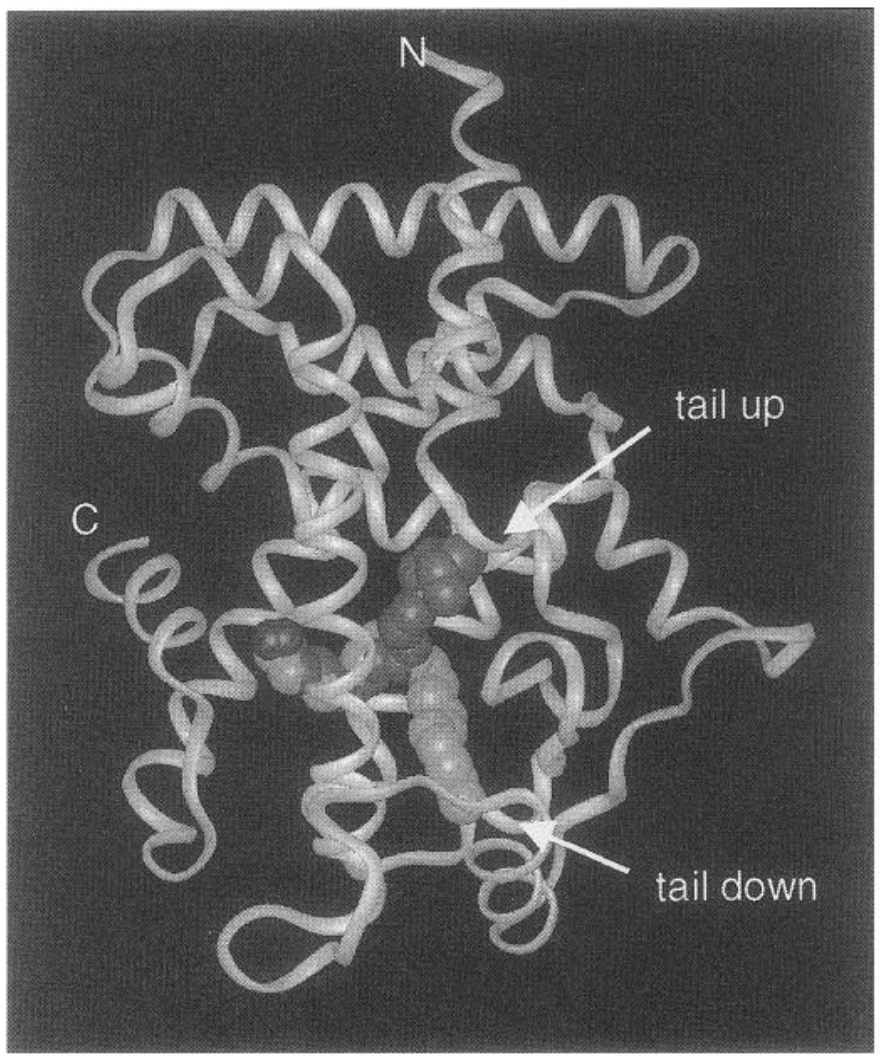

FIG. 6. Crystal structure of PPAR $\delta$ bound to the fatty acid EPA. The two different conformations of EPA seen in the crystal structure are indicated as "tail up" and "tail down."

acids $(C<14)$ could not make the hydrophobic interactions necessary for stable ligand binding. In contrast, long-chain fatty acids $(C>20)$ could not fit into the pocket in either binding mode and would be exposed to the destabilizing effects of the solvent. We suggest that the PPARs may have evolved their unique structures in order to detect a variety of different fatty acids and/or fatty acid metabolites and to regulate transcription accordingly.

\section{Selective PPAR $\gamma$ Modulators}

Synthetic antagonists have been identified for a number of the nuclear receptors that counter the effects of hormones. Notable examples include the antipro- 
gestin and antiglucocorticoid RU486, the antimineralocorticoid spironolactone, and the antiestrogens tamoxifen and raloxifene. Not only have these antagonists been useful tools for the dissection of nuclear reccptor signaling pathways but they also have important pharmacologic activities that make them useful for the treatment of various diseases or physiological conditions. Recent $x$-ray crystal structures of the estrogen receptor bound to either tamoxifen and raloxifene reveal that these antagonists bind into the ligand-binding pocket in such a way as to force the AF-2 helix into a conformation that prevents coactivator interactions (Brzozowski et al., 1997; Shiau et al., 1998).

The large volume of the PPAR ligand-binding cavities suggested that it might be possible to identify novel classes of ligands that bind to these receptors without activating them. We recently identified a nonthiazolidinedione acetamide, designated GW 0072 (Figure 2), that bound to PPAR $\gamma$ with high affinity $\left(\mathrm{K}_{\mathrm{i}}=70 \mathrm{nM}\right)$ (Oberfield et al., 1999). However, unlike other PPAR $\gamma$ ligands, GW 0072 was only a very weak partial agonist of PPAR $\gamma$, activating the full-length PPAR $\gamma$ with only about 15 percent of the efficacy of rosiglitazone and other PPAR $\gamma$ full agonists. Consistent with its weak transcriptional activity, GW 0072 did not promote efficient interactions between PPAR $\gamma$ and various coactivator proteins. Moreover, GW 0072 was able to effectively antagonize the recruitment of coactivators induced by rosiglitazone. Thus, the low efficacy of GW 0072 in transfection assays was paralleled by its inability to recruit coactivator proteins.

As already noted, efficacious PPAR $\gamma$ agonists promote the differentiation of various preadipocyte and stem cell lines into mature adipocytes. To further characterize the activities of GW 0072, we profiled its effects on the differentiation of $10 \mathrm{~T} 1 / 2$ cells to adipocytes. As expected, treatment of $10 \mathrm{~T} 1 / 2$ cells with rosiglitazone resulted in their efficient differentiation, as measured by the accumulation of neutral lipid droplets and expression of an adipocyte-specific pattern of gene expression. By contrast, treatment of 10T1/2 cells with GW 0072 resulted in very little conversion of the cells to mature adipocytes. Notably, GW 0072 completely blocked rosiglitazone-induced adipocyte differentiation. Thus, GW 0072 represents a novel class of high-affinity PPAR $\gamma$ ligands that can block glitazone-induced adipocyte differentiation in vitro.

In order to understand precisely how GW 0072 antagonized PPAR $\gamma$ activity, the co-crystal structure of GW 0072 bound to PPAR $\gamma$ was solved. Remarkably, GW 0072 bound exclusively in the righthand portion of the pocket bounded by helices 3, 6, and 7 (Figure 7) (Oberfield et al., 1999). Unlike the other PPAR ligands studied, the carboxylic acid of GW 0072 did not interact with the AF-2 helix or the adjacent histidine residues but was instead embedded in the loop separating helices $2^{\prime}$ and 3 . Residues Y473, H449, and H323, which make the network of hydrogen bonds that tether the AF-2 helix in the active configuration in the presence of full agonists, adopted positions similar to the apo PPAR $\gamma$ crystal 


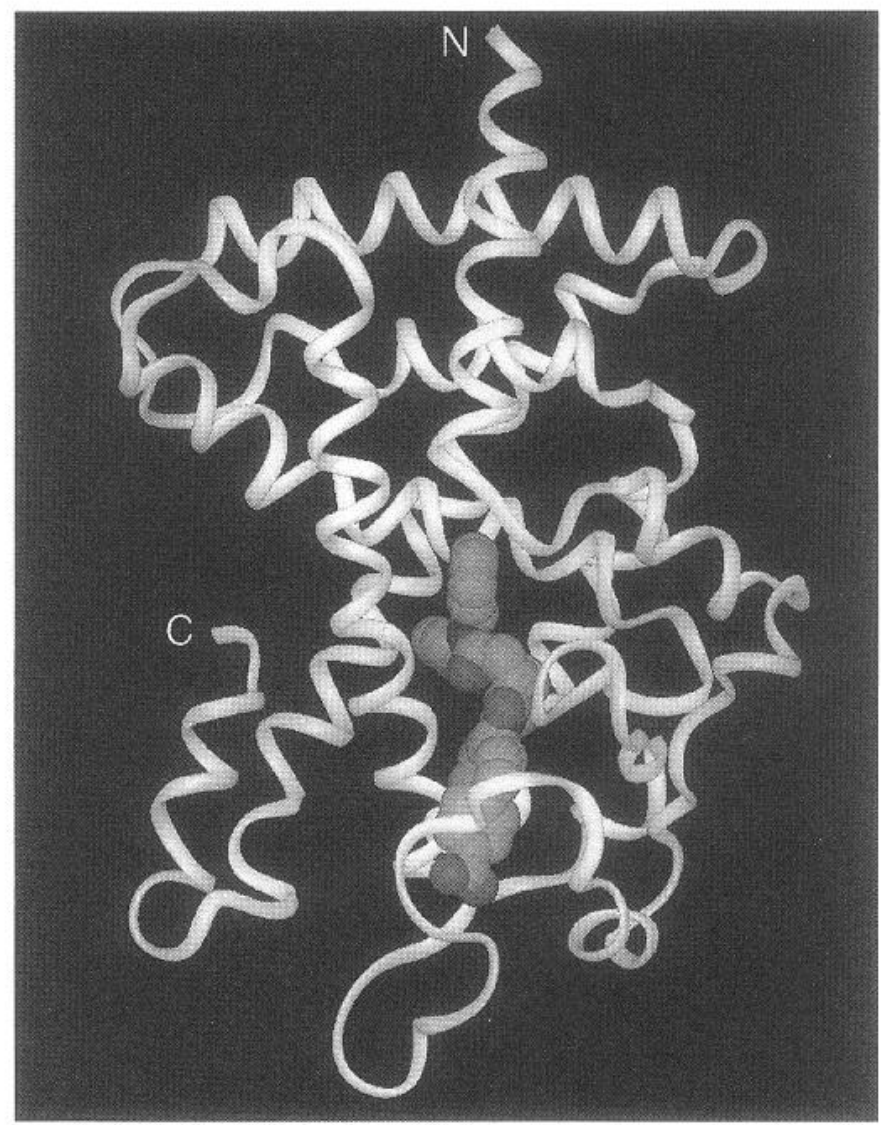

FIG. 7. Crystal structure of the partial agonist GW 0072 bound to the PPAR $\gamma$ LBD. GW 0072 is bound in the righthand portion of the LBD and does not interact with the AF-2 helix.

structure. These data suggest that GW 0072 binding results in a PPAR $\gamma$ conformation that is not permissive for the recruitment of coactivator proteins. Although GW 0072 is only a weak PPAR $\gamma$ agonist in vitro, it maintains many of the in vivo effects of full PPAR $\gamma$ agonists. For example, in insulin-resistant, nondiabetic Zucker fatty rats, GW 0072 showed equivalent efficacy to the tyrosine agonist GW 7845 for lowering insulin levels. Interestingly, in contrast to GW 7845, GW 0072 did not cause weight gain in these animals. These results raise the intriguing possibility the PPAR $\gamma$ modulators could be developed that maintain the antidiabetic properties of the glitazone drugs while reducing some of their adverse effects on weight gain. 


\section{Summary}

Over 30 years of classical pharmacology and medicinal chemistry have contributed to our understanding of the PPARs. These studies began with the synthesis of clofibrate as a metabolically stable fatty acid mimic with triglyceride-lowering activity. This, in turn, led to the synthesis of the glitazones as fibrate analogs with improved glucose-lowering activity. These advances laid the foundation for the discovery of PPAR $\alpha$ and PPAR $\gamma$ as the molecular targets for the fibrates and glitazones, respectively. We have now completed the first lap of this chemical excursion by demonstrating that a variety of natural fatty acids and fatty acid metabolites serve as PPAR ligands. The unique structure of the PPARs allows them to bind to this structurally diverse collection of natural and synthetic molecules and suggests that these ligand-activated transcription factors evolved as the body's fatty acid sensors in order to couple fatty acid levels to glucose and lipid homeostasis. Among the significant challenges that remain are the elucidation of the biological role of PPAR $\delta$ and the identification of selective PPAR modulators that maximize therapeutic effects and minimize detrimental side effects. As we begin the new millennium, we are confident that the intense research into the PPARs will continue to generate exciting new insights into human physiology and to yield new generations of safer and more-effective drugs for the treatment of atherosclerosis, dyslipidemia, type 2 diabetes, and other medical disorders.

\section{ACKNOWLEDGMENTS}

We thank our many colleagues at Glaxo Wellcome for their contributions to the work presented in this review. We acknowledge Jim Way, Wally Harrington, and Kathleen Brown for communicating unpublished results.

\section{REFERENCES}

Banner, C.D., Gottlicher, M., Widmark, E., Sjovall, J., Rafter, J.J., and Gustafsson, J.A. (1993). J. Lipid Res. 34, 1583-1591.

Bastie, C., Holst, D., Gaillard, D., Jehl-Pietri, C., and Grimaldi, P.A. (1999). J. Biol. Chem. 274, 21920-21925.

Berger, J., Leibowitz, M.D., Doebber, T.W., Elbrecht, A., Zhang, B., Zhou, G., Biswas, C., Cullinan, C.A., Hayes, N.S., Li, Y., Tanen, M., Ventre, J., Wu, M.S., Berger, G.D., Mosley, R., Marquis, R., Santini, C., Sahoo, S.P., Tolman, R.L., Smith, R.G., and Moller, D.E. (1999). J. Biol. Chem. 274, 6718-6725.

Bourguet, W., Ruff, M., Chambon, P., Gronemeyer, H., and Moras, D. (1995). Nature 375, 377-382.

Brown, K.K., Henke, B.R., Blanchard, S.G., Cobb, J.E., Mook, R., Kaldor, I., Kliewer, S.A., Lehmann, I.M., I enhard, J.M., Harrington, W.W., Novak, P.J., Faison, W., Binz, J.G., Hashim, M.A., Oliver, W.O., Brown, H.R., Parks, D.J., Plunket, K.D., Tong, W.Q., Menius, J.A., Adkison, K., Noble, S.A., and Willson, T.M. (1999). Diabetes 48, 1415-1424. 
Brown, P.J., Smith-Oliver, T.A., Charifson, P.S., Tomkinson, N.C., Fivush, A.M., Sternbach, D.D., Wade, L.E., Orband-Miller, L., Parks, D.J., Blanchard, S.G., Kliewer, S.A., Lehmann, J.M., and Willson, T.M. (1997). Chem. Biol. 4, 909-918.

Brown, P.J., Winegar, D.A., Plunket, K.D., Moore, L.B., Lewis, M.C., Wilson, J.G., Sundseth, S.S., Koble, C.S., Wu, Z., Chapman, J.M., Lehmann, J.M., Kliewer, S.A., and Willson, T.M. (1999). J. Med. Chem. 42, 3785-3788.

Brzozowski, A.M., Pike, A.C.W., Dauter, Z., Hubbard, R.E., Bonn, T., Engstrom, O., Ohman, L., Greene, G.L., Gustafsson, J.-A., and Carlquist, M. (1997). Nature 389, 753-758.

Chawla, A., Schwarz, E.J., Dimaculangan, D.D., and Lazar, M.A. (1994). Endocrinology 135, 798-800 Cobb, J.E., Blanchard, S.G., Boswell, E.G., Brown, K.K., Charifson, P.S., Cooper, J.P., Collins, J.L., Dezube, M., Henke, B.R., Hull-Ryde, E.A., Lake, D.H., Lenhard, J.M., Oliver, W., Jr., Oplinger, J., Pentti, M., Parks, D.J., Plunket, K.D., and Tong, W.Q. (1998). J. Med. Chem. 41, 5055-5069.

Collins, J.L., Blanchard, S.G., Boswell, G.E., Charifson, P.S., Cobb, J.E., Henke, B.R., Hull-Ryde, E.A., Kazmierski, W.M., Lake, D.H., Leesnitzer, L.M., Lehmann, J., Lenhard, J.M., Orband-Miller, L.A., Gray-Nunez, Y., Parks, D.J., Plunkett, K.D., and Tong, W.Q. (1998). J. Med. Chem. 41, 5037-5054.

Costet, P., Legendre, C., More, J., Edgar, A., Galtier, P., and Pineau, T. (1998). J. Biol. Chem. 273, 29577-29585.

Delerive, P., Furman, C., Teissier, E., Fruchart, J., Duriez, P., and Staels, B. (2000). FEBS Lett. 471, 34-38.

De Vos, P., Lefehvre, A.M., Miller, S.G., Guerre-Millo, M., Wong, K., Saladin, R., Hamann, L.G., Staels, B., Briggs, M.R., and Auwerx, J. (1996). J. Clin. Invest. 98, 1004-1009.

Dowell, P., Peterson, V.J., Zabriskie, T.M., and Leid, M. (1997). J. Biol. Chem. 272, 2013-2020.

Dreyer, C., Krey, G., Keller, H., Givel, F., Helftenbein, G., and Wahli, W. (1992). Cell 68, 879-887.

Fiedorek, F.T., Wilson, G.G., Frith, L., Patel, J., and Abou-Donia, M. (2000). Diabetes 49, 157.

Forman, B.M., Tontonoz, P., Chen, J., Brun, R.P., Spiegelman, B.M., and Evans, R.M. (1995). Cell 83, 803-812.

Forman, B.M., Chen, J., and Evans, R.M. (1997). Proc. Natl. Acad. Sci. U.S.A. 94, 4312-4317.

Gampe, R.T., Montana, V.G., Lambert, M.H., Miller, A.B., Bledsoe, R.K., Milburn, M.V., Kliewer, S.A., Willson, T.M., and Xu, H.E. (2000). Mol. Cell 5, 545-555.

Gottlicher, M., Widmark, E., Li, Q., and Gustafsson, J.A. (1992). Proc. Natl. Acad. Sci. U.S.A. 89, 4653-4657.

Guerre-Millo, M., Gervois, P., Raspe, E., Madsen, L., Poulain, P., Derudas, B., Herbert, J.M., Winegar, D.A., Willson, T.M., Fruchart, J.C., Berge, R.K., and Staels, B. (2000). J. Biol. Chem. 275, 16638-16642.

Harris, P.K., and Kletzien, R.F. (1994). Mol. Pharmacol. 45, 439-445.

Hawke, R.L., Chapman, J.M., Winegar, D.A., Salisbury, J.A., Welch, R.M., Brown, A., Franzmann, K.W., and Sigel, C. (1997). J. Lipid Res. 38, 1189-1203.

Ilenke, B.R., Blanchard, S.G., Brackeen, M.F., Brown, K.K., Cobb, J.E., Collins, J.L., Harrington, W.W., Jr., Hashim, M.A., Hull-Ryde, E.A., Kaldor, I., Kliewer, S.A., Lake, D.H., Leesnitzer, L.M., Lehmann, J.M., Lenhard, J.M., Orband-Miller, L.A., Miller, J.F, Mook, R.A., Jr., Noble, S.A., Oliver, W., Jr., Parks, D.J., Plunket, K.D., Szewczyk, J.R., and Willson, T.M. (1998). J. Med. Chem. 41, 5020-5036.

Hiragun, A., Sato, M., and Mitsui, H. (1988). J. Cell. Physiol. 134, 124-130.

Hofmann, C., Lorenz, K., Braithwaite, S.S., Colca, J.R., Palazuk, B.J., Hotamisligil, G.S., and Spiegelman, B. M. (1994). Endocrinology 134, 264-270.

Huang, J.T., Welch, J.S., Ricote, M., Binder, C.J., Willson, T.M., Kelly, C., Witztum, J.L., Funk, C.D., Conrad, D., and Glass, C.K. (1999). Nature 400, 378-382.

Issemann, I., and Green, S. (1990). Nature 347, 645-650. 
Jones, S.A., Moore, L.B., Shenk, J.L., Wisely, G.B., Hamilton, G.A., McKee, D.D., Tomkinson, N.C., LeCluyse, E.L., Lambert, M.H., Willson, T.M., Kliewer, S.A., and Moore, J.T. (2000). Mol. Endocrinol. 14, 27-39.

Kallen, C.B., and Lazar, M.A. (1996). Proc. Natl. Acad. Sci. U.S.A. 93, 5793-5796.

Kletzien, R.F., Foellmi, L.A., Harris, P.K.W., Wyse, B.M., and Clarke, S.D. (1992). Mol. Pharmacol. 42, 558-562.

Kliewer, S.A., Umesono, K., Noonan, D.J., Heyman, R.A., and Evans, R.M. (1992). Nature 358, 771-774.

Kliewer, S.A., Forman, B.M., Blumberg, B., Ong, E.S., Borgmeyer, U., Mangelsdorf, D.J., Umesono, K., and Evans, R.M. (1994). Proc. Natl. Acad. Sci. U.S.A. 91, 7355-7359.

Kliewer, S.A., Lenhard, J.M., Willson, T.M., Patel, I., Morris, D.C., and Lehmann, J.M. (1995). Cell 83, 813-819.

Kliewer, S.A., Sundseth, S.S., Jones, S.A., Brown, P.J., Wisely, G.B., Koble, C.S., Devchand, P., Wahli, W., Willson, T.M., Lenhard, J.M., and Lehmann, J.M. (1997). Proc. Natl. Acad. Sci. U.S.A. 94, 4318-4323.

Kliewer, S.A., Lehmann, J.M., and Willson, T.M. (1999). Science 284, 757-760.

Krey, G., Braissant, O., L'Horset, F., Kalkhoven, E., Perroud, M., Parker, M.G., and Wahli, W. (1997). Mol. Endocrinol. 11, 779-791.

Lee, S.S., Pineau, T., Drago, J., Lee, E.J., Owens, J.W., Kroetz, D.L., Fernandez-Salguero, P.M., Westphal, H., and Gonzalez, F.J. (1995). Mol. Cell. Biol. 15, 3012-3022.

Lehmann, J.M., Moore, L.B., Smith-Oliver, T.A., Wilkison, W.O., Willson, T.M., and Kliewer, S.A. (1995). J. Biol. Chem. 270, 12953-12956.

Lim, H., Gupta, R.A., Ma, W.G., Paria, B.C., Moller, D.E., Morrow, J.D., DuBois, R.N., Trzaskos, J.M., and Dey, S.K. (1999). Genes Devel. 13, 1561-1574.

Majer, M., Popov, K.M., Harris, R.A., Bogardus, C., and Prochazka, M. (1998). Mol. Gen. Metab. 65, 181-186.

Mangelsdorf, D.J., and Evans, R.M. (1995). Cell 83, 841-850.

Moore, L.B., Parks, D.J., Jones, S.A., Bledsoe, R.K., Consler, T.G., Stimmel, J.B., Goodwin, B., Liddle, C., Blanchard, S.G., Willson, T.M., Collins, J.L., and Kliewer, S.A. (2000). J. Biol. Chem. 275, 15122-15127.

Mukherjee, R., Davies, P.J.A., Crombic, D.L., Bischoff, E.D., Cesario, R.M., Jow, L., Hamann, L.G., Boehm, M.F., Mondon, C.E., Nadzan, A.M., Paterniti, J.R., Jr., and I leyman, R.A. (1997). Nature 386, 407-410.

Nagy, L., Tontonoz, P., Alvarez, J.G., Chen, H., and Evans, R.M. (1998). Cell 93, 229-240.

Nolte, R.T., Wisely, G.B., Westin, S., Cobb, J.E., Lambert, M.H., Kurokawa, R., Rosenfeld, M.G., Willson, T.M., Glass, C.K., and Milburn, M.V. (1998). Nature 395, 137-143.

Oberfield, J.L., Collins, J.L., Holmes, C.P., Goreham, D.M., Cooper, J.P., Cobb, J.E., Lenhard, J.M., Hull-Ryde, E.A., Mohr, C.P., Blanchard, S.G., Parks, D.J., Moore, L.B., Lehmann, J.M., Plunket, K., Miller, A.B., Milburn, M.V., Kliewer, S.A., and Willson, T.M. (1999). Proc. Natl. Acad. Sci. U.S.A. 96, 6102-6106.

Parks, D.J., Tomkinson, N.C., Villeneuve, M.S., Blanchard, S.G., and Willson, T.M. (1998). Bioorg. Med. Chem. Lett. 8, 3657-3658.

Peters, J.M., Hennuyer, N., Staels, B., Fruchart, J.C., Fievet, C., Gonzalez, F.J., and Auwerx, J. (1997). J. Biol. Chem. 272, 27307-27312.

Peters, J.M., Lee, S.S. T., Li, W., Ward, J.M., Gavrilova, O., Everett, C., Reitman, M.L., Hudson, L.D., and Gonzalez, F.J. (2000). Mol. Cell. Biol. 20, 5119-5128

Randle, P.J. (1998). Diabetes Metab. Rev. 14, 263-283.

Renaud, J.-P., Rochel, N., Ruff, M., Vivat, V., Chambon, P., and Moras, D. (1995). Nature 378, 681-689.

Repa, J.J., and Mangelsdorf, D.J. (1999). Curr. Opin. Biotech. 10, 557-563. 
Shiau, A.K., Barstad, D., Loria, P.M., Cheng, L., Kushner, P.J., Agard, D.A., and Greene, G.L. (1998). Cell 95, 927-937.

Shimkets, R.A., Lowe, D.G., Tai, J.T., Sehl, P., Jin, H., Yang, R., Predki, P.F., Rothberg, B.E., Murtha, M.T., Roth, M.E., Shenoy, S.G., Windemuth, A., Simpson, J.W., Simons, J.F., Daley, M.P., Gold, S.A., McKenna, M.P., Hillan, K., Went, G.T., and Rothberg, J.M. (1999). Nature Biotech. 17, 798-803.

Sohda, T., Mizuno, K., Tawada, H., Sugiyama, Y., Fujita, T., and Kawamatsu, Y. (1982). Chem. Pharm. Bull. 30, 3563-3573.

Sparks, R.L., Strauss, E.E., Zygmunt, A.I., and Phelan, T.E. (1991). J. Cell. Physiol. 146, 101-109.

Spiegelman, B.M. (1998). Diabetes 47, 507-514.

Sreenan, S., Keck, S., Fuller, T., Cockburn, B. and Burant, C.F. (1999). Am. J. Physiol. 276, E1119E1129.

Takada, I., Yu, R.T., Xu, H.E., Lambert, M.H., Montana, V.G., Kliewer, S.A., Evans, R.M., and Umesono, K. (2000). Mol. Endocrinol. 14, 733-740.

Tontonoz, P., Hu, E., Graves, R.A., Budavari, A.I., and Spiegelman, B.M. (1994a). Genes Devel. 8, 1224-1234.

Tontonoz, P., Hu, E., and Spiegelman, B.M. (1994b). Cell 79, 1147-1156.

Uppenberg, J., Svensson, C., Jaki, M., Bertilsson, G., Jendeberg, L., and Berkenstam, A. (1998). J. Biol. Chem. 273, 31108-31112.

Vamecq, J., and Draye, J.-P. (1989). Essays Biochem. 24, 115-225.

Willson, T.M., Cobb, J.E., Cowan, D.J., Wiethe, R.W., Correa, I.D., Prakash, S.R., Beck, K.D., Moore, L.B., Kliewer, S.A., and Lehmann, J.M. (1996). J. Med. Chem. 39, 665-668.

Willson, T.M., Brown, P.J., Sternbach, D.D., and Henke, B.R. (2000a). J. Med. Chem. 43, 527-550.

Willson, T.M., Cobb, J.E., Parks, D.J., and Blanchard, S.J. (2000b). Diabetes 49, 523.

Wilson, G.G., Abou-Donia, M., Frith, L., Patel, J., and Fiedorek, F.T. (2000). Diabetes 49, 525.

Xu, H.E., Lambert, M.H., Montana, V.G., Parks, D.J., Blanchard, S.G., Brown, P.J., Sternbach, D.D., Lehmann, J.M., Wisely, G.B., Willson, T.M., Kliewer, S.A., and Milburn, M.V. (1999). Mol. Cell 3, 397-403.

Yu, K., Bayona, W., Kallen, C.B., Harding, H.P., Ravera, C.P., McMahon, G., Brown, M., and Lazar, M.A. (1995). J. Biol. Chem. 270, 23975-23983. 



\title{
Insulin Resistance and Its Treatment by Thiazolidinediones
}

\author{
Harold E. Lebovitz and Mary Ann Baneru \\ State University of New York, Health Science Center at Brooklyn, Box 50, \\ 450 Clarkson Avenue, Brooklyn, New York 11203
}

\begin{abstract}
Insulin resistance is a change in physiologic regulation such that a fixed dose of insulin causes less of an effect on glucose metabolism than occurs in normal individuals. The normal compensatory response to insulin resistance is an increase in insulin secretion that results in hyperinsulinemia. If the hyperinsulinemia is sufficient to overcome the insulin resistance, glucose regulation remains normal; if not, type 2 diabetes ensues. Associated with insulin resistance, however, is a cluster of other metabolic abnormalities involving body fat distribution, lipid metabolism, thrombosis and fibrinolysis, blood pressure regulation, and endothelial cell function. This cluster of abnormalities is referred to as the insulin resistance syndrome or the metabolic syndrome. It is causally related not only to the development of type 2 diabetes but also to cardiovascular disease. A major unresolved issue is whether there is a single underlying cause of this syndrome and, if so, what might it be? Several promising hypotheses have been proposed. There are some data to support the hypothesis that fetal malnutrition imprints on metabolic regulatory processes that, in later adult life, predispose to the development of the insulin resistance syndrome. Visceral obesity also has been a candidate for the cause of the syndrome. Whatever mechanism is ultimately found to be responsible, it will undoubtedly have both genetic and environmental components. Among the biochemical mediators that are likely to be responsible for the interference with insulin's effects on intermediary metabolism are free fatty acids and other products from adipose tissue. Recent data suggest that the substances stimulate serine phosphorylation of molecules involved in the initial steps of insulin action, thereby blocking the ability of these molecules to be tyrosine phosphorylated and initiate the subsequent steps of the insulin action cascade.

The thiazolidinediones are a new class of agents that have been developed to treat type 2 diabetic patients. These drugs act as peroxisome proliferator-activated receptor gamma (PPAR $\gamma$ ) agonists. Following their binding to the receptor, the heterodimer molecule that contains the binding site is activated. The activated complex binds to the response elements of specific genes that regulate molecules that effect insulin action and lipid metabolism. These genes are either activated or inhibited. Specifically, the thiazolidinediones improve insulin action and decrease insulin resistance. The exact mechanism by which these agents decrease insulin resistance is not clear but they do decrease the elevated free fatty acid levels present in insulin-resistant patients and they appear to change the body distribution of adipose tissue. Treatment of insulin-resistant type 2 diabetic patients with thiazolidinediones not only improves glycemic control and decreases insulin resistance, it also improves many of the abnormalities that are part of the insulin resistance syndrome.
\end{abstract}




\section{Introduction}

Insulin resistance has been recognized since the 1930s. However, it was the development of sensitive assays for insulin and quantitative methods for estimating insulin action that made it possible to define the scope of the problem and its clinical implications (Reaven, 1988; Zavaroni et al., 1989). Most individuals appear to develop insulin resistance when environmental factors interact with specific genetic predispositions that confer susceptibility (Stern, 1997; Samaras and Campbell, 2000). The key environmental factors responsible for the development of insulin resistance are abnormalities of nutritional intake (Samaras et al., 1998), leading to fetal malnutrition and/or adult obesity and decreased physical activity. The genetic factors have yet to be clarified. Changing lifestyles throughout the world have resulted in as much as 16 to 25 percent of some adult populations having insulin resistance and an associated cluster of metabolic and cardiovascular risk factor abnormalities that have been termed "the metabolic syndrome." Individuals with the metabolic syndrome are at increased risk to develop type 2 diabetes, polycystic ovarian syndrome (PCOS), and/or accelerated atherosclerosis with its accompanying cardiovascular complications.

The metabolic syndrome and its associated diseases have engendered extensive research into understanding the molecular mechanisms involved in causing insulin resistance and to develop pharmacologic agents to specifically treat insulin resistance and the metabolic syndrome. The thiazolidinediones represent such a new class of drugs that have been developed to treat insulin resistance. Inquiries into the mechanism of action of these drugs have led to our greatly expanded knowledge of peroxisome proliferator activated receptors (PPARs) and their role in human physiology (Willson et al., 2000). This chapter focuses on some of our studies to define the mechanisms responsible for insulin resistance, the metabolic syndrome, and its clinical consequences in humans and to understand the role that the thiazolidinediones may play in treatment.

\section{Insulin Resistance and the Metabolic Syndrome: A Disease of Nutritional Intake?}

Insulin resistance and the metabolic syndrome have been the subject of hundreds of reviews. An area of major interest to us has been the role of nutrition and its effect on body composition as a cause of insulin resistance and its associated metabolic and cardiovascular risk factor abnormalities. Our identification of insulin-sensitive and insulin-resistant variants of type 2 diabetes in African American populations in the late 1980s (Banerji and Lebovitz, 1989) gave us the opportunity to dissociate the pathogenesis of hyperglycemia from that of insulin resistance in type 2 diabetic patients. We observed several major differences between these two variants. Insulin-sensitive type 2 diabetics had a mean body 
mass index (BMI) of $25.6 \mathrm{~kg} / \mathrm{m}^{2}$; normal mean fasting plasma insulin levels; and normal insulin sensitivity, as measured by the euglycemic-hyperinsulinemic clamp (Banerji and Lebovitz, 1992; Chaiken et al., 1991). They had no evidence of diabetic dyslipidemia or increased blood pressure (Chaiken et al., 1993). In contrast, individuals with the insulin-resistant variant had a mean BMI of 30.2 $\mathrm{kg} / \mathrm{m}^{2}$; were hyperinsulinemic and insulin resistant, as measured by the euglycemic-hyperinsulinemic clamp; and had classic diabetic dyslipidemia (Banerji and Lebovitz, 1989; Chaiken et al., 1991,1993). A more-detailed analysis of our populations revealed that insulin resistance was rarely observed in African American individuals with a BMI $<24.5 \mathrm{~kg} / \mathrm{m}^{2}$; was almost always observed in those with a BMI $>28.5 \mathrm{~kg} / \mathrm{m}^{2}$; and was present in about 50 percent of those with a BMI between 24.5 and $28.5 \mathrm{~kg} / \mathrm{m}^{2}$ (Banerji and Lebovitz, 1992). These data suggested that body fat distribution, rather than generalized obesity, might be responsible for insulin resistance. Similar data on insulin-sensitive and insulin-resistant diabetic subjects have been reported in Caucasian and Oriental populations (Groop et al., 1993; Haffner et al., 1999; Taniguchi et al., 2000). Table I summarizes the differences that have been reported between insulin-sensitive and insulin-resistant type 2 diabetic patients.

Utilizing a computerized tomography (CT) technique involving 22 to 24 scans of the trunk, abdomen, and extremities and a computer program analysis, we were able to show that insulin sensitivity, as measured by the euglycemic hyperinsulinemic clamp, was negatively correlated with visceral adipose tissue volume in both men and women (Figure 1) and had no significant correlation with subcutaneous adipose tissue volume (Figure 2) (Banerji et al., 1995a, 1997). We hypothesized that insulin sensitivity was determined to a significant degree by the volume of visceral adipose tissue mass. This relationship is a negative curvilinear one. The greatest impact in decreasing insulin resistance occurs when the visceral adipose tissue volume increases from 1.0 to 3.5 liters. The increase in visceral adipose tissue volume is significantly associated with the presence and magnitude of diabetic dyslipidemia.

Many other published studies support the hypothesis that visceral obesity plays a major role in causing insulin resistance (Lemieux et al., 1996; Boyko et al., 2000; Karter et al., 1996). Goodpaster and his associates (1997) initially reported in a cross-sectional study that insulin resistance correlated with subcutaneous and thigh muscle fat independently of visceral fat. However, in a follow-up study of a group of patients who had undergone significant weight loss, the improvement in insulin sensitivity correlated with the change in visceral adipose tissue (Figure 3), rather than the change in subcutaneous adipose tissue (Goodpaster et al., 1999). Longitudinal studies in Japanese Americans over a 5-year period showed that accumulation of visceral adipose tissue predicts the development of insulin resistance and type 2 diabetes (Boyko et al., 2000). Cross-sectional and longitudinal studies carried out by Despres and colleagues likewise have 
TABLE I

Differences in Metabolic Profiles Between Insulin-sensitive and Insulin-resistant Type 2 Diabetic Patients

\begin{tabular}{lll} 
Metabolic Parameter & Insulin Sensitive & Insulin Resistant \\
\hline Hyperglycemia & Yes & Yes \\
Hyperinsulinemia & No & Yes \\
Central obesity & No & Yes \\
Diabetic dyslipidemia & & \\
$\quad \begin{array}{l}\uparrow \text { Plasma triglycerides } \\
\quad \downarrow \text { Plasma HDL cholesterol }\end{array}$ & No & Yes \\
$\quad$ Small, dense LDL pattern & No & Yes \\
Pro-coagulant state & No & Yes \\
$\quad \begin{array}{l}\uparrow \text { Plasma fibrinogen } \\
\quad\end{array}$ & No & Yes \\
Hypertension & No & Yes \\
& Not $\uparrow(?)$ & $\uparrow$ in normal weight but
\end{tabular}

[Table reflects results of studies in Banerji, M.A., and Lebovitz, H.E. Diabetes 38, 784-782, 1989; Banerji, M.A., and Lebovitz, H.E. Diabetes Care 15, 1295-1302, 1992; Banerji, M.A., Chaiken, R.L., Gordon, D., Kral, J.G., and Lebovitz, H.E. Diabetes 44, 141-146, 1995; Banerji, M.A., Lebovitz, J., Chaiken, R.L., Gordon, D., Kral, J.G., and Lebovitz, H.E. Am. J. Physiol. 273, E425-E432, 1997; Chaiken, R.L., Banerji, M.A., Pasmantier, R.M., Huey, H., Hirsch, S., and Lebovitz, H.E. Diabetes Care 14, 1036-1042, 1991; Chaiken, R.L., Banerji, M.A., Huey, H., and Lebovitz, H.E. Diabetes 42 , 444-449, 1993; Haffner, S.M., Rewers, M., D’Agostino, R. Jr., Selby J., Mykkanen, L., Savage, P.J., Tracy, R., Saad, M.F., and Iloward, B.V. Diabetes Care 22, 562-568, 1999; Taniguchi, A., Fukushima, M., Sakai, M., Kataoka, K., Miwa, K., Nagata, 1., Doi, K., Arakawa, H., Nagasaka, S., Tokuyama, K., and Nakai, Y. Metabolism 49, 1001-1005, 2000.]

shown that insulin resistance and the metabolic syndrome appear to be caused by an increase in visceral adipose tissue mass (Lemieux et al., 1996).

Not all studies have supported the concept that visceral obesity is the cause of insulin resistance (Abate et al., 1995). Several technological reasons can account for the failure of a study to show an independent relationship between visceral adipose tissue mass and insulin resistance. The negative curvilinear relationship between insulin action and visceral adipose tissue volume shown in our studies and implied from the weight loss studies of Goodpaster (Figures 1 and 3) indicate that attempting to measure any relationship in severely obese people 


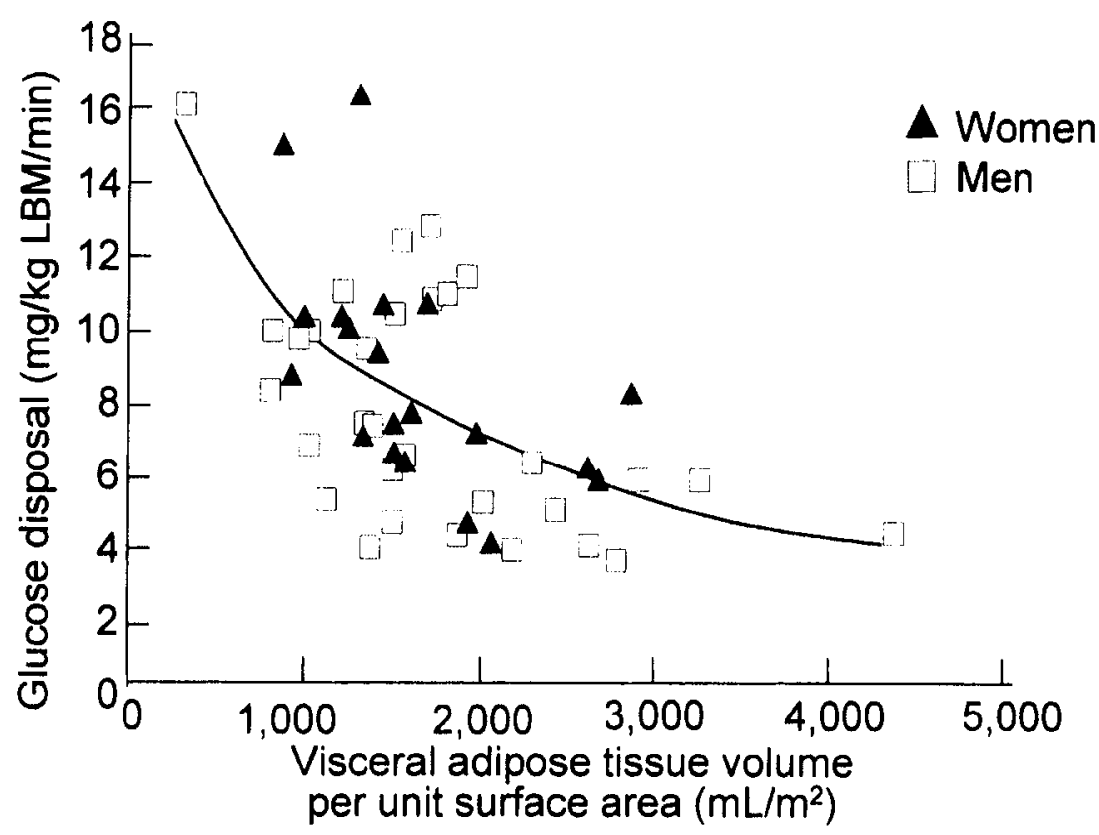

FlG. 1. Relationship between visceral adipose tissue volume and insulin action in AfricanAmerican type 2 diabetic men and women. Visceral adipose tissue volume was determined from multiple CAT scans through the abdominal region by a computer program that integrates the data from individual scans. Insulin sensitivity was determined by the euglycemic-hyperinsulinemic clamp technique. The relationship is highly statistically significant $(r=0.54 ; P<0.002)$ and is clearly curvilinear. [Reprinted with permission from Banerji, M.A., Lebovitz, J., Chaiken, R.L., Gordon, D., Kral, J.G., and Lebovitz, H.E. Am. J. Physiol, 273, E425-E432, 1997.]

would be meaningless, since the slope of the curve is likely to be flat. The use of a single CAT scan or magnetic resonance imaging (MRI) slice may give an imprecise estimate of the total visceral and subcutaneous adipose tissue volumes (Samaras and Campbell, 2000). Finally, it is important to recognize that it is the flux of active substances from the visceral adipose tissue that is likely to be causing the metabolic effects and that volume is a crude estimate of what may be the flux. It is important to note that visceral obesity does have a significant relationship to total obesity. However, there are striking differences among individuals in the percent of their total body fat deposited in their visceral adipose tissue. In our studies, this can vary by as much as three- to four-fold and appears to be genetically determined (Banerji et al., 1995a).

The mechanism by which an increase in visceral adipose mass causes insulin resistance and its associated metabolic and cardiovascular abnormalities has not been clearly defined. It is postulated that insulin resistance results from the direct 


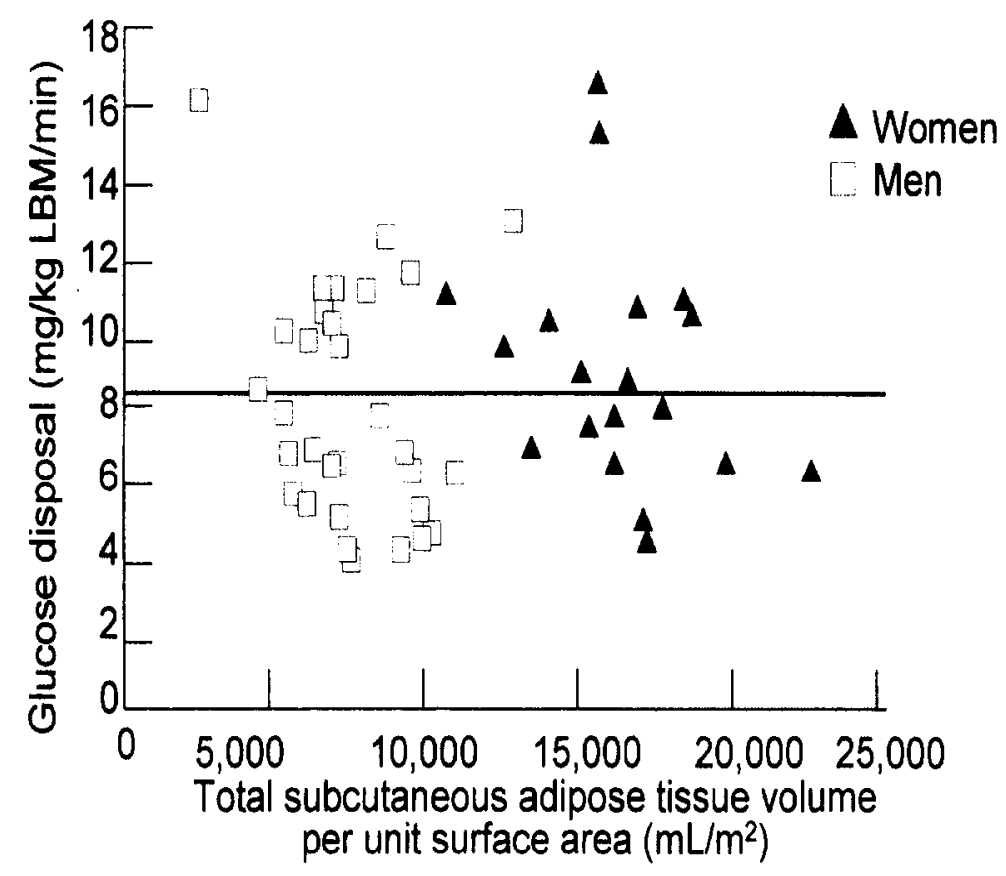

FIG. 2. Lack of relationship between total subcutaneous adipose tissue volume and insulin action in African-American type 2 diabetic men and women. Subcutaneous adipose tissue volume was determined from 22 to 24 CAT scans and calculated by a computer program that integrates the data. Insulin sensitivity was determined by the euglycemic-hyperinsulinemic clamp technique. The correlation coefficient was 0.24 , which was not statistically significant. [Reprinted with permission from Banerji, M.A., Lebovitz, J., Chaiken, R.L., Gordon, D., Kral, J.G., and Lebovitz, H.E. Am. J. Physiol. 273, E425-E432, 1997.]

flux of active factors such as free fatty acids and perhaps tumor necrosis factor alpha (TNF $\alpha$ ) from the visceral adipose tissue through the portal vein into the liver and beyond into the peripheral tissues (Figure 4). Abdominal adipose tissue is known to have a high rate of lipolysis and a rapid turnover of free fatty acids (Rebuffe-Scrive et al., 1989; Boden, 1996). Increased flux of free fatty acids into the liver increases very-low-density lipoprotein (VLDL) particle synthesis and hepatic triglyceride concentrations (Brunzell and Hokanson, 1999). It therefore would be expected - and, indeed, has been shown - that an increase in visceral adipose tissue mass is associated with an increase in hepatic (Figure 4) as well as intramuscular triglycerides and circulating plasma triglycerides (Banerji et al., 1995b). Hepatic sensitivity to insulin as well as hepatic clearance of insulin are thought to be reduced by increases in hepatic triglycerides. Free fatty acids and 


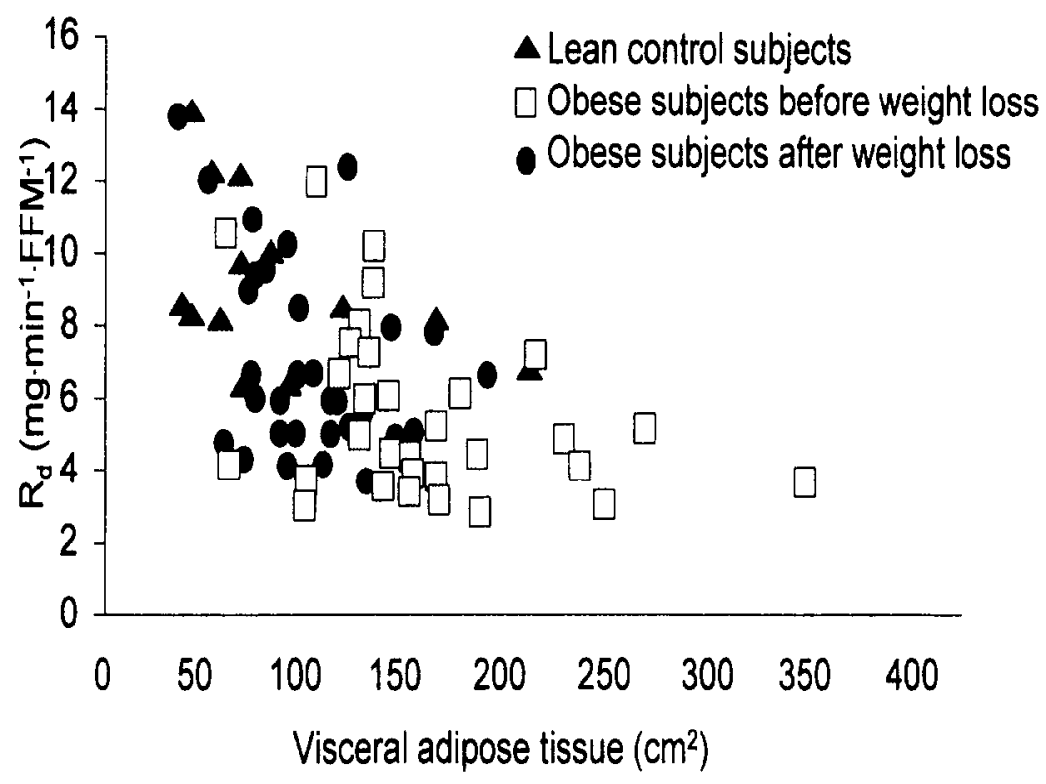

FIG. 3. Relationship between visceral adipose tissue and insulin sensitivity in lean control subjects, obese subjects before weight loss, and the same obese subjects after weight loss. Visceral adipose tissue area was determined as the area in a CAT scan through L4-5. Insulin sensitivity was determined by the euglycemic-hyperinsulinemic clamp technique. The data show that insulin sensitivity changes in a curvilinear fashion in relationship to visceral adipose tissue area. [Data from Goodpaster, B.H., Kelley, D.E., Wing, R.R., Meier, A., and Thaete, F.L. Diabetes 48, 839-847, 1999.]

TNF $\alpha$ both have been shown to cause peripheral insulin resistance. The exposure of the liver to these and other products of adipose tissue release is likely to be much greater from visceral that from subcutaneous adipose tissue. Free fatty acids cause insulin resistance in human skeletal muscle by interfering with the effect of insulin in increasing Glut-4-mediated glucose transport across the plasma membrane (Cline et al., 1999). The molecular mechanism for this interference in insulin action appears to be the consequence of a reduction in insulin receptor substrate-1 (IRS-1)-associated phosphatidylinositol 3-kinase (PI-3-kinase) activity (Dresner et al., 1999). It has been suggested that free fatty acids activate an intracellular serine kinase, leading to phosphorylation of serine sites on IRS-1 and/or the insulin receptor, reducing their abilities to be activated by tyrosine phosphorylation. The role of TNFo in the pathogenesis of human insulin resistance is controversial. Studies in rodents show unequivocally that TNF $\alpha$ is a mediator of insulin resistance. It appears to activate phosphorylases that phospho- 


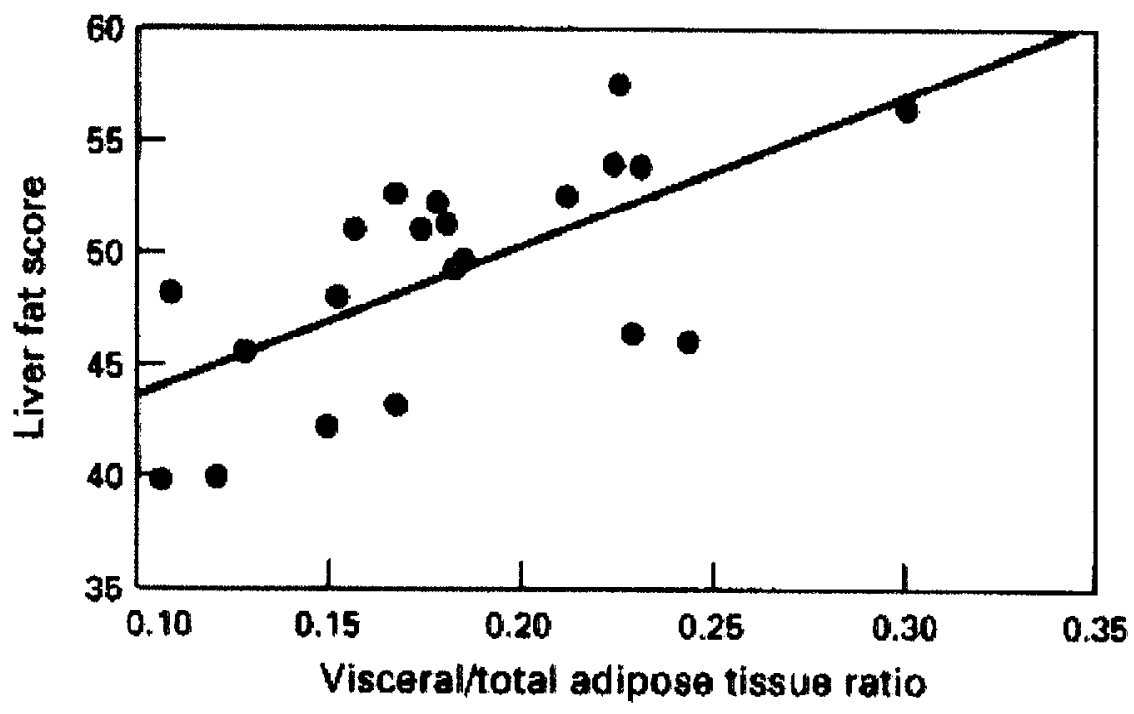

FIG. 4. Liver fat is highly correlated with visceral adipose tissue volume. [Data from Banerji, M.A., Buckley, M.C., Chaiken, R.L., Gordon, D., Lebovitz, H.E., and Kral, J.G., Intl. J. Obesity 19 , $846-850,1995$.

rylate serines on IRS-1 and/or PI-3-kinase and reduces their ability to be activated by insulin (Hotamisligil and Spiegelman, 1994). While the direct drainage of high concentrations of free fatty acids into the liver increases hepatic VLDL triglyceride synthesis, causes an increase in hepatic steatosis, and is likely to cause insulin resistance at the level of the hepatocyte, other effects on hepatic function have not been appropriately assessed. An overview of our hypotheses of the mechanisms by which visceral adiposity could cause insulin resistance and the insulin resistance syndrome is outlined in Figure 5.

Another factor that is assuming increasing importance in our attempts to understand the pathogenesis of insulin resistance and the metabolic syndrome is fetal malnutrition. Many epidemiologic studies have shown a significant relationship between low birth weight and the subsequent development of type 2 diabetes, insulin resistance, and the metabolic syndrome in later adult life (Barker et al., 1993; Bavdekar et al., 1999; Dabelea et al., 1999). These low birth weight babies have a tendency to be overweight adults. The hypothesis that has been formulated is that fetal malnutrition causes the induction of metabolic pathways in the fetus to maximally conserve nutrients (Phillips et al., 1998). These imprinted pathways then persist throughout the life of the individual. The lifestyle of our current society provides these individuals with excess food and reduced physical activity 
Increased visceral adipose tissue

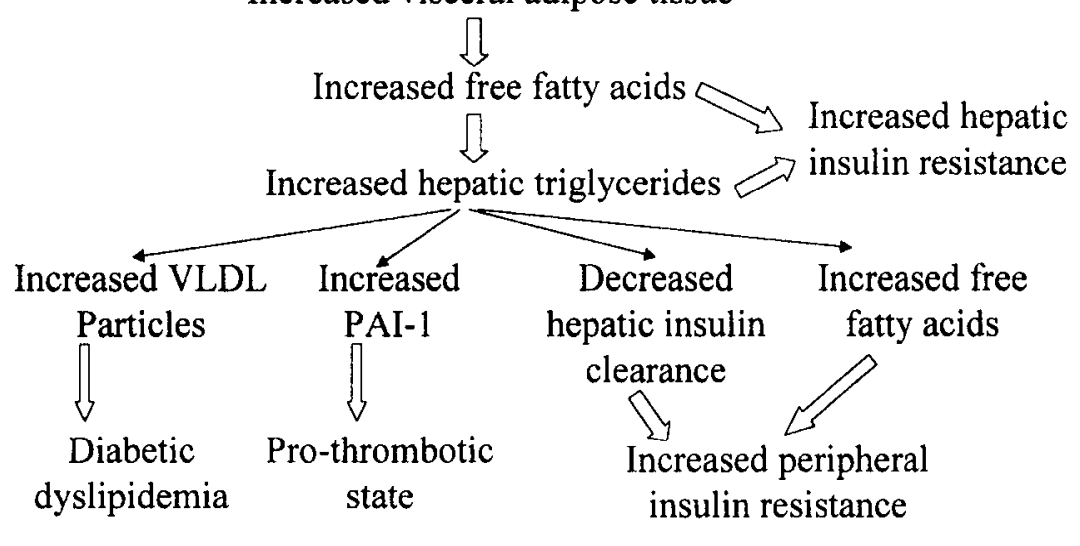

FIG. 5. A proposed scheme for the mechanisms by which visceral adipose tissue mass might cause insulin resistance and the insulin resistance syndrome.

such that they become obese and develop insulin resistance and the metabolic syndrome.

\section{Components of the Metabolic Syndrome}

The concept of the metabolic syndrome has evolved from a number of metabolic and cardiovascular epidemiologic studies that have identified a group of factors that cluster together and are associated with an increased risk of developing type 2 diabetes and/or macrovascular disease. This cluster consists of 1) insulin resistance and hyperinsulinemia; 2) central obesity; 3) a classic dyslipidemia that includes an elevated plasma triglyceride, a low plasma highdensity lipoprotein (HDL) cholesterol, and a small, dense low-density lipoprotein (LDL) cholesterol particle pattern; 4) a procoagulant state made up of elevated plasma fibrinogen and plasminogen activator inhibitor 1 (PAI-1); 5) elevated systolic and diastolic blood pressure; 6) hyperuricemia; and 7) microalbuminuria. Insulin resistance and/or hyperinsulinemia have been postulated to be the cause of the other abnormal metabolic and cardiovascular risk factors that occur in the metabolic syndrome. In all likelihood, it is the combination of visceral adiposity and its resultant insulin resistance that, together, create the other components of the syndrome.

Visceral obesity is highly correlated with the other components of the insulin resistance syndrome and, as noted above, with insulin resistance itself. Many epidemiologic studies have shown that visceral obesity, as estimated by waist/hip ratio or waist circumference, predicts the development of type 2 diabetes (Ohlson 
et al., 1985; Okosun et al., 1998), hypertension (Okosun et al., 1998), and cardiovascular disease (Larsson et al., 1984; Lapidus et al., 1984; Rexrode et al., 1998).

\section{Thiazolidinediones: A Class of Agents That Are PPAR $\gamma$ Agonists}

The thiazolidinediones were discovered during the screening of a number of compounds for lipid-lowering effects. They were noted to decrease hyperglycemia and hyperinsulinemia in several rodent models of insulin resistance. Three thiazolidinediones have been studied extensively and used clinically in humans: troglitazone, rosiglitazone, and pioglitazone. Troglitazone was introduced first, so more information is available about its effects. Because of severe idiosyncratic liver toxicity leading to hepatic failure and death, troglitazone was removed from the market in March 2000. Rosiglitazone and pioglitazone have been approved for clinical use since the spring and summer of 1999, respectively. The major structural differences in the three thiazolidinediones are in their side chains (Willson et al., 2000). Troglitazone combines a vitamin E structural moiety with a glitazone moiety. The thiazolidinediones (or glitazones, as they are commonly called) are ligands for a PPAR complex located within the nucleus (Lehmann $e t$ al., 1995). The PPAR family consists of three distinct types (PPAR $\alpha$, PPAR $\gamma$, PPAR 8 ), each of which has unique functions. All three thiazolidinediones have been shown to have only PPAR $\gamma$ agonist activity (Lehmann et al., 1995; Willson et al., 2000). None of them has been shown to have any significant PPAR $\alpha$ or PPAR $\delta$ activity in binding assays. The three thiazolidinediones have significantly different binding affinities for the PPAR $\gamma$ receptor, with rosiglitazone having the greatest binding affinity, troglitazone the least, and pioglitazone intermediate. The binding affinities correlate reasonably well with the therapeutic doses that are effective in treating hyperglycemia in insulin-resistant type 2 diabetes in humans.

The mechanism of action of thiazolidinediones involves their binding to the nuclear PPAR $\gamma$ receptor. PPAR $\gamma$ receptors are part of a heterodimer that includes an retinoid X receptor (RXR) (Olefsky, 2000). This heterodimer is involved in the control of various aspects of lipid and carbohydrate metabolism. When thiazolidinediones bind to the PPAR $\gamma$ receptor, the heterodimer attaches to the PPAR $\gamma$ response elements within the promoter domains of specific target genes and modifies their interactions with co-repressor and coactivator elements. This results in either activation or inhibition of specific gene transcription (Saltiel and Olefsky, 1996; Whitcomb and Saltiel, 1995). PPAR $\gamma$ receptors are of two subtypes. PPAR $\gamma 2$ is found in high concentrations in adipocytes. Muscle contains PPAR $\gamma 1$ and its concentration is about 10 to 15 percent that of PPAR $\gamma$ receptors in adipose tissuc (Kruszynska et al., 1998). PPAR $\gamma 2$ is a splice variant of PPAR $\gamma 1$ containing 
30 additional amino-terminal amino acids (Willson et al., 2000). PPAR $y$ agonists promote the differentiation of precursor cells to adipocytes as well as promote the differentiation of many other cell types. PPAR $\gamma$ receptors are abundant in other cells (e.g., macrophages, endothelium, vascular smooth muscle, colon epithelium).

The molecular mechanisms of thiazolidinedione action are quite complex. Recent studies have suggested that the various thiazolidinediones may have differential effects in some tissues (Walker et al., 1998; Camp et al., 2000). Studies with heterozygous PPAR $\gamma$ knockout mice show paradoxical effects to those that would be expected (Kadowaki, 2000; Miles et al., 2000). These mice have normal adipose tissue development and normal glucose metabolism. However, they have an enhanced sensitivity to insulin, rather than the expected insulin resistance. PPAR $\gamma$ activation by thiazolidinediones to improve insulin sensitivity and promote adipose cell differentiation must be more complex than previously thought. The potential implications of these data have been reviewed recently by Olefsky (2000).

\section{A. EFFECTS OF THIAZOLIDINEDIONES ON ADIPOSE TISSUE}

Specific effects of thiazolidinediones are most dramatic on adipose tissue cell differentiation. Incubation of 3T3 fibroblast cells with thiazolidinediones in tissue culture causes cells to differentiate into adipocytes (Kreutter et al., 1990). This differentiation causes the expression of a number of genes, including those coding for glycerol phosphate dehydrogenase, Glut 4 glucose transporter, fatty acid binding protein aP2, and adipsin (Wu et al., 1998). In obese Zucker rats, administration of troglitazone for 15 days increased the number of small adipocytes approximately four-fold in both subcutaneous and retroperitoneal adipose tissue (Okuno et al., 1998). At the same time, it decreased the number of large adipocytes by approximately 50 percent. These effects were associated with a 2.5 -fold increase in apoptotic nuclei in retroperitoneal adipose tissue and a reduction in adipose tissue expression of TNF $\alpha$ and leptin. Plasma triglyceride levels were significantly reduced. In humans, rosiglitazone has been shown to increase the differentiation of subcutaneous adipose tissue stem cells into adipocytes but to have no effect on the differentiation of omental (visceral) adipose tissue stem cells into adipocytes (Adams et al., 1997). These effects on adipose tissue are of considerable importance because the presence of so few PPAR $\gamma$ receptors in muscle has raised the speculation that the primary effect of PPAR $\gamma$ activation in decreasing insulin resistance may be secondary to its effects on adipose tissue, rather than a direct effect on muscle. This is unresolved, as there are some data to support a direct action on muscle. A small number of PPAR $\gamma$ receptors are found in muscle (Kruszynska $e t a l ., 1998$ ); thiazolidinediones do improve insulin 
action on glucose transport in muscle in vitro (Ciaraldi et al., 1990); and thiazolidinediones improve insulin sensitivity in insulin-resistant transgenic mice in which most adipose tissue has been ablated (Burant $e t$ al., 1997). Two human studies of short duration, with a limited number of patients and using CAT or MRI scans, have reported that troglitazone has a differential effect on body fat in type 2 diabetic patients. Mori and colleagues (1999) found that 6 months' treatment with $400 \mathrm{mg} /$ day increased subcutaneous fat but had either no effect or caused a small decrease in visceral fat mass. Kelly and coworkers (1999) reported that 12 weeks' treatment with $600 \mathrm{mg}$ daily decreased intra-abdominal fat mass but had no effect on either total fat or subcutaneous fat mass. Preliminary results of our large, ongoing investigation into the effects of rosiglitazone $8 \mathrm{mg}$ daily for six months on body composition in type 2 diabetic patients are shown in Table II. After 6 months of treatment, our first 16 patients had a $2.53-\mathrm{kg}$ mean increase in body weight. Total body fat increased as a result of an increase in subcutaneous adipose tissue mass. No significant change occurred in the visceral adipose tissue mass. Overall, the limited data available on the effects of thiazolidinediones on body composition in humans suggest that the thiazolidinediones cause a modest increase in subcutaneous adipose tissue mass but appear to have little or minimal effects on visceral fat mass. The effects on hepatic triglycerides independent of improved glycemic control are unknown.

\section{B. EFFECTS OF THIAZOLIDINEDIONES ON INSULIN RESISTANCE}

In animal models of insulin resistance, thiazolidinediones have been shown to be remarkably efficacious in improving insulin sensitivity. They frequently restore insulin sensitivity to normal. When thiazolidinediones have been admin-

TABLE II

Effect of 6 Months' Treatment with Rosiglitazone on Body Composition in 16 Type 2 Diabetic Patients

\begin{tabular}{llll} 
Parameter & Baseline & Change & Significance \\
\hline Weight $(\mathrm{kg})$ & $81.8 \pm 3.38$ & $2.53 \pm 0.87$ & 0.011 \\
$\mathrm{BMI}\left(\mathrm{kg} / \mathrm{m}^{2}\right)$ & $29.75 \pm 1.10$ & $0.99 \pm 0.34$ & 0.011 \\
Abdominal SQ adipose tissue (liters) & $8.69 \pm 0.91$ & $0.68 \pm 0.31$ & 0.046 \\
Visceral adipose tissue (liters) & $3.75 \pm 0.31$ & $0.04 \pm 0.09$ & 0.631 \\
Total body fat (percent) & $36.06 \pm 2.82$ & $1.05 \pm 0.62$ & 0.113 \\
Total lean body mass (liters) & $45.59 \pm 2.59$ & $0.70 \pm 0.28$ & 0.026 \\
\hline
\end{tabular}


istered to humans with type 2 diabetes and insulin resistance, the effects in reported studies have been more modest (Saleh et al., 1999; Olefsky, 2000). Insulin action in muscle, as measured by the euglycemic-hyperinsulinemic clamp technique, has been reported to increase anywhere from 30 to 54 percent but usually not into the normal range. Recently, the HOMA (Homeostasis Optimal Model Assessment) model, which assesses insulin resistance from a computer-derived model using simultaneously measured fasting plasma glucose and insulin, has been validated as an acceptable method for quantifying insulin action in population-based studies (Haffner et cl., 1996; Bonora et cl., 2000). We have used this model to analyze the effect of rosiglitazone monotherapy on insulin sensitivity in a large population of type 2 diabetic patients. In this population of patients with mean hemoglobin A lc (HbAlc) of approximately 7.8 percent, rosiglitazone $2 \mathrm{mg}$ twice a day improved insulin sensitivity by 23.9 percent and $4 \mathrm{mg}$ twice a day by 32.5 percent, when compared to the placebo-treated controls (Lebovitz et al., in press).

A major question that remains to be answered is what is the mechanism by which thiazolidinediones decrease insulin resistance? Since a major target of the thiazolidinediones is adipose tissue and the quantity of PPAR $\gamma$ receptors in muscle is one tenth that in adipose tissue (Kruszynska et al., 1998), it has been speculated that the improvement in insulin action in muscle is secondary to PPAR $\gamma$ action on adipose tissue. Several consequences of PPAR $\gamma$ action on adipose tissue could be involved. Thiazolidinediones cause a significant decrease in the release of free fatty acids and TNF $\alpha$ from adipose tissue. This results in a significant decline in plasma levels of these factors and, as might be expected from their known effects in causing insulin resistance, an improvement in insulin action. The impressive magnitude of suppression of fasting plasma free fatty acids by rosiglitazone treatment of type 2 diabetics is shown by our recent data summarized in Table III. A second and somewhat intriguing possibility is that of a redistribution of adipose tissue mass from the central compartment of visceral, hepatic, and intramuscular sites to the peripheral subcutaneous depot. Since insulin resistance is mediated by the central pool of adipose tissue, including hepatic and intramuscular triglycerides (Banerji et al., 1995b,1997; Pan et al., 1997), and not by the peripheral pool, this would reduce insulin resistance. These two mechanisms are not mutually exclusive. As discussed previously, a direct action of thiazolidinediones on muscle cannot be excluded.

The effects of thiazolidinediones in directly improving insulin sensitivity at the level of the liver, as assessed by improvement in insulin-mediated suppression of hepatic glucose production, are significantly less impressive than their effects in improving insulin-mediated glucose muscle uptake by skeletal muscle (Inzucchi et al., 1998; Maggs et al., 1998; Yu et al., 1999). Several studies in which type 2 diabetic patients were treated with as much as 400 to $600 \mathrm{mg}$ of troglitazone 
TABLE III

Effect of 26 Weeks' Treatment with Rosiglitazone on Plasma Free Fatty Acids in Type 2 Diabetic Patients

\begin{tabular}{llll}
\hline & Baseline $(\mathrm{mg} / \mathrm{dl})$ & $\begin{array}{l}\text { Mean Decrease with } \\
\text { Treatment }(\mathrm{mg} / \mathrm{dl})\end{array}$ & $\begin{array}{l}\text { Change with } \\
\text { Treatment }(\%)\end{array}$ \\
\hline $\begin{array}{l}\text { Placebo } \\
\text { Rosiglitazone, 2 mg twice }\end{array}$ & $19.0 \pm 6.8^{\mathrm{a}}$ & $-0.9 \pm 7.6$ & -4.7 \\
$\begin{array}{l}\text { daily } \\
\begin{array}{l}\text { Rosiglitazone, 4 mg twice } \\
\text { daily }\end{array}\end{array}$ & $18.5 \pm 7.8$ & $-4.2 \pm 7.9$ & -21.9 \\
\hline
\end{tabular}

[Data from Lebovitz, H.E., Dole, J.F., Patwardhan, R., Rappaport, E.B., and Freed, M.I. J. Clin. Endocrinol. Metab. 85 , in press.] ${ }^{\mathrm{a}} 19.0 \mathrm{mg} / \mathrm{dl}$ is approximately $670 \mu \mathrm{mol} / \mathrm{l}$.

per day were unable to show a significant improvement in insulin-mediated suppression of hepatic glucose production (Inzucchi et al., 1998; Maggs et al., 1998). These studies were done using the euglycemic-hyperinsulinemic clamp technique. The lack of a significant effect raises the possibility that the improvement in fasting plasma glucose seen during thiazolidinedione treatment of type 2 diabetes is not a primary effect on the liver but may be secondary to some of the other beneficial effects of these drugs.

\section{THIAZOLIDINEDIONE EFFECTS ON THE DYSLIPIDEMIA ASSOCIATED WITH TYPE 2 DIABETES AND INSULIN RESISTANCE}

The characteristic lipid abnormalities present in type 2 diabetic patients with insulin resistance are an increase in plasma triglycerides and a decrease in plasma HDL cholesterol (Brunzell and Ilokanson, 1999; Stern, 1997). Plasma LDL cholesterol concentration is within the range seen in nondiabetics; however, the size and buoyancy of the LDL particle is different in that it is smaller and more dense (Festa et al., 1999). The small, dense LDL particles are more susceptible to oxidation and glycation and penetrate the vascular endothelium more readily. These changes are all associated with an increase in the development of cardiovascular disease in general and coronary heart disease in particular. Additionally, poor glycemic control causes an increase in VLDL particles, contributing further to the rise in plasma triglycerides.

Treatment of human type 2 diabetic patients with thiazolidinediones ameliorates many of these lipid abnormalities. Published data from the various U.S. clinical trials with troglitazone demonstrated a consistent 15 to 20 percent decrease in fasting triglycerides, a variable but approximately 10 percent rise in 
plasma LDL cholesterol, and an inconsistent effect on plasma HDL cholesterol (Willson et al., 2000). LDL particle size was found to be increased by troglitazone treatment in one small study (Tack et al., 1998a). Troglitazone treatment has been reported to increase the resistance of LDL cholesterol to oxidation (Noguchi et al., 1996). Data presented in the package prescribing information for pioglitazone show that treating type 2 diabetic patients in the clinical trials with pioglitazone resulted in a small, though statistically insignificant, rise in plasma LDL cholesterol, approximately a 6 percent rise in plasma HDL cholesterol, and a 14 percent decrease in fasting plasma triglyceride, as compared to the placebo-treated control group. Rosiglitazone treatment of type 2 diabetic patients in the clinical trials showed approximately a 9 to 14 percent increase in plasma LDL cholesterol, a 5 to 15 percent increase in plasma HDL cholesterol, and no significant change in fasting plasma triglycerides (Fonseca et al., 2000; Lebovitz et al., in press).

Interpretation of data on the effects of various thiazolidinediones on plasma lipids from several clinical trials is confounded by a number of variables. VLDL particle synthesis is increased when glycemic control is poor. Thus, baseline fasting plasma triglycerides are a function of the degree of hyperglycemia as well as the level of insulin resistance and hyperinsulinemia. When evaluating the effects of different thiazolidinediones on fasting plasma triglycerides, data can be compared only if the baseline hyperglycemia and degree of improvement are comparable and if the baseline fasting plasma triglyceride levels are approximately in the same range. Patient populations with high baseline fasting plasma triglycerides and very poor glycemic control would be expected to show significant decreases in plasma triglycerides, while those with somewhat lower baseline plasma triglycerides and better baseline glycemic control would be expected to show little change. The interpretation of the changes in LDL cholesterol must consider whether the LDL cholesterol value is calculated by the Friedewald formula or is directly measured and the magnitude of the baseline LDL cholesterol value. Additionally, it is necessary to determine whether the change in plasma LDL cholesterol concentration is a change in the number of LDL particles or in particle size and composition (small, dense vs. large, fluffy). It appears most likely that the subtle differences in effects on plasma lipids reported for the various thiazolidinediones reflect differences in the characteristics of the populations studied, rather than differences in the properties of the drugs. None of the thiazolidinediones studied have any significant PPAR $\alpha$ activity.

Interpretation of the clinical relevance of the thiazolidinedione-mediated changes in plasma lipids is, at best, speculative. The lowering of plasma triglycerides, when elevated, as well as the consistent increase in plasma HDL, cholesterol and a decrease in LDL cholesterol/HDL cholesterol ratio would be expected to reduce cardiovascular risk. The rise in plasma LDL cholesterol concentration should increase cardiovascular risk but is likely to be offset by the 
improvement in the LDL particle pattern. The evidence supports the supposition that overall cardiovascular risk would be lower. However, clinical outcome data are essential to answer this key question. This is even more critical, since there are several reports that the thiazolidinediones increase lipoprotein a (Lp(a)) levels.

\section{EFFECTS OF THIAZOLIDINEDIONES ON GLYCEMIC CONTROL IN TYPE 2 DIABETIC PATIENTS}

A number of peer-reviewed publications have described the effects of troglitazone on glycemic control in type 2 diabetic patients. These have been reviewed in detail by Saleh and associates (1999). I will summarize the data briefly, since troglitazone is no longer available for clinical use, but the data do provide a background for evaluating new information on currently available agents. Several studies in which troglitazone was used as monotherapy for the treatment of type 2 diabetic patients who were not adequately controlled on dietary and other aspects of lifestyle management showed that troglitazone was only mildly effective in improving glycemic control (Fonseca et al., 1998; Maggs et al., 1998). When administered to drug-naïve patients, troglitazone 400 and $600 \mathrm{mg} /$ day caused 0.7 and 1.1 percent absolute decreases in baseline $\mathrm{HbAlc}$ values, respectively. When troglitazone or placebo was administered to patients who had previously been on sulfonylurea treatment and washed out, troglitazone 400 and 600 $\mathrm{mg}$ /day resulted in improved glycemic control, when compared to placebo. However, the baseline $\mathrm{HbAlc}$ in all groups rose a minimum of 1 percent, indicating that the doses of troglitazone administered were not able to maintain glycemic control as well as the sulfonylurea drugs that the patients had been taking (Fonseca et al., 1998). In contrast to its relatively weak effect in improving glycemic control as monotherapy, troglitazone, when added to the treatment program of type 2 diabetic patients taking sulfonylureas or insulin, had a very significant effect in lowering $\mathrm{HbAlc}$ an additional 1.75 or 1.4 percent, respectively, at the maximal dose of troglitazone administered (Horton et al., 1998; Schwartz et al., 1998). The major lessons concerning use of thiazolidinediones in improving glycemic control in type 2 diabetic patients that were learned from the troglitazone clinical trials were that thiazolidinediones are ineffective unless adequate endogenous insulin secretion is still present. If it is not, then insulin secretion must be stimulated by insulin secretagogues or replaced by some form of exogenous insulin.

Rosiglitazone and pioglitazone appear to be more potent as monotherapies than troglitazone but the same principles apply to their use in improving glycemic control (Lebovitz et al., in press). In order for any of the thiazolidinediones to be effective as monotherapy, endogenous insulin secretion needs to have been preserved. Monotherapy treatment of type 2 diabetic patients with thiazolidinediones is no more effective in improving glycemic control than is treatment with sulfonylureas, metformin, or insulin. The main advantages of thiazolidinediones as mono- 
therapy reside in their ability to reduce insulin resistance at the same time that they are improving glycemic control. The improvement in insulin resistance results in amelioration of many components of the metabolic syndrome: the dyslipidemia, the procoagulant state, and the hyperinsulinemia (Fonseca et al., 2000; Lebovitz et al., in press). Additional potential benefits of reducing insulin resistance are preservation of beta-cell function and anti-atherogenic effects on blood vessels (Mykkanen et al., 1997; Berkowitz et al., 1996; Li et al., 2000).

We have participated in studies of the effects of monotherapy treatment of type 2 diabetic patients inadequately controlled by diet and lifestyle changes with rosiglitazone $2 \mathrm{mg}$ twice a day and $4 \mathrm{mg}$ twice a day for 6 months (Lebovitz et al., in press). The patients selected for this large, multicenter trial were 27 percent drug naïve and 73 percent washed out from a previous therapy. Rosiglitazone 2 $\mathrm{mg}$ twice a day and $4 \mathrm{mg}$ twice a day decreased $\mathrm{HbAlc} 0.3$ and 0.6 percent from baseline and 1.2 and 1.5 percent from placebo treatment. The decrease in $\mathrm{HbAlc}$ observed in the individual patient will depend, in part, on the baseline $\mathrm{HbA}$ lc. Patients whose baseline $\mathrm{HbAlc}$ is between 9.0 and 10.0 percent are likely to have a decrease of 1.8 percent, while those with a baseline $\mathrm{HbAlc}$ between 7.0 and 8.0 percent are more likely to have a decrease from baseline of 0.5 to 0.6 percent. An important issue in treatment with thiazolidinediones is to recognize that it takes about 8 weeks of treatment to maximize the effect on fasting plasma glucose and 14 to 18 weeks on HbAlc. This is likely due to the mechanism of action of these drugs, which involves modifying the constituents and function of the cell. There are relatively few peer-reviewed data on the efficacy of pioglitazone. What are available suggest that, at the appropriate doses (i.e., $30-45 \mathrm{mg} /$ day), it has similar effects to rosiglitazone on glycemic control.

Both rosiglitazone and pioglitazone have been added to the therapeutic programs of type 2 diabetic patients who have had inadequate glycemic regulation on sulfonylurea, metformin, or insulin treatment (Fonseca et al., 2000). In each instance, the large, multicenter studies have shown an additional decrease of 1.0 to 1.5 percent in $\mathrm{HbAlc}$. The additional improvement in glycemic control (a decrease in $\mathrm{HbA}$ lc of 0.9 percent from baseline) that occurred when rosiglitazone 2 or $4 \mathrm{mg}$ twice a day was given to type 2 diabetics who had not achieved target glycemic goals on metformin $2.5 \mathrm{~g}$ per day supports earlier data that had suggested that metformin and the thiazolidinediones improve insulin action by different mechanisms and in different tissues

Clinical studies with all the thiazolidinediones indicate that the best glycemic responses to monotherapy are observed in type 2 diabetic patients who are 1) diet and lifestyle treatment failures and not previously drug treated; 2 ) female (usually have an 0.5 percent greater decrease in HbAlc than males); 3) overweight or obese; and 4) with good residual beta-cell function. 


\section{E. EFFECTS ON THE PROCOAGULANT STATE}

The insulin-resistant state is associated with a number of changes in the coagulation schema that favor thrombosis and interfere with fibrinolysis (Yudkin, 1999; Meigs et al., 2000). This procoagulant state is thought to be a significant factor in the increase in macrovascular disease that occurs in type 2 diabetes. A factor that helps to regulate fibrinolysis is plasminogen activator inhibitor 1 (PAI-1). PAI-1 interferes with the conversion of plasminogen to plasmin. This substance is synthesized by the liver and endothelial cells. In insulin-resistant states, plasma levels of PAI-1 are elevated. Treatment of type 2 diabetic patients by troglitazone or rosiglitazone decreases plasma PAI-1 levels, presumably by decreasing insulin resistance (Saleh et al., 1999; Kato et al., 2000).

\section{F. EFFECTS ON BETA-CELL FUNCTION}

One of the major factors causing progressive deterioration of glycemic control in type 2 diabetes is an unrelenting loss of beta-cell function. This deterioration is both functional and structural. The long-term treatment of type 2 diabetic patients with diet, metformin, or sulfonylureas does not alter this progressive loss of beta-cell function. The mechanisms responsible for this deterioration are not known. Several potential mechanisms have been postulated. Insulin resistance could be a significant factor in facilitating beta-cell loss through its continual demand for greater beta-cell function, leading to an increased rate of apoptosis and/or a concomitant increase in amylin secretion and increased amyloid deposits in the pancreatic islets. If either or both of those mechanisms were operative, the reduction of peripheral insulin resistance would be expected to preserve beta-cell function. While only long-term outcome studies can determine definitively whether such treatments will have a positive outcome, there are data indicating that treatment of type 2 diabetic patients with thiazolidinediones will decrease the elevated plasma proinsulin-to-insulin ratio observed in such patients. Some investigators hypothesize that the elevated plasma proinsulin-to-insulin ratio reflects dysfunctional beta-cell processes (Mykkanen et al., 1997) and that improvement in this ratio reflects improved beta-cell function.

\section{G. EFFECTS OF THIAZOLIDINEDIONES ON VASCULAR ENDOTHELIAL AND VASCULAR SMOOTH MUSCLE CELLS}

In vitro and in vivo animal studies have suggested that insulin resistance may significantly interfere with normal control of blood vessel vasodilatation, endothelial function, and smooth muscle cell proliferation. All of these processes are thought to be important in the pathogenesis of atherosclerosis. A relatively new area of research in thiazolidinedione pharmacology is the role that these agents might have in correcting abnormal vascular endothelial and smooth muscle cell 
abnormalities in insulin-resistant type 2 diabetic patients. Two studies have addressed the question of whether troglitazone treatment would increase the impaired vasodilatation that occurs in insulin-resistant individuals. Tack and colleagues (1998b) failed to find any effect of troglitazone $400 \mathrm{mg} /$ day for 8 weeks on impaired, insulin-induced vasodilation in obese, insulin-resistant subjects, even though insulin sensitivity was increased approximately 23 percent. In contrast, Avena and coworkers (1998) found a significant restoration of brachial artery vasodilatation in patients with impaired glucose tolerance and peripheral vascular disease after 4 months of treatment with troglitazone.

Minamikawa and colleagues (1998) have reported that treatment of type 2 diabetic patients with troglitazone $400 \mathrm{mg}$ daily for 6 months resulted in a statistically significant reduction in carotid arterial intimal and medial complex thickness (IMT), compared to control type 2 diabetic patients. The IMT was measured by a B-mode ultrasound technique. Though $\mathrm{HbAlc}$ and serum triglycerides were lowered by troglitazone treatment, there was no correlation between the decrease in IMT and reductions in HbAlc or triglycerides. Since IMT is thought to be a surrogate marker for atherosclerosis, these data have been interpreted as supportive of the concept that thiazolidinediones activate anti-atherogenic processes at the level of the vascular endothelium and smooth muscle cells.

\section{H. THIAZOLIDINEDIONES AND MICROALBUMINURIA}

The mesangial cell in the kidney glomerulus is a contactile cell involved in controlling the filtration process. Alterations in mesangial cell function are associated with microalbuminuria. Since microalbuminuria are associated with insulin resistance (Mykkanen et al., 1998), it is possible the mesangial cell function is affected by insulin resistance. Thiazolidinediones have been shown to decrease urinary albumin excretion and microalbuminuria in type 2 diabetic patients. Table IV shows the results of changes in urinary albumin excretion rates following 6 months treatment with either 2 or $4 \mathrm{mg}$ of rosiglitazone twice a day. A study comparing troglitazone to metformin treatment of type 2 diabetics showed that troglitazone significantly reduced microalbuminuria and metformin did not, despite the latter having a greater effect in reducing hyperglycemia (Imano et al., 1998). These data suggest that thiazolidinediones may directly influence mesangial cell function.

\section{SAFETY ISSUES WITH THIAZOLIDINEDIONE THERAPY}

Safety issues have and will determine the extent to which thiazolidinediones will be used in the treatment of type 2 diabetes and other insulin-resistant states. Safety issues include those that are unique characteristics of the individual thiazolidinedione, those that are common to all thiazolidinediones, and those that are 
TABLE IV

Effect of 26 Weeks' Treatment with Rosiglitazone on Type 2 Diabetic Patients

\begin{tabular}{llll} 
Treatment Group & Number & $\begin{array}{l}\text { Baseline AER } \\
(\mu \mathrm{g} / \mathrm{mg})^{\mathrm{a}}\end{array}$ & $\begin{array}{l}\text { Percent Change from Baseline } \\
(95 \% \text { Confidence Limits })\end{array}$ \\
\hline Placebo & 132 & 17.8 & $+3.6(-9.1,18.0)$ \\
Rosiglitazone, 2 mg twice daily & 142 & 21.0 & $-14.0(-25.3,-0.9)$ \\
Rosiglitazone, 4 mg twice daily & 145 & 16.0 & $-21.6(-30.6,-11.3)^{\mathrm{a}}$ \\
\hline
\end{tabular}

Patients had albumin excretion rates (AERs) determined by measurement of albumin and creatinine in random morning urine specimens. AER is defined as the albumin/creatinine ratio. ${ }^{a}$ Geometric mean; ${ }^{a} \mathrm{p}<0.001$. [Data from Lebovitz, H.E., Dole, J.F., Patwardhan, R., Rappaport, E.B., and Freed, M.I. J. Clin. Endocrinol. Metab. 85, in press.]

common to all PPAR $\gamma$ agonists. Hepatotoxicity appears to be specific to troglitazone, while random, unexplained, transient elevations of serum creatine phosphokinase (CPK) activity have been reported only with pioglitazone. Weight gain, increase in plasma volume, edema, and increased plasma LDL cholesterol concentration appear to be class effects of PPAR $\gamma$ agonists.

\section{Hepatotoxicity}

Idiosyncratic hepatotoxicity with troglitazone came to the attention of the regulatory agencies in the fall of 1997 , several months after marketing began in the United States. After several cases of liver failure and/or death, a letter dated October 31, 1997, was sent to all U.S. physicians alerting them to the possibility of troglitazone-induced hepatotoxicity and requiring routine testing of liver function in patients taking the drug. Seventy-four cases of jaundice and bilirubinemia were reported to the Food and Drug Administration (FDA) in the fourth quarter of 1997. Additional cases of jaundice, liver failure, and/or death in patients taking troglitazone were reported to the FDA throughout 1998 and into 1999, despite several additional warning letters and more-stringent requirements to monitor liver function at very-frequent intervals. The actual number of cases of liver failure requiring transplantation or having a fatal outcome that can actually be causally related to troglitazone idiosyncratic hepatotoxicity is still controversial. Diabetic patients are frequently on many different medications and are known to have a significant risk of developing various types of liver disease in long-term followup. The most-objective data were provided by Murray Lumpkin, Deputy Director of the FDA's Center for Drug Evaluation and Research, at a meeting of the agency's Endocrinology, Metabolism, and Diabetes Advisory Committee on May 19, 2000. The FDA defined liver failure as hepatic insufficiency that resulted in 
death, liver transplantation, being put on the transplantation waiting list, or recovering after clinical hepatic encephalopathy. Based on these criteria, the FDA concluded that 90 cases of liver failure were possibly or probably related to troglitazone therapy during the 36 months that it had been on the market. As a result of this analysis and the availability of rosiglitazone and pioglitazone, both of which appear not to have liver toxicity, the FDA encouraged Parke Davis to withdraw troglitazone from the market, which it did on March 22, 2000.

As the evidence for troglitazone-induced idiosyncratic hepatotoxicity accumulated (Gitlin et al., 1998; Herrine and Choudhary, 1999; Vella et al., 1998), the major question that arose was whether this was a class effect of all thiazolidinediones or perhaps all PPAR $\gamma$ agonists or whether it was unique to some aspect of troglitazone structure or metabolism. A number of characteristics of troglitazone are somewhat unique and suggested that its hepatotoxicity might be so also. Troglitazone combines the glitazone structure with a vitamin E-related moiety. This molecule generates a family of quinone metabolites that are unique among thiazolidinediones. Troglitazone is concentrated 15-20 fold in the liver, compared to plasma levels (Kawai et al., 1997). Troglitazone and its metabolites are secreted into the bile and undergo considerable biliary recirculation. The plasma half-life of troglitazone in humans is $16-34$ hours; less than 3 percent is excreted in the urine (Henry, 1997). In vitro troglitazone and its quinone metabolites have been toxic to cultured hepatocytes at concentrations comparable to those achieved in vivo near the normal dose range (Elcock et al., 1999). Finally, the dose of troglitazone required to achieve a therapeutic effect in type 2 diabetic humans is 100 -fold greater than, for example, rosiglitazone. During the clinical trials with rosiglitazone and pioglitazone, it was anticipated that they were much less likely to have a troglitazone-like hepatotoxicity.

When troglitazone hepatotoxicity began appearing in type 2 diabetic patients, it was widely publicized that this was totally unexpected and could not have been predicted from the data gathered during the clinical trial. This led to concerns that sample sizes during clinical trials would not be large enough to detect a potential hepatotoxicity in any thiazolidinedione or PPAR $\gamma$ agonist being developed. A careful review of the troglitazone clinical trial data and an analysis of the clinical cases of troglitazone hepatotoxicity allow us to identify the risk of hepatotoxicity from such compounds. In the combined North American clinical trials of troglitazone, 2510 patients received troglitazone and 475 received placebo. Elevations of serum alanine aminotransferase (ALT) greater than three times the upper limit of normal were detected in 48 (1.9 percent) of the troglitazone-treated patients and in only three $(0.6$ percent) of the placebo-treated ones (Watkins and Whitcomb, 1998). Seventeen (0.68 percent) troglitazone-treated patients had an ALT level greater than 10 times the upper limit of normal and five $(0.19$ percent $)$ had an ALT greater than 20 times the upper limit of normal. Twenty patients had 
troglitazone discontinued because of abnormal liver function. Two of those had developed jaundice. Liver function in the 20 patients returned to normal after a mean of 55 days. Liver biopsies were obtained in two patients, one of whom had jaundice. The biopsies were compatible with an idiosyncratic drug reaction. Thus, it is reasonable to conclude that the clinical trials with troglitazone were associated with evidence of a potential problem of hepatotoxicity. That provides some measure of confidence that hepatotoxicity of newer PPAR $\gamma$ agonists would likely be detected by a signal in the clinical trials.

The clinical course of the troglitazone hepatotoxicity is unclear. Initial reports suggested that the hepatic reaction occurred within the first few months of therapy and was unlikely to occur in individuals on therapy longer than 1 year (Watkins and Whitcomb, 1998). The FDA data show that new-onset hepatic abnormalities can occur in individuals who were on troglitazone in excess of 1 year. The hepatotoxicity due to troglitazone progresses for a period of time, even after the drug has been discontinued. In most instances, the liver recovers after several weeks to months but, in some individuals, there has been a steady progression to liver failure.

Examination of the clinical trial data presented to the FDA by the rosiglitazone and pioglitazone sponsors was remarkably devoid of any suggestion of hepatic toxicity. Figure 6 shows that the incidence of serum ALT elevations greater than three times the upper limit of normal in both rosiglitazone- and pioglitazone-treated patients was the same as in their appropriate placebo- or comparator-treated controls. To the present time, more than one million patients have been treated with rosiglitazone and there are no cases of hepatotoxicity in which rosiglitazone has been proven to be the cause (Lebovitz and Salzman, 2000). A similar situation occurs for pioglitazone. The available data indicate that hepatotoxicity is unique to troglitazone and is not a class effect of either thiazolidinediones or PPAR $\gamma$ agonists.

\section{Increase in Extracellular Volume and Edema}

Clinical trials with all of the thiazolidinediones and the new non-thiazolidinedione PPAR $\gamma$ agonists have shown small decreases in the hemoglobin and hematocrit of treated patients. This decrease appears to be the consequence of an expansion of the extracellular compartment. Preliminary studies have shown no signiticant changes in red cell mass in treated patients (Young et al., 1999). The mechanism for expansion of the plasma volume has not been defined. PPAR $\gamma$ agonists decrease peripheral resistance and lower diastolic blood pressure approximately 2 to $4 \mathrm{mmHg}$ (Ogihara et al., 1995; Saleh et al., 1999). The increase in the volume of the extracellular compartment could result from potentiation of insulin's actions to cause vasodilation and to increase sodium resorption. The 


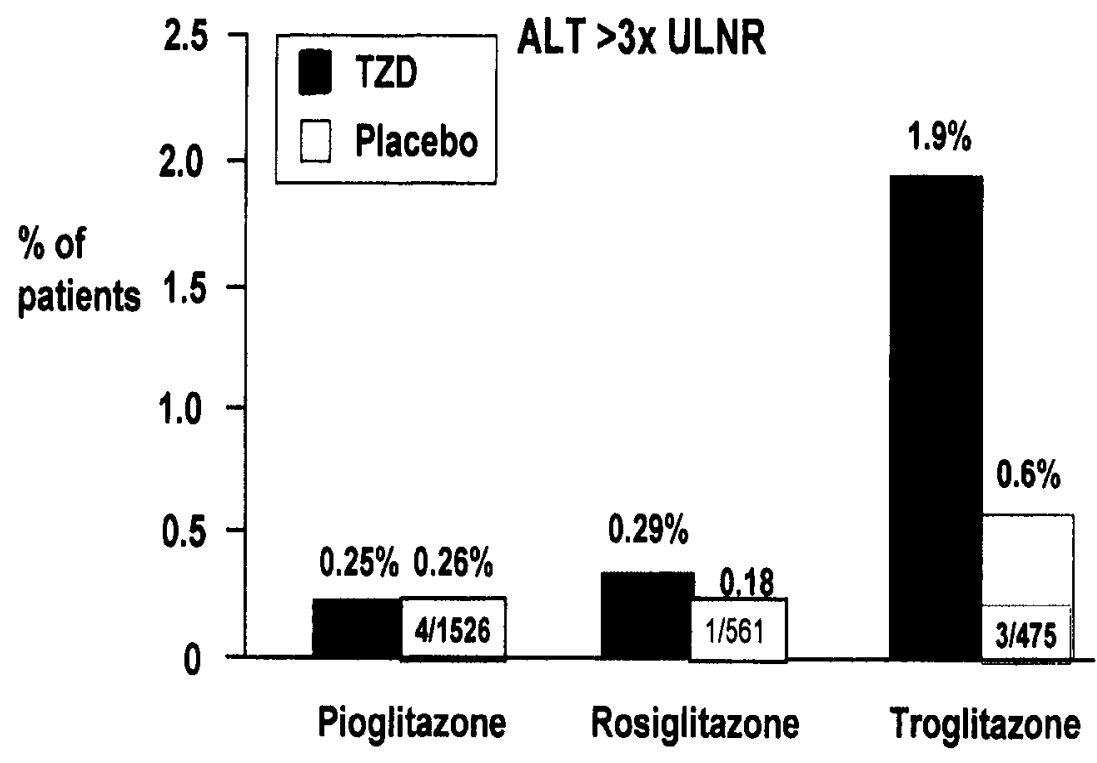

FIG. 6. Incidence of serum alanine aminotransferase elevations greater than three times the upper limit of normal in the clinical trials submitted to the FDA by the sponsors of troglitazone, rusiglitazone, and pioglitazone. The only thiazolidinedione that showed a potential for liver toxicity was troglitazone.

decrease in hemoglobin and hematocrit occurs during the first 12 weeks of treatment and the values are stable after 12 weeks.

Peripheral edema has been observed in 4 to 5 percent of patients treated with pioglitazone or rosiglitazone as monotherapy for type 2 diabetes (Table V). Placebo-treated or patients treated with sulfonylureas or metformin had an incidence of peripheral edema of 1 to 2 percent. Combination therapy of thiazolidinediones with insulin has been associated with more-significant rates of edema. For example, in clinical studies, edema was noted in 7 percent of insulin-treated type 2 diabetic patients and in 15.3 percent of those on pioglitazone plus insulin. The edema that develops during therapy with $\operatorname{PPAR} \gamma$ agonists is usually mild to moderate and responds to therapy with diuretics. A rare patient, however, may develop quite severe edema that does not respond well to diuretics. In these patients, thiazolidinedione may have to be discontinued. The reasons for the heterogeneous responses are unclear.

A likely related side effect that is receiving considerable attention is the precipitation of congestive heart failure. Although few formal data with comparator trials exist, there is a growing clinical awareness that some patients develop congestive heart failure when treated with $P P \Lambda R \gamma$ agonists. The magnitude and 
TABLE V

Frequency of Peripheral Edema Reported in Double-blind Clinical Trials with Rosiglitazone

\begin{tabular}{llll} 
Therapy & Number & Percent reporting edema & $\begin{array}{l}\text { Number withdrawing } \\
\text { because of edema }\end{array}$ \\
\hline Rosiglitazone & 2526 & 4.8 & 1 \\
Rosiglitazone + metformin & 226 & 2.5 to 3.5 & 1 \\
Rosiglitazone + sulfonylurea & 726 & 3.0 & 0 \\
Placebo & 601 & 1.3 & 1 \\
Metformin & 225 & 2.2 & 0 \\
Sulfonylureas & 626 & 1.0 & 0 \\
\hline
\end{tabular}

severity of the problem have not been adequately defined. The impression is that this phenomenon is the consequence of an increase in plasma volume in individuals who are in borderline heart failure. One- and 2-year echocardiographic studies in type 2 diabetic patients with normal baseline cardiac function taking troglitazone, rosiglitazone, and pioglitazone have shown no detrimental effects on cardiac function (Ghazzi et al., 1997). Those studies actually demonstrated an improvement in overall cardiac output and stroke volume, without an increase in cardiac mass. These data, however, may not apply to individuals who already have some degree of cardiac disease. Additional studies of dynamic cardiac function need to be carried out in individuals with cardiac disease and borderline compensation. An awareness of increasing concern about use of PPAR $\gamma$ agonists in patients with cardiac disease is exemplified by a recent letter sent to Japanese physicians by that country's Ministry of Health recommending that pioglitazone not be prescribed to patients with heart failure. This was prompted by reports of five patients with congestive heart failure who subsequently experienced nonfatal heart attacks while taking pioglitazone.

\section{Body Fat and Weight Gain}

Weight gain has been seen in experimental animals and humans treated with thiazolidinediones. The weight gain has two components: an increase in subcutaneous adipose tissue and an increase in extracellular water. The mechanisms responsible for each have been discussed. The severity of the weight gain in humans appears to be proportional to the level of improvement in glycemic control and is accentuated by drugs that increase the plasma insulin levels. Monotherapy with rosiglitazone for 26 weeks was associated with a mean increase in body 
weight of $1.8 \mathrm{~kg}$ at the dose of $4 \mathrm{mg}$ per day and $3.5 \mathrm{~kg}$ at the dose of $8 \mathrm{mg}$ per day. Similar weight gain was reported in the pioglitazone studies (Table VI). Concurrent therapy of thiazolidinediones with insulin for a year resulted in more weight gain, with an average mean of approximately $4.0 \mathrm{~kg}$. Similar degrees of weight gain accompanied concurrent thiazolidinedione and sulfonylurea therapy. Insufficient data are available to determine whether the weight gain stabilizes after 6 to 12 months or whether it is progressive with time. There are unusual patients who gain large amounts of weight when treated with thiazolidinediones. It then becomes a clinical judgment as to whether the improvement in glycemic control is sufficiently beneficial to justify the magnitude of the weight gain. In any individual patient, it may be difficult to determine the relative contributions that fluid retention vs. an increase in adipose tissue mass make to the weight gain.

\section{Potential for Drug Interactions}

The thiazolidinediones are metabolized by oxidative cytochrome pathways in the liver. Troglitazone, pioglitazone, and rosiglitazone are metabolized via different cytochrome pathways. Troglitazone is primarily metabolized by CYP 3A4 (Yamazaki et al., 1999). Additionally, it induces the metabolism of other drugs that are substrates for CYP 3A4. Pioglitazone is metabolized via CYP 3A4, CYP $2 \mathrm{C} 8$, and CYP $1 \mathrm{~A} 1$ pathways. Rosiglitazone is predominantly metabolized via CYP 2C8 and to a much-lesser degree by CYP 2C9. More than half of the

TABLE VI

Effect of Thiazolidinedione Treatment on Weight Gain in Type 2 Diabetic Patients

\begin{tabular}{llll} 
& \multicolumn{3}{c}{ Gain in weight $(\mathrm{kg})$} \\
\cline { 2 - 4 } Type of treatment & Pioglitazone* & Troglitazone & Rosiglitazone \\
\hline Monotherapy & 0.5 to 2.8 & - & 1.6 to $3.5^{\mathrm{a}}$ \\
With sulfonylurea & 1.9 to 2.9 & 2.6 to $5.9^{\mathrm{b}}$ & - \\
With metformin & 1.0 & - & 0.7 to $1.9^{\mathrm{c}}$ \\
With insulin & 2.3 to 3.7 & 1.9 to $3.6^{\mathrm{d}}$ & - \\
\hline
\end{tabular}

Data from clinical trials after 6 months of treatment. ${ }^{2} 2$ and $4 \mathrm{mg}$ twice daily [Lebovitz, H.E, Dole, J.F., Patwardhan, R., Rappaport, E.B., and Freed, M.I. J. Clin. Endocrinol. Metab. 85, in press.] $\mathrm{b}_{200}$ and $600 \mathrm{mg}$ daily [Horton, E.S., Venable, T.C., Whitehouse, F., Ghazzi, M.N., and Whitcomb, R.W. Diabetes Care 21, 1462-1469, 1998.] ${ }^{\mathrm{c}} 2$ and $4 \mathrm{mg}$ twice daily [Fonseca, V., Rosenstock, J., Parwardhan, R., and Salzman, A. J. Am. Med. Assn. 283, 1695-1702, 2000.] d 200 and $600 \mathrm{mg}$ daily [Schwartz, S., Raskin, P. Fonseca, V., and Graveline, J.F. N. Engl. J. Med. 338, 861-866, 1998.] *Pioglitazone patient information. 
commonly used drugs are metabolized via the CYP 3 A4 pathway. Thus, it would not be unreasonable to suspect that drugs such as troglitazone. which induce and are metabolized via CYP $3 \mathrm{~A} 4$, would have significant drug-drug interactions with other drugs metabolized via CYP 3A4. Indeed, troglitazone has been shown to induce the metabolism of the oral contraceptive constituents ethinylestradiol, northindrone, and levonorgestrel as well as atorvastatin, terfenadine, and cyclosporin. The potential for drug interactions and alterations in effectiveness and safety need to be considered when two drugs metabolized via CYP $3 \mathrm{~A} 4$ are administered together.

Co-administration of pioglitazone with warfarin, glipizide, metformin, or digoxin does not change the pharmacokinetic and pharmacodynamic profiles of these drugs. The effect of pioglitazone on induction or inhibition of CYP $3 \mathrm{~A} 4$ has not been reported. Some data suggest that ketoconizole may inhibit the metabolism of pioglitazone.

Because rosiglitazone does not interact with CYP $3 \mathrm{~A} 4$, it is unlikely to have many drug-drug interactions. In particular, co-administration with rosiglitazone does not alter the metabolism of oral contraceptives or nifedipine. No clinically relevant drug interactions have been found between rosiglitazone and digoxin, metformin, glyburide, ranitidine, or warfarin. The number of drugs metabolized via CYP 2 C8 are few; therefore, the chance of drug interactions between rosiglitazone and other concomitantly given drugs is quite low.

\section{Conclusions}

Insulin resistance is increasing in our society at a very rapid rate. This is the result of changing lifestyles superimposed upon genetic predisposition. Unfortunately, the development of insulin resistance is associated with the development of the insulin resistance syndrome, with its component abnormalities. The development of the insulin resistance syndrome predisposes individuals to increased risks of developing type 2 diabetes and cardiovascular disease. These detrimental outcomes have stimulated great interest in developing methods to prevent and treat insulin resistance. One such approach is to uncover the specific biochemical pathways responsible for mediating insulin resistance and to develop pharmacologic agents to block or reverse these pathways. The thiazolidinediones are the first effort in this approach. Treatment of insulin resistance with the thiazolidinediones improves insulin action, lowers plasma insulin levels, ameliorates many of the components of the insulin resistance syndrome, and improves glycemic control in type 2 diabetic patients. Studies are underway to determine whether these agents can prevent type 2 diabetes and what their impact will be on the development of cardiovascular disease. Insights from studies on their molecular mechanism of action have provided clues for developing new and perhaps moreeffective agents to treat insulin resistance. 


\section{REFERENCES}

Abate, N., Garg, A., Peshock, R.M., Stray-Gundersen, J., and Grundy, S.M. (1995). J. Clin. Invest. 96, 88-98.

Adams, M., Montague, C.T., Prins, J.B., Holder, J.C., Smith, S.A., Sanders, L., Digby, J.E., Sewter, C.P., Lazer, M.A., Chatterjee, V.K.K., and O'Rahilly, S. (1997). J. Clin. Invest. 100, 3149-3153.

Avena, R., Mitchell, M.E., Nylen, E.S., Curry, K.M., and Sidway, A.N. (1998). J. Vasc. Surg. 28, 1024-1032.

Banerji, M.A., and Lebovitz, H.E. (1989). Diabetes 38, 784-782.

Banerji, M.A., and Lebovitz, H.E. (1992). Diabetes Care 15, 1295-1302.

Banerji, M.A., Chaiken, R.L., Gordon, D., Kral, J.G., and Lebovitz, H.E. (1995a). Diabetes 44, 141-146.

Banerji, M.A., Buckley, M.C., Chaiken, R.L., Gordon, D., Lebovitz, H.E., and Kral, J.G. (1995b). Intl. J. Obesity 19, 846-850.

Banerji, M.A., Lebowitz, J., Chaiken, R.L., Gordon, D., Kral, J.G., and Lebovitz, H.E. (1997). Am. J. Physiol. 273, E425-E432.

Barker, D.J.P., Hales, C.N., Fall, C.H.D., Osmond, C., Phipps, K., and Clark, P.M.S. (1993). Diabetologia 36, 62-67.

Bavdckar, A., Yajnik, C.S., Fall, C.H.D., Bapat, S., Pandit, A.N., Deshpande, V., Bhave, S., Kellingray, S.D., and Joglekar, C. (1999). Diabetes 48, 2422-2429.

Berkowitz, K., Peters, R., Kjos, S.L., Goico, J., Marroquin, A., Dunn, M.E., Xiang, A., Azen, S., and Buchanan, T.A. (1996). Diabetes 45, 1572-1579.

Boden, G. (1996). Diabetes 45, 3-10.

Bonora, E., Saggiani, F., Targher, G., Zenere, M.B., Alberiche, M., Monauni, T., Bonadonna, R.C., and Muggeo, M. (2000). Diabetes Care 23, 57-63.

Boyko, E.J., Leonetti, D. I., Fujimoto, W.Y., and Newell-Morris, L. (2000). Diabetes Care 23, 465-471.

Brunzell, J.D., and Hokanson, J.E. (1999). Diabetes Care 22(suppl. 3), Cl0-Cl3.

Burant, C.F., Sreenan, S., Hirano, K.-I., Tai, T.-A.C., Lohmiller, J., Lukens, J, Davidson, N.O., Ross, S., and Graves, R.A. (1997). J. Clin. Invest. 100, 2900-2908.

Camp, H.S., Li, O., Wise, S.C., Hong, Y.H., Frankowski, C.L., Shen, X., Vanbogelen, R., and Leff, T. (2000). Diabetes 49, 539-547.

Chaiken, R.L., Banerji, M.A., Pasmantier, R.M., Huey, H., Hirsch, S., and Lebovitz, H.E. (1991). Diabetes Care 14, 1036-1042.

Chaiken, R.L., Banerji, M.A., Huey, H., and Lebovitz, H.E. (1993). Diabetes 42, 444-449.

Ciaraldi, T.P., Gilmore, A., Olefsky, J.M., Goldberg, M., and Heidenreich, K.A. (1990). Metabolism 39, 1056-1062.

Cline, G.W., Petersen, K.F., Krssak, M., Shen, J., Hundal, R.S., Trajanoski, Z., Inzucchi, S., Dresner, A., Rothman, D.L., and Shulman, G.I. (1999). N. Engl. J. Med. 341, 240-246.

Dabelea, D., Imperatore, G., Pettit, D.J., Bennett, P.H., Hanson, R.l., and Knowler, W.C. (1999). Diabetes Care 22, 944-950.

Dresner, A., Laurent, D., Marcucci, M., Griffin, M.E., Dufour, S., Cline, G.W., Slezak, L.A., Andersen, D.K., Hundal, R.S., Rothman, D.L., Petersen, K.F., and Shulman, G.I. (1999). J. Clin. Invest. 103, 253-259.

Elcock, F.J., Lyon, J.J., Hitchcock, J., Morgan, D.G., Bertram, T.A., and Bugelski, P.J. (1999). Diabetes 48(suppl. 1), A63.

Festa, A., Hales, C.N., D'Agostino, R. Jr., Howard, B.V., Mykkanen, L., Haffner, S.M., and Tracy, R.F. (1999). Diabetes Care 22, 1688-1693.

Fonseca, V.A., Valiquett, T.R., Huang, S. M., Ghazzi, M.N., and Whitcomb, R.W. (1998). J. Clin.Endocrinol. Metab. 83, 3169-3176.

Fonseca, V., Rosenstock, J., Parwardhan, R., and Salzman, A. (2000). J. Am. Med. Assn. 283, 1695-1702. 
Ghazzi, M.N., Perez, J.E., Antonucci, T.K., Driscoll, J.H., Huang, S.M., Faja, B.W., and Whitcomb, R.W. (1997). Diabetes 46, 433-439.

Gitlin, N., Julie, N.L., Spurr, C.L., Lim, K.N., and Juarbe, H.M. (1998). Ann. Intern. Med. 129, 36-38. Goodpaster, B.H., Thaete, F.L., Simoneau, J.-A., and Kelley, D.E. (1997). Diabetes 46, 1579-1585.

Goodpaster, B.H., Kelley, D.E., Wing, R.R., Meier, A., and Thaete, F.L. (1999). Diabetes 48, 839-847. Groop, L., Ekstrand, A., Forsblom, C., Widen, E., Groop, P.-H., Teppo, A.-M., and Eriksson, J. (1993). Diabetologia 36, 642-647.

Haffner, S.M., Kennedy, E., Gonzalez, C. Stern, M.P., and Miettinen, H. (1996). Diabetes Care 19, 1138-1141.

Haffner, S.M., Rewers, M., D'Agostino, R. Jr., Selby, J., Mykkanen, L., Savage, P.J., Tracy, R., Saad, M.F., and Howard, B.V. (1999). Diabetes Care 22, 562-568.

Henry, R.R. (1997). Endocrinol. Metab. Clin. N. Am. 26, 553-575.

Herrine, S.K., and Choudhary, C. (1999). Ann. Intern. Med 130, 163-164.

Horton, E.S., Venable, T.C., Whitehouse, F., Ghazzi, M.N., and Whitcomb, R.W. (1998). Diabetes Care $21,1462-1469$.

Hotamisligil, G.S., and Spiegelman, B.M. (1994). Diabetes 43, 1271-1278.

Imano, E., Motomura, M., Kanda, T., Kajimoto, Y., Nakatani, Y., Yamasaki, Y., Nishida, T., Hori, M., and Arai, K. (1998). Diabetes Care 21, 2135-2139.

Inzucchi, S.E., Maggs, D.G., Spollett, G.R., Page, S.L., Rife, F.S., Walton, V., and Shulman G. (1998). N. Engl. J. Med. 338, 867-872.

Karter, A.J., Mayer-Davis, E.J., Selby, J.V., D'Agostino, R.B. Jr., Haffner, S.M., Sholinsky, P., Bergman, R., Saad, M.F., and Hamman, R.F. (1996). Diabetes 45, 1547-1555.

Kato, K., Yamada, D., Midorikawa, S., Sato, W., and Watanabe, T. (2000). Metabolism 49, 662-665.. Kadowaki, T. (2000). J. Clin. Invest. 106, 459-465.

Kawai, K., Kawasaki-Tokui, Y., Odaka, T., Tsuruta, F., Kazui, M., Iwabuchi, H., Nakamura, T., Kinoshita, T., Ikeda, T., Yoshioka, T., Komai, T., and Nakamura, K. (1997). Arzneimittel-Forschung, 47, 356-368.

Kelly, I.R., Walsh, K., Han, T.S., and Lean, M.E.J. (1999). Diabetes Care 22, 288-293.

Kreutter, D.K., Andrews, K.M., Gibbs, E.M., Hutson, N.J., and Stevenson, R.W. (1990). Diabetes 39, 1414-1419.

Kruszynska, Y.T., Mukherjee, R., Jow, L., Dana, S., Paterniti, J.R., and Olefsky, J.M. (1998). J. Clin. Invest. 101, 543-548.

Lapidus, L., Bengtsson, C., Larsson, B., Pennert, K., Rybo, E., and Sjostrom, L. (1984). Br. Med. J. 289, 1257-1261.

Larsson, B., Svardsudd, K., Welin, L., Wilhelmsen, L., Bjorntorp, P., and Tibblin, G. (1984). Br. Med. J. 288, 1401-1404.

Lebovitz, H.E., and Salzman, A. (2000). Diabetes 49(suppl. 1), A39.

Lebovitz, H.E., Dole, J.F., Patwardhan, R., Rappaport, E.B., and Freed, M.I. (2001). J. Clin. Endocrinol. Metab. 85, in press.

Lehmann, J. M., Moore, L.B., Smith-Oliver, T.A., Wilkison, W.O., Willson, T.M., and Kliewer, S.A. (1995). J. Biol. Chem. 270, 12953-12956.

Lemieux, S., Tremblay, A., Prud'Homme, D., Bouchard, C., Nadeau, A., and Despres, J-P. (1996). Diabetes Care 19, 983-991.

Li, A.C., Brown, K.K., Silvestre, M.J., Willson, T.M., Palinski, W., and Glass, C.K. (2000). J. Clin Invest. 106, 523-531.

Maggs, D.G., Buchanan, T.A., Burant, C.F., Cline, G., Gumbiner, B., Isueh, W.A., Inzucchi, S., Kcllcy, D., Nolan, J., Olefsky, J.M., Polonsky, K.S., Silver, D., Valiquett, T.R., and Shulman, G.I. (1998). Ann. Intern. Med. 128, 176-185.

Meigs, J.B., Mittleman, M.A., Nathan, D.M., Tofler, G.H., Singer, D.E., Murphy-Sheehy, P.M., Lipinska, I., D'Agostino, R.B., and Wilson, P.W.F. (2000). J. Am. Med. Assn. 283, $221-228$. 
Miles, P.D.G., Barak, Y., He, W., Evans, R.M., and Olefsky, J.M. (2000). J. Clin. Invest. 105, 287-292. Miniamikawa, J., Tanaka, S., Yamauchi, M., Inoue, D., and Koshiyama H. (1998). J. Clin. Endocrinol. Metab. 83, 1818-1820.

Mori, Y., Yokoyama, J., Murakawa, Y., Tajima, N, Okada, K., Ikeda, Y., and Horikoshi, H. (1999). Diabetes Care 22, 908-912.

Mykkanen, L., Haffner, S.M., Hales, N., Ronnemaa, T., and Laakso, M. (1997). Diabetes 46, 1990-1995.

Mykkanen, L., Zaccaro, D.J., Wagenknecht, L.E., Robbins, D.C., Gabriel, M., and Haffner, S.M. (1998). Diabetes 47, 793-800.

Noguchi, N., Sakai, H., Kato, Y., 'Tsuchiya, J., Yamamoto, Y., Niki, E., Horikoshi, H., and Kodama T. (1996). Atherosclerosis 123, 227-234.

Ogihara, T., Rakugi, H., Ikegami, H., Mikami, H., and Masuo K. (1995). Am. J. Hypertens. 8, 316-320.

Ohison, L-O., Larsson, B., Svardsudd, K., Welin, L., Eriksson, H., Wilhelmsen, L., Bjorntorp, P., and Tibblin, G. (1985). Diabetes 34, 1055-1058.

Okosun, I.E., Osotimehin, B., Cooper, R. S., Forrester, T., and Rotimi, C.N. (1998). Diabetes Care 21, 1836-1842.

Okuno, A., Tamemoto, H., Tobe, K., Ueki, K., Mori, Y., Iwamoto, K., Umesono, K., Akanuma, Y., Fujiwara, T., Horikoshi, H., Yazaki, Y., and Kadowaki T. (1998). J. Clin. Invest. 101, 13541361

Olefsky, J.M. (2000). J. Clin. Invest. 106, 467-472

Pan, D.A., Lillioja, S., Kriketos, A.D., Milner, M.R., Bauer, L.A., Bogardus, C., Jenkins, A.B., and Storlien, L.H. (1997). Diabetes 46, 983-988.

Phillips, D.I.W., Barker, D.J.P., Fall, C.H.D., Seckle, J.R., Whorwood, C.B., Wood, P.J., and Walker, B.R. (1998). J. Clin. Endocrinol. Metab. 83, 757-760.

Reaven, G.M. (1988). Diabetes 37, 1595-1607.

Rebuffe-Scrive, K.M., Carey, V.J., Olbe, L., and Bjorntorp, P. (1989). Metabolism 38, 453-458.

Rexrode, K.M., Carey, V.J., Hennekens, C.H., Walters, E.E., Colditz, G.A., Stampfer, M.J., Willett, W.C., and Manson, J.E. (1998). J. Am. Med. Assn. 280, 1843-1848.

Saleh, Y.M., Mudaliar, S.R., and Henry, R.R. (1999). Diabetes Rev. 7, 55-76.

Saltiel, A.R., and Olefsky, J.M. (1996). Diabetes 45, 1661-1669.

Samaras, K., and Campbell, L.V. (2000). Diabetes Care 23, 443-444.

Samaras, K., Arden, N., Kelly, P.J., Spector, T.D., Chiano, M.N., and Campbell, L.V. (1998). Diabetes Care 21, 2069-2076.

Schwartz, S., Raskin, P., Fonseca, V., and Graveline, J.F. (1998). N. Engl J. Med. 338, 861-866.

Stern, M.P. (1997). In "International Textbook of Diabetes Mellitus," 2nd edit. (K.G.M.M. Alberti., P. 7.immet, R.A. DeFronzo, and H. Keen, eds.), pp. 255-283. John Wiley and Sons, Chichester, U.K

Tack, C.J.J., Smits, P., Demacker, P.N.M., and Stalenhoef, A.F.H. (1998a). Diabetes Care 21, 796-797.

Tack, C.J., Ong, M.K., Lutterman, J.A., and Smits, P. (1998b). Diabetologia 41, 569-576.

Taniguchi, A., Fukushima, M., Sakai, M., Kataoka, K., Miwa, K., Nagata, I., Doi, K., Arakawa, H., Nagasaka, S., Tokuyama, K., and Nakai, Y. (2000). Metabolism 49, 1001-1005.

Vella, A., de Growen, P.C., and Dinneen, S.F. (1998). Ann. Intern Med. 129, 1080.

Walker, A.D., Naderali, E.K., Chattington, P.D., Buckingham, R.E., and Williams, G. (1998). Diabetes 47, 810-814.

Watkins, P.B., and Whitcomb, R.W. (1998). N. Engl. J. Med. 338, 916-917.

Willson, T.M., Brown, P.J., Sternback, D.D., and Henke, B.R. (2000). J. Med. Chem. 43, 527-550.

Whitcomb, R.W., and Saltiel, A. R. (1995). Exp. Opin. Invest. Drugs 4, 1299-1309.

Wu, Z., Xie, Y., Morrison, R.F., Bucher, N.L.R., and Farma, S.R. (1998). J. Clin. Invest. 101, 22-32

Yamazaki, H., Shibata, A., Suzuki, M., Nakajima, M., Shimada, N., Guengerich, F.P., and Yokoi, T. (1999). Drug Metab. Dispos. 27, 1260-1266. 
Young, M.M., Squassante, L., Wemer, J., van Marle, S.P., Dogterom, P., and Johnkman, J.H. (1999). Eur. J. Clin. Pharmacol. 55, 101-104.

Yu, J.G., Kruszynska, Y.T., Mulford, M.I., and Olefsky, J.M. (1999). Diabetes 48, 2414-2421.

Yudkin, J.S. (1999). Diabetes Care 22(suppl. 3), C25-C30.

Zavaroni, I., Bonora, E., Pagliara, M., Dall'Aglio, E., Luchetti, L., Buonanno, G., Bonati, P.E., Bergonzani, M., Gnudi, L., Passeri, M., and Reaven, G. (1989). N. Engl. J. Med. 320, $702-706$. 


\title{
Insulin Resistance in Polycystic Ovary Syndrome: Progress and Paradoxes
}

\author{
Aradhana M. Venkatesan, Andrea Dunaif, and Anne Corbould \\ The Division of Women's Health, The Departments of Medicine and of Obstetrics and Gynecology, \\ Brigham and Women's Hospital, 75 Francis Street, Boston, Massachusetts 02115
}

\begin{abstract}
Over the past 20 years, it has been clearly documented that 1 ) polycystic ovary syndrome (PCOS) has major metabolic sequelae related to insulin resistance and 2) insulin resistance plays an important role in the pathogenesis of the reproductive abnormalities of the disorder. Women with PCOS are at significantly increased risk of developing type 2 diabetes mellitus (DM). Studies in isolated adipocytes and in cultured skin fibroblasts from PCOS women have demonstrated intrinsic postbinding defects in insulin-mediated glucose metabolism. In fibroblasts, the mitogenic pathway of insulin action is intact, consistent with a selective defect in insulin signaling. While PCOS skeletal muscle is resistant to insulin in vivo, cultured muscle cells have normal insulin sensitivity, consistent with a major role of extrinsic factors in producing insulin resistance in this tissue. Excessive serine phosphorylation of the insulin receptor or downstream signaling proteins may be involved in the pathogenesis of insulin resistance in PCOS. The putative serine kinase is extrinsic to the insulin receptor but its identity is unknown. The explanations for tissue-specific and signaling pathway-specific differences in insulin action in PCOS are unknown but may involve differential roles of insulin receptor substrate (IRS)-1 and IRS-2 in insulin signal transduction.
\end{abstract}

\section{PCOS - A Disorder of Insulin Action and a Risk Factor for Type 2 Diabetes Mellitus}

Polycystic ovary syndrome (PCOS) is among the most-common endocrinopathies of premenopausal women, with a prevalence estimated at approximately 5 percent of this population (Knochenhauer et al., 1998). PCOS is characterized by hyperandrogenism, anovulatory infertility, and profound insulin resistance. In addition to playing an important role in the pathogenesis of the reproductive abnormalities of PCOS, insulin resistance has major metabolic consequences. Women with PCOS have an approximately seven-fold increased risk of developing type 2 diabetes mellitus (DM), compared to that of unaffected women (Legro et al., 1999).

The association of hyperandrogenism and diabetes was first described by Achard and Thiers in 1921 as "la diabete des femmes a barbe" (diabetes of bearded women) (Achard and Thiers, 1921). In 1980, Burghen and colleagues 
reported that obese women with PCOS had significantly elevated basal and postglucose-load insulin levels, compared to weight-matched control women, suggesting that the PCOS women were resistant to insulin (Burghen et al., 1980). Subsequent studies determined that hyperinsulinemia was a characteristic feature of women with PCOS, independent of obesity (Dunaif et al., 1987). In addition, Dunaif and colleagues found that obese women with PCOS had significantly increased glucose levels in response to an oral glucose challenge, compared with age- and weight-matched ovulatory control women (Dunaif et al., 1987). A study of 254 women with PCOS found nearly 40 percent of them to be glucose intolerant, with 31 percent having impaired glucose tolerance and 7.5 percent having type 2 DM (Legro et al., 1999) (Figure 1). These prevalences were significantly higher than those in age-, weight-, and ethnicity-matched control women as well as those in US-based population studies of premenopausal women. Rates of impaired glucose tolerance and type 2 DM were similar in PCOS women of diverse ethnic backgrounds, suggesting that PCOS per se is a more-important risk factor for glucose intolerance in young women than either race or ethnicity (Legro et al., 1999; Ehrmann et al., 1999).

In women with PCOS, basal insulin secretion is increased and hepatic insulin clearance is reduced, resulting in hyperinsulinemia (O'Meara et al., 1993). Insulin-stimulated glucose utilization is decreased by 35 to 40 percent in women with PCOS, independent of obesity, a decrease similar in magnitude to that seen in type 2 DM (Dunaif, 1997). Obesity and PCOS have a synergistic negative impact

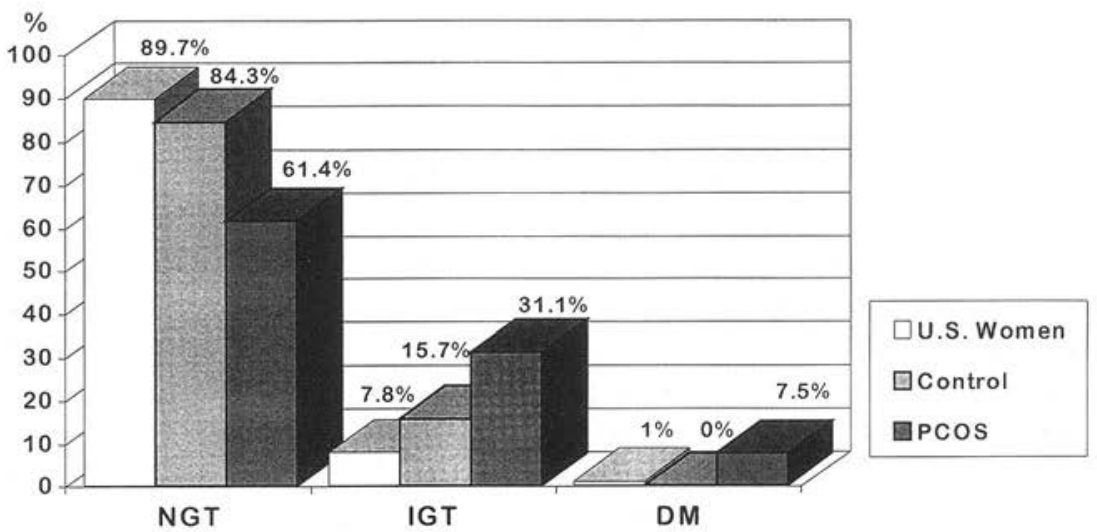

FIG. 1. Glucose tolerance in PCOS. Glucose tolerance was assessed by a 75-gram oral glucose tolerance test in women with PCOS; age-, weight-, and ethnicity-comparable reproductively normal control women; and US women, aged 20-44 years, from the Second National Health and Nutrition Survey (Legro et al., 1999). The prevalence rates for normal glucose tolerance (NGT), impaired glucose tolerance (IGT), and undiagnosed diabetes mellitus (DM) according to World Health Organization criteria are shown. [Reprinted with the permission of A. Dunaif.] 
on insulin sensitivity, so that hepatic insulin resistance is found only in PCOS women who are obese (Dunaif, 1997). Under normal circumstances, insulin secretion increases as insulin sensitivity decreases, in order to maintain glucose homeostasis. This relationship of insulin sensitivity and insulin secretion, known as the disposition index, is described by a hyperbolic curve (Bergman et al., 1996). In both nonobese and obese PCOS women, insulin secretion is inappropriately low for their degree of insulin resistance, suggesting the presence of pancreatic $\beta$-cell dysfunction in these patients (Ehrmann et al., 1995; Dunaif and Finegood, 1996) (Figure 2). Ehrmann and colleagues have demonstrated decreased postprandial insulin secretory responses as well as abnormalities in entrainment of insulin secretion to an oscillatory glucose infusion in PCOS women, consistent with $\beta$-cell dysfunction (Ehrmann et al., 1995). $\beta$-cell dysfunction can precede glucose intolerance in PCOS (Dunaif and Finegood, 1996).

Insulin resistance plays a role in the pathogenesis of the reproductive abnormalities characteristic of PCOS. Burghen and colleagues found a significant

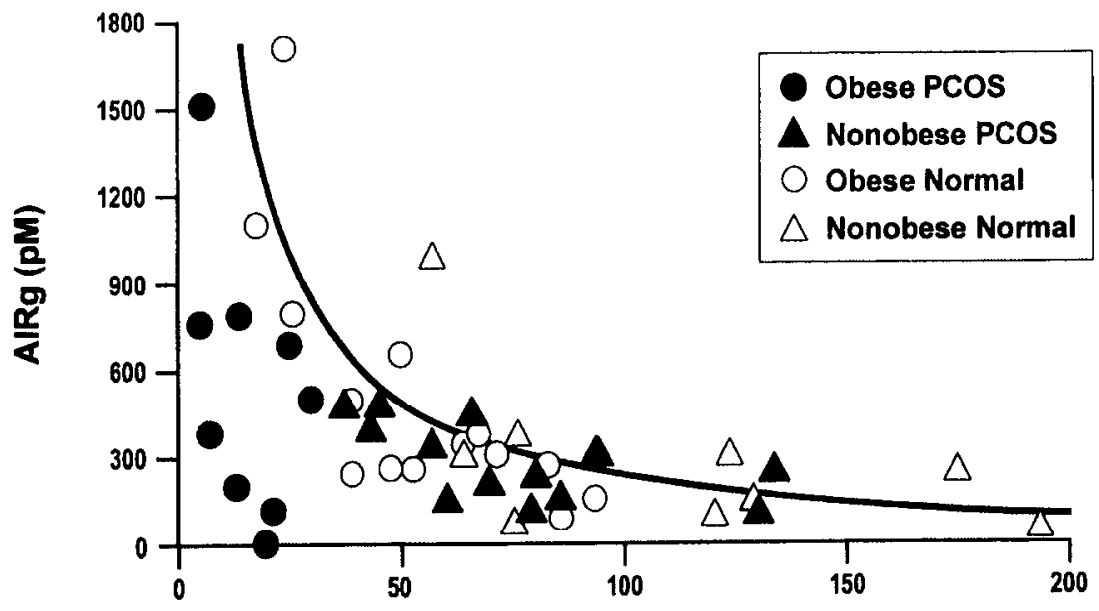

Insulin Sensitivity $\left(\mathrm{min}^{-1} /(\mathrm{nmol} / \mathrm{ml})\right)$

FIG. 2. The disposition index. Insulin sensitivity determined by minimal model analysis of a frequently sampled, intravenous glucose tolerance test is on the $x$-axis. Insulin secretion determined by the acute insulin response to an intravenous glucose load (AIRg) is on the $y$-axis. Under normal circumstances, the product of insulin sensitivity and insulin secretion, known as the disposition index, is constant and is described by a hyperbolic curve, as depicted. An early defect in the pathogenesis of diabetes is $\beta$-cell dysfunction, resulting in inappropriately low insulin secretion for the degree of insulin resistance. This is present in nonobese and obese women with PCOS, most of whom do not have DM, as can be seen in the figure where the disposition index is below the normal curve in PCOS women (solid symbols). [Reprinted with the permission of A. Dunaif.] 
positive correlation between insulin and androgen levels in their PCOS subjects, suggesting a possible causal relationship (Burghen et al., 1980). Insulin resistance is more severe in women with PCOS who are anovulatory than in women who are equally hyperandrogenemic but have normal cycles (Dunaif et al., 1987; Robinson et al., 1993), suggesting that insulin resistance contributes to anovulation. In obese women with PCOS who undergo weight loss, a reduction in basal and glucose-stimulated plasma insulin levels is associated with resumption of ovulatory cycles (Pasquali et al., 1989; Kiddy et al., 1992). Perhaps the most convincing of the lines of evidence linking insulin resistance and reproductive abnormalities have been studies utilizing insulin-sensitizing drugs to restore ovulation in women with PCOS (Dunaif et al., 1996; Nestler et al., 1998, 1999).

\section{Mechanisms of Insulin Resistance in PCOS}

\section{A. MOLECULAR EVENTS IN INSULIN SIGNALING}

Insulin is a hormone with diverse metabolic and mitogenic effects. In addition to promoting the synthesis and storage of carbohydrates, lipids, and proteins, insulin regulates cell growth and differentiation. The mechanisms controlling insulin receptor signal specificity are the subject of intense investigation (Virkamäki et al., 1999).

The insulin receptor belongs to a family of tyrosine kinase receptors, including the insulin-like growth factor-1 (IGF-1) receptor, with which it shares significant structural homology. (Other family members are epidermal growth factor, fibroblast growth factor, platelet-derived growth factor, and colony-stimulating factor-1 receptors) (De Fronzo, 1997). The insulin receptor is a heterotetramer consisting of two $\alpha, \beta$ dimers linked by disulfide bonds. The $\alpha$ subunit contains the ligand binding domain; the membrane-spanning $\beta$ subunit has an intracytoplasmic portion that possesses intrinsic tyrosine kinase activity (Cheatham and Kahn, 1995). Binding of insulin activates the receptor's kinase and leads to rapid autophosphorylation of specific tyrosine residues on the $\beta$ subunit (Saltiel, 1996). This results in tyrosine phosphorylation of insulin receptor substrate (IRS) molecules. The IRSs are a family of proteins, nine of which have been identified (Virkamäki et al., 1999; White and Yenush, 1998). These include IRS-1 through -4 , Gab-1, Shc (which has three isoforms), and p62 dok. All the proteins in this family bind transiently to the activated insulin receptor, during which time they are phosphorylated on several tyrosine sites. The tyrosine-phosphorylated sites on the insulin receptor substrates serve as binding sites for several SH2 domain proteins, including phosphatidylinositol 3-kinase (PI3-kinase). Activation of PI3kinase is a key step in the pathways ultimately resulting in stimulation of insulinmediated metabolic functions such as translocation of GLUT4 to the cell surface, 
leading to glucose uptake as well as glycogen synthesis. (Virkamäki et al., 1999; Saltiel, 1996; White and Yenush, 1998). PI3-kinase activation results in the phosphorylation of Akt, also known as protein kinase B (PKB), which, in turn, phosphorylates and inactivates glycogen synthase kinase-3 (GSK-3). GSK-3 tonically inhibits glycogen synthase activity. Thus, inhibition of GSK-3 activity results in glycogen synthase activation (Lawrence and Roach, 1997). Mitogenic actions of insulin (e.g., control of cell growth and differentiation) can occur independently of PI3-kinase activation via activation of the Ras-mitogen-activated protein (MAP) kinase pathway (Saltiel, 1996; White and Yenush, 1998) (Figure 3).

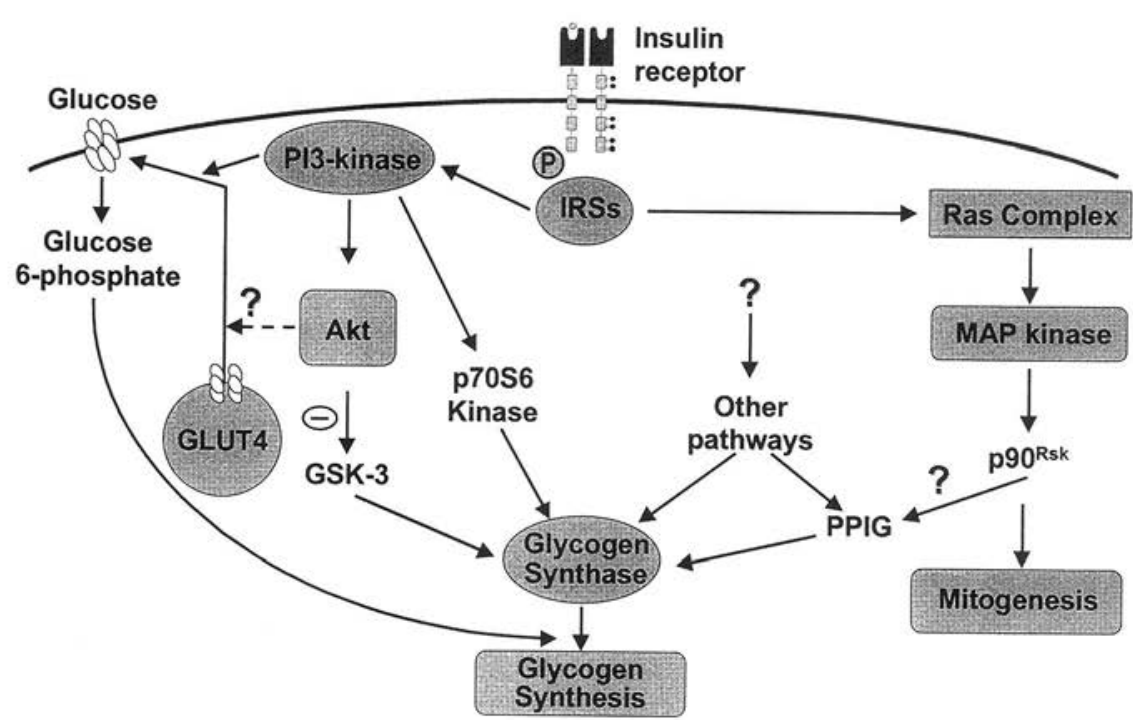

FIG. 3. Insulin receptor signaling. Insulin binding initiates signal transduction by inducing tyrosine autophosphorylation of the insulin receptor, which increases its protein kinase activity. The activated receptor tyrosine phosphorylates intracellular substrates such as insulin receptor substrates (IRSs) 1-4 and Shc (Src and collagen-homologous protein). Tyrosine phosphorylated sites on IRSs dock signaling and adaptor molecules propagating the signal. One important pathway is the activation of phosphatidylinositol 3-kinase (PI3-kinase), which ultimately leads to translocation of the insulinresponsive glucose transporter, GLUT4, and glucose uptake. PI3-kinase also results in the activation of Akt (also known as protein kinase B), which, in turn, phosphorylates and inactivates glycogen synthase kinase-3 (GSK-3). Inactivation of GSK-3 results in activation of glycogen synthase and glycogen synthesis. Other pathways (e.g., p70S6 kinase, glycogen-bound form of protein phosphatase 1 (PP1G)) may regulate glycogen synthesis. The insulin receptor can directly phosphorylate Shc, leading to the activation of the Ras signaling complex that results in MAP kinase activation and certain mitogenic actions of insulin. [Reprinted with the permission of A. Dunaif.] 


\section{B. INSULIN ACTION IN PCOS ADIPOCYTES}

Studies in adipocytes, a classic insulin target tissue, have demonstrated no abnormalities in insulin receptor number or affinity in PCOS women, when compared to appropriately weight-matched control women (Dunaif et al., 1992; Ciaraldi et al., 1992,1997). However, in the PCOS adipocytes, there was a striking and significant shift to the right in the dose-response curve for glucose uptake. Maximal rates of glucose transport were modestly but significantly decreased, compared to control women. This suggests the presence of a postbinding defect in insulin receptor-mediated signal transduction in PCOS. Decreased glucose transport in PCOS adipocytes has been shown to be secondary to a significant reduction in the abundance of GLUT4 glucose transporters (Rosenbaum et al., 1993). These defects in PCOS adipocytes occurred in the absence of glucose intolerance, obesity, or changes in waist-to-hip girth ratios. There was no significant relationship demonstrated with circulating levels of sex hormones (Dunaif et al., 1992). This suggests that the abnormalities of insulin action in PCOS adipocytes may reflect intrinsic rather than acquired defects.

\section{INSULIN ACTION IN PCOS FIBROBLASTS}

Although fibroblasts are not classic insulin target cells, defects in insulin receptor number and kinase activity in skin fibroblasts have reflected mutations in the insulin receptor in several of the rare syndromes of extreme insulin resistance (Taylor et al., 1992). Studies using cultured skin fibroblasts from women with PCOS have been performed to investigate the cellular mechanisms of insulin resistance in this disorder. Furthermore, by studying cells that have been removed from their in vivo environment for several generations, it is possible to assess insulin action without the confounding influences of the in vivo environment (e.g., hyperinsulinemia, hyperandrogenemia, hyperglycemia), all of which can produce insulin resistance (Dunaif, 1997). Presumably, signaling defects observed in cultured cells reflect intrinsic genetic defects in insulin action.

Consistent with experiments using isolated adipocytes, studies of insulin receptor binding in cultured skin fibroblasts from PCOS women have shown no differences in receptor number and affinity, compared with cultured cells from reproductively normal women (Dunaif et al., 1995). However, partially purified insulin receptors from cultured fibroblasts of nearly half of PCOS women (PCOSser) were noted to have markedly increased basal autophosphorylation with minimal further insulin-stimulated autophosphorylation. Phosphoamino acid analysis revealed that the increased basal phosphorylation represented primarily phosphoserine (Dunaif et al., 1995). Serine phosphorylation has been shown in several experimental systems to inhibit the intrinsic tyrosine kinase activity of the insulin receptor (White and Yenush, 1998). Consistent with this, the PCOS-ser insulin receptors demonstrated significantly reduced tyrosine kinase activity towards an 
artificial substrate, compared to partially purified insulin receptors from the fibroblasts of reproductively normal control women (Dunaif et al., 1995). This abnormal pattern of phosphorylation also has been detected in insulin receptors partially purified from PCOS skeletal muscle, a classic insulin target tissue, suggesting that this receptor abnormality may have relevance to the underlying pathogenesis of insulin resistance in PCOS (Dunaif et al., 1995).

No mutations have been detected in the coding region of the insulin receptor gene in women with PCOS (Sorbara et al., 1994; Dunaif et al., 1995; Talbot et al., 1996). Moreover, immunoprecipitation and mixing experiments have suggested that a factor extrinsic to the insulin receptor - presumably, a serine kinase - is responsible for the abnormal pattern of phosphorylation in PCOS fibroblasts (Dunaif et al, 1995). When control insulin receptors were mixed with wheat germ agglutinin eluates from the PCOS-ser fibroblast cell lines, a decrease in insulinstimulated tyrosine phosphorylation of the normal receptors was observed, along with an increase in serine phosphorylation of these receptors (Dunaif et al., 1995). These findings recently have been confirmed in studies of intact cells. $\mathrm{Li}$ and colleagues, using a highly sensitive ELISA technique to measure insulin receptor tyrosine phosphorylation in fibroblasts from PCOS and control women, showed a 33 percent decrease in insulin-stimulated receptor tyrosine phosphorylation in intact PCOS fibroblasts. However, no abnormality in insulin receptor tyrosine phosphorylation was observed when insulin receptors from solubilized PCOS fibroblasts were first immunocaptured and then stimulated with insulin. This finding is consistent with the existence of a factor extrinsic to the insulin receptor causing impaired signaling (Li et al., 2000).

Fibroblasts from approximately 50 percent of women with PCOS studied by Dunaif and colleagues (1995) manifested no abnormalities in insulin receptor phosphorylation, despite demonstrating in vivo insulin resistance comparable to PCOS-ser women. This observation suggests the existence of postreceptor abnormalities of insulin signaling in these women, perhaps at the level of IRS-1 phosphorylation or PI3-kinase activation. It is possible that the same factor responsible for excessive serine phosphorylation of the insulin receptor in the PCOS-ser women also phosphorylates downstream signaling proteins. Protein kinase $\mathrm{C}(\mathrm{PKC})$ is a candidate factor that can serine-phosphorylate the insulin receptor (White and Yenush, 1998) as well as IRS-l (De Fea and Roth, 1997).

The putative serine phosphorylation factor of PCOS is an example of insulin resistance secondary to extrinsic factors that interact with key proteins of the insulin-signaling cascade to inhibit signaling (Figure 4). Plasma cell differentiation factor-1 (PC-1) inhibits insulin receptor tyrosine kinase activity but is not associated with increased serine phosphorylation of the insulin receptor (Maddux et al., 1995). TNF- $\alpha$ appears to induce insulin resistance by serine phosphorylation of IRS-1, thus inhibiting propagation of the signal (Hotamisligil et al., 1996). 


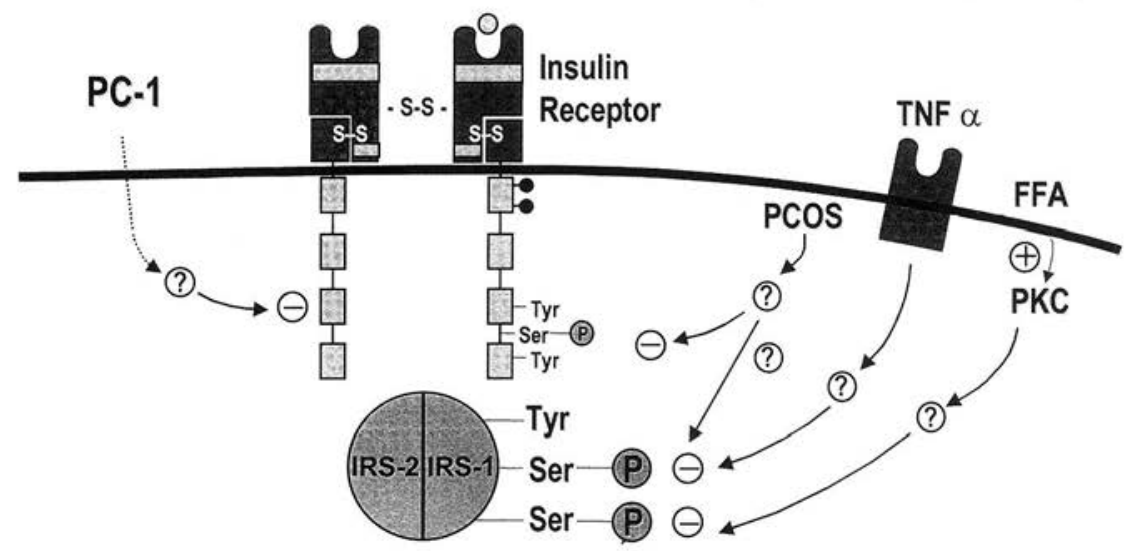

FIG. 4. Insulin resistance secondary to factors altering receptor signaling. Insulin resistance in cultured skin fibroblasts from approximately 50 percent of PCOS women appears to be secondary to a cell membrane-associated factor, presumably a serine/theronine kinase, that serine-phosphorylates the insulin receptor inhibiting signaling. Serine phosphorylation of IRS-1 appears to be the mechanism for TNF- $\alpha$-mediated insulin resistance. Free fatty acids can activate protein kinase C (PKC). PKCs can serine-phosphorylate IRS-1 and inhibit signaling. The membrane glycoprotein plasma cell differentiation factor also inhibits insulin receptor kinase activity but does not cause serine phosphorylation of the receptor. [Reprinted with the permission of A. Dunaif.]

Free fatty acids (FFAs) inhibit IRS-1 tyrosine phosphorylation and IRS-1-associated PI3-kinase activity, perhaps as a consequence of PKC-induced serine phosphorylation of IRS-1 (Griffin et al., 1999). There is evidence to suggest that hyperglycemia-induced insulin resistance is mediated by PKC-induced serine phosphorylation of the insulin receptor (Pillay et al., 1996).

In order to determine whether the abnormalities observed in insulin receptor phosphorylation in PCOS fibroblasts were physiologically relevant, studies of insulin action in intact cells were performed. Insulin-stimulated glycogen synthesis was found to be significantly decreased in PCOS fibroblasts, whereas thymidine incorporation was unchanged, compared with control fibroblasts (Book and Dunaif, 1999). Thus, insulin resistance in these cells appears to be selective, affecting metabolic, but not mitogenic, pathways of insulin signaling. Similar findings have been reported in the cultured fibroblasts of patients with type $2 \mathrm{DM}$ (Wells et al., 1993). IRS-1- and IRS-2-associated PI3-kinase activity was similar in cultured fibroblasts from PCOS and control women (Book and Dunaif, 1999; Venkatesan et al., 2000). Activation of Akt was similar in PCOS and control fibroblasts but GSK-3 phosphorylation was decreased in PCOS fibroblasts, consistent with an increase in GSK-3 activity (although this was not measured directly). Given that GSK-3 inhibits glycogen synthase, increased activity of GSK-3 
may account for the decreased glycogen synthesis observed in PCOS fibroblasts (Venkatesan et al., 2000). The relationship between abnormalities in phosphorylation of the insulin receptor and the downstream effects observed in PCOS fibroblasts is not clear.

\section{INSULIN ACTION IN PCOS SKELETAL MUSCLE}

Skeletal muscle is responsible for about 85 percent of insulin-stimulated glucose uptake in vivo (De Fronzo, 1997). Significant defects in insulin action have been demonstrated in the skeletal muscle of PCOS women, independent of obesity and hyperglycemia. In serial skeletal muscle biopsies obtained at baseline and during insulin infusion using the euglycemic clamp technique, insulin-stimulated activation of IRS-1-associated PI3-kinase was significantly decreased in PCOS skeletal muscle (Dunaif et al., in press). This occurred in parallel with decreased insulin-stimulated glucose uptake in vivo. The abundance of IRS- 1 and the p85 regulatory subunit of PI3-kinase was comparable in skeletal muscle from PCOS and control women. Thus, decreased IRS-1-associated PI3-kinase activation in PCOS muscle could not be accounted for by changes in the abundance of signaling proteins. Normal abundance of key insulin-signaling proteins also has been demonstrated in type $2 \mathrm{DM}$ without massive obesity (Bjornholm et al., 1997; Krook et al., 2000). However, alterations in abundance of insulin-signaling proteins have been described in other insulin-resistant states. In morbid obesity, the abundance of IRS-1 and p85 is decreased (Goodyear et al., 1995). In gestational $\mathrm{DM}$, the abundance of skeletal muscle insulin receptor is unchanged, whereas IRS- 1 abundance is decreased and IRS-2 and p85 are increased (Friedman et al., 1999).

These findings in PCOS skeletal muscle in vivo are consistent with inhibition of the insulin receptor tyrosine kinase by increased serine phosphorylation of the receptor that has previously been identified in PCOS cell-free systems (Dunaif $e t$ al., 1995). This mechanism may not be unique to PCOS. Recent studies suggest that increased insulin receptor serine/threonine phosphorylation in skeletal muscle may play a role in the pathogenesis of gestational DM (Shao et al., 2000). However, increased insulin receptor serine phosphorylation in the skeletal muscle of type 2 DM patients has not been demonstrated (Kellerer et al., 1995). Additionally, induction of serine phosphorylation of the insulin receptor in type $2 \mathrm{DM}$ may be secondary to hyperglycemia (Pillay et al., 1996), rather than an intrinsic defect.

All of these studies of PCOS and other insulin-resistant states used muscle biopsies or freshly isolated muscle strips. It is unclear whether the defects in insulin signaling that have been demonstrated in these studies are intrinsic to muscle tissue (i.e., genetically determined) or are acquired secondary to in vivo environmental factors such as hyperinsulinemia, increased circulating FFAs, or, 
in diabetic individuals, hyperglycemia. Another possibility is that both genetic and environmental influences on insulin signaling in skeletal muscle are important in the pathogenesis of insulin resistance, with underlying genetic defects in muscle conferring increased susceptibility to environmental factors.

Human skeletal muscle cells grown in culture show many of the characteristics of mature skeletal muscle in vivo. Therefore, this represents an excellent system for the study of muscle cells that have been removed from the in vivo environment (Sarabia et al., 1992; Henry et al., 1995). In contrast to the findings in PCOS skeletal muscle in vivo, our preliminary studies of culturcd muscle cclls of obese, nondiabetic PCOS women and weight-matched controls showed normal insulin responsiveness of glucose transport and glycogen synthesis in the PCOS muscle (unpublished data). These observations are consistent with a major role of environmental factors in the pathogenesis of insulin resistance in PCOS. Likewise, a recent study comparing insulin action and signaling in cultured skeletal muscle cells from normoglycemic, lean, insulin-resistant, and insulin-sensitive first-degree relatives of type $2 \mathrm{DM}$ patients failed to detect significant differences (Krützfeldt et al., 2000). However, a similar study using cultured skeletal muscle cells showed defects in insulin-stimulated glucose uplake in approximately half of overweight insulin-resistant, nondiabetic, first-degree relatives of type 2 diabetic patients, compared with lean control subjects (Jackson et al., 2000). Abnormalities in insulin action have been demonstrated in cultured skeletal muscle cells from patients with type 2 DM (Henry et al., 1995, 1996). However, it is not clear whether these defects are intrinsic or acquired, since it is possible that chronic hyperinsulinemia and/or hyperglycemia in vivo may induce nonreversible changes in insulin action. In insulin-resistant, nondiabetic Pima Indians, insulin action in cultured skeletal muscle cells was positively correlated with in vivo measures of insulin action, suggesting that genetic background may be relevant (Thompson et al., 1996; Mott et al., 1998; Youngren et al., 1999).

The candidate circulating factor that could produce skeletal muscle insulin resistance that has received the most attention is FFA. Circulating levels of FFAs correlate with in vivo measures of insulin resistance in first-degree relatives of individuals with type 2 DM (Gulli et al., 1992). Recent studies have shown that the administration of FFA to healthy subjects can produce insulin resistance and defects in muscle IRS-1-associated PI3-kinase activation (Dresner et al., 1999). Rodent studies suggest that this may be mediated via PKC-theta activation (Griffin et al., 1999). FFAs also appear to modulate hepatic glucose output (Bergman and Mittelman, 1998). These dual effects of FFA - to increase hepatic glucose production and to decrease muscle insulin sensitivity - have led Bergman to propose the "single-gateway hypothesis" (Bergman and Mittelman, 1998). According to this hypothesis, increased adipocyte lipolysis accounts for hepatic and peripheral insulin resistance. Adipocyte lipolysis could be increased by resistance to the antilipolytic actions of insulin and/or an increase in lipolytic stimuli (i.e., 
catecholamines), sensitivity to these stimuli, and/or the amount of lipolytically active visceral fat. FFA levels are increased in obese PCOS women and have not been studied in insulin-resistant, nonobese PCOS women (Holte et al., 1994; Robinson et al., 1996). Another circulating factor that could produce peripheral insulin resistance is TNF- $\alpha$ (Hotamisligil, 1999). One study has reported TNF- $\alpha$ clevations in PCOS (Naz et al., 1995).

\section{Insulin Resistance in PCOS: Progress and Paradoxes}

Insulin resistance in PCOS is associated with a postbinding defect in insulin receptor signaling that has been demonstrated in isolated adipocytes, cultured fibroblasts, and skeletal muscle in vivo. Current evidence suggests that constitutively increased serine phosphorylation of the insulin receptor or downstream key signaling proteins are involved in the pathogenesis of insulin resistance in PCOS. The factor responsible for this serine phosphorylation remains to be identified. Recent data suggest that this mechanism of induction of insulin resistance may not be unique to PCOS.

How this increase in serine phosphorylation of the insulin receptor or downstream proteins leads to a selective defect affecting only the metabolic pathway of insulin action in cultured PCOS skin fibroblasts is unclear. The mechanism may involve differential roles of IRS- 1 and IRS-2 in mediating the metabolic and mitogenic effects of insulin, in addition to abnormal interaction of the IRSs with the insulin receptor. The latter recently has been demonstrated in muscle cells expressing an insulin receptor containing a point mutation in the $\beta$ subunit (Arg1152Gln). This mutant receptor constitutively activates the metabolic pathway of insulin action, although mitogenic responses to insulin are normal (Petruzziello et al., 1993; Miele et al., 1999). In L6 muscle cells expressing the mutant receptor IRS-2, which was constitutively tyrosine phosphorylated and showed increased binding to the insulin receptor, mediated insulin regulation of glucose metabolism. In contrast, IRS-1 showed normal phosphorylation and mediated insulin mitogenic signals.

Tissue differences in insulin sensitivity may be present in PCOS, as reported by Ciaraldi and colleagues (1998). Isolated adipocytes and cultured skin fibroblasts from PCOS women demonstrate resistance to the metabolic effects of insulin. Conversely, preliminary studies of cultured PCOS skeletal muscle cells suggest that these cells are insulin sensitive, despite being insulin resistant in vivo. A possible explanation for these tissue-specific differences is that the abnormalities in insulin action in cultured fibroblasts and in isolated adipocytes reflect intrinsic defects. However, insulin resistance in skeletal muscle is either secondary to the in vivo environment or else interaction with environmental factors is required for an underlying genetic defect in insulin signaling to be manifested. The 
demonstration of abnormalities in insulin signaling in adipocytes of PCOS women is in keeping with the single-gateway hypothesis that proposes that increased adipocyte lipolysis, leading to increased circulating FFA levels, is the primary defect in insulin-resistant states. Tissue-specific differences in the roles of the IRS-1 and -2 in mediation of insulin signaling also may be relevant. Recent experiments using knockout mice have shown that IRS-1 plays a prominent role in insulin action in skeletal muscle and IRS-2 in liver (Higaki et al., 1999; Kido et al., 2000). Studies in human muscle appear to support these findings (Kim et al., 1999).

\section{Summary}

Women with PCOS are at substantially increased risk of developing impaired glucose tolerance and type $2 \mathrm{DM}$ at a young age. This risk is conferred by profound peripheral insulin resistance and defects in insulin secretion. The major abnormality in insulin action in PCOS is a postbinding defect in insulin signaling. This appears to be secondary to a factor extrinsic to the receptor that modulates its signaling and perhaps the signaling transduced by IRSs. Our preliminary studies suggest that not all tissues are intrinsically insulin resistant in PCOS. Skeletal muscle has acquired defects in insulin action. Hence, it is possible that insulin resistance in PCOS adipocytes leads to increased circulating FFA levels that, in turn, produce peripheral insulin resistance. Other circulating factors (e.g., TNF- $\alpha$ ) may contribute to insulin resistance in PCOS. Elucidating the pathogenesis of insulin resistance in PCOS will provide insight into an important cause of type $2 \mathrm{DM}$.

\section{REFERENCES}

Achard, C., and Thiers, J. (1921). Bull. Acad. Natl. Med. (Paris) 86, 51-64.

Bcrgman, R.N., and Mittclman, S.D. (1998). J. Basic. Clin. Physiol. Pharmacol. 9, $205-221$.

Bergman, R.N., Watanabe, R., Rebrin, K., Ader, M., and Steil., G. (1996). Diabet. Med. 13, S67-S77.

Björnholm, M., Kawano, Y., Lehtihet, M., and Zierath, J.R. (1997). Diabetes 46, 524-527.

Book, C.B., and Dunaif, A. (1999). J. Clin. Endocrinol. Metab. 84, 3110-3116.

Burghen, G.A., Givens, J.R, and Kitabchi, A.E. (1980). J. Clin. Endocrinol. Metab. 50, 113-116.

Cheatham, B., and Kahn, C.R. (1995). Endocr Rev. 16, 117-142.

Ciaraldi, T.P., El-Roeiy, A., Madar, Z., Reichart, D., Olefsky, J.M., and Yen, S.S.C. (1992). J. Clin. Endocrinol. Metab. 75, 577-583.

Ciaraldi, T.P., Morales, A.J., Hickman, M.G., Odom-Ford, R., Olefsky, J.M., and Yen, S.S.C. (1997). J. Clin. Endocrinol. Metab. 82, 1421-1425.

Ciaraldi, T.P., Morales, A.J., Hickman, M.G., Odom-Ford, R., Yen, S.S.C., and Olefsky, J.M. (1998). Metabolism 47, 940-946.

De Fea, K., and Roth, R.A. (1997). Biochemistry 36, 12939-12947.

De Fronzo, R.A. (1997). Diabetes Rev. 5, 177-269. 
Dresner, A., Laurant, D., Marcucci, M., Griffin, M.E., Dufour, S., Cline, G.W., Slezak, L.A., Andersen, D.K., Hundal, R.S., Rothman, D.L., Falk Petersen, K., and Shulman, G.I. (1999). J. Clin. Invest. $103,253-259$.

Dunaif, A. (1997). Endocr Rev. 18, 774-800.

Dunaif, A., and Finegood, D.T. (1996). J. Clin. Endocrinol. Metab. 81, 942-947.

Dunaif, A., Graf, M., Mandeli, J., Laumas, V., and Dobrjansky, A. (1987). J. Clin. Endocrinol. Metab. 65, 499-507.

Dunaif, A., Futterweit, W., Segal, K.R., Shelley, D.R., Green, G., Dobrjansky, A., and Licholai, T (1992). Diabetes 41, 1257-1266.

Dunaif, A., Xia, J., Book, C.B., Schenker, E. and Tang, Z. (1995). J. Clin. Invest. 96, 801-810.

Dunaif, A., Scott, D., Finegood, D., Quintana, B., and Whitcomb, R. (1996). J. Clin. Endocrinol. Metab. 81, 3299-3306.

Dunaif, A., Wu, X., Lee, A., and Diamanti-Kandarakis, E. (2001). Am J. Physiol., in press.

Fhrmann, D.A., Sturis, I, Ryrne, M.M., Karrison, T., Rosenfield, R.L., and Polonsky, K.S. (1995). J. Clin. Invest. 69, 520-527.

Ehrmann, D.A., Barnes, R.B., Rosenfield, R.L., Cavaghan, M.K., and Imperial, J. (1999). Diabetes Care $22,141-146$.

Friedman, J.E., Ishizuka, T., Shao, J., Huston, L., Highman, T., and Catalano, P. (1999). Diabetes 48, 1807-1814.

Goodyear, L.J., Giorgino, F., Sherman, L.A., Carey, J., Smith, R.J., and Dohm, G.L. (1995). J. Clin. Invest. 95, 2195-2204.

Griffin, M.E., Marcucci, M.J., Cline, G.W., Bell, K., Barucci, N., Lee, D., Goodyear, L.J., Kraegen, E.W., White, M.F., and Shulman, G.I. (1999). Diabetes 48, 1270-1274.

Gulli, G., Ferrannini E., Stern, M., Haffner, S., and DeFronzo, R.A. (1992). Diabetes 41, 1575-1586.

Henry, R.R., Abrams, L., Nikoulina, S., and Ciaraldi, T.P. (1995). Diabetes 44, 939-946.

Henry, R.R., Ciaraldi, T.P., Abrams-Carter, L., Mudaliar, S., Park, K.S., and Nikoulina, S.E. (1996). J. Clin. Invest. 98, 1231-1236.

Higaki, Y., Wojtaszewski, J.F., Hirshman, M.F., Withers, D.J., Towery, H., White, M.F., and Goodyear, L.J. (1999). J. Biol. Chem. 274, 20791-20795.

Holte, J., Bergh, T., Berne, C., and Lithell., H. (1994). Clin. Endocrinol. (Oxf.) 41, 463-471.

Hotamisligil, G.S. (1999). Exp. Clin. Endocrinol. Diabetes 107, 119-125.

Hotamisligil, G.S., Peraldi, P., Budavari, A., Ellis, R., White, M.F., and Spicgelman, B.M. (1996). Science 271, 665-668.

Jackson, S., Bagstaff, S.M., Lynn, S., Yeaman, S.J., Turnbull, D.M., and Walker, M. (2000). Diabetes 49, $1169-1177$.

Kellerer, M., Coghlan, M., Capp, E., Muhlhofer, A., Kroder, G., Mosthaf, L., Galante, P., Siddle, K., and Häring, H.U. (1995). J. Clin. Invest. 96, 6-11.

Kiddy, D.S., Hamilton-Fairley, D., Bush, A., Short, F., Anyaoku, V., Reed, M.J., and Franks, S. (1992). Clin. Endocrinol. 36, 105-111.

Kido, Y., Burks, D.J., Withers, D., Bruning, J.C., Kahn, C.R., White, M.F., and Accili, D. (2000). $J$. Clin. Invest. 105, 199-205.

Kim, Y.-B., Nikoulina, S.E., Ciaraldi, T.P., Henry, R.R., and Kahn, B.B. (1999). J. Clin. Invest. 104, 733-741.

Knochenhauer, E.S., Key, J., Kahsar-Miller, M., Waggoner, W., Boots, L.R., and Azziz, R. (1998). J. Clin. Endocrinol. Metab. 83, 3078-3082.

Krook, A., Bjornholm, M., Galuska, D., Jiang, X.J., Fahlman, R., Myers, M.G. Jr., Wallberg-Henriksson, H., and Zierath, J.R. (2000). Diabetes 49, 284-292.

Krützfeldt, J., Kausch, C., Volk, A., Klein, H.H., Rett, K., Häring, H.-U., and Stumvoll, M. (2000). Diabetes 49, 992-998.

Lawrence, J.C.J., and Roach, P.J. (1997). Diabetes 46, $541-547$. 
Legro, R.S., Kunselman, A., Dodson, W.C., and Dunaif, A. (1999). J. Clin. Endocrinol. Metab. 84, $165 \cdot 169$.

Li, M., Youngren, J.F., Maddux, B.A., Dunaif, A., Thomas, A., Zhang, B., and Goldfine, I.D. (2000). In "Program \& Abstracts of the 82nd Annual Meeting of The Endocrine Society," June 2000, Toronto, Canada, abstract 2331, p. 563.

Maddux, B.A., Sbraccia, P., Kumakura, S., Sasson, S., Youngren, J., Fisher, A., Spencer, S., Grupe, A., Henzel, W., Stewart, T.A., Reaven, G.M., and Goldfine, I.D. (1995). Nature 373, 448-451.

Miele, C., Caruso, M., Calleja, V., Auricchio, R., Oriente, F., Formisano, P., Condorelli, G., Cafieri, A., Sawka-Verhelle, D., Van Obberghen, E., and Beguinot, F. (1999). J. Biol. Chem. 274, 3094-3102.

Mott, D.M., Pratley, R.E., and Bogardus, C. (1998). J. Clin. Invest. 101, 2252-2256.

Naz, R.K., Thurston, D., and Santoro, N. (1995). Am. J. Reprod. Immunol. 34, 170-175.

Nestler, J.E., Jakubowicz, D.J., Evans, W.S., and Pasquali, R. (1998). N. Engl. J. Med. 338, 1876-1880.

Nestler, J.E., Jakubowicz, D.J., Reamer, P., Gunn, R.D., and Allan, G. (1999). N. Engl. J. Med. 340, 1314-1320.

O'Meara, N.M., Blackman, J.D., Ehrmann, D.A., Barnes, R.B., Jaspan, J.B., Rosenfield, R.L., and Polonsky, K.S. (1993). J. Clin. Endocrinol. Metab. 76, 1241-1247.

Pasquali, R., Antenucci, D., Casimirri, F., Venturoli, S., Paradisi, R., Fabbri, R., Balestra, V., Melchionda, N., and Barbara, L. (1989). J. Clin. Endocrinol. Metab. 68, 173-179.

Pillay, T.S., Xiao, S., and Olefsky, J.M. (1996). J. Clin. Invest. 97, 613-620.

Petruzziello, A., Formisano, P., Miele, C., Di Finizio, B., Riccardi, G., Ferrara, A., Beguinot, L., and Beguinot, F. (1993). J. Clin. Endocrinol. Metab. 77, 409-412.

Robinson, S., Kiddy, D., Gelding, S.V., Willis, D., Niththyananthan, R., Bush, A., Johnston, D.G., and Franks, S. (1993). Clin. Endocrinol. (Oxf.) 39, 351-355.

Robinson, S., Henderson, A.D., Gelding, S.V., Kiddy, D., Niththyananthan, R., Bush, A., Richmond, W., Johnston, D.G., and Franks, S. (1996). Clin. Endocrinol. (Oxf.) 44, 277-284.

Rosenbaum, D., Ilaber, R., and Dunaif, A. (1993). Am. J. Physiol. 264, E197-E202.

Saltiel, A.R. (1996). Am. J. Physiol. 270, E375-E385.

Sarabia, V., Lam, L., Burdett, E., Leiter, L.A., and Klip, A. (1992). J. Clin. Invest. 90, 1386-1395.

Shao, J., Catalano, P.M., Yamashita, H., Ruyter, I., Smith, S., Youngren, J., and Friedman, J.E. (2000). Diabetes 49, 603-610.

Sorbara, L.R., Tang, Z., Cama, A., Xia, J., Schenker, E., Kohanski, R.A., Poretsky, L., Koller, E., Taylor, S.I., and Dunaif, A. (1994). Metabolism 43, 1568-1574.

Talbot, J.A., Bicknell, E.J., Rajkhowa, M., Krook, A., O'Rahilly, S., and Clayton, R.N. (1996). J. Clin. Endocrinol. Metab. 81, 1979-1983.

Taylor, S.I., Cama, A., Accili, D., Barbetti, F., Quon, M.J., de la Luz Sierra, M., Suzuki, Y., Koller, E., Levy-Toledano, R., Wertheimer, E., Moncada, V.Y., Kadowaki, H., and Kadowaki, T. (1992). Endocr. Rev. 13, 566-595.

Thompson, D.B., Pratley, R., and Ossowski, V. (1996). J. Clin. Invest. 98, 2346-2350.

Venkatesan, A., Corbould, A., and Dunaif, A. (2000). In "Program \& Abstracts of the 82nd Annual Meeting of The Endocrine Society," June 2000, Toronto, Canada, abstract 330, p 88.

Virkamaki, A., Ueki, K., and Kahn, C.R. (1999). J. Clin. Invest. 103, 931-943.

Wells, A.M., Sutcliffe, I.C., Johnson, A.B., and Taylor, R. (1993). Diabetes 42, 583-589.

White, M.F., and Yenush, L. (1998). Curr. Top. Microbiol. Immunol. 228, 179-208.

Youngren, J.F., Goldfine, I.D., and Pratley, R.E. (1999). Am. J. Physiol. 276, E990-E994. 


\title{
The $\beta$-Adrenergic Receptors and the Control of Adipose Tissue Metabolism and Thermogenesis
}

\author{
Sheila Collins* ${ }^{\dagger}$ and Richard S. Surwit ${ }^{\dagger}$ \\ Departments of *Pharmacology and ${ }^{\dagger} p_{\text {sychiairy and Behavioral Sciences, }}$ \\ Duke University Medical Center, Durham, North Carolina 277J0
}

\begin{abstract}
The $\beta$-adrenergic receptors ( $\beta$ ARs) are members of the large family of $G$ protein-coupled receptors. There are three $\beta A R$ subtypes $\left(\beta_{1} A R, \beta_{2} A R \beta_{3} A R\right)$, each of which is coupled to $G \alpha$ and the stimulation of intracellular cAMP levels. While $\beta_{1} A R$ and $\beta_{2} A R$ are broadly expressed throughout tissues of the body, $\beta_{3} A R$ is found predominantly in adipocytes. Stimulation of the $\beta A R s$ leads to lipolysis in white adipocytes and nonshivering thermogenesis in brown fat. However, in essentially all animal models of obesity, the $\beta A R$ system is dysfunctional and the ability to stimulate lipolysis and thermogenesis is impaired. Nevertheless, we and others have shown that selective $\beta_{3} A R$ agonists are able to prevent or reverse obesity and the loss of $\beta A R$ expression and to stimulate thermogencsis. This chapter will review the current understanding of the role of the sympathetic nervous system and the adipocyte $\beta A R$ s in models of obesity; the physiologic impact of changes in $\beta A R$ expression on body composition and thermogenesis; and the regulation and unique properties of $\beta A R$ subtypes in brown and white adipocytes. The latter includes our recent discovery of novel signal transduction mechanisms utilized by $\beta_{3} A R$ to activate simultaneously the protein kinase $A$ and MAP kinase pathways. The impact of understanding these pathways and their potential role in modulating adaptive thermogenesis is discussed
\end{abstract}

\section{Thermogenesis: Control of Heat Generation and Body Composition}

\section{A. BROWN FAT THERMOGENESIS AND UNCOUPLING OF MITOCHONDRIAL RESPIRATION}

The rich and varied history of brown adipose tissue (BAT) as an anatomically discrete tissue type included speculations during the 17th century that it was part of the thymus and, a century later, that it was an endocrine organ involved in blood formation or a form of fat acting as a reservoir for certain nutrients (Lindberg, 1970). It wasn't until 1961 that BAT was proposed to be thermogenic (Ball and Jungas, 1961; Smith, 1961). Since that time, an immense body of work has shown that BAT is uniquely capable of responding to various environmental stimuli to generate heat from stored metabolic energy. In response to sympathetic 
nervous system (SNS) activation, noradrenergic stimulation of beta-adrenergic receptors ( $\beta A R s$ ) (with a lesser contribution from $\alpha 1 \mathrm{AR}$ ) triggers an orchestrated hyperplastic and hypertrophic expansion of BAT. There is also a dramatic increase in blood flow and recruitment of metabolic fuel (i.e., free fatty acids, glucose) to support a massive increase in oxidative metabolism (Bukowiecki et al., 1982; Géloën et al., 1992). A unique and critical element of this thermogenic machine was recognized to be the presence of a brown fat-specific mitochondrial uncoupling protein (UCP), originally called "thermogenin" (Cannon et al., 1982). This mitochondrial protein allows controlled proton "leakage" across the inner mitochondrial membrane, along its electrochemical potential gradient, for the purpose of heat generation at the expense of coupled adenosine triphosphate (ATP) production (Figure 1). Cloning of the gene for the brown fat UCP (now called UCP1) from rodents provided the opportunity to investigate the molecular mechanism of thermogenic uncoupling in mitochondria and regulation of the UCP gene and protein by hormonal and metabolic stimulation (Bouillaud et al., 1984; Kozak et al., 1994; Larose et al., 1996). Today, although there is still much debate concerning the specific mechanism by which UCP1 promotes proton movement (Garlid

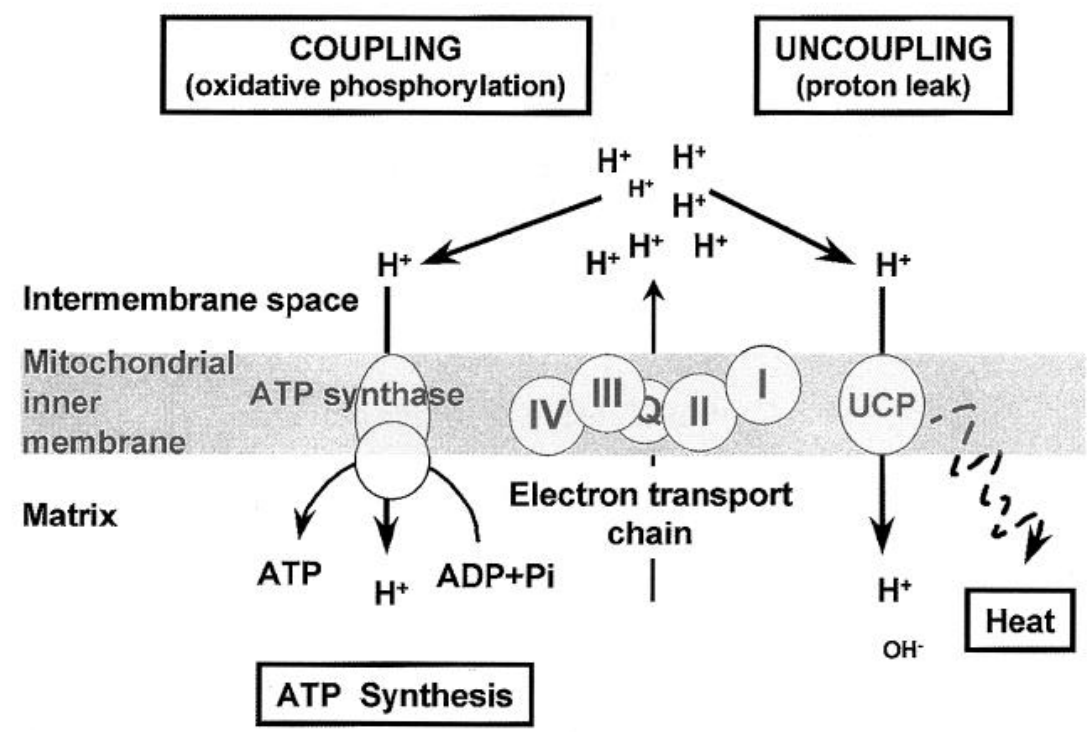

FIG. 1. Schematic model of mitochondrial "uncoupling." This simple cartoon compares the relationship between mitochondrial respiration that is "coupled" to the production of ATP via the ATP synthase, vs. the UCP-dependent transfer of protons across the inner membrane down the electrochemical gradient, whose action yields heat as a result of a need for increased fuel consumption to maintain ATP levels. 
et al., 1998; Bouillaud, 1999; Klingenberg et al., 1999), there is little dispute that this specialized member of the mitochondrial carrier family is responsible for the utilization of metabolic fuel to render mitochondrial metabolism inefficient, the byproduct of which is heat.

\section{B. THERMOGENIC DEFECTS IN OBESITY}

From the earliest studies of the ob/ob or "obese" mouse (now called leptindeficient $\mathrm{C} 57 \mathrm{BL} / 6 \mathrm{~J} L e p^{o b}$ ), there was evidence that these mice were not only obese, hyperglycemic, and hyperinsulinemic but that they also exhibited extreme sensitivity to the cold (reviewed in Bray and York, 1979). Histologically, BAT in these obese animals appears "inactive" in that it is infiltrated by white adipocytes and lacks the rich density of UCP1-containing mitochondria normally seen in lean animals (Cinti, 1999). The blunted capacity for adrenergic stimulation of lipolysis in adipose tissue of these animals (described in Section IIIA) probably also hinders the direct activation of UCP1 protein by free fatty acids. Other monogenic obesity models and hypothalamic lesioning studies in rodents all indicated a complex set of neural and endocrine abnormalitics, culminating in the loss of homeostatic mechanisms controlling both food intake and metabolic efficiency (reviewed in Bray et al., 1990).

The suggestive role for brown fat thermogenesis in body weight regulation was further strengthened by two sets of studies. First, mice lacking brown fat as a consequence of targeted expression of diptheria toxin in brown adipocytes (Lowell et al., 1993) became obese and somewhat hyperphagic. Since the adipocyte-derived hormone leptin (the product of the ob locus) (Zhang et al., 1994) regulates food intake, metabolic rate, and thermogenesis in brown fat (Campfield et al., 1995; Halaas et al., 1995; Pelleymounter et al., 1995; Collins et al., 1996), the obesity in brown fat-ablated mice may be a result of the inability of leptin to modulate brown fat thermogenesis. Second, Kopecky and Kozak showed that overexpression of UCPI in white adipose tissue (WAT) could ameliorate obesity syndromes resulting from either genetic or dietary manipulation (Kopecky et al., 1996a,1996b). In our studies, we have shown that high-fat feeding differentially increases plasma leptin, body temperature, and expression of UCP1 in BAT in the obesity-resistant A/J strain of mice (Surwit et al., 1997,1998). Since temperature is a surrogate measure for metabolic rate (Rising et al., 1992), this suggests that increased plasma leptin in response to high-fat feeding may serve to modulate the subsequent accumulation of body fat in rodents through increased metabolic activity in BAT. Recently, the notion that brown fat and UCP1 play a pivotal role in regulating body composition has been complicated by observations from specific targeted disruption of the UCP1 gene: these animals are completely intolerant of the cold, but are not obese (Enerback et al., 1997). Nevertheless, the inverse relationship between energy expenditure (particularly resting metabolic rate) and 
obesity has been observed repeatedly in laboratory animals and in humans (Ravussin et al., 1982; Landsberg and Young, 1984; Sims and Danforth, 1987; Spraul et al., 1993).

\section{UNUSUAL THERMOGENIC PROPERTIES OF ATYPICAL $\beta$ AR LIGANDS AND DISCOVERY OF THE $\beta_{3}$ AR}

\section{Studies in Laboratory Animal Models}

Study of lipolysis and thermogenesis in WAT and BAT by $\beta A R$ has witnessed episodic confusion and controversy, as the number of adrenergic receptor subtypes has grown by molecular cloning and new pharmacologic tools have become available. For many years, it was generally accepted that a single $\beta A R$ subtype controlled adipocyte metabolism (Fain and Garcia-Sainz, 1983; Bahouth and Malbon, 1988). This view began to erode as ever-more-selective sympathomimetic agents were developed that could discriminate between $\beta_{1} A R$ and $\beta_{2} A R$. Major evidence for a "new" $\beta A R$ subtype distinct from $\beta_{1}$ - or $\beta_{2} A R$ appeared when Arch and colleagues (1984) reported that a series of new $\beta$-adrenergic ligands that were clearly not "classical" $\beta_{1} A R$ or $\beta_{2}$ AR ligands had remarkable anti-obesity effects in the severely obese and diabetic C57BL/6J Lep ${ }^{o b}$ mouse. These novel compounds increased oxygen consumption, eliminated the classic cold intolerance of these animals, and, importantly, reduced their excess adipose tissue stores. In 1989 , the first report of a novel $\beta$ AR subtype, $\beta_{3} A R$, was published (Emorine et al., 1989), followed by reports of homologous genes in rodents (Granneman et al., 1991; Muzzin et al., 1991; Nahmias et al., 1991). It is now apparent that earlier results from $\beta A R$ radioligand-binding studies in adipose tissue were misleading, because the classical $\beta A R$ radioligands (e.g., cyanopindolol) exhibit a 20 - to 50 -fold weaker affinity for $\beta_{3} A R$ than for $\beta_{1} A R$ and $\beta_{2} A R$ (Tate et al., 1991). Therefore, $\beta_{3} A R$ levels were essentially undetected and any estimates made of $\beta_{1} A R$ and $\beta_{2} A R$ were distorted.

Many studies have documented the potent anti-obesity and antihyperglycemic properties of ever-more-selective $\beta_{3}$ AR agonists in a variety of animal models (Arch et al., 1984; Himms-Hagen et al., 1994; Largis et al., 1994; Collins and Surwit, 1996; Collins et al., 1997; Sasaki et al., 1998). In most species studied, including some studies in nonhuman primates, $\beta_{3}$ AR-agonist treatment is associated with increased density of brown adipocytes expressing UCP1 within typical WAT depots (Champigny et al., 1991; Himms-Hagen et al., 1994; Collins et al., 1997; Fisher et al., 1998; Sasaki et al., 1998). At least from our studies in various inbred strains of mice, the relative success of $\beta_{3}$ agonists as an anti-obesity therapy appears to depend upon genetic background and parallels the quantitative extent of this expansion of BAT (Collins et al., 1997). Others have reported similar 
effects of cold exposure as well as acute $\beta_{3}$-agonist stimulation in a series of recombinant inbred strains of mice (Guerra et al., 1998). Importantly, in our studies, we have observed, as shown in Figure 2A, that the beneficial effects of $\beta_{3} \mathrm{AR}$ agonists to decrease adipose tissue mass and improve glycemic control in mouse models of obesity and diabetes can persist, even after many weeks of chronic treatment (Collins et al., 1997). This apparent lack of functional desensitization is rather unusual, particularly since tachyphylaxis is a hallmark of most receptor systems. It is likely that $\beta_{3} A R$ activation and stimulation of downstream effectors can continue because $\beta_{3} A R$ is neither a target for phosphorylation (Liggett et al., 1993) nor does it bind $\beta$-arrestin (Cao et al., 2000; discussed in Section II), an accessory protein that is involved in desensitization of $\mathrm{G}$ protein-coupled receptors (Lefkowitz, 1998). In addition, as shown in Figure 2B, this decrease in body fat stores is not due to changes in food intake, dispelling the notion that these agents possess satiety properties.

\section{Controversy over the Existence of $\beta 3 A R$ in Humans}

The remarkable results from selective $\beta_{3} \mathrm{AR}$ agonist treatment in various laboratory animal models have fueled intense investigation of these drugs as potential obesity and/or diabetes therapies for humans. The presence of $\beta_{3} A R$ in adult human adipose tissue has been a subject of great debate. Part of the difficulty stems from the fact that $\beta_{3} A R$-selective agonists such as BRL37344 and CL316,243 initially were used to assess functional $\beta_{3} A R$ in fat samples from humans as well as nonhuman primates. The problem here is that these compounds are, at best, weak partial agonists at the primate homologues of $\beta_{3} \mathrm{AR}$ (Strosberg, 1997). Therefore, many studies with these compounds yielding negative results need to be carefully viewed in this light. There is now clear evidence of $\beta_{3} A R$ in human adipose tissue but the levels found in human fat cells are clearly much lower than those observed in rodents (Krief et al., 1993; Weyer et al., 1998) and are most evident in brown adipocytes found clustered amongst white fat cells, particularly within intra-abdominal depots. As a result of this visceral location, it has been difficult, to say the least, to quantify the relative abundance of these brown adipocytes among individuals and in response to drug treatments.

Humans are essentially like dogs and monkeys in that, as newborns, there are discrete depots of brown fat that "disappear" with growth into adulthood. However, upon treatment with selective $\beta_{3} A R$ agonists, dogs and rhesus monkeys have been found to exhibit increased metabolic rate, decreased fat mass, and the appearance of brown adipocytes scattered within the so-called typical "white" adipose depots, similar to what we and others have observed in mice and rats (Champigny et al., 1991; Fisher et al., 1998; Sasaki et al., 1998; Shih et al., 1999). These studies are promising in that they suggest that humans also may respond to 
$\mathbf{A}$

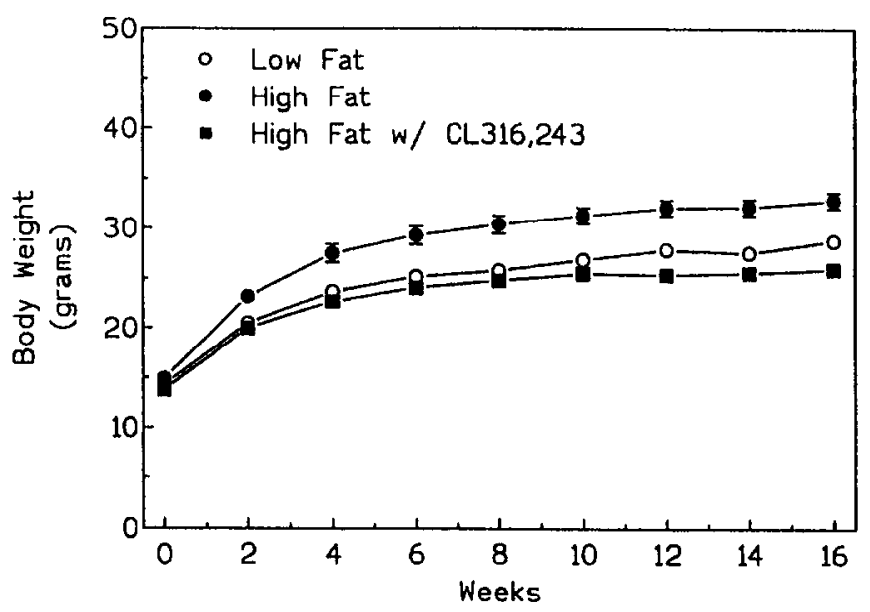

B

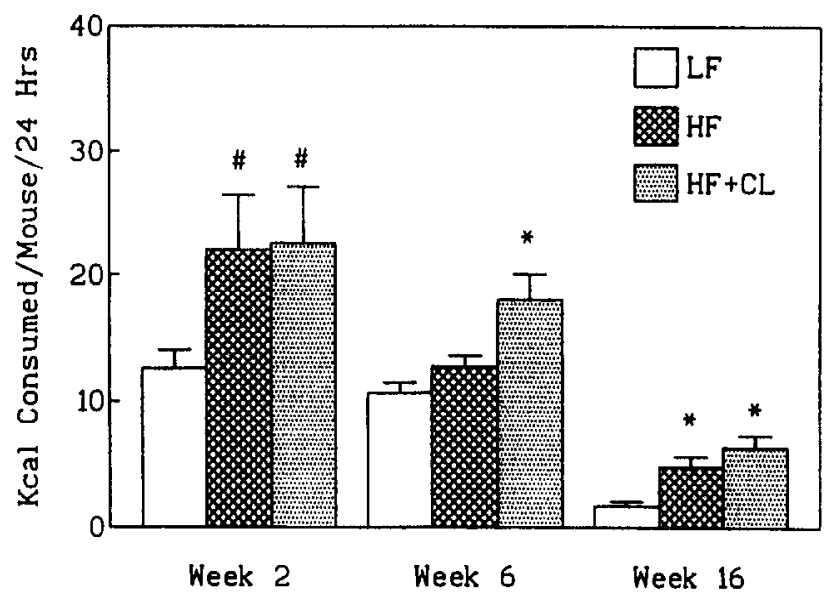

FIG. 2. Obesity induced by high-fat feeding and its prevention by a $\beta_{3} A R$ agonist. Thirty $A / J$ male mice were obtained from Jackson Laboratories (Bar Harbor, Maine) at 4 weeks of age. Groups of 10 mice were randomly assigned to a low-fat diet (open circles), high-fat diet (filled circles), or high-fat diet containing $0.001 \%$ CL316,243 (filled squares). (A) Animals were weighed at biweekly intervals throughout the 16 weeks on the diets. (B) Food intake measurements over a 24-hour period were made for individual animals at three different times during the study (after 2 weeks, 6 weeks, and 16 weeks on the diets). Each group of 10 animals was housed individually and grams of food consumed over 24 hours were measured. Caloric content of each diet was calculated based on 5.55 $\mathrm{kcal} / \mathrm{g}$ for the high-fat diets and $4.07 \mathrm{kcal} / \mathrm{g}$ for the low-fat diet. \#, significantly different from low-fat fed animals at $p<0.05 .^{*}$, significantly different from low-fat and high-fat animals at week 6 and from low-fat animals at week 16, p $<0.005$. [Adapted from Collins, S., and Surwit, R.S., J. Biol. Chem. 271, 9437-9440, 1996. Copyright 1996 The American Society for Biochemistry \& Molecular Biology.] 
these compounds. However, there is clearly a need for a more thorough, largescale analysis of intra-abdominal adipose tissue from humans, in order to obtain a more-accurate assessment of the extent to which brown adipocytes remain in adult humans. Likewise, the ability of newer compounds that are full agonists and antagonists for human $\beta_{3} \mathrm{AR}$ (Candelore et al., 1999) to directly modulate lipolysis in monkey or human fat cells should help to clarify these issues and to determine whether a similar recruitment of thermogenically active brown adipocytes will result from treatment of adult humans with $\beta_{3} \mathrm{AR}$ agonists.

\section{Why Three $\beta$ ARs in Adipocytes?}

The realization that adipocytes can express all three members of the $\beta A R$ family (van Liefde et al., 1992; Galitzky et al., 1993; Collins et al., 1994; Rohlfs et al., 1995), each coupled to the stimulation of adenylyl cyclase and the protein kinase A (PKA) pathway, raises the question as to whether these receptors are simply redundant or are responsible for regulating distinct cellular responses. Since the adipocyte-specific $\beta_{3}$ AR lacks sites for regulatory phosphorylation, a process that triggers rapid desensitization of most $G$ protein-coupled receptors, it was postulated that, during times of intense sympathetic stimulation, $\beta_{3} A R$ would remain functional while $\beta_{1} A R$ and $\beta_{2} A R$ became downregulated. However, even prior to discovery of $\beta_{3} A R$, studies in primary cultures of rodent brown adipocytes strongly suggested that $\beta A R$ subtypes may regulate discrete cellular functions in adipocytes. For example, $\beta_{1} \mathrm{AR}$ was shown to be linked to the proliferation of brown adipocytes in response to increased SNS stimulation (Bukowiecki et al., 1982; Géloën et al., 1992). Following the discovery of $\beta_{3} A R$ and the development of immortalized brown adipocyte cell systems (Kozak et al., 1992; Klaus et al., 1994), we examined the ability of individual $\beta A R$ subtypes to induce expression of UCP1 and to stimulate cell proliferation. We showed that induction of UCP1 transcripts could occur by any of the $\beta A R$ subtypes, although it appeared that the most-robust stimulation was achieved when the $\beta A R s$ were activated simultaneously (Rohlfs et al., 1995). By contrast, as shown in Figure 3, the norepinephrinestimulated proliferation and associated induction of c-fos and junB in B7 brown adipocytes were observed only in response to $\beta_{1} A R$ and, to a lesser extent, via $\beta_{2}$ AR but not via $\beta_{3}$ AR or $\alpha 1$ AR. As will be discussed in Section IIB, the ability of the $\beta A R s$ to couple to different signal transduction pathways, or to be physically localized in proximity to certain downstream targets, might contribute to nonoverlapping $\beta A R$ functions in the adipocyte. It should be noted that these immortalized brown adipocyte cell systems can provide certain significant advantages over primary cultures, particularly in terms of clonal purity and ease of growth. However, the maintenance of certain phenotypic characteristics - most notably, expression of UCP1 — can wane with passage (Irie et al., 1999). 


\section{Regulation and Signal Transduction Properties of the $\beta_{3} A R$}

\section{A. ADIPOCYTE-SPECIFIC EXPRESSION OF $\beta_{3}$ AR REQUIRES C/EBP $\alpha$}

Unlike $\beta_{1} A R$ and $\beta_{2} A R$, whose expression is quite ubiquitous, $\beta_{3} A R$ is confined to adipocytes. From studies in adipocyte cell lines - such as 3T3-F442A (Figure 4), 3T3-L1, and C3H10T1/2 - functional $\beta_{3} \mathrm{AR}$ appears relatively late during the process of adipogenesis (Feve et al., 1991); Dixon et al., in press). Two groups of transcription factors are responsible for initiating and maintaining adipocyte differentiation: CCAAT/enhancer binding protein (C/EBP) (Darlington et al., 1998) and peroxisome proliferator-activated receptor-gamma (PPAR $\gamma$ ) (Spiegelman, 1998). The C/EBPs are expressed in a cascade-like fashion during
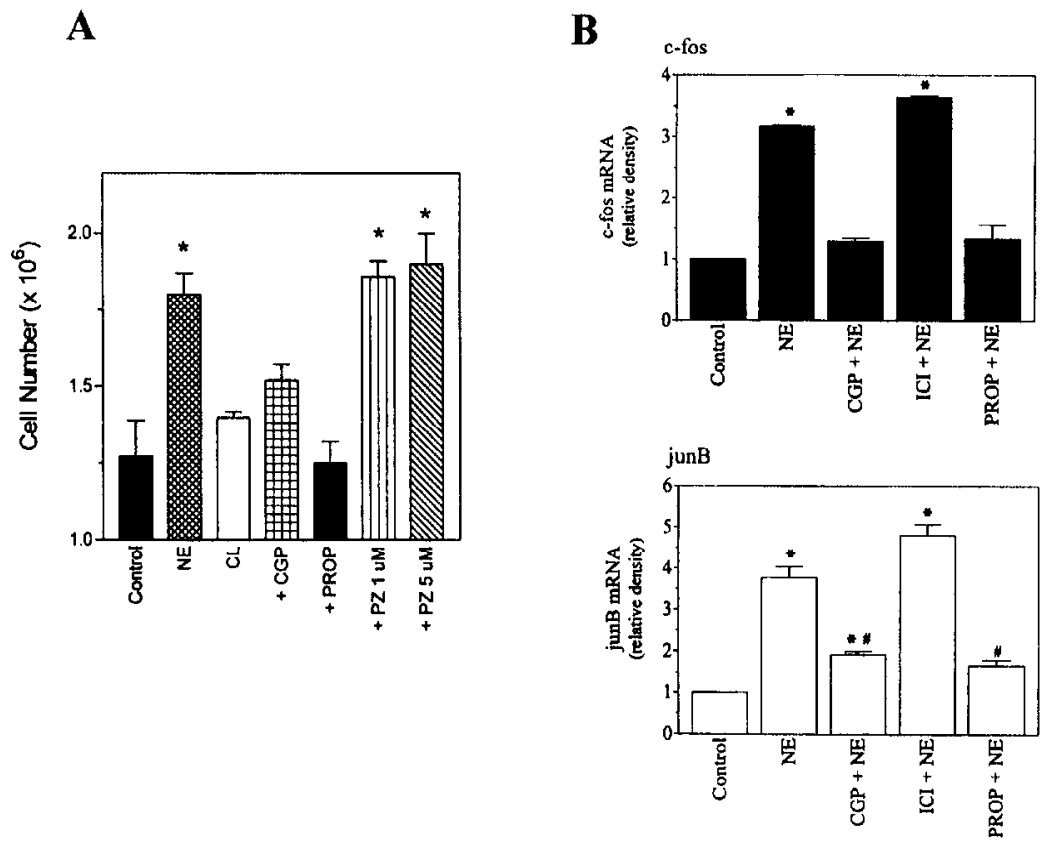

FIG. 3. Effect of selective activation of $\beta$-adrenergic receptor subtypes on proliferation and expression of fos/jun family members in $\mathrm{B} 7$ brown adipocytes. One day after seeding into six-well plates, the cells were treated with $1 \mu \mathrm{M} \mathrm{NE}$ or CL316,243 (CL) supplemented with or without $1 \mu \mathrm{M}$ of the following $\beta A R$ antagonists: CGP20712A (CGP) ICI118,551 (ICI) or propranolol (PROP), or the AR antagonist prazosin (PZ). Media with drugs were replaced every 2 days. (A) The data shown represent the cell number on day 6 (mean $\pm S . E . M . ; n=6$ ). ${ }^{*}$; values signiticantly different from untreated control $(p<0.001)$. All other groups were not different from the unstimulated control. (B) Cells were treated with NE $\pm \beta$ antagonists for 30 minutes. RNA was collected and analyzed by Northern blotting. RNA levels of cyclophilin served as a control for RNA/lane. *, $p<0.001$; \#, p < $0.05)$. 
the early stages of adipocyte differentiation, with C/EBP $\beta$ and C/EBP $\delta$ preceding the appearance of C/EBP $\alpha$ (Cao et al., 1991; Yeh et al., 1995). More recent studies indicate that the expression of PPAR $\gamma$ is partially under the control of the C/EBP family of transcription factors and vice versa (reviewed in Wu et al., 1999). By examining a series of adipogenic cell lines expressing various combinations of $\mathrm{PPAR} \gamma$ and $\mathrm{C} / \mathrm{EBPs}$, we find that $\mathrm{C} / \mathrm{EBP} \alpha$ is required for the adipocyte-dependent expression of the mouse $\beta_{3}$ AR gene (Figure 5). We have defined the C/EBP-binding site responsible for this regulation in the $\beta_{3} \mathrm{AR}$ promoter (Dixon et al., 2000).

\section{B. COUPLING OF $\beta_{3}$ AR IN ADIPOCYTES TO MULTIPLE SIGNALING PATHWAYS}

Long before the discovery of $\beta_{3} \mathrm{AR}$ and its recognition as a unique, adipocyte-specific receptor controlling lipolysis and thermogenesis, the Rodbell group made the observation that there was an unusual, biphasic stimulation of cyclic AMP (cAMP) production in adipocytes in response to the $\beta A R$ agonist isoproterenol (Cooper et al., 1979). Depending upon the concentration of guanosine triphosphate (GTP) in the assay, isoproterenol could either stimulate or inhibit adenylyl cyclase activity in adipocyte plasma membranes. Murayama and Ui (1983) showed that this inhibitory phase could be relieved by pretreating adipocytes with pertussis toxin (PTX). With the cloning of the $\beta_{3} \mathrm{AR}$ gene and the development of highly selective $\beta_{3} \mathrm{AR}$ agonists (Arch et al., 1984; Bloom et al.,

\section{Adenylyl Cyclase}

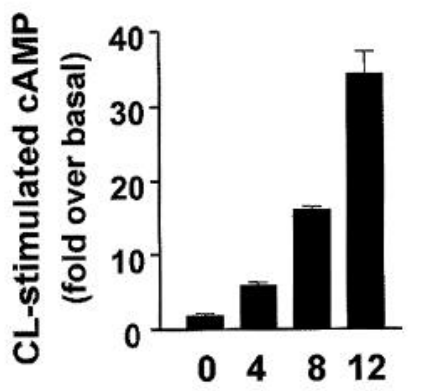

Days

\section{Northern Blot}

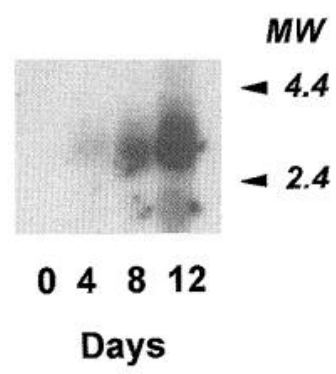

FIG. 4. Differentiation-dependent expression and activity of $\beta_{3} \mathrm{AR}$ in 3T3-F442A adipocytes. As adipogenic cell lines such as 3T3-F442A differentiate, the expression and functional activity of $\beta_{3} \mathrm{AR}$ appear. (Left panel) Cyclic AMP production in cells differentiated for the indicated periods. (Right panel) Northern blot analysis of $\beta_{3} \mathrm{AR}$ mRNA levels. 


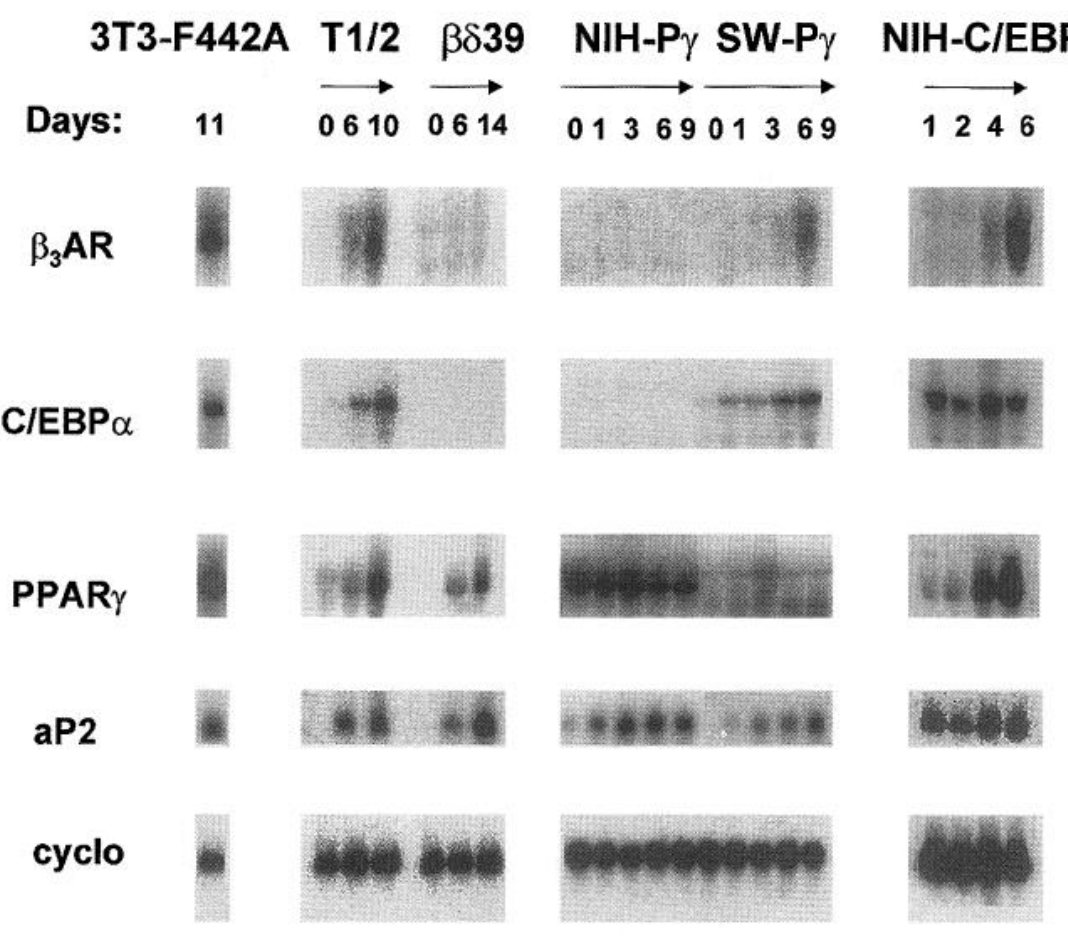

FIG. 5. Expression of $\beta_{3} A R$ during adipocyte differentiation is dependent on $C / E B P \alpha$. Cell lines indicated were differentiated and expression of $\operatorname{PPAR} \gamma, \mathrm{C} / \mathrm{EBP} \alpha$, the fatty acid binding protein aP2, and $\beta_{3}$ AR were examined by Northern blotting and adenylyl cyclase activity (not shown). Cyclophilin (cyclo) mRNA levels were monitored as a control for the amount of RNA applied to the gel.

1992), it was postulated that this novel, adipocyte-specific $\beta A R$ may be responsible for the biphasic adenylyl cyclase response in adipocytes (Begin-Heick, 1995). We previously noted that, despite the relatively high level of expression of $\beta_{3} \mathrm{AR}$ in adipocytes, the efficiency of coupling of $\beta_{3} \mathrm{AR}$ to stimulation of adenylyl cyclase is rather low (Collins et al., 1994). However, until recently, there had been no clear biochemical demonstration of physical coupling of $\beta_{3} \mathrm{AR}$ to $\mathrm{Gi}$, other than comparative functional experiments in the presence or absence of PTX. Nor had there been any indication of what additional second messenger pathway may be activated as a consequence of this putative coupling of $\beta_{3} \mathrm{AR}$ to Gi. We recently reported (Soeder et al., 1999) that $\beta_{3} A R$ is simultaneously coupled to $\mathrm{Gs}$ and $\mathrm{Gi}$, with the consequent activation of the PKA and mitogen-activated protein (MAP) kinase pathways, respectively (Figure 6). Physical coupling of $\beta_{3} \mathrm{AR}$ to $\mathrm{Gs}$ and $\mathrm{Gi}$ was demonstrated in cultured adipocytes using a photolabeling technique that relies upon the ability of agonist-activated receptor to trigger 
$\mathrm{G} \alpha$-subunit dissociation and binding of GTP. Thus, these data showed the restraining effect of Gi on cAMP production and the dependence of MAP kinase signaling on Gi activation by $\beta_{3} \mathrm{AR}$ (Soeder et al., 1999). More recent work in our laboratory shows that novel sequence elements within the $\beta_{3} \mathrm{AR}$ itself are responsible for the direct recruitment of $\mathrm{SH} 3$ domain-containing signaling molecules such as c-Src. This interaction is required to trigger the extracellular signalregulated kinase (ERK) cascade (Collins et al., 2000; Cao et al., 2000) (Figure 7). Further complicating this situation is the possibility that other members of the MAPK cascade may be involved.
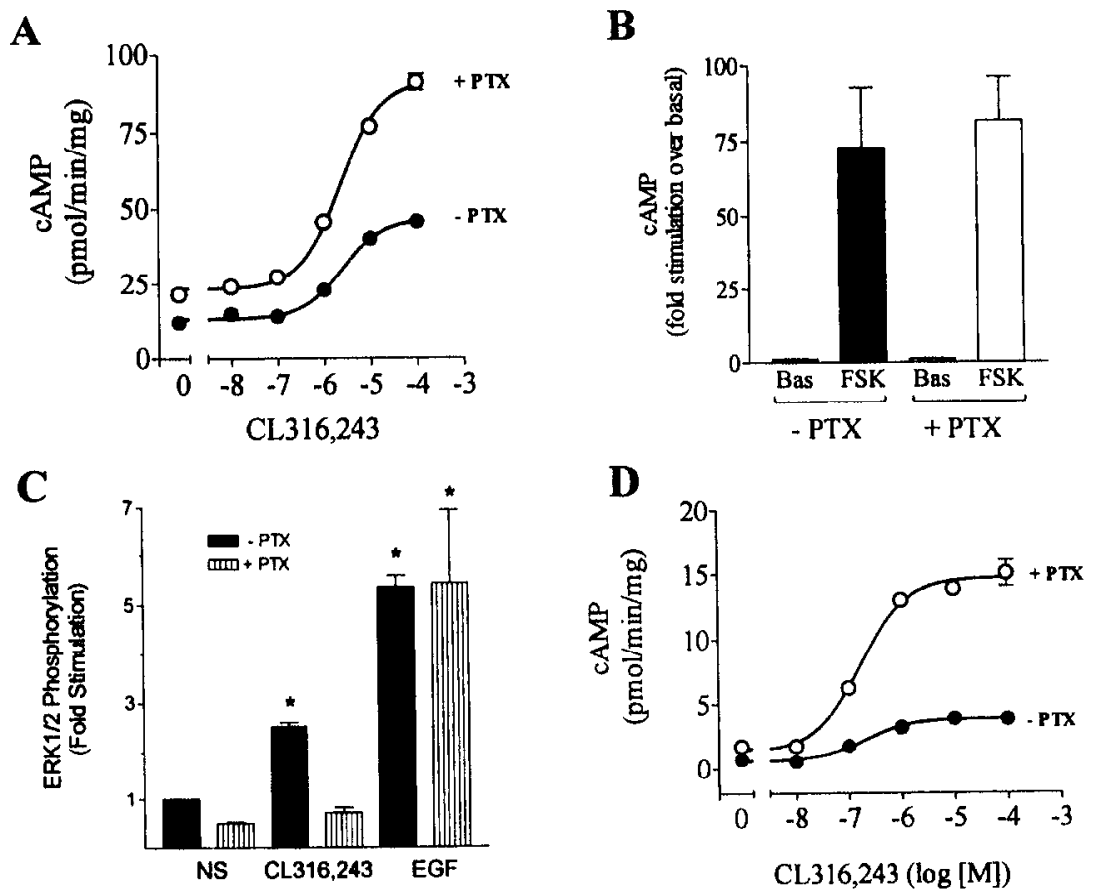

FIG. 6. $\beta_{3} \mathrm{AR}$ in adipocytes is coupled to $\mathrm{Gs}$ and $\mathrm{Gi}$, for stimulation of cAMP and MAP kinase. (A) Dose-response curve for agonist stimulation of cAMP production in differentiated 3T3-F442A adipocytes in response to CL316,243. Cells were pretreated or not with PTX, as indicated. (B) Basal and forskolin-stimulated cAMP production in membranes from untreated and PTX-pretreated cells. (C) Quantitation of ERK1/2 phosphorylation in response to CL316,243 $(5 \mu \mathrm{M})$ and EGF $(10 \mathrm{ng} / \mathrm{ml})$ after pretreatment with PTX, as indicated. *, one-way ANOVA, $p<0.001$. (D) Dose-response curve for agonist stimulation of cAMP production in CHO cells stably transfected with mouse $\beta_{3} \mathrm{AR}$ in response to CL316,243. Cells were pretreated or not with PTX, as indicated. [Adapted from Soeder, K.S., Snedden, K.S., et al., J. Biol. Chem. 274,12017-12022, 1999. Copyright 1999 The American Society for Biochemistry \& Molecular Biology.] 
This realization that $\beta_{3} \mathrm{AR}$ in adipocytes is coupled to multiple signaling pathways has potentially important implications for understanding the unique physiologic properties of selective agonists for this receptor. For example, the discoveries that hormone-sensitive lipase (HSL), a classic target of $\beta$ AR-mediated PKA activation, is phosphorylated by (an)other kinase (Anthonsen et al., 1998) and that HSL is probably not a single isozyme (Saltiel, 2000) require that we re-examine our thinking about how this regulation is achieved. In addition, the lipogenic transcription factor PPAR $\gamma$ can be phosphorylated by ERK1/2 on Ser 112. This modification serves to blunt its transcriptional activity (Hu et al., 1996; Adams et al., 1997). Alternatively, expression of the transcriptional coactivator PGC-1, which has been shown in model systems to stimulate mitochondriogenesis (Wu et al., 1999), is stimulated by cAMP (Puigserver et al., 1998). Thus, the possibility needs to be explored that the combined activation of the PKA and

A. Northern blot

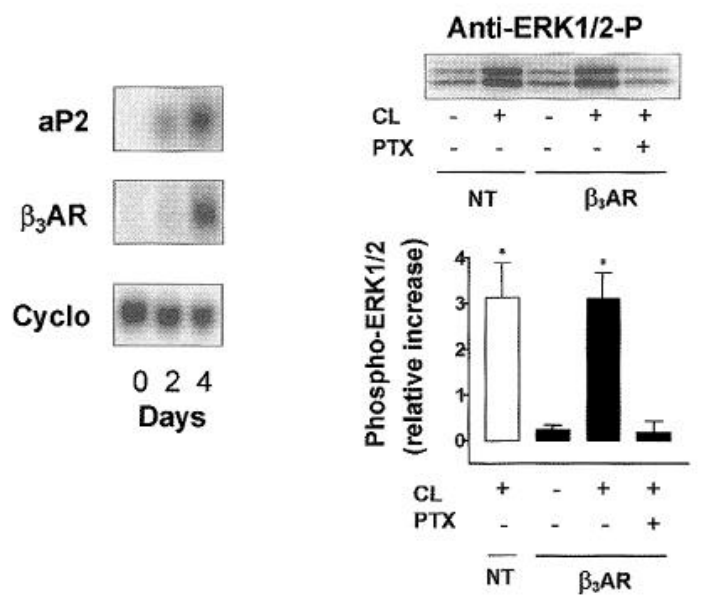

C. IP
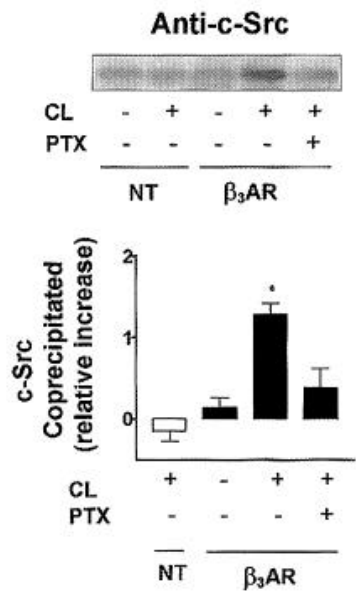

FIG. 7. $\beta_{3} \mathrm{AR}$ recruits c-Src in a Gi protein- and agonist-dependent manner in differentiated C3H10T1/2 adipocytes. C3H10T1/2 cells were transfected with HA- $\beta_{3} \mathrm{AR}$ and differentiated as described in "Experimental Procedures." Immunoprecipitation assays (IP) were performed with anti-HA antibody. (A) Expression of aP2 and $\beta_{3} \mathrm{AR}$ mRNA as a function of differentiation on the indicated days. Levels of aP2 are maximal by day 4. Cyclophilin RNA (cyclo) is a control in Northern blotting. (B) The levels of phosphorylated ERK1/2 (ERK1/2-P) in cell lysates and quantification of three independent experiments (mean $\pm S D$ ). (C) The level of c-Src co-precipitated with $\beta_{3} A R$ and quantification of three independent experiments (mean $\pm \mathrm{SD}$ ). [Reprinted with permission from Cao, W., Luttrell, L.M., Medvedev, A.V., Pierce, K.L., Daniel, K.W., Dixon, T.M., Lefkowitz, R.J., and Collins, S. J. Biol. Chem. 275, 38131-38134, 2000. Copyright 2000 The American Society for Biochemistry \& Molecular Biology.] 
MAPK pathways could underlie the $\beta_{3} A R$ agonist-dependent appearance of thermogenically active brown adipocytes and a change in energy balance away from lipid storage, although this is admittedly just speculation at this point.

\section{Regulation of Body Composition by Adipose Tissue $\beta$-Adrenergic Receptors}

\section{A. IMPAIRED $\triangle$ DIPOSE TISSUE $\triangle$ DRENERGIC SIGNALING IN OBESITY}

It was known for many years that obese C57BL/6J Lep $p^{o b}$ and C57KsJ Lep $R^{d b}$ mice exhibited a marked inability to effectively mobilize triglycerides from WAT and were unable to recruit BAT for thermogenesis in response to cold temperature. These deficiencies indicate that adrenergic mechanisms regulating metabolism in both white and brown fat are affected (discussed in Collins et al., 1994). While defects in SNS outflow have been shown to be associated with obesity in these models (Levin et al., 1983; Takeuchi et al., 1994), other experiments clearly indicated that there was impaired $\beta A R$ function at the level of the adipocyte itself, independent of the availability of catecholamines (Shepherd et al., 1977).

In the late 1970 s and early 1980 s, many investigators tried to determine the nature of the molecular defect in adipocytes from these obese animals. The components of the adrenergic signal transduction pathway (at least those which were known at the time; which did not include $\beta_{3} \mathrm{AR}$ ) were examined. Despite a severe blunting of $\beta$-adrenergic stimulation of cAMP production and lipolysis, the activity of adenylyl cyclase itself and other downstream effectors of the lipolytic process did not differ between lean and obese animals (Bégin-Heick and Heick, 1977; Shepherd et al., 1977). The discovery of $\beta_{3}$ AR as a adipocyte-specific $\beta$ AR subtype forced a re-evaluation of years of work on adipocyte adrenoceptors. Therefore, we examined the expression and functional activity of all three $\beta A R s$ in adipocytes from lean and genetically obese (C57BL/6J Lep $\left.{ }^{o b}\right)$ mice. Figure 8 shows the impaired ability of $\beta$ agonists to stimulate adenylyl cyclase activity in WAT from the $L e p^{o b}$ and the dramatic decrease in expression of $\beta_{3} A R$ as well as a 80 percent loss of $\beta_{1} A R$ in these obese mice, compared to lean littermates (Collins et al., 1994). In these studies, a series of detailed pharmacologic analyses allowed us determine the relative contributions of the three subtypes to the response and to conclude that these reductions in expression of specific $\beta A R$ subtypes are fully responsible for the defects in catecholamine-stimulated lipolysis and thermogenesis observed in the C57BL/6J Lep ${ }^{a b}$ mouse. Similar findings of depressed $\beta_{3}$ AR mRNA levels in the Zucker fatty (fa/fa) rat were reported by Muzzin et al. (1991) but their relationship to changes in the function of the receptor and tissue responses were not examined in that study. We extended these 

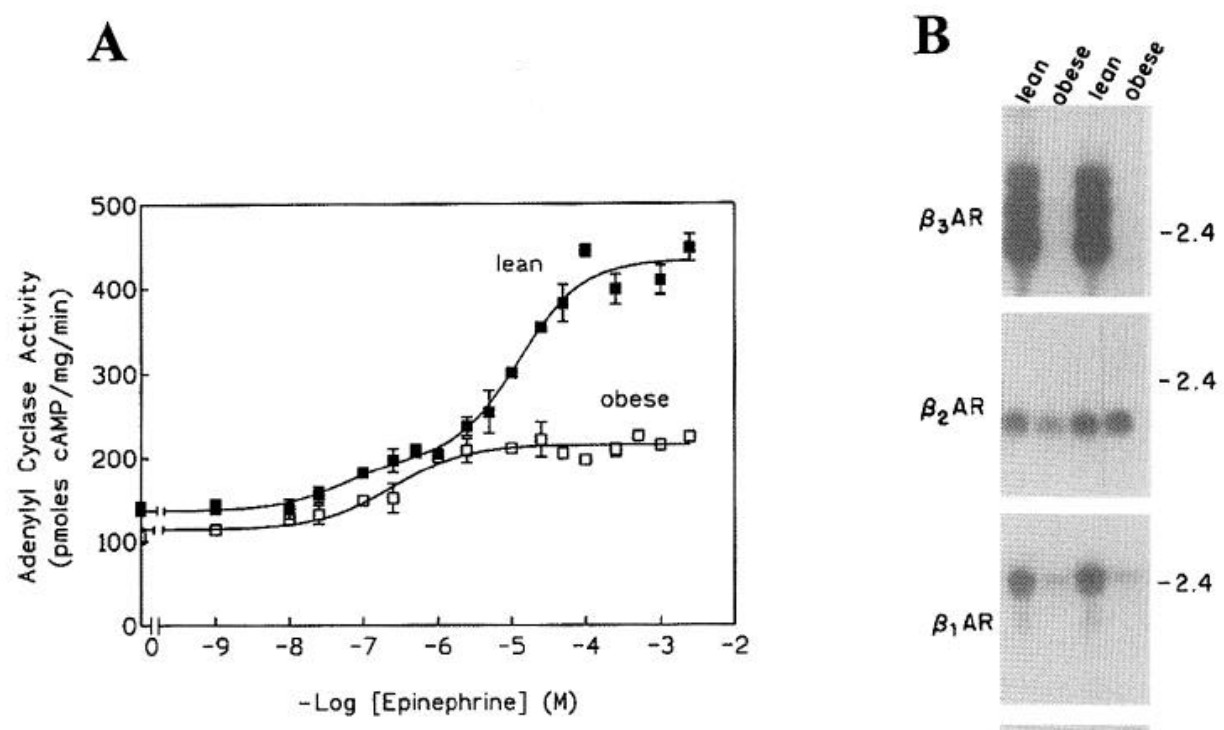

G3PDH $-1.4$

FIG. 8. $\beta$-agonist-stimulated adenylyl cyclase and expression of $\beta$ AR subtypes in WAT of C57BL/6J ("lean") and Lep ${ }^{o b}$ ("obese") mice. (Left panel) Dose-response curves of stimulation of cAMP production by epinephrine in WAT plasma membranes. (Right panel) Northern blot of $\beta_{1^{-}}, \beta_{2^{-}}$, and $\beta_{3} \mathrm{AR}$ mRNA levels in WAT. [Reprinted with permission from Collins, S., Daniel, K.W., et al., Mol. Endocrinol. 8, 518-527, 1994. Copyright The Endocrine Society.]

original findings in the $L e p^{o b}$ mouse to several other models of obesity. Significant deficits in the expression and function of adipocyte $\beta$ ARs were found in essentially every model of obesity that we have examined, including obesity induced by high-fat feeding in nonmutant mice (Collins et al., 1999) and in the slowly developed obesity associated with aging (Gettys et al., 1995).

The molecular basis for this dramatic impairment in $\beta A R$ expression and function may be related to the abnormal endocrine profiles observed in these models of obesity (reviewed in Bray et al., 1990). For example, in C57BL/6J $L e p^{o b}$ and C57BL/6J LepR $R^{d b}$ mice, hypercorticism is an important component in the development of obesity (Bray and York, 1979). However, manipulation of this system in the face of leptin deficiency is unable to fully reverse this phenotype. There is also significant adipose deposition in humans with Cushing's syndrome, particularly in intra-abdominal regions (Wilson et al., 1998). Despite this evidence for a role of the hypothalamic-pituitary-adrenal (HPA) axis in certain situations, most obese humans, as well as other mouse obesity mutants such as tubby and 
Cpe fat (Naggert et al., 1995; Noben-Trauth et al., 1996) and diet-induced obese mice (Surwit et al., 1988), have completely normal corticosteroid levels, yet clearly show reduced expression and function of adipocyte $\beta_{3}$ AR and $\beta_{1} A R$ (Collins et al., 1997,1999).

\section{B. OBESITY AND INSULIN RESISTANCE}

The single, most-common variable among all animal models of obesity, as well as a large percentage of obese humans, is the coexistence of hyperinsulinemia and insulin resistance. Due to this strong association between compromised glycemic control, obesity, and $\beta A R$ expression and function, we have proposed that this contributes, either directly or indirectly, to the inhibition of adipocyte $\beta \Lambda R$ expression and function (Collins et al., 1999). The result is impaired catecholamine-stimulated lipolysis, which exaggerates the excessive lipid storage in the adipocyte and consequent obesity. Evidence in support of this hypothesis includes the observation that $\beta_{3} A R$ expression rapidly declined in differentiated 3T3F442A mouse adipocytes treated with insulin (Fève et al., 1994). In addition, a role for insulin in affecting $\beta A R$ function in adipocytes is suggested by a series of studies showing that suppressing hyperinsulinemia with the $\mathrm{K}_{\text {ATP }}$ channel agonist, diazoxide, results in improved ability to stimulate lipolysis and a significant loss of adipose tissue mass (Alemzadeh et al., 1996,1998). The mechanism by which insulin may negatively impact $\beta A R$ function is not known but may be related to an insulin-dependent decrease in expression or function of $\mathrm{C} / \mathrm{EBP} \alpha$ (MacDougald et al., 1995), which we now know is critically required for proper expression of $\beta_{3} \mathrm{AR}$ in adipocytes (Dixon et al., 2000).

We provided additional support for the hypothesis that hyperinsulinemia directly affects adipocyte $\beta A R$ function in a recent study in which we used diazoxide to suppress diet-induced hyperinsulinemia in B6 mice. We observed a restoration of both $\beta_{3} \mathrm{AR}$ expression and function. Animals treated with diazoxide exhibit lower plasma insulin levels and increased cAMP production in adipocytes in response to a selective $\beta_{3} \mathrm{AR}$ agonist (Surwit et al., 2000). As a consequence of the restoration of $\beta_{3} A R$ expression by diazoxide, increased cAMP production was accompanied by significant loss of WAT mass. In combination with $\beta_{3} A R-$ agonist stimulation, there was a significant increase in UCP1 in brown fat. Because diazoxide could have some direct effects on $\mathrm{Ca}^{+2}$ influx through the adipocyte sulfonyl urea receptor 1 and $\mathrm{K}_{\mathrm{ATP}}$ to increase lipolysis (Shi et al., 1999), it is not clear to what extent the effects of diazoxide are due to decreased circulating insulin. In spite of this, the clinical efficacy is encouraging for future studies to explore the mechanisms responsible for these effects of diazoxide, with and without a selective $\beta_{3} A R$ agonist. 


\section{DISCOVERY OF UCP1 HOMOLOGS AND LINKAGE WITH METABOLIC FUEL HOMEOSTASIS}

Strong evidence of a proton "leak" in mitochondria from non-BAT tissues such as liver (Porter and Brand, 1993), coupled with sporadic observations that the brown fat UCP could be detected in other tissues such as muscle (Yoshida et al., 1994) (primarily when using antisera to measure protein levels), led Ricquier and colleagues to search for UCP homologues. In 1997, the isolation of UCP2, a novel gene with significant homology (59 percent) to the brown fat UCP (now named UCP1) was reported (Fleury et al., 1997). In addition to its ability to uncouple respiration as efficiently as UCP1 in model systems, UCP2 was broadly expressed in many tissues. These features of UCP2 led to the proposition that it was the long-sought explanation for the relative inefficiency of oxidative respiration seen in most cell types. In those initial studies, we noted that the UCP2 gene resides in a location on mouse chromosome 7 that is coincident with a quantitative trait locus (QTL) linked to hyperinsulinemia and high plasma leptin levels (reflective of body fat stores). In addition, we showed that consumption of a high-fat diet increased expression of UCP2 specifically in WAT in strains of mice that are relatively resistant to the development of diet-induced obesity and diabetes but not in obesity-prone strains of mice (Fleury et al., 1997; Surwit et al., 1998).This upregulation of UCP2 gene expression was postulated to be due to increased flux of fatty acids and a PPAR-dependent increase in UCP2 expression (Aubert et al., 1997; Camirand et al., 1998; Viguerie-Bascands et al., 1999). Subsequent discovcry of another UCP homologue, UCP3, which is expressed predominantly in skeletal muscle and brown fat, was reported by several groups (Boss et al., 1997; Vidal-Puig et al., 1997). The structural homology between these UCPs and basic features about their regulation and expression in various rodent models and human populations have been recently reviewed (Boss et al., 1998; Ricquier et al., 1999). Because the UCP3 gene is also located $8-10 \mathrm{~kb} \mathrm{5}$ to the UCP2 gene in both the mouse and human genomes (Solanes et al., 1997; Surwit et al., 1998), this close linkage relationship means that either or both (or neither) of these UCPs could be related to this QTL. However, we could find no evidence for changes, at least in expression of UCP3, in the mouse models that originally defined this QTL (Surwit et al., 1998). Confounding the concept that these novel UCPs may be involved in dissipation of excess caloric energy, it was reported that fasting led to increases, rather than decreases, in expression of UCP2 and UCP3. Dulloo and colleagues showed that blockade of the fasting-induced rise in free fatty acids completely prevented the increase in UCP2 and UCP3 mRNA (Samec et al., 1998,1999; Cadenas et al., 1999). Thus, in the aggregate, these novel UCPs would appear to be involved in the metabolic adaptations required during the fasted state, which requires a switch in fuel source from predominantly glucose to predominantly fatty acids. More importantly, since neither UCP2 nor UCP3 are expressed in 
hepatocytes under normal conditions, neither of these UCPs would appear to explain the well-documented proton leak in these cells (Porter and Brand, 1993).

Recently, several groups, including our own, have generated mice with targeted disruption of the UCP2 gene (Arsenijevic et al., 2000; Zhang et al., submitted) or the UCP3 gene (Gong et al., 2000; Vidal-Puig et al., 2000). At this point, there is no evidence of significant effects on either body temperature or body weight in either UCP2- or UCP3-deficient mice, even when challenged with a hyperlipidemic diet. However, since strain background can significantly affect the propensity to develop obesity and diabetes, the consequences of UCP2 or UCP3 deficiency may be revealed only under certain genetic or environmental situations. Nevertheless, novel phenotypes that are emerging in UCP2 -/- mice (Arsenijevic et al., 2000; unpublished observations) should help to shed light on the physiologic role of these mitochondrial proteins. Indeed, if we consider the fact that the mechanism of uncoupling by UCP1 is still debated many years after its discovery, perhaps we should not be surprised that efforts to determine the function of UCP2 and UCP3 at the physiologic and molecular level are unresolved.

\section{Summary}

During the past decade, the discovery of a host of new molecules involved in adipocyte metabolism (Friedman and Halaas, 1998), thermogenesis (Lowell and Spiegelman, 2000), and hypothalamic peptides and receptors (Schwartz et al., 2000 ) have led to new concepts and paradigms about the control of fuel intake and metabolism. While our understanding of the importance of catecholamines in controlling lipolysis and thermogenesis in the adipocyte is established, it is clear that we still have much work to do in order to understand the interplay between $\beta A R$ subtypes, their signaling pathways, and their regulation. Since pharmacologic treatments for obesity that focus on depressing food intake will likely never overcome the inevitable drop in energy expenditure that accompanies caloric restriction (Leibel et al., 1995), future discoveries in $\beta A R$ signaling mechanism and their targets in the adipocyte may provide not only insight into the "expenditure" side of the energy balance equation but also another therapeutic angle to exploit in the treatment of the growing obesity problem.

\section{ACKNOWLEDGMENTS}

This work was supported in part by National Institutes of Health grants R01DK53092 and R01DK54024 and an unrestricted gift from Novo Nordisk. We thank our collaborators and the members of our laboratories, which together have made this work possible. We also thank Claire Pecqueur for the original design of Figure 1. 


\section{REFERENCES}

Adams, M., Reginato, M., et al. (1997). J. Biol. Chem. 272(8), 5128-5132.

Alemzadeh, R., Jacobs, W., et al. (1996). Metabolism 45, 334-341.

Alemzadeh, R., Langley, G., et al. (1998). J. Clin. Endocrinol. Metab. 83, 1911-1915.

Anthonsen, M., Ronnstrand, L., et al. (1998). J. Biol. Chem. 273(1), 215-221.

Arch, J.R.S., Ainsworth, A.T., et al. (1984). Nature 309, 163-165.

Arsenijevic, D., Onuma, H., Pecqueur, C., Raimbault, S., Manning, B.S., Miroux, B., Couplan, E., Goubern, M., Alves-Guerra, M.-C., Surwit, R., Bouillard, F., Richard, D., Collins, S., and Ricquier, D. (2000). Nature Genet. 26, 435-439.

Aubert, J., Champigny, O., et al. (1997). Biochem. Biophys. Res. Comm. 238, 606-611.

Bahouth, S.W., and Malbon, C.C. (1988). Mol. Pharmacol. 34, 318-326.

Ball, E., and Jungas, R. (1961). Proc. Natl. Acad. Sci. U.S.A. 47, 932.

Begin-Heick, N. (1995). J. Cell. Biochem. 58, 464-473.

Bégin-Heick, N., and Heick, H.M.C. (1977). Can. J. Physiol. Pharmacol. 55, 1320-1329.

Bloom, J.D., Dutia, M.D., et al. (1992). J. Med. Chem. 35, 3081-3084.

Boss, O., Samec, S., et al. (1997). FEBS Lett. 408, 39-42.

Buss, O., Muzzin, P., et al. (1998). Eur. J. Endocrinol. 139, 1-9.

Bouillaud, F. (1999). Intl. J. Obes. 23(suppl. 6), S19-S23.

Bouillaud, F., Ricquier, D., et al. (1984). J. Biol. Chem. 259, 11583-11586.

Bray, G.A., and York, D.A. (1979). Physiol. Rev. 59, 719-809.

Bray, G.A., Fisler, J., et al. (1990). Front. Neuroendocrinol. 11, 128-181.

Bukowiecki, L., Collet, A., et al. (1982). Am. J. Physiol. 242, E353-E359.

Cadenas, S., Buckingham, J.A., et al. (1999). FEBS Lett. 462, 257-260.

Camirand, A., Marie, V., et al. (1998). Endocrinology 139, 428-431.

Campfield, L.A., Smith, F.J., et al. (1995). Science 269, 546-549.

Candelore, M.R., Deng, L., et al. (1999). J. Pharmacol. Exp. Ther. 290, 649-655.

Cannon, B., Hedin, A., et al. (1982). FEBS Lett. 150, 129-132.

Cao, Z., Umek, R.M., et al. (1991). Genes Devel. 5, 1538-1552.

Cao, W., Luttrell, L.M., Medvedev, A.V., Pierce, K.L., Daniel, K.W., Dixon, T.M., Lefkowitz, R.J., and Collins, S. (2000). J. Biol. Chem. 275, 38131-38134.

Champigny, O., Ricquier, D., et al. (1991). Proc. Natl. Acad. Sci. U.S.A. 88, 10774-10777.

Cinti, S. (1999). "The Adipose Organ." Editrice Kurtis, Milan, Italy.

Collins, S., and Surwit, R.S. (1996). J. Biol. Chem. 271, 9437-9440.

Collins, S., Daniel, K.W., et al. (1994). Mol. Endocrinol. 8, 518-527.

Collins, S., Kuhn, C.M., et al. (1996). Nature 380, 677.

Collins, S., Daniel, K.W., et al. (1997). Endocrinology 138, 405-413.

Collins, S., Daniel, K.W., et al. (1999). Intl. J. Obes. 23, 669-677.

Collins, S., Cao, W., et al. (2000). In "Adipocyte Biology and Hormone Signaling" (J.M. Ntambi, ed.), pp. 51-62. IOS Press, Washington, D.C.

Cooper, D., Schlegel, W., et al. (1979). J. Biol. Chem. 254, 8927-8931.

Darlington, G.J., Ross, S.E., et al. (1998). J. Biol. Chem. 273, 30057-30060.

Dixon, T.M., Daniel, K.W., Farmer, S.R., and Collins, S. (2000). J. Biol. Chem., in press.

Emorine, L.J., Marullo, S., et al. (1989). Science 245, 1118-1121.

Enerback, S., Jacobsson, A., et al. (1997). Nature 387, 90-94.

Fain, J., and Garcia-Sainz, J. (1983). J. Lipid Res. 24, 945-966.

Fève, B., Elhadri, K., et al. (1994). Proc. Nall. Acad. Sci. U.S.A. 91, 5677-5681.

Fisher, M.H., Amend, A.M., et al. (1998). J. Clin. Invest. 101, 2387-2393.

Fleury, C., Neverova, M., et al. (1997). Nature Genet. 15, 269-272.

Friedman, J.M., and Halaas, J.L. (1998). Nature 395, 763-770. 
Galitzky, J., Reverte, M., et al. (1993). Am. J. Physiol. 264, E403-E412.

Garlid, K.D., Jaburek, M., et al. (1998). FEBS Lett. 438, 10-14.

Géloèn, A., Collct, A.J., et al. (1992). Am. J. Physiol. 263, R1176-R1181.

Gettys, T.W., Rohlfs, E.M., et al. (1995). Endocrinology 136, 2022-2032.

Gong, D.W., Monemdjou, S., et al. (2000). J. Biol. Chem. 275, 16251-16257.

Granneman, J.G., Lahners, K.N., et al. (1991). Mol. Pharmacol. 40, 895-899.

Guerra, C., Koza, R.A., et al. (1998). J. Clin. Invest. 102, 412-420.

Halaas, J.L., Gajiwala, K.S., et al. (1995). Science 269, 543-546.

Himms-Hagen, J., Cui, J., et al. (1994). Am. J. Physiol. 266, R1371-R1382.

Hu, E., Kim, J.B., et al. (1996). Science 274, 2100-2103.

Irie, Y., Asano, A., et al. (1999). Biochem. Biophys. Res. Comm. 255, 221-225.

Klaus, S., Choy, L., et al. (1994). J. Cell Sci. 107, 313-319.

Klingenberg, M., Echtay, K.S., et al. (1999). Intl. J. Obes. 23(suppl. 6), S24-S29.

Kopecky, J., Hodny, Z., et al. (1996a). Am. J. Physiol. 270, E768-E775.

Kopecky, J., Rossmeisl, M., et al. (1996b). Am. J. Physiol. 270, E776-E786.

Kozak, U.C., Held, W., et al. (1992). Mol. Endocrinol. 6, 763-772.

Kozak, U.C., Kopecky, J., et al. (1994). Mol. Cell. Biol. 14, 59-67.

Krief, S., Lonnqvist, F., et al. (1993). J. Clin. Invest. 91, 344-349.

Landsberg, L., and Young, J.B. (1984). J. Clin. Endocrinol. Metab. 13, 475-499.

Largis, E.E., Burns, M.G., et al (1994). Drug Devel. Res. 32, 69-76.

Larose, M., Cassard-Doulcier, A.-M., et al. (1996). J. Biol. Chem. 271, 31533-31542.

Lefkowitz, R.J. (1998). J. Biol. Chem. 273, 18677-18680.

Leibel, R.L., Rosenbaum, M., et al. (1995). N. Engl. J. Med. 332, 621-628.

Levin, B.E., Triscari, J., et al. (1983). Am. J. Physiol. 244, R347-R371.

Liggett, S., Freedman, N.J., et al. (1993). Proc. Natl. Acad. Sci. U.S.A. 90, 3665-3669.

Lindberg, O. (1970). "Brown Adipose Tissue." American Elsevier Publishing Co., New York.

Lowell, B.B., and Spiegelman, B.M. (2000). Nature 404, 652-660.

Lowell, B.B., Susulic, V., et al. (1993). Nature 366, 740-742.

MacDougald, O.A., Cornelius, P., et al. (1995). J. Biol. Chem. 270, 647-654.

Murayama, T., and Ui, M. (1983). J. Biol. Chem. 258, 3319-3326.

Muzzin, P., Revelli, J.P., et al. (1991). J. Biol. Chem. 266, 24053-24058

Naggert, J.K., Fricker, L.D., et al. (1995). Nature Genet. 10, 135-142.

Nahmias, C., Blin, N, et al. (1991). EMBO J. 10,3721-3727.

Noben-Trauth, K., Naggert, J.K., et al. (1996). Nature 380, 534-538.

Pelleymounter, M.A., Cullen, M.J., et al. (1995). Science 269, 540-543.

Porter, R., and Brand, M. (1993). Nature 362, 628-630.

Puigserver, P., Wu, Z., et al. (1998). Cell 92, 829-839.

Ravussin, E., Burnand, B., et al. (1982). Am. J. Clin. Nutrit. 35, 566-573.

Ricquier, D., Fleury, C., et al. (1999). J. Intern. Med. 245, 637-642.

Rising, R., Keys, A., et al. (1992). Am. J. Physiol. 263, E730-E734.

Rohlfs, E.M., Daniel, K.W., et al. (1995). J. Biol. Chem. 270, 10723-10732.

Saltiel, A.R. (2000). Proc. Natl. Acad. Sci. U.S.A. 97, 535-537.

Samec, S., Seydoux, J., et al. (1998). FASEB J. 12, 715-724.

Samec, S., Seydoux, J., et al. (1999). Pfluger's Arch. 438, 452-457.

Sasaki, N., Uchida, E., et al. (1998). J. Vet. Med. Sci. 60, 465-469.

Schwartz, M., Woods, S., et al. (2000). Nature 404, 661-671.

Shepherd, R.E., Malbon, C.C., et al. (1977). J. Biol. Chem. 252, 7243-7248.

Shi, H., Moustaid-Moussa, N., et al. (1999). FASEB J. 13, 1833-1838.

Shih, T.L., Candelore, M.R., et al. (1999). Bioorg. Med. Chem. Lett. 9, 1251-1254.

Sims, E.A.H., and Danforth, E., Jr. (1987). J. Clin. Invest. 79, 1019-1025. 
Smith, R. (1961). Physiologist 4, 113.

Soeder, K.S., Snedden, S.K., et al. (1999). J. Biol. Chem. 274, 12017-12022.

Solanes, G., Vidal-Puig, A., et al (1997). J. Biol Chem. 272, 25433-25436.

Spiegelman, B. (1998). Diabetes 47, 507-514.

Spraul, M., Ravussin, E., et al. (1993). J. Clin. Invest. 92, 1730-1735.

Strosberg, A. (1997). Ann. Rev. Pharmacol. Toxicol. 37, 421-450.

Surwit, R.S., Kuhn, C.M., et al. (1988). Diabetes 37(9), 1163-1167.

Surwit, R., Petro, A., et al. (1997). Diabetes 46, 1516-1520.

Surwit, R.S., Wang, S., et al. (1998). Proc. Natl. Acad. Sci. U.S.A. 95, 4061-4065.

Surwit, R.S., Dixon, T.M., Petro, A., Daniel, K.W., and Collins, S. (2000). Endocrinology 141, 3630-3637.

Takeuchi, H., Matsuo, T., et al. (1994). J. Nutrit. 125, 920-925.

Tate, K.M., Briend-Sutren, M.-M., et al. (1991). Eur. J. Biochem. 196, 357-361.

van Liefde, I., van Witzenberg, A., et al. (1992). J. Pharmacol. Exp. Ther. 262, 552-558.

Vidal-Puig, A. (2000). Nature Genet. 26, 387-388.

Vidal-Puig, A., Solanes, G., et al. (1997). Biochem. Biophys. Res. Comm. 235, 79-82.

Vidal-Puig, A.J., Grujic, D., et al. (2000). J. Biol. Chem. 275, 16258-16266.

Viguerie-Bascands, N., Saulnier-Blache, J.S., et al. (1999). Biochem. Biophys. Res. Comm. 256, 138-141.

Weyer, C., Tataranni, P.A., et al. (1998). Diabetes 47, 1555-1561.

Wilson, J.D., Foster, D.W., et al. (eds.) (1998). "Williams Textbook of Endocrinology," edit 9. W.B. Saunders Company, Philadelphia.

Wu, Z., Puigserver, P., et al. (1999). Cell 98, 115-124.

Yeh, W.C., Cao, Z., et al. (1995). Genes Devel. 9, 168-181.

Yoshida, T., Sakane, N., et al. (1994). Life Sci. 54, 491-498.

Zhang, Y., Proenca, R., et al. (1994). Nature 372, 425-432. 


\title{
The Adipocyte as a Secretory Organ: Mechanisms of Vesicle Transport and Secretory Pathways
}

\author{
Richard L. Bradley, Kelly A. Cleveland, and Bentley Cheatham \\ Research Division, Joslin Diabetes Center and the Department of Medicine, \\ Harvard Medical School, Boston, Massachusetts 02215
}

\begin{abstract}
Obesity is a common problem in western society that is directly linked to several disease processes and is associated with significant morbidity and mortality. Adipocytes - the primary site for energy storage (as triglycerides) and release - were long suspected to have an active role in regulating body weight homeostasis and energy balance. As a result, many studies have focused on finding abnormalities in adipocyte physiology and metabolism. An cver-increasing body of cvidence indicates that, in addition to serving as a repository for energy reserves, adipocytes secrete a myriad of factors that comprise a complex network of endocrine, autocrine, and paracrine signals. Very little is known regarding the molecular mechanisms utilized by the adipocyte in regulating the biosynthesis and exocytosis of these secreted products. In order to gain a better understanding of these processes, we have examined the two classical secretory pathways: regulated and constitutive. Using leptin as a model adipocyte-secretory protein, this review focuses primarily on the latter pathway. This includes regulation of leptin synthesis and secretion by insulin and glucocorticoids and, more recently, the finding that the orexigenic neuropeptide, melanin-concentrating hormone $(\mathrm{MCH})$, can stimulate leptin synthesis and secretion. This chapter also incorporates new data describing the partial purification and effect of insulin on leptin-containing vesicles in rat adipocytes. These data indicate that the majority of leptin trafficking occurs via a constitutive secretory pathway and that the primary acute insulin effect on leptin secretion is to increase leptin protein content. In addition, we describe the identification and characterization of the vesicle-associated protein, pantophysin, which may play a multifunctional role in vesicle biogenesis and transport.
\end{abstract}

\section{Introduction}

The cloning of the $o b$ gene (Zhang et al., 1994) and the identification of its secreted product, leptin, as an adipocyte-derived hormone that communicates information regarding the size of adipose depots to the brain firmly established an active role for the adipocyte in regulating energy balance. These findings also triggered a renewed frenzy of research aimed at dissecting and analyzing the complex network of endocrine, paracrine, and autocrinc signals cmanating from adipocytes. Indeed, it has become increasingly apparent that the adipocyte functions as a global regulator of metabolic activity via this complex network. Adipocytes express and secrete a plethora of molecules, leptin being one of the morenotable ones. Two types of adipose tissue - white and brown - exist, each with 
distinct physiological roles. While there is evidence suggesting that brown adipose tissue (BAT) expresses some leptin (Frederich et al., 1995; Moinat et al., 1995; Deng et al, , 1997), the vast majority of leptin is synthesized and secreted by white adipose tissue (WAT). Furthermore, studies to date indicate that WAT is a primary source of key factors involved in the regulation of various cell biological processes. A summary of the various adipocyte secretory products is shown in Table I. The roles of many of these adipocyte-derived signals are still not fully understood. However, these proteins consist of a group of molecules that affect numerous cell biological and metabolic functions, such as glucose and lipid metabolism, insulin action, insulin secretion, energy balance, host defense, and reproduction. Hence, by virtue of the multitude of signals emanating from it, the adipocyte plays a critical role in regulating a multitude of biological processes. Furthermorc, it has become increasingly apparent that dysregulation of these signals in the obese state underlies the pathophysiology and sequelae of numerous diseases.

TABLE I

Known Adipocyte Secretory Proteins

- Leptin

- Alternative complement pathway proteins (adipsin, C3, B)

- Adipocyte complement related protein of $30 \mathrm{kDa}$ (ACRP30)

- Acylation-stimulating protein (ASP)

- Lipoprotein lipase (LPL)

- Nonesterified fatty acids (NEFAs)

- Cholesteryl ester transfer protein

- Apolipoprotein E (ApoE)

- Retinol-binding protein

- Vascular endothelial growth factor (VEGF)

- Monobutyrin

- Tumor necrosis factor-alpha (TNF- $\alpha)$

- Interleukin-6 (IL-6)

- Angiotensinogen

- Plasminogen activator inhibitor-1 (PAI-1)

- Transforming growth factor-beta (TGF- $\beta$ )

- Hepatocyte growth factor

- Insulin-like growth factor-1 (IGF-1)

- Adiponectin

[Data taken from Dobson, D., Kambc, A, Block, E., Dion, T., Lu, HI, Castellot, J., and Spiegelman, B. Cell 61, 223-230, 1990; Claffey, K.,Wilkison, W., and Spiegelman, B. J. Biol. Chem. 267, 16317-16322, 1992; Scherer, P., Williams, S., Fogliano, M., Baldini, G., and Lodish, H.F. J. Biol. Chem. 270, 26746-26749, 1995; Wei, S., Lai, K., Patel, S., Piantedosi, R., Shen, H., Colantuoni, V., Kraemer, F., and Blaner, W. J. Biol. Chem. 272, 14159-14165, 1997; Mohamed-Ali, V., Pinkney, J.H., and Coppack, S.W. Intl. J. Obesity Relat. Metab. Disord. 22, 1145-1158, 1998; Ouchi, N., Kihara, S., Arita, Y., Maeda, K., Kuriyama, H., Okamoto, Y., Hotta, K., Nishida, M., Takahashi, M., Nakamura, T., Yamashita, S., Funahashi, T., and Matsuzawa, Y. Circulation 100, 2473-2476, 1999.] 


\section{Functions of Adipocyte Secretory Products}

The exact roles of adipocyte-derived signals are not fully understood. Nonetheless, our current knowledge of the functions of these factors continues to expand rapidly. For example, leptin's ability to relay information regarding the size of adipose depots from the adipocyte to the brain is well established. However, the signaling pathways governing cross-talk between adipocytes and the brain are still not fully defined. In addition, the cytokines tumor necrosis factoralpha (TNF- $\alpha$ ) and interleukin-6 (IL-6) have important roles in host defense and may regulate both glucose and lipid metabolism (Castell et al., 1989; Grunfeld and Feingold, 1991). TNF- $\alpha$ also is reported to act as a mitogenic agent, to induce apoptosis of adipocytes, to stimulate leptin secretion, as well as to regulate mitochondrial function and gene expression (Prins et al., 1997). Acylation-stimulating protein (ASP) is involved in fatty acid metabolism, translocation of glucose transporters to the cell surface, as well as triglyceride synthesis and storage (Cianflone et al., 1992). Conversely, lipoprotein lipase (LPL) is known to be involved in the hydrolysis of triglycerides from circulating chylomicrons and very-low-density lipoproteins (Spooner et al., 1979). Cholesteryl ester transport protein facilitates uptake of cholesteryl esters from high-density lipoproteins into adipocytes (Benoist et al., 1998). Both vascular endothelial growth factor (VEGF) and monobutyrin have angiogenic effects in vivo and stimulate mitogenesis of vascular endothelial cells in vitro (Dobson et al., 1990; Claffey et al., 1992). Retinol-binding protein is a plasma transport protein for retinol (Wei et al., 1997), while angiotensinogen promotes adipocyte differentiation (Wciglc, 1997). Very recently, a new adipocyte secretory product, adiponectin, was reported (Ouchi et $a l ., 1999)$. Adiponectin is a plasma protein abundantly present in the systemic circulation. It apparently accumulates in vascular walls in response to endothelial injury and is thought to modulate the endothelial inflammatory response (Ouchi et al., 1999). These are only a few examples of the variety of signals governed by adipocyte-secretory products.

\section{Consequences of Obesity on Adipose Tissue Function}

Obesity is regarded as a pathological state that results in dysregulation of the endocrine function of adipose tissue. This dysfunction, in turn, can lead to numerous cardiovascular and metabolic disease processes, including hypertension, hyperlipidemia, hyperglucocorticoidemia, hyperinsulinemia, and type 2 diabetes. Obesity results in increased secretion of several adipocyte-derived factors (e.g., leptin, TNF- $\alpha$, IL-6, NEFA) (Mohamed-Ali et al., 1998). The initial effects of increased secretion of these factors serve to limit further weight gain by a variety of mechanisms, including 1) reduction of food intake, 2) increase in energy expenditure, 3) induction of insulin resistance, 4) stimulation of lipolysis, 5) 
suppression of lipogenesis, and 6) induction of adipocyte de-differentiation and reduction of preadipocyte differentiation (Prins et al., 1997; Mohamed-Ali et al., 1998). However, in an obese state, chronic exposure to elevated concentrations of these agents can have deleterious effects. For example, chronically elevated TNF$\alpha$ directly induces insulin resistance in both adipose tissue and skeletal muscle, which, in turn, can lead to type 2 diabetes and cardiovascular anomalies (Prins $e t$ al., 1997). Similarly, increased NEFA as a consequence of obesity may play a role in the pathogenesis of insulin resistance and hyperinsulinemia (Mohamed-Ali et al., 1998). Interestingly, adiponectin has been shown to inhibit TNF- $\alpha$-induced responses. Adiponectin levels are decreased in the obese state (Ouchi et al., 1999). Thus, obesity-induced decreases in adiponectin may further facilitate the adverse effects of TNF- $\alpha$ on various metabolic processes. In addition, decreased secretion of adiponectin in obesity has been linked to thrombotic vascular disease (Funahashi et al., 1999). Furthermore, plasminogen activator inhibitor-1 (PAI-1), which is overexpressed in obesity, may play a role in vascular disease (Funahashi $e t$ al., 1999), while increased IL-6 secretion in obesity may result in increased hepatic synthesis of pro-coagulant factors (Mohamed-Ali et al., 1998).

Despite the relatively large volume of secreted factors from adipocytes, the pathways and molecular components governing intracellular vesicular traffic and exocytosis of these factors remain relatively obscure. Like neuroendocrine cells, adipocytes have two primary secretory pathways: regulated and constitutive. This chapter will briefly discuss the components and current model for insulin-stimulated Glut 4 translocation as an example of a regulated pathway. As an example of a constitutive pathway, we will describe the regulation of leptin biosynthesis and secretion and its intracellular vesicle transport pathways. Furthermore, pantophysin was very recently identified as a phosphoprotein component of adipocyte transport vesicles and is present on both Glut4 vesicles and on a population of leptin-containing vesicles (Brooks et al., 2000). We will summarize the characterization of pantophysin and discuss the potential impact of these findings on the study of vesicle trafficking and secretion in adipocytes.

\section{Leptin and Signaling via the Leptin Receptor}

Many systematic studies on the pathophysiology of obesity have focused on mice homozygous for the obese $(o b)$ mutation. The $o b / o b$ mice display severe obesity and type 2 diabetes as well as a host of other metabolic and cardiovascular anomalies. Zhang et al. (1994) identified the gene responsible for the ob/ob mouse phenotype and its encoded protein, leptin, a 16-kDa protein secreted primarily by adipocytes. Numerous studies using a variety of rodent models have firmly established leptin as a key factor for regulating body weight and energy expenditure. Several excellent reviews on leptin and its role in regulating energy homeostasis 
are available (Caro et al., 1996; Auwerx and Staels, 1998; Trayhurn et al., 1999). The ability of leptin to regulate energy balance is based primarily on its capacity for relaying information regarding the size of adipose depots from the adipocyte to the brain. These effects of leptin are believed to be mediated through a closedloop feedback system involving the hypothalamus, within which leptin receptors have been identified (Mercer et al., 1996). In humans, however, the role of leptin in regulating adiposity appears to be more complex than in rodent models of obesity. Most obese individuals have higher levels of circulating leptin than their lean counterparts and appear to be resistant to their cndogenous leptin production. This observation has led to the "leptin resistance" hypothesis (Auwerx and Staels, 1998). A recent study showed that a leptin-inducible member of the suppression of cytokine signaling (SOCS) family, SOCS-3, inhibits leptin signaling in mammalian cell lines. It was proposed that SOCS-3 may be a potential mediator of leptin resistance in obesity (Bjorbaek et al., 1998). Overall, though, unlike in the $o b / o b$ mouse, human obesity is not a consequence of absolute leptin deficiency per se. Nonetheless, although cases are rare, deficiencies in leptin production and/or the leptin receptor have been linked to human obesity (Montague et al., 1997; Ravussin et al., 1997; Clement et al., 1998; Strobel et al., 1998).

Aside from regulating energy homeostasis, leptin is reported to have several other effects (Casanueva and Dieguez, 1999). Leptin is implicated in regulating fertility and is a permissive factor for the onset of puberty. The placenta is a source of leptin and leptin is present in human milk, suggesting that it may play a role in gestation and infant development. Furthermore, leptin is reported to have a role in hemopoiesis, to regulate cortisol secretion, and to facilitate activation and proliferation of human circulating $\mathrm{T}$ lymphocytes. Leptin is also involved in the neuroendocrine control of growth hormone secretion and there is some evidence that leptin may regulate thyrotropin-releasing hormone (TRH)-thyrotropin (TSH) and prolactin secretion (Casanueva and Dieguez, 1999).

In addition to being present in the hypothalamus, leptin receptors have been detected at lower amounts in several peripheral tissues, including heart, lung, liver, kidney, skeletal muscle, adipose tissue, and pancreatic $\beta$ cells (Tartaglia et al., 1995; Lee et al., 1996; Emilsson et al., 1997). This suggests that leptin signals to a variety of tissue and organ systems. The physiological significance of this expression, however, remains unclear. The leptin receptor gene is complex and encodes at least five different isoforms (Tartaglia et al., 1995; Lee et al., 1996). The "long-form" leptin receptor is considered the main signal-transducing form. It is abundantly expressed in the hypothalamus and, to a lesser extent in peripheral tissues, where the short form predominates (Lee et al., 1996; Tartaglia et al., 1995). The leptin receptor belongs to the class I cytokine receptor family (Tartaglia et al., 1995). Leptin receptor signaling is accomplished via the JAKSTAT pathway and involves transmission of the leptin signal via JAK-2 to STATs 
3, 5, and 6 (Baumann et al., 1996; Ghilardi et al., 1996; Vaisse et al., 1996). The leptin receptor is mutated in several rodent models of obesity, including the $d b / d b$ mouse, the Zucker fatty rat $(f a / f a)$, and the Koletsky rat (Auwerx and Staels, 1998). As previously mentioned, leptin receptors are present on pancreatic $\beta$ cells. Studies using both $o b / o b$ and wild-type mice have shown that leptin can effectively inhibit secretion of insulin (Emilsson et al., 1997; Kieffer et al., 1997), indicating that leptin may play a physiological role as an inhibitor of insulin secretion. The so-called adipoinsular axis postulates the existence of a feedback loop between adipose tissue and the endocrine pancreas (Kieffer et al., 1996). Furthermore, a number of studies have indicated a potential role for leptin in the pathogenesis of peripheral insulin resistance. It has been shown that both acute and chronic in vitro treatment of Hep G2 cells or rat fibroblasts with leptin caused attenuation of insulin-stimulated phosphorylation of insulin receptor substrate-1 (IRS-1). Exposure of primary rat adipocytes to leptin in vitro has been shown to decrease insulin-stimulated glucose metabolism (Cohen et al., 1996; Muller et al., 1997; Kroder et al., 1996).

\section{Regulation of Leptin Biosynthesis and Secretion}

In an effort to better understand secretory pathways utilized by the adipocyte, we have studied leptin secretion (and Glut4 translocation, to be discussed later in this chapter). Synthesis and secretion of leptin is regulated by a complex series of neuroendocrine, endocrine, and paracrine signals to which the adipocyte is exquisitely sensitive. The underlying biochemical and molecular mechanisms that regulate leptin synthesis and secretion are not fully defined. However, several positive and negative regulators of leptin production by adipocytes have been identified. Positive effectors include insulin, glucocorticoids, TNF- $\alpha$, prolactin, A1 adenosine receptor agonists, and estrogens (Mohamed-Ali et al., 1998; Casanueva and Dieguez, 1999; Gualillo et al., 1999; Rice et al., 2000). Conversely, negative regulators of leptin production include catecholamines, androgens, cAMP agonists, $\beta$-adrenergic receptor agonists, peroxisome proliferator-activated receptor gamma (PPAR- $\gamma$ ) agonists, phorbol esters, and possibly thyroid hormone (Mohamed-Ali et al., 1998; Casanueva and Dieguez, 1999). It is noteworthy that, depending on their location, adipose tissues respond differently to these agents. As examples, subcutaneous adipocytes apparently respond more to insulin and less to glucocorticoids than do omental adipocytes, while female adipose tissue responds more robustly to steroid hormones than does male adipose tissue (Casanueva and Dieguez, 1999). Many systematic studies of factors regulating leptin production by adipocytes have focused on two principal candidates, glucocorticoids and insulin. With rare exception (Rentsch and Chiesi, 1996), glucocorticoids have more or less consistently been shown to increase $o b$ gene expression 
and leptin secretion in vivo in both normal rats and humans (Larsson and Ahren, 1996; De Vos et al., 1995; Papaspyrou-Rao et al., 1997), as well as in vitro in primary cultures of adipocytes (Bradley and Cheatham, 1999; Murakami et al., 1995; Hardie et al., 1996; Halleux et al., 1998). With regard to the effect of insulin, the vast majority of studies indicate that insulin modulates leptin synthesis and secretion from adipocytes (Bradley and Cheatham, 1999; Saladin et al., 1995; Gettys et al., 1996; Hardie et al., 1996). However, a few studies have disagreed with these findings, leading to the suggestion that these disparate reports may have resulted from the use of different culture models. In support of an in vivo stimulatory effect, it was reported that $o b$ mRNA and leptin protein secretion in rats are tightly regulated by diet and insulin (Saladin et al., 1995). Under fasting conditions, $o b$ mRNA and leptin protein levels decreased to barely detectable levels but were restored to normal within 4 hours after re-feeding or administration of insulin. In addition, rats rendered hyperinsulinemic in clamp studies showed a marked increase in $o b$ mRNA, compared with controls.

\section{A. REGULATION OF LEPTIN MRNA AND LEPTIN SECRETION BY INSULIN AND DEXAMETHASONE}

To gain further insight into hormonal regulation of $o b$ gene expression and leptin secretion, we have studied the acute effects of insulin and dexamethasone (Dex), a synthetic glucocorticoid on leptin synthesis and secretion. In a recent study (Bradley and Cheatham, 1999), a 2-hour incubation of rat adipocytes with insulin had no effect on $a b$ mRNA levels (Figure 1A) but stimulated a two-fold increase in leptin secretion (Figure 1B). Dex, on the other hand, stimulated both a two- to four-fold increase in $o b$ mRNA (Figure 1A) and a two-fold increase in leptin secretion (Figure 1B), indicating that Dex acutely regulates $o b$ gene transcription in isolated rat adjpocytes. As shown in Figure 1A, concurrent treatment with both hormones did not stimulate $o b$ gene expression to any greater extent than did the individual agents. In contrast to its lack of effect on $o b$ mRNA, insulin stimulated a two-fold increase in leptin protein secretion. Similarly, incubation of adipocyte preparations with Dex showed a two-fold increase in leptin secretion. These data suggested that insulin regulates leptin synthesis/secretion via a potential post-transcriptional mechanism. Dex appears to do so via a transcriptionally mediated process.

To verify this hypothesis, we used the transcriptional inhibitor actinomycin D. Consonant with a post-transcriptional and transcriptional regulatory mechanism for insulin- and Dex-stimulated leptin secretion, respectively, actinomycin D blocked Dex-stimulated leptin secretion but did not affect insulin-stimulated leptin secretion (Bradley and Cheatham, 1999). Furthermore, as illustrated in Figure $2 \mathrm{~A}$, inhibition of protein synthesis with cycloheximide did not affect $o b$ mRNA accumulation but reduced total secreted leptin (Figure 2B). Interestingly, 

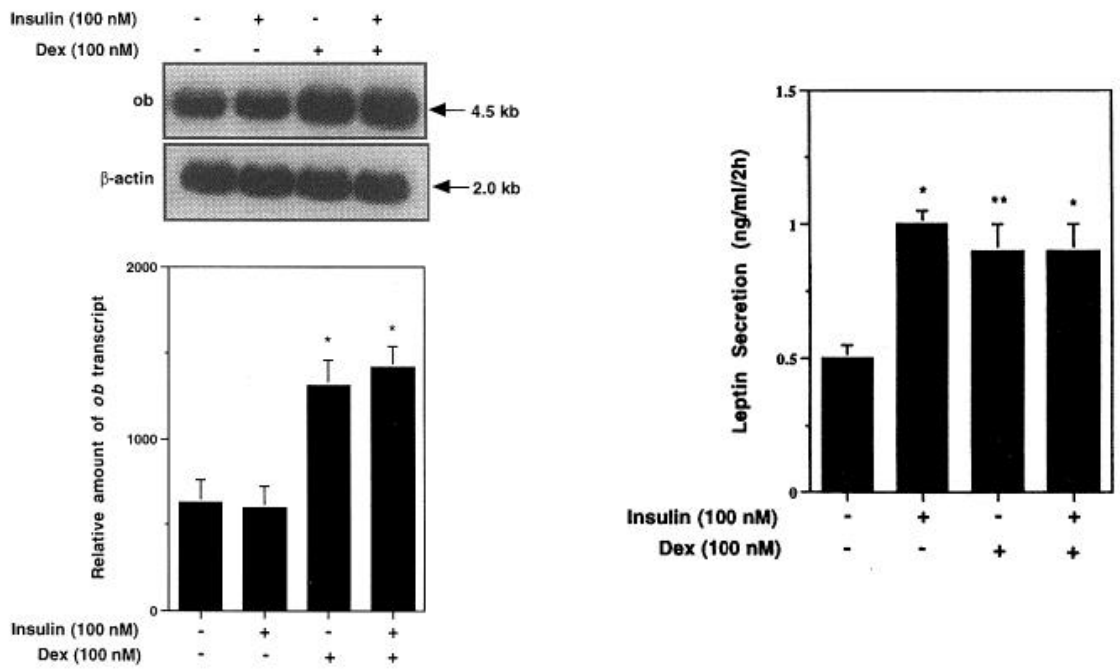

FIG. 1. (Left panels) Northern blot showing the individual and combined effects of insulin and dexamethasone on $o b$ gene expression in freshly isolated rat adipocytes. Adipocytes were incubated with and without either $100 \mathrm{nM}$ insulin, $100 \mathrm{nM}$ dexamethasone, or both simultaneously for 2 hours. Each lane was loaded with $10 \mu \mathrm{g}$ total RNA and hybridized with a mouse $o b$ cDNA probe. Densitometric scanning was used to determine the relative amount of $o b$ transcript. Blots were stripped and reprobed with a human $\beta$-actin cDNA probe as a control for RNA integrity and loading. (Right panels) Individual and combined effects of insulin and dexamethasone on leptin secretion from freshly isolated rat adipocytes. Incubation conditions were those described above. Leptin secreted into the conditioned medium was measured using a commercially available rat leptin radioimmunoassay (RIA) kit. All results are expressed as the mean \pm S.E.M. and are representative of three independent experiments $\left(\mathrm{n}=8\right.$ per assay). ${ }^{* \mathrm{P}}<0.05$ vs. control $(-/-) ;{ }^{* *} \mathrm{P}<0.02$ vs. control $(-/-)$. [Reprinted with permission from Bradley, R.L., and Cheatham, B. Diabetes 48, 272-278, 1999. Copyright by the American Diabetes Association.]

despite this decrease, insulin (but not Dex) was still able to stimulate a two-fold increase in leptin secretion by rat adipocytes relative to control cells in the presence of cycloheximide (Figure 2B). These data suggest that insulin, but not Dex, is able to stimulate secretion of a small, pre-existing, intracellular pool of leptin-containing vesicles. However, de novo protein synthesis is required for the full insulin-stimulated response.

\section{B. INSULIN-REGULATED SIGNALING PATHWAYS AND LEPTIN SECRETION}

Insulin regulates a variety of intracellular growth and metabolic processes (Cheatham and Kahn, 1995). Early events in insulin action involve the insulin- 
(A)

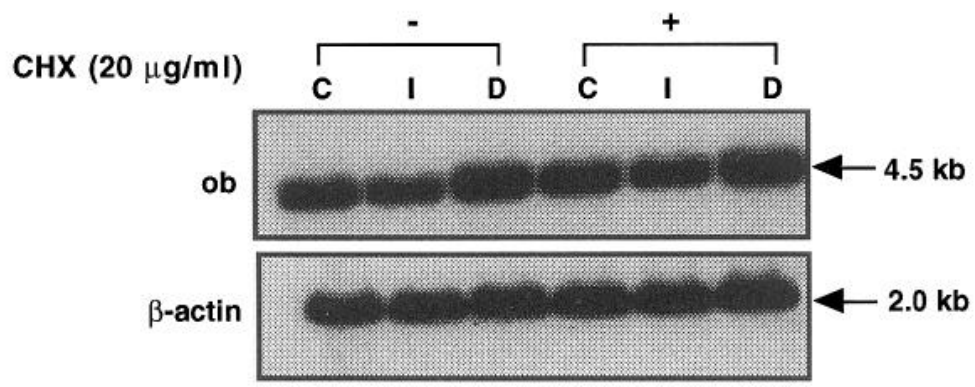

(B)

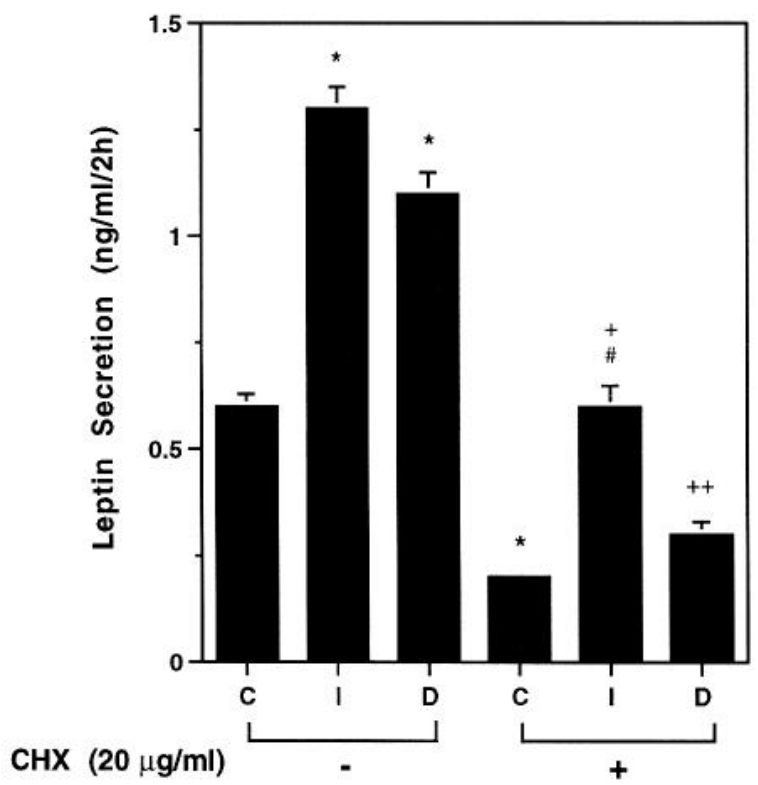

FIG. 2. (A) Northern blot showing the effect of the protein synthesis inhibitor cycloheximide (CHX) on $o b$ gene expression in control (C), insulin- (I), and dexamethasone- (D) treated rat adipocytes. Freshly isolated adipocytes were preincubated in the absence and presence of $20 \mu \mathrm{g} / \mathrm{ml} \mathrm{CHX}$ for 30 minutes. The conditioned medium was changed and adipocytes were incubated for 2 hours with and without $100 \mathrm{nM}$ insulin or $100 \mathrm{nM}$ dexamethasone in the absence and presence of CHX (20 $\mu \mathrm{g} / \mathrm{ml}$ ). Each lane was loaded with $10 \mu \mathrm{g}$ total RNA and membranes hybridized with a mouse $o b$ cDNA probe. Membranes were stripped and reprobed with a human $\beta$-actin cDNA probe as a control for RNA integrity and loading. (B) Effect of CHX $(20 \mu \mathrm{g} / \mathrm{ml})$ on leptin secretion. Secreted leptin contained in the conditioned medium from the 2-hour incubation described above was measured by RIA. Results are the mean \pm S.E.M. for 12 rats. ${ }^{*} \mathrm{P}<0.05$ vs. control minus $\mathrm{CHX} ;+\mathrm{P}<0.05$ vs. insulin treated minus $\mathrm{CHX}$; $\mathrm{P}<0.02$ vs. control plus $\mathrm{CHX} ;++\mathrm{P}<0.05$ vs. dexamethasone treated minus CHX. [Reprinted with permission from Bradley, R.L., and Cheatham, B. Diabetes 48, 272-278, 1999. Copyright by the American Diabetes Association.] 
stimulated activation of the insulin receptor and phosphorylation of intracellular substrates, including insulin receptor substrates 1 and 2 (IRS- 1 and IRS-2) and Shc. These tyrosine-phosphorylated proteins then act as docking sites for proteins containing SH2 domains such as Grb2, phosphatidylinositol (PI) 3-kinase, and the tyrosine phosphatase, SHP2. These protein-protein interactions, in turn, lead to regulation of more-distal events, including activation of Ser/Thr phosphorylation cascades that result in activation of mitogen-activated protein kinase (MAPK), pp90 S6 kinase, and pp70 S6 kinase. The end result of this series of events is the modulation of many of insulin's final biological responses (Cheatham and Kahn, 1995). Using specific inhibitors of PI 3-kinase, MAPK kinase (MEK), and pp70 S6 kinase, we have evaluated signaling pathways involved in the acute phase of insulin- and Dex-stimulated leptin synthesis/secretion. The effects of the PI 3-kinase inhibitor LY294002; the MEK inhibitor PD98059; and the immunosuppressant rapamycin on both insulin- and Dex-stimulated leptin mRNA secretion were determined. During a 2-hour incubation, basal levels of secreted leptin were not affected by any of the inhibitors (Figure 3 ). However, all three inhibitors markedly decreased both insulin- and Dex-stimulated leptin secretion (Figure 3). These findings suggest that a complex set of signaling pathways mediate insulin- and Dex-stimulated leptin production. These pathways involve multiple steps that are PI 3-kinase dependent as well as steps that are sensitive to the MEK inhibitor PD98059 and the immunosuppressant rapamycin.

\section{MELANIN-CONCENTRATING HORMONE REGULATES LEPTIN SECRETION}

More recently, the orexigenic (appetite-stimulating) neuropeptide, melaninconcentrating hormone $(\mathrm{MCH})$, has emerged as a regulator of leptin production by rat adipocytes (Bradley et al., 2000). MCH is a hypothalamic peptide important in the regulation of feeding behavior, primarily via uncharacterized pathways in the central nervous system (CNS). Leptin is known to mediate some of its actions through several hypothalamic neuropeptides (Elmquist et al., 1998). Indeed, $\mathrm{MCH}$-expressing neurons may be an important target of leptin action in the CNS, as $\mathrm{MCH}$ levels rise dramatically in the absence of leptin and also respond to fasting ( $\mathrm{Qu}$ et al., 1996). In addition, adipocytes express a number of receptors for both hormones and neuropeptides, including peptides involved in the regulation of appetite, and it is possible that other peptides may regulate leptin responses. Very-recent studies show MCH to modulate leptin production (Bradley et al., 2000 ). In these studies, $\mathrm{MCH}$ stimulated a two-fold increase in leptin secretion by isolated rat adipocytes after 4 hours of treatment. This increase in secreted leptin was preceded by a rapid and transient increase in $o b$ mRNA levels. As shown in Figure 4, MCH stimulated a 2.5 -fold increase in $o b$ mRNA within 1 hour of treatment, followed by a decline to basal levels within 4 hours. Thus, 


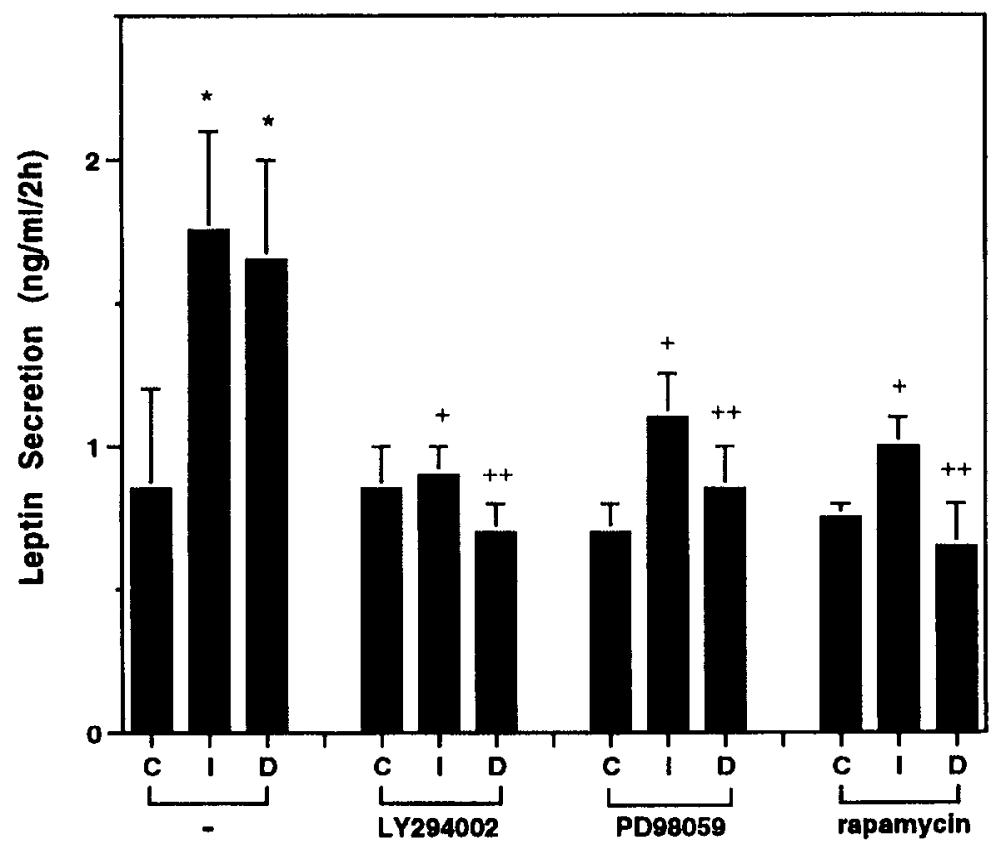

FIG. 3. Effects of the PI 3-kinase inhibitor LY294002, the MEK inhibitor PD98059, and the immunosuppressant rapamycin on leptin secretion from control (C), insulin- (I), and dexamethasone(D) treated rat adipocytes. Adipocytes were incubated in the absence and presence of $50 \mu \mathrm{M}$ LY294002, $10 \mu \mathrm{M}$ PD98059, or $20 \mathrm{nM}$ rapamycin for 30 minutes. The conditioned medium was changed and adipocytes incubated for 2 hours with and without $100 \mathrm{nM}$ insul in or $100 \mathrm{nM}$ dexamethasone in the absence and presence of each inhibitor at the aforementioned concentrations. Secreted leptin contained in the conditioned medium was measured by RIA. Results are the mean \pm S.E.M. for three independent experiments $\left(n=14\right.$ per assay). ${ }^{*} P<0.05$ vs. control $(-) ;+P<0.05$ vs. insul in treated (-); ++ P<0.05 vs. dexamethasone treated (-). [Reprinted with permission from Bradley, R.L., and Cheatham, B. Diabetes 48, 272-278, 1999. Copyright by the American Diabetes Association.]

$\mathrm{MCH}$ acutely stimulates an increase in $o b$ gene expression in rat adipocytes, resulting in an increase in leptin protein production and secretion (Bradley et al., 2000). In addition, we found that the $\mathrm{MCH}$ receptor, a seven-transmembrane domain G-protein-coupled receptor termed SLC-1 (Chambers et al., 1999; Saito et al., 1999), expressed in adipocytes (Figure 5). These data suggest that fat cells are targets of $\mathrm{MCH}$ or an $\mathrm{MCH}$-like peptide under physiological conditions. These observations also represent the first cultured-cell model for signaling via endogenous $\mathrm{MCH}$ receptors. We did not find $\mathrm{MCH}$ expressed in WAT (Bradley et al., 2000). At present, the exact physiological role of $\mathrm{MCH}$-stimulated leptin secretion is unclear. The cellular origin of $\mathrm{MCH}$ in peripheral tissues is unknown but circulating levels of $\mathrm{MCH}$ or an $\mathrm{MCH}-$ like peptide can be detected in rat plasma 

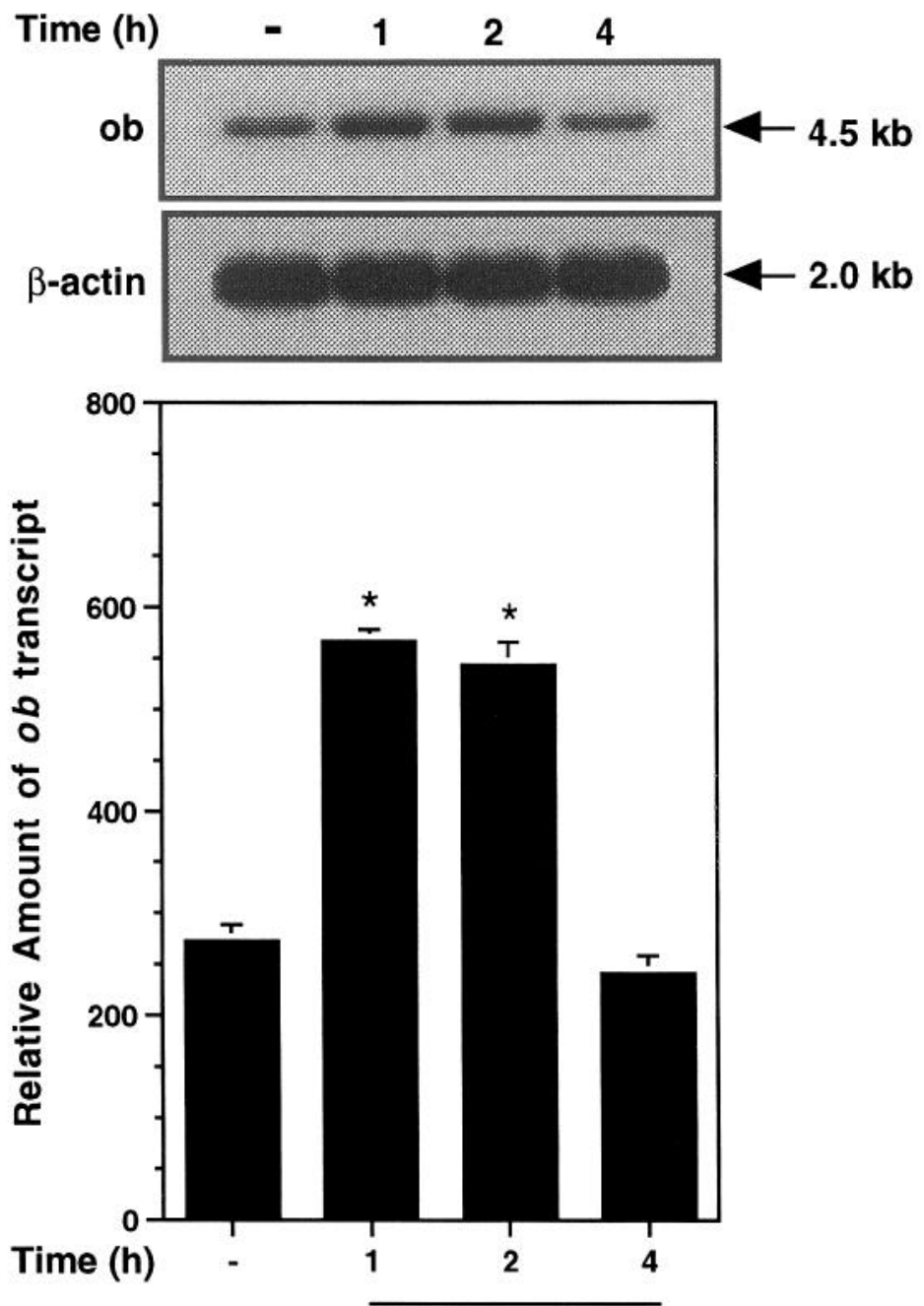

$\mathrm{MCH}$

FIG. 4. Northern blot showing the effect of $\mathrm{MCH}$ on $o b$ gene expression in isolated rat adipocytes derived from epididymal fat pads. Cells were incubated in the absence (-) and presence of $1 \mu \mathrm{M} \mathrm{MCH}$ as indicated for up to 4 hours. Total RNA was isolated from cells collected at each time-point. Each lane was loaded with $10 \mu \mathrm{g}$ total RNA and hybridized with a mouse $o b$ cDNA probe. Densitometric scanning was used to determine the relative amount of $o b$ transcript. Blots were stripped and reprobed with a human $\beta$-actin cDNA as a control for RNA integrity and loading. Results are means \pm S.E.M. for two independent experiments ( $\mathrm{n}=8$ per assay). ${ }^{*} \mathrm{P}<0.05 \mathrm{vs}$. control $(-)$. [Reprinted with permission from Bradley, R.L., Kokkotou, E.G., Maratos-Flier, E., and Cheatham, B. Diabetes 49, 1073-1077, 2000. Copyright by the American Diabetes Association.] 


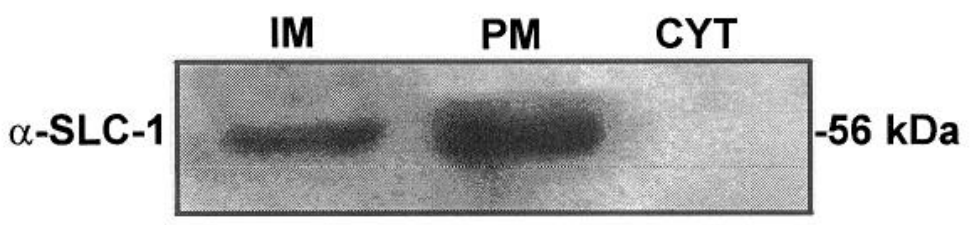

FIG. 5. Immunoblot showing the presence of SLC-1 protein in rat adipocytes. Membrane fractions were prepared from rat adipocytes derived from epididymal fat pads. Aliquots $(50 \mu \mathrm{g})$ of purified plasma membrane (PM), total internal membrane (IM), or cytosol (CYT) were solubilized in SDS-PAGE sample buffer, resolved by SDS-PAGE, transferred to nitrocellulose, and subjected to immunoblotting with a polyclonal $\alpha$-SLC-1 peptide antibody. Immunoreactive material was detected using $\left[{ }^{125} \mathrm{I}\right]$-labeled protein $\mathrm{A}$, followed by autoradiography. Results are representative of three independent experiments. [Reprinted with permission from Bradley, R.L., Kokkotou, E.G., MaratosFlier, E., and Cheatham, B. Diabetes 49, 1073-1077, 2000. Copyright by the American Diabetes Association.]

(Bradley et al., 2000). Finally, these data suggest a potential feedback mechanism between the brain and adipose tissue and further underscore the complexity of the cross-talk between the neuroendocrine systems mediating energy homeostasis as well as the ability of the adipocyte to respond to a wide range of afferent signals.

\section{Vesicle Transport Pathways in Adipocytes}

It is clear that adipocytes secrete a wide variety of proteins that affect numerous cell biological and metabolic pathways. However, very little is known regarding the components of the intracellular secretory pathways through which these proteins transit and ultimately exit the adipocyte. The following section summarizes some of the known components of the regulated pathway, discusses a newly identified protein (pantophysin) that may play a multifunctional role in vesicle biogenesis and transport, and describes the characteristics of intracellular trafficking of leptin-containing vesicles.

\section{A. COMPONENTS OF THE REGULATED SECRETORY PATHWAY IN ADIPOCYTES}

Insulin's primary physiological role is maintenance of glucose homeostasis via inhibition of hepatic glucose production and stimulation of glucose uptake into insulin-sensitive tissues: adipose, skeletal, and cardiac muscle (Birnbaum, 1992). Insulin stimulates the rate of glucose uptake into these tissues 10- to 20 -fold. This is accomplished by an insulin-stimulated translocation of the Glut4 glucose transporter protein from a unique, intracellular vesicular compartment to the plasma 
membrane (Birnbaum, 1992). The mechanism underlying translocation of Glut4 is the best-characterized, acutely regulated vesicle transport process in adipocytes. Approaches to define this mechanism have focused on determining the insulinregulated signaling pathways involved and identifying the molecular components utilized in directing movement and fusion of the Glut4-vesicle to the plasma membrane. A detailed review of this process by Pessin and colleagues can be found in this volume (Chapter 9); therefore, we will only touch on some highlights, emphasizing the components in this vesicle transport pathway.

Multiple lines of evidence have established a requirement for insulin-stimulated activation of PI 3-kinase as an upstream regulator or Glut4 translocation. More recently, protein kinase $\mathrm{B}(\mathrm{PKB})$ and the atypical $\mathrm{PKCs}(\mathrm{PKC} \lambda$ and $\mathrm{PKC} \zeta$ ) have been implicated as potential downstream targets of PI 3-kinase. However, their exact roles in signaling to the Glut 4 vesicles are unknown. Clearly, much remains to be learned regarding the exact signaling cascade. However, in an effort to link signaling pathways to the Glut4 vesicle, several laboratories have focused on identifying proteins in adipocytes that are involved in regulated exocytosis. These studies found that adipocytes express proteins previously shown to mediate acute regulated vesicle transport events in neuroendocrine tissues. The research suggested that translocation of Glut4 may utilize machinery similar to that found in these systems (St-Denis and Cushman, 1998). These proteins include soluble N-ethylmaleimide-sensitive fusion (NSF) protein attachment protein receptors, or SNARE proteins. Through defined protein-protein interactions, members of the SNARE family of membrane-associated proteins can selectively mediate homotypic and heterotypic membrane fusion (Pfeffer, 1999; Hay and Scheller, 1997). The current model for SNARE-mediated docking and fusion states that a vesicleassociated SNARE ( $v$-SNARE) interacts with cognate target-membrane SNAREs (t-SNARE) to mediate the interaction between two separate membrane populations. This minimal complement of a v-SNARE and t-SNAREs is sufficient to mediate fusion in vitro (Weber et al., 1998).

VAMP-2 is a v-SNARE in adipocytes associated with the Glut4 vesicle (St-Denis and Cushman, 1998). Syntaxin-4 and SNAP-23 are the cognate tSNARE molecules expressed in adipocytes and found almost exclusively on the plasma membrane (St-Denis and Cushman, 1998). Studies from this lab and others have provided multiple lines of evidence for a specific role of these proteins in Glut4 translocation (Cheatham et al., 1996; St-Denis and Cushman, 1998; Pessin et al., 1999). The current minimal model for SNARE-mediated Glut4 vesicle trafficking suggests that VAMP-2 on the Glut4 vesicle forms a stable, heterotrimeric complex with syntaxin-4 and SNAP-23 present at plasma membrane and brings the Glut4 vesicle in contact with the plasma membrane driving fusion. Disruption of the heterotrimeric complex and regeneration of the individual SNARE proteins are thought to involve the ATPase activity associated with NSF 
and the soluble NSF attachment protein, $\alpha$-SNAP. This "priming step" prepares the SNARE proteins for further fusion events (Pfeffer, 1999; Rice and Brunger, 1999; Hay and Scheller, 1997).

The regulation of SNARE-complex formation is currently an area of intense focus. Munc $18 \mathrm{c}$ and Synip have been identified as potential regulators of SNAREcomplex formation in adipocytes. Munc $18 \mathrm{c}$ is a member of a family of syntaxin-4binding proteins and, when bound to syntaxin-4, inhibits the binding of VAMP-2. Thus, Munc18c is thought to be a negative regulator of SNARE-complex formation (Tellam et al., 1997; Tamori et al., 1998). Synip was isolated as a syntaxin4-binding protein in a yeast two-hybrid screen (Min et al., 1999). It contains several potential functional domains, including a PDZ domain, a coiled-coil region, and a WW domain. Synip also is enriched in adipose and skeletal muscle. Synip is associated with syntaxin- 4 in the basal state. The current model for Synip function involves the interaction of its $\mathrm{COOH}$-terminal domain with syntaxin-4, while the $\mathrm{NH}_{2}$-terminal domain responds to an as-yet-undefined insulin-generated signal. It should be noted that, other than Glut4 vesicles, a functional role for SNARE proteins in mediating exocytosis of other adipocyte-derived products has not been reported.

\section{B. PANTOPHYSIN IS A PHOSPHOPROTEIN COMPONENT OF ADIPOCYTE TRANSPORT VESICLES}

We have recently identified and have partially characterized pantophysin as another potential regulator of SNARE-complex formation and as a component of numerous intracellular vesicles (Brooks et al., 2000). Pantophysin is a homologue of the neuroendocrine-specific protein synaptophysin, sharing a 43 percent overall amino acid sequence identity (Haass et al., 1996). It contains four predicted transmembrane domains that are highly homologous to synaptophysin. In contrast, the $\mathrm{NH}_{2}$ - and $\mathrm{COOH}$-termini are quite divergent. Synaptophysin contains an extended $\mathrm{COOH}-$ terminus that includes a tyrosine-rich domain not present in pantophysin. Synaptophysin is fairly well characterized and, although its exact function is unclear, has been implicated in regulating SNARE-SNARE interactions, formation of fusion pores, vesicle biogenesis, and formation of lipid microdomains at the cell surface (Elferink and Scheller, 1995; Thiele et al., 2000). Thus, based upon these data and the homology between the proteins, pantophysin is an intriguing molecule that may play a multifunctional role in vesicle biogenesis and transport. Therefore, we have included a description of some of the characteristics of pantophysin.

\section{Expression of Pantophysin and Its Subcellular Distribution}

Pantophysin mRNA is widely expressed in mouse tissues and is relatively abundant in adipose tissue. Pantophysin mRNA increases (approximately two- 
fold) during adipogenesis of 3T3-L1 cells (Brooks et al., 2000). Immunoblot analysis indicates pantophysin is present exclusively in membrane fractions and relatively evenly distributed in the plasma membrane and two internal membrane fractions, the low (LDM)- and high (HDM)-density microsomes. Like synaptophysin, pantophysin is glycosylated and migrates as a broad band on SDS-PAGE. Following deglycosylation with $\mathrm{N}$-glycosidase $\mathrm{F}$, pantophysin migrates at its predicted molecular mass of $28.5 \mathrm{kDa}$ (Brooks et al., 2000). Interestingly, its pattern of glycosylation changes during differentiation of 3T3-L/ cells to adipocytes. In addition, we observed that the glycosylation pattern of pantophysin varies considerably in a tissue-dependent manner. The role of this variable glycosylation is unclear but may be involved in membrane targeting or perhaps in functional differences between tissues.

\section{Pantophysin Is a Component of Glut4 Vesicles}

Compared to insulin-stimulated Glut4 translocation, insulin stimulates only a modest, but significant, redistribution of pantophysin from an intracellular vesicular compartments to the plasma membrane. Thus, some pantophysin trafficks in an insulin-dependent manner similar to that of Glut4, although to a lesser extent. Further analysis of pantophysin vesicles by sucrose gradient ultracentrifugation demonstrated that pantophysin and Glut4 exhibited overlapping distribution profiles. Analysis of immunopurified vesicles revealed that Glut4 vesicles contain pantophysin. Both Glut4 and pantophysin were depleted from this vesicle population following treatment with insulin (Figure 6). However, only a subpopulation of the total immunopurified pantophysin vesicles contained insulin-responsive Glut4, consistent with the modest, insulin-stimulated redistribution of pantophysin (Brooks et al., 2000). Taken together, these data suggest pantophysin is present on a variety of intracellular vesicles.

\section{Pantophysin Interacts with VAMP-2}

Synaptophysin has been shown to interact with the v-SNAREs in neuroendocrine tissues (Calakos and Scheller, 1994). Consistent with this observation, we found pantophysin associated with VAMP-2 but not VAMP-3 in adipocytes (Figure 7). The interaction of VAMP with synaptophysin has been shown to block the interaction of VAMP with syntaxin (Calakos and Scheller, 1994). Thus, it has been suggested that the synaptophysin/VAMP interaction may be a regulatory mechanism in SNARE-SNARE interaction or perhaps is involved in recycling of VAMP into a newly formed regulated vesicle. Our data suggest that pantophysin may play a similar role; however, much more work is necessary. Currently, we are mapping domains on VAMP-2 that are involved in the interaction with pantophysin. 


\title{
IPVs
}
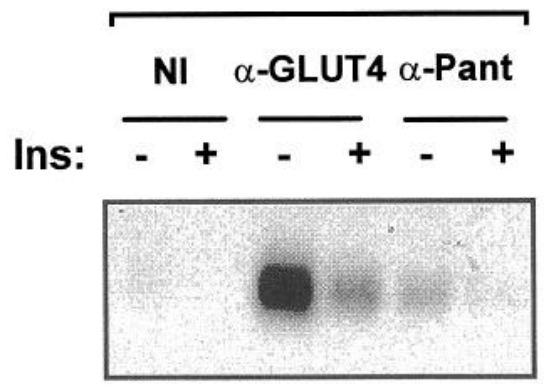

\section{$\alpha$-GLUT4}

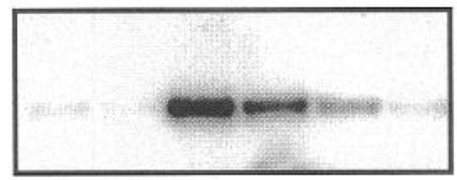

\author{
$\alpha-$ IRAP
}

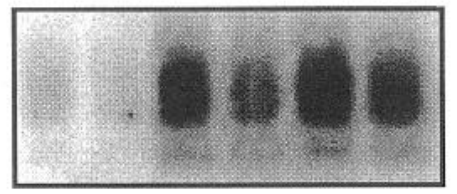

$\alpha$-Pant

FIG. 6. Immunopurified GLUT4- and pantophysin-containing vesicles analyzed by immunoblot with $\alpha$-GLUT4, $\alpha$-pantophysin, and $\alpha$-IRAP. Fully differentiated 3T3-L1 adipocytes were incubated in DMEM/ $0.1 \%$ bovine serum albumin (BSA) overnight and treated for 10 minutes in the absence (-) or presence $(+)$ of $100 \mathrm{nM}$ insulin. Immunopurified vesicles (IPVs) were prepared from LDM fractions using nonimmune (NI) serum, $\alpha$-GLUT4-, or $\alpha$-pantophysin (pant). Immunopurified vesicles were solubilized in SDS-PAGE sample buffer, separated on $10 \%$ SDS-PAGE, and electrophoretically transferred to nitrocellulose for immunoblotting with $\alpha$-GLUT4 (top), $\alpha$-IRAP (middle), or $\alpha$-pantophysin (bottom). Immunoreactive material was detected by $\left[{ }^{125} \mathrm{I}\right]-$ labeled protein $\mathrm{A}$ and subsequent autoradiography. Results are representative of three or more independent experiments. [Reprinted with permission from Brooks, C.C., Scherer, P.E., Cleveland, K., Whittemore, J.L., Lodish, H.F., and Cheatham, B. J. Biol. Chem. 275, 2029-2036, 2000. Copyright The American Society for Biochemistry \& Molecular Biology.]

\section{Pantophysin Is Phosphorylated}

Finally, another interesting characteristic of pantophysin is its phosphorylation. In [ $\left.{ }^{32} \mathrm{P}\right]$-orthophosphate-labeled 3T3-L1 adipocytes, pantophysin is phosphorylated in the basal state. Insulin or serum does not affect this phosphorylation. However, we did observe an insulin-stimulated phosphorylation of a $77-\mathrm{kDa}$ protein associated with $\alpha$-pantophysin immunoprecipitates (Brooks et al., 2000). 


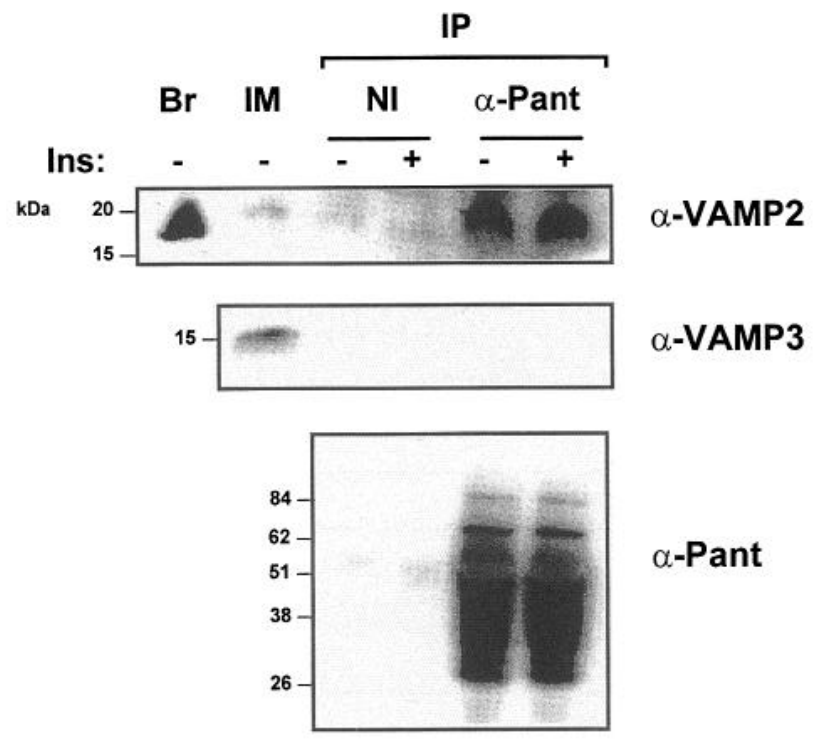

FIG. 7. Interaction of pantophysin and VAMP2 in $\alpha$-pantophysin immunoprecipitates. Fully differentiated 3T3-L1 adipocytes were incubated in DMEM/0.1\% BSA overnight. Cells were incubated for 10 minutes in the absence (-) or presence (+) of $100 \mathrm{nM}$ insulin, lysed, and immunoprecipitates using nonimmune (NI) or $\alpha$-pantophysin (pant) serum prepared. Samples were solubilized in SDS-PAGE sample buffer, separated on SDS-PAGE, and electrophoretically transferred to nitrocellulose membranes for immunoblotting with $\alpha$-VAMP2, $\alpha$-VAMP3, or $\alpha$-pantophysin. Immunoreactive material was detected by $\left[{ }^{125} \mathrm{I}\right]$-labeled protein $\mathrm{A}$ and subsequent autoradiography. IP $=$ immunoprecipitate; $\mathrm{Br}=40 \mu \mathrm{g}$ brain lysate; $\mathrm{IM}=20 \mu \mathrm{g}$ 3T3-L1 adipocyte internal membranes (LDM + HDM). The data presented are representative of three or more independent experiments. [Reprinted with permission from Brooks, C.C., Scherer, P.E., Cleveland, K., Whittemore, J.L., Lodish, H.F., and Cheatham, B. J. Biol. Chem. 275, 2029-2036, 2000. Copyright The American Society for Biochemistry \& Molecular Biology.]

The role of phosphorylation of pantophysin is unclear. Identification of sites of phosphorylation and a potential regulation of this phosphorylation are underway.

Although the functional role of pantophysin in vesicle trafficking is unclear, its presence on Glut4 vesicles is consistent with the emerging role of SNAREcomplex and related proteins in regulated vesicle transport in adipocytes. Recently, we observed that microinjection of antibodies against pantophysin or microinjection of a $\mathrm{COOH}$-terminal fragment of pantophysin inhibited insulinstimulated Glut4 translocation (L. Foster, B. Cheatham, and A. Klip, unpublished data). The exact mechanism of this inhibition is unclear but is consistent with a role of this protein in vesicle transport/biogenesis. In addition, since pantophysin is present on a variety of intracellular vesicles, we have begun using it as a marker to help define the trafficking and contents of uncharacterized vesicles in adipo- 
cytes. As will be discussed, we show that leptin is a cargo protein in a subpopulation of pantophysin-containing vesicles that is distinct from Glut4-containing vesicles. In addition, the predicted roles of synaptophysin in a variety of vesicle transport functions, vesicle biogenesis, and formation of lipid microdomains at the cell surface suggest that pantophysin may play an important role in one or more of these processes.

\section{A CONSTITUTIVE SECRETORY PATHWAY IN ADIPOCYTES: INTRACELLULAR TRAFFICKING OF LEPTIN-CONTAINING VESICLES}

As discussed earlier, insulin stimulates leptin secretion by primary rat adipocytes. However, the extent to which this effect is mediated by a regulated secretory pathway vs. a constitutive pathway remains unclear. In one study, insulin appeared to acutely stimulate exocytosis of leptin directly from an endoplasmic reticulum (ER)-enriched compartment, bypassing accumulation in identifiable, regulated secretory vesicles (Barr et al., 1997). Our initial studies suggested that the major component of insulin-stimulated leptin secretion trafficks along a constitutive path requiring de novo protein synthesis. However, we also showed that, in the presence of cycloheximide, insulin can stimulate exocytosis of a small, preformed pool of leptin-containing vesicles (Bradley and Cheatham, 1999). These data suggest that leptin secretion is primarily via a constitutive pathway; however, there is a small population of leptin-containing vesicles able to undergo acutely regulated exocytosis. These observations are similar to the characteristics for the stimulated secretion of Acrp30 (Bogan and Lodish, 1999). To better define these pathways, we have analyzed and characterized leptin-containing vesicles in rat adipocytes.

\section{Subcellular Distribution of Leptin in Rat Adipocytes}

To analyze intracellular pools of leptin, rat adipocytes were homogenized and the nuclear/mitochondrial supernatant subjected to subcellular fractionation by differential centrifugation to yield two internal membrane fractions, the LDM and HDM, respectively. The LDM is enriched with Golgi-derived membranes as well as the insulin-sensitive pool of Glut4 vesicles, whereas the HDM is enriched with ER-derived markers. As shown in Figure 8A - and consistent with the expected enrichment of specific organellar membranes in the HDM and LDM - both ER-specific proteins translocon-associated protein- $\alpha$ (TRAP- $\alpha$ ) and calnexin were highly enriched in the HDM and were undetectable in the LDM. The LDM was enriched with the Golgi markers mannosidase II and coat protein, $\beta$-COP. Furthermore, as shown in Figure 8B, intracellular leptin associates primarily with the HDM and insulin stimulates an increase in intracellular leptin exclusively in this compartment. This increased intracellular leptin was accompanied by a corresponding increase in secreted leptin. Under these conditions, there were no detectable changes in leptin protein levels in the LDM. These results also indicate 

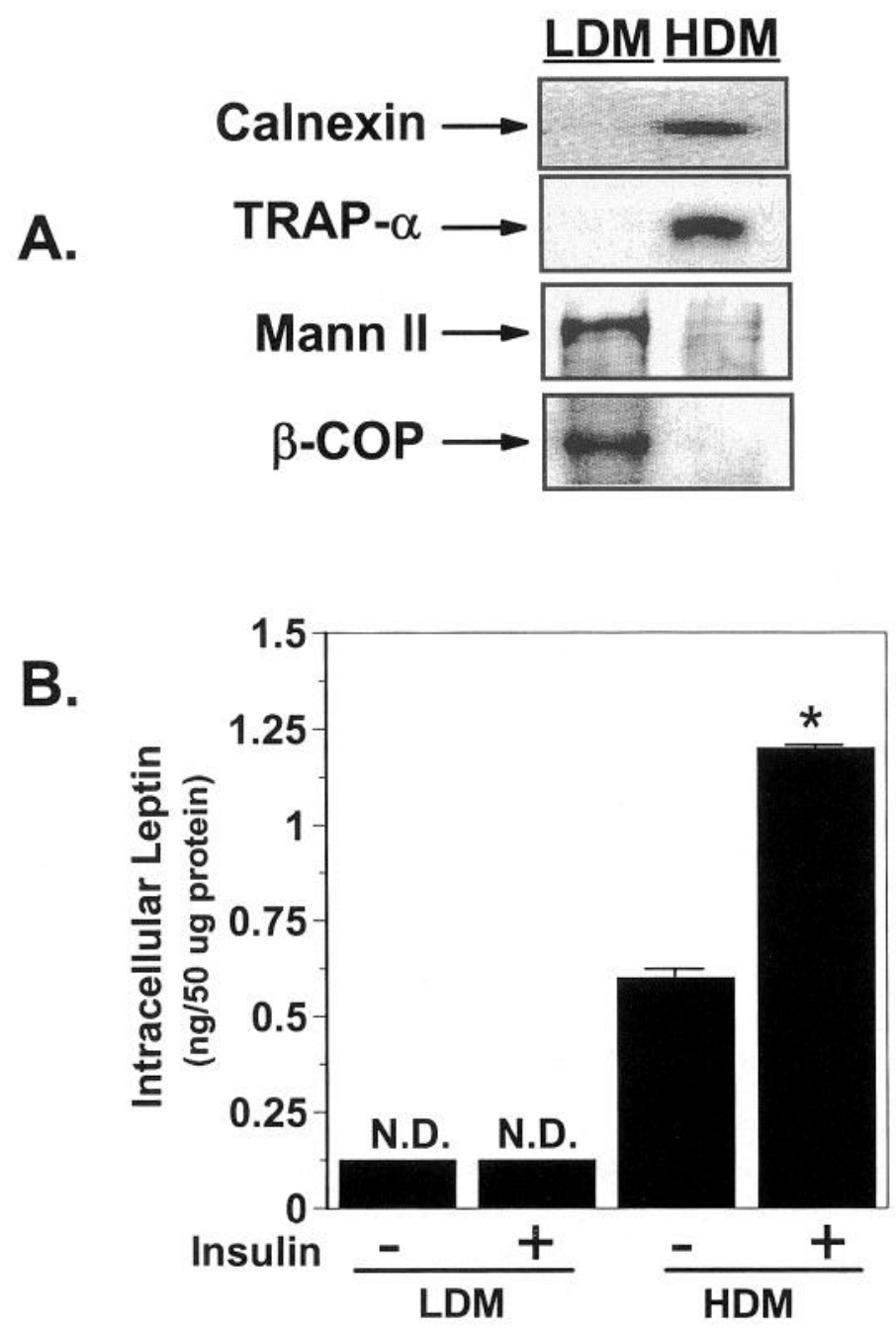

FIG. 8. Effect of insulin on the subcellular distribution of leptin. (A) Membrane fractions were prepared from freshly isolated rat adipocytes. An aliquot containing an equal amount of protein (10-25 $\mu \mathrm{g}$ ) from LDM or HDM was solubilized in SDS-PAGE sample buffer, resolved by SDS-PAGE, and electrophoretically transferred to nitrocellulose membranes. For marker enzyme assays, membranes were subjected to immunoblotting using primary antibodies for calnexin, TRAP- $\alpha$, mannosidase II (Mann II), or $\beta$-COP. (B) Leptin content was determined in HDM and LDM fractions from rat adipocytes incubated in the absence or presence of $100 \mathrm{nM}$ insulin for 2 hours. The leptin content of each sample was measured using a commercially available rat leptin RIA kit. Results are representative of three independent experiments ( $n=8$ per assay) and are expressed as the mean \pm S.E.M. N.D. $=$ below the limit of detection. ${ }^{*} \mathrm{P}<0.05 \mathrm{vs}$. HDM in the absence of insulin. 
that leptin-containing vesicles are distinct from the insulin-sensitive, Glut4-containing vesicles that are present in the LDM. Our findings are consistent with immunofluorescence data of Barr and coworkers (1997) that showed co-localization of leptin with the ER-specific protein calnexin and insulin stimulation of the release of leptin from this compartment.

\section{Effect of Brefeldin A on Leptin Secretion and Accumulation of Leptin in Golgi Compartments at $20^{\circ} \mathrm{C}$}

It is generally accepted that proteins destined for secretion leave the ER and transit through the Golgi for final processing on their way to the cell surface. Barr and colleagues (1997) showed that insulin stimulated the transport of leptin from the ER without any observable accumulation in secretory vesicles. They hypothesized that since leptin is not glycosylated, it is not retained and rapidly transits the Golgi. Similarly, we were unable to detect changes in leptin content in the Golgi-enriched LDM. To test this hypothesis, and to further define the intracellular localization of leptin and its transit through the secretory pathway, adipocytes were incubated in the absence or presence of brefeldin A, which causes Golgi disassembly. Brefeldin A treatment blocked both basal and insulin-stimulated leptin secretion from rat adipocytes (Figure 9A). As indicated in Figure 9B, this resulted in a dramatic accumulation of intracellular leptin (i.e., a 12-fold increase) in the HDM from basal cells. In the presence of brefeldin A, insulin stimulated an even-greater accumulation of intracellular leptin (i.e., a 24-fold increase). These findings suggest a role of intact Golgi membranes in secretion of leptin and further confirm that insulin stimulates an increase in intracellular leptin content. In addition, these data suggest that secretion of the proposed preformed, insulinsensitive pool of leptin-containing vesicles requires the presence of intact Golgi membranes. Thus, these preformed, insulin-sensitive, leptin-containing vesicles are most likely pre-Golgi or are derived from an intermediate Golgi compartment. Furthermore, the primary insulin-stimulated component is to increase leptin protein levels. This model is similar to that shown for another adipocyte-derived protein, Acrp30, in which a portion of Acrp30 is apparently sorted into regulated secretory vesicles whose initial exocytosis is stimulated by insulin. The remainder are sorted into vesicles that undergo constitutive exocytosis (Bogan and Lodish, 1999). The exact nature of this model of secretion in adipocytes is unclear and requires further analysis.

The brefeldin A experiments described here provide evidence for a role of the Golgi complex in leptin secretion. In an effort to show transit of leptin through the Golgi, we determined the extent of leptin secretion and its subcellular distribution in experiments performed at $20^{\circ} \mathrm{C}$. At this temperature, secretory proteins exit the ER and enter the Golgi, where they accumulate in late Golgi compartments (Matlin and Simons, 1983). At $20^{\circ} \mathrm{C}$, leptin secretion was completely 
A.

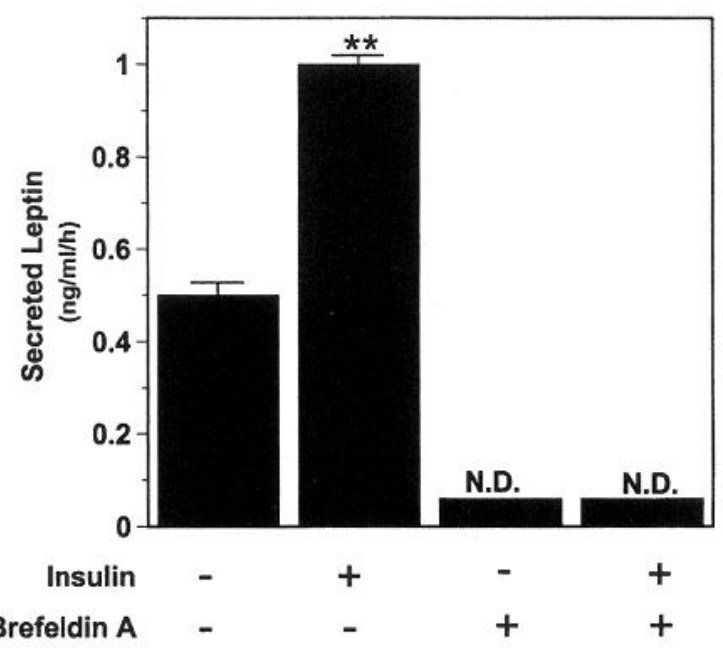

B.

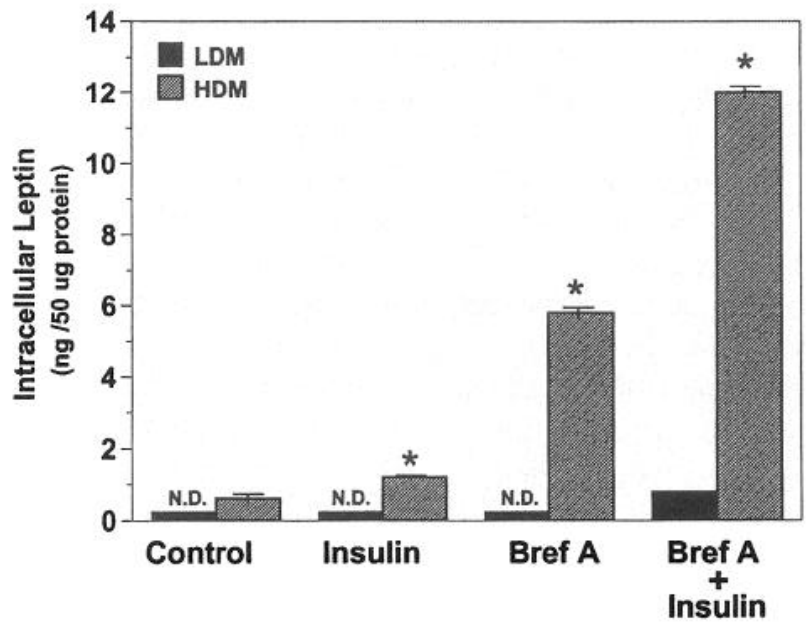

FIG. 9. Effect of brefeldin A on basal and insulin-stimulated leptin secretion and intracellular leptin content. Adipocytes were preincubated in the absence and presence of $20 \mu \mathrm{g} / \mathrm{ml}$ brefeldin A for 30 minutes. The conditioned medium was changed and the adipocytes were incubated for 2 hours with or without $100 \mathrm{nM}$ insulin in the absence or presence of $20 \mu \mathrm{g} / \mathrm{ml}$ brefeldin A. (A) Secreted leptin contained in the conditioned medium was measured by RIA. (B) HDM and LDM were prepared and the leptin content of each was measured by RIA. Results are means \pm S.E.M. for three independent experiments $(\mathrm{n}=10$ per assay). N.D. $=$ below the limit of detection. $* \mathrm{P}<0.05 \mathrm{vs}$. relative to control $\mathrm{HDM} ; * * \mathrm{P}<0.02$ vs. control. 
A.

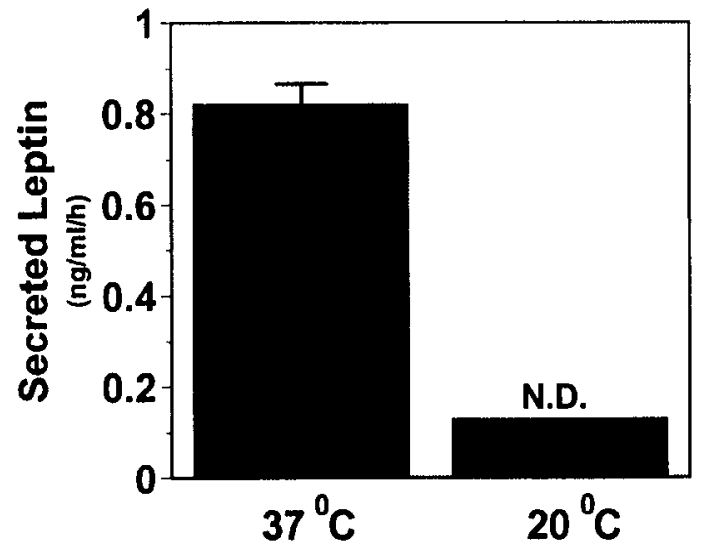

B.

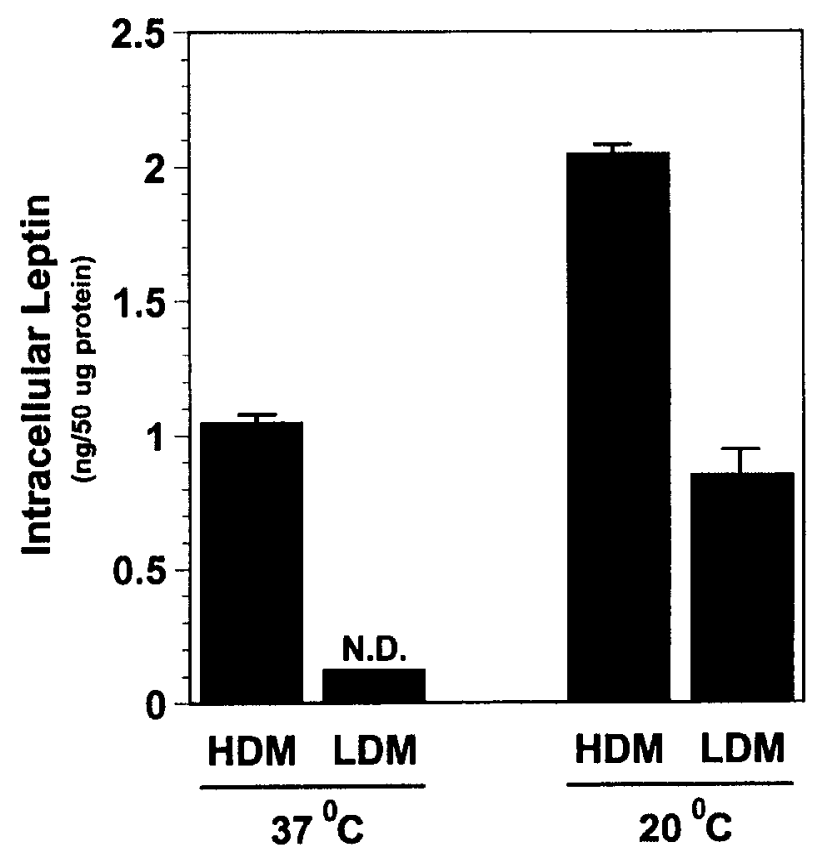

FIG. 10. Secretion and subcellular distribution of leptin at $20^{\circ} \mathrm{C}$. Isolated rat adipocytes were subjected to incubation at $37^{\circ} \mathrm{C}$ or $20^{\circ} \mathrm{C}$ for 1.5 hours. (A) Conditioned medium was subjected to RIA for leptin. (B) Cells were homogenized and HDM and LDM fractions prepared and analyzed for leptin content by RIA. Results are means \pm S.E.M. for two independent experiments. N.D.= below the limit of detection. 
inhibited (Figure 10A). Analysis of intracellular leptin showed a two-fold increase in HDM-derived leptin. Consistent with a block in exit from the Golgi at $20^{\circ} \mathrm{C}$, there was an approximately 10-fold increase in leptin content in the Golgi-enriched LDM compartment (Figure 10B).

\section{Analysis of Leptin-containing Vesicles by Sucrose Gradient Ultracentrifugation}

To further characterize LDM- and HDM-derived, leptin-containing intracellular vesicles, we performed velocity and density gradient ultracentrifugation. The distribution of total protein in LDM or HDM, as resolved by velocity gradient centrifugation, is shown in Figure 11A. Figure 11B shows a typical immunoblot for pantophysin contained in fractions derived from the gradients (fractions are shown from bottom to top, i.e., fraction 1 is bottom of the gradient). Figures $11 \mathrm{C}$ and 11D depict the velocity gradient profiles for leptin and pantophysin from the LDM and HDM, respectively. LDM-derived vesicles containing leptin fractionated near the bottom of the gradient and were largely separated from the total protein and pantophysin vesicles, suggesting there is little overlap of these markers in this subcellular compartment. In contrast, the profiles of pantophysin and

A.

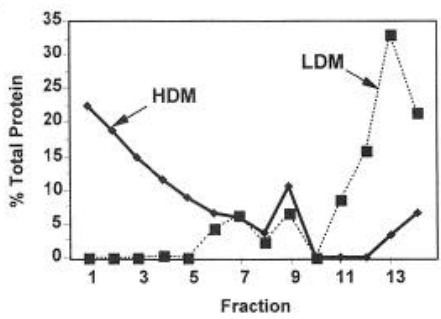

B.

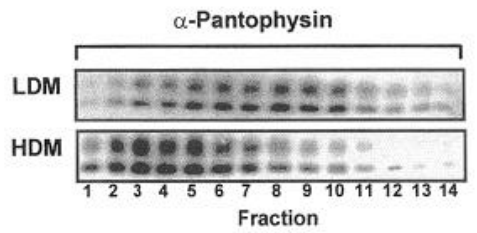

C.

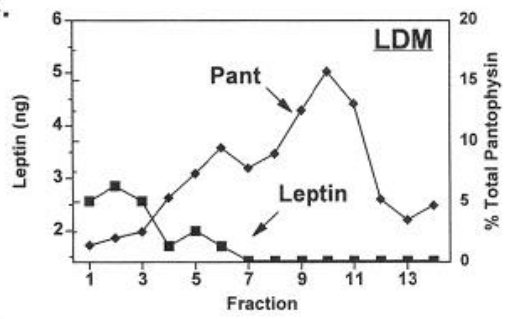

D.

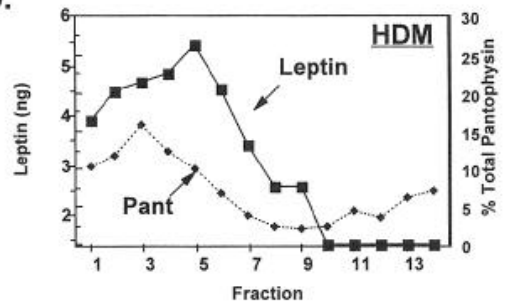

FIG. 11. Velocity gradient centrifugation analysis of subcellular fractions. LDM and HDM subcellular fractions were subjected to velocity gradient centrifugation. Fractions were collected from bottom (Fraction 1) to top (Fraction 14) and subjected to protein analysis (A). (B) Aliquots of each fraction were subjected to immunblot analysis with $\alpha$-pantophysin. (C) and (D) show a typical profile for pantophysin, as determined by densitometric scans of autoradiograms (dotted line) and leptin content as determined by RIA (solid line) from LDM and HDM, respectively. Results are representative of three or more independent experiments. 
leptin from HDM-derived vesicles indicate a significant overlap (Figure 11D) and suggest that these vesicles are of similar size.

\section{Immunopurified Pantophysin Vesicles Contain Leptin}

Gradient profiles of leptin and pantophysin from HDM-derived vesicles showed a significant overlap, which suggested that these vesicles were of similar size and buoyant density. The similar profiles of these proteins and the presence of pantophysin on various intracellular vesicles raised the possibility that leptin is potentially a cargo protein in a population of pantophysin-containing vesicles. To address this, pantophysin-containing vesicles were immunopurified. In these studies, internal membrane fractions were incubated with a nonrelated $\operatorname{IgG}$ or with an antibody specific for the C-terminus of pantophysin, then immunopurified vesicles (IPVs) were analyzed for leptin content and pantophysin. As illustrated in Figure 12A, immunoblotting showed that vesicles containing the two isoforms of pantophysin (native, $28.5 \mathrm{kDa}$, and glycosylated, $38 \mathrm{kDa}$ ) detected in rat adipocytes were specifically immunoadsorbed with $\alpha$-pantophysin but not with non-immune IgG. Subsequent analysis of the same IPVs by radioimmunoassay (Figure 12B) showed that leptin is, indeed, a resident cargo protein in a subpopulation of pantophysin-containing vesicles. Leptin was not detected in the nonimmune sample. Thus, using pantophysin as a marker and $\alpha$-pantophysin as an affinity reagent to purify these vesicles, we have been able to partially purify and enrich for leptin-containing vesicles. The exact origin of these vesicles and their content require further investigation.

\section{Summary and Conclusions}

The adipocyte is an important site of synthesis and secretion of factors that regulate a variety of metabolic processes. Alterations in the production of these factors are associated with - and can play a primary role in - the pathophysiology of several disease states, including obesity, hypertension, cardiovascular anomalies, hyperglycemia, insulin resistance, and type 1 and type 2 diabetes. Despitc the volume of protein cargo and vesicle traffic within the adipocyte, very little is known regarding the synthesis and secretion of adipocyte-secretory products. There have been significant advances in our understanding of the acute regulated translocation of Glut 4 . These include the signaling pathways downstream of IRS proteins and PI 3-kinase as well as SNARE proteins that comprise some of the molecular machinery necessary for docking and fusion of the Glut4 vesicles (see Figure 13 for a summary model). However, the pathways utilizcd by other secreted proteins have not been investigated to any significant extent. In an attempt to better define these pathways, we have analyzed the agonist-stimulated biosynthesis and secretion of leptin and characterized the intracellular vesicle 

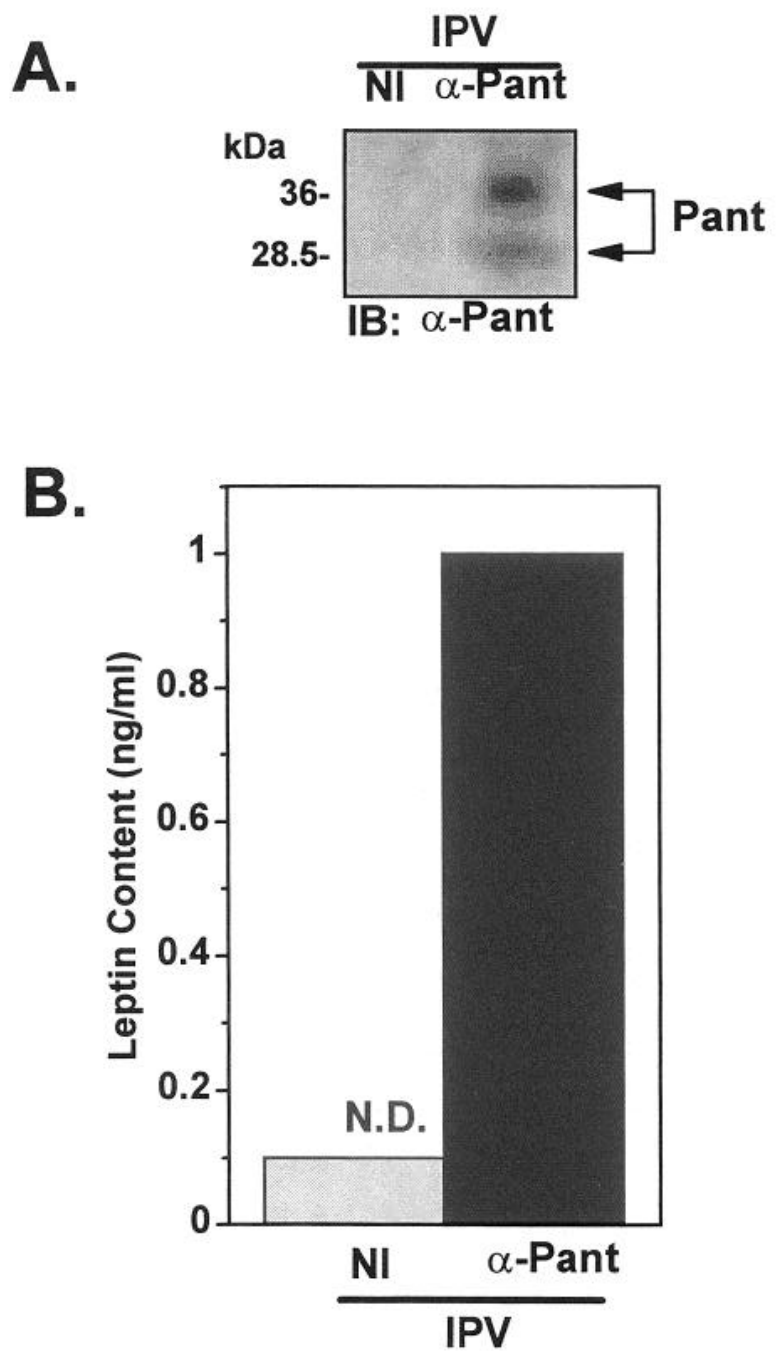

FIG. 12. Immunopurification of pantophysin-containing vesicles. Pantophysin-containing vesicles were purified from an intracellular membrane preparation by immunoadsorption using a non-immune IgG $(\mathrm{NI})$ or specific pantophysin antisera ( $\alpha$-Pant). Associated proteins were analyzed for pantophysin by immunoblot (A) or for leptin content (B) by RIA. Results are representative of two independent experiments. N.D. $=$ below the limit of detection. 


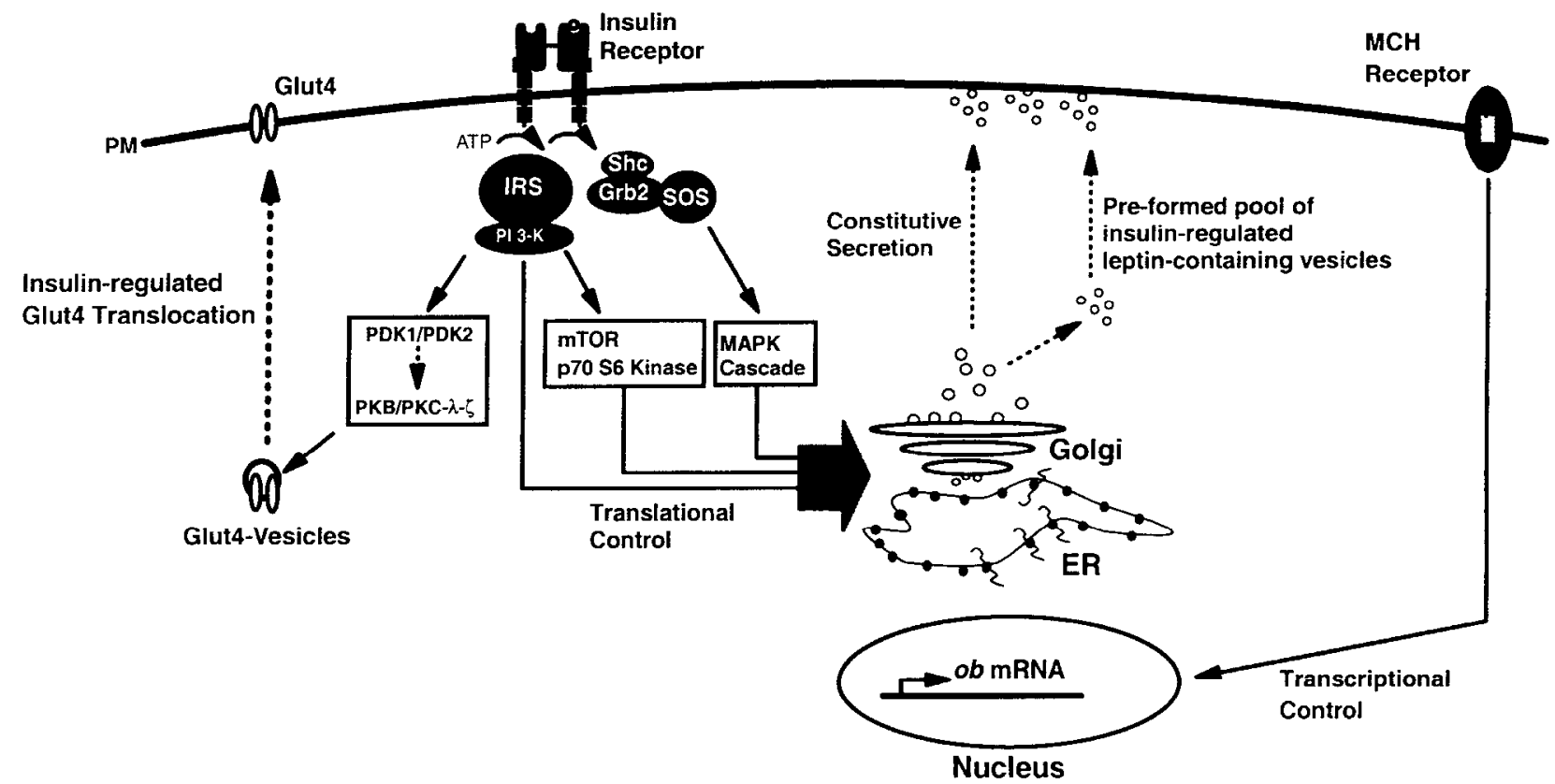

FIG. 13. A model of insulin-regulated vesicle transport pathways in adipocytes. Insulin stimulates the translocation of Glut4-containing vesicles from an intracellular compartment to the plasma membrane (PM). This involves signaling pathways requiring IRS proteins, PI 3-kinase, and the downstream kinases PDK1/PDK2, PKB, and the atypical PKCs $\lambda$ and $\zeta$. This process also requires SNARE proteins such as VAMP-2 on the Glut 4 vesicle and syntaxin-4 and SNAP-23 at the PM. Insulin-stimulated leptin secretion occurs via at least two components. The major component involves increases in the intracellular content of leptin, while a minor component is the insulin-stimulated release of a small, preformed pool of leptin-containing vesicles. These mechanisms require intact signaling downstream of PI 3-kinase, pp70 S6 kinase, and the MAPK cascade but are not dependent on ongoing transcription. In contrast, MCH-stimulated or glucocorticoid-stimulated leptin secretion occurs primarily via transcriptional mechanisms. 
transport of leptin-containing vesicles. Our findings suggest that insulin-stimulated leptin secretion involves at least two components. The major component is the ability of insulin to stimulate an increase in intracellular leptin content that appears to traffick out of the cell in a constitutive manner. A second, relatively minor component is the ability of insulin to stimulate the secretion of a small, preformed pool of leptin-containing vesicles. These pathways are independent of ongoing transcription but do require intact signaling pathways through PI 3-kinase, pp70 S6 kinase, and the MAPK cascade. We also report that leptin secretion is stimulated by treatment of adipocytes with glucocorticoids or $\mathrm{MCH}$. These pathways appear primarily to utilize a transcriptional mechanism to increase $o b$ mRNA, with subsequent increases in leptin protein production. Continued elucidation of the molecular components involved in secretion of leptin should provide a more-comprehensive understanding of the vesicle transport and secretory pathways in adipocytes.

\section{ACKNOWLEDGMENTS}

This work was supported by National Institutes of Health grants R01 DK-51668 (B.C.) and National Research Service Award DK-09745 (R.L.B.).

\section{REFERENCES}

Auwerx, J., and Staels, B. (1998). Lancet 351, 737-742.

Barr, V.A., Malide, D., Zarnowski, M.J., Taylor, S.I, and Cushman, S.W. (1997). Endocrinology 138, 4463-4472.

Baumann, H., Morella, K.K., White, D.W., Dembski, M., Bailon, P.S., Kim, H., Lai, C.F., and Tartaglia, L.A. (1996). Proc. Natl. Acad. Sci. U.S.A. 93, 8374-8378.

Bcnoist, F., Paulina, L., McDonncl, M., Doclle, Il., Milnc, R., and McPhcrson, R. (1998). J. Biol. Chem. 272, 23572-23577.

Birnbaum, M.J. (1992). Intl. Rev. Cytol. 137, 239-297

Bjorbaek, C., Elmquist, J.K., Frantz, J.D., Shoelson, S.E., and Flier, J.S. (1998). Mol Cell 1, 619-625.

Bogan, J.S., and Lodish, H.F. (1999). J. Cell Biol. 146, 609-620.

Bradley, R.L., and Cheatham, B. (1999). Diabetes 48, 272-278.

Bradley, R.L., Kokkotou, E.G., Maratos-Flier, E., and Cheatham, B. (2000). Diabetes 49, 1073-1077.

Brooks, C.C., Scherer, P.E., Cleveland, K., Whittemore, J.L., Lodish, H.F., and Cheatham, B. (2000). J. Biol. Chem. 275, 2029-2036.

Calakos, N., and Scheller, R.H. (1994). J. Biol. Chem. 269, 24534-24537.

Caro, J.F., Sinha, M.K., Kolaczynski, J.W., Zhang, P.L., and Considine, R.V. (1996). Diabetes 45, 1455-1462.

Casanueva, F.F., and Dieguez, C. (1999). Front. Neuroendocrinol. 20, 317-363.

Castell, J.V., Gomez-Lechon, M.J., David, M., Andus, T., Geiger, T., Trullenque, R., Fabra, R., and Heinrich, P.C. (1989). FEBS Lett. 242, 237-239.

Chambers, J., Ames, R.S., Bergsma, D., Muir, A., Fitzgerald, L.R., Hervieu, G., Dytko, G.M., Foley, J.J., Martin, J., Liu, W.S., Park, J., Ellis, C., Ganguly, S., Konchar, S., Cluderay, J., Leslie, R., Wilson, S., and Sarau, H.M. (1999). Nature 400, 261-265.

Cheatham, B., and Kahn, C.R. (1995). Endocr. Rev. 16, 117-142. 
Cheatham, B., Volchuk, A., Kahn, C.R., Wang, L., Rhodes, C.J., and Klip, A. (1996). Proc. Natl. Acad. Sci. U.S.A. 93, 15169-15173.

Ciantlone, K.M., Sniderman, A.D., Dallongeville, J., Bertrand, M., Raffai, E., and Davignon, J. (1992). Clin. Invest. Med. 15, 132-140.

Claffey, K., Wilkison, W., and Spiegelman, B. (1992). J. Biol. Chem. 267, 16317-16322.

Clement, K., Vaisse, C., Lahlou, N., Cabrol, S., Pelloux, V., Cassuto, D., Gourmelen, M., Dina, C., Chambaz, J., Lacorte, J.M., Basdevant, A., Bougneres, P., leBouc, Y., Froguel, P., and GuyGrand, B. (1998). Nature 392, 398-401.

Cohen, B., Novick, D., and Rubinstein, M. (1996). Science 274, 1185-1188.

Deng, C., Moinat, M., Curtis, L., Nadakal, A., Preitner, F., Boss, O., Assimacopoulos-Jeannet, F., Seydoux, J., and Giacobino, J.P. (1997). Endocrinology 138, 548-552.

De Vos, P., Saladin, R., Auwerx, J., and Staels, B. (1995). J. Biol. Chem. 270, 15958-15961.

Dobson, D., Kambe, A., Block, E., Dion, T., Lu, H., Castellot, J., and Spiegelman, B. (1990). Cell 61, 223-230.

Elferink, L.A., and Scheller, R.H. (1995). Prog. Brain Res. 105, 79-85.

Elmquist, J.K., Maratos-Flier, E., Saper, C.B., and Flier, J.S. (1998). Nat. Neurosci. 1, 445-450.

Emilsson, V., Liu, Y.L., Cawthorne, M.A., Morton, N.M., and Davenport, M. (1997). Diabetes 46, 313-316.

Frederich, R.C., Lollmann, B., Hamann, A., Napolitano-Rosen, A., Kahn, B.B., Lowell, B.B., and Flier, J.S. (1995). J. Clin. Invest. 96, 1658-1663.

Funahashi, T., Nakamura, T., Shimomura, I., Macda, K., Kuriyama, H., Takahashi, M., Arita, Y., Kihara, S., and Matsuzawa, Y. (1999). Intern. Med. 38, 202-206.

Gettys, T.W., Harkness, P.J., and Watson, P.M. (1996). Endocrinology 137, 4054-4057.

Ghilardi, N., Ziegler, S., Wiestner, A., Stoffel, R., Heim, M.H., and Skoda, R.C. (1996). Proc. Natl. Acad. Sci. U.S.A. 93, 6231-6235.

Grunfeld, C., and Feingold, K.R. (1991). Biotherapy 3, 143-158.

Gualillo, O., Lago, F., Garcia, M., Menendez, C., Senaris, R., Casanueva, F.F., and Dieguez, C. (1999). Endocrinology 140, 5149-5153.

Haass, N.K., Kartenbeck, J., and Leube, R.E. (1996). J. Cell Biol. 134, 731-746.

Halleux, C.M., Servais, 1., Reul, B.A., Detry, R., and Brichard, S.M. (1998). J. Clin. Endocrinol. Metab. 83, 902-910.

Hardie, L.J., Guilhot, N., and Trayhurn, P. (1996). Horm. Metab. Res. 28, 685-689.

Hay, J.C., and Scheller, R.H. (1997). Curr. Opin. Cell Biol. 9, 505-512.

Kieffer, T.J., Heller, R.S., and Habener, J.F. (1996). Biochem. Biophys. Res. Commun. 224, 522-527.

Kieffer, T.J., Heller, R.S., Leech, C.A., Holz, G.G., and Habener, J.F. (1997). Diabetes 46, 1087-1093.

Kroder, G., Kellerer, M., and Haring, H.U. (1996). Exp. Clin. Endocrinol. Diabetes 104(suppl. 2), 66 (abstr.).

Larsson, H., and Ahren, B. (1996). J. Clin. Endocrinol. Metab, 81, 4428-4432.

Lee, G.H., Proenca, R., Montez, J.M., Carroll, K.M., Darvishzadeh, J.G., Lee, J.l., and Friedman, J.M. (1996). Nature 379, 632-635.

Matlin, K., and Simons, K. (1983). Cell 34, 233-243.

Mercer, J.G., Hoggard, N., Williams, L.M., Lawrence, C.B., Hannah, L.T., and Trayhurn, P. (1996). FEBS Lett. 387, 113-116.

Min, J., Okada, S., Kanzaki, M., Elmendorf, J.S., Coker, K.J., Ceresa, B.P., Syu, L.J., Noda, Y., Saltiel, A.R., and Pessin, J.E. (1999). Mol. Cell 3, 751-760.

Mohamed-Ali, V., Pinkney, J.H., and Coppack, S.W. (1998). Int. J. Obes. Relat. Metab. Disord. 22 , 1145-1158

Moinat, M., Deng, C., Muzzin, P., Assimacopoulos-Jeannet, F., Seydoux, J., Dulloo, A.G., and Giacobino, J.P. (1995). FEBS Lett. 373, 131-134. 
Montague, C.T., Farooqi, I.S., Whitehead, J.P., Soos, M.S., Rau, H., Wareham, N.J., Sewter, C.P., Digby, J.E., Mohammed, S.N., Hurst, J.A., Cheetham, C.H., Early, A.R., Barnett, A.H., Prins, J.B., and O'Rahilly, S. (1997). Nature 387, 903-908.

Muller, G., Ertl, J., Gerl, M., and Preibisch, G. (1997). J. Biol. Chem. 272, 10585-10593.

Murakami, T., Iida, M., and Shima, K. (1995). Biochem. Biophys. Res. Commun. 214, 1260-1267.

Ouchi, N., Kihara, S., Arita, Y., Maeda, K., Kuriyama, H., Okamoto, Y., Hotta, K., Nishida, M., Takahashi, M., Nakamura, T., Yamashita, S., Funahashi, T., and Matsuzawa, Y. (1999). Circulation 100, 2473-2476.

Papaspyrou-Rao, S., Schneider, S.H., Petersen, R.N., and Fried, S.K. (1997). J. Clin. Endocrinol. Metab. 82, 1635-1637.

Pessin, J.E., Thurmond, D.C., Elmendorf, J.S., Coker, K.J., and Okada, S. (1999). J. Biol. Chem. 274, 2593-2596.

Pfeffer, S.R. (1999). Nat. Cell Biol. 1, E17-E22

Prins, J.B., Niesler, C.U., Winterford, C.M., Bright, N.A., Siddle, K., O'Rahilly, S., Walker, N.I., and Cameron, D.P. (1997). Diabetes 46, 1939-1944.

Qu, D., Ludwig, D.S., Gammeltoft, S., Piper, M., Pelleymounter, M.A., Cullen, M.J., Mathes, W.F., Przypek, J., Kanarek, R., and Maratos-Flier, E. (1996). Nature 380, 243-247.

Ravussin, E., Pratley, R.E., Maffei, M., Wong, H., Friedman, J.M., Bennett, P.H., and Bogardus, C. (1997). Nature Med. 3, 238-240.

Rentsch, J., and Chiesi, M. (1996). FEBS Lett. 379, 55-59.

Rice, A.M., Fain, J.N., and Rivkees, S.A. (2000). Endocrinology 141, 1442-1445

Rice, L.M., and Brunger, A.T. (1999). Mol. Cell 4, 85-95.

Saito, Y., Nothacker, H.P., Wang, Z., Lin, S.H., Leslie, F., and Civelli, O. (1999). Nature 400, 265-269.

Saladin, R., De Vos, P., Guerre-Millo, M., Leturque, A., Girard, J., Staels, B., and Auwerx, J. (1995). Nature 377, 527-529.

Scherer, P., Williams, S., Fogliano, M., Baldini, G., and Lodish, H.F. (1995). J. Biol. Chem. 270, 26746-26749.

Spooner, P., Chernick, S., Garrison, M., and Scow, R. (1979). J. Biol. Chem. 254, $10021-10029$.

St-Denis, J.F., and Cushman, S.W. (1998). J. Basic. Clin. Physiol. Pharmacol. 9, 153-165.

Strobel, A., Issad, T., Camoin, L., Ozata, M., and Strosberg, A.D. (1998). Nature Genet. 18, 213-215.

Tamori, Y., Kawanishi, M., Niki, T., Shinoda, H., Araki, S., Okazawa, H., and Kasuga, M. (1998). J. Biol. Chem. 273, 19740-19746.

Tartaglia, L.A., Dembski, M., Weng, X., Deng, N., Culpepper, J., Devos, R, Richards, G.J., Campfield, L.A., Clark, F.T., Deeds, J., Muir, C., Sanker, S., Moriarty, A., Moore, K.J., Smutko, J.S., Mays, G.G., Woolf, E.A., Monroe, C.A., and Tepper, R.I. (1995). Cell 83, 1263-1271.

Tellam, J.T., Macaulay, S.L., McIntosh, S., Hewish, D.R., Ward, C.W., and James, D.E. (1997). J. Biol. Chem. 272, 6179-6186.

Thiele, C., Hannah, M.J., Fahrenholz, F., and Huttner, W.B. (2000). Nature Cell Biol. 2, 42-49.

Trayhurn, P., Hoggard, N., Mercer, J.G., and Rayner, D.V. (1999). Int. J. Obes. Relat. Metab. Disord. 23(suppl. 1), 22-28.

Vaisse, C., Halaas, J.L., Horvath, C.M., Darnell, J.E., Jr., Stoffel, M., and Friedman, J.M. (1996). Nature Genet. 14, 95-97.

Weber, T., Zemelman, B.V., McNew, J.A., Westermann, B., Gmachl, M., Parlati, F., Sollner, T.H., and Rothman, J.E. (1998). Cell 92, 759-772.

Wei, S., Lai, K., Patel, S., Piantedosi, R., Shen, H., Colantuoni, V., Kraemer, F., and Blaner, W. (1997). J. Biol. Chem. 272, 14159-14165.

Weigle, D. (1997). Ann. Endocrinol. Paris 58, 132-136.

Zhang, Y., Proenca, R., Maffei, M., Barone, M., Leopold, L., and Friedman, J. (1994). Nature 372, 425-432. 


\title{
Central Melanocortins and the Regulation of Weight During Acute and Chronic Disease
}

\author{
Daniel L. Marks* and Roger D. Cone ${ }^{\dagger}$ \\ *Department of Pediatric Endocrinology, 'The Vollum Institute, \\ Oregon Health Sciences University, Portland, Oregon 97201
}

\begin{abstract}
Recent advances in our understanding of the regulation of body weight, appetite, and metabolic rate have highlighted the role of the adipose-derived hormone leptin and its receptor as fundamental modulators of these processes. Investigations of the neural targets for leptin action - as well as characterization of the agouti obesity syndrome - have, in turn, led to the discovery of fundamental neural pathways involved in the central regulation of energy homeostasis. In particular, the central melanocortin system has been shown to regulate appetite and metabolic rate in rodents; mutations in this system have been demonstrated to result in obesity in humans. Overall, the melanocortin system appears to function as a bidirectional rheostat in the regulation of energy intake and expenditure in rodents and potentially in humans. The first section of this chapter will focus on the development of our understanding of melanocortin physiology in the context of obesity. In particular, recent data regarding the interplay between melanocortin and neuropeptide Y (NPY) signaling at a cellular level will be discussed. The following section will discuss the hypothesis that melanocortin signaling plays a role in pathological weight luss and hypermetabolism observed in murine cachexia models. The potential role of this system in integrating a variety of anorexic and cachexic signals, as well as the potential for its pharmacological manipulation in the treatment of human cachexia, will be discussed.
\end{abstract}

\section{The Central Melanocortin System and Energy Homeostasis}

\section{A. INTRODUCTION}

The last decade has witnessed significant advances in our understanding of energy homeostasis and regulation of body weight. The recent cloning and characterization of the genes responsible for obesity syndromes in the mouse - fatty, tubby, obesity, diabetes, and agouti - had led to our current understanding of the role of the adipocyte hormone leptin and its receptor in the feedback regulation of appetite and metabolic rate (Chua et al., 1996; Tartaglia et al., 1995; Zhang et al., 1997). Furthermore, we have started to unravel the neuronal pathways that mediate many of the effects of leptin and exert central control over processes fundamental to the intake, use, and storage of body fuels. In particular, the cloning of the agouti gene (Buitman et al., 1992; Miller et al., 1993) and the discovery of 
its mechanism of action (Fan et al., 1997; Huszar et al., 1997; Lu et al., 1994) led to the identification of a set of neuronal pathways that we will refer to as the melanocortin system. In general, the melanocortin system can be defined as the hypothalamic and brainstem neurons expressing pro-opiomelanocortin (POMC), the hypothalamic neurons coexpressing neuropeptide Y (NPY) and the melanocortin antagonist agouti-related protein (AGRP), and the neurons downstream of these systems. For the purposes of this chapter, these downstream neurons will be defined by the presence of the two primary central melanocortin receptors, melanocortin 3 receptor (MC3-R) (Roselli-Rehfiss et al., 1993) and melanocortin 4 receptor (MC4-R) (Mountjoy et al., 1994).

\section{B. LEPTIN AND ENERGY HOMEOSTASIS}

Leptin is secreted by adipocytes. It regulates adiposity and metabolic rate by reducing food intake and increasing energy expenditure (Campfield et al., 1995; Halaas et al., 1995; Pelleymounter et al., 1995; Zhang et al., 1994). Experimental elevation of leptin within the physiological range produces weight loss and relative anorexia, while decreased leptin levels lead to the complex neuroendocrine response that occurs during starvation (Ahima et al., 1996; Halaas et al., 1997). Numerous studies of leptin levels in humans of various age groups and disease states have been published (for review, see Roemmich and Rogol, 1999). In normal infants, school-aged children, and adolescents, the normative range of serum leptin varies with age and becomes sexually dimorphic during adolescence. Within groups matched for age and gender, leptin levels are directly proportional to body fat mass, as reflected in the calculated body mass index (BMI) or measured with other methods (Blum et al., 1997; Clayton et al., 1997; Roemmich and Rogol, 1999). Given that elevations in circulating leptin would normally result in anorexia and weight loss, the elevated leptin concentrations consistently found in obese individuals have been interpreted as an indication of leptin resistance (Considine et al., 1996). Diseases associated with malnutrition - including anorexia nervosa, Crohn's disease, cystic fibrosis, and acquired immune deficiency syndrome (AIDS) - confer low leptin levels. This highlights the energy-conserving role of leptin in regulating the neuroendocrine response to starvation (Ahima et al., 1996). The possibility that a primary defect in leptin expression may lead to human disease was confirmed in studies of highly inbred kindreds with extreme obesity (Montague et al., 1997; Ozata et al., 1999).

However, human leptin deficiency is extremely rare. The obesity syndrome that results bears little resemblance to the polygenic obesity that has become epidemic in our society. In humans, leptin deficiency results primarily in a combination of extreme hyperphagia, hypogonadism, and decreased sympathetic tone, with some suggestion of immune dysfunction as well (Farooqi et al., 1999; Montague et al., 1997; Ozata et al., 1999). In the mouse, hypercorticosteronemia, 
hypometabolism, and hyperinsulinemia also are observed and result in impaired growth of bone and lean tissue (Ahima et al., 1996; Ozata et al., 1999).

\section{MELANOCORTIN OBESITY SYNDROMES AND CENTRAL MELANOCORTIN RECEPTORS}

Several observations have led to the proposal that disruption of appropriate melanocortin signaling may be a more-common cause of obesity in humans. First, as will be described, the disruption of melanocortin signaling in various rodent models recapitulates the various features observed in obese individuals. Second, obesity syndromes involving defects in two different steps in the melanocortin pathway have been described in humans. One of these syndromes represents the most-common monogenic form of obesity yet described. Specifically, families with rare, deleterious mutations in the POMC gene have been described in whom the defect results in a syndrome of red hair, corticotropin (ACTH) deficiency, and obesity (Krude et al., 1998). Heterozygous mutations in MC4-R have been reported to be associated with common pediatric obesity (Vaisse et al., 1998; Yeo et al., 1998). Another group has demonstrated strong genetic linkage of obesity to the POMC locus on chromosome 2p21, although no defects in the coding region of the gene were found (Comuzzie et al., 1997). Collectively, these data argue strongly that the melanocortin system plays a role in the regulation of energy homeostasis in mice and humans.

Among the multiple gene mutations that lead to an obese phenotype in mice, the dominantly inherited lethal yellow mouse $\left(A^{Y} /-\right)$ exhibits a phenotype that is most reminiscent of common human obesity. These mice are hyperphagic, have an efficient metabolism, and develop insulin resistance as they age (Frigeri et al., 1983,1988; Michaud et al., 1994; Yen et al., 1994). These animals have normal reproductive and adrenal axies (Wolff and Flack, 1971) and increased somatic growth (Wolff, 1963), features that are found in the development of obesity in humans. The basis for this phenotype was found to be due to a genetic rearrangement that led to the constitutive and ubiquitous expression of the agouti peptide, a paracrine factor that is normally involved in regulation of pigmentation (Bultman et al., 1992). Agouti was shown to be an antagonist of melanocortin signaling at the MC-1 receptor on pigment cells. There, it functioned to block the melanocyte-stimulating hormone (MSH)-stimulated synthesis of eumelanin ( $\mathrm{Lu}$ et al., 1994), thereby explaining the yellow coat color seen in this strain (Bultman et al., 1992; Duhl et al., 1994; Miller et al., 1993). Remarkably, agouti also was found to be a specific, high-affinity antagonist of the hypothalamic MC4-R ( $\mathrm{Lu}$ et al., 1994). In this model, both agouti and MC4 receptor mRNAs were present in areas of the hypothalamus known to be involved in feeding behavior. This led to our proposal that aberrant antagonism of the central MC4-R was the direct cause of the agouti obesity syndrome. Investigations using a $\mathrm{MC} 4$ receptor antagonist, 
SHU9119, confirmed this hypothesis by demonstrating an increase in feeding behavior in animals injected intracerebroventricularly (ICV) with this compound (Fan et al., 1997). Furthermore, ICV injection of the small $\alpha-\mathrm{MSH}$ agonist, MTII, inhibited feeding behavior. This inhibition of feeding behavior could be reversed by administration of SHU9119. The antagonist data argue that the endogenous POMC neurons exert a tonic inhibitory effect on feeding and energy storage via their release of desacetyl- $\alpha$-MSH, the primary melanocortin cleavage product in the brain, at downstream sites containing MC4-R.

The importance of melanocortinergic neurons and the MC4-R in the regulation of feeding behavior was reinforced with the targeted disruption of the MC4-R (Huszar et al., 1997). Homozygous knockout animals recapitulated all of the primary metabolic, growth, and behavioral phenotypes of the agouti obesity syndrome. Furthermore, mice heterozygous for the MC4-R are more obese than wild-type mice, suggesting that a decrease in MC4-R number would lead to obesity. Thus, the MC4-R appears to function as a rheostat that can provide the bidirectional tuning necessary to maintain relative energy stability in a variety of environmental conditions.

Investigation of the agouti obesity syndrome led to the cloning and characterization of AGRP, an endogenous, hypothalamic, melanocortin receptor antagonist (Fong et al., 1997; Ollmann et al., 1997). The function of AGRP as an endogenous melanocortin receptor antagonist was demonstrated when its ectopic expression in transgenic mice was found to cause an obesity syndrome analogous to that seen in the MC4-RKO animal (Ollmann et al., 1997). The pattern of AGRP expression and release is also consistent with its role as a melanocortin antagonist. It is expressed in high levels almost exclusively in the arcuate nucleus cell bodies that project to MC4-R-expressing neurons in areas such as the dorsomedial hypothalamus (DMH), the paraventricular nucleus (PVN), and the septal region (Haskell-Luevano et al., 1999). Furthermore, AGRP expression is robustly stimulated by fasting and inhibited by leptin (Broberger et al., 1998; Chen et al., 1999; Mizuno and Mobbs, 1999; Shutter et al., 1997), bolstering the argument that its primary role is as an endogenous melanocortin antagonist.

\section{CELLULAR INTEGRATION OF OREXIGENIC AND ANOREXIGENIC SIGNALS}

Interestingly, it appears that most AGRP neurons coexpress NPY (Broberger et al., 1998; Smith, 1993), a molecule with well-described effects on energy homeostasis (for review, see Kalra et al., 1999). NPY is produced in hypothalamic nuclei known to regulate appetite and metabolism. It is a potent orexigen when injected centrally (Baskin et al., 1999; Billington et al., 1991; Blasquez et al., 1995; Gerald et al., 1996; Larsen et al., 1997; Stanley et al., 1985). The role of NPY in the feedback effects of leptin first was indicated by observations that leptin 
deficiency resulted in a significant upregulation of NPY expression, while leptin administration caused a decrease in expression (Stephens et al., 1995; Wilding et al., 1993). The role of NPY in mediating leptin feedback was bolstered further by the observation that genetic deletion of NPY in leptin-deficient animals resulted in a significant decrease in their degree of obesity (Erickson et al., 1996). In general, it appears that NPY synthesis and secretion are upregulated in most models of energy deficiency or increased metabolic demand (Inui, 1999b; Smith, 1993). Thus, a decrease in the level of NPY expression was proposed as one potential mechanism for the anorexic effects of leptin. The demonstration that the arcuate POMC (Cheung et al., 1997) and NPY/AGRP (Hakansson et al., 1996) neurons express the leptin receptor strengthened this argument and led us to propose that effector neurons downstream of leptin receptors would functionally integrate NPY and melanocortin signals (Cowley et al., 1999). The output of these effector neurons would vary, depending on their identity, with the overall effect being the complex behavioral and metabolic response seen with alterations in leptin levels.

Anatomical support for this idea was derived from confocal microscopic analysis, which clearly demonstrated neuronal fibers containing both NPY and AGRP and neuronal fibers containing $\alpha$-MSH adjacent to single-cell bodies within the $\mathrm{mpPVH}$, as well as the presence of MC4-R immunoreactivity (IR) within the same region (Cowley et al., 1999). Interestingly, MC4-R IR was found primarily on the terminals of axons in the section of the PVH that was studied, implying that MC4-R activity may be presynaptic.

Functional antagonism of melanocortin agonists and NPY within the PVH also was demonstrated in this study. Here, microinjections of SHU9119 (a MC3/MC4-R antagonist) and MTII (a MC3/MC4 agonist) into the PVH were first shown to have opposing actions on food intake and oxygen consumption. As expected from previous work, SHU9119 not only prevented the MTII-induced inhibition of feeding but also produced a stimulation of feeding when injected alone. This is consistent with the idea that melanocortin peptides provide a tonic inhibition of food intake via their binding to MC4-R in the hypothalamus. Demonstration of the potent effect of unilateral PVH injections of these compounds was followed by an analysis of the functional interaction between NPY and MTII in this brain region. PVH microinjections of NPY produced the expected potent orexigenic activity. However, preinjection of MTII 10 minutes prior to the NPY injection completely suppressed the orexigenic effect of NPY. The ability of MTII microinjection into the PVH to stimulate oxygen consumption was demonstrated in this study. This observation, combined with previous demonstration of a decrease in energy expenditure with PVN NPY microinjection (Billington et al., 1991; Egawa et al., 1991), argues strongly that information from POMC and 
NPY/AGRP neurons can be functionally integrated within the PVH to regulate both feeding and energy expenditure.

To further explore the cellular basis for this interaction, whole-cell recordings were made from mpPVH neurons in brain slice preparations. The neurons were chosen on the basis of a predominant inhibitory synaptic response to local electrical stimulation, recorded as an outward synaptic current (IPSC). In the region of the PVH studied, nearly 80 percent of neurons showed a modification of this IPSC in response to bath application of NPY or a melanocortin agonist. The application of MTII (10 nM) resulted in a significant increase in current amplitude $(25 \%+4 \%)$, with an $\mathrm{EC}_{50}$ of $1.4 \mathrm{nM}$. In 23 of 24 of the cells responsive to MTII, NPY caused a significant inhibition of the IPSC $(29 \%+3 \%)$. These effects were shown to be specific, as bath application of SHU9119 blocked the effect of MTII but had no effect on the inhibition brought about by NPY application. Furthermore, the effects of the synthetic melanocortin agonist and antagonist were identical to those seen with applications of the endogenous ligands, $\alpha-\mathrm{MSH}$ and AGRP, respectively. Further studies employed specific channel agonists and antagonists to demonstrate that the IPSC being studied in these cells was due to alterations in gamma aminobutyric acid (GABA) release and that this effect was mediated at a presynaptic level. Collectively, these observations led to the construction of a theoretical general model for the cellular integration of NPY/AGRP signals with melanocortin signals. Obviously, other orexigenic and anorexigenic neuropeptides and neurotransmitters will play a role in modulating the effector neurons. The area of the PVH studied is unlikely to be the sole site of integration of these signals, since melanocortin effects on feeding and metabolism have been demonstrated within the brainstem (Grill et al., 1998). Nonetheless, these data provide the first direct evidence of a functional cellular integration of these two crucial neural mediators of appetite and metabolic efficiency.

\section{The Role of Melanocortins in Anorexia and Cachexia}

\section{A. THE ROLE OF CYTOKINES}

The role of nutrition and balanced metabolism in normal growth and development is well known. Individuals affected with either acute or chronic diseases often show disorders of nutrient balance. Furthermore, children may have no obvious organic disease, yet gain weight poorly, resulting in the observational diagnosis of failure to thrive. In some cases, a devastating state of malnutrition known as cachexia arises, brought about by a synergistic combination of a dramatic decrease in appetite and an increase in metabolism of fat and lean body mass. This combination is found in a number of disorders, including cancer, cystic fibrosis, AIDS, rheumatoid arthritis, congestive heart failure, and renal failure 
(Tisdale, 1997). The severity of cachexia in these illnesses is often the primary determining factor in both quality of life and eventual mortality (Larkin, 1998; Tisdale, 1997). Indeed, body mass retention in AIDS patients has a stronger correlation with survival than any other current measure of the disease (Kotler et al., 1989). This has led to intense investigation of cachexia and the proposal of numerous hypotheses regarding its etiology. At this point, most authors suggest that cytokines released during inflammation and malignancy act on the central nervous system (CNS) to alter the release and function of a number of key neurotransmitters, thereby altering both appetite and metabolic rate (Inui, 1999a; Plata-Salaman 1989,1998; Tisdale, 1997).

Support for this mechanism is derived from numerous sources. One model that is particularly useful in studying cachexia is administration of a purified product found in the cell wall of gram-negative bacteria known generically as lipopolysaccharide (LPS). Early experiments focused on the ability of LPS injections to reliably produce anorexia in experimental animals (Baile et al., 1981; Murray and Murray, 1979). It is now known that LPS potently stimulates the release of numerous cytokines from immune cells in the periphery and glia within the CNS (Cavaillon and Haeffner-Cavaillon, 1990; Hillhouse and Mosley, 1993; Molloy et al., 1993; Van Dam et al., 1995). Furthermore, both peripheral and central injections of purified recombinant cytokines can reproduce the behavioral, feeding, and autonomic responses seen with LPS administration (Dinarello, 1996; Masotto et al., 1992; Plata-Salaman, 1998; Plata-Salaman and Borkoski, 1994). Chronic overproduction of cytokines may lead to cachexia when an illness is prolonged. Cytokines such as interleukin-1beta (IL-1 $\beta$ ), interleukin-6 (IL-6), and tumor necrosis factor-alpha (TNF- $\alpha$ ) are upregulated in various animal cachexia models. Neutralization of these factors by genetic or pharmacological methods leads to attenuation of cachexia (Plata-Salaman, 1998; Plata-Salaman and Borkoski, 1994; Sherry et al., 1989; Vallieres and Rivest, 1997; Yasumoto et al., 1995). Finally, chronic infusion of IL-1 $\beta$ or TNF- $\alpha$ causes anorexia, rapid weight loss, and catabolism of body protein stores, analogous to the state observed with chronic illness (Fong et al., 1989; Plata-Salaman et al., 1996).

\section{B. LEPTIN AS A CYTOKINE}

As outlined in section I, leptin is secreted by adipocytes and regulates adiposity and metabolic rate by reducing food intake and increasing energy expenditure (Campfield et al., 1995; Halaas et al., 1995; Pelleymounter et al., 1995; Zhang et al., 1994). Remarkably, leptin is a member of the IL-6 superfamily of proteins and has many biochemical features of a cytokine molecule (Zhang et al., 1994,1997). Using reporter gene analysis, Baumann and colleagues demonstrated that the signaling cascade events downstream of leptin treatment are nearly identical to those of the cytokine IL- 6 and that leptin can act synergistically with other 
cytokines in regulating gene expression (Baumann et al., 1996; Wang et al., 1997). Experimental elevation of leptin within the physiological range produces weight loss and relative anorexia that is similar in character to that seen with administration of other cytokines (Ahima et al., 1996; Halaas et al., 1997). Leptin secretion is increased by both central and systemic immunological challenge and has therefore been proposed as a potential mediator of inflammation-induced anorexia (Finck and Johnson, 1999; Finck et al., 1998; Grunfeld et al., 1996; Sarraf et al., 1997). The mechanism whereby leptin expression and secretion is enhanced during inflammation is complex but has been shown to be mediated by both IL-1 $\beta$ and TNF- $\alpha$ (Grunfeld et al., 1996; Sarraf et al., 1997). Conversely, leptin induces production and release of $1 \mathrm{~L}-1 \beta$ in the brains of normal rats and the release of both IL-1 $\beta$ and TNF- $\alpha$ from mouse macrophages (Loffreda et al., 1998; Luheshi et al., 1999). Collectively, these observations suggest a potential interplay between leptin and other cytokines in the regulation of metabolism and appetite during acute and chronic illness and that they activate similar signaling pathways. However, the observation that both leptin-deficient and leptin receptor-deficient mice have a robust anorexic response to LPS injections highlights the complexity of this system and indicates that cytokines may regulate leptin but also clearly act downstream of the leptin receptor (Faggioni et al., 1997).

\section{CENTRAL REGULATION OF ANOREXIA AND CACHEXIA}

Leptin is thought to exert its effects on feeding and metabolism primarily via regulation of hypothalamic neurons (Baskin et al., 1999; Cheung et al., 1997; Elmquist et al., 1997; Glaum et al., 1997; Jacob et al., 1997; Woods and Stock, 1996). Several neuronal pathways have been identified as targets of leptin action within the hypothalamus. As discussed in section I, several lines of evidence point toward a direct regulation of NPY synthesis and release by alterations in leptin levels brought about by fasting or by experimental manipulation (for review, see Inui, 1999a). This has led, in turn, to the hypothesis that a decrease in the level of NPY expression is responsible for the cytokine-mediated anorexia seen in animal models of inflammation and chronic disease. However, the observations that there was no change - or even an increase in NPY mRNA and peptide expression in tumor-bearing rats, rats with dehydration-induced anorexia, and rats treated with LPS - indicates that downregulation of NPY expression is not a primary stimulus for anorexia in these models (Gayle et al., 1998; Jensen et al., 1998; Plata-Salaman et al., 1998; Watts et al., 1999). A decrease in the response to exogenous NPY in anorexic, tumor-bearing animals has been demonstrated but it is unclear if this represents an effect that is specific to NPY signaling or is a reflection of increased inhibitory tone exerted by another neurotransmitter system (Chance et al., 1996). 
POMC neurons in the arcuate nucleus express the leptin receptor and MC4RKO mice are leptin resistant, leading several investigators to propose that melanocortin neurons mediate the anorexic effects of elevated leptin (Friedman, 1997; Gura, 1997). However, in other studies, it clearly has been shown that the melanocortin system has effects on weight and metabolism that are independent of leptin feedback and that leptin-deficient animals with disrupted melanocortin signaling remain sensitive to leptin administration (Boston et al., 1997; Marsh et al., 1999). Nonetheless, POMC neurons represent a logical target for cytokinemediated feedback on feeding behavior and metabolic control. Preliminary data suggest a role in anorexia and cachexia as well (Huang et al., 1999; Marks et al., 2000 ).

As outlined earlier, POMC neurons are thought to provide an important tonic inhibition of food intake and energy storage, primarily via production and release of $\alpha-\mathrm{MSH}$ from the POMC precursor. Central administration of MC4-R agonists can inhibit energy intake, increase energy expenditure (Cowley et al., 1999; Fan et al., 1997), lower serum insulin (Fan et al., 2000), and reduce body weight (Stair et al., 1999; Patterson et al., 1999). Thus, MC4-R activation results in a physiological response that mirrors that observed when various cytokines, including leptin, are administered or elevated in animals and humans. In addition, the leptin-deficient $o b / o b$ mouse and the leptin receptor-deficient $d b / d b$ mouse remain sensitive to the hypophagic effects of LPS. This implies that the site of integration of this anorexic signal lies downstream of the hypothalamic leptin receptor (Faggioni et al., 1997).

Studies of melanocortin function in cytokine-induced anorexia are complicated by the fact that $\alpha$-MSH is a potent anti-inflammatory and antipyretic when administered centrally. Therefore, it may have effects on feeding that are not specific to the hypothalamus (Adan and Gispen, 1997; Catania and Lipton, 1993; Catania et al., 1992). Furthermore, investigations of the impact of central melanocortins in transducing anorexic signals from cytokines have had mixed results. Blockade of MC4-R signaling has been shown to enhance the anorexia observed when IL $1-\beta$ is administered peripherally (Shimomura et al, 1991) and to diminish the response when administered centrally (Tatro et al., 1999). Huang and colleagues investigated the impact of central administration of $\alpha-\mathrm{MSH}$ or the melanocortin receptor subtype3/subtype4 antagonist SHU-9119 on LPS-induced anorexia and fever in rats (Huang et al., 1999). In this study, the investigators found a significant potentiation of the suppressive effects of LPS on food intake with administration of $\alpha$-MSH and a reversal of LPS-induced anorexia with SHU-9119 administration. These same treatments reduced and increased LPS-induced fever, respectively.

AGRP is an endogenous antagonist of the hypothalamic MC4-R but its role in limiting inhibition of feeding by cytokines has not been reported. However, 
AGRP is robustly regulated in other models of energy imbalance and its anatomical distribution and function make it a logical candidate for transduction of anorexic signals during both acute and chronic illness. We have demonstrated that AGRP administration can prevent the anorexia and hypodipsia brought about with LPS administration (Marks et al., 2000). Furthermore, we have demonstrated that MC4-RKO animals resist LPS-induced anorexia, hypodipsia, and decreased locomotion. Our feeding data are in agreement with those of Huang and colleagues and demonstrate that both genetic and pharmacologic blockade of central MC4-R signaling can prevent the response to induction of a complex and pleotropic cytokine response (Marks et al., 2000). Additionally, we have demonstrated that in young, rapidly growing mice, the weight loss that accompanies LPS-induced illness can be reversed, allowing the animals to continuc to follow a normal growth curve (Marks et al., 2000).

\section{MLLANOCORTINS AND CANCER CACIIEXIA}

Cachexia commonly is observed in patients with cancer, particularly in children and the elderly (Bruera, 1997). The resulting malnutrition and loss of lean body mass reduces the quality of life for the affected individual and compromises recovery by decreasing tolerance to therapy and increasing postsurgical complications (Inui, 1999a; Larkin, 1998). Attempts at drug therapy for cachexia with a variety of agents have met with limited success (DeConno et al., 1998; Windisch et al, 1998; Rivandeneria et al, 1999; McCarthy, 1999). The most-widely utilized agent, megestrol acetate, has shown some promise in reversing weight loss. However, this is primarily due to increases in fat mass and water retention rather than to preservation of lean body mass (Strang, 1997). Various murine models of cancer cachexia exist that recapitulate the anorexia, rapid weight loss, and catabolism of body protein stores found in human cancer patients. Subcutaneous injections of Lewis lung adenocarcinoma (LLA) or various types of methylcholanthrene-induced sarcomas reliably produce cachexigenic tumors in mice, therefore providing useful models for genetic and pharmacologic analysis of this disorder and its potential treatment (Matthys et al, 1991; Llovera et al, 1998; Ushmorov et al, 1999; Svaninger et al., 1987).

NPY has a duration of action that is limited to hours. Its role in multiple other neural processes including seizure kindling may limit the usefulness of NPY agonists in cachexia therapy (Levine and Morley, 1984; Marksteiner and Sperk, 1988). In contrast, several investigators have demonstrated prolonged duration of activity of AGRP, with feeding effects being observed for as long as 2 to 3 days (Dinulescu, 2000; Rossi et al., 1998). $\Lambda$ GRP is not known to produce illness behavior or aversive consequences after injection, perhaps due to the relatively small number of potential activity sites in the brain. These features highlight the potential of AGRP analogs as therapeutic agents in prolonged human illness. 
Overall, if melanocortin signaling is necessary for anorexia or cachexia, then the MC4-R may be an outstanding target for the development of antagonist drugs that would be useful in preventing and treating these conditions.

Recently, we have studied the role of melanocortin receptors in transducing the prolonged metabolic derangement observed in experimental cancer. Previous investigators have demonstrated that tumor-bearing animals frequently die from cachexia and exhaustion of metabolic fuels, rather than from metastasis or infection (Svaninger et al., 1987,1989; Emery et al., 1984; Emery, 1999). Our observations demonstrate that hypophagia and carcass weight loss induced by sarcoma growth can be both reversed and prevented by administration of the endogenous MC3/MC4 antagonist, AGRP (Marks et al., 2000). In these experiments, the duration of the experiment in the AGRP-treated animals was limited only by ethical concerns due to the size of the tumor, rather than by anorexia or lack of physical activity and grooming. Prevention of tumor-induced hypophagia with early and repeated AGRP injections resulted in maintenance of normal food intake. This enhancement of feeding was much greater than the relative hyperphagia observed in the sham-tumor-implanted animals. The inability to completely mimic the feeding observed in the sham-tumor group is likely to reflect, in part, the energy drain imposed by the rapid growth of a metabolically active tissue. However, our observation that the rate of tumor growth was identical between AGRP and a vehicle-treated group argues strongly that tumor growth produces a global metabolic derangement that is mediated to a significant extent by central melanocortin receptor activation. If the tumor simply represented a metabolic sink, increased nutrient intake would be expected to result in increased tumor growth at the expense of nontumor body mass.

\section{Summary}

Recently, great strides have been made in understanding endocrine and neural control of appetite, metabolism, and body weight. In particular, we now know that disorders of leptin feedback or hypothalamic melanocortin signaling can lead to pathological weight gain and diabetes in humans (Clement et al., 1998; Krude $e t$ al., 1998; Montague et al., 1997). While obesity is clearly a major health challenge, other disorders affecting body weight are found in humans, particularly in pediatric populations. Several chronic diseases - including renal failure, cystic fibrosis, AIDS, and various forms of cancer - are associated with weight loss and growth failure. In children with chronic diseases, there appears to be a dysfunctional change in the regulation of appetite and metabolism. Indeed, cachexia, characterized by weight loss and progressive tissue wasting out of proportion to the level of anorexia, is a feature of many of these diseases and contributes significantly to mortality (Tisdale, 1997). Early studies of the role of melanocortin signaling in illness-related anorexia have demonstrated that this 

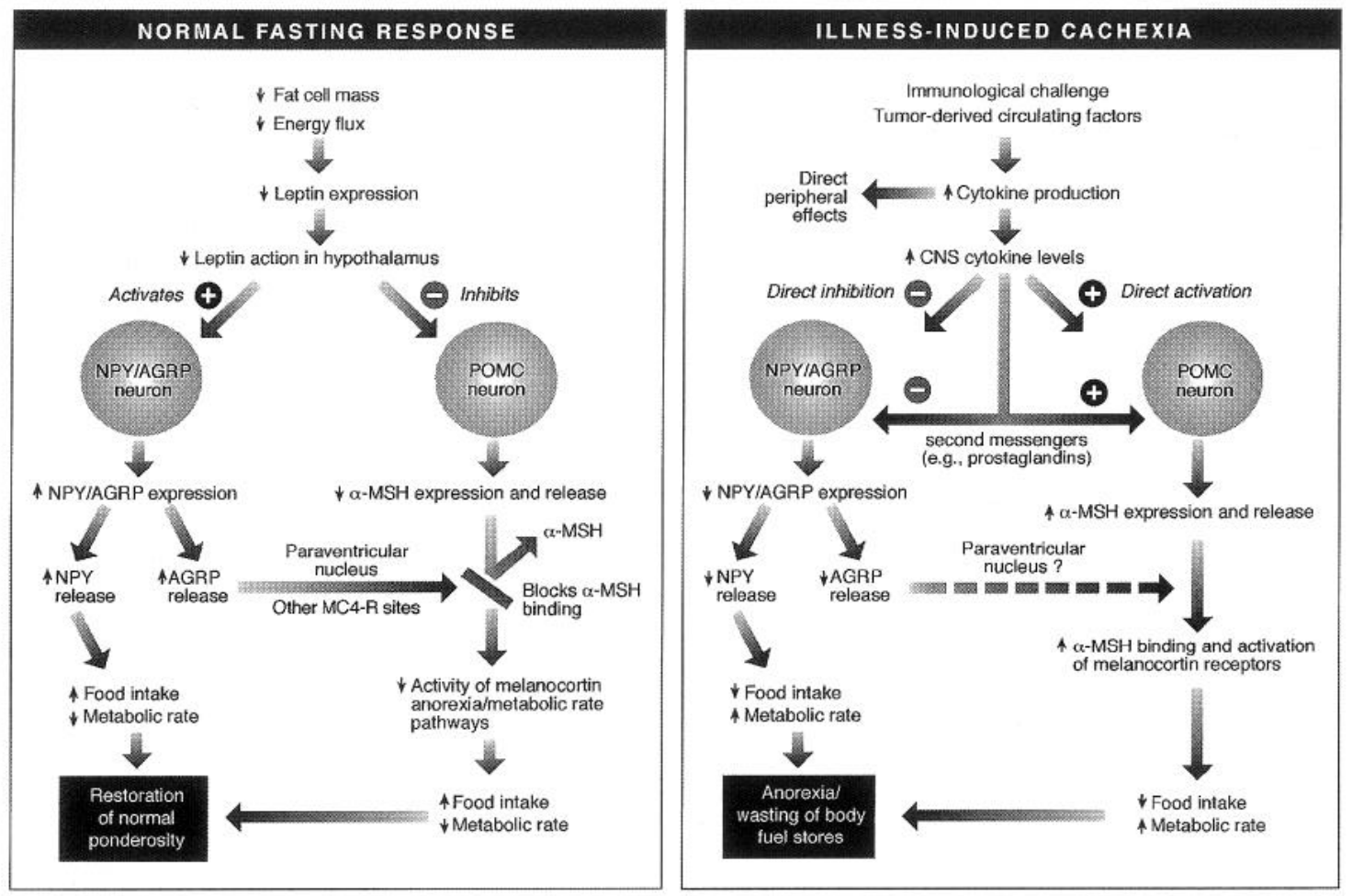

FIG. 1. Hypothetical models for the regulation of feeding and metabolic rate during normal fasting (left panel) and illness-induced cachexia. During illness, cytokines and other circulating factors result in activation of central anorexic/metabolic pathways, resulting in decreased energy intake and pathological wasting of body fuels, even in a relatively starved state (right panel). 
system may play a key role in the development of this pathological state (Huang, 1999). By analogy with the role of melanocortins in CNS-mediated obesity syndromes, we propose that stimuli that decrease feeding and cause a pathological wasting of body fuel stores exert their effects in part by enhancing signaling at hypothalamic melanocortin receptors (Figure 1). However, further studies will be required to elucidate the hormonal and neural pathways involved and to demonstrate the potential therapeutic role of melanocortin antagonists in treating anorexia and cachexia.

\section{ACKNOWLEDGMENTS}

The authors are grateful for the commentary and editorial assistance of Dr. Michael Cowley and for the laboratory assistance of Kathy Khong and Katie Miles.

\section{REFERENCES}

Adan, R.A.H., and Gispen, W.H. (1997). Peptides 18(6), 1-7.

Ahima, R., Prabakaran, D., Mantzoros, C., Qu, D., Lowell, B., Maratos-Flier, E., and Flier, J.S. (1996). Nature 382, 250-252.

Baile, C.A., Naylor, J., McLaughlin, C.L., and Catanzaro, C.A. (1981). Physiol. Behav. 27, 271-277. Baskin, D.G., Breininger, J.F., and Schwartz, M.W. (1999). Diabetes 48, 828-833.

Baumann, H., Morella, K.K., White, D.W., Dembski, M., Bailon, P.S., Kim, H., Lai, C., and Tartaglia, L.A. (1996). Proc. Natl. Acad. Sci.U.S.A. 93, 8374-8378.

Billington, C.J., Briggs, J.E., Grace, M., and Levine, A.S. (1991). Am. J. Physiol. 260, R321-R327.

Rlasquez, C., Jegou, S., Friard, O., Tonon, M.C., Fournier, A., and Vaudry, H. (1995). Neuroscience 68(1), 221-227.

Blum, W.F., Englaro, P., Hanitsch, S., Juul, A., Hertel, N.T., Muller, J., Skakkebaek, N.E., Heiman, M.L., Birkett, M., Attanasio, A.M., Kiess, W., and Rascher, W. (1997). J. Clin. Metab. 82, 2094-2100.

Boston, B.A., Blaydon, K.M., Varnerin, J., and Cone, R.D. (1997). Science 278, 1641-1644.

Broberger, C., Johansson, C., Schalling, M., and Hokfelt, T. (1998). Proc. Natl. Acad. Sci. U.S.A. 95, 15043-15048.

Bruera, E. (1997). Br. Med. J. 315, 1219-1222.

Bultman, S.J., Michaud, E.J., and Woychik, R.P. (1992). Cell 71, 1195-1204.

Campfield, L.A., Smith, F.J., Guisez, Y., Devos, R., and Burn, P. (1995). Science 269, 546-549.

Catania, A., and Lipton, J.M. (1993). Endocr. Rev. 14(5), 564-576.

Catania, A., Manfredi, M.G., Airaghi, L., and Lipton, J.M. (1992). Pharmacol. Res. 26(suppl. 2), $72-73$.

Cavaillon, J.M., and Haeffner-Cavaillon, N. (1990). Cytokine 2, 313-329.

Chance, W.T., Balasubramaniam, A., Thompson, H., Mohapatra, B., Ramo, J, and Fischer, J.E. (1996). Peptides 17, 797-801.

Chen, P., Li, C., Haskell-Luevano, C., Cone, R.D., and Smith, M.S. (1999). Endocrinology 140(6), 2645-2650

Cheung, C.C., Clifton, D.K., and Steiner, R.A. (1997). Endocrinology 138, 4489-4492.

Chua, S.C., Chung, W.K., Wu-Peng, X.S., Zhang, Y., Liu, S.-M., Tartaglia, L., and Leibel, R.L. (1996) Science 271, 994-996.

Clayton, P.E., Gill, M.S., Hall, C.M., Tillman, V., Whatmore, A.J., and Price, D.A. (1997). Clin. Endocrinol. 46, 727-733. 
Clement, K., Vaisse, C., Lahlou, N., Cabrol, S., Pelloux, V., Cassuto, D., Gourmelen, M., Dina, C., Chambaz, J., Lacorte, J.M., Basdevant, A., Bougneres, P., Lebouc, Y., Froguel, P., and Guy-Grand, B. (1998). Nature 392, 398-401.

Comuzzie, A.G., Hixson, J.E., Almasy, L., Mitchell, B.D., Mahaney, M.C., Dyer, T.D., Stern, M.P., MacCluer, J.W., and Blangero, J. (1997). Nature Genet. 15, 273-276.

Considine, R.V., Sinha, M.K., Heiman, M.L., Kriauciunas, A., Stephens, T.W., Nyce, M.R., Ohannesian, J.P., Marco, C.C., McKee, L.J., Bauer, T.L., and Caro, J.F. (1996). N. Engl. J. Med. 334, 292-295.

Cowley, M.A., Pronchuk, N., Fan, W., Dinulescu, D.M., Colmers, W.F., and Cone, R.D. (1999). Neuron 24(1), 155-163.

DeConno, F., Martini, C., Zecca, E., Balzarini, A., Venturino, P., Groff, L., and Caraceni, A. (1998). Eur. J. Cancer 34(11), 1705-1709.

Dinarello, C.A. (1996). Blood 87, 2095-2147.

Dinulescu, D.M. (2000). "The role of mahagony in melanocortin signaling," $\mathrm{PhD}$ dissertation, Oregon Health Sciences University, Portland, Ore.

Duhl, D.M., Stevens, M.E., Vrieling, H., Saxon, P.J., Miller, M.W., Epstein, C.J., and Barsh, G.S. (1994). Development 120(6), 1695-1708.

Egawa, M., Yoshimatsu, H., and Bray, G.A. (1991). Am. J. Physiol. 260, R328-R334.

Elmquist, J.K., Ahima, R.S., Maratos Flier, E., Flier, J.S., and Saper, C.B. (1997). Endocrinology 138, 839-842.

Emery, P.W. (1999). Nutrition 15, 600-603.

Emery, P.W., Lovell, L., and Rennie, M.J. (1984). Cancer Res. 44, $2779-2784$.

Erickson, J., Hollopeter, G., and Palmiter, J.D. (1996). Science 274, 1704-1707.

Faggioni, R., Fuller, J., Moser, A., Feingold, K.R., and Grunfeld, C. (1997). Am. J. Physiol. 273(42), R181-R186.

Fan, W., Boston, B.A., Kesterson, R.A., Hruby, V.J., and Cone, R.D. (1997). Nature 385, 165-168.

Fan, W., Dinulescu, D.M., Butler, A.A., Zhou, J., Marks, D.L., and Cone, R.D. (2000). Endocrinology 141(9), 3072-3079.

Farooqi, I.S., Jebb, S.A., Langmack, G., Lawrence, E., Cheetham, C.H., Prentice, A.M., Hughes, I.A., McCamish, M.A., and O'Rahilly, S. (1999). N. Engl. J. Med. 341(12), 879-884.

Finck, B.N., and Johnson, R.W. (1999). NeuroReport 10, 153-156.

Finck, B.N., Kelly, K.W., Dantzer, R., and Johnson, R.W. (1998). Endocrinology 139(5), 2278-2283.

Fong, T.M., Mao, C., MacNeil, C., Kalyani, R., Smith, T., Weinberg, D., Tota, M.R., and Van der Ploeg, L.H. (1997). Biochem. Biophys. Res. Commun. 237, 629-631.

Fong, Y., Moldawer, I.I., Marann, M., Wei, H, Barher, A., Manogue, K., Tracey, K.I., Kun, G., Fischman, D.A., Cerami, A., and Lowry, S.F. (1989). Am. J. Physiol. 256(25), R659-R665.

Friedman, J.M. (1997). Nature 385, 119-120.

Frigeri, L.G., Wolff, G.L., and Robel, G. (1983). Endocrinology 113, 2097-2105.

Frigeri, L.G., Wolff, G.L., and Teguh, C. (1988). Intl. J. Obesity 12, 305-320.

Gayle, D., Ilyin, S.E., Flynn, M.C., and Plata-Salaman, C.R. (1998). Brain Res. 795, 77-86.

Gerald, C., Walker, M.W., Criscione, L., Gustafson, E.L., Batzl-Hartmann, C., Smith, K.E., Vaysse, P., Durkin, M.M., Laz, T.M., Linemeyer, D.L., Schauffhauser, A.O., Whitebread, S., Hofbauer, K.G., Taber, R.I., Branchek, T.A., and Weinshank, R.L. (1996). Nature 382, 168-171.

Glaum, S.R., Hara, M., Bindokas, V.P., Lee, C.C., Polonsky, K.S., Bell, G.I., and Miller, R.J. (1997). Mol. Pharmacal. 50, 230-235.

Grill, H.J., Ginsberg, A.B., Seeley, R.J., and Kaplan, J.M. (1998). J. Neurosci. 18(23), 10128-10135.

Grunfeld, C., Zhao, C., Fuller, J., Pollock, A., Moser, A., Friedman, J., and Feingold, K.R. (1996). J. Clin. Invest. 97, 2152-2157.

Gura, T. (1997). Science 275, 751-753.

Hakansson, M.L., Hulting, A.L., and Meister, B. (1996). NeuroReport 7(18), 3087-3092. 
Halaas, J., Gajiwala, K., Maffei, M., Cohen, S., Chait, B., Rabinowitz, D., Lallone, R., Burley, S., and Friedman, J.M. (1995). Science 269, 543-546.

Halaas, J.L., Boozer, C., Blair-West, J, Fidahusein, N., Denton, D. A., and Friedman, J.M. (1997). Proc. Natl. Acad. Sci. U.S.A. 94, 8878-8883.

Haskell-Luevano, C., Chen, P., Li, C., Chang, K., Smith, M.S., Cameron, J.L., and Cone, R.D. (1999). Endocrinology 140, 1408-1415.

Hillhouse, E.W., and Mosley, K. (1993). Br. J. Pharmacol. 109, 289-290.

Huang, Q.H., Hruby, V.J., and Tatro, J.B. (1999). Am. J. Physiol. 276 (3 pt. 2), R864-R871.

Huszar, D., Lynch, C.A., Fairchild-Huntress, V., Dunmore, J.H., Fang, Q., Berkemeier, L.R., Gu, W., Kesterson, R.A., Boston, B.A., Cone, R.D., Smith, F.J., Campfield, L.A., Burn, P., and Lee, F. (1997). Cell 88, 131-141.

Inui, A. (1999a). Cancer Res. 59, 4493-4501.

Inui, A. (1999b). Trends Neurosci. 22(2), $62-67$.

Jacob, R.J., Dziura, J., Madwick, M., Leone, P., Caprio, S., During, M., Shulman, G.1., and Sherwin, R.S. (1997). Diabetes 46, 150-153.

Jensen, P.B., Blume, N., Mikkelsen, J.D., Larsen, P.J., Jensen, H.I., Holst, J.J., and Madsen, O.D. (1998). J. Clin. Invest. 101(2), 503-510.

Kalra, S.P., Dube, M.G., Pu, S., Xu, B., Horvath, T.L., and Kalra, P.S. (1999). Endocr. Rev. 20(1), 68-100.

Kotler, D.P., Tierney, A.R., Wang, J., and Pierson, R.N.J. (1989). Am. J. Clin. Nutrit. 50(3), 444-447.

Krude, H., Biebermann, H., Luck, W., Horn, R., Brabant, G., and Gruters, A. (1998). Nature Genet. 19, 155-157.

Larkin, M. (1998). Lancet 351, 1336.

Larsen, P.J., Tang-Christensen, M., and Cameron, J.L. (1997). Soc. Neurosci. Abst. 1997, 528.14.

Levine, A.S., and Morley, J.E. (1984). Peptides 5(6), 1025-1029.

Llovera, M., Garcia-Martinez, C., Lopez-Soriano, J., Agell, N., Lopez-Soriano, F.J., Garcia, I., and Argiles, J.M. (1998). Cancer Lett. 130, 19-27.

Loffreda, S., Yang, S.Q., Lin, II.Z., Karp, C.L., Brengman, M.L., Wang, D.J., Klein, A.S., Bulkley, G.B., Bao, C., Noble, P.W., Lane, M.D., and Diehl, A.M. (1998). FASEB J. 12(1), 57-65.

Lu, D., Willard, D., Patel, I.R., Kadwell, S., Overton, L., Kost, T., Luther, M., Chen, W., Woychik, R.P., Wilkison, W.O, and Cone, R.D. (1994). Nature 371, 799-802.

Luheshi, G.N., Gardner, J.D., Rushforth, D.A., Loudon, A.S., and Rothwell, N.J. (1999). Proc. Nat. Acad. Sci. U.S.A. 96(12), 7047-7052.

Marks, D.L., Miles, K.E., and Cone, R.D. (2000). In "Program \& Abstracts of the 1 lth International Congress of Endocrinology," October 30-November 2, 2000, Sydney, Australia

Marksteiner, J., and Sperk, G. (1988). Neuroscience 26(2), 379-385.

Marsh, D.J., Hollopeter, G., Huszar, D., Laufer, R., Yagaloff, K.A., Fisher, S.L., Burn, P., and Palmiter, R.D. (1999). Nature Genet. 21, 119-122.

Masotto, C., Caspani, G., Grazia de Simoni, M., Mengozzi, M., Scatturin, M., Sironi, M., Carenzi, A., and Ghezzi, P. (1992). Brain Res. Bull. 28, 161-165.

Matthys, P., Heremans, H., Opdenakker, G., and Billiau, A. (1991). Eur. J. Cancer 27(2), 182-187.

McCarthy, D.O. (1999). Res. Nurs. Health 22(5), 380-387.

Michaud, E.J., Bultman, S.J., Klebig, M.L., van Vugt, M.J., Stubbs, L.J., Russell, L.B., and Woychik, R.P. (1994). Proc. Natl. Acad. Sci. U.S.A. 91(7), 2562-2566.

Miller, M.W., Duhl, D. M. J., Vrieling, H., Cordes, S.P., Ollmann, M.M., Winkes, B.M., and Barsh, G.S. (1993). Genes Devel. 7, 454-467.

Mizuno, T.M., and Mobbs, C.V. (1999). Endocrinology 140(2), 814-817.

Molloy, R.G., Mannick, J.A., and Rodrick, M.L. (1993). Br. J. Surg. 80, 289-297. 
Montague, C.T., Farooqi, I.S., Whitehead, J.P., Soos, M.A., Rau, H., Wareham, N.J., Sewter, C.P., Digby, J.E., Mohammed, S.N., Hurst, J.A., Cheetham, C.H., Earley, A.R., Barnett, A.H., Prins, J.B, and O'Rahilly, S. (1997). Nature 387, 903-908.

Mountjoy, K.G., Mortrud, M.T., Low, M.J., Simerly, R.B., and Cone, R.D. (1994). Mol. Endocrinol. 8, 1298-1308

Murray, M.J., and Murray, A.B. (1979). Am. J. Clin. Nutrit. 32, 593-596.

Ollmann, M.M., Wilson, B.D., Yang, Y.-K., Kerns, J.A., Chen, Y., Gantz, I., and Barsh, G.S. (1997). Science 278, 135-137.

Ozata, M., Ozdemir, C., and Licinio, J. (1999). J. Clin. Endocrinol. Metab. 84(10), 3686-3695.

Patterson, T.A., Hedde, J.R., She, L., Newsome, W.P., and Cornelius, P. (1999). Soc. Neurosci. Abst. 25,618 .

Pelleymounter, M., Cullen, M., Baker, M., Hecht, R., Winters, D., Boone, T., and Collins, F. (1995). Science 269, 540-543.

Plata-Salaman, C.R. (1989). Brain Behav. Immunol. 3, 193-213.

Plata-Salaman, C.R. (1998). Psychoneuroendocrinology 24, 25-41.

Plata-Salaman, C.R., and Borkoski, J.P. (1994). Am. J. Physiol. 266, R1711-R1715.

Plata-Salaman, C.R., Sonti, G., Borkoski, J.P., Wilson, C.D., and Ffrench-Mullen, J.M.H. (1996). Phys. Behav. 60, 867-875.

Plata-Salaman, C.R., Ilyin, S.E., and Gayle, D. (1998). Am. J. Physiol. 275, R566-R573.

Rivandeneria, D.E., Naama, H.A., McCarter, M.D., Fujita, J., Evoy, D., Mackrell, P., and Daly, J.M. (1999). Nutrit. Cancer 35(2), 202-206.

Roemmich, J.N., and Rogol, A.D. (1999). Endocrinol. Metab. Clin. N. Am. 28(4), 749-764.

Roselli-Rehfuss, L., Mountjoy, K.G., Robbins, L.S., Mortrud, M.T., Low, M.J., Tatro, J.B., Entwistle, M.L., Simerly, R., and Cone, R.D. (1993). Proc. Natl. Acad. Sci. U.S.A. 90, 8856-8860.

Rossi, M., Kim, M.S., Morgan, D.G., Small, C.J., Edwards, C.M., Sunter, D., Abusnana, S., Goldstone, A.P., Russell, S.H., Stanley, S.A., Smith, D.M., Yagaloff, K., Ghatei, M.A., and Bloom, S.R. (1998). Endocrinology 139(10), 4428-4431.

Sarraf, P., Frederich, R.C., Turner, E.M., Ma, G., Jaskowiak, N.T., Rivet, D.J.I., Flier, J.S., Lowell, B.B., Fraker, D.L., and Alexander, H.R. (1997). J. Exp. Med. 185(1), 171-175.

Sherry, B.A., Gelin, J., Fong, Y., Marano, M., Wei, H., Cerami, A., Lowry, S.F., Lundholm, K.G., and Moldawer, L.L. (1989). FASEB J. 3, 1956-1962.

Shimomura, Y., Inukai, T., Kuwabara, A., Shimizu, H., Sato, N., Uehara, Y., Kobayashi, I., and Kobayashi, S. (1991). Eur. J. Pharmacol. 209, 15-18.

Shutter, J.R., Graham, M., Kinsey, A.C., Scully, S., Luthy, R., and Stark, K.L. (1997). Genes Devel. $11,593-602$.

Smith, M.S. (1993). Endocrinology 133, 1258-1265.

Stair, J.N., Shu, J., Camacho, R., Murphy, B., Hickey, G.J., MacIntyre, D.E., and Strack, A.M. (1999). Soc. Neurosci Abst. 25, 619.

Stanley, B.G., Chin, A.S., and Leibowitz, S.F. (1985). Brain Res. Bull. 14, 521-524.

Stephens, T.W., Basinsky, M., Bristow, P.K., Bue-Valleskey, J.M., Burgett, S.G., Craft, L., Hale, J., Hoffmann, J., Hsiung, H.M., Kriauciunas, A., Mackeller, W., Rosteck, P.R., Schoner, B., Smith, D., Tinsley, F.C., Zhang, X.-Y., and Heiman, M. (1995). Nature 377, 530-532.

Strang, P. (1997). Anticancer Res. 17(1b), 657-662.

Svaninger, G., Drott, C., and Lundholm, K. (1987). J. Natl. Cancer Inst. 78, 943-950.

Svaninger, G., Gelin, J., and Lundholm, K. (1989). Eur. J. Cancer Clin. Oncol. 25(9), 1295-1302.

Tartaglia, L.A., Dembski, M., Weng, X., Deng, N., Culpepper, J., Devos, R., Richards, G.J., Campfield, L.A., Clark, F.T., Deeds, J., Muir, C., Sanker, S., Moriarty, A., Moore, K.J., Smutko, J.S., Mays, G.G., Woolf, E.A., Monroe, C.A., and Tepper, R.I. (1995). Cell 83, 1263-1271.

Tatro, J.B., Huszar, D., Fairchild-Huntress, V., and Huang, Q.H. (1999). Soc. Neurosci. Abst. 25(624.4), 1558. 
Tisdale, M.J. (1997). J. Natl. Cancer Inst. 89, 1763-1773.

Ushmorov, A., Hack, V., and Droge, W. (1999). Cancer Res. 59, 3527-3534.

Vaisse, C., Clement, K., Guy-Grand, B., and Froguel, P. (1998). Nature Genet. 20, 113-114.

Vallieres, L., and Rivest, S. (1997). J. Neurochem. 69, 1668-1683.

Van Dam, A.M., Bauer, J., Tilders, F.J., and Berkenbosch, F. (1995). Neuroscience 65, 815-826.

Wang, Y., Kuropatwinski, K.K., White, D.W., Hawley, T.S., Hawley, R.G., Tartaglia, L.A., and Baumann, H. (1997). J. Biol. Chem. 272(26), 16216-16233.

Watts, A.G., Sanchez-Watts, G., and Kelly, A.B. (1999). J. Neurosci. 19(14), 6111-6121.

Wilding, J.P.H., Gilbey, S.G., Bailey, C.J., Batt, R.A.L., Williams, G., Ghatei, M.A., and Bloom, S.R. (1993). Endocrinology 132, 1939-1944.

Windisch, P., Papatheofanis, F.J., and Matuszewski, K.A. (1998). Ann. Pharmacother. 32(4), 437-445.

Wolff, G.L. (1963). Genetics 48, 1041-1058.

Wolff, G.L., and Flack, J.D. (1971). Nature 232, 181-182.

Woods, A.J., and Stock, M.J. (1996). Nalure 381, 745 .

Yasumoto, K., Mukaida, N., Harada, A., Kuno, K., Akiyama, M., Nakashima, E., Fujioka, N., Mai, M., Kasahara, T., and Fujimoto-Ouchi, K. (1995). Cancer Res. 55(4), 921-927.

Yen, T.T., Gill, A.M., Frigeri, L.G., Barsh, G.S., and Wolff, G.L. (1994). FASEB J. 8(8), 479-488.

Yeo, G.S.H., Farooqi, I.S., Aminian, S., Halsall, D.J., Stanhope, R.G., and O'Rahilly, S. (1998). Nature Genet 20,111-112.

Zhang, F.M., Basinski, M.B., and Beals, J.M. (1997). Nature 387, 206-209.

Zhang, Y., Procnca, R., Maffci, M., Baronc, M., Lcopold, L., and Fricdman, J.M. (1994). Nature 372, 425-432. 



\title{
Glucagon-Like Peptide-1
}

\author{
Máire E. Doyle and Josephine M. Egan \\ Diabetes Section, National Institute on Aging, National Institutes of Health, \\ 5600 Nathan Shock Drive, Baltimore, Maryland 21224
}

\begin{abstract}
There is a progressive impairment in beta-cell function with age. As a result, 19 percent of the U.S. population over the age of 65 is diagnosed with type 2 diabetes mellitus (DM). Glucagon-like peptide-1 (GLP-1) is a potent insulin secretagogue that has multiple synergetic effects on the glucosedependent insulin secretion pathways of the $\beta$-cell. This peptide and its longer-acting analog exendin- 4 are currently under review as treatments for type $2 \mathrm{DM}$. In our work on the rodent model of glucose intolerance in aging, we found that GLP-1 is capable of rescuing the age-related decline in $\beta$-cell function. We have shown that this is due to the ability of GLP-1 to 1) recruit $\beta$-cells into a secretory mode; 2 ) upregulate the genes of the $\beta$-cell glucose-sensing machinery; and 3 ) cause $\beta$-cell differentiation and neogenesis.

Our investigations into the mechanisms of action of GLP-1 began by using the reverse hemolytic plaque assay to quantify insulin secretion from individual cells of the RIN 1046-38 insulinoma cell line in response to acute treatment with the peptide. GLP-1 increases both the number of cells secreting insulin and the amount secreted per cell. This response to GLP-1 is retained even in the $\beta$ cell of the old (i.e., 22-month), glucose-intolerant Wistar rat, which exhibits a normal, first-phase insulin response to glucose following an acute bolus of GLP-1. Preincubation with GLP-1 (24 hours) potentiates glucose- and GLP-1-dependent insulin secretion and increases insulin content in the insulinoma cells. Treatment of old Wistar rats for $\mathbf{4 8}$ hours with GLP-1 leads to normalization of the insulin response and an increase in islet insulin content and mRNA levels of GLUT 2 and glucokinase. PDX-1, a transcriptional factor activator of these three genes, also is upregulated in the insulinoma cell line in aged rats and diabetic mice following treatment with GLP-1. Administration of GLP-1 to old rats leads to pancreatic cell proliferation, insulin-positive clusters, and an increase in $\beta$-cell mass. This evidence led us to believe that GLP-1 is an endocrinotrophic factor. We used an acinar cell line to show that GLP-1 can directly cause the conversion of a putative pro-endocrine cell into an endocrine one. 'Thus, the actions of GLP-I on the $\beta$-cell are complex, with possible benefits to the diabetic patient that extend beyond a simple glucose-dependent increase in insulin secretion. The major limitation to GLP-1 as a clinical treatment is its short biological half-life. We have shown that the peptide exendin-4, originating in the saliva of the Gila monster, exhibits the same insulinotropic and endocrinotrophic properties as GLP-1 but is more potent and longer acting in rodents and humans.
\end{abstract}

\section{Introduction}

Type 2 diabetes mellitus (DM) is one of the most-prevalent metabolic disorders in the United States. Almost 6 percent of the population carries the diagnosis 
(Centers for Disease Control and Prevention, 1998), while approximately another three percent are estimated to be unaware that they have the condition (Harris et $a l ., 1998)$. It results from a failure of the $\beta$-cell to produce sufficient insulin to meet the demands of the body. Medications currently in use have limited success in controlling blood glucose levels, hence, the complications of the disease (United Kingdom Prospective Diabetes Study, 1998). An ongoing failure in $\beta$-cell function is another aspect of this disease that none of the currently used medications is capable of reversing (United Kingdom Prospective Diabetes Study, 1995). The Diabetes Section at the National Institute on Aging (NIA) became interested in glucagon-like peptide-1 (GLP-1) as a treatment for diabetes when it was reported to effectively lower blood glucose levels in diabetic subjects (Nathan et al., 1992; Gutniak et al., 1992). We set out to investigate its mechanisms of action and to develop its potential and that of agonists at the GLP-1 receptor as treatments for type $2 \mathrm{DM}$. In both instances, we have placed a special emphasis on the aged diabetic condition.

\section{Background}

GLP-1 is secreted by intestinal L-cells in response to nutrient ingestion (Mojsov et al., 1986). Exogenous administration of GLP-1 reduces blood glucose levels in both normal and diabetic subjects (Nathan et al., 1992) by stimulating insulin secretion and synthesis in concert with glucose (Fehmann et al., 1992) and by suppressing glucagon secretion (Komatsu et al., 1989). (For a more-comprehensive review of glucagon-like peptides, see Fehmann et al., 1995, and Drucker, 1998. This chapter will confine its background discussion to effects on acute insulin secretion.)

GLP- 1 action in the $\beta$-cell is mediated by binding of the peptide to a specific, seven-membered transmembrane receptor (Thorens et al., 1993). Activation of this G-coupled receptor causes an increase in intracellular cAMP concentration (Drucker et al., 1987) and activation of protein kinase A (PKA). GLP-1 acts directly through the cAMP/PKA pathway to enhance and sensitize $\beta$-cells to glucose-stimulated insulin secretion (Figure 1). The metabolism of glucose in the $\beta$-cell causes an increase in the amount of adenosine triphosphate (ATP) and a rise in the cytoplasmic ATP:adenosine diphosphate (ADP) ratio, leading to closure of the $\mathrm{K}_{\mathrm{ATP}}$ channels. GLP-1 may close the $\mathrm{K}_{\mathrm{ATP}}$ channels via a PKA-mediated phosphorylation. These channels possess sites that are phosphorylated by G-protein stimulated PKA (Béguin et al., 1999). The ability of the specific PKA inhibitor Rp-cAMP to reverse the GLP-1 action on the $\mathrm{K}_{\mathrm{A} \text { TP }}$ channels confirms the involvement of PKA in this step (Holz et al., 1993).

Subsequent membrane depolarization results in the activation of voltage-dependent L-type $\mathrm{Ca}^{2+}$ channels, an influx of $\mathrm{Ca}^{2+}$, and exocytosis of the insulin- 


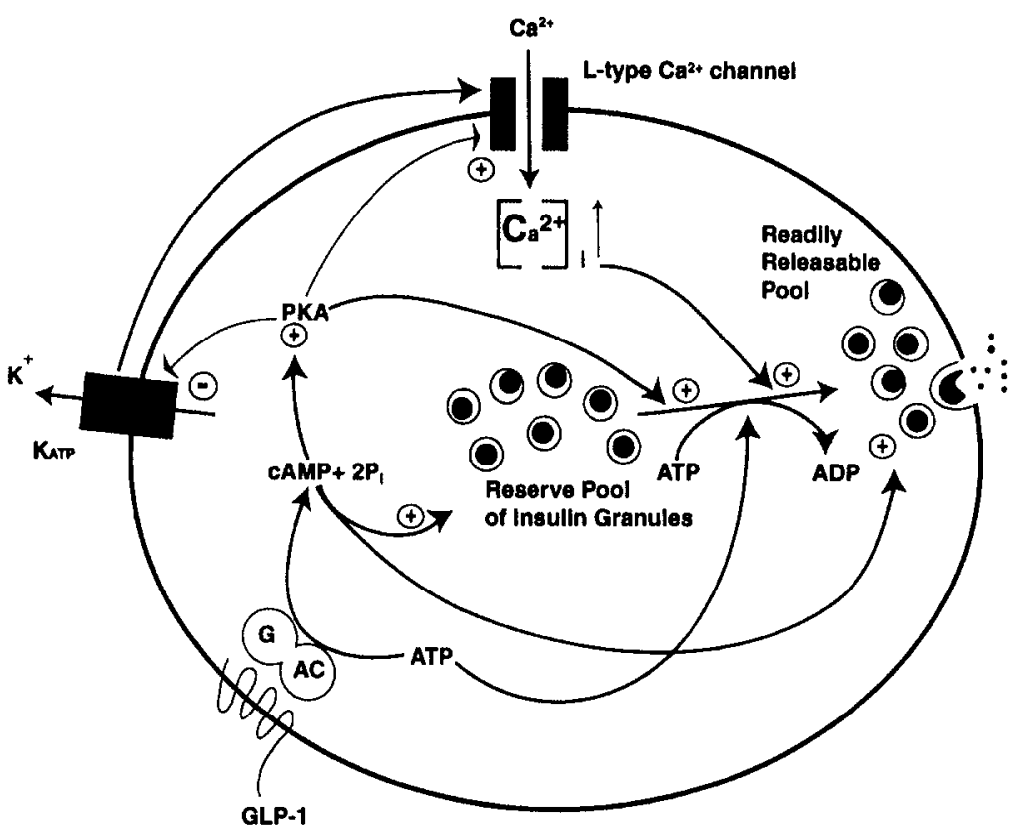

FIG 1. Known intercellular events that occur upon binding of GLP-1 to its specific G-coupled protein receptor. Adenylyl cyclase is activated, with a consequential rise in cAMP that, in turn, results in PKA activation. Then, by PKA-dependent and -independent means, insulin release results.

containing granules. GLP-1 is known to cause a rise in intracellular $\mathrm{Ca}^{2+}$ concentration $\left[\mathrm{Ca}^{2+}\right]_{i}$ (Yada et al., 1993). This effect is attributable both to greater activation of the L-channel, which could be due to its phosphorylation by PKA, and also to a mobilization of the intercellular stores of $\mathrm{Ca}^{2+}$, which may or may not be PKA dependent (Bode et al., 1999). GLP-1 also increases insulin secretion in a calcium-independent manner by mobilizing secretory vesicles to enter the readily releasablc pool. Again, this is by both PKA-dependent and -independent means (Gromada et al., 1998).

\section{GLP-1 Acute Effects on Insulin Secretion in Vitro}

GLP-1 enhances glucose-dependent insulin secretion in the insulinoma cell line RIN 1046-38 (Montrose-Rafizadeh et al., 1994). The presence of GLP-1 (10 $\mathrm{nM}$ ) decreases the concentration of glucose required for half-maximal insulin secretion ( 0.72 vs. $0.19 \mathrm{mM}$, control vs. treated). During the course of a 1-hour static insulin-release experiment, the total amount of insulin secreted en masse is increased by the addition of GLP-1 $(10 \mathrm{nM})$ from 10.33 to $25.18 \mathrm{ng}$ insulin $/ \mathrm{mg}$ 
protein/hour. This occurs as GLP-1 increases 1) the amount of insulin secreted per cell and 2) the number of cells that are responsive to glucose. This has been demonstrated by the use of the reverse hemolytic plaque assay, the only means of determining both the relative amount of insulin secreted by an individual $\beta$-cell and the percentage of cells secreting insulin (Egan et al., 1991).

\section{A. INCREASE IN THE AMOUNT OF INSULIN SECRETED}

Under conditions of maximal glucose stimulation, the addition of GLP-1 results in a 1.3-fold increase in the relative amount of insulin secreted per cell (Table I, compare conditions 5 and 6). Priming the cells by adding potassium (20 $\mathrm{mM}$ ) results in membrane depolarization and the secretion of insulin, even in the absence of glucose. That the glucose requirement for GLP-1 action can be replaced by cell membrane depolarization (condition 5 vs. 7) suggests that a rise of intracellular $\mathrm{Ca}^{2+}$ may be an early step required for GLP-1 action. When GL.P-1

TABLE I

Insulin Secretion from Single RIN 1046-38 Cells, Measured by Reverse Hemolytic Plaque Assay

\begin{tabular}{llllll}
$\begin{array}{l}\text { Condi- } \\
\text { tion }\end{array}$ & $\begin{array}{l}\text { Glucose } \\
(\mathrm{mM})\end{array}$ & $\begin{array}{l}\mathrm{KCl} \\
(\mathrm{mM})\end{array}$ & $\begin{array}{l}\text { GLP-1 } \\
(\mathrm{nM})\end{array}$ & $\begin{array}{l}\text { Percent of } \\
\text { Secreting Cells }\end{array}$ & $\begin{array}{l}\text { Mean Plaque Area } \\
\left(\mu \mathrm{m}^{2)}\right.\end{array}$ \\
\hline 1 & 0 & 0 & 0 & $12.5 \pm 1.5$ & $\mathrm{ND}$ \\
2 & 0 & 0 & 10 & $14.7 \pm 1.1$ & $\mathrm{ND}$ \\
3 & 0 & 20 & 0 & $41.2 \pm 1.4$ & $506 \pm 29$ \\
4 & 0 & 20 & 10 & $67.6 \pm 1.3$ & $2185.9 \pm 111.2$ \\
5 & 20 & 0 & 0 & $55.9 \pm 3.3$ & $1586.5 \pm 86.2$ \\
6 & 20 & 0 & 10 & $83.5 \pm 1.2$ & $1990.8 \pm 88.4$ \\
7 & 20 & 20 & 0 & $93.8 \pm 1.2$ & $3076.1 \pm 124.5$ \\
8 & 20 & 20 & 10 & $92.7 \pm 10.9$ & $4825.0 \pm 194.5$ \\
\hline
\end{tabular}

Insulin secretion from individual cells and the number of cells secreting insulin were measured by the reverse hemolytic plaque assay in either normal $\mathrm{K}^{+}(5 \mathrm{mM} \mathrm{KCl})$ or high $\mathrm{K}^{+}(20 \mathrm{mM} \mathrm{KCl}$; i.e., membrane depolarization), in the presence or absence of $20 \mathrm{mM}$ glucose and in the presence or absence of $10 \mathrm{nM}$ GLP-1. The percentage of cells with plaques (cells secreting insulin) was quantified by counting 50-60 cells in each treatment group. Plaque area was measured as described in 15-20 cells in each treatment group. Results are presented as the mean \pm S.E. of measurements from three independent cell preparations. ND, not determined because plaques were too small for reliable measurements. [Adapted with permission from Montrose-Rafizadeh, C., Egan, J.M., and Roth, J Endocrinology 135, 589-594, 1994. Copyright The Endocrine Society.] 
is added to the combination of glucose and membrane depolarization, there is no additional increase in the number of cells secreting insulin but there is a 1.6-fold increase in the relative amount of insulin secreted per cell (compare conditions 7 and 8).

The increase in the amount of insulin secreted by the $\beta$-cell can be attributed to the action of GLP-1-stimulated second messengers on the process of exocytosis of the insulin granules (Figure 1). GLP-1 accelerates insulin secretion by stimulating exocytosis of the readily releasable pool of insulin granules. These are the granules close to the surface of the $\beta$-cell. Their release is stimulated by the action of cAMP (Renström et al., 1997). Pretreatment of RIN cells with the cAMP analog 8-Br-cAMP renders the cells more glucose competent. Basal levels of cAMP are elevated in RIN 1046-38 cells, which overexpress the GLP-1 receptor (MontroseRafizadeh et al., 1997) relative to the parental cells (18.6 vs. $35.96 \mathrm{nmol} \mathrm{dm}^{-3} / \mathrm{mg}$ protein hour, parental vs. transfected). This is reflected by increased insulin secretion both in the absence of glucose ( $1.62 \mathrm{vs.} 2.66 \mathrm{pmol} \mathrm{dm}^{-3} / \mathrm{mgprotein}$ hour; parental $v s$. transfected) and in response to $0.5 \mathrm{mM}$ glucose $(2.21$ vs. $4.52 \mathrm{pmol}$ /mgprotein hour; parental $v s$. transfected). Cells overexpressing the receptor respond to GLP-1 even in the absence of glucose, indicating that an increased cAMP level is permissive to the glucose response (Montrose-Rafizadeh et al., 1997).

Treatment of RIN 1046-38 cells with GLP-1 (10 nM) increases tyrosine phosphorylation of proteins (Zhou and Egan, 1997), specifically, phosphorylation of the SNARE (soluble NSF attachment protein receptor) protein, SNAP-25 (Figure 2A). SNARE proteins are requircd for the docking and fusion of vesicles with their target membrane in a variety of organisms and tissues (Jahn and Södhof, 1999). Cleavage of SNAP-25 results in an inhibition of $\mathrm{Ca}^{2+}$-stimulated insulin release in $\beta$-cell lines and primary rat islets (Sadoul et al., 1995). Tyrosine phosphorylation of SNAP-25 and, correspondingly, GLP-1-induced insulin secretion are attenuated by the tyrosine kinase inhibitor genistein and enhanced by vanadate, a tyrosine phosphatase inhibitor. Thus, activation of SNAP-25 is probably another aspect of the mechanism by which GLP-1 stimulates exocytosis and consequently the amount of insulin secreted by the $\beta$-cell.

\section{B. INCREASE IN THE NUMBER OF CELLS SECRETING INSULIN}

Virtually all RIN cells secreted insulin when glucose $(20 \mathrm{nM})$ and membrane depolarization were applied simultaneously (Table I, compare conditions 5 and 6). GLP-1 sensitizes more cells for secretion in the presence of glucose or membrane depolarization but not in the absence of glucose (Montrose-Rafizadeh et al., 1994). Thus, GLP-1 renders more cells glucose competent (Holz et al., 1993) and recruits more cells into a secretory mode. This can be understood in terms of the action of GLP-1 on the $\mathrm{K}_{\mathrm{ATP}}$ channels. The greater membrane depolarization increases the number of these channels that are closed and, consequently, the $\mathrm{Ca}^{2+}$ 
A
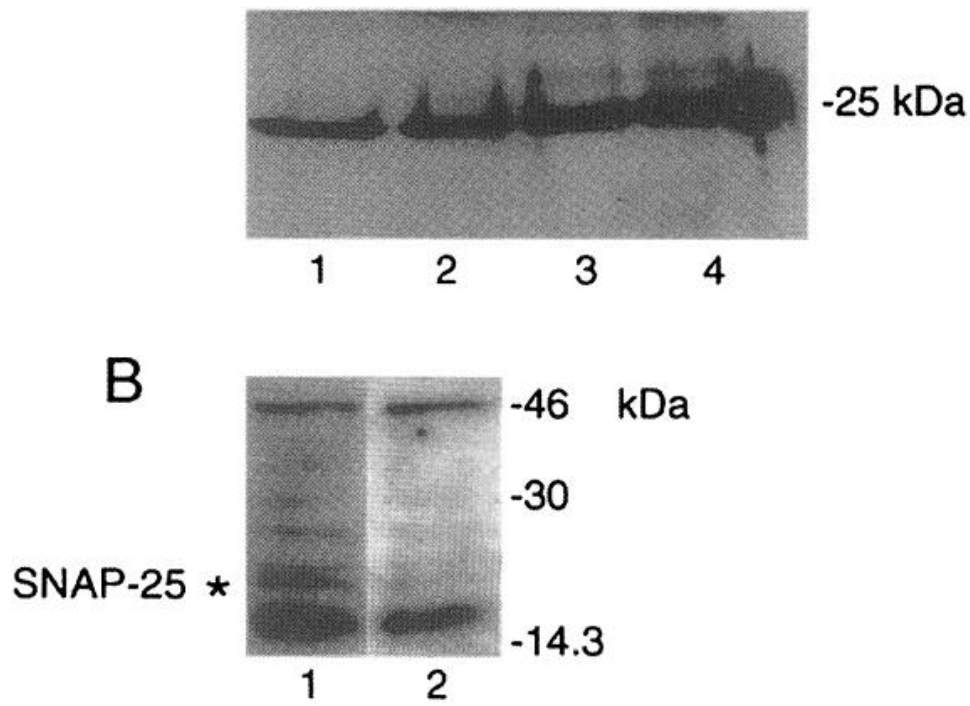

FIG. 2. Western blot illustrating the effects of the insulin secretagogues, GLP-1 and glucose, on tyrosine phosphorylation of SNAP-25. (A) RIN 1046-38 cells treated as follows: 1) no treatment, 2) glucose $(20 \mathrm{mM}), 3) \mathrm{GLP}-1(20 \mathrm{nM})$, and 4) glucose $(20 \mathrm{mM})$ and GLP-1 $(20 \mathrm{nM})$. Cells were lysed and the phosphoproteins immunoprecipitated using a monoclonal antiphosphotyrosine antibody. The immunoprecitates were separated by SDS-PAGE and proteins transferred to polyvinylidene difluoride (PVDF) membrane, which was incubated with a monoclonal anti-SNAP-25 antibody. (B) Western blot of immunoprecipitates from cells treated with glucose and GLP-1 and probed for an antiphosphotyrosine anitbody in absence 1) or presence 2) of a 1000-fold excess of phosphorylated tyrosine. [Adapted from Zhou J., and Egan J.M. Biochem. Biophys. Res. Commun. 238, 297-300, 1997. Copyright Academic Press, Inc.]

current undergoes more-extensive inactivation before the cells start repolarizing (Gromada et al., 1998). The interburst membrane potential does not fall back to resting membrane potential, so the cells can begin depolarizing even before they completely recover from inactivation. Therefore, the silent interburst intervals are shorter and the periods for which the $\mathrm{Ca}^{2+}$ channels are active are longer (Gromada et al., 1998).

\section{GLP-1 ACUTE EFFECTS ON INSULIN SECRETION IN VIVO}

Aging is an etiological factor in non-insulin-dependent diabetes. While impaired insulin secretion, reduced insulin sensitivity, and obesity are the predisposing factors for this disease, a general decline in $\beta$-cell function with age is noted 
even in lean subjects (United Kingdom Prospective Diabetes Study, 1995). The Wistar rat is an ideal model for studying the effects of aging on the progression of diabetes, as these rats develop glucose intolerance and have a significantly lower insulin response to a glucose load with aging (Reaven et al., 1979). As in the insulinoma cell line, individual pancreatic $\beta$-cells harvested from young (3month) and old (22-month) Wistar rats display an improved response to glucose across a range of glucose concentrations, when co-incubated with GLP-1, as illustrated by the data in Table II. In the absence of GLP-1, the number of cells secreting insulin is significantly reduced in the population of cells from old animals. The addition of GLP-1 redeems the glucose responsiveness of the older cells. Hence, the number of cells secreting insulin are on a par with the values observed in the younger animals.

The capacity of GLP-1 to render $\beta$-cells glucose competent is dramatically illustrated by the improved glucose tolerance in old Wistar rats that have been subjected to an intravenous glucose tolerance test (IVGTT) following a bolus of GLP-1 (De Ore et al., 1997). Old (22-month) animals have a delayed and attenuated acute insulin response during an IVGTT, when compared to 3-month-old rats (Figure 3). Of the range of GLP-1 concentrations tested (i.e., 0.05, 0.1, 0.2, 0.4, and $0.5 \mathrm{nmol} \mathrm{kg}$ ), the maximum response in both young and old animals was attained with a $0.4 \mathrm{nmol} \mathrm{kg}^{-1}$ bolus of GLP-1. Serum insulin levels peaked at 2 minutes in the young animals $(859 \mathrm{pmol} / \mathrm{l})$. The old animals did not reach peak insulin levels until 7 minutes after completion of the intravenous glucose administration and were deficient when compared to the younger ones $(656 \mathrm{pmol} / \mathrm{l})$.

TABLE II

Percentage of Plaque-forming $\beta$-Cells Isolated from Young (3-Month) and Old (22-Month) Wistar Rats in Response to Varying Concentrations of Glucose and with GLP-I

Glucose (mM)

\begin{tabular}{llccc} 
& \multicolumn{4}{c}{ Glucose (mM) } \\
\cline { 2 - 5 } Rat & 3.5 & 5 & 11 & 20 \\
\hline Young (3 month) & $7 \pm 1$ & $10 \pm 1$ & $17 \pm 1$ & $17 \pm 2$ \\
Old (22 month) & $2 \pm 1$ & $7 \pm 1$ & $11 \pm 2$ & $10 \pm 2$ \\
Old + GLP-1 (10 nm) & $6 \pm 1$ & $11 \pm 4$ & $15 \pm 5$ & $15 \pm 7$ \\
\hline
\end{tabular}

Islets were extracted as described previously (Egan et al., 1991) and dissociated into individual $\beta$-cells that were then incubated for 1 hour in the absence of glucose (Montrose-Rafizadeh et al,, 1994). The cells were then challenged with varying concentrations of glucose, as shown, for a period of 10 minutes. $\beta$-cells originating from old islets were also treated with GLP-1 concomitantly with glucose to assess the impact of this treatment on the number of cells secreting insulin. 

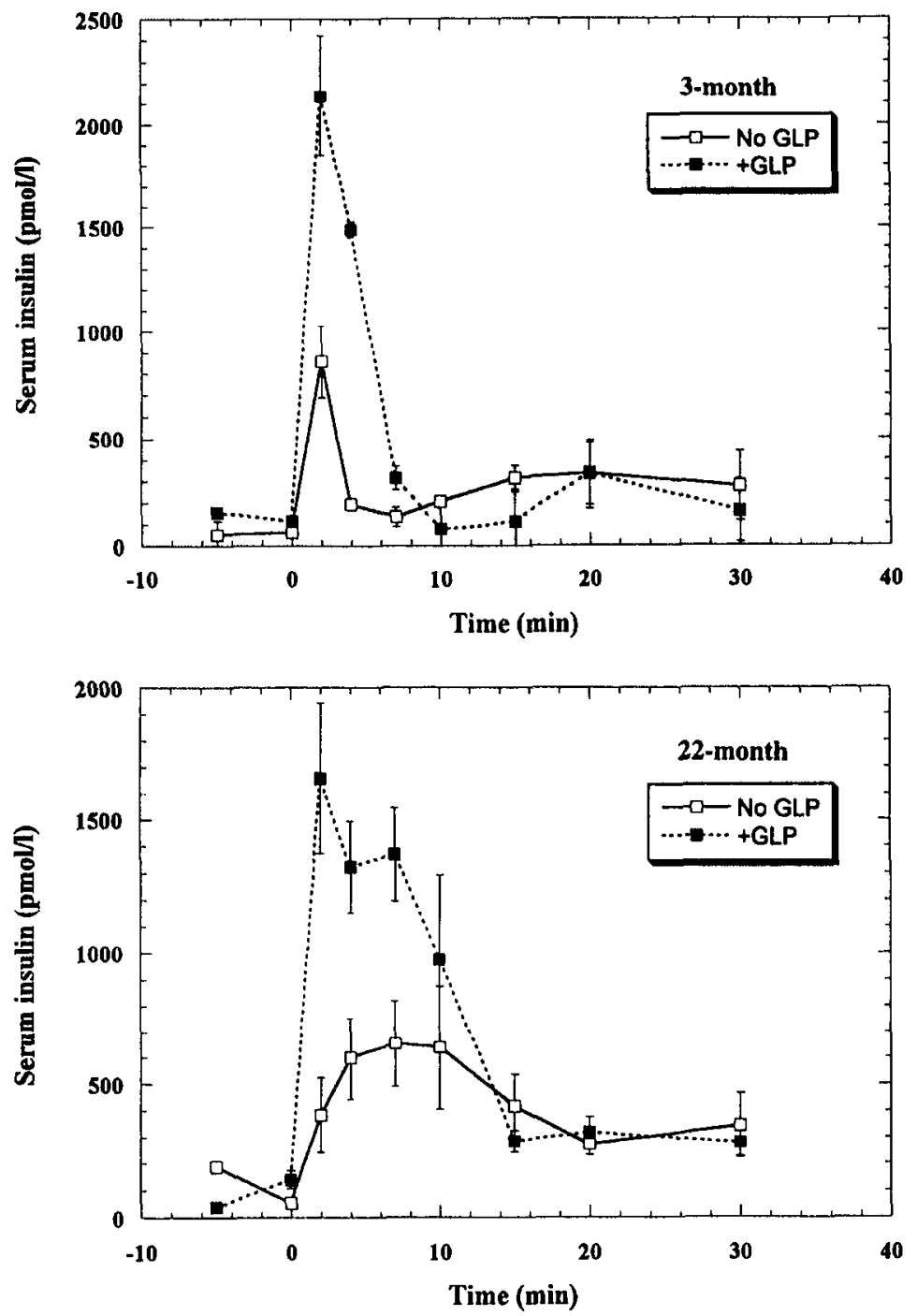

FIG. 3. Insulin levels in young (top panel) and old (bottom panel) rats after an IVGTT $(0.5 \mathrm{~g} / \mathrm{kg}$ glucose $)$ in the presence or absence of a bolus of GLP-1 $(0.4 \mathrm{nmol} / \mathrm{kg})$. Each timepoint represents the mean \pm S.E.M. of six animals. Blood was drawn at the times plotted on the graph. In young rats (top panel), $\mathrm{AUC}_{0-20}$ was $2504 \pm 251$ for IVGTT alone vs. $8180 \pm 1043 \mathrm{pmol} / \mathrm{l}$ for IVGTT with $0.4 \mathrm{nmol} / \mathrm{kg}$ GLP-1; $p=0.006$ by one-way ANOVA. In old rats (bottom panel), $\mathrm{AUC}_{0-15}$ was $7973 \pm 2096$ for IVGTT alone vs. $16,160 \pm 3232 \mathrm{pmol} / \mathrm{l}$ for IVGTT with $0.4 \mathrm{nmol} / \mathrm{kg} \mathrm{GLP-1} ; p=0.012$ by one-way ANOVA. [Reprinted with permission from De Ore K., Greig N.H., Holloway H.W., Wang Y., Perfetti R., and Egan J.M...J. Gerontol. Biol. Sci. 52A, B245-B249, 1997. Copyright The Gerontological Society of America.] 
When GLP-1 $(0.4 \mathrm{nmol} / \mathrm{kg})$ was administered prior to the IVGTT, the amount of insulin secreted by the old animals increased two-fold relative to that seen in the absence of GLP-1. First-phase insulin response to glucose was restored, as demonstrated by the higher blood insulin levels at 2 minutes in those treated with GLP-1 vs. the controls $(1,659$ vs. $384 \mathrm{pmol} / \mathrm{l}, p=0.002)$ and the significantly increased $\mathrm{AUC}_{0-15}$ for insulin (16,160 vs. $\left.7973 \mathrm{pmol} / \mathrm{l}, p=0.012\right)$.

Thus, the ability to respond to the insulinotropic influence of GLP-1 is retained even in old, glucose-intolerant rats. The magnitude of the response is the same regardless of age and there is a reversal of the age-related decline in $\beta$-cell function. This is compatible with the in vitro observations and can be explained by the ability of GLP-1 to sensitize previously unresponsive $\beta$-cells to glucose and to increase the amount of insulin secreted per cell.

\section{GLP-1 Administered Chronically: Effects on Insulin Secretion}

This parity of response between young and old animals is exhibited following a 48-hour infusion of GLP-1 (Wang et al., 1997). Administration of GLP-1 (1.5 pmol kg-1 $\mathrm{min}^{-1}$ ) by means of an Alzet micro-osmotic pump implanted in the intracapular region dramatically improved the response of both 3- and 22-monthold Wistar rats to an intraperitoneal glucose tolerance test (IPGTT), performed 120 minutes after removal of the pumps (Figures 4 and 5).

As in the acute study, the striking feature of the IPGTT is the reversal of the glucose intolerance in the old animals. The rise in blood glucose levels was identical to that in the young treated animals and significantly lower than the saline-treated controls (Figure 4). The insulin response peaked at 15 minutes in the treated animals vs. a peak insulin response at 30 minutes for the controls (Figure 5). This accounts for the lower blood glucose levels at 15 minutes in the treated animals. Intra-islet insulin content following acid ethanol extraction was, on average, three-fold greater in the treated old animals than in the control old animals (5.31 vs. $19.68 \mathrm{ng}$ of insulin per $\mu \mathrm{g}$ of total pancreatic protein).

The in vivo results correspond with observations in vitro (Wang et al., 1995) when RIN 1046-38 insulinoma cells were incubated for 24 hours with GLP-1 (10 $\mathrm{nM}$ ). During this extended exposure to GLP-1, there was a 3.5 -fold increase in the cumulative amount of insulin secreted in the 24-hour incubation period and a 1.5-fold increase in intracellular insulin content, relative to untreated controls. Following this incubation, cells were washed extensively to remove GLP-1 and glucose, incubated in a glucose-free buffer for a period of 1 hour, then subjected to a 1-hour insulin-release experiment in response to varying concentrations of glucose. Glucose responsiveness was significantly improved in the cells preincubated with GLP-1: at $10 \mathrm{mM}$ glucose, the untreated cells increased insulin secretion by 1.9 -fold, compared with $0 \mathrm{mM}$ glucose, whereas the GLP-1 preincubated 

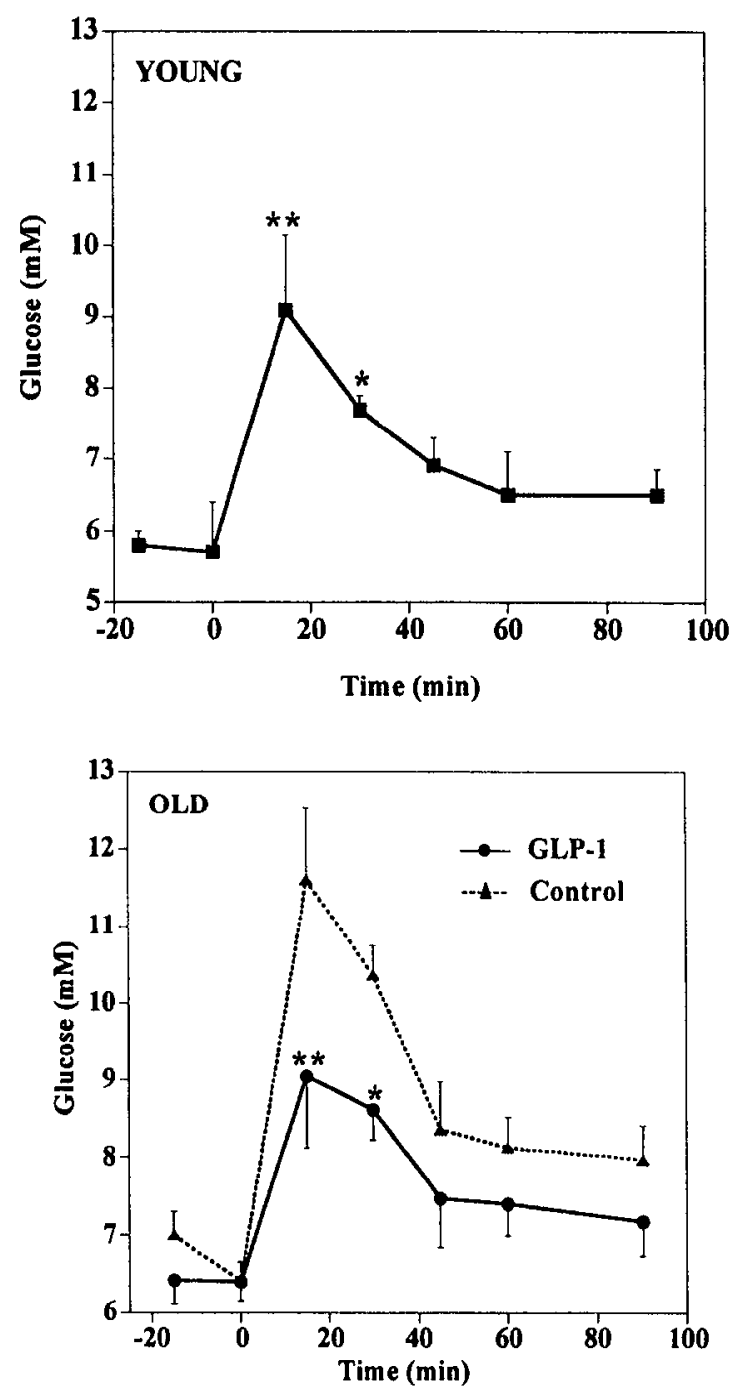

FIG. 4. Plasma glucose levels during an IPGTT $(1 \mathrm{~g} / \mathrm{kg})$ in young (3-month) (top panel) and old (22-month) rats (bottom panel). GLP-1-treated old animals received $1.5 \mathrm{pmol} \mathrm{kg}^{-1} \mathrm{~min}^{-1}$ for 48 hours by subcutaneous infusion. All other animals were infused for 48 hours with saline. The results are shown as mean \pm S.E.M. of six animals in each group. Repeated measures analysis of variance from 0 to 30 minutes showed a value of $P<0.05$ for the GLP-1-treated vs. control old animals. ${ }^{*} P<0.05$, ${ }^{* *} \mathrm{P}<0.01$, as determined by unpaired Student's $t$ test for control young vs. control old animals and or GLP-1-treated vs. control old animals. [Reprinted with permission from Wang, Y., Perfetti, R., Greig, N.H., Holloway, H.W., DeOre, K., Montrose-Rafizadeh, C., Elahi, D., and Egan, J.M. J. Clin. Invest. 99, 2883-2889, 1997. Copyright Rockefeller University Press.] 


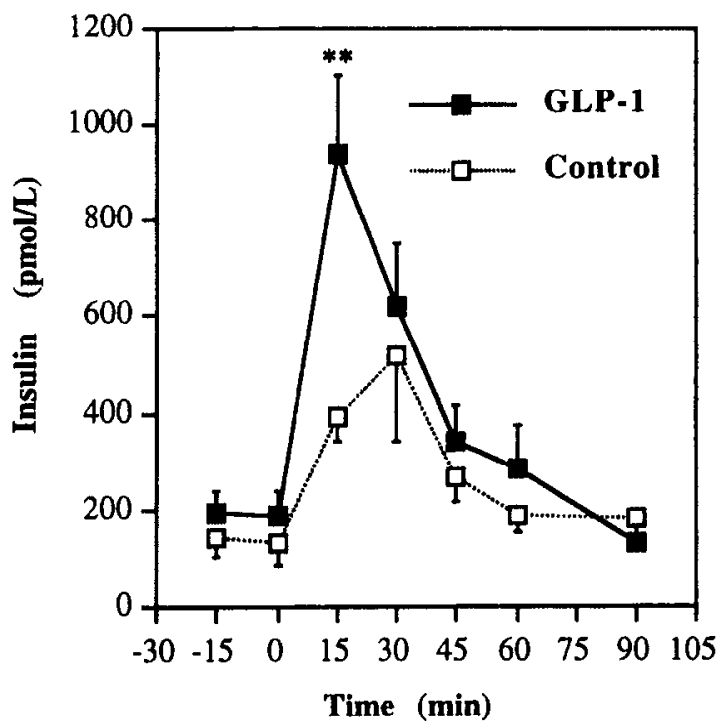

FIG 5. Plasma insulin levels during an intraperitoneal glucose $(1 \mathrm{~g} / \mathrm{kg})$ tolerance test in 22 month-old animals. GLP-1-treated animals received $1.5 \mathrm{pmol} / \mathrm{kg}^{-1} \mathrm{~min}^{-1}$ for 48 hours by subcutaneous infusion. Controls were infused with saline. The results are mean \pm S.E.M. of six treated and six control animals. Repeated measures analysis of variance from 0 to 30 minutes showed a value of *P $<0.05,{ }^{* *} P<0.01$, as determined by unpaired Student's $t$ test. [Reprinted with permission from Wang, Y., Perfetti, R., Greig, N.H., Holloway, H.W., DeOre, K., Montrose-Rafizadeh, C., Elahi, D., and Egan, J.M. J. Clin. Invest. 99, 2883-2889, 1997. Copyright Rockefeller University Press.]

cells increased insulin secretion by 2.8 -fold. This indicates that the preincubation period with GLP-1 had potentiated the glucose sensitivity of the cells.

The time lapse between termination of treatment and the ongoing effect of GLP-1 observed in vitro and in vivo indicate that the effect of the peptide extends beyond an impact on insulin release. This is of particular importance, given the short half-life (6-8 minutes) in vivo of the peptide (Elahi et al., 1994). Therefore, we examined the effects of GLP-1 on the mRNA levels of insulin and the glucose-sensing components of the $\beta$-cell in the islets of the treated rats, specifically, the high $\mathrm{K}_{\mathrm{m}}$ glucose transporter GLUT2 and the high $\mathrm{K}_{\mathrm{m}}$ glucose phosphorylating enzyme, glucokinase (GCK).

\section{GLP-1 Administered Chronically: Effects on $\beta$-cell Gene Expression}

The mRNA levels of insulin and GLUT2 measured in total RNA extracted from mice pancreata decrease progressively with age (Perfetti et al., 1995,1996). 
In contrast, GCK levels increase significantly with age, which we interpret as a compensation within the glucostat for the diminishing levels of GLUT2. A similar age-related pattern in mRNA levels was observed in the untreated animals (Wang et al., 1997) in the 48-hour study on the Wistar rats. Levels of insulin mRNA were decreased by 50 percent, GLUT2 mRNA levels by 70 percent, with no change observed in GKC mRNA levels in old (22-month) relative to young (3-month) animals (Figure 6). Not only did GLP-1 elevate the levels of all three of these mRNAs in the young animals but it also completely reversed the decrease observed in GLUT2 and insulin mRNA levels in the old animals. When GLP-1 infusion was accompanied by an infusion of a competitive antagonist, exendin (9-39), no enhancement of the mRNA expression of these genes was observed. Treatment with the antagonist alone decreases mRNA levels, indicating an inhibition of endogenous GLP-1 effects. Significantly, this was the first reported observation of a pharmacologically induced elevation of GLUT2 mRNA. Sulfonylurea agents, for example, increase insulin secretion by inhibition of the $\mathrm{K}_{\mathrm{ATP}}$ channels. A recent report (Porzio et al., 1999) shows that glimepiride, a sulfonylurea derivative, upregulates GLUT2 and GKC mRNA following a 24-hour treatment of the insulinoma cells $\beta$ TC6-F7. However, at the 48- and 72-hour timepoints, GLUT2 and GKC mRNA and protein levels were reduced relative to basal levels, indicating a downregulation of these genes by the drug.

\section{GLP-1 Upregulates PDX-1 in Vitro and in Vivo}

One method of influencing transcriptional regulation of the insulin gene is directly mediated by the cAMP-response element-binding protein (CREB). However, this is not the case for GLUT2, as it does not possess a CREB-binding site. Therefore, in the GLP-1 upregulation of these genes, a master regulatory transcription factor may be downstream of CREB that is responsible for the regulation of all three genes; GLUT2, insulin, and GKC. The most-probable candidate is the homeodomain transcription factor PDX-1, which is known to regulate insulin (Petersen et al., 1994), GLUT2 (Waeber et al., 1996), GKC, and islet amyloid precursor genes (Watada et al., 1996a,1996b). PDX-1 is essential for the early development of the pancreas (Jonsson et al., 1994) and the maintenance of the $\beta$-cell phenotype (Ahlgren et al., 1998). Under conditions of sustained hyperglycemia, such as in the diabetic state, there is a downregulation of PDX-1 expression and a decrease in insulin secretion (Olson et al., 1995). PDX-1 expression, as measured by immunofluorescence staining of sections taken from islets extracted from the Wistar rats of various ages, decreases by approximately 50 percent in animals older than 13 months. Therefore, one might expect this downregulation would lead to a downregulation of the genes under the transcriptional control of 


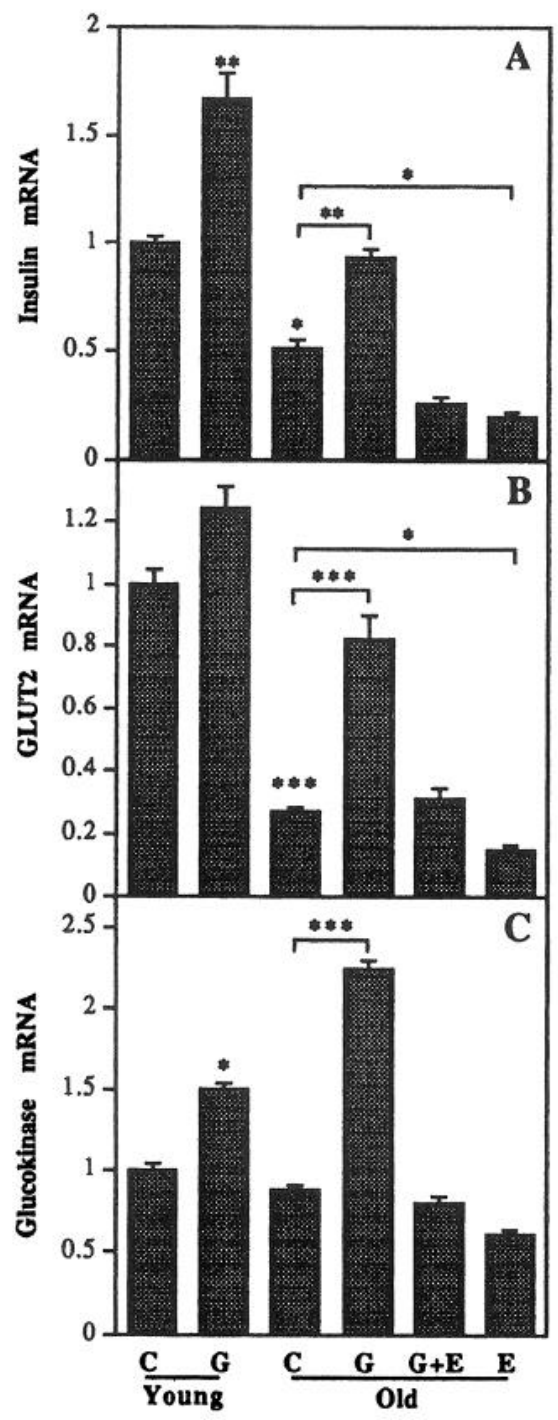

FIG. 6. Effects of normal saline (C), GLP-1 (G), GLP-1 and exendin (9-39) (G and E), and exendin (9-39) (E) treatment for 48 hours on mRNA levels of insulin (panel A), GLUT2 (panel B), and glucokinase (panel C) using pancreatic poly-A RNA in young and old animals. Images of cDNA hybridization were quantified using densitometry and normalized with those of poly-A. Data are shown as mean \pm S.E.M. $\left(n=6\right.$ animals per group), with control data assigned a value of $1 .{ }^{* P}<0.05,{ }^{* *} \mathrm{P}$ $<0.01,{ }^{* * *} \mathrm{P}<0.001$ by unpaired Student's $t$ test. [Reprinted with permission from Wang, Y., Perfetti, R., Greig, N.H., Holloway, H.W., DeOre, K., Montrose-Rafizadeh, C., Elahi, D., and Egan, J.M.. J. Clin. Invest.. 99, 2883-2889, 1997. Copyright Rockefeller University Press.] 
PDX-1 and that GLP-1 treatment should lead to an upregulation of PDX-1 expression.

Returning to the insulinoma cell line RIN 1046-38 (Wang et al., 1999), we have demonstrated a functionally significant upregulation of the PDX-1 gene in response to GLP-1 (10 nM). Following 1 hour of treatment, PDX-1 mRNA levels increased 1.3-fold (Figure 7) above basal levels $(p<0.01$ ), with a maximum 1.4 -fold increase at 2 hours $(p<0.01)$. This coincides with a rise in insulin mRNA expression that reaches a maximum level at 3 hours. For the entire duration of the 12-hour treatment, levels of PDX-1 mRNA and protein remained significantly above basal. PDX-1 protein levels were 1.6-fold above basal in nuclear and whole-cell extracts $(p<0.001)$. Treating the cells with glucose $(6$ and $15 \mathrm{mM})$ alone resulted in translocation of, but not transcription or translation of, $p d x-1$. This, at least in part, explains the ability of GLP-1 to stimulate insulin biosynthesis and provides a mechanism for the long-term effects of GLP-1 on $\beta$-cell insulin content and insulin secretion. The functional significance of the GLP-1-induced PDX-1 expression was demonstrated by the increase in binding of PDX-1 to the A1 element of the insulin promoter (Macfarlane et al., 1994) consequent upon GLP-1 treatment (Figure 8). Maximum binding occurs at the 2-hour timepoint. The specificity of this binding was confirmed by supershift analysis and competitive binding studies. These results have been substantiated in a recent report by Buteau and colleagues (1999) demonstrating a similar upregulation of PDX-1 by GLP-1 in the insulinoma cell line INS-1.

This led us to believe that restoration of $\beta$-cell function in the glucose-intolerant Wistar rats upon treatment with GLP-1 is mediated by the transcription factor activator PDX-1 and prompted us to examine PDX-1 protein and mRNA content in whole pancreata and islets extracted from Wistar rats treated with GLP-1 (Perfetti et al., 2000). The trend in the level of expression of PDX-1 paralelled that of the insulin and GLUT2 genes (i.e., a decrease in expression with age but an increase of comparable magnitude observed in both young (6-month) and old (22-month) pancreata upon treatment with GLP-1). GLP-1 treatment (1.5 pmol kg-1 $\mathrm{min}^{-1} ; 48$-hour infusion using a micro-osmotic pump) upregulated $p d x-1$ mRNA in both the islets and in whole pancreatic extracts. The amount of $p d x$ ImRNA in the whole pancreas was twice that seen in the untreated pancreas, which is an interesting phenomenon, given that the islets comprise only about 0.5 to 1 percent of the total pancreatic mass. PDX-1 protein levels also were increased in response to treatment in islets and whole pancreas. This fits with what we see in mice that have been treated with continuous subcutaneous GLP- 1 infusion for 14 days. Western blot analysis reveals a 4.6-fold increase in PDX-1 levels in total pancreata (Stoffers et al., 2000).

Following 2 days of treatment, the extent of proliferative activity in the pancreata of the treated rats (both young and old) incrcased, as determined quali- 


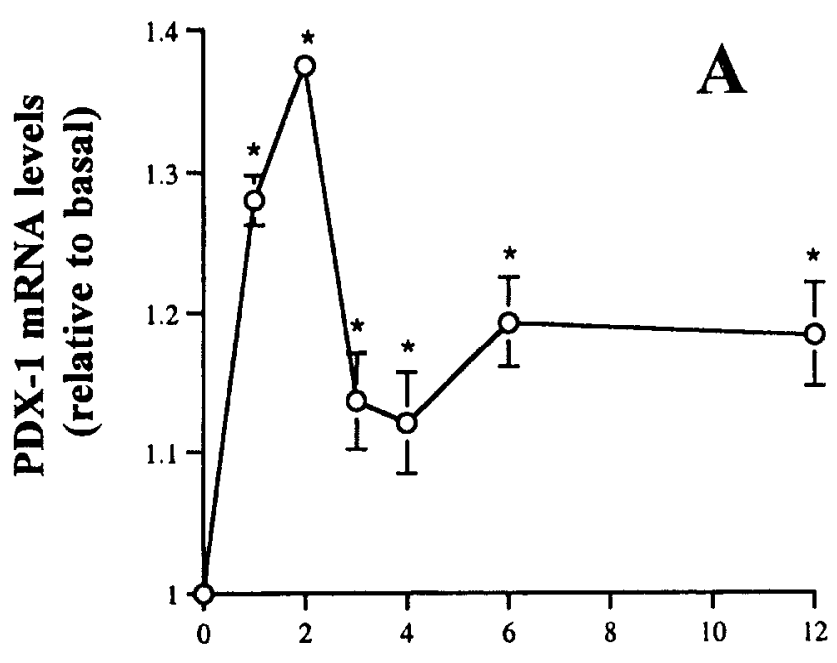

Time (h)

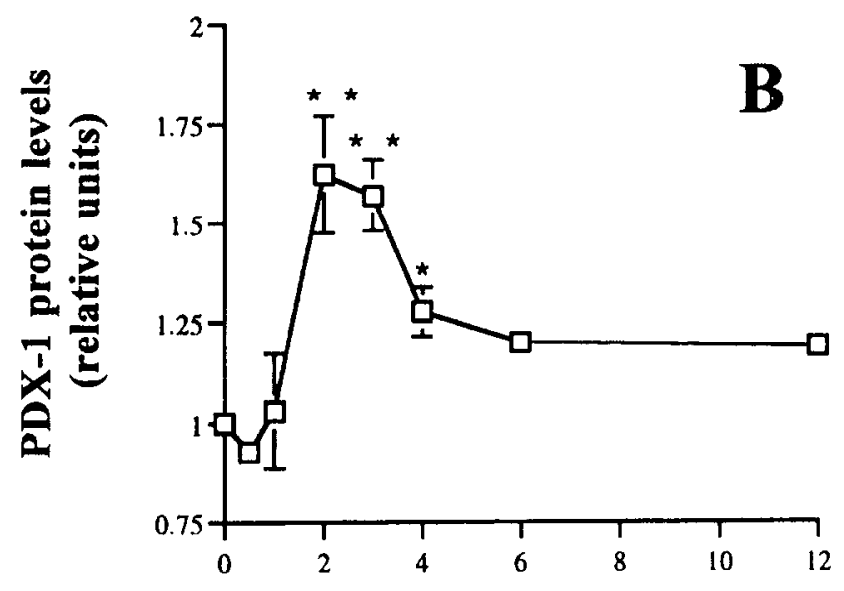

Time (h)

FIG. 7. Timecourse of the effect of GLP-1 (10 nM) on PDX-1 mRNA (panel A) and protein levels (panel B) in RIN 1046-38 cells. Nuclear extracts were used for the Western blot. [Adapted with permission from Wang, X., Cahill, C.M., Pineyro, M.A., Zhou, J., Doyle, M.E., and Egan, J.M. Endocrinology 140, 4904-4907, 1999. Copyright The Endocrine Society.] 

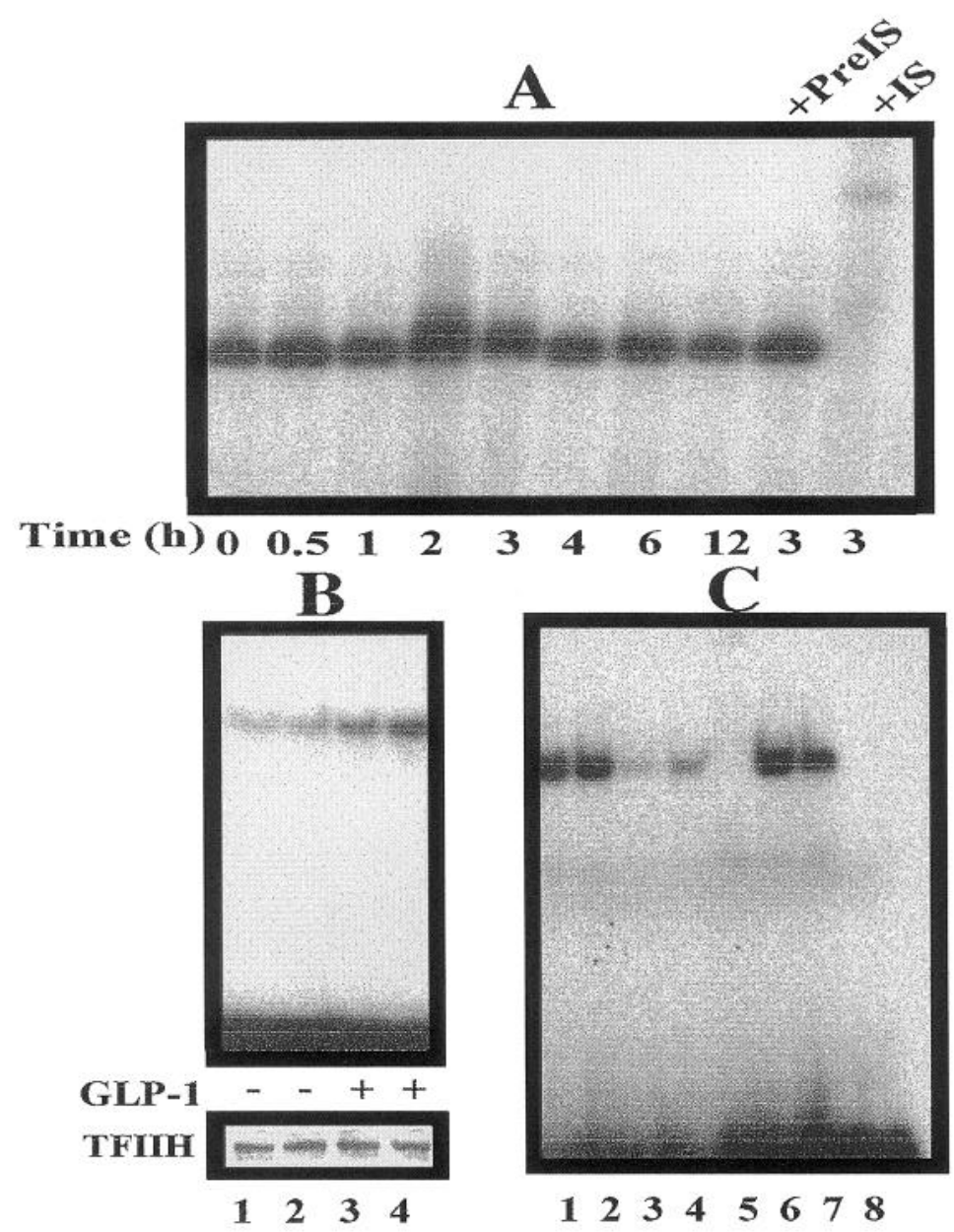

FIG. 8. Timecourse and effect of GLP-1 on PDX-1 protein binding to the rat insulin promoter A1 element using nuclear extract from RIN cells that had been treated \pm GLP-1 (10 nM). (A) Cells were cultured in glucose $(6 \mathrm{mM})$ and GLP-1 for the indicated times. Equal concentrations of the proteins were analyzed by electrophoretic mobility shift assays (EMSA) for binding to Al element of rat insulin II promoter ${ }^{32} \mathrm{P}$-labeled probe. The last lane indicates a shift in the binding with PDX-1 antibody (IS). (B) EMSA of protein from cells treated \pm GLP-1 for 2 hours. Western blot analysis of transcription factor IIH (TFIIH) was used as a control of protein levels loaded onto the gel in EMSA. (C) Competition and supershift analysis of A1 binding complex. Lanes 1 and 2; addition of unlabeled HSE promoter $(10 \times, 5 \times)$ : Lanes 3 and 4; addition of unlabeled $A 1$ element $(10 \times, 5 \times)$ : Lane 5 ; addition of the PDX-1 antibody, causing a shift in the binding: Lane 6; addition of PDX-1 preimmune serum: Lane 7; nuclear extract and labeled A1 element: Lane 8; Al element alone. [Reprinted with permission from Wang X., Cahill C.M., Pineyro M.A., Zhou J, Doyle M.E., and Egan J.M. Endocrinology 140, 4904-4907, 1999. Copyright The Endocrine Society.] 
tatively by proliferative cell nuclear antigen (PCNA) staining. The contrast between the treated and untreated pancreata was very distinct. There was extensive PCNA staining in acinar tissue as well as in ducts in the treated pancreata, with very little positive staining seen in these areas of the untreated pancreata. This proliferative response had dissipated following 5 days of treatment with GLP-1; at this timepoint, there was no difference between the treated and control tissues. There was a substantial increase in the $\beta$-cell mass of the treated pancreata $(7.02$ vs. $4.88 \mathrm{mg}$ : treated vs. control, respectively, $p=0.02$, after 5 days of treatment). This was reflected by the distribution of insulin staining in the pancreata that had been exposed to GLP-1 treatment. In the saline-treated animals, discrete islets can be seen with no insulin staining elsewhere. In contrast, sections from GLP-1 animals exhibit insulin staining in small clusters and ducts as well as in the mature islets. These insulin-positive clusters were obvious by 5 days. A few insulin-positive cells could be seen in ducts as early as 3 days. Clearly, the induction of proliferation by GLP-1 is followed by differentiation of some of these cells into small clusters of $\beta$ cells.

The increase in gene expression of the key $\beta$-cell regulatory factor $p d x-1$ in the acinar and ductular tissue, and the subsequent increase in $\beta$-cell mass, all point to GLP-1 being an endocrinotrophic factor. This property of GLP-1 also is shared with exendin- 4 and has been further explored in mice and in an in vitro acinar cell model (see section VII).

\section{The Long-acting GLP-1 Agonist, Exendin-4}

As mentioned briefly earlier, the half-life of GLP-1 in vivo is very short, necessitating either continuous infusion or frequent subcutaneous injection, making the administration of this medicine as cumbersome as the daily treatment of type 1 diabetic subjects with insulin. Consequently, for a type 2 diabetic subject, the potential benefits of GLP-1, not withstanding $\beta$-cell regeneration, are oulweighed by the disadvantages of the treatment regimen. Means of circumventing this inconvenience are currently under investigation in several laboratories but one therapy appears to offer all of the physiological and pharmacological benefits of GLP-1 without the disadvantage of a short biological half-life.

In 1993, the capacity of exendin-4 as an agonist at the GLP-1 receptor was first reported (Goke et al., 1993; Thorens et al., 1993). Exendin-4 is a 39-amino acid peptide with a 53 percent amino acid homology with GLP-1 (Eng et al., 1992) found in salivary glands of the Gila monster lizard (Heloderma suspectum). Attempts to find the mammalian homolog of this gene have failed so far (Chen and Drucker, 1997; Pohl and Wank, 1998). Despite the nonmammalian nature of this protein and its implied potential antigenicity, we have demonstrated it to be a very-potent insulin secretagogue in rodents and humans, possessing all of the 
pharmacological and physiological properties of GLP-1 with two vital exceptions, its duration of action in vivo and potency.

Exendin- 4 , when administered acutely $(0.4 \mathrm{nmol} / \mathrm{kg})$, increases insulin secretion in the Wistar rats more than two-fold above the levels obtained with GLP-1 at the same concentration (Greig et al., 1999). The $\mathrm{ED}_{50}$ values were found to be 0.0143 and $0.19 \mathrm{nmol} / \mathrm{kg}$ for exendin- 4 and GLP-1, respectively. Thus, exendin- 4 is 10 times more potent than GLP-1 in vivo. The increased insulin secretion arises from the higher intracellular cAMP concentrations generated by exendin- 4 , as comparcd to GLP-1. In islets isolated from the Wistar rats, a dose of $1 \mathrm{nmol}$ of exendin- 4 produced a three-fold greater cAMP response relative to an equivalent concentration of GLP-1.

Long-term treatment with exendin-4 significantly improved glucose tolerance and hemoglobin Alc $\left(\mathrm{HbA}_{l \mathrm{c}}\right)$ levels in $d b / d b$ mice (Grieg et al., 1999). Following 13 weeks of once-daily intraperitoneal exendin- $4(24 \mathrm{nmol} / \mathrm{kg})$ injections, the treated mice had a lower mean $\mathrm{HbA}_{\mathrm{Ic}}$ than did the saline-injected controls ( $4.7 \mathrm{vs.}$ 8.8 percent). The response to an IPGTT was significantly improved, as insulin concentrations were higher ( 4600 vs. $707.2 \mathrm{pmol} / \mathrm{l}$ ) in exendin-4 treated mice, compared with saline-treated controls. Young and coworkers have extended the observations on the antidiabetic properties of exendin- 4 in animal models to include Zucker diabetic fatty (ZDF) rats and diabetic rhesus monkeys (Young et al., 1999).

Using the Zucker fatty (ZF) rat as a model, we have shown that twice-daily injections of exendin-4 led to a deceleration in weight gain and fat deposition as well as amelioration of diabetic symptoms (Szayna et al., 2000). The ZF rat is a good model for obesity, as it has a defect in the leptin receptor (Lepr) due to a Gln269Pro mutation (Chua et al., 1996). Therefore, plasma leptin levels are elevated but the rat is unresponsive to the satiety effect of this protein. As a consequence, it overeats, gains weight quickly, and becomes obese and diabetic. These rats, when treated with exendin- $4(10 \mu \mathrm{g} / \mathrm{kg})$ via a daily intraperitoneal injection, showed an initial drop (for the first 5 days) in food intake and weight. Subsequently, however, these parameters were indistinguishable from those of the saline-treated controls. Following 2 weeks of this treatment, a second daily injection was added to the protocol. This resulted in a sustained reduction in food intake and weight gain relative to controls for the remainder of the 56-day treatment regimen. Using magnetic resonance imaging (MRI), it was possible to image the areas of adipose tissue in the rats and hence monitor the rate at which fat was laid down in the abdomen. At the completion of the study (56 days), the amount of fat in both the visceral and subcutaneous compartments of the treated animals was significantly less than in the controls. Thus, despite the high leptin levels, food intake could be influenced by exendin- 4 treatment. Should this chronic effect be 
transferable to humans, it would be beneficial in the management of type 2 diabetes.

As the data from our rodent experiments showed the potential of exendin- 4 as a treatment for type $2 \mathrm{DM}$, we proceeded with acute experiments with exendin4 in nondiabetic and type 2 diabetic subjects (Egan et al., 1999). We demonstrated in both groups of volunteers that exendin-4 is a potent insulinotropic agent. Plasma insulin levels achieved in response to intravenous infusion of $0.15 \mathrm{pmol}$ $\mathrm{kg}^{-1} \mathrm{~min}^{-1}$ for 1 hour were comparable to those achieved with $1.5 \mathrm{pmol} \mathrm{kg}{ }^{-1} \mathrm{~min}^{-1}$ of GLP-1. Additionally, the duration of its biological effects far exceeded those of GLP-1. Insulin levels did not even begin to decrease for at least 3 hours following termination of exendin-4 infusion, unlike GLP-1 infusion, where insulin levels begin to decrease within minutes of termination. Indeed, 6.5 hours after completion of the exendin-4 infusion, plasma insulin levels were still double the fasting values, even in subjects with type 2 diabetes.

The nondiabetic subjects, but not the diabetic subjects, required intravenous glucose for up to 8 hours after termination of exendin- 4 to prevent blood glucose from dropping below $80 \mathrm{mg} / \mathrm{dl}$. This was despite having eaten a meal containing 550 calories 3.5 hours after termination, which, again, points to its sustained biological effect. There was no rise in postprandial levels of plasma insulin relative to those measured just prior to eating. However, as stated earlier, the fasting levels in both nondiabetic and diabetic subjects were twice basal values for several hours following treatment. As there was no postprandial rise in blood glucose after eating, our assessment is that the food was not being utilized. We also measured GLP-1 levels and could not demonstrate any rise after eating, which indicates that food was probably not leaving the stomach. It is therefore evident that exendin-4 prevents postprandial rise in blood glucose, most probably via inhibition of gastric emptying.

Changes seen in other metabolic parameters are consistent with increased insulin secretion in response to exendin-4. Glucagon and nonesterified fatty acid levels decreased as insulin levels increased. As exendin- 4 increases endogenous insulin secretion, one would expect that intra-islet levels of insulin, though not quantifiable, would be far greater than plasma levels. Yet, glucagon levels in diabetic subjects did not suppress to levels seen in nondiabetic subjects. A comparison of the magnitude of suppression, as opposed to absolute levels, indicates that glucagon levels were approximately half the fasting levels in both nondiabetic and diabetic subjects in the 3 hours following exendin- 4 treatment. Therefore, the suppression observed as a result of exendin- 4 is the insulin-dependent component of glucagon regulation. This also indicates that there is an insulin-independent component to glucagon regulation that is altered in type 2 diabetes and is not influenced by endogenous insulin secretion. We conclude that exendin-4 is a 
powerful insulinotropic agent in humans. Its effects on the $\beta$-cells of the pancreas appear long-lived, when compared to GLP-1.

It is feasible that exendin- 4 could be administered in an oral formulation. The stability of the compound in vivo can be attributed to the absence of a dipeptidyl peptidase IV cleavage site in N-terminal sequence (Mentlein, 1999) and to a reduced susceptibility to cleavage by neutral endodpeptidase 24.11 (Hupe-Sodmann et al., 1995) at sites throughout the GLP-1 sequence that are not found in exendin-4. We are currently investigating chimeric derivatives to assess the importance of these sites and to examine which ones confer the greater potency to exendin-4.

\section{Endocrinotrophic Properties of GLP-1 and Exendin-4}

Expansion and subsequent differentiation of the ductal epithelium into clusters of $\beta$-cells has been postulated as the route to $\beta$-cell neogenesis in adult life (Bonner-Weir et al., 1993; Gu et al., 1994). PDX-1 expression, as quantified by immunofluorescence and western blot, is upregulated in the ductular tissue of pancreatectomized rats (Sharma et al., 1999). This upregulation follows a burst of proliferation (Sharma et al., 1999). Thus, PDX-1 is an integral transcription factor in the process of $\beta$-cell neogenesis. We also observe a burst of proliferation in GLP-1-treated animals and upregulation of PDX-1 by western blot of whole pancreas. Using immunofluorescence, enhanced expression of PDX-1 was observed in the ducts and the exocrine tissue of both nondiabetic and diabetic $d b / d b$ mice treated with exendin-4 for 2 weeks (Stoffers et al., 2000). Furthermore, Xu and colleagues have shown $\beta$-cell neogenesis in response to exendin- 4 treatment ( $1 \mathrm{nmol} / \mathrm{kg}, 10$ days), wherein $\beta$-cell mass was increased by 40 percent (Xu et al., 1999). Collating these data allows us to surmise that GLP-1 and exendin-4 cause pancreatic cell proliferation. Some of the proliferating cells become PDX-1 positive. These, in turn, depending on the milieu of the PDX-1-containing cells, become endocrine cells.

The acinar cell line AR42J was chosen as the model to examine the ability of GLP-1 and exendin- 4 to cause the differentiation of putative pancreatic precursor cells into islet cells (Zhou et al., 1999). They are derived from a chemically induced rat pancreatic tumor (Rosewicz et al., 1992) and have the potential to be convertcd into cndocrine (Mashima et al., 1996) or exocrine cells (Christophe, 1994). AR42J cells do not express the islet hormones, insulin, glucagon, and pancreatic polypeptide under usual culture conditions. Upon treatment with both GLP-1 (10 nM) and exendin-4 $(0.1 \mathrm{nM})$ for 3 days, 20 percent of the cells were positive for insulin and 50 percent were glucagon positive. While the percentage of glucagon-positive cells began to decrease on the fourth day, the number of insulin-positive cells remained the same during this time. The extent of 5'-bromo- 
$2^{\prime}$-deoxyuridine (BrdU) incorporation was used to measure proliferation of the cells. In untreated cells, 60 percent of the population were dividing but, after 3 days, only 12 percent of the cells were in S phase. Hence, GLP-1 reduced replication in a greater proportion of the cells than were staining positive for the endocrine hormones. The mRNA transcripts of insulin, glucagon, pancreatic polypeptide, glucokinase, and GLUT2 were detected upon reverse transcriptionpolymerase chain reaction (RT-PCR) analysis of total RNA extracts of the treated cells. None of these transcripts were present in the samples extracted from AR42J cells under usual culture conditions. Protein expression of another important $\beta$-cell transcription factor, Neuro D/Beta 2, was only evident in treated cells. When the cells were co-incubated with either GLP-1 or exendin- 4 and the antagonist exendin (9-39), there was no conversion; hence, the differentiation process occurs via direct activation of the GLP-1 G-coupled protein receptor in the case of both peptides. Finally, although PDX-1 normally is present in cells, expression of the protein was enhanced 1.7-fold as a consequence of exendin-4 treatment.

\section{Conclusion}

Our interest in GLP-1 began with an investigation of the compound as an insulin secretagogue. Our observations of the prolonged effect of GLP- 1 following chronic treatment and the reprise of glucose responsivity in the $\beta$-cells of old animals led us to investigate the regulation of key $\beta$-cell transcription factors by GLP-1. We showed that GLP-1 upregulates the master $\beta$-cell transcription factor PDX-1. Our evidence indicates that elevation of this transcription factor is a key element in $\beta$-cell differentiation as induced by GLP-1 and exendin- 4 . Thus, we have uncovered a new benefit to chronic treatment with these peptides. The discovery of GLP-1- and exendin-4-induced $\beta$-cell neogenesis has implications for the use of these peptides in the treatment of the diabetic condition.

\section{REFERENCES}

Ahlgren, U., Jonsson, J., Jonsson, L., Simu, K, and Edlund, H. (1998). Genes Devel. 12, 1763-1768.

Beguin, P., Nagashima, K., Nishimura, M., Gonoi, T., and Seino, S. (1999). EMBO J. 18, 4722-4732.

Bode, H.-P., Moorman, B., Dabew, R., and Goke, B. (1999). Endocrinology 140, 3919-3927.

Bonner-Weir, S., Baxter, L.A., Schuppin, G.T., and Smith, F.E. (1993). Diabetes 42, 1715-1720.

Buteau, J., Roduit, R., Susini, S, and Prentki, M. (1999). Diabetologia 42, 856-864

Centers for Disease Control and Prevention. (1998). "National Diabetes Fact Sheet: National Estimates and General Information on Diabetes in the U.S." (rev. edit.). U.S. Department of Health and Human Services, Atlanta, Ga.

Chen, Y.E., and Drucker, D.J. (1997). J. Biol. Chem. 272, 4108-4115.

Christophe, J. (1994). Am. J. Physiol. 266, G963-G971.

Chua, S.C., Jr., White, D.W., Wu-Peng, S., Liu, S.-M., Okada, N., Kershaw, E.E., Chung, W.K., Power-Kehoe, L., Chua, M., Tartaglia, L.A., and Leibel, R.L. (1996). Diabetes 45, 1141-1143. 
De Ore, K., Greig, N.H., Holloway, H.W., Wang, Y., Perfetti, R., and Egan, J.M. (1997). J. Gerontol. A Biol. Sci. Med. Sci. 52, B245-B249.

Drucker, D.J. (1998). Diabetes 47, 159-169.

Drucker, D.J., Philippe, J., Mojsov, S., Chick, W.L., and Habener, J.F. (1987). Proc. Natl. Acad. Sci. U.S.A. 84, 3434-3438.

Egan, J.M., Asplin, C.M., Drumheller, M.A., Kerrigan, J.R., Scott, J., Martha, P.M., Jr., and Evans, W.S. (1991). Proc. Soc. Exp. Biol. Med. 196, 203-209.

Egan, J.M., Clocquet, A., and Elahi, D. (1999). Diabetologia 42, 148A (abstract).

Elahi, D., McAloon-Dyke, M., Fukagawa, N.K., Meneilly, G.S., Sclater, A.L., Minaker, K.L., Habener, J.F., and Anderson, D.K. (1994). Regul. Peptides 51, 63-74.

Eng, J., Kleinman, W.A., Singh, G., and Raufman, J.P. (1992). J. Biol. Chem. 267, 7402-7405.

Fehmann, H.C., Goke, R., and Goke, B. (1992). Mol. Cell. Endocrinol. 85, C39-C44.

Fehmann, H.C., Goke, R., and Goke, B. (1995). Endocr. Rev. 16, 390-410.

Goke, R., Fehmann, H.-C., Linn ,T., Schmidt, H., Krause, M., Eng, J., and Goke, B. (1993). J. Biol. Chem. 268, 19650-19655.

Greig, N.H., Holloway, H.W., De Ore, K.A., Jani, D., Wang, Y., Zhou, J., Garant, M.J., and Egan, J.M. (1999). Diabetologia 42, 45-50.

Gromada, J., Holst, J.J., and Rorsman, P. (1998). Pflugers Arch. 435, 583-594.

Gu, D., Lee, M.S., Krahl, T., and Sarvetnick, N. (1994). Development 120, 1873-1881

Gutniak, M., Ørskov, C., Holst, J.J., Ahren, B., and Efendic, S. (1992). N. Engl. J. Med. 326, 1316-1322.

Harris, M.I., Flegal, K.M., Cowie, C.C., Fherhardt, M.S., Goldstein, D.F., I.ittle, R.R., Wiedmeyer, H.M., and Byrd-Holt, D.D. (1998). Diabetes Care 21, 518-524

Holz, G.G., Kuhtreiber, W.M., and Habener, J.F. (1993). Nature 361, 362-365.

Hupe-Sodmann, K., McGregor, G.P., Bridenbaugh, R., Goke, R., Goke, B., Thole, H., Zimmermann, B., and Voigt, K. (1995). Regul. Peptides 58, 149-156.

Jahn, R., and Sodhof, T.C. (1999). Annu. Rev. Biochem. 68, 863-911.

Jonsson, J., Carlsson, L., Edlund, T., and Edlund, H. (1994). Nature 371, 606-609.

Komatsu, R., Matsuyama ,T., Namba, M., Watanabe, N., Itoh, H., Kono, N., and Tarui, S. (1989). Diabetes 38, 902-905.

Macfarlane, W.M., Read, M.L., Gilligan, M., Bujalska, I., and Docherty, K. (1994). Biochem. J. 303, 625-631.

Mashima, H., Ohnishi, H., Wakabayashi, K., Mine, T., Miyagawa, J.-I., Hanafusa, T., Seno, M., Yamada, H., and Kojima, I. (1996). J. Clin. Invest. 97, 1647-1654.

Mentlein, R. (1999). Regul. Peptides. 85, 9-24.

Mojsov, S., Heinrich, G., Wilson, I.B., Ravazzola, M., Orci, L., and Habener, J.F. (1986). J. Biol. Chem. 261, 11880-11889.

Montrose-Rafizadeh, C., Egan, J.M., and Roth, J. (1994). Endocrinology 135, 589-594.

Montrose-Rafizadeh, C., Wang, Y., Janczewski, A.M., Henderson, T.E., and Egan, J.M. (1997). Mol. Cell. Endocrinol.130, 109-117.

Nathan, D.M., Schreiber, E., Fogel, H., Mojsov, S., and Habener, J.F. (1992). Diabetes Care 15, 270-276.

Olson, L.K., Sharma, A., Peshavaria. M., Wright, C.V., Towle, H.C., Rodertson, R.P., and Stein, R. (1995). Proc. Natl. Acad. Sci. U.S.A. 92, 9127-9131.

Perfetti, R., Rafizadeh, C.M., Liotta, A.S., and Egan, J.M., (1995). Am. J. Phys. Endocrinol. Meiab. 32, E983-E990.

Perfetti, R., Wang, Y., Shuldiner, A.R., and Egan, J.M., (1996). Gerontol. A Biol. Sci. Med. Sci. 51, B331-B336.

Perfetti, R., Zhou, J., Doyle, M.E., and Egan, J.M. (2000). Endocrinology 141, in press.

Petersen, H.V., Serup, R., Leonard, J., Michelsen, B.K., and Madsen, O.D. (1994). Proc. Natl. Acad. Sci. U.S.A. 91, 10465-10469. 
Pohl, M., and Wank, S.A. (1998). J. Biol. Chem. 273, 9778-9784.

Porzio, O., Marlier, L.N., Federici, M., Hribal, M.L., Magnaterra, R., Lauro, D., Fusco, A., Sesti, G., Borboni, P. (1999). Mol. Cell. Endocrinol. 153, 155-161

Reaven, E.P., Gold, G., and Reaven, G.M. (1979). J. Clin. Invest. 64, 591-599.

Renstrom, E., Eliasson, L., and Rorsman, P.J. (1997). J. Physiol. (Lond). 502, 105-118.

Rosewicz, S., Riecken, E.O., and Wiedenmann, B. (1992). Clin. Invest. 70, 205-209.

Sadoul, K., Lang, J., Montecucco, U., Regazzi, R., Catsicas, S., Wolheim, C.B., and Halban P.A. (1995). J. Cell Biol. 128, 1019-1028.

Sharma, A., Zangen, D.H., Reitz, P., Taneja, M., Lissauer, M.E., Miller, C.P., Weir, G.C., Habener, J.F., and Bonner-Weir, S. (1999). Diabetes 48, 507-513.

Stoffers, D.A., Kieffer, T.J., Hussain, M.A., Drucker, D.J., Bonner-Weir, S., Habener, J.F., and Egan, J.M. (2000). Diabetes 49, 741-748.

Szayna, M., Doyle, M.E., Betkey, J.A., Holloway, H.A., Spencer, R.G.S., Greig, N.H., and Egan, J.M. (2000). Endocrinology 141, 1936-1941.

Thorens, B., Porret, A., Bühler, L., Deng, S.P., Morel, P., and Widmann, C. (1993). Diabetes 42 , 1678-1682

United Kingdom Prospective Diabetes Study. (1995). Diabetes 44, 1249-1258.

United Kingdom Prospective Diabetes Study. (1998). Lancet 352, 837-853.

Waeber, G., Thompson, N., Nicod, P., and Bonny, C. (1996). Mol. Endocrinol. 10,1327-1334.

Wang, X., Cahill, C.M., Pineyro, M.A., Zhou, J., Doyle, M.E., and Egan, J.M. (1999). Endocrinology 140, 4904-4907.

Wang, Y., Egan, J.M., Raygada, M., Nadiv, O., Roth, J., and Montrose-Rafizadeh, C. (1995). Endocrinology 136, 4910-4917.

Wang, Y., Perfetti, R., Greig, N.H., Holloway, H.W., DeOre, K.A., Montrose-Rafizadeh, C., Elahi, D., and Egan, J.M. (1997). J. Clin. Invest. 99, 2883-2889.

Watada, H., Kajimoto, Y., Miyagawa, J., Hanafusa, T., Hamaguchi, K., Matsuoka, T., Yamamoto, K., Matsuzawa, Y., Kawamori, R., and Yamasaki, Y. (1996a). Diabetes 45,1826-1831.

Watada, H, Kajimoto, Y., Kaneto, H., Matsuoka, T., Fujitani, Y., Miyazaki, J., and Yamasaki, Y. (1996b). Biochem. Biophys. Res. Commun. 229, 746-751.

Xu, G., Stoffers, D.A., Habener, J.F., and Bonner-Weir, S. (1999). Diabetes 48, 2270-2276

Yada, T., Itoh, K., and Nakata, M. (1993). Endocrinology 133, 1685-1692.

Young, A.A., Gedulin, B.R., Bhavsar, S., Bodkin, N., Jodka, C., Hansen, B., and Denaro, M. (1999). Diabetes 48, 1026-1034.

Zhou, J., and Egan, J.M. (1997). Biochem. Biophys. Res. Commun. 238, 297-300.

Zhou, J., Wang, X., Pineyro, M.A., and, Egan, J.M. (1999). Diabetes 48, 2358-2366. 



\title{
Leptin Controls Bone Formation Through a Hypothalamic Relay
}

\author{
GERARD KaRSENTY \\ Baylor College of Medicine, One Baylor Plaza, Houston, Texas 77030
}

\begin{abstract}
$\Lambda \mathrm{BSTR} \wedge \mathrm{CT}$
Menopause favors osteoporosis and obesity protects from it. In an attempt to decipher the molecular bases of these two well-known clinical observations, we hypothesized that they meant that bone remodeling, body weight, and reproduction are controlled by identical endocrine pathways. We used mouse genetics as a tool to translate these clinical observations into a molecular hypothesis. The $\mathrm{ob} / \mathrm{ob}$ and $\mathrm{db} / \mathrm{db}$ mice were valuable models, since two of the three functions thought to be co-regulated are affected in these mice: they are obese and hypogonadic. Surprisingly, given their hypogonadism, both mouse mutant strains have a high bone mass phenotype. Subsequent analysis of the mechanism leading to this high bone mass revealed that it was due to an increase of bone formation. All data collected indicate that, in vivo, leptin does not act directly on osteoblasts but rather through a central pathway following binding to its specific receptors located on hypothalamic nuclei. This result revealed that bone remodeling, like most other homeostatic functions, is under hypothalamic control. The nature of the signal downstream of the hypothalamus is unknown but current experiments are attempting to identify it.
\end{abstract}

\section{Use of Mouse Genetics in Physiologic Studies}

When homologous recombination in embryonic stem (ES) cells made its entry on the scene of biology, it was heralded, and rightly so, as a breakthrough of historical importance in developmental biology (Capecchi, 1989). It allowed one to study the function of any given gene in vivo by simply deleting it from the mouse genome, thereby giving to mouse genetics the power of yeast, worm, or fly genetics. This was only 10 or 12 years ago, so we have just begun to accept the revolutionary notion that genes important for worm or fruit fly development also may be important during higher organism development. We knew very little about the genetic control of early or even late vertebrate development; the power of this technology to decipher vertebrate embryology seemed limitless. Moreover, it was as a given that what is learned in mice will apply to other vertebrates, including humans. As a matter of fact, the many instances where a mutation of the same gene in mice and in humans led to the same phenotypic consequences demonstrated that this assumption was true overall. For all of these reasons, 
gene-deletion technology was received with an extraordinary enthusiasm as a means to understand mouse, and thereby human, embryology. Retrospectively, it is hard to recount the euphoria surrounding the early success of gene-deletion technology at the beginning of the 1990s.

With time, the technique became even more sophisticated. It is, for instance, now possible to delete genes at a specific time and/or in a specific cell type. Moreover, with the progress on mouse genome mapping, it has become increasingly easier to clone the genes mutated in spontaneously occurring mutations. This approach has contributed significantly to the success of mouse genetics. Yet, with time, the fascination for gene-targeting technology and, more generally, for the power of mouse genetics to study questions relevant to all mammals has partly dissipated. Overall, it has lost most of its magical right of a priori approval.

Yet, mouse genetics is much more powerful and useful that it was a decade ago. With all the technical improvement mentioned above, it is possible to study more than embryologic development. One can, for instance, analyze the molecular bases of tumor formation (Kumar et al., 1995), a rather poorly understood field, or areas of vertebrate physiology for which there are more beliefs than data (Carmeliet et al., 1998; Kappel et al., 1994). In particular, in these latter areas, the use of mouse genetics may potentially revolutionize our understanding of the physiopathology and possibly the management of degenerative diseases. This new - and, so far, only - way to explore untested questions should be welcomed with the same enthusiasm as the one manifested by developmental biologists 10 years ago. Indeed, what is at stake is at least of the same biologic importance and possibly of more-immediate impact therapeutically. But for that to happen, one has to take mouse genetics at face value. If a gene deletion gives a clear phenotypic abnormality that is rigorously studied, it should be accepted. Especially if it challenges previous beliefs, it should be exploited to reanalyze our view of a physiologic process.

This statement may sound a bit defensive and rightly so. It is defensive because, when it comes to physiology, mouse genetics faces several obstacles, even though its potential usefulness is tremendous. One of these obstacles is that phenotypic abnormalities may be subtle and hard to uncover. Another one, possibly harder to overcome, is that it may contradict some untested textbook assumptions. Nevertheless, mouse genetics is in physiology what it is in developmental biology: the best-available approach to study the biologic function of a given gene, in a given cell type, at a given time. This is the assumption that supports the various projects summarized in this chapter.

\section{Bone Remodeling as a Complex Function}

In humans, and in vertebrates in general, bone mass is maintained constant between the end of the period of linear skeletal growth and gonadal failure by a 
complex and dynamic process termed bone remodeling (Karsenty, 1999). Initially, there is resorption of pre-existing bone by a bone-specific cell type, the osteoclast. This is a relatively rapid step that occurs in a few weeks. It is followed by de novo bone formation through another bone-specific cell type, the osteoblast. This latter step is slower and may take several months, so that a typical cycle of bone remodeling requires at least 3 months. This continuous destruction/formation cycle occurs in multiple locations at the same time in such a way that the bone mass of a given individual remains constant until late in life. In women, bone mass remains constant until menopause, when the sex steroid deprivation characterizing this life stage leads to a relative increase in bone resorption over bone formation, decreased bone mass (osteopenia), and possible risk of fractures after a minimum traumatism (osteoporosis) (Karsenty, 1999). The biologic importance of bone remodeling is illustrated by the fact that osteoporosis is the most-frequent degenerative disease in developed countries, affecting 28 million people in the United States alone. Moreover, its incidence will only increase with overall aging of the population.

The fact that bone remodeling occurs in multiple locations at the same time has long been viewed as a suggestive argument in favor of a local, autocrine and/or paracrine regulation (Manolagas, 2000). There are several experimental reasons to believe that this type of regulation must exist. One of them, and not the least persuasive, is that differentiation of osteoclasts, the bone-resorbing cells, has been shown to depend on the presence of genes expressed in the osteoblasts, the bone-forming cells. For instance, genetic experiments in mice have shown that osteoprotegerin (OPG), a soluble TNF receptor present in the bone extracellular matrix, inhibits osteoclast differentiation. However, OPG ligand, a growth factor also present in the bone micro-environment, favors bone resorption (Bucay et al., 1998; Kong et al., 1999; Lacey et al., 1998; Simonet et al., 1997). To a certain extent, another cytokine, interleukin-6 (IL-6), is involved in controlling bone resorption (Poli et al., 1994). It is likely that other secreted molecules will be demonstrated to contribute to this type of regulation of bone remodeling.

The same observation that bone remodeling occurs in multiple locations at the same time can be taken as a suggestive argument in favor of an endocrine regulation of bone remodeling. Such regulation should not be surprising, as most homeostatic functions of the body are under endocrine regulation, superimposed on local regulation. Here, also, the evidence for this type of regulation is overwhelming. As mentioned earlier, sex steroid hormones play a critical role in controlling tightly osteoclast differentiation and bone resorption (Couse and Korach, 1999). We do not understand fully their mechanisms of action but it is clear that gonadal failure in females and, to a certain extent, in males favors bone resorption in humans, rats, and, to a lesser extent, mice. Other hormones favor bone resorption physiologically: parathyroid hormone (PTH), whose mechanism of action in bone is still poorly understood (Potts and Juppner, 1998), and calci- 
tonin that has receptors on the osteoclasts (Nicholson et al., 1986). The endocrine control of bone resorption by already-differentiated osteoclasts plays a crucial role and does not exclude that local factors could contribute or amplify the resorptive action of hormones. It is important from the beginning to emphasize that these two types of regulation, local and endocrine, are not exclusive and can, in fact, be synergistic.

\section{Control of Bone Formation Is Poorly Understood}

All the regulatory events described above, regardless of their nature, affect the bone-resorption aspect of bone remodeling, thus pointing out a blatant lack of knowledge about the molecular control of bone formation by osteoblasts. The only molecule known to affect the rate of bone formation by the osteoblast is a transcription factor, Cbfal (Ducy et al., 1999). Intuitively, however, it seems reasonable that the same type of regulations that apply to bone resorption, local and endocrine, also must apply to bone formation. One experiment from our laboratory suggested even more strongly that there was a tight and critical endocrine regulation of bone formation. Curiously, the most-important meaning of this experiment escaped us initially. We generated transgenic mice expressing the thymidine kinase ( $t k$ ) gene specifically in differentiated osteoblasts, the only cells that synthesize the bone matrix (Corral et al., 1998). The product of this gene is innocuous until cells expressing $t k$ are treated with gancyclovir. This leads to a disruption of DNA replication in the $t k$-expressing cells and to cell death. This cell-ablation process is reversible at the arrest of the gancyclovir treatment.

In absence of any detectable bone formation, bone resorption continued unaffected in these $o s c-t k$ (osteocalcin promoter driving $t k$ expression) mice. As a result, their bones were empty and osc-tk mice stopped growing, as osteoblasts are required for longitudinal growth of the skeleton. Surprisingly, after the gancyclovir treatment was stopped, within a month (a very short period of time according to bone-remodeling standards), the bones of the transgenic mice had recovered a normal appearance and the $o s c-t k$ mice reached a normal size. What was most remarkable in this recovery was its extreme precision. The osc-tk transgenic mice regained a normal bone volume, neither less nor more than their wild-type mice littermates. This extreme precision in the recovery process, together with its speed, suggested that differentiated osteoblasts were able to sense how much bone matrix should be synthesized in different circumstances and that they had at least two speeds to synthesize the bone matrix. Indeed, osteoblasts could, for several days after gancyclovir withdrawal, synthesize large amounts of bone matrix to repopulate the bones. Then, when the bone volume had reached the level observed in wild-type mice, the osteoblasts of the osc-tk mice seemed able to decrease the amount of bone matrix deposited so that bone volume remained normal. We interpreted this ability of the osteoblasts to sense the need of protein synthesis as 
an indication of the existence of endocrine control of bone formation. This led us to search for putative hormones regulating osteoblast function under physiological circumstances.

\section{Evidence for a Common Control of Bone Mass, Body Weight, and Reproduction}

In an effort to identify circulating molecules that could regulate bone formation, we decided to use the clinical literature as a tool to identify one endocrine pathway. As mentioned earlier, the most-frequent bone-remodeling disease is osteoporosis, which is characterized by a relative increase of bone resorption over bone formation. Among the multitude of clinical features characterizing osteoporosis, we were struck by two of them: 1) gonadal failure favors bone loss and 2) obesity protects from bone loss, even after menopause (Felson et al., 1993; Raven et al., 1999; Riggs and Khosla, 1998; Riggs and Melton, 1986; Tremollieres et al., 1993). We hypothesized that the fact that bone mass was affected by a gonadal function and body weight controls bone mass was an indication that these three homeostatic functions - bone mass, body weight, and reproduction - were under the control of the same hormone(s). Given the recent progress in our molecular understanding of the control of appetite and body weight, this hypothesis could be tested in vivo. One has to be aware, however, that this hypothesis has one implication that should be accepted a priori, if one decides to test it. The control of both body weight and reproduction is primarily, although not exclusively, of neuroendocrine nature (i.e., it relies on hormones acting through, or secreted by, the hypothalamus). Therefore, it was a distinct possibility that this investigation might uncover a neuro(endo)crine regulation of bone remodeling and, more specifically, of bone formation.

\section{Leptin as One Regulator of Bone Formation}

In the hypothetical framework of a common regulation for bone mass, body weight, and reproduction, leptin was certainly an attractive - although not the only - candidate molecule (Friedman and Halaas, 1998; Spiegelman and Flier, 1996). Indeed, in mice, rats, and human beings deficient in leptin or its receptor, two of the functions allegedly co-regulated in this model are affected: these animals or patients are obese and hypogonadic (Chen et al., 1996; Clement et al., 1998; Lee et al., 1996; Montague et al., 1997; Strobel et al., 1998; Tartaglia et al., 1995; Zhang et al., 1994). Before going further in describing leptin's action on bone, it is important to emphasize that no animal model has been described in which there is a co-existence of hypogonadism and high bone mass.

The expectations were that the existence of a hypogonadism in these two mutant mouse strains should lead to a low bone mass phenotype whose severity 
may or may not be limited by obesity. Thus, it was a complete surprise to notice that these mice had a high bone mass phenotype (Ducy et al., 2000). The phenotypic changes were so important that they could be detected with a low-sensitivity technique such as x-ray. Obviously, these phenotypic changes were subsequently confirmed by histologic analysis. The phenotypic abnormalities affected the entire skeleton, including long bones, vertebrae, and the skull, as one would expect from an endocrine effect. The initial groups of mice that were analyzed were 6 months old with a full-blown obesity, raising the question whether this phenotypic abnormality was secondary to the obesity. To address this concern, we used ob/ob mice that were put on a low-fat diet at birth. This regimen can postpone the appearance of the obesity in these mice until the sixth week of life. At 4 weeks of age, these ob/ob mice had a normal weight but already a high bone mass phenotype, albeit less severe than the one observed in adult ob/ob animals. This phenotype seemed specific to the leptin signaling pathway, as it could not be observed in Agouti yellow mice. Agouti yellow mice are another mutant strain that develops an obesity phenotype, secondary to the binding of the Agouti protein to the melanocortin receptor 4 (MC-R4) in the hypothalamus (Dinulescu and Cone, 2000). The Agouti protein acts as an antagonist of MC-R4 signal transduction (Lu et al., 1994). Neither in this Agouti yellow mouse model nor in wild-type mice fed a high-fat, high-carbohydrate diet for 1 month to achieve obesity a high bone mass was observed.

There are three possible mechanisms to achieve a high bone mass phenotype. The first is to have an increase in bone formation itself secondary to an increase in osteoblast differentiation and/or function. The second is to have a partial failure of bone resorption. The third possible mechanism is a combination of these two. To study bone formation dynamically, one can rely on histomorphometry, a technique based on the fact that calcein will bind at the front of mineralization in bone. Thus, when injected 8 days apart, calcein allows one to measure the mineral apposition rate, the rate of bone formation, and other parameters (Parfitt $e t$ al., 1987). Using this technique, we could demonstrate that there was a clear increase in all the parameters of bone formation in ob/ob and $\mathrm{db} / \mathrm{db}$ mice prior to the appearance of obesity but not in any of our control animals. All the parameters of bone formation were increased, except an important one. There was a normal number of osteoblasts in ob/ob and db/db mice, demonstrating that leptin does not affect osteoblast proliferation but only their function. This is important because, if one considers using an antagonist of the leptin pathway to favor bone formation, this absence of a mitogenic function is certainly a great advantage.

To study the bone-resorption aspect of bone remodeling in mice deprived of leptin signaling, we took advantage of their hypogonadism. Hypogonadism always leads to an increase in osteoclast numbers (Marcus et al., 1996) and this rule holds true in ob/ob and $\mathrm{db} / \mathrm{db}$ mice as well. Thus, we reasoned that if the osteoclasts of these mice were defective, correcting their hypogonadism would decrease 
their osteoclast numbers but not worsen their phenotype. If, on the other hand, the osteoclasts of the ob/ob and $\mathrm{db} / \mathrm{db}$ mice were working normally, correcting the hypogonadism of these mice should worsen their bone phenotype by decreasing their osteoclast numbers. To test which of these two models was correct, we treated male and female ob/ob mice with testosterone or estradiol implants, respectively, for 4 months. This treatment corrected their hypogonadism and decreased the number of osteoclasts in these mice but also worsened considerably their high bone mass phenotype. These results demonstrated, along with other cell-based, in vitro assays, that leptin did not act on bone resorption. At that stage, regardless of the mechanism of action of leptin, this high bone mass phenotype was quite extraordinary, since it occurred in hypogonadal mice. The uniqueness of the coexistence of high bone mass and hypogonadism required us to identify one general mechanism of action of leptin in the control of bone formation.

\section{High Bone Mass Phenotype Observed in Absence of Leptin Signaling Is Dominant}

Although the high bone mass phenotype precedes the appearance of obesity, it is still conceivable that it is secondary to the existence of other endocrine abnormalities in the ob/ob and $\mathrm{db} / \mathrm{db}$ mice. This possibility is, theoretically at least, rather unlikely, as no hormones are known to favor bone formation to the extent observed in eugonadal ob/ob mice. Nevertheless, it was tested thoroughly. Most of the endocrine abnormalities characterizing $\mathrm{ob} / \mathrm{ob}$ and $\mathrm{db} / \mathrm{db}$ mice should have, at best, marginal effects on bone formation or bone resorption. The exception is that these mice are hypercortisolic, a condition that favors a decrease, not an increase, in bone formation (Ahima et al., 1996; Reid, 1997).

But there is a more-convincing and more-important argument to exclude any endocrine dysregulation at the origin of this phenotype. This argument is that the high bone mass phenotype can be observed in $\mathrm{ob} /+$ and $\mathrm{db} /+$ animals that not only are not obese but also have none of the known endocrine abnormalities observed in the ob/ob and db/db animals. This latter aspect of the bone phenotype observed in the absence of leptin has considerable therapeutic potential, as it implies that one could modulate the leptin pathway to affect bone mass without affecting body weight. This dominant effect also indicates that bone is a major target organ of leptin, as there are very few examples of hormones whose partial deficiency causes such a dramatic phenotype.

\section{Absence of Evidence for a Local or Endocrine Effect of Leptin on Osteoblasts in Vivo}

If the high bone mass phenotype observed in absence of leptin signaling is not secondary to the obesity or any endocrine abnormalities, then how does it 
develop? As for every secreted molecule, leptin could signal to the osteoblasts through three possible and not necessarily exclusive pathways. It could act locally through an autocrine/paracrine mechanism, it could act through a classical endocrine pathway, and, finally, it could act - as it does for the control of appetite - through a neuro(endo)crine pathway. The point here was not so much to push one mode of action and to exclude the others a priori but rather to analyze, in the most-neutral way, the abnormalities of the ob/ob and db/db mice and to take full advantage of what mouse genetics can teach us, especially if it opens new directions for research and establishes new concepts of physiology.

Because the absence of leptin does not affect the osteoblast number, one has to assume that, regardless of the mechanism involved, leptin affects the function of the osteoblast. Therefore, all experiments aimed at demonstrating an autocrine, paracrine, or endocrine effect of leptin should be conducted using differentiated osteoblasts (i.e., cells able to synthesize and deposit a bone matrix). By definition, an autocrine regulation requires that leptin is expressed in osteoblasts. Northern hybridization failed to detect any leptin mRNA expression in bone or primary cultures of osteoblasts, even after a long exposure, thus excluding the possibility of an autocrine mechanism of action as leptin's main mode of action on bone formation. A paracrine or endocrine mechanism of action has the same requirement: differentiated osteoblasts must have signal-transducing leptin receptors on their surface. There are multiple isoforms of the leptin receptor but one only, ObRb, has been shown to have signal-transduction ability (Chen et al., 1996; Lee et al., 1996). ObRb is expressed predominantly in four nuclei of the hypothalamus. We could not detect any expression of ObRb in primary osteoblast cultures. This result was in contradiction to the result of Thomas and coworkers (1999) using transformed cells; moreover, it was based on a reverse transcription-polymerase chain reaction (RT-PCR) analysis. For these two reasons, we felt that more-reliable assays of a biochemical, genetic, and physiologic nature were needed to establish this important point in vivo.

Signal transduction through ObRb leads to phosphorylation of Stat 3, a transcription factor, and increased expression of early genes such as Tis 11 and C-fos (Baumann et al., 1996; Elmquist et al., 1997; Ghilardi et al., 1996; Moitra et al., 1998; Vaisse et al., 1996). One cytokine, oncostatin M, has a receptor that belongs to the same superfamily as the leptin receptor. Binding of oncostatin M to this receptor leads to phosphorylation of Stat 3 and early-response gene expression (Ahn et al., 1998; Levy et al., 1996). Moreover, oncostatin M receptors are present and abundant on the surface of differentiated osteoblasts (Bellido et al., 1996). Thus, to determine whether there were signal-transducing leptin receptors on osteoblasts, we used primary cultures of wild-type mouse osteoblasts that were treated with vehicle, various amounts of leptin, or with oncostatin $M$. While oncostatin M invariably induces Stat 3 phosphorylation and Tis 11 and $C$-fos expression, various doses of leptin, ranging from 8 to $100 \mathrm{ng} / \mathrm{ml}$, failed to induce 
any phosphorylation of Stat 3 or to affect Tis 11 and $C_{-f}$ fos expression. A genetic argument also supports the notion that the osteoblasts are not the direct target of leptin. One can assume that if the bone phenotype of the ob/ob and $\mathrm{db} / \mathrm{db}$ mice was due primarily to the absence of signal-transducing leptin receptors on the surface of differentiated osteoblasts, then primary osteoblast cultures of $\mathrm{db} / \mathrm{db}$ mice, which have an inactivating mutation in ObRb, should produce more bone matrix than primary osteoblast cultures of wild-type mice. This was not the case, demonstrating that, at least in culture, the absence of ObRb on osteoblasts does not cause any increase in bone formation. Taken together, all these evidences if they do not formally prove, because of their negative nature, that there is no leptin-signaling receptor on differentiated osteoblasts - strongly suggested that we should look elsewhere for a mechanism of action of leptin on bone formation. In any case, one additional piece of evidence, this time of a positive nature (see below), indicates that leptin can control bone formation without interacting physically with osteoblasts.

The observation that leptin signaling controls bone mass was made in mutant animals; therefore, it could be some type of genetic artifact. Indeed, it was possible that the absence of leptin signaling somehow allowed the adipocytes to secrete a molecule that they normally do not secrete and whose function is to favor bone matrix deposition by differentiated osteoblasts. To test this hypothesis, we used another mouse model, the "fat-free" mice (Moitra et al., 1998). These transgenic mice express a dominant-negative form of the leucine zipper family of transcription factors in their adipocytes (Ahn et al., 1998). Among the leucine zipper type of transcription factors expressed in adipocytes are the CCAAT/enhancer binding proteins (C/EBP), which play a critical role during adipocyte differentiation (Bellido $e t$ al., 1996). As a result, these mice have no adipocytes, no white fat, and a 20-fold reduction in leptin level (Ahn et al,, 1998). If the adipocytes, and not the absence of leptin signaling, are the cause for the high bone mass phenotype observed in ob/ob and $\mathrm{db} / \mathrm{db}$ mice, then these fat-free mice should have a normal bone mass. If, on the other hand, it is the decrease or absence of leptin expression that causes the phenotype, regardless of the number of adipocytes, then fat-free mice should have the same high bone mass phenotype as the ob/ob or ob/+mice. This was precisely the case, indicating that it is the absence of leptin signaling that is causing the phenotype, not the adipocytes. The fat-free mice are very important for another reason. They are an animal model of a human disease, generalized lypodistrophy, that is characterized, among many other features, by the existence of a high bone mass phenotype (Westvik, 1996).

\section{Leptin Acts Centrally to Control Bone Formation in Vivo}

The next general mechanism to study was the central or neuroendocrine control of bone formation. This was a valid pathway to test, since leptin is believed 
to control appetite, body weight, and the onset of puberty, primarily through its binding to specific receptors localized in four nuclei of the hypothalamus. For this pathway to be the one regulating bone mass, two criteria should be met; one genetic, one physiologic. First, intracerebroventricular (icv) infusion of leptin at a dose that does not cross the blood-brain barrier should correct the high bone mass phenotype of the ob/ob mice. Second, the same icv infusion of leptin, this time in wild-type mice, should lead to osteopenia.

When delivering a small amount of leptin (i.e., $8 \mathrm{ng} / \mathrm{h}$ ) through icv infusion in ob/ob mice, we noticed, as has been shown before, that these mice lost considerable weight. The dose of leptin used for icv infusion is the same that was used to demonstrate that leptin acts centrally to control body weight (Halaas et al., 1997). We made sure that there was no detectable leptin in the serum of these animals. This icv treatment lasted 1 month, a long time in terms of body weight control but a relatively short time in terms of bone remodeling, as a cycle of bone remodeling may last more than 3 months. Yet, at the end of this 1 -month period, the ob/ob animals treated with leptin had lost a considerable amount of bone mass, to the point that it was slightly lower than in all of the wild-type animals. This experiment demonstrated that leptin can affect bone formation without being present in the serum and therefore in the bone microenvironment. This positive result, along with the absence of signaling leptin receptor in primary osteoblast cultures, uncovered a novel level of regulation of bone remodeling through a central control.

As mentioned above, for this pathway to be of physiological relevance, one has to demonstrate that this type of regulation occurs not only in genetically modified animals but also in normal ones. As predicted, icv infusion of the same low dose of leptin in wild-type mice for 1 month led to the appearance of osteopenia in these animals and demonstrated the physiologic relevance of leptin's regulation of bone remodeling (Ducy et al., 2000).

No clear and simple model exists to explain how leptin controls appetite following its binding to ObRb in the hypothalamus (Elmquist et al., 1999; Marsh et al., 1999). Clearly, the mode of action of leptin on bone formation is not more advanced. However, one can attempt to take advantage of the current knowledge about the molecular control of appetite to address an initial question: is the leptin action affected by the same neuropeptides involved in the control of appetite/body weight and of bone mass? Neuropeptide Y (NPY) is one of the many neuropeptides that favor appetite and body weight gain. NPY expression is increased in ob/ob mice; NPY deficiency partially corrects the obesity phenotype of the ob/ob mice (Erickson et al., 1996; Schwartz et al., 1998). Thus, in terms of body weight and appetite control, leptin and NPY appear to have antagonistic functions. Nevertheless, icv infusion of NPY in wild-type mice caused a bone loss, indicating that leptin and NPY do not antagonize each other's function in the control of bone 
formation. This result may be viewed as an indication that leptin uses different sets of mediators to control body weight and bone mass.

\section{Neuroendocrine Regulation of Bone Mass Is Limited Neither to Mice nor to Leptin}

The entire study originally reported was performed in ob/ob and $\mathrm{db} / \mathrm{db}$ mice that are deficient in leptin or its receptor, respectively. It has subsequently been expanded to rats, with essentially identical results. This study was obviously not performed in humans for several reasons. First of all, the number of patients is too small. Second, ethical considerations will prevent anyone from performing invasive manipulations such as bone biopsies in these patients. Third, and not any less important, we have shown that the bone phenotype caused by the absence of leptin signaling worsens over time and is quite severe in adult animals. Human patients are children that were treated before the putative bone phenotype may have fully developed. Nevertheless, patients affected by generalized lypodistrophy (i.e., absence of adipocytes) have accelerated bone growth and high bone mass. Moreover, the recent demonstration that children deficient in MC-R4 are not only obese but also have a high bone mass gives further credence to the concept that body weight, bone mass, and reproduction share common neuroendocrine regulators (Farooqi et al., 2000). This latter result in humans is not in contradiction with our own observation that Agouti yellow mice, a mouse model of late obesity due to the binding of an antagonist protein to the MC-R4, had no bone abnormalities. Indeed, it is known that MC-R4 mutation causes a more-severe phenotype than the one observed in Agouti yellow mice (Huszar et al., 1997).

\section{Implications}

The finding that leptin regulates the bone formation arm of bone remodeling has important implications for leptin biology as well as for bone biology. The fact that $\mathrm{ob} /+$ and $\mathrm{db} /+$ mice already have a high bone mass phenotype is rather exceptional as, in most cases, hormone deficiencies do not have dominant phenotypes. This, together with our failure to demonstrate a peripheral action of leptin on osteoblasts, has several implications that can be tested experimentally. One implication is that if the apparent lack of effect of leptin on osteoblasts is true, then raising the serum concentrations of leptin should induce a state of leptin resistance but should not lead to osteoporosis. Another implication of the apparent predilection of leptin for bone could be that repcating the infusion of leptin with a lower dose should result in bone loss at doses that cannot affect body weight. A third and more-important implication of the dominant nature of the leptin function on bone formation is that it would be feasible to antagonize only partially the leptin signaling pathway, so that animals would have a higher bone mass 
without becoming obese or sterile. Finally, if leptin uses different pathways to control body weight and bone formation, then in animal models of obesity with leptin resistance, it may still be possible to induce bone loss through icv infusion of leptin.

In terms of bone biology, the implications are tremendous. Not only do these findings in mice, rats, and humans establish a new physiologic concept and provide a molecular basis for the well-known protective effect exerted by obesity on bone mass, they also open new directions of physiologic and pharmacologic research. One should first wonder, why do vertebrates need such a powerful brake to be applied on bone formation? This is a very speculative question. Although the consequences of an incorrect control of bone remodeling are not as immediately apparent as the consequences of an incorrect control of body weight, onc could argue that it is a critical function to regulate tightly during evolution. We already know that abnormalities in the regulation of bone resorption may result in osteopetrosis, a disease lethal during early childhood. Likewise, an uncontrolled increase in bone formation results in a massive increase in bone mass, a failure of bone marrow to maintain a normal hematopoiesis, and possibly a drop in serum calcium concentration, all abnormalities that lead to early death (Jochum et al., 2000). If regulating bone formation is so important, then it is likely that leptin is not the only regulator and a positive regulation of bone formation probably exists to counteract leptin's function. Likewise, if one adopts a unifying view of bone remodeling, then it is conceivable and testable that the bone-resorption arm of bone remodeling may be under central hormonal regulation.

Finally, it is still too early to determine whether this novel regulation of bone remodeling will result in novel therapeutic approaches but at least this a reasonable route to explore. As mentioned throughout this review, three aspects of leptin regulation of bone mass are particularly attractive in that regard. The first aspect is that modulating the leptin signaling pathway offers the possibility to increase bone formation that is defective in osteoporosis. Conceivably, a treatment that would increase bone formation could be viewed as a cure, if not a preventive treatment, for osteoporosis. The second aspect is that, in absence of leptin signaling, there is an increase in bone formation without any increase in osteoblast numbers. In other words, there is no mitogenic effect of the absence of leptin signaling on the osteoblast, which is always an advantage if one thinks of a long-term drug treatment. The final aspect is that the bone phenotype of the leptin-deficient mice is dominant, whereas the obesity is recessive, thus raising the possibility that partial inhibitors of leptin action may have beneficial and selective effects on bone mass. 


\section{ACKNOWLEDGMENTS}

The author is grateful to Drs. T. Schinke and P. Ducy for critical reading of the manuscript. This work was supported by National Institutes of Health R01 R01 DK58883.

\section{REFERENCES}

Ahima, R.S., Prabakaran, D., Mantzoros, C., Qu, D., Lowell, B., Maratos-Flier, E., and Flier, J.S. (1996). Nature 382, 250-252.

Ahn, S., Olive, M., Aggarwal, S., Krylov, D., Ginty, D.D., and Vinson, C. (1998). Mol. Cell. Biol. 18, 967-977.

Baumann, H., Morella, K.K., White, D.W., Dembski, M., Bailon, P.S., Kim, H., Lai, C.F., and Tartaglia, L.A. (1996). Proc. Natl. Acad. Sci. U.S.A. 93, 8374-8375.

Bellido, T., Stahl, N., Farruggella, T.J., Borba, V., Yancopoulos, G.D., and Manolagas, S.C. (1996). J. Clin. Invest. 2, 431-437.

Bucay, N., Sarosi, I., Dunstan, C.R., Morony, S., Tarpley, J., Capparelli, C., Scully, S., Tan, H.L., Xu, W., I acey, D. L., Royle, W.J., and Simonet, W.S. (1998). Genes Devel. 12, 1260-1268.

Capecchi, M.R. (1989). Science 4910, 1288-1292.

Carmeliet, P., Moons, L., and Collen, D. (1998). Cardiovasc. Res. 1, 8-33.

Chen, H., Charlat, O., Tartaglia, L.A., Woolf, E.A., Weng, X., Ellis, S.J., Lakey, N.D., Culpepper, J., Moore, K.J., Breitbart, K.E., Duyk, G.M., Tepper, R.I., and Morgenstern, J.P. (1996). Cell 84, 491-495.

Clement, K., Vaisse, C., Lahlou, N., Cabro, S., Pelloux, V., Cassuto, D., Gourmelen, M., Dina, C., Chambaz, J., Lacorte, J.M., Basdevant, A., Bougneres, P., Lebouc, Y., Froguel, P., and Guy-Grand, B. (1998). Nature 6674, 398-401.

Corral, D.A., Amling, M., Priemel, M., Loyer, E., Fuchs, S., Ducy, P., Baron, R., and Karsenty, G. (1998). Proc. Natl. Acad. Sci. U.S.A. 95, 13835-13840.

Couse, J.F., and Korach, K.S. (1999). Endocr. Rev. 3, 358-417.

Dinulescu, D.M., and Cone, R.D. (2000). J. Biol. Chem. 275, 6695-6698.

Ducy, P., Starbuck, M., Priemel, M., Shen, J., Pinero, G., Geoffroy, V., Amling, M., and Karsenty, G. (1999). Genes Devel. 13, 1025-1036.

Ducy, P., Amling, M., Takeda, S., Priemel, M., Schilling, A.F., Beil, F.T., Shen, J., Vinson, C., Rueger, J.M., and Karsenty, G. (2000). Cell 2, 197-207.

Elmquist, J.K., Ahima, R.S., Maratos-Flier, E., Flier, J.S., and Saper, C.B. (1997). Endocrinology 138, 839-842.

Elmquist, J.K., Elias, C.F., and Saper, C.B. (1999). Neuron 2, $221-232$.

Erickson, J.E., Hollopeter, G., and Palmiter, R.D. (1996). Science 274, 1704-1707.

Farooqi, I.S., Yeo, G.S., Keogh, J.M., Aminian, S., Jebb, S.A., Butler, G., Cheetham, T., and O'Rahilly, S. (2000). J. Clin. Invest. 2, 271-279.

Felson, D.T., Zhang, Y., Hannan, M.T., and Anderson, J.J. (1993). J. Bone Miner. Res. 8, 567-573.

Friedman, J.M., and Halaas, J.L. (1998). Nature 395, 763-770.

Ghilardi, N., Ziegler, S., Wiestner, A., Stoffel, R., Heim, M.Il, and Skoda, R.C. (1996). Proc. Natl. Acad. Sci. U.S.A. 93, 6231-6235.

Halaas, J.L., Boozer, C., Blair-West, J., Fidahusein, N., Denton, D.A., and Friedman, J.M. (1997). Proc. Natl. Acad. Sci. U.S.A. 94, 8878-8883.

Huszar, D., Lynch, C.A., Fairchild-Huntress, V., Dunmore, J.H., Fang, Q., Berkemeier, L.R., Gu, W., Kesterson, R.A., Boston, B.A., Cone, R.D., Smith, F.J., Campfield, L.A., Burn, P., and Lee, F. (1997). Cell 1, 131-141.

Jochum, W., David, J.P., Elliott, C., Wutz, A., Plenk, H. Jr., Matsuo, K., and Wagner, E.F. (2000). Nature Med. 6, 980-984. 
Kappel, C.A., Bieberich, C.J., and Jay, G. (1994). FASEB J. 8, 583-592.

Karsenty, G. (1999). Genes Devel. 13, 3037-3051.

Kong, Y.Y., Yoshida, H., Sarosi, I., Tan, H.L., Timms, E., Capparelli, C., Morony, S., Oliveira-dosSantos, A.J., Van, G., Itie, A., Khoo, W., Wakeham, A., Dunstan, C.R., Lacey, D.L., Mak, T.W., Boyle, W.J., and Penninger, J.M. (1999). Nature 397, 315-323.

Kumar, T.R., Donehower, L.A., Bradley, A., and Matzuk, M.M. (1995). J. Intern. Med. 3, 233-238.

Laccy, D.L., Timms, E., Tan, I.L., Kelley, M.J., Dunstan, C.R., Burgess, T., Elliott, R., Colombero, A., Elliott, G., Scully, S., Hsu, H., Sullivan, J., Hawkins, N., Davy, E., Capparelli, C., Eli, A., Qian, Y.X., Kaufman, S., Sarosi, I., Shalhoub, V., Senaldi, G., Guo, J., Delaney, J., and Boyle, W.J. (1998). Cell 93, 165-176

Lee, G.H., Proenca, R., Montez, J.M., Carroll, K.M., Darvishzadeh, J.G., Lee, J.I., and Friedman, J.M. (1996). Nature 379, 632-635.

Levy, J.B., Schindler, C., Raz, R., Levy, D.E., Baron, R., and Horowitz, M.C. (1996). Endocrinology 137, 1159-1165.

Lu, D., Willard, D., Patel, I.R., Kadwell, S., Overton, L., Kost, T., Luther, M., Chen, W., Woychik, R.P., and Wilkison, W.O. (1994). Nature 371, 799-802.

Manolagas, S.C. (2000). Endocr. Rev. 2, 115-137.

Marcus, R., Feldman, D., and Kelsey, J. (1996). "Osteoporosis." Academic Press, San Diego.

Marsh, D.J., Hollopeter, G., Huszar, D., Laufer, R., Yagaloff, K.A., Fisher, S.L., Burn, P., and Palmiter, R.D. (1999). Nature Genet. 1, 119-122.

Moitra, J., Mason, M.M., Olive, M., Krylov, D., Gavrilova, O. Marcus-Samuels, B., Feigenbaum, L., Lee, E., Aoyama, T., Eckhaus, M., Reitman, M.L., and Vinson, C. (1998). Genes Devel. 12, 3168-3181.

Montague, C.T., Farooqi, I.S., Whitehead, J.P., Soos, M.A., Rau, H., Wareham, N.J, Sewter, C.P., Digby, J.E., Mohammed, S.N., Hurst, J.A., Cheetham, C.H., Earley, A.R., Barnett, A.H., Prins, J.B., and O'Rahilly, S. (1997). Nature 387, 903-908.

Nicholson, G.C., Moseley, J.M., Sexton, P.M., Mendelsohn, F.A., and Martin, T.J. (1986). J. Clin. Invest. 2, 355-360.

Parfitt, A.M., Drezner, M.K., Glorieux, F.H., Kanis, H.A., Malluche, H., Meunier, P.J., Ott, S.M., and Recker, R.R. (1987). J. Bone Miner. Res. 6, 595-610.

Poli, V., Balena, R., Fattori, E., Markatos, A., Yamamoto, M., Tanaka, H., Ciliberto, G., Rodan, G.A., and Costantini, F. (1994). EMBO J. 5, $1189-1196$.

Potts, J.T., and Juppner, H. (1998). "Parathyroid Hormone and Parathyroid Hormone-related Peptide in Calcium Homeostasis, Bone Metabolism, and Bone Development: the Proteins, Their Genes, and Receptors." Academic Press, San Diego.

Raven, P., Cizza, G., Bjarnason, N.H., Thompson, D., Daley, M., Wasnich, R.D., McClung, M., Hosking, D., Yates. A.J., and Christiansen, C. (1999). J. Bone Miner. Res. 14, 1622-1627.

Reid, I.R. (1997). N. Engl. J. Med. 337, 420-421.

Riggs, B., and Khosla, S. III. (1998). J. Bone Miner. Res. 13, 763-773.

Riggs, B.L., and Melton, L.J. (1986). N. Engl. J. Med. 314, 1676-1686.

Schwartz, M.W., Erickson, J.C., Baskin, D.G., and Palmiter, R.D. (1998). Endocrinology 5, 2629-2635.

Simonet, W.S., Lacey, D.L., Dunstan, C.R., Kelley, M., Chang, M.S., Lithy, R., Nguyen, H.Q., Wooden, S., Bennett, L., Boone, T., Shimamoto, G., DeRose, M., Elliott, R., Colombero, A., Tan, H.L., Trail, G., Sullivan, J., Davy, E., Bucay, N., Renshaw-Gegg, L., Hughes, T.M., Hill, D., Pattison, W., Campbell, P., and Boyle, W.J. (1997). Cell 89, 309-319.

Spiegelman, B.M., and Flier, J.S. (1996). Cell 87, 377-389.

Strobel, A., Issad,T., Camoin, L., Ozata, M., and Strosberg, A.D. (1998). Nature Genet. 3, 213-215.

Tartaglia, L.A., Dembski,M., Weng, X., Deng, N., Culpepper, J., Devos, R., Richards, G.J., Campfield, L.A., Clark, F.T., Deeds, J., Muir, C., Sanker, S., Moriarty, A., Moore, K.J., Smutko, J.S., Mays, G.G., Woolf, E.A., Monroe, C.A., and Tepper, R.I. (1995). Cell 83, 1263-1271. 
Thomas, T., Gori, F., Khosla, S., Jensen, M.D., Burguera, B., and Riggs, B.L. (1999). Endocrinology 140, 1630-1638.

Tremollieres, F.A., Pouilles, J.M., and Ribot. C. (1993). J. Clin. Endocrinol. Metab. 77, 683-686.

Vaisse, C., Halaas, J.L., Horvath, C.M., Darnell, J.E.J., Stoffel, M., and Friedman, J.M. (1996). Nature Genet. 14, 95-97.

Westvik, J. (1996). Acta Paediatr. Suppl. 413, 44-51.

Zhang, Y., Proenca, R., Maffei, M., Barone, M., Leopold, L., and Friedman, J.M. (1994). Nature 372, 425-432. 



\title{
Mechanisms of Inhibin Signal Transduction
}

\author{
Daniel J. Bernard, Stacey C. Chapman, and Teresa K. Woodruff \\ Department of Neurobiology and Physiology, Northwestern University, \\ 2153 N. Campus Drive, Evanston, Illinois 60208
}

\begin{abstract}
Inhibin was first identified as a gonadal hormone that potently inhibits pituitary follicle-stimulating hormone (FSH) synthesis and secretion. Although the notion of a nonsteroidal, gonadally derived inhibitory substance was realized in the early 1930s (McCullagh, 1932), identification of the hormone was not accomplished until more than $\mathbf{5 0}$ years later. At that time, inhibin was purified from bovine and porcine follicular fluid and was shown to be produced in two forms through dimeric assembly of an $\alpha$ subunit $(18 \mathrm{kDa}$ ) and one of two closely related $\beta$ subunits ( $\beta A$ and $\beta B$, approximately $14 \mathrm{kDa}$ ) (Ling et al., 1985; Miyamoto et al., 1985; Rivier et al., 1985; Robertson et al., 1985). Dimers of $\alpha$ and $\beta A$ and $\alpha$ and $\beta B$ subunits form inhibin $A$ and inhibin $B$, respectively. In the process of purifying inhibin, two groups also identified homo- and heterodimers of the inhibin $\beta$ subunits (Ling et al., 1986; Vale et al., 1986). These hormoncs, the activins, werc shown to potently stimulate FSH secretion from primary pituitary cultures and are now known to play important roles in growth and development (Woodruff, 1998; Pangas and Woodruff, 2000). Inhibins and activins are considered members of the transforming growth factor-beta (TGF- $\beta$ ) superfamily of growth and differentiation factors, based on a pattern of conserved cysteine residues in the $\alpha$ and $\beta$ subunits, similar to other ligands in the family.

Identification of the subunit proteins led to the cloning of their cDNAs and subsequently to their chromosomal mapping in several species (Mason et al., 1985,1986, Forage et al., 1986; Mayo et al., 1986; Esch et al., 1987; Woodruff et al., 1987; Barton et al., 1989; Hiendleder et al., 2000). Three additional activin-related $\beta$ subunits ( $\beta \mathrm{C}$ and $\beta \mathrm{E}$ in mammals and $\beta \mathrm{D}$ in Xenopus laevis) also have been identified but do not appear to play a role in FSH regulation (Hotten et al., 1995; Oda et al., 1995; Fang et al., 1996,1997; Loveland et al., 1996; Schmitt et al. 1996; O'Bryan et al., 2000; Lau et al., 2000). To date, only one $\alpha$ subunit has been reported. The inhibin subunits are expressed in various tissues (Meunier et al., 1988a,1988b) but the gonads are clearly the primary source of circulating inhibins (Woodruff et al., 1996). While inhibins act in a paracrine role in some tissues (Hsueh et al., 1987), their best-understood roles are as endocrine regulators of pituitary FSH. Activins also were purified from follicular fluid but because circulating activin levels generally are low, most actions of the hormones are likely to be paracrine in nature (Woodruff, 1998). Several reviews in the past decade have clearly and thoroughly addressed the characterization and regulation of the inhibins and activins and their roles in reproductive function (Vale et al., 1988; Ying, 1988; Woodruff and Mayo, 1990; Mayo, 1994; Woodruff and Mather, 1995).

In this chapter, we focus our attention on more-recent developments in inhibin research. First, we discuss differential regulation of inhibin isoforms. Specifically, we describe patterns of inhibin A and $B$ secretion in the context of the female reproductive cycle. Second, we review molecular mechanisms of inhibin subunit regulation. Third, while inhibins are best known for their role in
\end{abstract}


pituitary FSH regulation, other functions of the ligands are becoming better understood. We review the animal and human literature addressing the possible role of inhibins in gonadal cancers. While we know "what" inhibins do in various contexts, we have a very limited understanding of "how" the ligands have their effects on target cells. Recently, candidate inhibin receptor molecules have been identified (Draper et al., 1998; Hertan et al., 1999; Lewis et al., 2000; Chung et al., 2000). Next, we detail our current understanding of inhibin signal transduction. Finally, in light of the data reviewed here, we pose questions and outline future directions for inhibin research. While this review is concerned primarily with expression and function of inhibin, activin function and mechanisms of action are described where necessary to shed light on inhibin function. Several reviews of activin's role in reproductive and other processes can be found elsewhere (Woodruff, 1998; Pangas and Woodruff, 2000).

\section{Physiological Regulation of Inhibin Isoforms}

Inhibin A and B circulate in the female rat; however, the two hormones are differentially secreted across the 4-day estrous cycle (Woodruff et al., 1996). Inhibin B levels are elevated from the morning of metestrus and decline gradually through the morning of proestrus. At or shortly after the time of the primary gonadotropin surges on the afternoon of proestrus, inhibin B levels decline to their lowest levels until the morning of estrus, when they again begin to increase. Serum inhibin A levels, on the other hand, are low on metestrus morning and increase progressively through the afternoon of proestrus, where they peak at the time of the primary FSH and luteinizing hormone (LH) surges. Thereafter, serum inhibin A plummets to undetectable levels until the morning of estrus, when levels begin to increase, in response to FSH. These changes parallel changes in subunit mRNA levels in developing follicles (Meunier et al., 1988a; Woodruff et al., 1988; Woodruff and Mayo, 1990). In light of the fact that both forms of inhibin are elevated at the time of the primary FSH surge, the potent stimulatory effect of the hypothalamic gonadotropin-releasing hormone $(\mathrm{GnRH})$ surge is able to overcome the inhibitory effects of both inhibins. The subsequent declines in both inhibins then provide a permissive endocrine milieu for the secondary FSH surge on the morning of estrus. The elevated inhibin B levels early in the cycle likely function to keep FSH levels low following the secondary FSH surge. In fact, the very high negative correlation between inhibin B and FSH across the entirety of the cycle $(\mathrm{r}=-0.89)$ (Woodruff et al., 1996) suggests that, at least in rodents, inhibin B is the primary regulator of serum FSH. What, then, is the function of inhibin A across the rodent estrous cycle? It is likely that both inhibin A and B are required to suppress FSH secretion from the metestrus to proestrus transition, although the specific role of each ligand during this period has yet to be determined. Immunoneutralization studies with isoform-specific antibodies may help determine whether or not the two inhibins have different functions during the cycle.

Similar to the case in rodents, female primates show dissociated patterns of inhibin A and B secretion during their reproductive cycle (Groome et al., 
1994,1996; Welt et al., 1997b,1999; Sehested et al., 2000). Inhibin B levels are elevated during the early to midfollicular phase, then decline in the late follicular phase just prior to the primary gonadotropin surges. Inhibin $B$ then rises again around the time of the gonadotropin surges and declines to low levels during the luteal phase. Inhibin A levels are low during early to midfollicular phase but increase around the time of the preovulatory gonadotropin surges. Inhibin A levels remain elevated through the majority of the luteal phase, then decrease in synchrony with declining progesterone just prior to the luteal-follicular phase transition. Changes in serum inhibin A and B levels across the menstrual cycle in stump-tailed macaques are nearly identical to those in humans, except increases in inhibin A appear to occur only after the midcycle gonadotropin surges (Fraser et al., 1999).

The data in rats and humans indicate that changes in serum FSH are instrumental in driving these changes in inhibin production. In rats, unilateral ovariectomy produces a transient increase in serum FSH but not LH. After 24 hours, there is a compensatory increase in inhibin subunit mRNA levels in the follicles of the remaining ovary (D'Agostino et al., 1989). Serum FSH and inhibin B levels are highly correlated across the rat estrous cycle (Woodruff et al., 1996). While elevated inhibin B early in the cycle appears to keep FSH levels low, increased serum FSH during the secondary surge then seems to increase both inhibin A and $B$ production on estrus morning. Although increasing FSH levels stimulate inhibin expression during most phases of the cycle, primary gonadotropin surges drive the declines in inhibin A and B production (Woodruff et al., 1989). Blockade of the primary gonadotropin surges with a GnRH antagonist prevents decreases in inhibin subunit mRNA levels on the afternoon of proestrus. Exogenous FSH or LH replacement in antagonist-treated animals decreases ovarian inhibin expression, showing a direct role for gonadotropins in this process. Clearly, different mechanisms control FSH-regulated inhibin expression during the primary surges and at other times of the cycle (see below).

In primates (including humans), a strong correlation exists between inhibin $\mathrm{B}$ and FSH during the follicular phase and the preovulatory gonadotropin surges. In both cases, increases in FSH are followed within 1-2 days by increases in inhibin B (Sehested et al., 2000). The increasing inhibin B levels are almost invariably associated with declining FSH levels. Thus, it appears that increasing FSH levels stimulate inhibin B secretion, which then feeds back to the pituitary to downregulate FSH secretion. In humans, FSH administration during the early follicular phase results in a robust and dose-dependent response by both inhibin B and inhibin A, although inhibin B is more responsive to a lower dose of gonadotropin (Burger et al., 1998).

The relationship between inhibin B and FSH has been demonstrated most clearly in GnRH antagonist-treated stump-tailed macaques where exogenous FSH stimulates inhibin B, but not inhibin A, secretion (Fraser et al., 1999). In addition, 
exogenous inhibin A treatment during the early follicular phase fails to decrease serum FSH (Fraser and Tsonis, 1994); immunoneutralization with $\alpha$-subunit antisera during the midluteal phase (when inhibin $\mathrm{A}$ is elevated) does not increase serum FSH in this species (Fraser et al., 1992). These data suggest that inhibin A may neither regulate nor be regulated by FSH in stump-tailed macaques. Similarly, treatment of metestrous female rats with rh-inhibin A fails to inhibit FSH levels, while administration of an identical dose on proestrus results in a prompt (i.e., within 1 hour) and sustained suppression of serum FSH (Woodruff et al., 1993b). In contrast, when porcine follicular fluid is administered on metestrus, serum FSH levels can be suppressed, eliminating the mundane interpretation that FSH levels are already at their lowest levels (Grady et al., 1981). Instead, these data suggest that something else contained within porcine follicular fluid, perhaps inhibin B, is a more-potent regulator of FSH on metestrus than is inhibin A.

In contrast to the stump-tail macaque and rat, administration of rh-inhibin $A$ to rhesus monkeys during the early follicular as well as the midluteal phase suppresses FSH, but not LH, release (Stouffer et al., 1994; Molskness et al., 1996). Because inhibin B rather than inhibin A is the dominant ligand of the follicular phase, adminstration of any amount of the A-isoform can be called supraphysiological, raising the possibility that the response in the rhesus monkey was achieved because of dose. Is it possible that the pituitary integrates an inhibin $B$ signal differently than an inhibin A signal? Interestingly, both primate and rodent males produce inhibin B rather than inhibin A (de Kretser et al., 1989; LambertMesserlian et al., 1994; Illingworth et al., 1996; Woodruff et al., 1996; Plant et al., 1997). This isoform is thought to be the primary regulator of FSH (Illingworth et al., 1996; Ramaswamy et al., 2000). Until inhibin A and inhibin B can be administered to or specifically eliminated from rodent models, subhuman primates, and humans, the specific contribution of these ligands to control of the various phases of the reproductive cycle remains to be clarified.

In summary, inhibin A and B are differentially regulated across the female reproductive cycle in rodents and primates. In most cases, there is a negative correlation between FSH and inhibin levels, consistent with the feed-forward/feed-back relationship proposed for these hormones. In both rats and primates, this relationship appears to be stronger for inhibin B than for inhibin A, although this can be seen more clearly in primates, where there is a greater temporal dissociation between patterns of inhibin A and B secretion. Preferential isoform release may reflect differential regulation of $\beta$-subunit transcription or translation as well as regulation of dimer assembly and secretion. An emerging concept is that inhibin A and inhibin B may serve different biological functions, particularly with respect to FSH regulation. This might be accomplished by a number of different mechanisms, chief among them, the presence of specific inhibin A or inhibin B receptors in the pituitary. Next, we turn to a discussion of 
the possible role of differential inhibin subunit transcription, then to the issue of inhibin isoform-specific receptors.

\section{Molecular Regulation of Inhibin Subunits}

The mechanisms regulating differential inhibin $A$ and $B$ synthesis and secretion are not thoroughly understood. With the cloning of promoters for the different subunits in rats and humans (Feng et al., 1989; Pei et al., 1991; Dykema and Mayo, 1994; Tanimoto et al., 1996; Ardekani et al., 1998), we are beginning to understand transcriptional regulation of inhibin subunit genes. As discussed previously, gonadotropins are potent regulators of inhibin subunit gene expression. Both LH and FSH signal in target cells through G protein-coupled receptors and increase intracellular cAMP levels via activation of adenylyl cyclase. In fact, many of the effects of FSH and LH on inhibin subunit expression can be mimicked by treatment with 8-bromo-cAMP or forskolin (Mayo, 1994). Because all three subunits are stimulated by gonadotropins, how can differential patterns of inhibin $\mathrm{A}$ and $\mathrm{B}$ production be manifested? Transcriptional analyses have revealed some clues as to how the three subunits may be regulated differentially in the face of similar hormonal stimulation.

Currently, transcriptional regulation is best understood for the $\alpha$ subunit. A nonconsensus cAMP response element (CRE) in the rat inhibin $\alpha$ promoter appears to mediate gonadotropin (cAMP)-dependent gene expression (Pei et al., 1991). Gonadotropins stimulate cAMP response element binding protein (CREB) phosphorylation in rat primary granulosa cells (Mukherjee et al., 1996) and stimulate trancription via CREB-mediated interaction with the CRE in the $\alpha$ promoter (Pei et al., 1991). This provides an explanation for how the gonadotropins (specifically, FSH) stimulate inhibin $\alpha$ expression throughout the majority female reproductive cycle. However, after the primary gonadotropin surges on the afternoon of proestrus in rats, there is a rapid decline in $\alpha$-subunit production and in secretion of both inhibin A and B (Meunier et al., 1988a; Woodruff $e t$ al., 1988,1996). These declines are dependent on the gonadotropin surges (Woodruff et al., 1989). How can the same stimuli have seemingly opposite effects? In a series of elegant experiments, Mukherjee and colleagues (1998) provide at least one potential explanation. During the time of the primary surges, a robust and transient expression of the inducible cAMP early repressor (ICER) occurs in the ovary. ICER is the product of an intronic promoter in the CRE-modulatory protein (CREM) gene (Molina et al., 1993; Stehle et al., 1993). The protein contains the DNA binding and dimerization domains of the CREM protein but lacks the $\mathrm{N}$-terminal transactivation domain. Thus, ICER downregulates cAMP-dependent signaling, at least in part, by competitively binding CREs in target genes. ICER is not upregulated in proestrous animals treated with a GnRH antagonist, demon- 
strating the importance of gonadotropin surges in its expression. Thus, the decrease in inhibin $\alpha$ expression at this time may be explained by the transient expression of ICER in response to the LH (and FSH) surge. This proposed mechanism of action is supported by several observations. First, human chorionic gonadotropin (hCG, an LH-like molecule of placental origin), but not pregnant mare serum gonadotropin (PMSG, a source of FSH-like activity), stimulates ICER expression in cultured rat granulosa cells. Second, ICER binds the CRE in the $\alpha$-subunit promoter in a mouse granulosa cell line (GRMO2). Third, ICER attenuates basal and forskolin-stimulated $\alpha$-subunit promoter activity in GRMO2 cells.

Thus, at least for rats, the transient expression of ICER provides one mechanism whereby gonadotropins (e.g., FSH) can stimulate $\alpha$-subunit expression through the majority of the cycle but can inhibit expression just after the primary gonadotropin surges. While both LH and FSH stimulate cAMP production, only the very large cAMP signal generated specifically by LH may be sufficient to induce ICER expression (Mukherjee et al., 1998). Unlike the case in rats, however, in humans, $\alpha$-subunit and dimeric inhibin A are produced prior to and after the preovulatory gonadotropin surges. Does this suggest that ICER or some other repressor molecule is not similarly expressed in the human ovary following the LH surge? A close inspection of the data in humans indicates that inhibin A levels peak at the time of the LH surge, transiently decline, and then increase to elevated luteal levels (Welt et al., 1999; Sehested et al., 2000). Therefore, a transient repression may occur in humans, just as it does in rats. The primary difference, then, lies in the fact that human, but not rat, corpora lutea produce inhibin subunit mRNAs ( $\alpha$ and $\alpha \mathrm{A}$ ) and dimeric inhibin A (Davis et al., 1987; Woodruff et al., 1987; Meunier et al., 1988a; Roberts et al., 1993). The mechanisms controlling this interesting and important species difference are not well understood. However, there clearly is strong selective pressure in rodents to suppress luteal inhibin production. Continued inhibin production by ovulatory follicles after the LH surge would block the secondary FSH surge and recruitment of the next cohort of follicles (Schwartz and Channing, 1977).

While we are beginning to get a handle on inhibin $\alpha$ expression during the cycle (particularly in rats), mechanisms of $\beta$-subunit regulation are less clear. In rats, $\beta A$ and $\beta B$ subunit mRNA levels decrease around the time of the primary gonadotropin surges (Meunier et al., 1988a; Woodruff et al., 1988). The role of repressor molecules such as ICER in these declines has not been ascertained. In fact, while mRNA levels of both $\beta$ subunits are increased by FSH or forskolin (Turner et al., 1989; Dykema and Mayo, 1994; Woodruff et al., 1987), it is not clear that these increases reflect classical cAMP-dependent signal transduction. The rat $\beta A$ promoter is stimulated by forskolin and by the phorbol ester, 12-Otetradecanoylphorbol-13-acetate (TPA), in mouse GRMO2 cells (Ardekani et al., 
1998). Similar data have been reported for the human $\beta A$ promoter in HT1080 cells (Tanimoto et al., 1996). These data suggest that $\beta$ A, unlike $\alpha$, may be a target of both protein kinase $A(P K A)$ - and protein kinase $C$ (PKC)-mediated signal transduction. Interestingly, the rat $\beta A$ promoter contains a novel, nonconsensus $\mathrm{CRE}$ that is critical for both forskolin- and TPA-mediated transcription. However, signaling appears to be mediated via AP-1 (Jun-B and fos-B), and not by $\mathrm{CREB} /$ activating transcription factor (ATF), transcription factors. It is not clear how FSH, a stimulator of CAMP and the PKA pathway, stimulates the PKC pathway in granulosa cells, although FSH has been shown to stimulate jun-B in rat Sertoli cells (Hamil et al., 1994). Thus, one mechanism of differential $\alpha$ - and $\beta A$-subunit regulation may be accounted for by the use of PKA- vs. PKA/PKCdependent signaling (Tuuri et al., 1996; Ardekani et al., 1998). While this may provide a mechanism through which activin $A$ and inhibin $A$ may be differentially produced, it does not account for how inhibin $\Lambda$ and $B$ are differentially regulated.

Unlike $\alpha$ and $\beta A$, the $\beta B$ gene gives rise to two predominant transcripts in both ovaries and testes (see Table I) (Dykema and Mayo, 1994; Feng et al., 1995). These transcripts arise from use of two different promoters within the $5^{\prime}$ flanking region of the gene, which initiate transcription from sites spaced about $1 \mathrm{~kb}$ apart. Despite the fact that both transcripts are upregulated by FSH, forskolin, or 8bromo-cAMP in primary granulosa cells, neither promoter is stimulated by forskolin in these same cells (Dykema and Mayo, 1994). Similar results have been

\section{TABLE I}

Inhibit Subunit Characteristics

\begin{tabular}{llllll} 
Subunit & Chromosome & $\begin{array}{l}\text { Major mRNA } \\
\text { Transcript }(\mathrm{kb})\end{array}$ & $\begin{array}{l}\text { \# of } \\
\text { Exons }\end{array}$ & $\begin{array}{l}\text { Size } \\
\text { (Amino Acids) }\end{array}$ & $\begin{array}{l}\text { Mature Subunit } \\
\text { Size (a.a.) }\end{array}$ \\
\hline$\alpha$ & $2^{\mathrm{h}}, 1^{\mathrm{m}}, 2^{\mathrm{o}}$ & $1.5^{\mathrm{r}}$ & 2 & $366^{\mathrm{r}}$ & $133^{\mathrm{r}}$ \\
$\beta A$ & $7^{\mathrm{h}}, 13^{\mathrm{m}}, 4^{\mathrm{o}}$ & $6.8^{\mathrm{r}}$ & 3 & $424^{\mathrm{r}}$ & $116^{\mathrm{r}}$ \\
$\beta B$ & $2^{\mathrm{h}}, 1^{\mathrm{m}}, 2^{\mathrm{o}}$ & $4.3-4.4$ and $3.3^{\mathrm{r}}$ & 2 & $411^{\mathrm{r}}$ & $115^{\mathrm{r}}$ \\
\hline
\end{tabular}

The three subunit genes have been chromusonally mapped in humans (h), mice (m), and sheep (o). Note that in all three species, the $\alpha$ and $\beta B$ genes map to the same chromosome (Barton et al., 1989; Hiendleder et al., 2000). The predominant mRNA transcript sizes in the gonads are indicated for rats (r) (e.g., Woodruff et al., 1987; Feng et al., 1989; Dykema and Mayo, 1994). The mRNAs are assembled from two exons for $\alpha$ and $\beta B$ and from three exons for $\beta A$ (see Mayo, 1994, for review). All three subunits are produced as prepro-hormones. The inhibin ligands are produced from the heterodimeric assembly of the so-called mature $\alpha$ subunit with one of the two $\beta$ subunits, which are cleaved from the C-terminal portion of the prepro-hormones. The sizes of the full-length and mature subunit proteins (in amino acids) are shown for rats (r) (Esch et al., 1987; Woodruff et al., 1987). 
observed in MA-10 Leydig tumor cells treated with cAMP or TPA (Feng et al., 1995). In human granulosa-luteal cells, $\beta B$ mRNA levels are unaffected by 8bromo-cAMP or TPA (Tuuri et al., 1996). Examination of both promoters fails to reveal consensus or variant CREs, which may account for the failure of cAMP or forskolin to stimulate these promoter constructs. However, anecdotal (Dykema and Mayo, 1994) and published reports (Najmabadi et al., 1993) indicate that these or similar promoter constructs are cAMP reponsive in different cell lines. Clearly, more work needs to be done to determine the role of cAMP-dependent signal transduction in $\beta \mathrm{B}$-subunit transcription.

In rats, $\beta A$ and $\beta B$ mRNA and protein are expressed in the same follicles and are regulated similarly across the cycle, with few exceptions (Meunier et al., 1988a). Therefore, regardless of the mechanisms regulating basal or stimulated expression of the $\beta$-subunit genes, it seems clear that some other, nontranscriptional mechanism must underlie the differential pattern of inhibin $A$ and inhibin $B$ secretion. Translational processing may not be involved, as both $\beta A$ and $\beta B$ protein levels mirror their mRNA levels (Meunier et al., 1988a). Instead, regulation is likely post-translational, involving dimerization and secretion. Unfortunately, relatively nothing is known about the regulation of these processes for inhibin. In humans, $\beta A$ - and $\alpha$-, but not $\beta B$-, subunit mRNAs are detected in human corpora lutea (Roberts et al., 1993). Therefore, unlike the case in rats, a transcriptional mechanism may participate in the differential release of inhibin A and B (particularly during the luteal phase) across the cycle.

In summary, all three subunits appear to be regulated by FSH in vivo and in vitro. Because the FSH receptor stimulates cAMP production following ligand binding, it appears that inhibin subunit transcription may be regulated by PKAdependent signaling. Promoter-reporter studies in primary granulosa cell culture indicate that both inhibin $\alpha$ and $\beta$ subunits are cAMP inducible. However, while the $\alpha$ subunit appears to be stimulated through conventional, PKA-mediated mechanisms, both PKA- and PKC-mediated pathways promote $\beta A$ transcription via AP-1 transcription factors that bind to a novel CRE. The role of cAMP in regulating the $\beta B$-subunit gene in rat granulosa cells is ambiguous. However, this pathway may be involved in $\beta B$ transcription in testicular Sertoli cells as well as in the ovary in vivo. The downregulation of inhibin production following gonadotropin surges in rats (and perhaps in humans) may be mediated by an LH-induced increase in ICER-mediated repression of cAMP-dependent gene expression. While this mechanism may account for the decline in all inhibin production at this time, the mechanisms through which inhibin A and B are differentially regulated throughout the cycle continue to be the subject of active investigation. 


\section{Inhibin and Cancer}

In addition to their roles in normal reproductive function, inhibins (and activins) have been implicated in pathologies of the reproductive system, including gonadal oncogenesis. This association is demonstrated most clearly in $\alpha$ subunit knockout mice. Both male and female mice homozygous for the null allele develop sex-cord gonadal tumors with 100 percent penetrance as early as 4 weeks of age (Matzuk et al., 1992). Castrated inhibin a knockout mice eventually develop adrenal tumors, indicating that inhibin acts as a tumor suppressor in multiple tissues (Matzuk et al., 1994). Importantly, inhibin $\alpha$ knockout mice engineered to produce inhibin $A$ through a mifeprisone-inducible system do not develop gonadal tumors, indicating that it is the absence of inhibin postnatally and not during development that causes tumor development (Pierson et al., 2000). In the absence of the $\alpha$ subunit, knockout mice produce very high levels of activin. However, these increased activin levels do not appear to cause the tumorigenic phenotype. Compound homozygous mutant mice deficient in both the $\alpha$ subunit and the ligand-binding activin receptor, actRII (Mathews and Vale, 1991), develop gonadal sex-cord tumors similar to those of the $\alpha$-subunit knockout animals (Coerver et al., 1996). However, it should be noted that activins may act via a second form of the receptor, actRIIB (Attisano et al., 1992), in these mice.

Another consequence of inhibin deficiency and activin overproduction is increased serum FSH levels (Matzuk et al., 1992). It is therefore possible that increased gonadotropin levels contribute to tumor development. Consistent with this argument, mice deficient in both the inhibin $\alpha$ subunit and GnRH (hpg) do not develop gonadal or adrenal tumors and live beyond 1 year of age (Kumar et al., 1996). While these data suggest that increased gonadotropin levels (FSH in particular) may drive the tumor development in $\alpha$-subunit knockouts, FSH alone does not appear to be responsible. Inhibin $\alpha$ and FSH- $\beta$ double-knockout mice develop gonadal tumors, although tumor onset is delayed, tumor phenotype is less extreme, and animals live longer than $\alpha$-subunit knockouts (Kumar et al., 1999). The role of LH in these animals is unclear, although it should be noted that transgenic mice overexpressing LH develop ovarian cysts and tumors (Risma et al., 1995).

There is also a relationship between ovarian cancer and inhibin in humans. Sex-cord stromal tumors are rare in humans, accounting for less than 10 percent of all cases of ovarian cancer. If inhibin acts as a tumor suppressor in humans as it does in mice, one would predict a decrease or absence of inhibin expression in these tumors. The opposite, in fact, appears to be the case. Several reports indicate inhibin $\alpha$ - and $\beta$-subunit expression in gonadal sex-cord tumors (Gurusinghe et al., 1995; Flemming et al., 1995,1996; Costa et al., 1997; Stewart et al., 1997; Yamashita et al., 1997; Zheng et al., 1997). In some cases, these tumors are 
associated with elevated circulating inhibin levels that are decreased following surgical resection (Lappohn et al., 1989; Silverman et al., 1994; Cooke et al., 1995; Yamashita et al., 1997). Serum FSH levels are inversely related to serum inhibin levels in some of these patients, indicating that the circulating inhibins are biologically active. Inhibin immunoreactivity also appears to be a good marker of testicular sex-cord stromal tumors (McCluggage et al., 1998). Thus, in apparent contrast to the case in mice, human sex-cord tumorigenesis is almost always associated with the presence, rather than the absence, of inhibin. Along these lines, it is interesting to note that, in human granulosa tumors, there is almost invariably no loss of heterozygosity ( $\mathrm{LOH}$ ) in the vicinity of the inhibin $\alpha$ gene on chromosome $2 \mathrm{q}$ (Watson et al., 1997), suggesting that inhibin is not aberrantly produced in these tumors. Interestingly, there is a $\mathrm{LOH}$ in this region in approximately 33 percent of the epithelial ovarian tumors examined. Thus, dysregulation of inhibin expression may be associated not with sex-cord stromal tumors but rather with epithelial ovarian cancer (EOC), the most-pervasive and deadly form of the disease.

In postmenopausal women, circulating inhibin levels are by-and-large undetectable (Burger et al., 1999). However, in many cases, postmenopausal women with EOC have elevated serum inhibin (Burger et al., 1996a; Blaakaer et al., 1993; Healy et al., 1993; Cooke et al., 1995; Frias et al., 1999). In one study (Blaakaer et al., 1993), increased survival was associated with elevated inhibin levels in postmenopausal women with malignant epithelial ovarian tumors, consistent with inhibin's proposed role as a tumor suppressor (Matzuk et al., 1992). However, a more-recent study indicates that elevated inhibin A levels may be a predictor of poor survival outcomes. In women with primary EOC, preoperative serum inhibin A levels above the median are associated with decreased disease-free survival and decreased overall survival in the 5 years after surgery (Frias et al., 1999). The apparent difference between these two studies may be attributed to the assays used to detect serum inhibins. The earlier study measured total serum inhibin (free $\alpha$ subunit as well as dimeric inhibin) (Blaakaer et al., 1993), while the later study used assays that measure both the free (pro- $\alpha \mathrm{C}$ ) and dimeric forms of inhibin (A and B) (Frias et al., 1999). There is some suggestion that free $\alpha$ subunit may be a better marker of EOC than dimeric inhibin A (Lambert-Messerlian et al., 1997; Burger et al., 1996b). The study by Frias and coworkers (1999) acknowledged both that overall inibin A levels were low in their patient population and that patients with pro- $\alpha \mathrm{C}$ levels above the median had a 2.4-fold increased chance of survival. Therefore, if the increased survival of patients with elevated inhibin levels in the Blaakaer study (1993) is related specifically to increased pro- $\alpha \mathrm{C}$, then the two studies may be consistent after all. In this case, elevated pro- $\alpha \mathrm{C}$ may provide a good survival marker, while increased inhibin A may be a marker of increased morbidity. 
What do these circulating hormone levels say about the underlying disease process? As discussed earlier, the lack of inhibin in $\alpha$-subunit knockout mice leads to sex-cord stromal tumorigenesis. One suggestion, therefore, is that, in the absence of the $\alpha$ subunit, unopposed activin dimer assembly occurs and activin may have a proliferative (oncogenic) effect on gonadal cells. Activin levels are, indeed, elevated in these animals (Matzuk et al., 1994). Activin production and secretion also are upregulated in EOC tumors, relative to normal ovarian epithelial cells (Welt et al., 1997a). However, deletion of actRII in $\alpha$-subunit knockouts does not alter the tumorigenic phenotype (Coerver et al., 1996). While activin stimulates proliferation in human ovarian cancer cell lines (that do not produce follistatin) (Di Simone et al., 1996), it does not consistently affect normal or EOC epithelia in primary culture (Welt et al., 1997a). Thus, the potential role of activin as a proliferative factor (and inhibin as an antiproliferative factor) in human EOC remains unclear.

In summary, inhibin acts as a sex-cord stromal tumor suppressor in mice but is upregulated in human sex-cord stromal tumors. While inhibin levels also are increased in human epithelial ovarian cancer, the source of inhibin and the role of the hormone in tumor development and progression are not thoroughly understood.

\section{Evidence for the Existence of an Inhibin Receptor}

Although we have learned a great deal about the regulation of inhibin production and secretion and about its endocrine and paracrine roles in reproductive physiology, we still know very little regarding how the hormone exerts its effects at a cellular and molecular level. This lack of knowledge stems mainly from the failure to identify and clone an inhibin receptor(s) and to characterize its underlying signal transduction pathway. This stands in sharp contrast to what has been learned about activin and its mechanisms of action during this same time period (Woodruff, 1998; Pangas and Woodruff, 2000). Given the failure to clone any inhibin receptors, one could speculate that none, in fact, exist. In this case, inhibin's actions may be mediated not by separate signaling mechanisms but rather via perturbation of activin signaling. There are at least two obvious ways in which inhibin could accomplish this task in the absence of its own receptor.

First, as described above, activins and inhibins share $\beta$ subunits. In many tissues, particularly the gonads, $\alpha$-subunit production is greater than that of the $\beta$ subunits. In these cases, inhibin production exceeds activin production. Therefore, where the $\alpha$ subunit is more abundant, activin production and, therefore, action is abrogated simply because less activin is available to act on target cells. Second, activin, like other members of the TGF- $\beta$ superfamily, binds a type II receptor (actRII or actRIIB), a receptor serine-threonine kinase (Mathews and Vale, 1991; 
Attisano et al., 1992; Mathews et al., 1992). Upon ligand binding, the type II receptor recruits and phosphorylates the activin type I receptor (Alk4 or actRIB), which is also a serine-threonine kinase (Attisano et al., 1993; Ebner et al., 1993; Tsuchida et al., 1993; Carcamo et al., 1994; ten Dijke et al., 1994). The type I receptor then phosphorylates intracellular signaling molecules in the Smad family (Smad2 or Smad3), which bind to another Smad protein (Smad4) before translocating to the nucleus to affect transcription of target genes. Inhibin binds to actRII via its $\beta$ subunit, albeit with lower affinity than does activin (Mathews and Vale, 1991; Xu et al., 1995; Martens et al., 1997). This binding does not stimulate recruitment or phosphorlyation of the type I receptor (LeBrun and Vale, 1997; Martens et al., 1997). Thus, in those situations where the concentration of inhibin exceeds that of activin, inhibin can competitively bind the type II receptor and block activin signaling. ${ }^{1}$

Do these two modes of antagonism describe the mechanisms for all inhibin action? If so, this would indicate that inhibin functions solely by regulating activin signaling. This may be the case but has it become increasingly clear that there are inhibin-specific binding proteins and mechanisms to modify or amplify an inhibin signal. Supporting this notion are the following experimental observations. First, high-affinity binding sites for inhibin have been identified in ovine pituitary cells in primary culture (Hertan et al., 1999). Second, several studies have demonstrated inhibin-specific binding sites in adult tissues. For example, iodinated inhibin A, but not iodinated activin A, binds to rat Leydig cells (Krummen et al., 1994). In addition, the adrenal, spleen, and bone marrow bind iodinated inhibin $A$ at much higher levels than they bind iodinated activin A (Woodruff et al., 1993a). Gonadal tumors derived from inhibin $\alpha$-subunit knockout mice bind iodinated inhibin $\mathrm{A}$ but bind iodinated activin $A$ at much lower levels. This inhibin binding is not displaced by excess unlabeled activin (Draper et al., 1998). These data indicate that the association between inhibin and target cells is mediated by an inhibinspecific receptor or binding protein and not solely via the activin type II receptors, or at least that an accessory protein forms a stable association between inhibin and actRII that is not easily disrupted by activin (Lewis et al., 2000).

Third, inhibin is not able to antagonize activin action in all contexts; in some situations, the two ligands appear to have similar effects. In KAR6 cells, erythroleukemia cells that inducibly overexpress actRII and Alk4, inhibin is unable to antagonizc activin-stimulated erythrodifferentiation, cell proliferation, activin receptor complex formation, and reporter gene activity, even at 128-fold higher concentrations of inhibin (LeBrun and Vale, 1997). Inhibin does not block

\footnotetext{
${ }^{\mathrm{I}}$ Functional antagonism also has been described between activin and the BMP OP-1 (BMP-7) during mesoderm formation, where OP-1 has been shown to bind to the activin type II receptor (Yamashita et al., 1995; Piek et al., 1999; Candia et al., 1997).
} 
activin-stimulated apoptosis in hepatocytes (Schwall et al., 1993) nor does it affect activin's suppression of corticotropin (ACTH) from AtT20 corticotrope cells (Bilezikjian et al., 1991). Both inhibin A and activin A stimulate primate oocyte maturation in vitro and cytochrome $\mathrm{P}_{50} \mathrm{O}_{17 \alpha}$ and $\mathrm{LH}$ receptor mRNA levels in porcine Leydig cells (Alak et al., 1996; Lejeune et al., 1997). Fourth, inhibin binding proteins have been identified in gonadal tumors from inhibin $\alpha$-subunit knockout mice, bovine pituitaries, and human erythroleukemia cells (K562) (LeBrun and Vale, 1997; Draper et al., 1998; Chong et al., 2000). Thus, several pieces of data converge to indicate the presence of an inhibin receptor or binding protein distinct from the activin type II receptors. As a result, both we and others embarked to identify this protein or proteins.

\section{Identification of Inhibin Binding Protein}

Initially, we employed an approach that had proven successful in the cloning of ligand binding receptors for TGF- $\beta$ ligands (Attisano et al., 1992). We used degenerate oligonucleotides directed against conserved portions of the activin type II (and later type I) receptors and attempted to amplify novel type II-like receptors from inhibin $\alpha$ knockout tumors and rat pituitaries by polymerase chain reaction (PCR). Given that inhibin and activin share common $\beta$ subunits and because inhibin binds activin type II receptors with low affinity (Mathews and Vale, 1991; $\mathrm{Xu}$ et al., 1995; Martens et al., 1997), it seemed reasonable to speculate that inhibin might signal through a type II-like receptor. However, this approach failed to produce any novel serine-threonine kinase receptors.

We next attempted to identify the inhibin receptor by expression cloning. We demonstrated that inhibin A, but not activin A, bound to gonadal tumors from inhibin $\alpha$ knockout mice (Draper et al., 1998). We therefore prepared a cDNA expression library from RNA extracted from these tumors, then screened transfected COS cells for their ability to bind iodinated inhibin A. An approach similar to this was used successfully to clone the first receptor in the TGF- $\beta$ superfamily, actRII (Matthews and Vale, 1991). Unfortunately, this approach also failed to generate any candidate inhibin binding molecules (see further discussion below).

With our third approach, we struck gold (Chong et al., 2000)! We used affinity chromatography to purify inhibin binding proteins. Operating under the assumption that the pituitary (the primary target of gonadally derived inhibin) would provide an enriched source of inhibin receptors, we purified membrane proteins from kilogram quantities of bovine pituitaries and ran them through a recombinant human ( $\mathrm{rh}$ ) inhibin $\mathrm{A}$ affinity column. The proteins retained within the column were then eluted and subjected to sodium dodecyl sulfate-polyacrylamide gel electrophoresis (SDS-PAGE) under reducing conditions. About 20 to 25 proteins were purified in this fashion; however, one protein with an apparent 
molecular mass of $142 \mathrm{kDa}$ was decreased significantly in abundance when the membrane proteins were pre-incubated with rh-inhibin A prior to column purification. The membrane proteins were purified to homogeneity using reverse-phase high-performance liquid chromatography (HPLC), subjected to SDS-PAGE, transferred to polyvinylidene difluoride (PDVF), and stained with Coomassie blue. The $142-\mathrm{kDa}$ protein was cut from the membrane and microsequenced. A 17-amino acid oligopeptide sequence was determined and used to search GenBank using the BLASTP algorithm. The sequence corresponded to two published human cDNAs in the database (AF034198 and Y10523) (Frattini et al., 1998; Mazzarella et al., 1998), mapping to a gene on human chromosome Xq25. The gene is comprised of 19 exons and spans approximately $20 \mathrm{~kb}$ of genomic sequence (Frattini et al., 1998). The two reported sequences are virtually identical; however, one appears to contain an additional exon (exon 3) encoding nine amino acids predicted to be part of the N-terminal signal peptide (Frattini et al., 1998). Both reports identified at least two transcripts in polyA+ RNA from different human tissues, suggesting that different forms of the receptor may be expressed. We have identified at least three transcripts in total RNA from rat pituitary and testes and are in the process of characterizing the different forms (Chong et al., 2000; unpublished results).

The full-length human cDNAs are predicted to encode a single transmembrane-spanning protein of approximately $147 \mathrm{kDa}$. The protein, called immunoglobulin domain-containing gene 1 (IGDC1) (Frattini et al., 1998) or immunoglobulin superfamily gene 1 (IGSF1) (Mazzarella et al., 1998), contains an N-terminal signal peptide followed by 12 Ig-like domains organized into motifs of five and seven Ig loops, separated by a short, hydrophobic linker region. The protein has a transmembrane domain following the 12th Ig loop and a short, serine/threonine rich cytoplasmic tail. Unlike the type I and II receptors in the TGF- $\beta$ family, this protein does not contain any apparent kinase domain. Cleavage of the signal peptide produces a protein with a predicted molecular mass of $142 \mathrm{kDa}$, which corresponds to the $142-\mathrm{kDa}$ protein purified in the affinity column. The oligopeptide sequence was used to generate polyclonal antisera. In western blot analyses of bovine pituitary membrane proteins, these antisera detect a single protein of $142 \mathrm{kDa}$. In the original purification of proteins from the affinity column, we erroneously identified the protein to have a molecular mass of $120 \mathrm{kDa}$ and referred to the protein as p120 (Chong et al., 2000). Subsequent measurements confirmed a molecular mass of approximately $142 \mathrm{kDa}$. We now refer to this protein as inhibin binding protein (InhBP). 


\section{Criteria for Inhibin Receptor Designation}

During our cloning effort, we established four criteria that we believe must be satisfied in order to call InhBP or any other candidate molecule an inhibin receptor (Woodruff, 1999). First, it must be expressed in known inhibin target tissues. In particular, it must be expressed in pituitary gonadotropes. Second, the receptor must bind inhibin with high affinity. Third, this binding must be of high specificity but not necessarily exclusivity. That is, the molecule should bind inhibin with higher affinity than other ligands in the TGF- $\beta$ superfamily, although some low-affinity association with other ligands is possible (as is the case for inhibin $A$ and bone morphogenic protein (BMP)-7 with actRII) (Matthews and Vale, 1991; Yamashita et al., 1995). Fourth, the molecule must provide a mechanism for functional antagonism of activin signaling.

Consistent with the first criterion, we initially identified InhBP in bovine pituitaries. However, this result did not demonstrate expression of the protein specifically within FSH-producing gonadotropes. We addressed this issue during more-extensive studies in rats. RNA blot analyses show a high level of InhBP expression in rat pituitary and a lower, but still detectable, level of expression in the testis (Chong et al., 2000). We since have observed high levels of InhBP expression in pituitaries of a variety of species, including mice, sheep, and cows. In situ hybridization analyses in rats indicate that InhBP mRNA is expressed exclusively within the anterior pituitary in a pattern similar to that of the FSH- $\beta$ subunit mRNA (unpublished observation). Immmunohistochemical studies using the polyclonal antisera described above have identified InhBP protein within the anterior pituitary, where it co-localizes with FSH protein. In addition, InhBP protein is abundantly expressed in testicular Leydig cells (Chong et al., 2000). Importantly, Leydig cells previously have been shown to be targets of inhibin, but not activin, binding (Krummen et al., 1994). Thus, InhBP satisifies the first criterion for designation as an inhibin receptor or binding protein.

To explore binding affinity and specificity of inhibin to InhBP, we have performed transient transfection of human InhBP and ligand binding studies in monkey kidney epithelial cells, CV-1. These cells, which do not endogenously express InhBP, do not bind iodinated inhibin A. However, transfection of fulllength InhBP is sufficient to confer binding to these cells. We currently are using this cell culture system to determine binding affinity and specificity. Preliminary observations indicate that inhibin $A$ binds these transfected cells with high affinity. In addition, while other TGF- $\beta$ ligands (e.g., activin A, TGF- $\beta 1$ ) appear to bind InhBP, they do so with lower affinity than inhibin $A$. We are currently confirming and extending these preliminary observations. Thus, while the data look promising, we cannot yet confirm that InhBP satisfies that second and third criteria stipulated previously. The fourth criterion holds that the candidate inhibin receptor must provide a mechanism for antagonism of activin signaling in target 
cells. We have dedicated much effort to the elucidation of InhBP's role in inhibinmediated antagonism of activin action; these data are reviewed below.

As we were characterizing InhBP as a candidate inhibin receptor, the TGF- $\beta$ type III receptor, betaglycan, was identified as a potential inhibin receptor (Lewis et al., 2000). This molecule appears to satisfy all of the criteria for designation as an inhibin receptor. First, betaglycan is expressed in inhibin target tissues, including testicular Leydig cells and pituitary gonadotropes (Lewis et al., 2000; MacConnell and Vale, 2000). Second, unlike InhBP, betaglycan alone does not bind inhibin A or at least does so with low affinity. However, in the presence of actRII, betaglycan confers high-affinity inhibin A binding to cells that do not normally bind inhibin. Third, this binding appears to be specific (although not exclusive). Excess activin $A$ is unable to disrupt the association among betaglycan, actRII, and inhibin A, although TGF- $\beta 1$ does disrupt formation of the complex. Fourth, betaglycan provides a mechanism for inhibin A antagonism of activin signaling via competition for actRII. That is, by binding betaglycan and actRII, inhibin blocks binding of activin to the type II receptor and subsequent recruitment of the signaling type I receptor. Because it requires actRII for high-affinity inhibin A binding and antagonism, betaglycan may best be thought of as a co-receptor (Lewis et al., 2000).

Next, we will review canonical signaling mechanisms within the TGF- $\beta$ superfamily, in order to provide the foundation for an understanding of how inhibin may antagonize activin signaling via InhBP.

\section{The Canonical TGF Holo-receptor Complex}

During the last 15 years, the signal transduction pathways initiated by TGF- $\beta$, activin, BMP, and Mullerian-inhibiting substance (MIS) have been well characterized. In comparison, understanding the mechanism of inhibin action has proceeded at a slower pace. The first of a large number of TGF- $\beta$ superfamily binding, signaling, and accessory receptors was described by Massague in 1985 (reviewed in Massague et al., 1987). Through affinity labeling using iodinated TGF- $\beta$, three distinct TGF- $\beta$ binding proteins were identified and designated type I, type II, and type III receptors, based on relative molecular weights (Massague, 1985; Cheifetz et al., 1986; Massague et al., 1987). Native gels suggested that these proteins existed as a heteromeric protein complex (Massague, 1985). The type II TGF- $\beta$ receptor resembled a newly identified type II activin receptor and shared a region of high homology within the intracellular serine-threonine kinase domain (Mathews and Vale, 1991; Lin and Wang, 1992). This activin receptor was identified in an AtT20 corticotroph library screen by ${ }^{125}$ I-activin A binding (Mathews and Vale, 1991). The resultant full-length positive clone was predicted to contain a single transmembrane domain, a cysteine-rich extracellular ligand 
binding domain, an intracellular serine-threonine kinase domain, and potential autophosphorylation sites. Identification of additional type II and type I activin and TGF- $\beta$ receptors was accomplished by cloning of proteins homologous within the predicted serine-threonine kinase domain (Attisano et al., 1992,1993; Mathews et al., 1992; Franzen et al., 1993; He et al., 1993; ten Dijke et al., 1993; Tsuchida et al., 1993). A growing number of type I and type II serine-threonine kinase receptors specific for other members of the TGF- $\beta$ superfamily, including the BMPs and MIS, have since been identified (Paralkar et al., 1991; di Clemente et al., 1994). All of these receptors are highly conserved within the serine-threonine kinase region and the functions of these receptors are highly conserved through evolution (Newfeld et al., 1999).

A series of TGF- $\beta$-resistant mink lung epithelial $(\mathrm{MvLu})$ cell lines deficient in either or both of the type I and II TGF- $\beta$ receptors provided initial insight into the role of these proteins in TGF- $\beta$ signal transduction (Boyd and Massague, 1989; Laiho et al., 1990,1991). Cells expressing only the type II receptor bound ligand but regained TGF- $\beta$ responsiveness upon reintroduction of the type I receptor protein, suggesting that the type I receptor was necessary for TGF- $\beta$ signal transduction but not for ligand binding (Laiho et al., 1991; Wrana et al., 1992). Introduction of a kinase-deficient type II receptor did not restore TGF- $\beta$ responsiveness or signal transduction. Thus, kinase activity as well as presence of the type I receptor was needed for TGF- $\beta$ signaling (Wrana et al., 1992). Accordingly, transphosphorylation of the TGF- $\beta$ and activin type I receptor by ligandbound type II receptor was found to be absolutely required for the assembly of the heteromeric receptor complex and signal transduction (Attisano et al., 1993,1996; Wrana et al., 1994; Tsuchida et al., 1995). Collectively, these data established the prevailing dual-receptor model of TGF- $\beta$ superfamily signal transduction involving heteromeric receptor complexes comprised of at least one type I receptor and one type II receptor.

Once activated, the heteromeric receptor complex phosphorylates and activates the downstream mediators of the signal, the Smad proteins (reviewed in Piek et al., 1999). Smad proteins are classified into three functional groups. Receptorspecific Smads are phosphorylated and activated by the heteromeric receptor complex: Smad 2 and Smad3 are phophorylated by TGF- $\beta$ and activin receptor complexes, while Smadl, Smad5, and Smad8 are activated by BMP type I receptors. Once activated, receptor-specific Smads associate with a common mediator Smad, Smad4, and together translocate to the nucleus, where they act as transcriptional co-factors (reviewed in Piek et al., 1999). The inhibitory Smad6 and Smad7 disrupt TGF- $\beta$ and activin signaling by preventing the phosphorylation, heteromerization, and translocation of the receptor-specific Smads (Imamura et al., 1997; Nakao et al., 1997; Hayashi et al., 1997). Smad6 interacts with the type I 
TGF- $\beta$, activin, and BMP receptors and interferes specifically with Smad2 and Smad1, but not Smad3, phosphorylation (Imamura et al., 1997; Ishisaki et al., 1999). Smad7 associates with TGF- $\beta$ and activin type I receptors and is capable of blocking Smad1, Smad2, and Smad3 phosphorylation (Nakao et al., 1997; Hayashi et al., 1997; Ishisaki et al., 1999; Kitamura et al., 2000). In Xenopus, Smad6 competes with Smad4 for binding to Smad1, thus blocking BMP signaling by preventing Smad1/Smad4 heteromerization and translocation to the nucleus (Hata et al., 1998).

The sharing of receptors by ligands and the formation of nontraditional heteromeric protein complexes add another dimension to the dual-receptor TGF- $\beta$ superfamily signaling model. For example, activin signals are transduced through the traditional type I/type II activin receptor complex and carried intracellularly by Smad2, Smad3, and Smad4. However, OP-1 (BMP-7) is also able to bind the type II activin receptor, which then forms a complex with the BMP type I receptor and transduces signals via Smad5 (Yamashita et al., 1995; Piek et al., 1999). Likewise, the ligand binding type II receptor for MIS (AMHRII) was found to form a functional receptor complex with the BMP type IB receptor and activate Smadl (di Clemente et al., 1994; Gouedard et al., 2000). Finally, the type I receptor TSR-1 (Tsk 7L) is capable of transducing either activin or TGF- $\beta$ signals in the presence of the appropriate type II receptor (Ebner et al., 1993). Thus, signaling specificity among TGF- $\beta$ superfamily members is determined in part by the complement of receptors and Smad proteins expressed in particular tissues.

\section{Modulation of TGF- $\beta$ Superfamily Signaling}

Modulation of TGF- $\beta$ superfamily signal transduction can occur through the action of the type III receptors, now referred to as the accessory signaling proteins. (Fig. 1). Cell surface accessory proteins are capable of binding ligand and interacting with the heteromeric receptor complex but have short intracellular domains with no intrinsic enzymatic activity (reviewed in Piek et al., 1999). Therefore, these accessory proteins modulate TGF- $\beta$ superfamily signaling by regulating delivery of ligand to the heteromeric signaling receptor complex or by modulating the assembly of the receptor complex. Because accessory proteins have restricted tissue expression patterns, it is hypothesized that responsiveness of a particular cell type to TGF- $\beta$ family members depends on the availability of not only the type $I$ and type II receptors but also on the availability of accessory signaling molecules.

The best-characterized accessory proteins are betaglycan and endoglin. Betaglycan, once called the TGF- $\beta$ type III receptor, is a membrane proteoglycan consisting of a core protein of approximately $100-120 \mathrm{kDa}$ with two regions of glycosylaminoglycan (GAG) attachment sites; a transmembrane domain; and a 


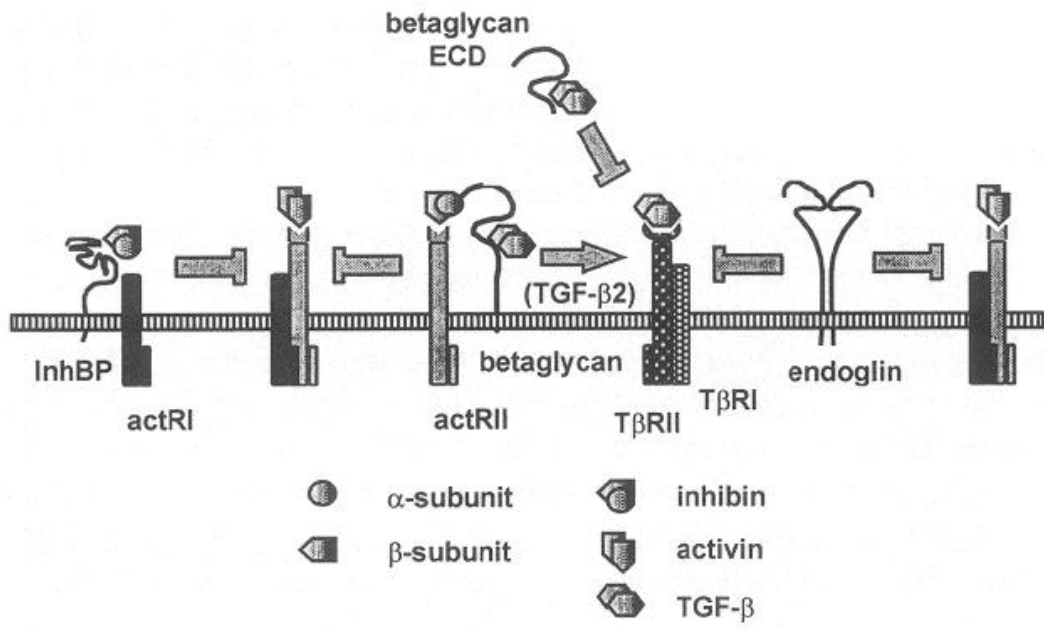

FIG. 1. Accessory proteins modify TGF- $\beta$ superfamily interactions with cell-surface receptors. The inhibin accessory protein, InhBP, can dissociate activin receptor complexes in the presence of inhibins (primarily inhibin B) and antagonize the action of that activin by rendering the receptor complex physically unable to associate. Other accessory proteins in the TGF- $\beta$ superfamily (e.g., endoglin, soluble betaglycan) act to disrupt TGF- $\beta$ signals by preventing stable receptor complex assembly. Accessory proteins also can facilitate signal transduction. For example, membrane-bound betaglycan facilitates TGF- $\beta 2$ access to TGF- $\beta$ receptors, enabling this ligand to act. Thus, an emerging theme for the mechanism of TGF- $\beta$ superfamily signal transduction is that accessory proteins are available to modify the net signal received by a cell for a given class of ligands. In addition, cells may use differential accessory protein expression to modify an existing signal. This idea is supported by the pattern of InhBP expression during the rat estrous cycle. Specifically, InhBP expression levels are highest during the follicular phase (when inhibin acts) and lowest at the time of the secondary FSH surge (when inhibin signals must be eliminated). Thus, the response of a cell to a given ligand may be modified by the presence of pre-existing or regulated receptor components at the cell surface.

short, serine-rich cytoplasmic tail (Cheifetz et al., 1998a; Lopez-Casillas et al., 1991,1994; Moren et al., 1992; Ponce-Castaneda et al., 1998). Betaglycan binds all three isoforms of TGF- $\beta$ and associates with ligand-bound type II receptor (Massague, 1985, Cheifetz et al., 1988a), increases the affinity of the type II receptor for TGF- $\beta$, and enhances cell responsiveness to TGF- $\beta 2$ (Lopez-Casillas et al., 1993). In fact, the affinity of the TGF- $\beta$ type II receptor is lower for TGF- $\beta 2$ than TGF- $\beta 1$ and -3 (Chiefetz, et al., 1990), yet cell types that express betaglycan respond to all three isoforms equally. Cells that do not express betaglycan respond poorly to TGF- $\beta 2$ (Ohta et al., 1987; Cheifetz et al., 1990). Thus, expression of this accessory protein can provide a degree of TGF- $\beta$ isoform signaling specific- 
ity. Betaglycan also exists in a soluble form that binds and sequesters TGF- $\beta$ and attenuates signaling (Andres et al., 1989, Lopez-Casillas et al., 1994). Therefore, betaglycan has been characterized as both a positive and negative regulator of TGF- $\beta$ signal transduction. As previously mentioned, betaglycan associates with actRII and, in the presence of inhibin, modifies the actions of activin. This function will be discussed in more detail later.

The betaglycan transmembrane and intracellular domains share 63 percent identity and 71 percent similarity with another signaling accessory protein, endoglin (Lopez-Casillas et al., 1991, Gougos and Letarte, 1990). Endoglin is a dimer consisting of a large, glycosylated, extracellular domain; one predicted transmembrane domain; and a serine-rich cytoplasmic tail. Endoglin was first identified as a vascular endothelial cell marker with an expression pattern similar to TGF- $\beta 1$ (Gougos and Letarte, 1988,1990). Indeed, endoglin was found to bind TGF- $\beta 1$ and TGF- $\beta 3$ but, unlike betaglycan, endoglin does not interact with TGF- $\beta 2$ (Cheifetz et al., 1992). Furthermore, endoglin assembles with the heteromeric receptor complexes of several members of the TGF- $\beta$ superfamily via the ligandbinding receptor component and acts as a negative regulator of signal transduction by these ligands (Barbara et al., 1999). Thus, endoglin appears to play a general role of a negative regulator of TGF- $\beta$ superfamily signal transduction.

\section{Variations on the TGF- $\beta$ Receptor Theme}

The inhibin $\alpha$ subunit sits on a distant branch of the TGF- $\beta$ superfamily tree (reviewed in Newfeld et al., 1999). However, because the inhibin heterodimer also includes an activin $\beta$ subunit, it was hypothesized (and hoped) that an inhibin receptor would resemble the traditional serine-threonine kinase receptors of activin and other TGF- $\beta$ superfamily proteins. Indeed, inhibin binds to the activin type II receptor, albeit at 10 -fold lower affinity than the activin dimer (Mathews and Vale, 1991, Mathews et al., 1992). However, initial attempts at cloning an inhibin receptor based on homology to the receptor serine-threonine kinase domain failed and it became clear that the mechanism of inhibin signal transduction would be unique.

Like inhibin, glial-derived neurotrophic growth factor (GDNF) is a distant member of the TGF- $\beta$ superfamily (reviewed in Newfeld et al., 1999). GDNF is involved in growth and differentiation of midbrain and peripheral neurons in vertebrates (Lin et al., 1993; Trupp et al., 1995). GNDF signals through a dualreceptor mechanism; however, the receptors are not serine-threonine kinases. GDNF binds to a GPI-anchored, cell-surface protein called GDNFR- $\alpha$, which has no intrinsic kinase activity (Jing et al., 1996; Treanor et al., 1996). Affinity-labeling experiments established that GDNFR- $\alpha$ binds GDNF and that this binding is 
abolished upon cleavage of the GPI anchor (Jing et al., 1996; Treanor et al., 1996). However, because GDNF is localized to the extracellular surface of the membrane and has no signaling capacity, it was hypothesized that another component was necessary to transduce a GDNF signal intracellularly. The proto-oncogene and receptor tyrosine kinase c-ret was identified as the second component of the GDNF signaling system, based on the overlapping patterns of GDNF and c-ret protein expression in the kidney and gut and on the similar phenotypes of mice deficient in either GDNF or c-ret (Schuchardt et al., 1994; Pichel et al., 1996; Sanchez et al., 1996; Moore et al., 1996). Once bound by GDNF, GDNFR- $\alpha$ associates with the Ret tyrosine kinase (Jing et al., 1996; Treanor et al., 1996). The ability of GDNF to promote axonal outgrowth in nephrogenic explants of $\mathrm{Ret}^{+/-}$but not $\mathrm{Ret}^{-1-}$ mice verified that Ret is necessary for GDNF signaling (Durbec et al., 1996). Thus, although the GDNF receptors do not utilize serinethreonine kinase receptors, the general dual-receptor mechanism of the superfamily is maintained. More intriguing is the fact that the GDNF signaling system incorporates elements of the two major growth factor families: 1) the dual-receptor mechanism of the TGF- $\beta$ superfamily and 2 ) the tyrosine kinases utilized by the large family of growth and differentiation factors, including epithelial growth factor (EGF) and insulin-like growth factor-1 (IGF-1) (reviewed in van der Geer et al., 1994).

GDF-9 is another distant member of the TGF- $\beta$ superfamily, although no receptor for this ligand has been identified. GDF-9 is expressed exclusively in the ovary and is absolutely required for preantral follicle devclopment (Dong et al., 1996; Hayashi et al., 1999). Treatment of cultured granulosa cells with recombinant GDF-9 stimulates the expression of several granulosa cell products, providing strong evidence for the existence of a GDF-9 receptor (Elvin et al., 1999). The C-terminal regions of GDF-9 and BMP-4 are highly homologous. However, unlike other TGF- $\beta$ superfamily proteins, GDF-9 lacks the conserved cysteine residues necessary for the formation of dimers (McPherron and Lee, 1993). Thus, although GDF-9 signals may be transduced through a traditional heteromeric receptor complex, it is also plausible that a unique receptor mediates GDF-9 effects on early folliculogenesis.

\section{Mechanism of Activin Antagonism}

With open minds, then, we have undertaken the task of elucidating either a novel and unique mechanism of inhibin signal transduction or one that partially resembles the dual-receptor model. Although we had a putative inhibin receptor in hand (i.e., InhBP), we had another obstacle to overcome: the lack of established inhibin responsive genes prevented a full understanding of inhibin signal transduction. For this reason, we and others (Lewis et al., 2000) have characterized the 
mechanism of inhibin action in terms of its ability to antagonize activin action. Of course, the best-known example of the opposing relationship between inhibin and activin is FSH production by the pituitary (Rivier et al., 1986). However, there is increasing evidence that inhibin also antagonizes the autocrine/paracrine actions of activin within the gonads. For instance, inhibin opposes inhibition of testosterone synthesis by activin in rat testicular and thecal cell cultures and inhibits activin-stimulated $3 \beta$-hydroxysteroid dehydrogenase (HSD) expression in porcine Leydig cells (Hsueh et al., 1987, Hillier et al., 1991, Lejeune et al., 1997, Lin et al., 1989). We are currently characterizing potential inhibin-responsive genes from each of these target tissues and anticipate that these will permit a more-detailed analysis of inhibin-specific signal transduction pathways. In the meantime, we have proceeded using the tools found on the activin toolbelt.

\section{Modulation of Activin Signaling by InhBP}

As described in an earlier section, InhBP has no intrinsic kinase domain. Based on this observation, and because inhibin is known to bind weakly to the activin type II receptor, we investigated assembly of InhBP with the activin receptors. Through immunoprecipitation/immunoblotting experiments, we demonstrated a strong InhBP interaction with the type IB activin receptor and little, if any, assembly of InhBP with the type II or type IIB activin receptors. This was somewhat unexpected, given that inhibin has been shown to bind the type II activin receptor and because inhibin binds betaglycan in the presence of the activin type II receptor (Mathews and Vale, 1991; Mathews et al., 1992; Lewis et al., 2000). InhBP also was able to assemble with the dual-specificity type I receptor TSR1 and the BMP type IB receptor (Alk6). It should be noted that InhBP shares with endoglin the ability to associate with several TGF- $\beta$ superfamily receptors. Thus, we predict that, as an accessory receptor, InhBP may have a similarly broad role in modulation of TGF- $\beta$ superfamily signaling.

InhBP-actRIB assembly was observed in vivo in a ligand-independent manner in sheep and human pituitary lysates. In vitro, InhBP-actRIB association was stabilized in the presence of activin $\mathrm{A}$. This interaction dropped to 60 percent of basal upon addition of inhibin B, but not inhibin A, to the system. ActRIB was shown to interact with both InhBP and actRIIB; however, it is not yet known whether InhBP exists as part of a heterotrimeric protein complex with both type $I$ and type II receptors. Nevertheless, treatment of cells with activin $A$ and inhibin $B$, but not activin $A$ and inhibin $A$, resulted in a dissociation of actRIB and InhBP as well as disassembly of the type IB/type IIB activin receptor complex. Finally, InhBP supported inhibin B-, but not inhibin A-, mediated antagonism of activin A-stimulated gene transcription. Functional antagonism of activin-stimulated gene expression was observed when cells were treated with activin A and either form 
of inhibin. However, cells transfected with InhBP and treated with inhibin B resulted in greater than 90 percent antagonism of activin-stimulated p3TP-luc expression. Importantly and necessarily, inhibin A or B alone or in the presence of InhBP was unable to antagonize TGF- $\beta$-stimulated gene expression.

Our working model describes an inhibin B-InhBP-mediated dissociation of the heteromeric activin receptor complex, which leads to abrogation of activin signal transduction and activin-stimulated gene expression. We propose that the presence of InhBP within the activin receptor complex renders it sensitive to inhibin $B$, such that low levels of inhibin B are capable of disrupting activin signaling. Cells in which InhBP is not expressed contain activin receptor complexes that are not as sensitive to inhibin treatment. In these cases, only a large excess of inhibin would be able to antagonize activin action.

The mechanism of inhibin B-InhBP-mediated dissociation of the heteromeric signaling complex is unknown. It is possible that InhBP may recruit endocytic machinery to the cell surface or that InhBP initiates receptor internalization by moving or tagging the activin receptor complex. Studies are currently underway to determine whether InhBP and inhibin B stimulate the ubiquitinization of the activin receptors. We are hesitant to rule out other means of antagonism of activin action by inhibin B and InhBP. For instance, inhibin and InhBP may initiate intracellular antagonism of activin signal transduction by activating the inhibitory Smad proteins (i.e., Smads 6 and 7) or through cross-talk with other pathways (e.g., the MAPK system), which has been shown to negatively regulate TGF- $\beta$ stimulated Smad activity (Kretzschmar et al., 1999).

The characterization of InhBP as a transducer of an independent inhibin signal has yet to be carried out, primarily due to the lack of available inhibinstimulated genes. It is possible that InhBP is a dual-function receptor involved in both inhibin signaling and inhibin-mediated activin antagonism. Indeed, several immunoglobulin domain-containing proteins are receptors. For example, the growth factor receptors of EGF and platelet-derived growth factor (PDGF) exhibit intrinsic tyrosine kinase activity and activate the MAPK signal transduction pathway (reviewed in Buck, 1992), while the cell adhesion molecule, N-CAM, is believed to transduce signals by initiating protein phosphorylation and activation of intracellular second messengers (reviewed in Goridis and Brunet, 1992). More interestingly, the receptors for two cysteine knot-containing proteins, vascular endothelial growth factor (VEGF) and nerve growth factor (NGF), contain immunoglobulin-like domains. Furthermore, the co-crystal structure of BMP-2 and the ectodomain of its ligand binding receptor, BMPRI, recently was solved and reveals a surprising and striking similarity to the co-crystal structures of VEGF and NGF with their respective receptors, Flt-1 and TrkA (Kirsch et al., 2000; Wiesmann and de Vos, 2000). These findings provide a compelling link between 
immunoglobulin domain-containing receptors and their corresponding cysteine knot-containing ligands.

Nevertheless, it is also possible that the positive and antagonistic actions of inhibin are supported by mutually exclusive receptors. The identification of inhibin-regulated genes is necessary to answer this question. Likewise, the discovery of the signaling molecules that lie downstream of InhBP is essential to understanding the mechanism(s) of inhibin signal transduction. Our finding that InhBP interacts with other TGF- $\beta$ receptors indicates that inhibin may signal through the Smad proteins.

\section{Future Directions}

Clearly, these are exciting times in inhibin research. The development of ligand-specific assays has revealed that inhibin A and B are differentially regulated across primate and rodent reproductive cycles, inhibin has been implicated in cancers of the reproductive system, and we are finally on the threshold of understanding how inhibin signals are conveyed to target cells.

\section{A. DIFFERENTIAL REGULATION OF INHIBIN ISOFORMS}

The data clearly indicate that inhibin A and B are differentially secreted across the female reproductive cycle. In addition, adult males appear to secrete inhibin B but not inhibin A (Woodruff et al., 1996). These observations beg the question, what is the functional significance of differential inhibin isoform secretion? Past research on inhibin action has relied heavily upon recombinant inhibin A, while little work on inhibin B has been reported. Therefore, we do not currently know the different roles or biological activities of the two ligands. There is certainly precedent for the belief that the two hormones will function differently. For example, we have shown that inhibin B, but not inhibin A, may use InhBP to antagonize activin actions. Inhibin $\mathrm{B}$ is more effective than inhibin $\mathrm{A}$ in blocking TGF- $\beta 1$ binding to $\mathrm{GH}_{3}$ rat pituitary cells (Cheifetz et al., 1988b). In addition, the different isoforms of activin (which share $\beta$ subunits with inhibin) have some redundant functions but clearly have different activities (Vassalli et al., 1994; Matzuk et al., 1995; Brown et al., 2000). The challenge for the immediate future is to develop model systems in which to assess the different functions of inhibin $\mathrm{A}$ and $\mathrm{B}$.

While the development of new assays to measure dimeric inhibins has revealed differential secretion of the two isoforms, we know little regarding how this is controlled. Although we are beginning to gain an appreciation for transcriptional regulation of the subunit genes, it is clear that transcriptional mechanisms alone cannot account for the different patterns of inhibin $A$ and $B$ release (at least not in rats). Perhaps there is regulation of dimer assembly but, at this point, we 
know little about how this is controlled. One report indicates that only when $\alpha$ subunit is expressed in excess of $\beta$ subunits is inhibin produced preferentially over activin (Mason, 1988). However, this does not address how different forms of inhibin may be differentially assembled. The signals controlling inhibin secretion may be the same for both ligands, with the differences in serum levels then reflecting different intracellular concentration of the two forms. Alternatively, different mechanisms may drive secretion of one form over the other. There is certainly precedent for this latter mechanism within the reproductive system. Both $\mathrm{LH}$ and FSH are produced and stored in the same gonadotrope cells in the anterior pituitary but the two hormones (which, like the inhibins, share a common $\alpha$ subunit) are differentially released. The frequency of $\mathrm{GnRH}$ pulses from the brain provides one mechanism governing this differential regulation of the two gonadotropins (Haisenleder et al., 1994). Future experiments need to address both the control of $\alpha \beta \mathrm{A}$ vs. $\alpha \beta \mathrm{B}$ assembly as well as the nature of the signals stimulating inhibin A vs. inhibin B release.

\section{B. INHIBIN AND CANCER}

Inhibin is normally expressed in the granulosa cells of the ovarian follicle and is abundantly expressed in granulosa cell tumors. It is not expressed in the surface epithelial layer of the ovary under normal conditions; however, it is detected in approximately 40 percent of pathology specimens from women with surface EOC. Therefore, production of inhibin by cancerous epithelial cells represents a failure of the molecular mechanisms governing cell-specific gene expression. An apparent conundrum exits between the relationship of inhibin and gonadal oncogenesis: a clear link has been demonstrated in animal models, yet the relationship in humans is less obvious. Does this contradict the proposed role of the ligands as tumor suppressors?

Inhibin production and secretion are often (although not always) associated with postmenopausal EOC. The role of the hormones, if any, in the disease process is not clear. Do aberrant inhibin production and signaling cause tumor development or growth, or do they merely reflect altered function of previously transformed, cancerous cells? At a minimum, it should be recognized that no mouse model has been generated that mimics human epithelial cell-derived ovarian cancer. The surface epithelia of the mouse and the human are similar in their role as the final boundary to the oocyte as it is expelled from the follicle into the oviduct. However, the epithelia of the rodent is protected from the peritoneal cavity by an extension of the oviduct, the bursal sac. One might conjecture that the rodent epithelia that must seal and re-seal six to eight times every 4 days is somehow resistant to the impacts of neoplasia. Alternatively, EOC is a product of an aging ovary and the mouse does not provide a suitable model for gerontological studies. An emerging line of thinking is that inhibin, similar to other 
cell-regulating molecules such as E2F1, may be functioning as both an oncogene and a tumor cell suppressor in certain tissues (Johnson, 2000). This dual action may depend on the ccll context (i.e., granulosa cell vs. surface epithelial cell) and may be responsive to the endocrine milieu at the time of altered expression (i.e., from birth or following follicular exhaustion). Clearly, new diagnostics and molecular tools are urgently needed to more fully understand EOC and then to intervene in disease. Inhibin may be one of many serum diagnostics that can be used but that may not be sufficiently predictive of disease at an earlier (moretreatable) stage. Alternatively, inhibin may participate in the onset or progression of disease; it is this latter point that bears examination.

\section{INHIBIN RECEPTORS}

While we have known for some time that inhibins can bind activin type II receptors, InhBP and betaglycan recently have been identified as putative inhibin receptors. While both molecules have provided novel insights into how inhibins abrogate activin signaling, many questions remain. First, can betaglycan and InhBP be described as receptors, despite the inability of these proteins to directly transduce a signal? To our way of thinking, the accessory proteins rightfully can be described as receptors. Like the cytokine Jak receptor system that depends upon extrinsic tyrosine kinases, the Stats, for signal transduction, the TGF- $\beta$ superfamily accessory proteins betaglycan and InhBP require association with the type II/type I complex in order to mediate a signal. Thus, the mechanism of signal transduction by the TGF- $\beta$ superfamily has expanded far beyond the traditional dual-receptor model to now include a role for accessory proteins in the modulation of signaling. Are InhBP and betaglycan the only inhibin receptors? If so, then all of inhibin action may be accounted for by perturbation or gating of activin signaling (Fig. 1). That is, in the models presented earlier, neither InhBP nor betaglycan interface with a signaling pathway. Rather, they describe mechanisms whereby inhibin can block access of activin to its signaling or ligand binding receptors. However, there are still some cases described in the literature where activin and inhibin appear to have similar effects (Lejeune $e t$ al., 1997). How can this occur if inhibin acts only by disrupting activin signaling? Perhaps InhBP and betaglycan interface with currrently unidentified signaling molecules or maybe other inhibin receptors exist. Future studies using protein-protein interaction methology (e.g., the yeast 2-hybrid system) will help identify potential signaling partners (and other interacting proteins) for these receptors.

Inhibin $A$ and $B$ are differentially regulated. Data suggest that the two ligands may serve different physiological roles. Are betaglycan and InhBP specific receptors for inhibin A and B, respectively? The data for InhBP indicate that the protein can bind both ligands but that it is superior in conveying inhibin B signals, in terms of antagonizing activin-stimulated gene transcription. Thus, InhBP may be 
an inhibin B receptor. Does betaglycan preferentially mediate inhibin A signals? There is certainly precedent for betaglycan mediating signals differently for closely related TGF- $\beta$ ligands (Mitchell et al., 1992; Lopez-Casillas et al., 1993). Given the established model system for betaglycan-mediated antagonism of activin signaling (Lewis et al., 2000), it should be straightforward to determine the relative efficacy of inhibin $A$ and $B$.

The cloning of these two receptors doubtlessly will clarify many existing questions within the field. This has already been demonstrated in several instances. For example, when working in K562 cells, LeBrun and Vale (1997) identified an inhibin-specific binding protein that forms a complex with inhibin $A$ and actRII. The data indicate that this binding protein is betaglycan (Lewis $e t$ al., 2000). In AtT20 cells, activin suppresses ACTH release, an effect that is not inhibited by inhibin. Interestingly, these cells do not express betaglycan endogenously (Wang et al., 1991). It will be important to demonstrate that transfection of betaglycan into these cells confers inhibin responsiveness and therefore abrogation of activin-induced suppression of ACTH release. Along these lines, inhibin attenuates activin-stimulated p3TP-luc activity in these cells but only after transfection of betaglycan. With relative ease, we should now be able to determine whether the inhibin binding proteins in $\alpha$-knockout tumors and ovine pituitaries (Draper et al., 1998; Hertan et al., 1999) correspond to InhBP or to betaglycan. Notably, we have identified high InhBP mRNA and protein levels in sheep pituitaries (unpublished observervation). If betaglycan, and not InhBP, confers inhibin binding to the tumors, this may account for our failure to identify an inhibin receptor by expression cloning. Betaglycan requires actRII for inhibin binding (Lewis et al., 2000; LeBrun and Vale, 1997). COS cells do not express actRII; thcrefore, even in transfected cells expressing betaglycan, we would have only seen negligible binding, if any at all.

Inhibin has relatively few defined target tissues, yet betaglycan (unlike InhBP) is ubiquitously expressed. Why, then, does inhibin not have morc wideranging effects? Because inhibin binding requires both betaglycan and actRII, inhibin action may be confined to those cells that express both receptors. Because InhBP does not appear to require other proteins for ligand binding, InhBP-mediated actions of inhibin may be defined by the narrow range of tissues in which the protein is expressed (most notably, the anterior pituitary).

In conclusion, we have learned much regarding inhibin regulation and function but many fascinating questions remain. Pursuit of the answers will keep inhibin researchers busy for some time to come.

\section{REFERENCES}

Alak, B.M., Smith, G.D., Woodruff, T.K., Stouffer, R.L., and Wolf, D.P. (1996). Fertil. Steril. 66, 646-653. 
Andres, J.L., Stanley, K., Cheifetz, S., and Massague, J. (1989). J. Cell Biol. 109, 3137-3145.

Ardekani, A.M., Romanelli, J.C., and Mayo, K.E. (1998). Endocrinology 139, 3271-3279.

Attisano, L., Wrana, J.L., Cheifetz, S., and Massague, J. (1992). Cell 68, 97-108.

Attisano, L., Carcamo, J., Ventura, F., Weis, F.M., Massague, J., and Wrana, J.L. (1993). Cell 75, $671-680$.

Attisano, L., Wrana, J.L., Montalvo, E., and Massague, J. (1996). Mol. Cell. Biol. 16, 1066-1073.

Barbara, N.P., Wranla, J.L., and Letarte, M. (1999). J. Biol. Chem. 274, 584-594.

Barton, D.E., Yang-Feng, T.L., Mason, A.J., Seeburg, P.H., and Francke, U. (1989). Genomics 5, 91-99.

Bilezikjian, L.M., Blount, A.L., Campen, C.A., Gonzalez-Manchon, C., and Vale, W. (1991). Mol. Endocrinol. 5, 1389-1395.

Blaakaer, J., Micic, S., Morris, I.D., Hording, U., Bennett, P., Toftager-Larsen, K., Djursing, H., and Bock, J.E. (1993). Eur. J. Obstet. Gynecol. Reprod. Biol. 52, 105-110.

Boyd, F.T., and Massague, J. (1989). J. Biol. Chem. 264, 2272-2278.

Brown, C.W., Houston-Hawkins, D.E., Woodruff, T.K., and Matzuk, M.M. (2000). Nature Genet. 25, 453-457.

Buck, C.A. (1992). Semin. Cell Biol. 3, 179-188.

Burger, H.G., Robertson, D.M., Cahir, N., Mamers, P., Healy, D.L., Jobling, T., and Groome, N. (1996a). Clin. Endocrinol. (Oxf.) 44, 413-418.

Burger, H., Hee, J., Bangah, M., Prince, M., McCloud, P., Ohara, A., Iwai, T., and Mori, T. (1996b). Clin. Endocrinol. (Oxf.) 45, 431-434.

Burger, H.G., Groome, N.P., and Robertson, D.M. (1998). J. Clin. Endocrinol. Metab. 83, 4167-4169.

Burger, H.G., Dudley, E.C., Hopper, J.L., Groome, N., Guthrie, J.R., Green, A., and Dennerstein, L. (1999). J. Clin. Endocrinol. Metab. 84, 4025-4030.

Candia, A.F., Watabe, T., Hawley, S.H., Onichtchouk, D., Zhang, Y., Derynck, R., Niehrs, C., and Cho, K.W. (1997). Development 124, 4467-4480.

Carcamo, J., Weis, F.M., Ventura, F., Wieser, R., Wrana, J.L., Attisano, L., and Massague, J. (1994). Mol. Cell. Biol. 14, 3810-3821.

Cheifetz, S., Like, B., and Massague, J. (1986). J. Biol. Chem. 261, 9972-9978.

Cheifetz, S., Andres, J.L., and Massague, J. (1988a). J. Biol. Chem. 263, 16984-16991.

Cheifetz, S., Ling, N., Guillemin, R., and Massague, J. (1988b). J. Biol. Chem. 263, 17225-17228.

Cheifetz, S., Hernandez, H., Laiho, M., ten Dijke, P., Iwata, K.K., and Massague, J. (1990). J. Biol. Chem. 265, 20533-20538.

Cheifetz, S., Bellon, T., Cales, C., Vera, S., Bernabeu, C., Massague, J., and Letarte, M. (1992). J. Biol. Chem. 267, 19027-19030.

Chong, H., Pangas, S.A., Bernard, D.J., Wang, E., Gitch, J., Chen, W., Draper, L.B., Cox, E.T., and Woodruff, T.K. (2000). Endocrinology 141, 2600-2607.

Coerver, K.A., Woodruff, T.K., Finegold, M.J., Mather, J., Bradley, A., and Matzuk, M.M. (1996). Mol. Endocrinol. 10, 534-543.

Cooke, I., O'Brien, M., Charnock, F.M., Groome, N., and Ganesan, T.S. (1995). Br. J. Cancer 71, 1046-1050.

Costa, M.J., Ames, P.F., Walls, J., and Roth, L.M. (1997). Hum. Pathol. 28, 1247-1254.

D'Agnstino, J., Woodruff, T.K., Mayn, K.E., and Schwartz, N.B (1989). Endocrinolngy 124, 310-317.

Davis, S.R., Krozowski, Z., McLachlan, R.I., and Burger, H.G. (1987). J. Endocrinol. 115, R21-R23.

de Kretser, D.M., McLachlan, R.I., Robertson, D.M., and Burger, H.G. (1989). J. Endocrinol. 120, 517-523.

di Clemente, N., Wilson, C., Faure, E., Boussin, L., Carmillo, P., Tizard, R., Picard, J.Y., Vigier, B., Josso, N., and Cate, R. (1994). Mol. Endocrinol. 8, 1006-1020.

Di Simone, N., Crowley, W.F., Jr., Wang, Q.F., Sluss, P.M., and Schneyer, A.L. (1996). Endocrinology 137, 486-494. 
Dong, J., Albertini, D.F., Nishimori, K., Kumar, T.R., Lu, N., and Matzuk, M.M. (1996). Nature 383, 531-535.

Draper, L.B., Matzuk, M.M., Roberts, V.J., Cox, E., Weiss, J., Mather, J.P., and Woodruff, T.K. (1998). J. Biol. Chem. 273, 398-403.

Durbec, P., Marcos-Gutierrez, C.V., Kilkenny, C., Grigoriou, M., Wartiowaara, K., Suvanto, P., Smith, D., Ponder, B., Costantini, F., Saarma, M., et al. (1996), Nature 381, 789-793.

Dykema, J.C., and Mayo, K.E. (1994). Endocrinology 135, 702-711.

Ebner, R., Chen, R.H., Lawler, S., Zioncheck, T., and Derynck, R. (1993). Science 262, 900-902.

Elvin, J.A., Clark, A.T., Wang, P., Wolfman, N.M., and Matzuk, M.M. (1999). Mol. Endocrinol. 13, 1035-1048.

Esch, F.S., Shimasaki, S., Cooksey, K., Mercado, M., Mason, A.J., Ying, S.Y., Ueno, N., and Ling, N. (1987). Mol. Endocrinol. 1, 388-396.

Fang, J., Yin, W., Smiley, E., Wang, S.Q., and Bonadio, J. (1996). Biochem. Biophys. Res. Commun. 228, 669-674.

Fang, J., Wang, S.Q., Smiley, E., and Bonadio, J. (1997). Biochem. Biophys. Res. Commun. 231, 655-661.

Feng, Z.M., Li, Y.P., and Chen, C.L. (1989). Mol. Endocrinol. 3, 1914-1925.

Feng, Z.M., Wu, A.Z., and Chen, C.L. (1995). Endocrinology 136, 947-955.

Flemming, P., Wellmann, A., Maschek, H., Lang, H., and Georgii, A. (1995). Am. J. Surg. Pathol. 19, 927-933.

Flemming, P., Grothe, W., Maschek, H., Petry, K.U., Wellmann, A., and Georgii, A. (1996). Histopathology 29, 465-468.

Forage, R.G., Ring, J.M., Brown, R.W., McInemey, B.V., Cobon, G.S., Gregson, R.P., Robertson, D.M., Morgan, F.J., Hearn, M.T., Findlay, J.K., et al. (1986). Proc. Natl. Acad. Sci. U.S.A. 83, 3091-3095.

Franzen, P., ten Dijke, P., Ichijo, H., Yamashita, H., Schulz, P., Heldin, C.H., and Miyazono, K. (1993). Cell 75, 681-692.

Fraser, H.M., and Tsonis, C.G. (1994). J. Endocrinol. 142, 181-186.

Fraser, H.M., Smith, K.B., Lunn, S.F., Cowen, G.M., Morris, K., and McNeilly, A.S. (1992). J. Endocrinol. 133, 341-347.

Fraser, H.M., Groome, N.P., and McNeilly, A.S. (1999). J. Clin. Endocrinol. Metab. 84, 1365-1369.

Frattini, A., Faranda, S., Redolfi, E., Allavena, P., and Vezzoni, P. (1998). Gene 214, 1-6.

Frias, A.E., Jr., Li, H., Keeney, G.L., Podratz, K.C., and Woodruff, T.K. (1999). Cancer 85, 465-471

Goridis, C., and Brunet, J.F. (1992). Semin. Cell. Biol. 3, 189-197.

Gouedard, L., Chen, Y.G., Thevenet, L., Racine, C., Borie, S., Lamarre, I., Josso, N., Massague, J., and di Clemente, N. (2000). J. Biol. Chem. 275, 27973-27978.

Gougos, A., and Letarte, M. (1988). J. Immunol. 141, 1934-1940.

Gougos, A., and Letarte, M. (1990). J. Biol. Chem. 265, 8361-8364.

Grady, R.R., Savoy-Moore, R.T., and Schwartz, N.B. (1981). In "Bioregulators of Reproduction" (G. Jagiello and H.J. Vogel, eds.), pp. 359-369. Academic Press, New York.

Groome, N.P., Illingworth, P.J., O'Brien, M., Cooke, I., Ganesan, T.S., Baird, D.T., and McNeilly, A.S. (1994). Clin. Endocrinol. (Oxf.) 40, 717-723.

Groome, N.P., Illingworth, P.J., O'Brien, M., Pai, R., Rodger, F.E., Mather, J.P., and McNeilly, A.S. (1996). J. Clin. Endocrinol. Metab. 81, 1401-1405.

Gurusinghe, C.I., Healy, D.L., Jnhling, T., Mamers, P., and Burger, H.G. (1995). Gynecol. Oncol. 57, 27-32.

Haisenleder, D.J., Dalkin, A.C., and Marshall, J.C. (1994). In "The Physiology of Reproduction" (E. Knobil and J.D. Neill, eds.), pp. 1793-1813. Raven Press, New York.

Hamil, K.G., Conti, M., Shimasaki, S., and Hall, S.H. (1994). Mol. Cell. Endocrinol. 99, 269-277.

Hata, A., Lagna, G., Massague, J., and Hemmati-Brivanlou, A. (1998). Genes Dev. 12, 186-197. 
Hayashi, H., Abdollah, S., Qiu, Y., Cai, J., Xu, Y.Y., Grinnell, B.W., Richardson, M.A., Topper, J.N., Gimbrone, M.A., Jr., Wrana, J.L., and Falb, D. (1997). Cell 89, 1165-1173.

Hayashi, M., McGee, E.A., Min, G., Klein, C., Rose, U.M., van Duin, M., and Hsueh, A.J. (1999). Endocrinology 140, 1236-1244.

He, W.W., Gustafson, M.L., Hirobe, S., and Donahoe, P.K. (1993). Dev, Dynam. 196, 133-142.

Healy, D.L., Burger, H.G., Mamers, P., Jobling, T., Bangah, M., Quinn, M., Grant, P., Day, A.J., Rome, R., and Campbell, J.J. (1993). N. Engl. J. Med. 329, 1539-1542.

Hertan, R., Farnworth, P.G., Fitzsimmons, K.L., and Robertson, D.M. (1999). Endocrinology 140, 6-12. Hiendleder, S., Dodds, K.G., and Wassmuth, R. (2000). J. Hered. 91, 343-345.

Hillier, S.G., Yong, E.L., Illingworth, P.J., Baird, D.T., Schwall, R.H., and Mason, A.J. (1991). Mol. Cell. Endocrinol. 75, R1-R6.

Hotten, G., Neidhardt, H., Schneider, C., and Pohl, J. (1995). Biochem. Biophys. Res. Commun. 206, 608-613.

Hsueh, A.J., Dahl, K.D., Vaughan, J., Tucker, E., Rivier, J., Bardin, C.W., and Valc, W. (1987). Proc. Natl. Acad. Sci. U.S.A. 84, 5082-5086.

Illingworth, P.J., Groome, N.P., Byrd, W., Rainey, W.E., McNeilly, A.S., Mather, J.P., and Bremner, W.J. (1996). J. Clin. Endocrinol. Metab. 81, 1321-1325.

Imamura, T., Takase, M., Nishihara, A., Oeda, E., Hanai, J., Kawabata, M., and Miyazono, K. (1997). Nature 389, 622-626.

Ishisaki, A., Yamato, K., Hashimoto, S., Nakao, A., Tamaki, K., Nonaka, K, ten Dijke, P., Sugino, H., and Nishihara, T. (1999). J. Biol. Chem. 274, 13637-13642.

Jing, S., Wen, D., Yu, Y., Holst, P.L., Luo, Y., Fang, M., Tamir, R., Antonio, L., Hu, Z., Cupples, R., Louis, J.C., Hu, S., Altrock, B.W., and Fox, G.M. (1996). Cell 85, $1113-1124$.

Johnson, D.G. (2000). Mol. Carcinog. 27, 151-157.

Kirsch, T., Sebald, W., and Dreyer, M.K. (2000). Nature Struct. Biol. 7, 492-496.

Kitamura, K., Aota, S., Sakamoto, R., Yoshikawa, S.I., and Okazaki, K. (2000). Blood 95, 3371-3379

Kretzschmar, M., Doody, J., Timokhina, I., and Massague, J. (1999). Genes Dev. 13, 804-816.

Krummen, L.A., Moore, A., Woodruff, T.K., Covello, R., Taylor, R., Working, P., and Mather, J.P. (1994). Biol, Reprod. 50, 734-744.

Kumar, T.R., Wang, Y., and Matzuk, M.M. (1996). Endocrinology 137, 4210-4216.

Kumar, T.R., Palapattu, G., Wang, P., Woodruff, T.K., Boime, I., Byrne, M.C., and Matzuk, M.M. (1999). Mol. Endocrinol. 13, 851-865.

Laiho, M., Weis, M.B., and Massague, J. (1990). J. Biol. Chem. 265, 18518-18524.

Laiho, M., Weis, F.M., Boyd, F.T., Ignotz, R.A., and Massague, J. (1991). J. Biol. Chem. 266, 9108-9112.

Lambert-Messerlian, G.M., Hall, J.E., Sluss, P.M., Taylor, A.E., Martin, K.A., Groome, N.P., Crowley, W.F., Jr., and Schneyer, A.L. (1994). J. Clin. Endocrinol. Metab. 79, 45-50.

Lambert-Messerlian, G.M., Steinhoff, M., Zheng, W., Canick, J.A., Gajewski, W.H., Seifer, D.B., and Schneyer, A.L. (1997). Gynecol. Oncol. 65, 512-516.

Lappohn, R.E., Burger, H.G., Bouma, J., Bangah, M., Krans, M., and de Bruijn, H.W. (1989). N. Engl. J. Med. 321, 790-793.

Lau, A.L., Kumar, T.R., Nishimori, K., Bonadio, J., and Matzuk, M.M. (2000). Mol. Cell. Biol. 20, 6127-6137.

Lebrun, J.J., and Vale, W.W. (1997). Mol. Cell. Biol. 17, 1682-1691.

Lejeune, H., Chuzel, F., Sanchez, P., Durand, P., Mather, J.P., and Saez, J.M. (1997). Endocrinology 138, 4783-4791.

Lewis, K.A., Gray, P.C., Blount, A.L., MacConell, L.A., Wiater, E., Bilezikjian, L.M., and Vale, W. (2000). Nature 404, 411-414.

Lin, H.Y., and Wang, X.F. (1992). Mol. Reprod. Dev. 32, 105-110.

Lin, L.F., Doherty, D.H., Lile, J.D., Bektesh, S., and Collins, F. (1993). Science 260, 1130-1132. 
Lin, T., Calkins, J.K., Morris, P.L., Vale, W., and Bardin, C.W. (1989). Endocrinology 125, 2134-2140.

Ling, N., Ying, S.Y., Ueno, N., Esch, F., Denoroy, L., and Guillemin, R. (1985). Proc. Natl. Acad. Sci. U.S.A. 82, 7217-7221.

Ling, N., Ying, S.Y., Ueno, N., Shimasaki, S., Esch, F., Hotta, M., and Guillemin, R. (1986). Nature 321, 779-782.

Lopez-Casillas, F., Cheifetz, S., Doody, J., Andres, J.L., Lane, W.S., and Massague, J. (1991). Cell 67, 785-795.

Lopez-Casillas, F., Wrana, J.L., and Massague, J. (1993). Cell 73, 1435-1444.

Lopez-Casillas, F., Payne, H.M., Andres, J.L., and Massague, J. (1994). J. Cell. Biol. 124, 557-568.

Loveland, K.L., McFarlane, J.R., and de Kretser, D.M. (1996). J. Mol. Endocrinol. 17, 61-65.

MacConell, L.A., and Vale, W. (2000). In "Program \& Abstracts of the 82nd Annual Meeting of The Endocrine Society," Toronto, Ontario, Canada, June 2000, abstract 552, p. 139.

Martens, J.W., de Winter, J.P., Timmerman, M.A., McLuskey, A., van Schaik, R.H., Themmen, A.P., and de Jong, F.H. (1997). Endocrinology 138, 2928-2936.

Mason, A.J. (1988). In "Nonsteroidal Gonadal Factors: Physiological Roles and Possibilities in Contraceptive Development" (G.D. Hodgen, Z. Rosenwaks, and J.M. Spieler, eds.), pp. 12-29. Jones Institute Press, Norfolk, Va.

Mason, A.J., Hayflick, J.S., Ling, N., Esch, F., Ueno, N., Ying, S.Y., Guillemin, R., Niall, H., and Seeburg, P.H. (1985). Nature 318, 659-663.

Mason, A.J., Niall, H.D., and Seeburg, P.H. (1986). Biochem. Biophys. Res. Commun. 135, 957-964.

Massague, J. (1985). J. Biol. Chem. 260, 7059-7066.

Massague, J., Cheifetz, S., Ignotz, R.A., and Boyd, F.T. (1987). J. Cell. Physiol. 5(suppl.), 43-47.

Mathews, L.S., and Vale, W.W. (1991). Cell 65, 973-982.

Mathews, I.S., Vale, W.W., and Kintner, C.R. (1992). Science 255, 1702-1705.

Matzuk, M.M., Finegold, M.J., Su, J.G., Hsueh, A.J., and Bradley, A. (1992). Nature 360, 313-319.

Matzuk, M.M., Finegold, M.J., Mather, J.P., Krummen, L., Lu, H., and Bradley, A. (1994). Proc. Natl. Acad. Sci. U.S.A. 91, 8817-8821.

Matzuk, M.M., Kumar, T.K., Vassalli, A., Bickenbach, J.K., Koop, D.R., Jaenisch, R., and Bradley, A (1995). Nature 374, 354-356.

Mayo, K.E. (1994). Trends Endocrinol. Metab. 5, 407-415.

Mayo, K.E., Cerelli, G.M., Spiess, J., Rivier, J., Rosenfeld, M.G., Evans, R.M., and Vale, W. (1986) Proc. Natl. Acad. Sci. U.S.A. 83, 5849-5853.

Mazzarella, R., Pengue, G., Jones, J., Jones, C., and Schlessinger, D. (1998). Genomics 48, 157-162.

McCluggage, W.G., Shanks, J.H., Whiteside, C., Maxwell, P., Banerjee, S.S., and Biggart, J.D. (1998). Am. J. Surg. Pathol. 22, 615-619.

McCullagh, E.P. (1932). Science 76, 19-20.

McPherron, A.C., and Lee, S.J. (1993). J. Biol. Chem. 268, 3444-3449.

Meunier, H., Cajander, S.B., Roberts, V.J., Rivier, C., Sawchenko, P.E., Hsueh, A.J., and Vale, W. (1988a). Mol. Endocrinol. 2, 1352-1363.

Meunier, H., Rivier, C., Evans, R.M., and Vale, W. (1988b). Proc. Natl. Acad. Sci. U.S.A. 85, 247-251

Mitchell, E.J., Fitz-Gibbon, L., and O'Connor-McCourt, M.D. (1992). J. Cell. Physiol. 150, 334-343.

Miyamoto, K., Hasegawa, Y., Fukuda, M., Nomura, M., Igarashi, M., Kangawa, K., and Matsuo, H. (1985). Biochem. Biophys. Res. Commun. 129, 396-403.

Molina, C.A., Foulkes, N.S., Lalli, E., and Sassone-Corsi, P. (1993). Cell 75, 875-886.

Molskness, T.A., Woodruff, T.K., Hess, D.L., Dahl, K.D., and Stouffer, R.L. (1996). J. Clin. Endocrinol. Metab. 81, 4002-4006.

Moore, M.W., Klein, R.D., Farinas, I., Sauer, H., Armanini, M., Phillips, H., Reichardt, L.F., Ryan, A.M., Carver-Moore, K., and Rosenthal, A. (1996). Nature 382, 76-79.

Moren, A., Ichijo, II, and Miyazono, K. (1992). Biochem. Biophys. Res. Commun. 189, 356-362.

Mukherjee, A., Park-Sarge, O.K., and Mayo, K.M. (1996). Endocrinology 137, 3234-3245. 
Mukherjee, A., Urban, J., Sassone-Corsi, P., and Mayo, K.E. (1998). Mol. Endocrinol. 12, 785-800.

Najmabadi, H., Rosenberg, L.A., Yuan, Q.X., Reyaz, G., and Bhasin, S. (1993). Mol. Endocrinol. 7, 561-569.

Nakao, A., Afrakhte, M., Moren, A., Nakayama, T., Christian, J.L., Heuchel, R., Itoh, S., Kawabata, M., Heldin, N.E., Heldin, C.H., and ten Dijke, P. (1997). Nature 389, 631-635.

Newfeld, S.J., Wisotzkey, R.G., and Kumar, S. (1999). Genetics 152, 783-795.

O'Bryan, M.K., Sebire, K.L., Gerdprasert, O., Hedger, M.P., Hearn, M.T., and de Kretser, D.M. (2000). J. Mol. Endocrinol. 24, 409-418.

Oda, S., Nishimatsu, S., Murakami, K., and Ueno, N. (1995), Biochem. Biophys. Res. Commun. 210, 581-588.

Ohta, M., Greenberger, J.S., Anklesaria, P., Bassols, A., and Massague, J. (1987). Nature 329, 539-541.

Pangas, S.A., and Woodruff, T.K. (2000). Trends Endocrinol. Metab. 11, 309-314.

Paralkar, V.M., Hammonds, R.G., and Redd, A.M. (1991). Proc. Natl Acad. Sci. U.S.A. 88, 3397-3401.

Pei, L., Dodson, R., Schoderbek, W.E., Maurcr, R.A., and Mayo, K.E. (1991). Mol. Endocrinol. 5, 521-534.

Pichel, J.G., Shen, L., Sheng, H.Z., Granholm, A.C., Drago, J., Grinberg, A., Lee, E.J., Huang, S.P., Saarma, M., Hoffer, B.J., Sariola, H., and Westphal, H. (1996). Nature 382, 73-76.

Piek, E., Heldin, C.H., and Ten Dijke, P. (1999). FASEB J. 13, 2105-2124.

Pierson, T.M., Wang, Y., DeMayo, F.J., Matzuk, M.M., Tsai, S.Y., and O'Malley, B.W. (2000). Mol. Endocrinol. 14, 1075-1085.

Plant, T.M., Padmanabhan, V., Ramaswamy, S., McConnell, D.S., Winters, S.J., Groome, N., Midgley, A.R., Jr., and McNeilly, A.S. (1997). J. Clin. Endocrinol. Metab. 82, 2617-2621.

Ponce-Castaneda, M.V., Esparza-Lopez, J, Vilchis-Landeros, M.M., Mendoza, V., and Lopez-Casillas, F. (1998). Biochim. Biophys. Acta 1384, 189-196.

Ramaswamy, S., Marshall, G.R., McNeilly, A.S., and Plant, T.M. (2000). Endocrinology 141, 18-27.

Risma, K.A., Clay, C.M., Nett, T.M., Wagner, T., Yun, J., and Nilson, J.H. (1995). Proc. Natl. Acad. Sci. U.S.A. 92, 1322-1326.

Rivier, C., Rivier, J., and Vale, W. (1986). Science 234, 205-208.

Rivier, J., Spiess, J., McClintock, R., Vaughan, J., and Vale, W. (1985). Biochem. Biophys. Res. Commun. 133, 120-127

Roberts, V.J., Barth, S., el-Roeiy, A., and Yen, S.S. (1993). J. Clin. Endocrinol. Metab. 77, 1402-1410.

Robertson, D.M., Foulds, L.M., Leversha, L., Morgan, F.J., Hearn, M.T., Burger, H.G., Wettenhall, R.E., and de Kretser, D.M. (1985). Biochem. Biophys. Res. Commun. 126, 220-226.

Sanchez, M.P., Silos-Santiago, I., Frisen, J., He, B., Lira, S.A., and Barbacid, M. (1996). Nature 382, 70-73.

Schmitt, J., Hotten, G., Jenkins, N.A., Gilbert, D.J., Copeland, N.G., Pohl, J., and Schrewe, H. (1996). Genomics 32, 358-366.

Schuchardt, A., D'Agati, V., Larsson-Blomberg, L., Costantini, F., and Pachnis, V. (1994). Nature 367, 380-383.

Schwall, R.H., Robbins, K., Jardieu, P., Chang, L., Lai, C., and Terrell, T.G. (1993). Hepatology 18, 347-356.

Schwartz, N.B., and Channing, C.P. (1977). Proc. Natl. Acad. Sci. U.S.A. 74, 5721-5724.

Sehested, A., Juul, A.A., Andersson, A.M., Petersen, J.H., Jensen, T.K., Muller, J., and Skakkebaek, N.E. (2000). J. Clin. Endocrinol. Metab. 85, 1634-1640.

Silverman, L.A., Gitelman, S.E., Woodruff, T.K., and Grumbach, M.M. (1994). Pediat. Res. 35, A107.

Stehle, J.H., Foulkes, N.S., Molina, C.A., Simonneaux, V., Pevet, P., and Sassone-Corsi, P. (1993). Nature 365, 314-320.

Stewart, C.J., Jeffers, M.D., and Kennedy, A. (1997). Histopathology 31, 67-74.

Stouffer, R.L., Dahl, K.D., Hess, D.L., Woodruff, T.K., Mather, J.P., and Molskness, T.A. (1994). Biol. Reprod. 50, 888-895. 
Tanimoto, K., Yoshida, E., Mita, S., Nibu, Y., Murakami, K., and Fukamizu, A. (1996). J. Biol. Chem. 271, 32760-32769.

ten Dijke, P., Ichijo, H., Franzen, P., Schulz, P., Saras, J., Toyoshima, H., Heldin, C.H., and Miyazono, K. (1993). Oncogene 8, 2879-2887.

ten Dijke, P., Yamashita, H., Ichijo, H., Franzen, P., Laiho, M., Miyazono, K., and Heldin, C.H. (1994). Science 264, 101-104.

Treanor, J.J., Goodman, L., de Sauvage, F., Stone, D.M., Poulsen, K.T., Beck, C.D., Gray, C., Armanini, M.P., Pollock, R.A., Hefti, F., Phillips, H.S., Goddard, A., Moore, M.W., Buj-Bello, A., Davies, A.M., Asai, N., Takahashi, M., Vandlen, R., Henderson, C.E., and Rosenthal, A. (1996). Nature 382, 80-83.

Trupp, M., Ryden, M., Jornvall, H., Funakoshi, H., Timmusk, T., Arenas, E., and Ibanez, C.F. (1995). J. Cell. Biol. 130, 137-148.

Tsuchida, K., Mathews, L.S., and Vale, W.W. (1993). Proc. Natl. Acad. Sci. U.S.A. 90, 11242-11246.

Tsuchida, K., Vaughan, J.M., Wiater, E., Gaddy-Kurten, D., and Vale, W.W. (1995). Endocrinology 136, 5493-5503.

Turner, I.M., Saunders, P.T., Shimasaki, S., and Hillier, S.G. (1989). Endocrinology 125, 2790-2792.

Tuuri, T., Framaa, M., Van Schaik, R.H., and Ritvos, O. (1996). Mol. Cell. Endocrinol. 121, 1-10.

Vale, W., Rivier, J., Vaughan, J, McClintock, R., Corrigan, A., Woo, W., Karr, D., and Spiess, J. (1986). Nature 321, 776-779.

Vale, W., Rivier, C., Hsueh, A., Campen, C., Meunier, H., Bicsak, T., Vaughan, J., Corrigan, A., Bardin, W., Sawchenko, P., et al. (1988). In "Recent Progress in Hormone Research," vol. 44, pp. 1-34. Academic Press, San Diego, Calif.

van der Geer, P., Hunter, T., and Lindberg, R.A. (1994). Anmu. Rev. Cell. Biol. 10, 251 -337.

Vassalli, A., Matzuk, M.M., Gardner, H.A., Lee, K.F., and Jaenisch, R. (1994). Genes Dev. 8, 414-427.

Wang, X.F., Lin, H.Y., Ng-Eaton, E., Downward, J., Lodish, H.F., and Weinberg, R.A. (1991). Cell 67, 797-805.

Watson, R.H., Roy, W.J., Jr., Davis, M., Hitchcock, A., and Campbell, J.G. (1997). Gynecol. Oncol. 65, 387-390.

Welt, C.K., Lambert-Messerlian, G., Zheng, W., Crowley, W.F., Jr., and Schneyer, A.L. (1997a). $J$. Clin. Endocrinol. Metab. 82, 3720-3727.

Welt, C.K., Martin, K.A., Taylor, A.E., Lambert-Messerlian, G.M., Crowley, W.F., Jr., Smith, J.A., Schoenfeld, D.A., and Hall, J.E. (1997b). J. Clin. Endocrinol. Metab. 82, 2645-2652.

Welt, C.K., McNicholl, D.J., Taylor, A.E., and Hall, J.E. (1999). J. Clin. Endocrinol. Metab. 84, 105-111.

Wiesmann, C., and de Vos, A.M. (2000). Nature Struct. Biol. 7, 440-442.

Woodruff, T.K. (1998). Biochem. Pharmacol. 55, 953-963.

Woodruff, T.K. (1999). Endocrinology 140, 3-5.

Woodruff, T.K., and Mather, J.P. (1995). Annu. Rev. Physiol. 57, 219-244.

Woodruff, T.K., and Mayo, K.E. (1990). Annu. Rev. Physiol. 52, 807-821.

Woodruff, T.K., Meunier, H., Jones, P.B., Hsueh, A.J., and Mayo, K.E. (1987). Mol. Endocrinol. 1, 561-568.

Woodruff, T.K., D'Agostino, J., Schwartz, N.B., and Mayo, K.E. (1988). Science 239, 1296-1299.

Woodruff, T.K., D'Agostino, J., Schwartz, N.B., and Mayo, K.E. (1989). Endocrinology 124, 2193 2199.

Woodruff, T.K., Krummen, L., Chen, S.A., Lyon, R., Hansen, S.E., DeGuzman, G., Covello, R., Mather, J., and Cossum, P. (1993a). Endocrinology 132, 725-734.

Woodruff, T.K., Krummen, L.A., Lyon, R.J., Stocks, D.L., and Mather, J.P. (1993b). Endocrinology 132, 2332-2341.

Woodruff, T.K., Besecke, L.M., Groome, N., Draper, L.B., Schwartz, N.B., and Weiss, J. (1996). Endocrinology 137, 5463-5467. 
Wrana, J.L., Attisano, L., Carcamo, J., Zentella, A., Doody, J., Laiho, M., Wang, X.F., and Massague, J. (1992). Cell 71, 1003-1014.

Wrana, J.L., Attisano, L., Wieser, R., Ventura, F., and Massague, J. (1994). Nature 370, 341-347.

Xu, J., McKeehan, K., Matsuzaki, K., and McKeehan, W.L. (1995). J. Biol. Chem. 270, 6308-6313.

Yamashita, H., ten Dijke, P., Huylebroeck, D., Sampath, T.K., Andries, M., Smith, J.C., Heldin, C.H., and Miyazono, K. (1995). J. Cell. Biol. 130, 217-226.

Yamashita, K., Yamoto, M., Shikone, T., Minami, S., Imai, M., Nishimori, K., and Nakano, R. (1997). Am. J. Obstet. Gynecol. 177, 1450-1457.

Ying, S.Y. (1988). Endocr. Rev, 9, 267-293.

Zheng, W., Sung, C.J., Hanna, I., DePetris, G., Lambert-Messerlian, G., Steinhoff, M., and Lauchlan, S.C. (1997). Intl. J. Gynecol. Pathol. 16, 263-271. 


\section{AUTHOR INDEX}

A

Aguilar-Bryan, Lydia, 47 Avruch, Joseph, 127

B

Banerji, Mary Ann, 265

Bernard, Daniel J., 417

Bradley, Richard L., 329

Brady, Matthew J., 157

Bryan, Joseph, 47

C

Cerami, Anthony, 1

Chapman, Stacey C., 417

Cheatham, Bentley, 329

Chu, Khoi, 23

Cleveland, Kelly A., 329

Collins, Sheila, 309

Cone, Roger D., 359

Corbould, Anne, 295

D

Doyle, Maire E., 377

Dunaif, Andrea, 295
E

Egan, Josephine M., 377

Eisenbarth, George S., 69

F

Fain, Pamela R., 69

Froguel, Philippe, 91

K

Karsenty, Gerard, 401

Khokhlatchev, Andrei, 127

Kliewer, Steven A., 239

Kyriakis, John M., 127

L

Lambert, Millard H., 239

Lebovitz, Harold E., 265

Luo, Zhijun, 127

\section{M}

Magnuson, Mark A., 195

Marks, Daniel L., 359 
Nakazaki, Mitsuhiro, 47

Nemoz-Gaillard, Eric, 23

Tsai, Ming-Jer, 23

Tzivion, Guri, 127

$\mathbf{P}$

U

Pelaez, Fernando, 107

Pessin, Jeffrey E., 175

Petersen, Kitt Falk, 219

Postic, Catherine, 195

$\mathbf{R}$

Vavvas, Demetrios, 127

Velho, Gilberto, 91

Venkatesan, Aradhana M., 295

Redondo, Maria J., 69

Roden, Michael, 219

W

$\mathbf{S}$

Watson, Robert T., 175

Willson, Timothy M., 239

Salituro, Gino M., 107

Woodruff, Teresa K., 417

Saltiel, Alan R., 157

Shiota, Masakazu, 195

Shulman, Gerald I., 219

Surwit, Richard S., 309

$\mathbf{X}$

Xu, H. Eric, 239

Z

Zhang, Bei B., 107

Zhang, Xian-feng, 127 


\section{SUBJECT INDEX}

$\boldsymbol{A}$

Activin, 417

Adipocyte, 309

Adipose

cell biology, 329

tissue, 309

b3-Adrenergic, 309

Aging, 1

Agouti-related peptide, 359

Amadori product, 1

Amino guanidine hydrochloride, 1

Androgens, 295

Animal models, 195

Antidiabetic agent, 107

Anti-islet autoantibodies, 69

ATP-sensitive potassium channels, 47

Autoimmune polyendocrine syndrome, 69

Autoimmunity, 69

B

Basic helix-loop-helix, 23

Beta2, 23

Body weight, 401

Bone

formation, 401

remodeling, 401

C

Cachexia, 359

Cancer, 359

Catecholamines, 309
beta-Cell

neogenesis, 377

transcription factors, 377

Cell-specific knuckouts, 195

Central

control, 401

nervous system, 359

Cerebellum, 23

Cirrhosis, 219

Congenital hyperinsulinism, 47

Cre/loxP strategy, 195

Cross-linking, 1

Cytokine, 359

D

Demethylasterriquinone B-1, 107

Diabetes, 1, 23, 47, 107, 157, 175, 219, 265, 309,377

animal models, 69

prediction, 69

type $1,69,219$

type $2,91,219,239,295$

Dimethylphenacylthiazolium chloride, 1

Dyslipidemia, 239, 265

E

Endocrine pancreas, 23

Endocytosis, 175

Exocytosis, 175 
$\mathbf{F}$

Fatty acid, 239

Feeding behavior, 359

Fetal malnutrition, 265

Fibrate, 239

Follicle, 417

-stimulating hormone, 417

G

G protein-coupled receptors, 309

Gene targeting, 195

Genetics, 69,91

Genome scans, 91

Glitazone, 239

Glucokinase, 91, 195

Gluconeogenesis, 219

Glucose

homeostasis, 157, 195

lowering, 107

sensing, 195

transport, 175

GLUT4, 175

Glycation, 1 end-products, advanced, 1

Glycogen

cycling, 219

synthase, 157

synthesis, 29,157

Glycogenolysis, 157;, 219

GM/GL , 157 [SPELL OUT]

Gonadotrope, 417

Gonadotropins, 295

Growth factor signaling, 127

GTPases, 127

Gut peptides, 377

\section{H}

Hippocampus, 23

Hormone action, 329

Human leukocyte antigen, 69

Hypoglycemia, 47

Hypothalamus, 401

\section{I}

Inhibin, 417

Insulin, 219, 265, 377

action, 157, 175, 329

autoantibodies, 69

receptor, 107, 295

receptor activator, 107

receptor substrates, 295

receptor tyrosine kinase, 107

resistance, $175,265,295$

secretion, 47, 377

signaling, 107

Islets of Langerhans, 377

$\mathbf{K}$

$K_{\text {ATP }}, 47$

Knockout/transgenic animals, 23

$\mathbf{L}$

Leptin, 401

biosynthesis and secretion, 329

Ligand, 239

Lipopolysaccharide, 359

M

Maillard reaction, 1

MAP kinase cascade, 127

Mature onset diabetes of the young, 91,219

type 2, 195

Melanocortin, 359

Metabolic syndrome, 265

Metabolism, 309, , 359

Mitochondria, 309

Mitogenesis, 127

Multifactorial diseases, 91

Mutations, 91 
$\mathbf{N}$

Natural products, 107

Nonpeptidyl small molecule, 107

Nuclear

magnetic resonance, 219

receptor, 239

$\mathbf{0}$

Obesity, 309, 329

Osteoporosis, 401

Ovary, 417

$\mathbf{P}$

Pancreas, 377

development, 23

Pancreatic beta cells, 195, 377

Pantophysin, 329

PDX-1, 377

Peroxisome proliferator-activated receptor, 239 -gamma agonists, 265

Persistent hyperinsulinemic hypoglycemia of infancy, 47

Pituitary, 417

Polycystic ovary syndrome, 295

Positional cloning, 91

Post-translational modification, 1

Proliferation/differentiation, 23

Proopiomelanocortin, 359

Protein

dephosphorylation, 157

kinases, 127

phosphorylation, 295

target to glycogen, 157

targeting, 157

14-3-3 Proteins, 127
$\mathbf{R}$

Raf, 127

Ras, 127

effectors, 127

Reproduction, 401, 417

Rosiglitazone, 265

S

Secretion, 329

Signal transduction, $127,175,295,417$

SNARE, 175, 329

Sulfonylurea receptors, 47

SurlkO, 47

T

Thiazolidinedione, 239, 265

Transcription, 239

factors, 91, 23

Tranforming growth factor beta, 417

Transgenic mice, 195

Twins, 69

U

Uncoupling proteins, 309

V

Vesicle

biogenesis, 329

transport, 329

Vesicular trafficking, 175

Visceral adiposity, 265 
